\title{
A taxonomic revision of the Neotropical electric fish genus Brachyhypopomus (Ostariophysi: Gymnotiformes: Hypopomidae), with descriptions of 15 new species
}

\author{
William G. R. Crampton ${ }^{1}$, Carlos D. de Santana ${ }^{1,2}$, Joseph C. Waddell ${ }^{1}$ and \\ Nathan R. Lovejoy ${ }^{3}$
}

The bluntnose knifefish genus Brachyhypopomus Mago-Leccia, 1994, is diagnosed from other Rhamphichthyoidea (Rhamphichthyidae + Hypopomidae) by the presence of a disk-like ossification in the anterior portion of the palatoquadrate, and by the following external characters: short snout, $18.7-32.6 \%$ of head length (vs. $33.3-68.6 \%$ in Hypopomus, Gymnorhamphichthys, Iracema, and Rhamphichthys), absence of a paired accessory electric organ in the mental or humeral region (vs. presence in Hypopygus and Steatogenys), presence of 3-4 pectoral proximal radials (vs. 5 in Akawaio), presence of the antorbital + infraorbital, and the preopercular cephalic lateral line canal bones (vs. absence in Racenisia). Brachyhypopomus cannot be diagnosed unambiguously from Microsternarchus or from Procerusternarchus on the basis of external characters alone. Brachyhyроротиs comprises 28 species. Here we describe 15 new species, and provide redescriptions of all 13 previously described species, based on meristic, morphometric, and other morphological characters. We include notes on ecology and natural history for each species, and provide regional dichotomous keys and distribution maps, based on the examination of 12,279 specimens from 2,787 museum lots. A lectotype is designated for Brachyhypopomus pinnicaudatus (Hopkins, Comfort, Bastian \& Bass, 1990). Brachyhypopomus species are abundant in shallow lentic and slow-flowing freshwater habitats from southern Costa Rica and northern Venezuela to Uruguay and northern Argentina. Species diversity is highest in Greater Amazonia, where 20 species occur: $B$. alberti, new species, $B$. arrayae, new species, and $B$. cunia, new species, in the upper rio Madeira drainage; $B$. batesi, new species, in the central Amazon and rio Negro; $B$. beebei, B. brevirostris, B. regani, new species, B. sullivani, new species, and $B$. walteri, widespread through the Amazon and Orinoco basins and the Guianas; $B$. belindae, new species, in the central Amazon basin; $B$. benjamini, new species, and $B$. verdii, new species, in the upper Amazon basin; $B$. bennetti, in the upper, central, and lower Amazon, lower Tocantins, and upper Madeira basins; B. bullocki in the Orinoco, Negro and Essequibo drainages; $B$. diazi in the Orinoco Llanos; B. flavipomus, new species, and B. hamiltoni, new species, in the central and upper Amazon basin; B. hendersoni, new species, in the central Amazon, lower Negro and Essequibo basins; $B$. pinnicaudatus in the central and lower Amazon, lower, upper Madeira, lower Tocantins and Mearim basins, and coastal French Guiana; and B. provenzanoi, new species, in the upper Orinoco and upper Negro basins. Five species are known from the Paraná-Paraguay-Uruguay basin and adjacent southern Atlantic drainages: B. bombilla in the lower Paraná, upper, central, and lower Paraguay, Uruguay and Patos-Mirim drainages; B. brevirostris in the upper Paraguay basin; B. draco in the lower Paraná, lower Paraguay, Uruguay, Patos-Mirim, and Tramandaí basins; B. gauderio in the lower Paraná, upper, central, and lower Paraguay, Uruguay, Patos-Mirim and Tramandaí basins; and $B$. walteri in the lower Paraná and upper Paraguay basins. Two species occur in small Atlantic drainages of southern Brazil: B. janeiroensis in the São João, Paraíba and small intervening drainages; and B. jureiae in the Ribeira de Iguape and Una do Prelado. One species occurs in the middle and upper São Francisco basin: B. menezesi, new species. Three species occur in trans-Andean drainages: $B$. diazi in Caribbean drainages of northern Venezuela; $B$. occidentalis in Atlantic and Pacific drainages of southern Costa Rica and Panama to Darién, and the Maracaibo, Magdalena, Sinú and Atrato drainages; and B. palenque, new species, in Pacific drainages of Ecuador.

\footnotetext{
${ }^{1}$ Department of Biology, University of Central Florida, PO Box 162368, Florida, 32816-2368, USA. (WGRC) crampton@ucf.edu (corresponding author); (JCW) joecwaddell@knights.ucf.edu

${ }^{2}$ (current address) Division of Fishes, Smithsonian Institution, National Museum of Natural History, 10th and Constitution Avenue, NW, Washington, DC 20560-0159, USA. apteronotidae@ig.com.br

${ }_{3}^{3}$ Department of Biological Sciences, University of Toronto Scarborough, 1265 Military Trail, Toronto, ON M1C 1A5, Canada. lovejoy@ utsc.utoronto.ca
} 
Peixes elétricos do gênero Brachyhypopomus Mago-Leccia, 1994, são diagnosticados dos outros Rhamphichthyoidea (Rhamphichthyidae + Hypopomidae) pela presença de uma ossificação discóide na porção anterior do palatoquadrado, e pelos seguintes caracteres externos: focinho curto, 18,7-32,6\% do comprimento da cabeça ( $v s .33,3-68,6 \%$ em Hypopomus, Gymnorhamphichthys, Iracema e Rhamphichthys), ausência de um órgão elétrico acessório pareado na região mental ou humeral (vs. presença em Hypopygus e Steatogenys), presença de 3-4 proximais peitorais radiais (vs. 5 em Akawaio), presença do antiorbital + infraorbital, e dos canais ossificados da linha lateral da região cefálica do pré-opérculo (vs. ausência em Racenisia). Brachyhypopomus não pode ser diagnosticado de maneira não-ambígua de Microsternarchus ou Procerusternarchus, com base em caracteres de morfologia externa. Brachyhypopomus compreende 28 espécies válidas. Aqui nós descrevemos 15 espécies novas, e fornecemos a redescrição de 13 espécies previamente descritas, baseado em caracteres merísticos, morfométricos e outros caracteres morfológicos. Nós incluímos notas sobre à ecologia e história natural para cada uma das espécies, e fornecemos chaves dicotômicas regionais e mapas de distribuição baseado no exame de 12.279 espécimes de 2.787 lotes de museus. Um lectótipo é designado para Brachyhypopomus pinnicaudatus (Hopkins, Comfort, Bastian \& Bass, 1990). Espécies de Brachyhypopomus são abundantes em habitats de águas rasas lênticas e com correntes fracas, ocorrendo do sul da Costa Rica e norte da Venezuela ao Uruguai e norte da Argentina. A diversidade de espécies é maior na Grande Amazônia, onde 20 espécies ocorrem: $B$. alberti, espécie nova, B. arrayae, espécie nova e $B$. cunia, espécie nova, na drenagem do alto rio Madeira; $B$. batesi, espécie nova, na Amazônia central e rio Negro; $B$. beebei, $B$. brevirostris, $B$. regani, espécie nova, $B$. sullivani, espécie nova e $B$. walteri, amplamente distribuídas nas bacias Amazônicas e do Orinoco, e nas Guianas; $B$. belindae, espécie nova, bacia Amazônica central; $B$. benjamini, espécie nova e $B$. verdii, espécie nova, na bacia do alto Amazonas; $B$. bennetti, no alto, médio e porções baixas da bacia Amazônica, baixo Tocantins e alto rio Madeira; $B$. bullocki nas drenagens do Orinoco, Negro e Essequibo; $B$. diazi nos Llanos do Orinoco; $B$. flavipomus, espécie nova e $B$. hamiltoni, espécie nova, no médio e alto Amazonas; $B$. hendersoni, espécie nova, na Amazônia central, baixo Negro e Essequibo; B. pinnicaudatus no médio e baixo Amazonas, baixo e alto Madeira, baixo Tocantins, bacia do Mearim e rios costeiros da Guiana Francesa; e B. provenzanoi, espécie nova, nas bacias do alto Orinoco e alto Negro. Cinco espécies são conhecidas das bacias Paraná-Paraguai-Uruguai e bacias adjacentes das drenagens do sul do Brasil: $B$. bombilla no alto, médio e baixo Paraguai, baixo Paraná, Uruguai e drenagens Patos-Mirim; B. brevirostris da bacia do alto Paraguai; $B$. draco das bacias do baixo Paraguai, baixo Paraná, Uruguai, Patos-Mirim e Tramandaí; $B$. gauderio das bacias do alto, médio e baixo Paraguai, baixo Paraná, Uruguai, Patos-Mirim e Tramandaí; e $B$. walteri das bacias do alto Paraguai e baixo Paraná. Duas espécies ocorrem nas drenagens costeiras do sudeste do Brasil: B. janeiroensis no São João, Paraíba e em drenagens menores nas adjacências; e $B$. jureiae no Ribeira de Iguape e Una do Prelado. Uma espécie ocorre no médio e alto rio São Francisco: B. menezesi, espécie nova. Três espécies ocorrem nas drenagens trans-Andinas: $B$. diazi nas drenagens do Caribe no norte da Venezuela; B. occidentalis nas drenagens do Atlantico e Pacífico do sul da Costa Rica e Panamá até Darién, e nas drenagens do Maracaibo, Magdalena, Sinú e Atrato; e B. palenque, espécie nova, nas drenagens do Pacífico no Equador.

Keywords: Biogeography, Bluntnose knifefish, Electroreception, Identification key, Rhamphichthyoidea.

\section{Introduction}

The weakly electric "bluntnose knifefish" genus Brachyhypopomus Mago-Leccia, 1994 occurs in lowland tropical and subtropical freshwater habitats from southern Costa Rica to southern Uruguay (Albert \& Crampton, 2003; Crampton, 2011). Brachyhypopomus species, listed in Table 1 , are small to medium-sized, cryptically pigmented, and nocturnally active predators of aquatic invertebrates. They occur in a wide variety of lentic or slowly-flowing lotic environments, including streams, river margins, swamps, and floodplains - where they often constitute a species-rich and abundant component of the local ichthyofauna (AlvesGomes, 1997; Crampton, 1996a,b; 1998a; 2011; Crampton $\&$ Albert, 2006). The genus is conspicuously absent from the bottoms of deep river channels (Crampton, 2007). Brachyhyроротиs and other small hypopomids are not exploited as food fish, but they sometimes feature in the diet of Amerindians (Ellis, 1913), and are occasionally exported as ornamental fish (Chao, 2001). Like all gymnotiform fish, Brachyhypopomus generate electric organ discharges (EODs), which, in combination with a cutaneous array of tuberous electroreceptors, facilitate active electrolocation and electrocommunication (Bullock et al., 2005; Kramer, 1995; Moller, 1995). The head-to-tail recorded EODs of Brachyhypopomus are weak $(<1 \mathrm{~V})$, pulsed (at rates of $<1$ $110 \mathrm{~Hz}$ ) waveforms of $c a$. 0.5-5 msec duration, comprising from one to four phases of alternating polarity (Crampton \& Albert, 2006; Waddell et al., 2016). Crampton et al. (2016) provide a phylogenetic reconstruction of Brachyhypopomus (including all 28 species described or redescribed herein) based on a combination of morphological data and sequence data from the mitochondrial cytochrome $b$ and nuclear rag2 genes.

The first reported species of Brachyhypopomus was described as Rhamphichthys brevirostris by Steindachner (1868a, b), based on specimens collected by Johann Natterer from the rio Guaporé. Thenceforth and until 1994, five additional species of Brachyhypopomus were described: B. beebei, B. diazi, B. janeiroensis, B. occidentalis, and B. pinnicaudatus (see Table 1 for authors and type localities). These were all assigned to the genus Hyроротиs, which was erected earlier by Gill (1864); see 'Taxonomic remarks' in the generic redescription herein. 


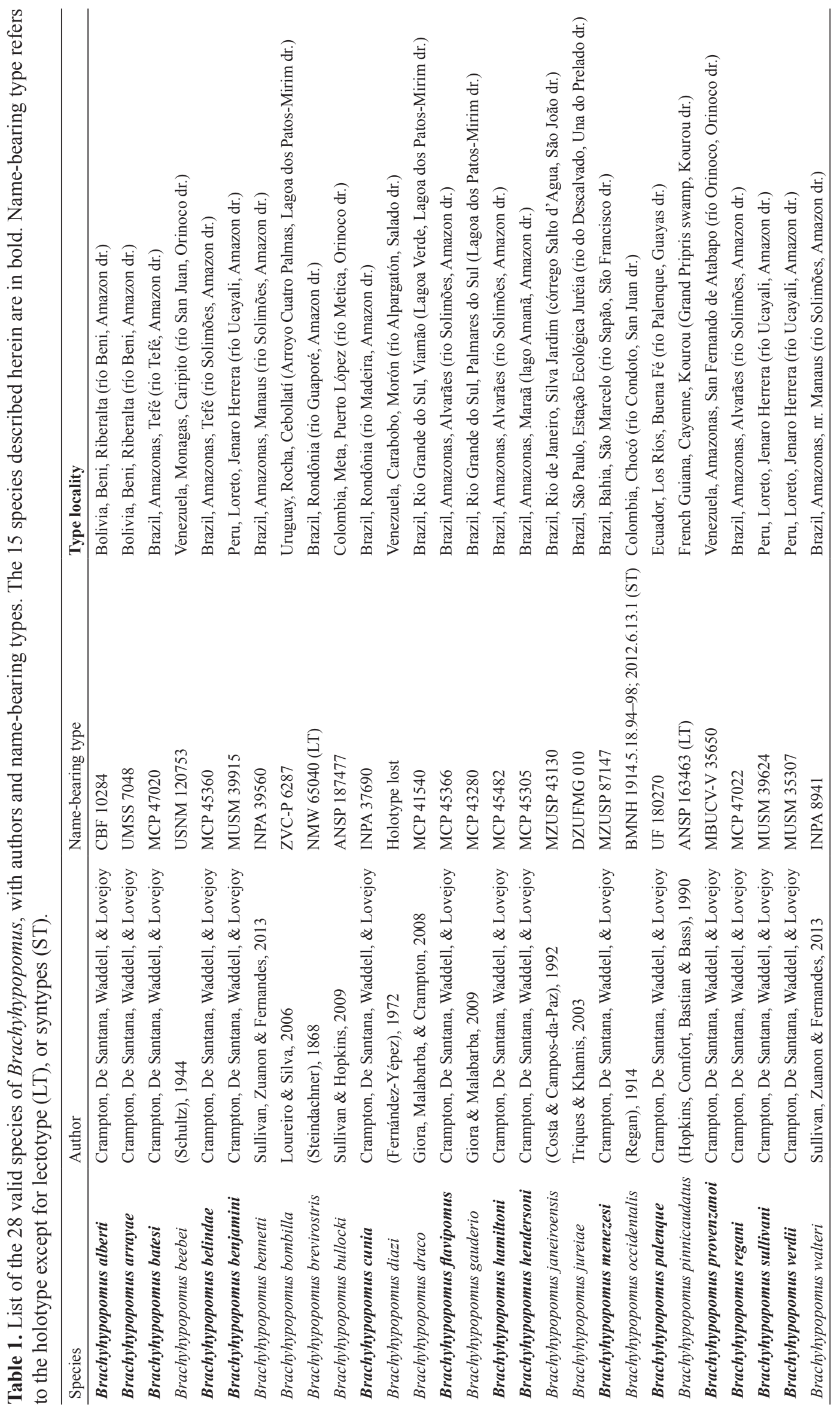


Mago-Leccia (1994) divided Hypopomus sensu Gill (1864) into two genera: Hypopomus, and a new genus Brachyhypopomus. Hyроротиs was restricted to Hypopomus artedi (Kaup, 1856) and its now junior synonym $H$. mulleri (Kaup, 1856) based on a longer, "projected" snout, and mouth in inferior position (among other characters). Brachyhypopomus was erected to accommodate $B$. beebei, $B$. brevirostris, $B$. diazi, B. janeiroensis, $B$. occidentalis, and $B$. pinnicaudatus based on a short snout and included mouth, the absence of a mesocoracoid bridge, a short and crescentshaped maxillary bone, and posterior nares closer to eye.

Subsequent to Mago-Leccia (1994), seven additional species of Brachyhyроротиs have been described, bringing the total to 13: B. bennetti, B. bombilla, B. bullocki, B. draco, $B$. gauderio, B. jureiae, and $B$. walteri (see Table 1 for authors and type localities). Two of these, $B$. bennetti and $B$. walteri, were assigned to a subgenus, Odontohypopomus Sullivan, Zuanon \& Cox Fernandes, 2013, based on the shared presence of premaxillary teeth in adult specimens (Sullivan et al., 2013).

Brachyhyроротиs belongs to the family Hypopomidae Mago-Leccia, which sensu Maldonado-Ocampo et al. (2014) contains six additional species in five genera: Akawaio penak Maldonado-Ocampo, López-Fernández, Taphorn, Bernard, Crampton \& Lovejoy, Hypopomus artedi, and the tribe Microsternarchini Albert - comprising Microsternarchus bilineatus Fernández-Yépez, $M$. brevis Fernandes, Nogueira, Williston \& Alves-Gomes, Procerusternarchus pixuna Fernandes, Nogueira \& Alves-Gomes, and Racenisia fimbriipinna MagoLeccia. Two genera, Hypopygus Hoedeman, 1962, and Steatogenys Boulenger, 1898, were until recently placed in the Hypopomidae, but were relocated to the family Rhamphichthyidae Regan by Maldonado-Ocampo et al. (2014) based on molecular phylogenetic evidence. The Hypopomidae and the Rhamphichthyidae together constitute the superfamily Rhamphichthyoidea.

The general biology of Brachyhypopomus is well known in comparison to other gymnotiforms, as reviewed below (see 'Functional biology and ecology', in the generic description), and one species, B. gauderio (formerly ascribed to $B$. pinnicaudatus), has served as a model species for understanding electroreception, electrogenesis, neuroethology, and the influence of steroid and peptide hormones on the electric organ and EOD communication signals; for recent reviews see Gavassa et al. (2013), Markham (2013), Salazar et al. (2013), Silva et al. (2013), and Giora et al. (2014). Nonetheless, only 13 valid species of Brachyhypopomus have been described to date (Table 1), despite knowledge of considerable additional species diversity - especially in the Amazon basin (Albert \& Crampton, 2003; Alves-Gomes, 1997; Crampton, 2011; Crampton \& Albert, 2006; Crampton \& Ribeiro, 2013; Heiligenberg \& Bastian, 1980; Schwassmann, 1978; Sullivan, 1997; Sullivan et al., 2013); see Fig. 1 for a local assemblage of 12 species from the central Amazon.
Here we provide a taxonomic revision of Brachyhypopomus. We confirm the validity of all 13 previouslydescribed species, describe 15 new species (Table 1), and provide descriptions of morphology, ecology, and geographical distributions based on a thorough examination of available museum material. We also provide regional dichotomous identification keys to the species of Brachyhypopomus.

\section{Material and Methods}

Specimens and collections. Examined specimens of Brachyhypopomus are listed in the species descriptions and redescriptions. Examined specimens of other gymnotiforms, with emphasis on other Rhamphichthyoidea are listed in Supplement 1 . Many of the specimens examined in this study were collected by us (WGRC, NRL, JCW) using fine mesh dip nets or small seine nets, often with the aid of an electric fish detector (Crampton et al., 2007). Specimens were euthanized in a $600 \mathrm{mgl}^{-1}$ solution of eugenol or in ice-ethanol slurry until apnea and EOD cessation, fixed in 10\% formalin, and preserved in $70 \% \mathrm{EtOH}$. Sex was assessed via examination of the gonads through a short incision in the ventral part of the body cavity of fixed specimens; this permitted an evaluation of sexual dimorphism of caudal filament structure and body length.

Osteological characters were assessed from specimens cleared and counterstained (CS) for cartilage and bone using the method outlined by Taylor \& Van Dyke (1985). In some specimens with weak ossification, bones were stained with alizarin red in ethanol solution instead of $\mathrm{KOH}$ solution (Springer \& Johnson, 2000). Examined cleared and stained specimens are listed in Supplements 1 and 2. Radiographs served as additional sources of osteological data for some species. We limit the presentation of osteological characters herein to those necessary to diagnose species where characters from non-invasive analyses were insufficient.

Species accounts. Species descriptions/redescriptions are presented in alphabetical order. Many taxonomic, ecological, behavioral and neurobiological papers make reference to Brachyhypopomus, and inevitably the high diversity of the genus coupled with the relatively small fraction of previously described species has resulted in incorrect identifications in the literature, assignment to cheironyms, or identification by alphabetic/numerical schemes. The synonymy sections herein provide an opportunity to correct many of these errors. Nonetheless, we adopt a conservative approach by confirming or correcting the identity of species mentioned in nontaxonomic papers only where photographs or other illustrations, or exclusive geographical distributions permit unequivocal identification. The differential diagnoses serve to unambiguously separate each species from all congeners and are confined where possible to external characters, or characters that can be analyzed without invasive procedures (e.g., vertebral counts from radiographs). 


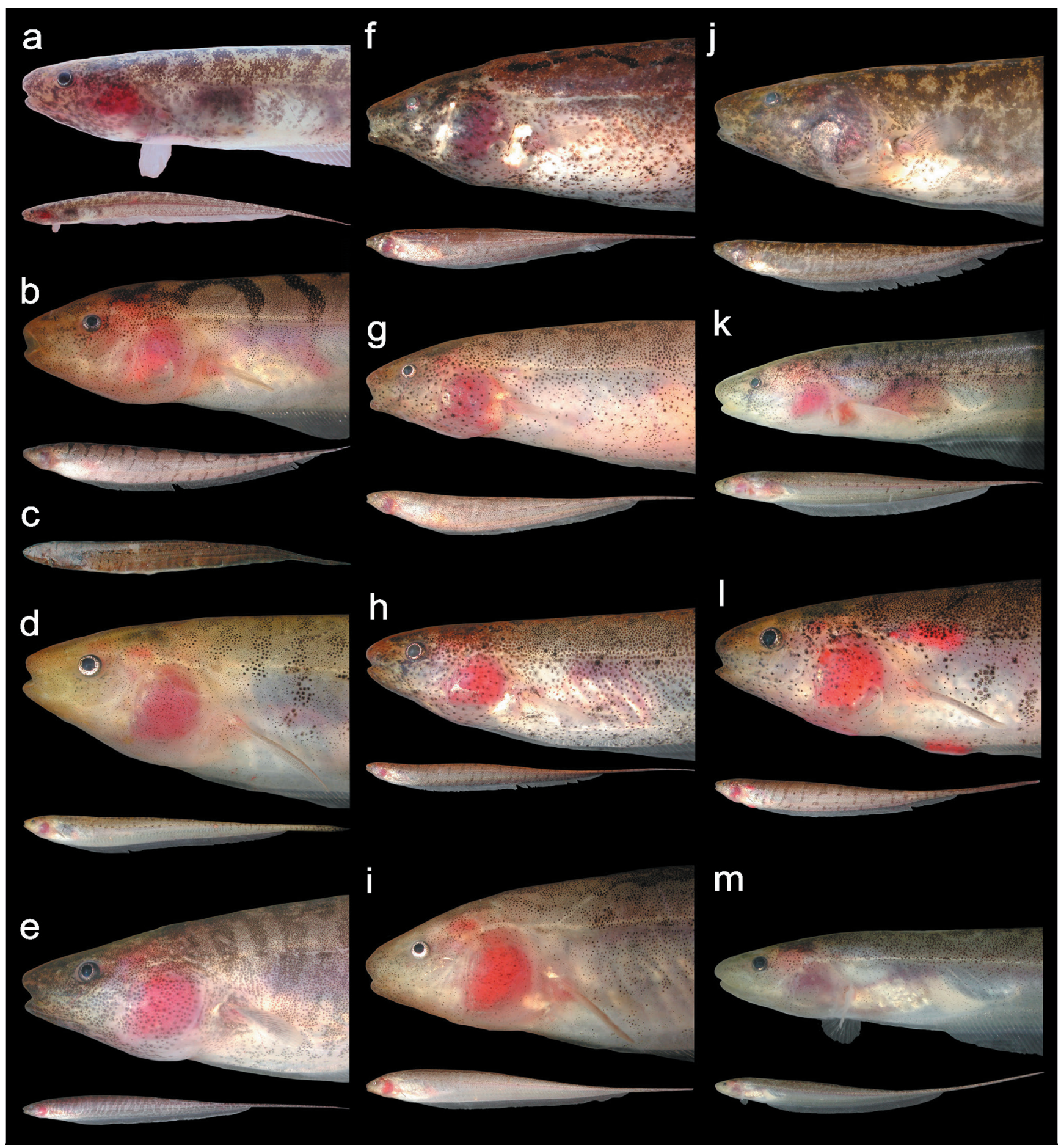

Fig. 1. Live individuals of 12 sympatric species of Brachyhypopomus and one species of Microsternarchus from the vicinity of Tefé, Amazonas, Brazil (Amazonas dr.). a. Brachyhypopomus batesi MCP 45312 (WC01.191293b), immature, $102 \mathrm{~mm}$ TL. b. Brachyhypopomus beebei head - uncat., immature, $75 \mathrm{~mm}$ TL; body - MCP 45450 (WC04.090600), female, $178 \mathrm{~mm}$ TL. c. Brachyhypopomus belindae MCP 45430, paratype, immature, 104 mm TL (photographed out of water, close up of head not available). d. Brachyhypopomus bennetti MCP 45451, male, 196 mm TL. e. Brachyhypopomus brevirostris uncat., immature, 224 mm TL. f. Brachyhypopomus flavipomus MCP 45453 (WC09.090600), female, 98 mm TL. g. Brachyhypopomus hamiltoni MCP 45482 (WC05.080301), holotype, female, 97 mm TL. h. Brachyhypopomus hendersoni MCP 45489, female, 164 mm TL. i. Brachyhypopomus pinnicaudatus MCP 45455, female, 135 mm TL. j. Brachyhypopomus regani, MCP 45285 (WC02.100301), male, 119 mm TL. k. Brachyhypopomus sullivani MCP 45464 (WC04.210201), immature, 79 mm TL. 1. Brachyhypopomus walteri MCP 45458 (WC 03.090600), male, 161 mm TL. m. Microsternarchus bilineatus uncat., $85 \mathrm{~mm}$ TL. 
Material examined are ordered alphabetically by country, state or department (in bold), collection code following Sabaj-Pérez (2014), and then numerically by catalog number. Institutions not listed in Sabaj-Pérez are: SINCHI-CIACOL (Colección Ictiológica de la Amazonia Colombiana del Instituto Amazónico de Investigaciones Científicos, Leticia, Colombia), and ZOOA (Universidad de Azuay, Azuay, Ecuador).

Holotypes of new species are deposited in the following institutions: CBF, Colección Boliviana de Fauna, Museo Nacional de Historia Natural, Instituto de Ecología, Academia Nacional de Ciencias de Bolivia, La Paz; INPA, Instituto Nacional de Pesquisas da Amazônia, Manaus; MBUCV-V, Museo de Biología de la Universidad Central de Venezuela, Caracas; MCP, Museu de Ciências e Tecnologia da Pontifícia Universidade Católica do Rio Grande do Sul, Porto Alegre; MUSM, Museo de Historia Natural, Universidad Nacional Mayor de San Marcos, Lima; MZUSP, Museu de Zoologia da Universidade de São Paulo, São Paulo; UF, University of Florida, Florida Museum of Natural History; UMSS, Universidad Mayor de San Simón, Facultad de Ciencias y Tecnología, Centro de Biodiversidad, Zoología, Laboratorio de Ictiología, Cochabamba.
Lots without reliable geographical coordinates are listed but excluded from the distribution maps. Geographical coordinates were taken from museum labels and corrected if necessary, or are given as approximate where a position within $30 \mathrm{~km}$ could be determined. Abbreviations are: affl. - affluent; confl. - confluence; dr/s. - drainage/s; hwy. highway; mun. - county/municipality; nr. - near; prov. province; rd. - road. Square parentheses denote corrections to and/or comments on localities.

Distribution maps.Localitieswereprojectedontocartographic projections of South and Central America (Fig. 2) using the ArcGIS geographical information system software, version 9.3.1 (ESRI, Redlands, CA). River networks, and elevation data were obtained from HydroSHEDS (Hydrological Data and Maps based on Shuttle Elevation Derivatives at Multiple Scales) (Lehner et al., 2006), from http://hydrosheds.cr.usgs. gov. HydroSHEDS derives from elevation data of the US NASA Space Shuttle Radar Topography Mission. We used the 30 arc-second resolution data set referenced to the WGS84 projection. Species distribution maps are presented in alphabetical order, and arranged to maximize cartographic magnification and ensure that species with overlapping geographical distributions are presented in separate figures.

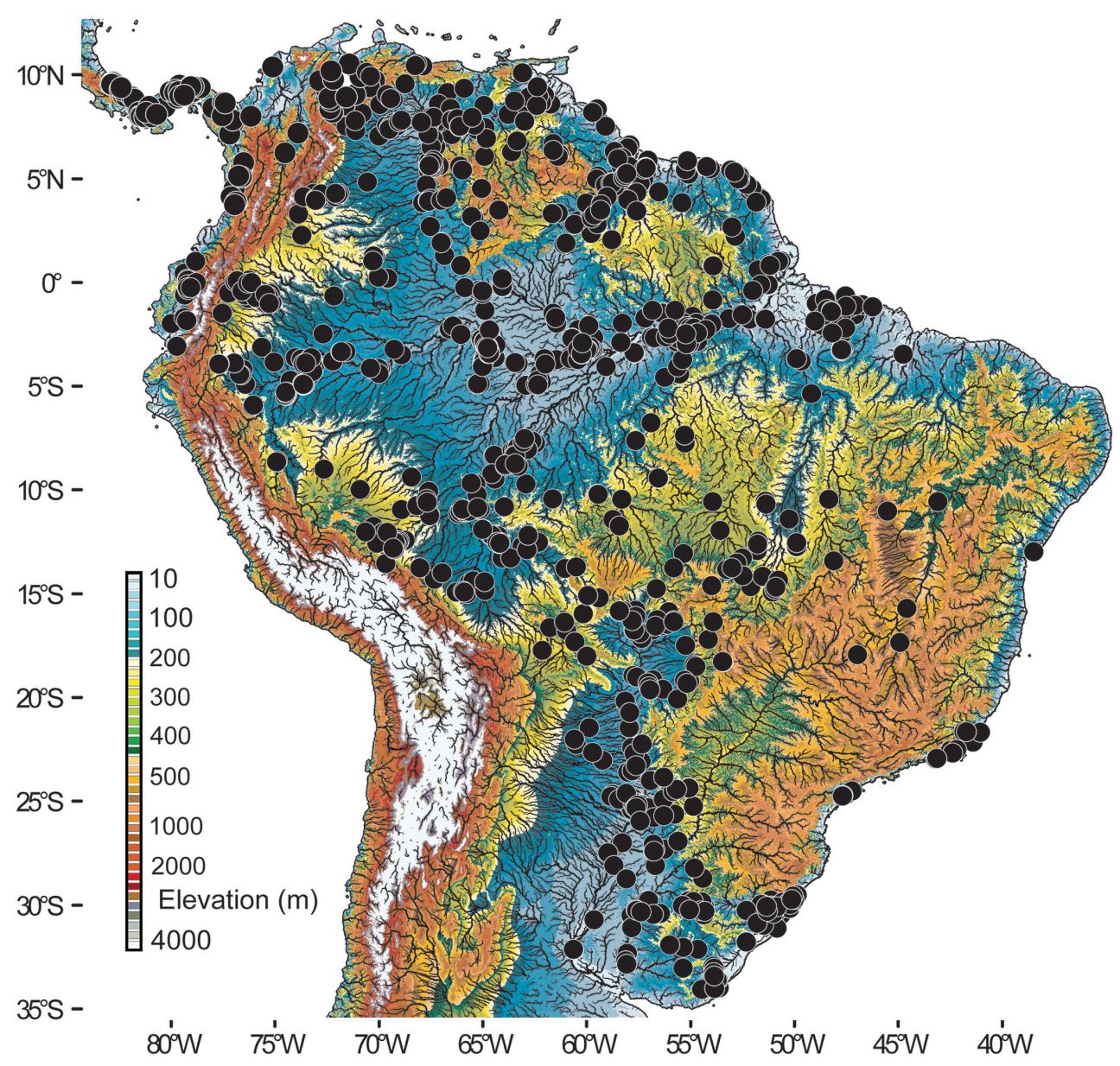

Fig. 2. Collection records for Brachyhypopomus based on 11,750 specimens from 2,642 georeferenced museum lots. Map of South and southern Central America based on HydroSHEDS data. Elevation data refers to altitude above mean sea level. 
Morphometric measurements. Body size measurements reported in this paper refer to total length (TL) - the distance from the tip of the snout to the end of the caudal filament, unless specified as length to end of anal fin (LEA) - the distance from the tip of the snout to the base of the last anal fin. Morphometric measurements are summarized in the schematic diagram in Fig. 3. Due to non-linear growth allometry we analyzed morphometric data only from specimens of reproductive size (above the minimum size at which gonadal development occurs for each species) (Crampton et al., 2011; Hulen et al., 2005). Measurements reported as a percentage of TL are given only for specimens with no damage or regeneration. Measurements reported as a percentage of LEA are given only for specimens with no damage or regeneration anterior to the anal-fin terminus. The following morphometrics represent the horizontal distance parallel to the long axis of the specimen, with the specimen extended on a flat surface - measured either with a ruler to the nearest $1 \mathrm{~mm}$ or, for smaller specimens, a digital caliper to the nearest $0.1 \mathrm{~mm}$ : Anal-fin length (AF) - the distance between the bases of the first and last rays of the anal fin. Caudal-filament length $(\mathrm{CF})$ - the distance from the base of the last anal-fin ray to the tip of the caudal filament; we describe the caudal filament as "short" if $<15 \%$ TL, "moderate" if $\geq 15<25 \%$ $\mathrm{TL}$, and "long" if $\geq 25 \% \mathrm{TL}$.

All other morphometrics, listed below, were measured using the ocular micrometer of a Meiji Techno RZ stereomicroscope, to the nearest $0.001 \mathrm{~mm}$, as planar distances orthogonal to the body axis (i.e. point-to-point as distances between imaginary lines drawn vertically upwards from the specimen towards the horizontal plane of the micrometer). We chose ocular micrometer measurements instead of point-to-point caliper measurements because the latter lacked sufficient precision and replicability for characterizing morphometrics in specimens smaller than $c a .100 \mathrm{~mm}$ TL. We were unable to obtain ocular micrometer measurements from the holotype specimens of some previously-described species (all previous descriptions employ caliper-based measurements). In these cases we excluded the holotype from the tabulated morphometric ranges we present in the species redescription; this is clarified in the appropriate table legends.

The following measurements (Figs. 3a,c) were taken from the left side of the fish, with the lateral line held parallel to the measuring surface: Body depth at analfin origin (BD1) - body depth measured vertically from dorsal midline to ventral midline at the anal-fin origin; we describe the body depth as "shallow" if $<10 \% \mathrm{TL}$, "moderate" if $\geq 10<14 \% \mathrm{TL}$, and "long" if $\geq 14 \% \mathrm{TL}$. Body depth at anal-fin middle (i.e. middle of the length of the anal fin) (BD2) - body depth measured vertically from dorsal midline to ventral midline at a point half-way between the bases of the first and last rays of the anal fin. Branchial aperture (opening) (BO) - the distance from the posterodorsal to anteroventral extents of the branchial aperture. Caudal-filament depth (CD) - the vertical depth of the caudal filament measured at the base of the last anal-fin ray. Caudal-filament length (CF) - the distance from the base of the last anal-fin ray to the tip of the caudal filament. Head depth at eye (HD1) - the maximum head depth measured at the center of the eye; we describe the head as "shallow" if head depth $<72 \%$ HL, "moderate" if $\geq 72<87 \%$ HL, and "deep" if $\geq 87 \%$ HL. Head depth at occiput (HD2) - the maximum head depth measured at the occiput. Head length (HL) - the distance from the tip of the snout to the posterior-most margin of the bony operculum; we describe the head as "short" if head length is $<11 \%$ LEA, "moderate" if $\geq 11<14 \%$ LEA, and "long" if $\geq 14 \%$ LEA. Internarial distance (AE) - the distance from the posterior margin of the anterior naris to the anterior margin of the posterior naris. Longest anal-fin ray (ARL) - the distance from the base to the distal tip of the longest anal-fin ray (or estimated longest anal-fin ray). Orbital diameter (OD) - the horizontal diameter of the eye; we describe the eye as "small" if the orbital diameter $<9 \%$ HL, "moderate" if $\geq 9<14 \% \mathrm{HL}$, and "large" if $\geq$ $14 \%$ HL. Pectoral-fin base (PB) - the distance from the base of the dorsal most ray of the pectoral fin to the base of the ventral-most ray; we describe the pectoral fin as "narrow" if its base is $<10 \% \mathrm{HL}$, "moderate" if $\geq 10<15 \%$ $\mathrm{HL}$, and "broad" if $\geq 15 \%$ HL. Posterior naris to eye (PE) - the distance from the posterior margin of the posterior naris to the anterior margin of the eye. Longest pectoralfin ray (PRL) - the distance from the base to the end of the longest pectoral-fin ray. Postorbital distance (PO) the distance from the posterior margin of the eye to the posterodorsal angle of the branchial opening. Preorbital distance (snout length) (PR) - the distance from the tip of the snout to the anterior margin of the eye. Snout to analfin origin (SA) - the distance from the tip of the snout to the base of the first anal-fin ray. Snout to occiput (SO) the distance from the tip of the snout to the occiput. Snout to pectoral-fin base (PP) - the distance from the tip of the snout to the base of the dorsal most ray of the pectoral fin.

The following measurements (Figs. 3b,d-e) were taken with the dorsal surface of the specimen facing up or down, while holding the lateral line parallel to the measuring surface: Body width at anal-fin origin (BW1) - the maximum body width at anal-fin origin. Body width at anal-fin middle (i.e. middle of the length of the anal fin) (BW2) - the maximum body width at a point halfway between the bases of the first and last rays of the anal fin. Caudal-filament width $(\mathrm{CW})$ - the horizontal width of the caudal filament measurement at the base of the last anal-fin ray. Head width at eye (HW1) - the maximum head width measured at the center of the eye; head width at occiput (HW2) - the maximum head width measured at the occiput; interorbital distance (IO) - the minimum width between the dorsal margins of the orbits; mouth width (MW) - the distance between the ricti. 

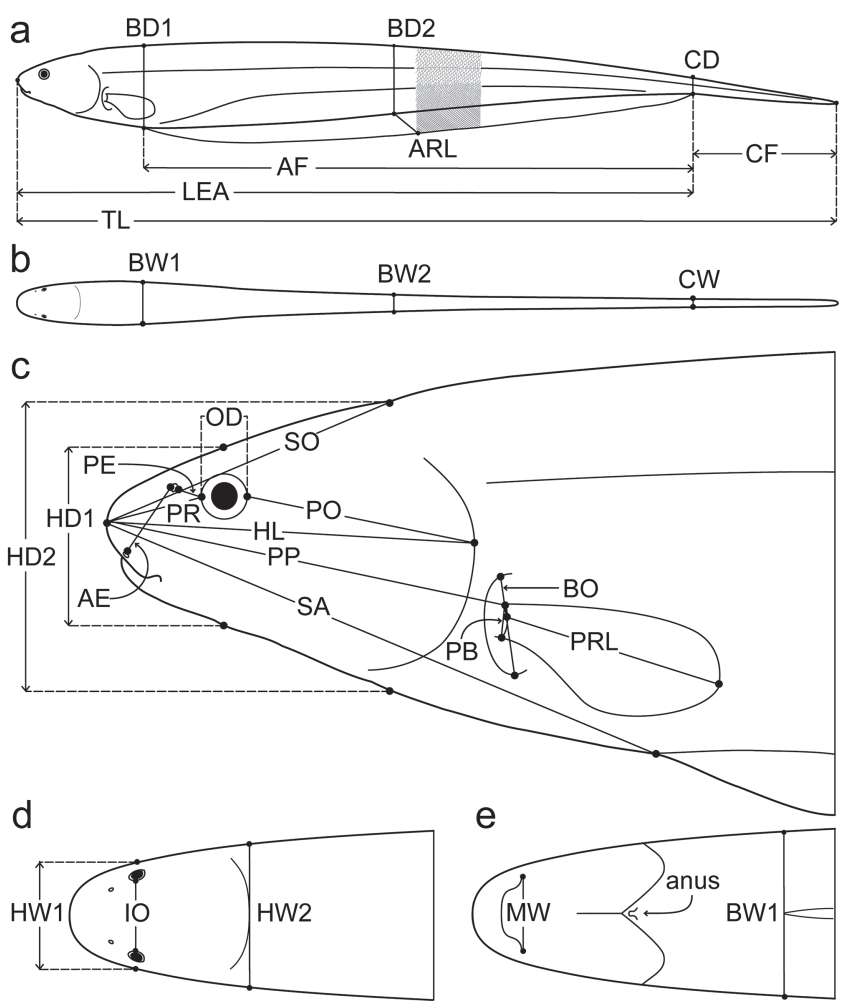

Fig. 3. Measurements used for morphometric analysis of Brachyhypopomus. See text for details. a. Body, lateral view: $\mathrm{AF}=$ Anal-fin length; $\mathrm{ARL}=$ Longest anal-fin ray; $\mathrm{BD} 1=$ Body depth at anal-fin origin; BD2 = Body depth at anal-fin middle; $\mathrm{CD}=$ Caudal-filament depth; $\mathrm{CF}=$ Caudal-filament length; LEA = Length to end of anal fin; $\mathrm{TL}=$ Total length. b. Body, dorsal view: BW1 = Body width at anal-fin origin; $\mathrm{BW} 2=$ Body width at anal-fin middle; $\mathrm{CW}=$ Caudal-filament width. c. Head, lateral view: $\mathrm{AE}=$ Internarial distance; $\mathrm{BO}$ $=$ Branchial aperture; HD1 $=$ Head depth at eye; HD2 = Head depth at occiput; $\mathrm{HL}=$ Head length; $\mathrm{PRL}=$ Longest pectoral-fin ray; $\mathrm{OD}=$ Orbital diameter; $\mathrm{PB}=$ Pectoral-fin base; $\mathrm{PE}=$ Posterior naris to eye; $\mathrm{PO}=$ Postorbital distance; $\mathrm{PP}=$ Snout to pectoral-fin base; PR $=$ Preorbital distance (snout length); $\mathrm{SA}=$ Snout to anal-fin origin; $\mathrm{SO}=$ Snout to occiput. d. Head, dorsal view: HW1 = Head width at eye; HW2 = Head width at occiput; IO = Interorbital width. e. Head, ventral view: MW $=$ Mouth width. Schematics based on B. diazi (UF 174333).

Meristics. Counts were taken only from specimens of reproductive size. Anal-fin ray number - the total number of branched and unbranched rays counted from ethanol preserved specimens or from cleared and stained (CS) specimens using a stereomicroscope with backlitillumination. Electrocyte columns at anal-fin terminus - the number of horizontally aligned bilateral columns of electrocytes in the hypaxial electric organ at a vertical with the anal-fin terminus; counted from ethanol preserved specimens using a stereomicroscope and backlit illumination, with skin and scales overlying the electric organ peeled off if necessary. Electrocyte columns at mid-caudal filament - the number of horizontally aligned bilateral columns of electrocytes in the hypaxial electric organ at the midpoint between the anal-fin terminus and tip of the caudal filament (in intact, non-regenerated specimens only). Pectoral-fin rays - total number of branched and unbranched rays; counted from ethanol preserved specimens using a stereomicroscope with transmitted light, or from cleared-and-stained specimens. Precaudal vertebrae - all precaudal vertebrate including the first four vertebrae (which form part of the Weberian apparatus), the second and third of which often exhibit fused centra (Campos-da-Paz, 2000: 524; Hopkins, 1991: 152), and all transitional vertebrae (which lack ribs and hemal spines); counted from digital X-ray images or from CS specimens. The number of precaudal vertebrae is a proxy for the length of the body cavity (Albert, 2001). Radiographs were taken with a Kodak DXS Pro digital X-ray system. Scales above the lateral line - counted as number of rows from the lateral line to the dorsal midline (but excluding the lateral-line series) at midpoint from snout to anal-fin terminus using a stereomicroscope with transmitted light. Gill filaments on first gill arch - counted as the number of filaments on the first gill arch (including only the lateral, i.e. outer, hemibranch).

\section{Results}

\section{Brachyhypopomus Mago-Leccia, 1994}

Brachyhypopomus Mago-Leccia, 1994:47(descriptionanddiagnosis for genus). -Alves-Gomes et al., 1995: 307-309, figs. 6-8, 312314, figs. 9-10 (position in phylogeny of the Gymnotiformes). -Sullivan, 1997 (diagnosis for genus, position in phylogeny of the Rhamphichthyoidea). -Albert \& Campos-da-Paz, 1998: 423, fig. 2, 426, 436, table 1, 439, table 2 (diagnosis for genus, position in phylogeny of the Gymnotiformes). -Albert, 2001: 12 , table 2, 56, table 3, 68-69 (diagnosis for genus, position in phylogeny of the Gymnotiformes). -Albert \& Crampton, 2003: 494 (catalog of hypopomids). -Carvalho, 2013: 78 (diagnosis for genus, position in phylogeny of the Rhamphichthyoidea). -Fernandes et al., 2014: 98, fig. 1 (position in phylogeny of the Rhamphichthyoidea). -Maldonado-Ocampo et al., 2014: 6,8 , fig. 6 (position in phylogeny of the Rhamphichthyoidea). -Tagliacollo et al., 2016: 10 (position in phylogeny of the Gymnotiformes). -Crampton et al., 2016: 1-66, table 1, 3-4, figs. 1-20 (phylogeny, biogeography and ecology of Brachyhypopomus).

Odontohypopomus (subgenus of Brachyhypopomus) Sullivan et al., 2013: 6 (erected to contain B. bennetti and B. sullivani).

Type species. Rhamphichthys brevirostris Steindachner (1868a). Type by original designation (Mago-Leccia, 1994: 47).

Diagnosis. Brachyhypopomus is diagnosed unambiguously from all other Gymnotiformes, including all other rhamphichthyoid species, by a single unreversed shared and 
derived character: the presence of a disk-like ossification in the anterior portion of the palatoquadrate cartilage in adult specimens. See Sullivan et al. (2013: 7, fig. 1) for a photograph of the ossified palatoquadrate cartilage in a cleared and stained specimen of $B$. walteri. The disk-like ossification of the palatoquadrate cartilage is present in all species of Brachyhypopomus, but in some specimens is relatively hard to discern due to poor uptake of stain. Likewise, in many immature specimens the ossification is incomplete. This may explain why Sullivan (1997) did not observe this character in some species of Brachyhypopomus (B. brevirostris, B. bullocki, B. bombilla, B. regani, B. sullivani), where we did. Moreover, because the ossification of the palatoquadrate cartilage is visible only in cleared and stained specimens, this character is inadequate for determining whether whole specimens belong to the genus Brachyhypopomus. The following combination of characters that do not require invasive techniques serves to delimit members of the genus from all other gymnotiforms.

Brachyhypopomus is diagnosed from the Apteronotidae by the absence of a caudal fin and dorsal electroreceptive filament and from the Gymnotidae and Sternopygidae by the absence of complete rows of teeth on the premaxilla in adults. Brachyhypopomus is diagnosed from the Rhamphichthyidae sensu Maldonado-Ocampo et al. (2014) (which includes Hypopygus and Steatogenys in the tribe Steatogeni) either by the short snout, $18.7-32.6 \%$ of HL, vs. 40.0-68.6\% in Gymnorhamphichthys, 53.8-55.4\% in Iracema, and 49.8-61.6\% in Rhamphichthys (Carvalho \& Albert, 2011; Carvalho et al., 2011; Géry \& Vu, 1964; Nijssen et al., 1976; Schwassmann, 1976; Schwassmann, 1989; Triques, 1999), or by the absence of a paired accessory electric organ in the mental or humeral region, vs. presence in the Steatogeni (de Santana \& Crampton, 2010).

Within the Hypopomidae sensu Maldonado-Ocampo et al. (2014), Brachyhypopomus is diagnosed from Akawaio by the presence of three or four pectoral proximal radials, vs. five in Akawaio (Maldonado-Ocampo et al., 2014). Brachyhypopomus is diagnosed from Hypopomus by the shorter snout, $18.7-32.6 \%$ of HL, vs. $33.3-40.1 \%$ of HL in adult $H$. artedi (based on measurements taken from 30 specimens of $H$. artedi from diverse localities in the Guyana Shield). Brachyhypopomus is diagnosed from Racenisia by the presence of the antorbital + infraorbital, and the preopercular cephalic lateral line canal bones, vs. complete absence in Racenisia; these canal bones are clearly visible in alcohol preserved specimens.

Brachyhypopomus cannot be diagnosed unambiguously from Microsternarchus or from Procerusternarchus on the basis of external characters alone. However, with the exception of B. benjamini and B. provenzanoi, species of Brachyhyроротиs are diagnosed from Microsternarchus by the presence of scales on the middorsal portion of the anterior third of the body, vs. absence in M. bilineatus and in M. brevis (Fernandes et al., 2015).
Previous generic diagnoses. Brachyhypopomus was assigned to the new family Hypopomidae sensu MagoLeccia (1978) based on the following combination of characters: absence of teeth in both oral jaws; relatively short snout; nares tubular in shape and well-separated, the anterior nares at the corner of the upper lip; 3-4 pectoralfin radials; supraorbital canal embedded with frontal bone; posttemporal and supracleithrum not fused; fixed anus position; and anal-fin origin posterior to the pectoral fin base. Two of these characters are now known to apply variably to members of Brachyhypopomus: two species, B. bennetti and $B$. walteri, possess premaxillary teeth in adults (Sullivan $e t$ al., 2013); and the supraorbital canal is independent from the frontal bone in B. flavipomus and B. verdii. Albert (2001) provided a revised diagnosis for the Hypopomidae, but many of the characters fail to accommodate recent increases in the species diversity of Brachyhypopomus (including many of the species described herein). A new formal generic diagnosis for the family - one which accommodates the recentlydescribed genera Akawaio and Procerusternarchus, and one which excludes the Steatogeni, following MaldonadoOcampo et al. (2014) - is necessary, but is beyond the scope of this paper.

Mago-Leccia (1994: 47) diagnosed six species of Brachyhypopomus from Hypopomus (but not from other genera) by "their short snout and included mouths, absence of mesocoracoid bridge, short and crescent-shaped maxillary bone" (Mago-Leccia, 1994: 165, figs. 65d,f), and "posterior nares closer to eyes." Of these characters, only "short snout" is able to diagnose all 28 species of Brachyhypopomus recognized herein from Hypopomus. Albert and Camposda-Paz (1998: 426) and Albert (2001: 68) diagnosed the (as-then) six valid species of Brachyhypopomus (and seven undescribed species) from other Gymnotiformes based on four characters others than those listed previously by MagoLeccia (1994): 1. "Premaxilla gracile with a curved anterior margin and forming a distinct angle with the maxilla in lateral view (... except B. beebei; also present in Steatogenys ...)"; 2. "Dentary gracile"; 3. "Body cavity with 16 - 17 PCV [precaudal vertebrae]"; 4. "Single transitional vertebrae (sic)." However, each of these four characters fails to unambiguously diagnose all 28 species of Brachyhypopomus recognized herein from other gymnotiforms.

Sullivan (1997) proposed a single character diagnostic character for the genus, "medial portion of upper jaw (premaxillary portion) forms distinct angle with sides of upper jaw (maxillary portion) in lateral and frontal view" (not present in Microsternarchus and Hypopomus), but acknowledged that this character also occurs in Steatogenys and some sternopygid genera. Sullivan et al. (2013) revisited this character and considered it to be a "single possible synapomorphy" for Brachyhypopomus - contrasting with the condition in most other Rhamphichthyoidea, in which the "maxilla is straight to slightly curved, and the medial and upper portions of the upper jaw form a continuous curve with little to no inflection point, viewed externally". 
We found that the curvature of the area of the jaws in which the premaxilla and maxilla join is highly variable, and is an unreliable means to distinguish individuals of all 28 species of Brachyhypopomus from individuals of Microsternarchus bilineatus (and from specimens of additional undescribed species of Microsternarchus). For example, Brachyhypopomus batesi, B. benjamini, and $B$. provenzanoi are indistinguishable from Microsternarchus in jaw structure, viewed externally.

Description. Members of the genus exhibit relatively little variation in body and head shape, and are generally delimited by a combination of morphometric and meristic characters. For brevity, we describe here external characters common to all congeners rather than repeating these in each of the species descriptions and redescriptions presented below.

Maximum known body size $461 \mathrm{~mm}$ TL (see redescription of $B$. brevirostris). Body elongate and laterally compressed (body width as percentage of body depth 36.4$82.1 \%$ and $18.9-51.7 \%$ at anal-fin origin and anal-fin middle respectively), reaching maximum width and depth in posterior region of body cavity and tapering gently posteriorly; short to very long caudal filament located posterior to the analfin terminus $(4.2-54.5 \%$ TL; 7.4-83.1\% LEA in intact specimens) - comprising mostly electrocytes, vertebral centra or a cartilaginous rod-like structure, and associated blood vessels and nerves (Albert, 2001). Greatest body depth and width located in area of body cavity or slightly posterior to body cavity, lateral sides of body more or less straight in dorsal view posterior to abdominal cavity. Dorsal profile of body straight or nearly straight in lateral view.

Single hypaxial electric organ (EO) extending just posterior to the urogenital orifice to the end (or near end) of the caudal filament; 1-6 bilateral horizontal (length way) columns of electrocytes along its length, 3-6 columns at analfin terminus, and 2-6 columns at a midpoint between analfin terminus and end of caudal filament. Paired subdermal accessory EO of unknown function in the opercular region are known from three species: $B$. bombilla, B. menezesi, and B. regani.

Anal fin elongate (71.6-94.0\% LEA) with origin located in anterior portion of body cavity, posterior to cleithrum of pectoral girdle; 143 to 293 rays (branched and unbranched combined). Each anal-fin ray connected via a ball-and-socket joint to a proximal anal-fin pterygiophore, and articulated in circular movements by the pinnalis analis muscles (Ellis, 1913). Anal-fin pterygiophores shorter than hemal spines at midbody.

Pectoral fin short to moderate (longest pectoral ray 3.2$8.5 \%$ LEA), broad and distally rounded or pointed, with 10-21 rays (branched and unbranched combined). Dorsal, adipose, and pelvic fins, and pelvic girdle absent, in common with all gymnotiforms. Caudal fin absent in common with all Rhamphichthyoidea, Sternopygidae, and Gymnotidae -although see de Santana et al. (2013) for comments on the caudal skeleton of Electrophorus.
Body cavity restricted to no more than 2.5 head-lengths posterior to occiput, with viscera rotated so that the anus and urogenital orifice are positioned below the operculum, near the isthmus. Anus and urogenital orifice in close juxtaposition. Urogenital papilla developed into elevated tube in breeding males and females. Transparent and thinwalled non-vascularized and non-physostomous swim bladder, comprising a smaller anterior chamber, and a larger posterior chamber (Ellis, 1913: 189, fig. 32). Body cavity short, with 15-26 precaudal vertebrae (including up to 3 transitional vertebrae). 1-3 slender displaced hemal spines embedded in hypaxial musculature posterior to body cavity, sometimes forked with bifurcated portions facing anteriorly.

Scales cycloid, with irregular ovoid shape; smaller dorsally and ventrally and larger laterally, especially in posterior region of body; partially or completely covered with skin and distributed over all of post-cranial portion of body except fins (exceptions are the absence of scales along the middorsal region of anterior third of body and over the analfin pterygiophores in B. benjamini and B. provenzanoi). 4-9 rows of lateral line scales above lateral line to dorsal midline at midbody. Anterior-most perforated lateral line scale located near vertical through pectoral-fin origin. Lateral line continuous in most species anterior to anal-fin terminus, but not continuing to end of caudal filament; usually terminating in the middle portion of the caudal filament. Dorsal branch of posterior lateral line nerve separate from recurrent ramus of anteroventral lateral line nerve (Albert, 2001: character 117 therein). No fleshy dorsal electroreceptive organ (as in the Apteronotidae). Body variably pigmented with dark bands and mottled patterns of stellate chromatophores and subcutaneous pigment, but never translucent as in several sternopygid taxa, notably many Eigenmannia and Rhabdolichops species (Crampton, 2007). Chromatophores contract at night resulting in a striking pale appearance of most Brachyhypopomus under artificial illumination; even those that are very darkly pigmented during the day. Ampullary electroreceptors in rosettes, with highest density on snout and around mouth. Schreckstoff club cells and observable fright response absent (Fink \& Fink, 1981: character 117 therein). Head widest in opercular region and deepest in occipital region. Eye small to relatively large in size $(5.9-20.6 \% \mathrm{HL})$, laterally positioned on dorsal half of head, visible in dorsal view, and completely covered by thin translucent epidermis (orbital margin not free). Mouth small and terminal or slightly to moderately sub-terminal; closed lips meet ventral to a horizontal through ventral margin of eye. 3-7 small, needle-like premaxillary teeth present in all examined small juveniles ( $<50 \mathrm{~mm}$ TL) but absent in adults except in B. bennetti and B. walteri (Sullivan et al., 2013). Dentary edentate in adults, although small conical dentary teeth present in all examined small juveniles ( $<50 \mathrm{~mm}$ TL). Maxilla edentate. Premaxillary and maxillary portions of upper jaw gently convex anteriorly, joining to form a slight concave angle or moderate to acute sigmoidal profile to upper jaw. Anterior and posterior nares present; both either 
ellipsoid or circular in shape. Anterior nares located at upper lip. Posterior nares located near eye (1.1-10.4\% HL from anterior margin of orbit). Dorsal and ventral profile of head from almost straight to strongly convex. Snout short $(18.7-32.6 \% \mathrm{HL})$ and rounded. Cephalic lateral line canals and pores complete in adults, with pores conspicuous (de Santana \& Crampton, 2011: 1104, fig. 2) - with the following two exceptions: i. The antorbital is absent in $B$. bennetti and $B$. walteri; ii. The branch of the infraorbital canal over the frontal is absent in B. alberti, B. arrayae, B. belindae, $B$. hamiltoni, and $B$. verdii. Branchial opening restricted to a short vertical aperture at posterior margin of opercle, branchial membranes joined at isthmus. 25-63 gill filaments on first arch. Sexual dimorphism of cranial morphology absent in all species in contrast to its presence in many apteronotid taxa (Albert \& Crampton, 2009; de Santana \& Vari, 2010; Fernandes et al., 2002) and in the sternopygid genus Archolaemus (Vari et al., 2012).

Functional biology and ecology. Specializations associated with the Electrogenic and Electrosensory system: As with other Gymnotiformes, Brachyhypopomus species exhibit a suite of morphological specializations associated with active electroreception and nocturnal activity. The eyes are reduced in size and retinal projections to the brain are much reduced (Lázár et al., 1987). A culteriform (knife-shaped) body plan facilitates maximum dipole separation of the fish as a dipole source (and therefore maximizes field strength per unit body mass, Stoddard et al., 1999), and optimizes use of the body surface as a relatively rigid electroreceptive array. Locomotion by undulation of the elongated anal-fin permits backwards-forwards electroreceptive rostral probing ('scan swimming' sensu Julian et al., 2003) (Albert \& Crampton, 2005; Lannoo \& Lannoo, 1993; Lissmann, 1961; Nanjappa et al., 2000; Stoddard et al., 1999) (in addition to anal-fin locomotion, Brachyhypopomus also swim in rapid bursts by anguilliform movement of the body when startled, Ellis, 1913). Body rigidity is augmented by ossified intermuscular bones (Schlesinger, 1910). Most of the post-cranial body and caudal filament is specialized for electrogenesis, with the body cavity confined to an area extending no more than 2.5 head-lengths posterior to the occiput (Ellis, 1913: 189, fig. 32).

Electric Organs: The hypaxial electric organ (EO) comprises myogenic electrocytes (derived from muscle cells). Studies of B. gauderio demonstrate that the EO develops from the ventral hypaxial musculature in larvae, and is retained through development (Franchina, 1997). Initially the EO comprises cylindrical, overlapping stalk-less electrocytes, but these later shorten along the rostrocaudal axis, separate into vertically aligned "rows", and form stalklike caudal projections onto which the spinal electromotor neurons terminate (Bass, 1986: 16, fig. 1; Franchina, 1997: 115-117, figs. 7-10). EODs are first generated six days after hatching, at $7 \mathrm{~mm}$ TL in B. gauderio (Franchina, 1997), and at 6-8 $\mathrm{mm}$ in B. beebei (Westby, 1988). Beyond the late larval stage (TL $>37 \mathrm{~mm}$ ), the $\mathrm{EO}$ of $B$. gauderio extends parallel to the body axis along the entire ventrolateral margin, from near the gill isthmus to the tip of the caudal filament (although in some species the distal portions of the caudal filament are sometimes free of electrocytes, especially in breeding males; see descriptions and redescriptions herein).

The adult EO of Brachyhypopomus comprises 3-6 bilateral longitudinal (horizontal) columns of cylindrical or box-shaped electrocytes bound by connective sheaths (Bass, 1986; Bennett, 1961; 1971a; Franchina, 1997; Hopkins et al., 1990; Stoddard et al., 1999). An opercular accessory electric organ distinct from the main hypaxial EO was first reported by Sullivan (1997) for B. regani (listed as B. electropomus), and has also been discussed by Crampton \& Albert (2006) and Carvalho (2013). Caputi (1999) and Caputi et al. (1998) review how the synchronized activity of electrocytes in the EO of B. gauderio - combined with the filtering properties of the skin and tissue of the fish, and the load of the surrounding water - determine in ensemble the spatiotemporally complex "near field" discharge that sums to the head-to-tail recorded EOD recorded in the far field.

Impedance matching: Impedance matching of electric organ anatomy to narrow ranges of conductivity has been noted in several species (Crampton, 1998a; Hopkins, 1999). Brachyhypopomus bullocki and B. brevirostris, which are restricted to low conductivity systems $\left(<30 \mu \mathrm{Scm}^{-1}\right)$ exhibit a predominantly serial arrangement of electrocytes in the caudal portion of the organ (longer caudal filaments comprising relatively few horizontal bilateral columns of electrocytes). In contrast, $B$. bennetti, $B$. diazi, and $B$. occidentalis (and also B. palenque, see description herein), which are restricted to high conductivity systems $(>60$ $\left.\mu \mathrm{Scm}^{-1}\right)$, exhibit a parallel arrangement of electrocytes (short caudal filaments with more electrocyte columns). The possibility that conductivity may serve as a barrier to the dispersal of some species, and consequently play a role in reproductive isolation, has been suggested by Crampton (1998a; 2011) and Hopkins (1999).

Electric Organ Discharges: Crampton \& Albert (2006) reviewed the diversity of head-to-tail recorded EODs in Brachyhypopomus. Of 18 species for which EOD data have been presented, 12 generate biphasic or nearly biphasic EODs with durations varying from $c a$. $0.5-5 \mathrm{~ms}$. One species, B. bennetti, generates a monophasic EOD. Five species generate relatively short $(0.5-1.5 \mathrm{~ms})$, more complex triphasic or tetraphasic EODs (Crampton \& Albert, 2006). Monophasic larval EODs have been documented in $B$. beebei, B. brevirostris, B. gauderio, and B. occidentalis, and this is presumed to be the case in other congeners, as in all other gymnotiforms (Crampton \& Albert, 2006). The EOD pulse rate of Brachyhypopomus varies from $c a$. 2 to $110 \mathrm{~Hz}$, and typically increases from a lower resting day-time rate to a higher nocturnal active rate (Crampton \& Albert, 2006). Assad et al. (1999), and Stoddard et al. (1999) map heterogeneity of the near-field recorded EOD of $B$. beebei, B. gauderio, and B. walteri. 
There is a rich and rapidly growing literature on the behavior, neuroethology, physiology, energetics, and hormonal basis of EOD generation and EOD plasticity in Brachyhypopomus - focused primarily on the model species B. gauderio, which is reviewed in part by Assad et al. (1999, 1998), Stoddard (2006), Gavassa et al. (2013), Markham (2013), Silva et al. (2013), and Salazar et al. (2013).

Electroreceptors: In common with all other gymnotiforms, Brachyhypopomus possess a cutaneous array of ampullary and tuberous electroreceptors. Ampullary electroreceptors are tuned to low frequencies and facilitate passive electroreception of weak, lowfrequency bioelectric fields. Tuberous electroreceptors are typically tuned to higher frequencies and facilitate both the active electrolocation of objects, and the passive detection of electric fields from other fish - an important component of electrocommunication (Hopkins, 2005; Hopkins et al., 1997). The morphology of ampullary and tuberous electroreceptors in Brachyhypopomus, their distribution over the body surface, and their directional sensitivity are among the best known of all gymnotiforms, having been described by Bullock et al. (1961), Hagiwara et al. (1962), Szamier \& Wachtel (1970), Bennett (1971b), Szabo (1974), Yager \& Hopkins (1993), and McKibben et al. (1993). Tuberous electroreceptor frequency responses in the genus, showing a correspondence of electroreceptor tuning with peak frequency characteristics of the EOD, are described by Bastian $(1976 ; 1977)$ and Hopkins \& Heiligenberg (1978).

Groove-like depigmented epidermal canals leading to tuberous electroreceptors, and which therefore may play a role in active electroreception are known from all members of the genus. Sullivan (1997) noted for B. diazi and $B$. occidentalis that "Microscopic examination and histological sections of these canals show them to be narrow tubes overlying one or more scales with an external pore anteriorly and a tuberous electroreceptor at the posterior end (J. Sullivan and C. Hopkins unpubl.)." The epidermal canals of Brachyhypopomus vary in abundance between species (see species descriptions and redescriptions herein) but are mostly located in the posterior half of the body - where they are restricted to nearby and either side of the lateral line, either side of the dorsal midline, and approximately midway between the lateral line and dorsal midline. In $B$. diazi the epidermal canals cover a larger area of the body than in congeners, including much of the dorsal and ventral flank of the anterior half of the body. Groovelike epidermal canals are also observable in all other hypopomid genera sensu Maldonado-Ocampo (2014), including Akawaio, Hypopomus, Procerusternarchus, and (in considerably lower densities), in Microsternarchus and Racenisia.

Auditory sensory apparatus: In addition to providing buoyancy, the swim bladder of Brachyhypopomus is involved in the ostariophysan hearing system, which involves a connection from the anterior chamber of the swim bladder to the stato-acoustic organ of the inner ear via the Weberian ossicles encased by the first four vertebrae. A pseudotympanum, a window-like thinning of the hypaxial muscles lateral to the anterior portion of the swim bladder is present, and comprises three distinct hiatuses in the obliquus inferioris and obliquus superioris muscles (Dutra et al., 2015). The pseudotympanum may enhance sound detection by acting as a window through which sound waves reach the anterior chamber of the swim bladder (Dutra et al., 2015).

Adaptations to hypoxia: The influence of dissolved oxygen on the distributions of Brachyhypopomus is discussed by Crampton $(1998 b ; 2011 ; 2008)$ and Crampton $\&$ Albert (2006). Species that occur in seasonally or perennially dysoxic habitats such as whitewater floodplains and terra firme swamps are adapted to survive protracted periods of hypoxia $\left(<1 \mathrm{mgl}^{-1}\right)$ or complete anoxia, while those that occur in permanently normoxic habitats, such as terra firme streams, are not (Crampton, 1998a,b). Some species are able to tolerate hypoxia by undertaking aerial gill respiration - either by periodically aspirating air bubbles into their gill chambers, or by opening their mouths at the surface meniscus to expose the gill lamellae to air (Crampton, 1998b; Hopkins, 1991). Carter \& Beadle (1931) reported an expanded gill chamber, unusually long gill lamellae, and greatly expanded secondary folds of the gill lamellae in B. "brevirostris" (probably B. gauderio) from the Paraguayan Chaco, and also concluded that the air bladder and gill chamber epithelium play no significant role in air breathing. Crampton et al. (2008) reported that species endemic to seasonally anoxic whitewater floodplain habitats exhibit significantly larger gills than species endemic to permanently normoxic terra firme stream systems. Many species of Brachyhypopomus also exhibit a reduction of activity and EOD pulse rate in response to declining oxygen levels (Crampton, 1998b).

Regeneration: Brachyhypopomus species, as in other Rhamphichthyoidea and Sternopygidae, are able to regenerate the entire post-coelomic portion of the body following damage from predators, with the vertebrae replaced by a rigid cartilaginous rod (Albert, 2001; Albert \& Crampton, 2005; Ellis, 1913; Sullivan et al., 2013). The proportion of individuals with caudal filament damage in natural populations has been estimated for three species: $B$. brevirostris $-8 \%$ (Ellis, 1913); B. draco-6.7\%, with $22.1 \%$ exhibiting regeneration from earlier damage (Cognato et al., 2007); B. occidentalis - varying among populations from $12 \%$ to $46 \%$ (Dunlap et al., 2016). Hopkins et al. (1990) and Sullivan et al. (2013) documented how damage to portions of the body posterior to the body cavity can influence the EOD waveform.

Reproductive anatomy: In $B$. gauderio, the males develop a paired, lobular testis of the unrestricted spermatogonial category, and females develop a paired saccular cystovary. In both sexes the gonads are positioned 
ventrally, and expand posteriorly until they reach the posterior wall of the abdominal wall (Quintana et al., 2004). Gonadal morphology appears to be similar in other species. França et al. (2007) and Giora \& Burns (2011) describe the ultrastructure of spermatozoa in Brachyhypopomus.

Reproductive biology and life history: Detailed accounts of the reproductive ecology and life history of Brachyhypopomus are available for $B$. occidentalis from Panama (Hagedorn, 1986; 1988), and for three species from southern subtropical systems: $B$. bombilla (Giora et al., 2011), B. draco (Schaan et al., 2009), and B. gauderio (Giora et al., 2014; Miranda et al., 2008; Quintana et al., 2004; Silva et al., 2007; Silva et al., 2002; Silva et al., 2008) (and see species descriptions herein). The reproductive ecology of Amazon and Orinoco species is largely undocumented, although Alves-Gomes (1997) noted spawning preceding seasonal flooding in the rio Branco system of Roraima, Brazil, and Crampton (1996a) observed spawning coincident with the rising-water period and high water period in species from Amazonian floodplains.

At extreme southern latitudes Brachyhypopomus exhibit several adaptations to the austral winter - including gonadal quiescence, a general reduction in activity and feeding, and an accompanying reduction of the EOD pulse-rate (Giora et al., 2011; 2014; Schaan et al., 2009; Silva et al., 2002). Some species of Brachyhypopomus appear to live for only one year, with mortality following a terminal reproductive effort - including $B$. bennetti (Crampton, 1996a) (listed therein as ' $B$. sp. 3'), $B$. gauderio (Silva et al., 2003), B. occidentalis (Hagedorn, 1988), and B. pinnicaudatus (Kirschbaum \& Schugardt, 2002). Fractional spawning has been observed in $B$. bombilla (Giora et al., 2011), B. brevirostris (Kirschbaum et al., 2008), B. draco (Schaan et al., 2009), B. gauderio (Giora et al., 2014; Miranda et al., 2008), B. occidentalis (Hagedorn, 1988), and B. pinnicaudatus (Kirschbaum et al., 2008). Kirschbaum \& Schugardt (2002) reported egg diameters of approximately $1.7 \mathrm{~mm}$ in $B$. pinnicaudatus, with hatching on day three after spawning, and exogenous feeding beginning on day eight.

Neither nesting nor parental care are known for the genus, including species bred and observed carefully in captivity (pers. comm. P. K. Stoddard, Florida International University, USA, for $B$. gauderio; $\mathrm{F}$. Kirschbaum, Humboldt University, Germany, for $B$. brevirostris and B. pinnicaudatus). Westby (1988) reported small aggregations of larval Brachyhypopomus beebei in a coastal stream of French Guiana but these were apparently not attended by a parent. However, Giora et al. (2014) reported aggregations of larval B. gauderio near single mature males.

Cytogenetics. Chromosomal rearrangements in gymnotiforms are suspected to play a significant role in reproductive isolation (Milhomem et al., 2008;
Nagamachi et al., 2010). Nonetheless, despite many descriptive cytogenetic studies of gymnotiform fishes (Oliveira et al., 2009), Brachyhypopomus remains poorly studied - with karyotypes available for only four species. Brachyhypopomus brevirostris exhibits a diploid number of 36, fundamental number (FN) of 42, and karyotypic formula $4 \mathrm{~m} / 2 \mathrm{sm} / 8 \mathrm{st} / 22 \mathrm{a}$ (Almeida-Toledo, 1987, cited in Cardoso et al., 2011). Brachyhypopomus gauderio exhibits a diploid number of 42 in females (all acrocentric), and 41 for males (40 acrocentric, 1 metacentric), a FN of 42, and a karyotypic formula of $1 \mathrm{~m} / 42 \mathrm{a}$ in females and $1 \mathrm{~m} / 41 \mathrm{a}$ in males (Almeida-Toledo et al., 2000; Mendes et al., 2012). Brachyhypopomus pinnicaudatus exhibits a diploid number of 42 in females (all acrocentric) and 41 in males (40 acrocentric, 1 metacentric), and a FN of 42 , as is the case in B. gauderio, and exhibits a karyotypic formula of 42 st-a in females and $1 \mathrm{~m}-\mathrm{sm} / 40$ st-a in males (Cardoso et al., 2015). Brachyhypopomus flavipomus (listed as B. n. sp. FLAV) exhibits a diploid number of 44 in females and 43 in male and a karyotypic formula of 44st-a in females and 1m-sm/42st-a in males (Cardoso et al., 2015). Cardoso et al. (2011) highlighted the conservancy of the diploid number in the Steatogeni and Rhamphichthys (invariably $2 \mathrm{n}=50$ ), in contrast to its variability among Brachyhypopomus spp. $(2 \mathrm{n}=36,41-42$, or $43-44)$ and Hypopomus artedi $(2 \mathrm{n}=38)$, and noted that this pattern is consistent with the phylogenetic scheme of Alves-Gomes et al. (1995) (and also Carvalho, 2013; MaldonadoOcampo et al., 2014; Tagliacollo et al., 2016), in which the Steatogeni is placed within the Rhamphichthyidae.

Three of the four Brachyhypopomus with cytogenetic analyses exhibit morphologically differentiated sex chromosomes. Brachyhypopomus flavipomus (Cardoso et al., 2015), B. gauderio (Almeida-Toledo et al., 2000; Mendes et al., 2012), and B. pinnicaudatus (Cardoso et al., 2015) all exhibit an unusual multiple sex chromosome system of the $\mathrm{X}_{1} \mathrm{X}_{1} \mathrm{X}_{2} \mathrm{X}_{2}: \mathrm{X}_{1} \mathrm{X}_{2} \mathrm{Y}$ type. In contrast, $B$. brevirostris is chromosomally homomorphic, and sex determination mechanisms are unknown in this species (Cardoso et al., 2011).

Taxonomic remarks. Here we provide comments on the early assignment of species now listed as Brachyhypopomus to the genus Hypopomus. Previous to the description of B. brevirostris (as Rhamphichthys brevirostris, see Introduction), the genus Rhamphichthys was erected by Kaup (1856) to include two relatively short-snouted species - $R$. artedi (now Hypopomus artedi) and $R$. mulleri - now recognized as a junior synonym of Hypopomus artedi (Albert \& Crampton, 2003), and also five long-snouted species - now recognized as Rhamphichthys spp.

In a brief address to the Academy of Natural Sciences of Philadelphia, Gill (1864) proposed a new genus Hyроротиs to accommodate the relatively shortsnouted Rhamphichthys mulleri, and separate this from 
the long-snouted species now assigned to the genus Rhamphichthys. Nonetheless, Gill's proposal was overlooked until 1903. For instance, Günther's (1870) catalog of fishes in the British Museum instead divided the genus Rhamphichthys into two subgenera. The first subgenus, Rhamphichthys (Brachyrhamphichthys), diagnosed by "snout not tubiform; vent behind the eyes; anal fin commencing below the pectorals", corresponds to the modern genera Hyроротиs and Brachyhypopomus (at the time this comprised $H$. artedi and its now junior synonym $H$. mulleri, and B. brevirostris). The second subgenus Rhamphichthys (Rhamphichthys), diagnosed by "snout produced into a tube; vent below or in advance of the eyes; anal fin commencing at the throat", corresponded to the modern genus Rhamphichthys. With respect to Brachyhypopomus brevirostris, Günther's (1870) scheme was followed by Steindachner (1880) and Eigenmann \& Eigenmann (1891), and later modified by Eigenmann (1894), who listed Rhamphichthys (Brachyrhamphichthys) brevirostris as simply Brachyrhamphichthys brevirostris.

The first authors to abandon Günther's (1870) scheme for short-snouted Rhamphichthyoidea, and instead adopt Gill's (1864) proposed genus Hyроротиs, which takes taxonomic priority, were Eigenmann \& Kennedy (1903) for "Hypopomus brevirostris" (referring in fact to $B$. gauderio, see synonymy section for $B$. gauderio), and later Eigenmann \& Ward (1905) for B. brevirostris. Only in 1914, with Regan's description of Brachyhypopomus occidentalis was a second species added to Hyророти sensu Gill (1864). Thereafter, the name Hyроротиs was used consistently until Mago-Leccia (1994) divided Gill's Hypopomus into Hypopomus and Brachyhypopomus (see Introduction).

Keys to species. Here we exclude the following regions represented by only one species: Caribbean drainages of northern Venezuela (B. diazi); rio São Francisco ( $B$. menezesi); Atlantic and Pacific drainages of southern Costa Rica and Panama to Darién, and Maracaibo, Magdalena, Sinú and Atrato (B. occidentalis); Pacific drainages of Ecuador (B. palenque).

Key to the species of Brachyhypopomus occurring in the río Orinoco, Essequibo River, coastal drainages of the Guianas, and the rio Negro, including the rio Branco

1A. Absence of accessory electric organ (AEO) over opercular region . .2

1B. Presence of AEO over opercular region......... B. regani (lower and middle Orinoco, upper Essequibo, some coastal drainages of Guianas)

2A. Presence of prominent pale uninterrupted middorsal stripe from occipital region to base of caudal filament ..................3

2B.Absence of pale prominent pale uninterrupted middorsal stripe from occipital region to base of caudal filament..... 4

3A. Anal-fin rays $214-230$ B. beebei (upper and lower Orinoco, Essequibo, coastal drainages of the Guianas, Negro including Branco).

3B. Anal-fin rays 176-196 B. pinnicaudatus

(small drainages of coastal French Guiana)

4A. Presence of scales in entire middorsal region ............... 5

4B. Absence of scales in middorsal region of anterior third of body..... B. provenzanoi (upper Orinoco, upper Negro)

5A. Presence of dark suborbital stripe 6

5B.Absence of dark suborbital stripe. 7

6A.Presence of horizontal dark-pigmented band along entire anal-fin base, over distal anal-fin pterygiophores

B. hendersoni (lower Negro, Essequibo)

6B. Absence of horizontal dark-pigmented band along analfin base. B. walteri (middle and lower Negro, Essequibo).

7A. Absence of series of diffuse horizontal dash-like dark markings along lateral line in posterior third of body, anterior to anal-fin terminus ........................................... 8

7B. Presence of series of diffuse horizontal dash-like dark markings along lateral line in posterior third of body, anterior to anal-fin terminus .......................... B. hamiltoni (middle Negro)

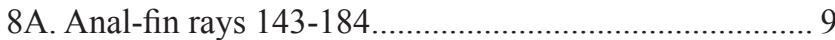

8B.Anal-fin rays 190-293 ................................................ 10

9A. Presence of prominent dark flecks on flank ............ B. sullivani (upper, middle and lower rio Negro, Essequibo, central Orinoco)

9B. Absence of prominent dark flecks on flank ...... B. batesi (upper Negro)

10A. Absence of prominent wide brown saddles on flank, or saddles if present pale and restricted to dorsal region, not extending undisrupted across lateral line .................... 11

10B.Presence of prominent wide brown saddles on flanks, which extend undisrupted across lateral line

B. brevirostris (upper, central, and lower Orinoco, Essequibo, and coastal drainages of the Guianas).

11A. Orbital diameter 14.2-18.5\% HL................ bu bullocki (upper, middle and lower Orinoco, Negro including upper Branco, upper and middle Essequibo)

11B. Orbital diameter 9.6-13.0\% HL......................... diazi (middle Orinoco in Llanos region, lower Orinoco)

Key to the species of Brachyhypopomus occurring in the upper Amazon, central Amazon, lower Amazon, and upper Madeira

1A. Absence of accessory electric organ (AEO) over opercular region........................................................ 2

1B. Presence of AEO over opercular region ....................... 3

2A. Presence of prominent pale uninterrupted middorsal stripe from occipital region to base of caudal filament............... 4

2B.Absence of pale prominent pale uninterrupted middorsal stripe from occipital region to base of caudal filament..... 8

3A. Dorsal surface dorsal surface speckled with small brown chromatophores on light brown background; opercular 
accessory electric organ overlaid with dense scattering of chromatophores............. B. bombilla (upper Madeira)

3B. Dorsal surface with large dark blotches on pale background; opercular accessory electric organ overlaid with depigmented skin

B. regani

(upper, central, and lower Amazon, lower Madeira, upper Purus, Tapajós, Araguaia, lower Tocantins)

4A. Precaudal vertebrae 24-26. 5

4B. Precaudal vertebrae $18-23$

5A. Bilateral columns of electrocytes at anal-fin terminus 3 B. verdii (upper Amazon)

5B.Bilateral columns of electrocytes at anal-fin terminus 4-5 B. belindae (central Amazon)

6A. Anal-fin rays $175-212$. ... 7

6B. Anal-fin rays 214-230 B. beebei (upper, central, lower Amazon, lower Madeira, lower Tocantins)

7A. Pectoral-fin rays 16-19 ....... B. arrayae (upper Madeira)

7B.Pectoral-fin rays 13-15 ........ B. pinnicaudatus (central and lower Amazon, lower, upper Madeira, lower Tocantins, Mearim)

8A. Presence of scales in entire middorsal region . 9

8B.Absence of scales in middorsal region of anterior third of body

B. benjamini (upper Amazon)

9A. Bilateral columns of electrocytes at anal-fin terminus 3-4.

10

9B. Bilateral columns of electrocytes at anal-fin terminus 6.........B. bennetti (upper, central, lower Amazon, upper Madeira, lower Tocantins)

10A. Presence of scattered conspicuous black or charcoal flecks on flank.

10B. Absence of scattered conspicuous black or charcoal flecks on flank......

11A. Presence of horizontal dark band or heavy concentration of dark flecks along entire anal-fin base 12

11B. Absence of horizontal dark band or heavy concentration of dark flecks along entire anal-fin base.........B. sullivani (upper, central, and lower Amazon, upper Madeira, upper Tapajós, lower Tocantins)

12A. Mouth width 24.8-38.0\% HL

B. cunia (upper Madeira)

12B. Mouth width $15.7-22.6 \% \mathrm{HL}$ B. hendersoni (central Amazon)

13A. Absence of dark suborbital stripe 14

13B. Presence of dark suborbital stripe B. walteri

(upper, central, and lower Amazon, upper Madeira, Tapajós, upper Xingu, Araguaia, upper and lower Tocantins).

14A. Anal-fin rays $163-208$. .. 15

14B. Anal-fin rays 226-293. B. brevirostris (upper, central, and lower Amazon, upper Madeira, upper Xingu)

$15 \mathrm{~A}$. Head depth at occiput $69.9-87.9 \% \mathrm{HL}$ 16

15B. Head depth at occiput $61.0-67.9 \% \mathrm{HL}$ B. batesi (central Amazon)

16A.Mouth width 15.9-20.7\% HL, absence of conspicuous patches of shiny yellow guanine on operculum and anterior to pectoral-fin base in live individuals.
16B. Mouth width 21.2-37.8\% HL, presence of conspicuous patches of shiny yellow guanine on operculum and anterior to pectoral-fin base in live individuals.

B. flavipomus

(upper and central Amazon)

17A. Pectoral-fin rays 15-16 (mode 16)

B. alberti (upper Madeira)

17B. Pectoral-fin rays 12-15 (rarely 15$)$, (mode 13 )

B. hamiltoni (upper and central Amazon)

Key to the species of Brachyhypopomus occurring in the Paraná, Paraguay and Uruguay drainages, the PatosMirim lagoon system and nearby Atlantic drainages of Rio Grande do Sul, and coastal drainages of Brazil from states of São Paulo to Rio de Janeiro

1A. Absence of electric accessory organ over opercular region. 2

1B. Presence of electric accessory organ over opercular region. B. bombilla

(lower Paraná, upper, central, and lower Paraguay, Uruguay, Patos-Mirim)

2B. Absence of pale prominent pale uninterrupted middorsal stripe from occipital region to base of caudal filament.... 3

2A. Presence of prominent pale uninterrupted middorsal stripe from occipital region to base of caudal filament... ..................... B. gauderio (lower Paraná, upper, central, and lower Paraguay, Uruguay, Patos-Mirim, Tramandaí)

3A. Absence of dark suborbital stripe... B. walteri

3B. Presence of dark suborbital stripe (lower Paraná, upper Paraguay)

4A. Anal-fin rays $155-223$ 5

4B. Anal-fin rays 226-293 .......B. brevirostris (upper Paraguay)

$5 \mathrm{~A}$. Dorsal rami of the recurrent branch of the anterior lateral line nerve visible

5B.Dorsal rami of the recurrent branch of the anterior lateral line nerve not visible............ B. draco (lower Paraná, central, and lower Paraguay, Uruguay, Patos-Mirim, Tramandaí)

6A. Snout to pectoral-fin base 10.7-12.3\% LEA

B. janeiroensis (São João, Paraíba and small intervening coastal drainages)

6B.Snout to pectoral-fin base 12.3-15.7\% LEA..... B. jureiae (Ribeira de Iguape, Una do Prelado)

\section{Brachyhypopomus alberti, new species}

urn:1sid:zoobank.org:act:4791D07C-1995-453E-99EEA87D60544613

\section{(Fig. 4; Tables 2-6)}

Brachyhypopomus sp. "alb". - Crampton, 2011: 176, table 10.2, species list; 179, figs. 10.2-10.3 (phylogeny, geographical and ecological distributions, gymnotiform biology).

Brachyhypopomus sp. "alberti". - Crampton et al., 2016: 1-66, table 1, 3-4, figs. 1-7, 11, 18-20 (phylogeny, biogeography and ecology of Brachyhypopomus). 


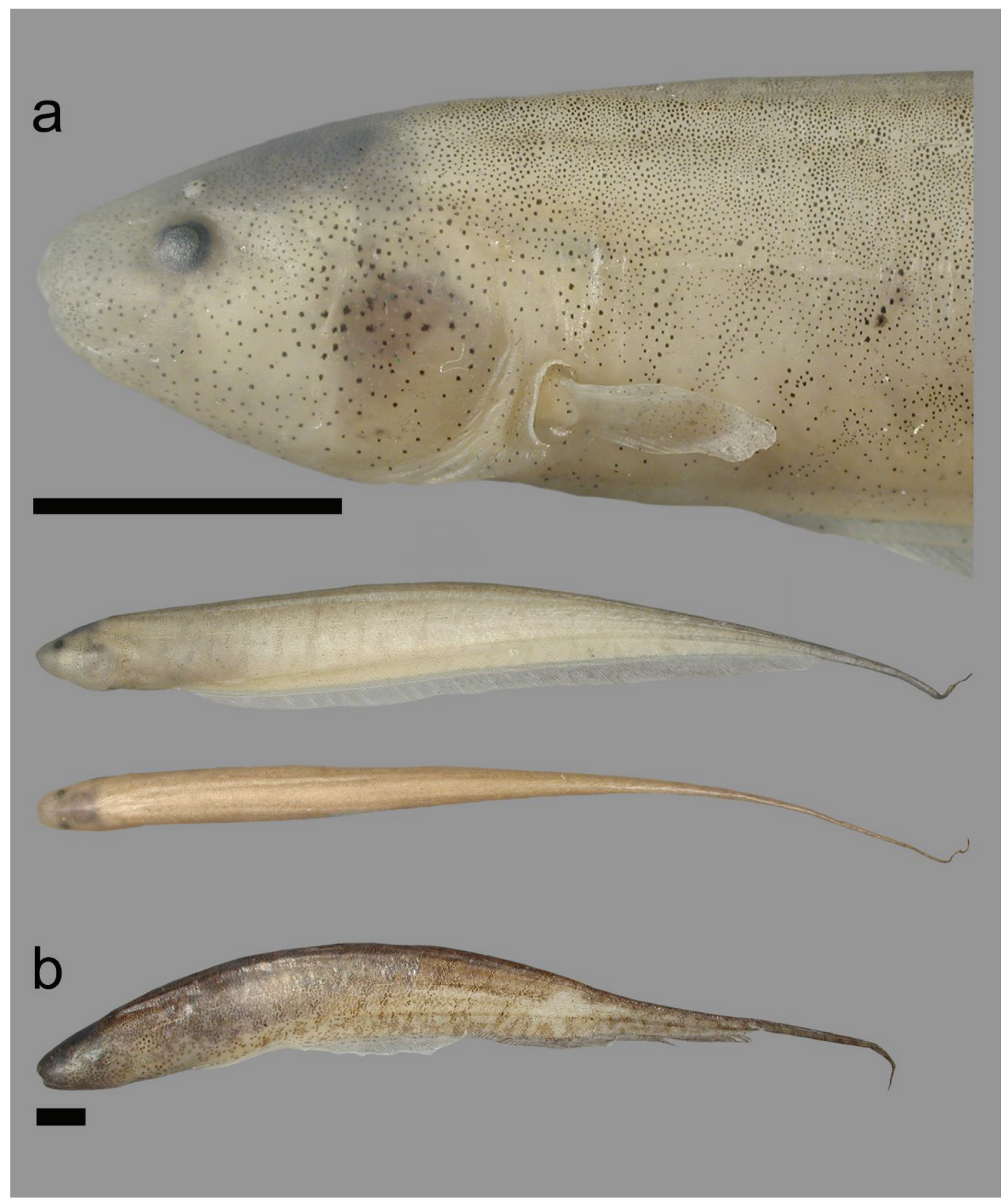

Fig. 4. Brachyhypopomus alberti. a. CBF 10284 (WC24.280607), holotype, male, $97 \mathrm{~mm}$ TL (head in lateral view, and body in lateral and dorsal views); b. ANSP 197573 (WC03.250607), paratype, female, $91 \mathrm{~mm}$ TL (body in lateral view); Bolivia, río Beni, Amazonas dr. Note the sexual dimorphism in body pigmentation, in which females are darker. Specimens fixed in formalin and preserved in EtOH. Scale bars $=5 \mathrm{~mm}$. 


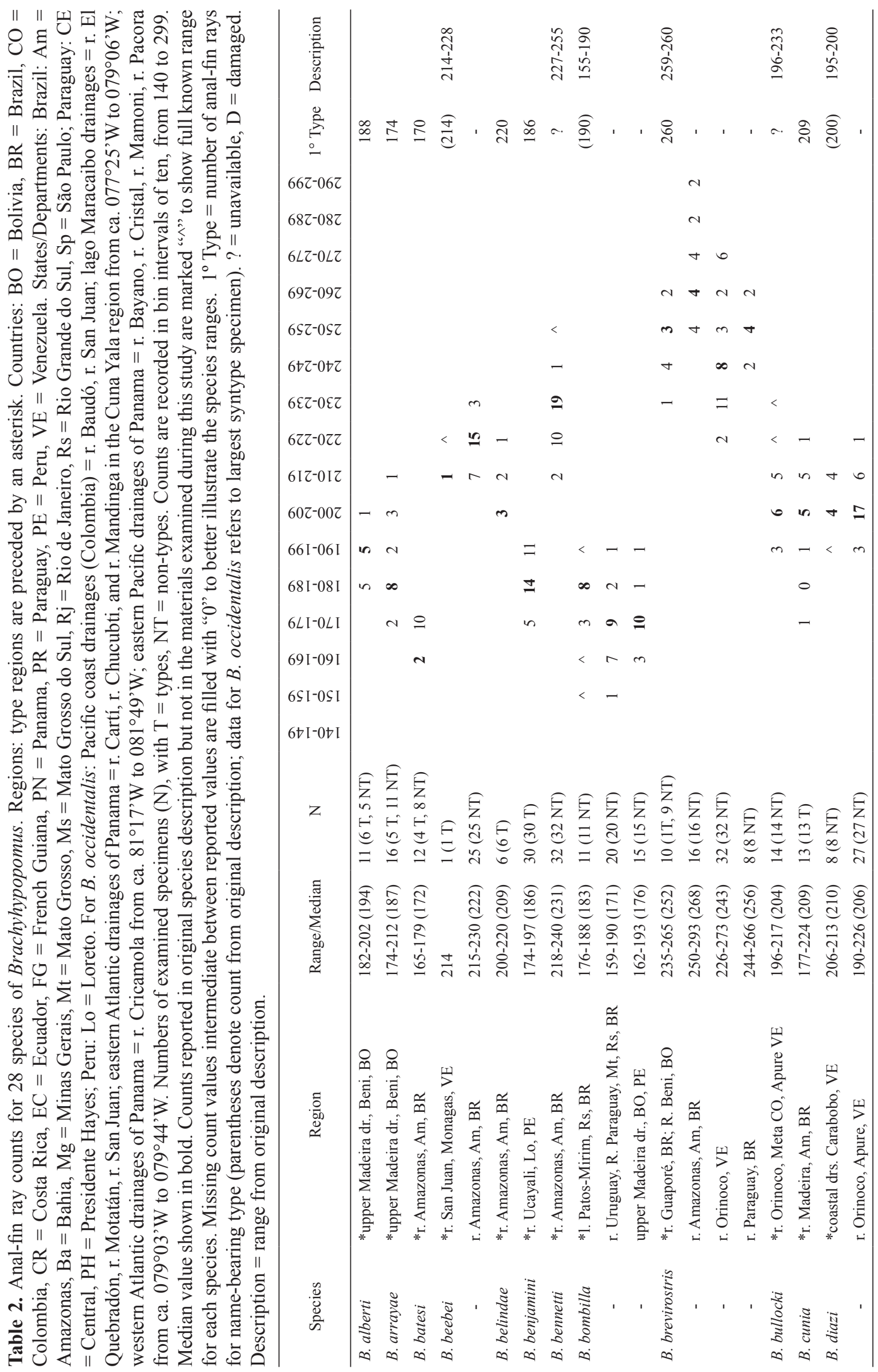




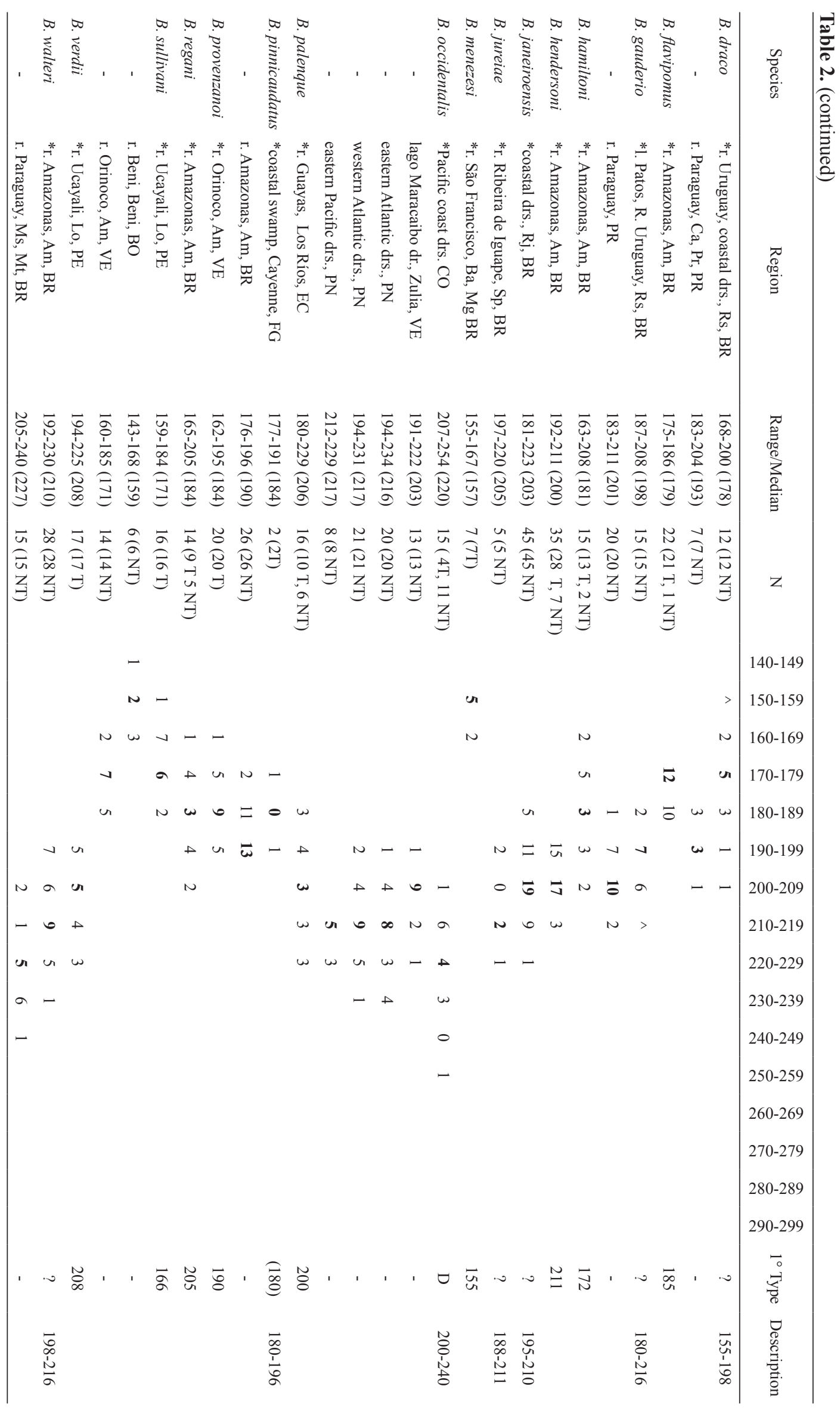


Neotropical Ichthyology, 14(4): e150146, 2016

W. G. R. Crampton, C. D. de Santana, J. C. Waddell \& N. R. Lovejoy

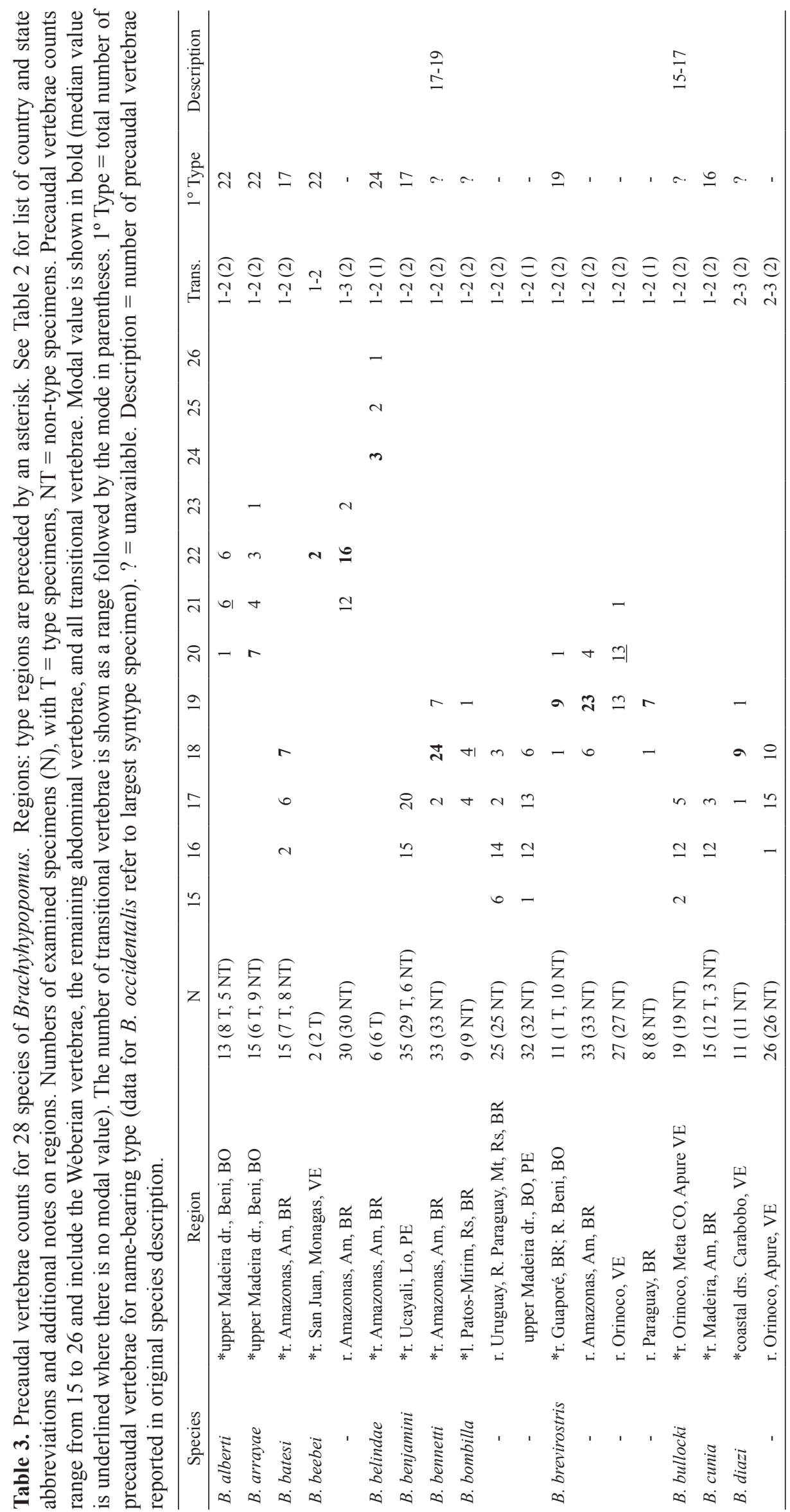




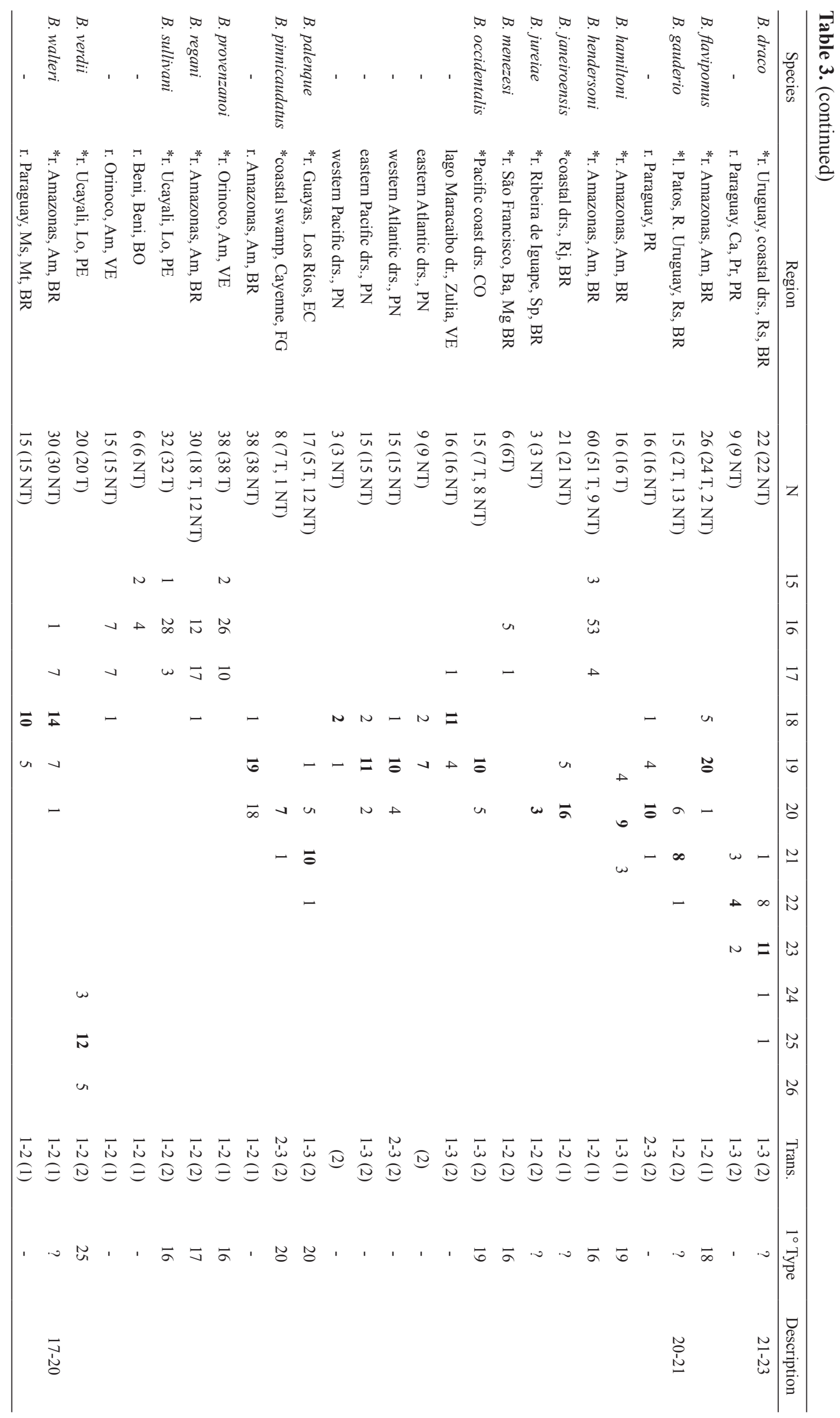




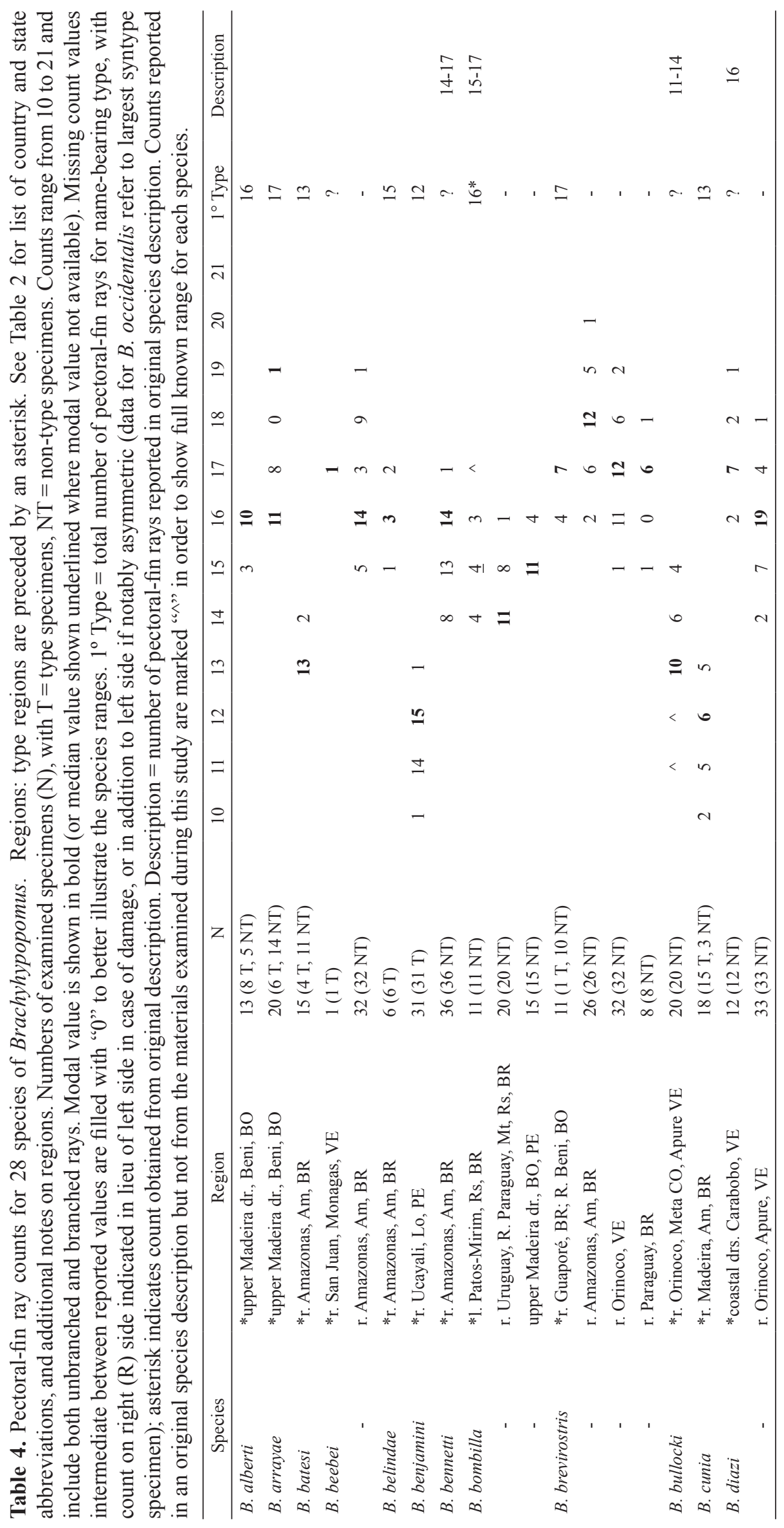




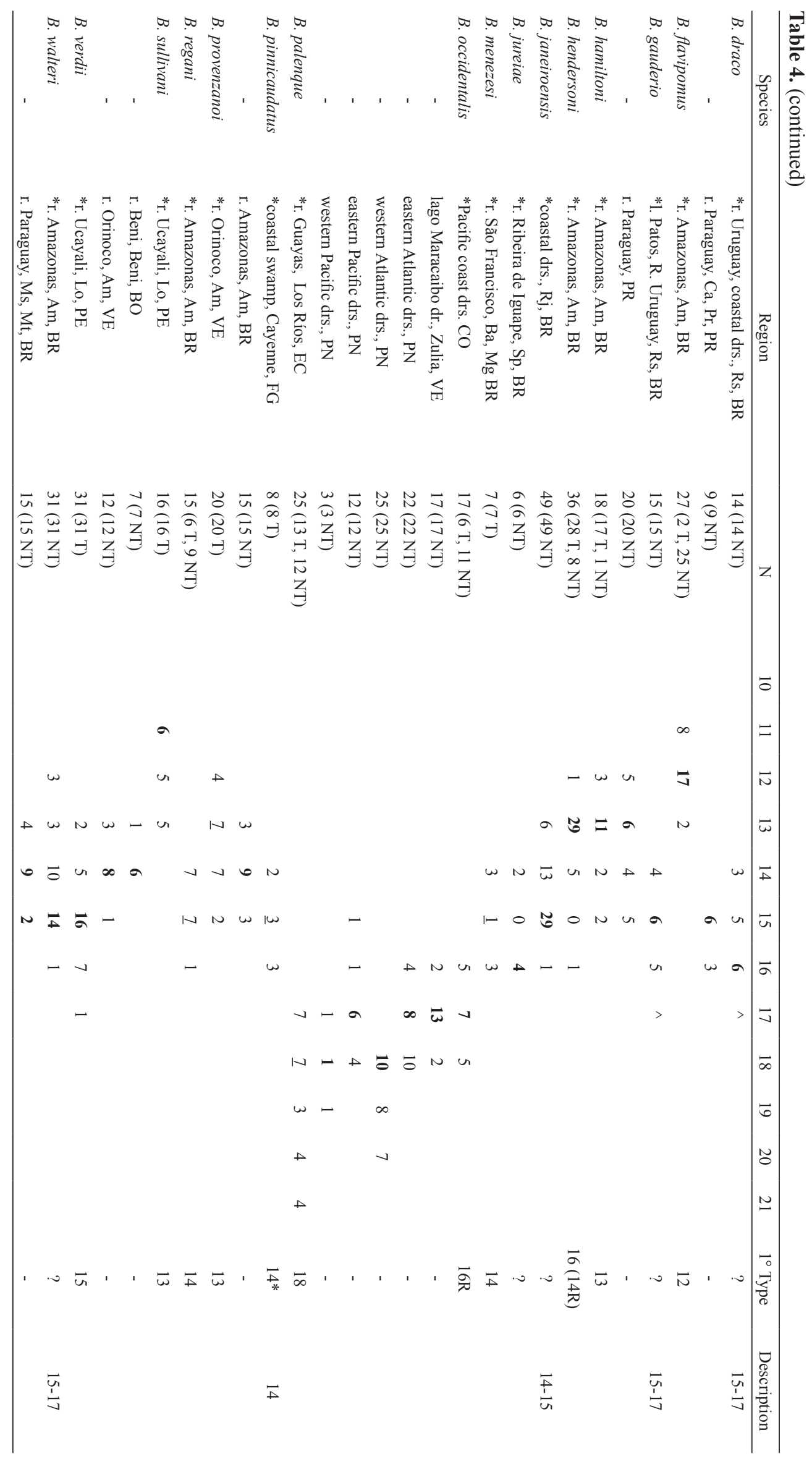




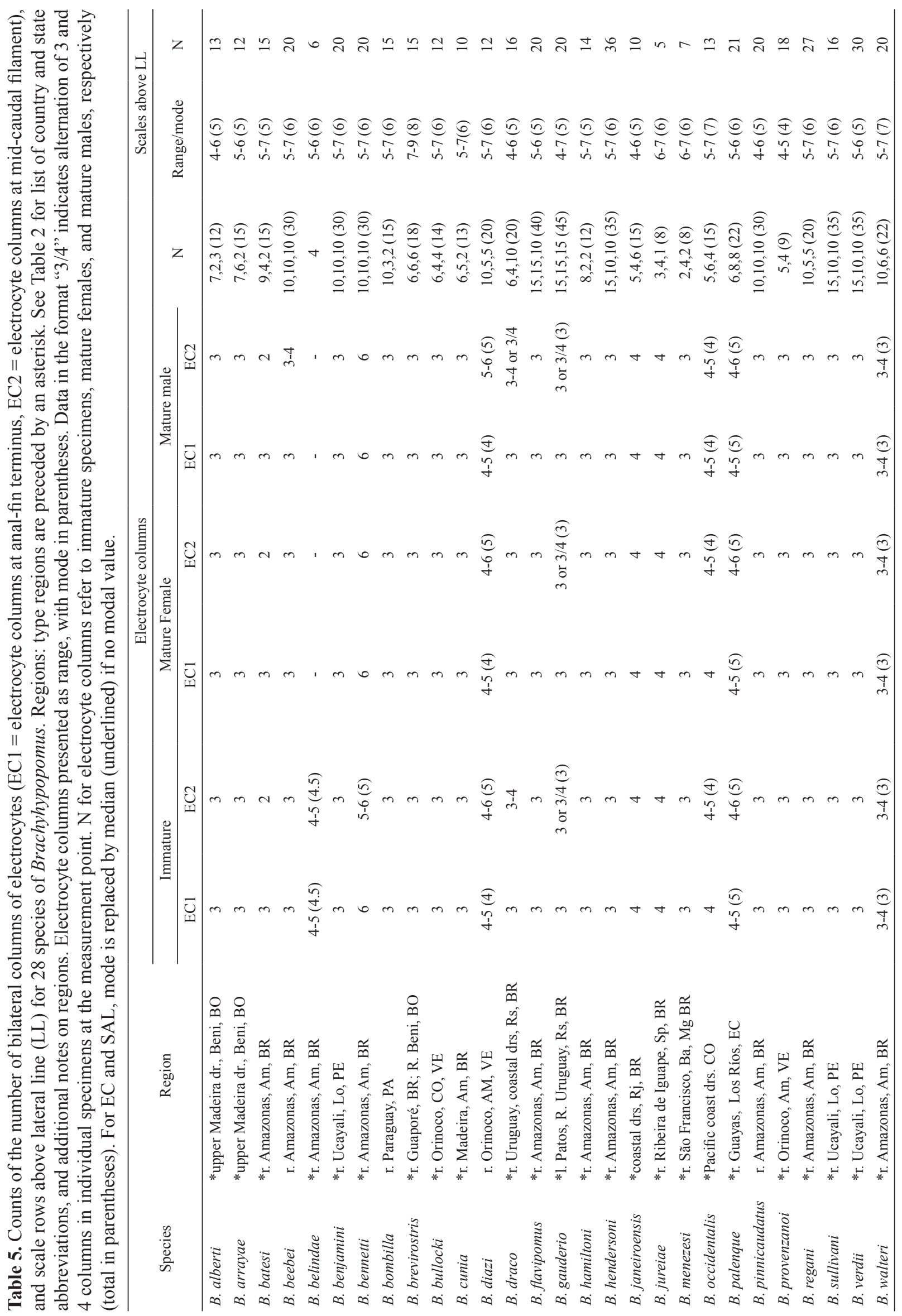


Table 6. Morphometrics for Brachyhypopomus alberti, Brachyhypopomus arrayae, and Brachyhypopomus batesi. HT, holotype; SD, standard deviation. Ranges for each species refer to the holotype and to paratype specimens from the type region.

\begin{tabular}{|c|c|c|c|c|c|c|c|c|c|c|c|c|c|c|c|}
\hline & \multicolumn{5}{|c|}{ B. alberti } & \multicolumn{5}{|c|}{ B. arrayae } & \multicolumn{5}{|c|}{ B. batesi } \\
\hline & HT & Range & Mean & SD & $\mathrm{n}$ & HT & Range & Mean & SD & $\mathrm{n}$ & HT & Range & Mean & SD & $\mathrm{n}$ \\
\hline Total length (TL) (mm) & 97 & $64-111$ & 83 & 17.5 & 10 & 77 & $65-133$ & 94 & 21.3 & 16 & 104 & $73-104$ & 86 & 9.6 & 15 \\
\hline Head length (HL) (mm) & 8.7 & $5.5-9.5$ & 7.8 & 1.2 & 10 & 7.7 & $6.4-10.3$ & 8.0 & 1.3 & 13 & 9.0 & $6.2-9.0$ & 7.6 & 0.9 & 13 \\
\hline \multicolumn{16}{|c|}{ Proportion of TL (\%) } \\
\hline Caudal filament length (CF) & 18.6 & $13.5-23.8$ & 17.7 & 3.3 & 8 & 14.3 & $9.5-24.0$ & 19.6 & 4.3 & 15 & 21.2 & $12.3-21.2$ & 16.2 & 3.7 & 15 \\
\hline Head length (HL) & 11.0 & $10.0-12.2$ & 11.0 & 0.8 & 8 & 11.6 & $10.1-12.1$ & 11.0 & 0.7 & 10 & 11.0 & $10.9-11.8$ & 11.3 & 0.4 & 11 \\
\hline Snout to occiput (SO) & 8.7 & $8.7-9.9$ & 9.5 & 0.5 & 8 & 10.2 & $8.5-10.4$ & 9.3 & 0.7 & 10 & 8.3 & $8.3-10.4$ & 9.5 & 1.1 & 11 \\
\hline Snout to anal-fin origin (SA) & 18.2 & $17.0-21.1$ & 18.4 & 1.4 & 8 & 20.9 & $16.7-23.6$ & 20.0 & 2.4 & 10 & 27.3 & $18.6-27.3$ & 21.7 & 3.8 & 11 \\
\hline Snout to pectoral-fin base (PP) & 12.7 & $11.5-13.6$ & 12.7 & 0.7 & 8 & 12.9 & $11.2-13.5$ & 12.4 & 0.7 & 15 & 12.0 & $12.0-13.1$ & 12.5 & 0.6 & 11 \\
\hline Longest pectoral-fin ray (PRL) & 4.9 & $4.1-5.1$ & 4.7 & 0.4 & 8 & 5.5 & $4.6-5.5$ & 4.9 & 0.2 & 15 & 4.9 & $4.9-5.8$ & 5.3 & 0.4 & 11 \\
\hline Body width at anal-fin origin (BW1) & 6.5 & $5.0-6.5$ & 5.8 & 0.6 & 8 & 6.6 & $5.7-7.4$ & 6.5 & 0.5 & 15 & 4.6 & $4.3-5.3$ & 4.6 & 0.5 & 11 \\
\hline Body depth at anal-fin middle (BD2) & 11.0 & $9.2-11.8$ & 10.5 & 0.9 & 8 & 11.6 & $8.3-12.3$ & 10.0 & 1.1 & 15 & 7.6 & $7.6-8.4$ & 8.1 & 0.3 & 11 \\
\hline Body width at anal-fin middle (BW2) & 4.1 & $3.1-4.3$ & 3.7 & 0.4 & 8 & 3.9 & $2.3-5.1$ & 3.4 & 0.8 & 15 & 2.1 & $2.1-3.2$ & 2.4 & 0.5 & 11 \\
\hline Caudal filament length (CF) & 22.8 & $15.7-31.2$ & 21.6 & 5.1 & 8 & 15.2 & $10.5-31.6$ & 24.8 & 6.3 & 15 & 26.8 & $14.1-26.8$ & 19.6 & 5.4 & 11 \\
\hline \multicolumn{16}{|c|}{ Proportion of HL (\%) } \\
\hline Preorbital distance $(\mathrm{PR})$ & 24.5 & $20.5-30.6$ & 25.2 & 2.6 & 10 & 24.0 & $23.9-27.6$ & 25.5 & 1.4 & 13 & 22.5 & $21.0-27.0$ & 23.4 & 2.5 & 13 \\
\hline Mouth width (MW) & 32.6 & $22.6-36.9$ & 29.2 & 4.5 & 10 & 35.9 & $17.3-37.0$ & 27.7 & 5.9 & 13 & 30.0 & $24.2-30.8$ & 27.8 & 3.1 & 13 \\
\hline Internarial distance (AE) & 24.2 & $20.5-30.1$ & 24.4 & 2.6 & 10 & 23.3 & $19.0-27.2$ & 24.1 & 1.5 & 13 & 20.4 & $19.2-22.6$ & 20.4 & 1.5 & 13 \\
\hline Posterior naris to eye (PE) & 2.5 & $2.5-5.6$ & 3.6 & 0.9 & 10 & 2.8 & $1.5-4.2$ & 3.0 & 0.8 & 13 & 5.9 & $3.0-5.9$ & 4.5 & 1.3 & 13 \\
\hline Branchial aperture (BO) & 18.7 & $16.6-21.4$ & $18 . .7$ & 1.6 & 10 & 25.0 & $17.6-25.0$ & 21.2 & 2.0 & 13 & 17.2 & $16.2-19.6$ & 17.5 & 1.5 & 13 \\
\hline Pectoral-fin base (PB) & 11.0 & $9.0-11.7$ & 10.3 & 0.8 & 10 & 12.8 & $7.5-13.8$ & 11.0 & 1.7 & 13 & 9.8 & $7.4-12.3$ & 9.2 & 2.3 & 13 \\
\hline Caudal filament depth (CD) & 15.3 & $12.8-17.9$ & 15.0 & 1.8 & 8 & 14.2 & $14.2-18.3$ & 16.0 & 1.5 & 11 & 15.4 & $12.8-15.4$ & 13.8 & 1.2 & 13 \\
\hline Caudal filament width (CW) & 6.1 & $5.4-7.8$ & 6.3 & 0.9 & 8 & 5.2 & $5.2-8.9$ & 6.4 & 1.2 & 11 & 8.6 & $3.7-8.6$ & 6.4 & 2.3 & 13 \\
\hline \multicolumn{16}{|c|}{ Other proportions } \\
\hline BW1 (\% of BD1) & 61.4 & $49.0-61.5$ & 54.0 & 4.5 & 10 & 56.1 & $44.6-63.8$ & 56.3 & 5.8 & 18 & 57.3 & $47.7-57.3$ & 51.9 & 4.2 & 13 \\
\hline BW2 (\% of BD2) & 36.8 & $31.0-44.1$ & 35.8 & 4.5 & 10 & 33.9 & $24.7-42.8$ & 34.5 & 5.5 & 18 & 27.8 & $26.3-37.8$ & 29.8 & 5.3 & 13 \\
\hline
\end{tabular}

Holotype. CBF 10284, male, $97 \mathrm{~mm}$ TL, $79 \mathrm{~mm}$ LEA, Bolivia, Beni, mun. Riberalta, stream nr. San José, nr. Riberalta, affl. río Beni, affl. rio Madeira, Amazonas dr., $10^{\circ} 55^{\prime} 32^{\prime \prime} \mathrm{S}, 066^{\circ} 00^{\prime} 36^{\prime \prime} \mathrm{W}, 28$ Jun 2007, W. Crampton, J. Albert \& M. Arraya.

Paratypes. 7 specimens, localities from mun. Riberalta, río Beni dr., affl. rio Madeira, Amazonas dr., collected by W. Crampton, J. Albert \& M. Arraya. Bolivia. Beni. ANSP
197573, 1, female, $91 \mathrm{~mm}, 25$ Jun 2007, CBF 10279, 1, female, $70 \mathrm{~mm}$, 25 Jun 2007, CBF 10280, 1, immature, 64 mm, 25 Jun 2007, stream nr. lago San José, 10 $54^{\prime} 47^{\prime \prime} \mathrm{S}$, $065^{\circ} 59^{\prime} 49^{\prime \prime} \mathrm{W}$. CBF 10281, 1, female, $72 \mathrm{~mm}, 28$ Jun 2007, CBF 10282, 1, immature, 48 mm, 28 Jun 2007, CBF 10283, 1, immature, $58 \mathrm{~mm}, 28$ Jun 2007, stream nr. San José, $10^{\circ} 55^{\prime} 32^{\prime \prime} \mathrm{S}, 066^{\circ} 00^{\prime} 36^{\prime \prime} \mathrm{W}$. UMSS 07042, 1, male (CS), 84 mm, 6 Jul 2007, stream nr. Bocerón, on RiberaltaGuayaramerín rd., $11^{\circ} 02^{\prime} 51^{\prime \prime} \mathrm{S}, 065^{\circ} 50^{\prime} 06^{\prime \prime} \mathrm{W}$. 
Non-types. 9 specimens, localities from rio Madeira dr., Amazonas dr. Bolivia. Beni (localities from mun. Riberalta, río Beni dr.). UF 177345, 1, immature, $68 \mathrm{~mm}$, RiberaltaGuayaramerín rd., nr. km 43, $11^{\circ} 00^{\prime} 30^{\prime \prime} \mathrm{S}, 065^{\circ} 39^{\prime} 49^{\prime \prime} \mathrm{W}$. UMSS 7041, 1, male, $96 \mathrm{~mm}$, stream nr. Bocerón, on Riberalta-Guayaramerín rd., $1^{\circ} 02^{\prime} 51^{\prime \prime} \mathrm{S}, 065^{\circ} 50^{\prime} 06^{\prime \prime} \mathrm{W}$. UMSS 7043, 1, immature, $70 \mathrm{~mm}$, stream nr. Hormiga, on Riberalta-Guayaramerín rd., $1^{\circ} 01^{\prime} 34^{\prime \prime} \mathrm{S}, 065^{\circ} 52^{\prime} 58^{\prime \prime} \mathrm{W}$. UMSS 7044, 1, male, 111 mm, UMSS 7045, 1, female, 100 $\mathrm{mm}$, Riberalta- Guayaramerín rd., nr. km 43, $11^{\circ} 01^{\prime} 58^{\prime \prime} \mathrm{S}$, $065^{\circ} 44^{\prime} 53^{\prime \prime}$ W. Brazil. Rondônia. UFRO-I 6482, 4, 53-57 $\mathrm{mm}$, rio Guaporé upstream community Pedras Negras, affl. rio Mamoré, $12^{\circ} 52^{\prime} 03^{\prime \prime} \mathrm{S} 62^{\circ} 52^{\prime} 38^{\prime \prime} \mathrm{W}$.

Diagnosis. Brachyhypopomus alberti is diagnosed from congeners by the following combination of characters: depigmented stripe along middorsal region of body absent, $v s$. prominent pale uninterrupted middorsal stripe from occipital region to base of caudal filament in $B$. arrayae, $B$. beebei, B. belindae, B. gauderio, B. pinnicaudatus, and $B$. verdii; precaudal vertebrae 20-22 vs. 15-19 in $B$. batesi, B. benjamini, B. bennetti, B. bombilla, $B$. bullocki, B. cunia, B. diazi, B. hendersoni, B. menezesi, $B$. provenzanoi, $B$. regani, and $B$. sullivani; anal-fin rays 182-202 vs. 226-293 in B. brevirostris; continuous or discontinuous dark vertical or diagonally oriented bands or saddles present on body surface dorsal to lateral line, often extending across lateral line into ventral lateral surface, $v s$. absence of oblique bands or saddles on body surface dorsal to lateral line in B. draco, B. flavipomus $B$. jureiae, and B. palenque; bilateral columns of electrocytes at the anal-fin terminus 3 vs. 4-5 in B. janeiroensis and $B$. occidentalis (except some populations in Colombia and Venezuela, see redescription of $B$. occidentalis); dark suborbital stripe absent, vs. present in B. walteri. Brachyhypopomus alberti can be distinguished from most but not all specimens of $B$. hamiltoni by a higher number of pectoral-fin rays - 15-16 (mode 16) vs. 12-15 (mode 13) (only 2 of 18 measured specimens of $B$. hamiltoni exhibited an overlapping number of pectoral-fin rays with $B$. alberti). Brachyhypopomus alberti can be further distinguished from $B$. hamiltoni by the absence of the first of five branchiostegal rays $v s$. presence in $B$. hamiltoni Mago-Leccia (1994: 175, fig. 77).

Description. Head and body shape, and pigmentation illustrated in Fig. 4. Meristic and morphometric data for examined specimens presented in Tables 2-6. Body shallow to moderate in depth. Head short to moderate in length and shallow to moderate in depth. Dorsal profile of head slightly convex from occiput to snout, ventral profile of head straight to slightly convex between operculum and snout, snout rounded and bulbous. Eye moderate in size. Upper jaw with slight to moderate sigmoidal angle between premaxillary and maxillary portions in lateral view. No accessory electric organ over operculum. Gill filaments on first gill arch 30-37 (median 36, $\mathrm{n}=4$ ). Precaudal vertebrae 20-22 (mode 22) including 1-2 (mode 2) transitional vertebrae. Pectoral fin narrow to moderate in width, pectoral-fin rays 16 in all specimens examined. Anal-fin origin slightly $(<0.25 \mathrm{HL}$ distance) anterior to, or near, tip of pectoral fin. Anal-fin rays 172-191 (median 184). Dorsal rami of recurrent branch of anterior lateral line nerve not visible. Middorsal region of body scaled. Rows of scales above lateral line 4-6 (mode 5). Lateral line discontinuous in all examined specimens; area in midbody with no pored lateral-line scales for $c a$. 1-2 head lengths. Sparse groove-like depigmented epidermal canals found mainly in posterior half of body - as parallel lines either side of lateral line, on flank midway from lateral line to dorsal midline, and as a pair of long irregular lines either side of dorsal surface in dorsal portion of flank. Three bilateral horizontal columns of electrocytes at analfin terminus and at mid-point between anal-fin terminus and tip of caudal filament in immature, mature female, and mature male specimens. Caudal filament short to moderate in length.

Coloration. (Fig. 4). Immature specimens and males with pale straw to tan background. Several diffuse and poorly defined vertical darker vertical bands comprising higher chromatophore densities located on lateral surface of body, traversing lateral line without disrupting ventrally. Dorsal region approximately uniformly pigmented with a speckling of brown chromatophores, without uninterrupted pale stripe along dorsal midline from occipital region to base of caudal filament. Irregular dark markings near upper portion of lateral surface but not on dorsal midline in some specimens. Very poorly defined light chocolate darker bands also located over anal-fin pterygiophores, sometimes forming inverted Y-shaped markings. Series of diffuse horizontal dash-like dark markings often present along lateral line in posterior third of body, anterior to anal-fin terminus. Head with evenly scattered dark chromatophores, darker dorsally. Eye without prominent suborbital patch, or stripe, of chromatophores/subcutaneous pigmentation. Pectoral and anal-fin membranes hyaline. Pectoral and anal-fin rays hyaline with light scattering of brown chromatophores. Anal-fin ray pigmentation darker in posterior half of fin. Mature female specimens distinctly darker, with much higher density of brown chromatophores over entire body, head and dorsal regions, background color of lateral surface of body light brown with indistinct oblique darker bands of dark brown. Color in live individuals similar to preserved specimens, with opercular region usually rosy due to underlying gills.

Size. Small adult size, largest specimen examined 111 mm TL, 89 mm LEA $(\mathrm{n}=17)$. Largest male specimen examined $111 \mathrm{~mm}$ TL, $89 \mathrm{~mm}$ LEA $(\mathrm{n}=4)$. Largest female specimen examined $100 \mathrm{~mm}$ TL, $77 \mathrm{~mm}$ LEA $(\mathrm{n}=4)$. 
Sexual dimorphism. Brachyhypopomus alberti exhibits an unusual sexual difference in pigmentation, with sexually mature females exhibiting a much darker overall coloration (a character shared with B. arrayae) (Figs. $4 a-b)$. No other secondary sexually dimorphic characters known.

Geographic distribution. Bolivia and Brazil (Fig. 5). Known from the upper rio Madeira (Amazonas dr.), above its series of falls beginning at the Cachoeira de Santo Antônio, near Porto Velho, Rondônia, Brazil; in the lower río Beni near the town of Riberalta, Bolivia; and from the rio Guaporé of Brazil.

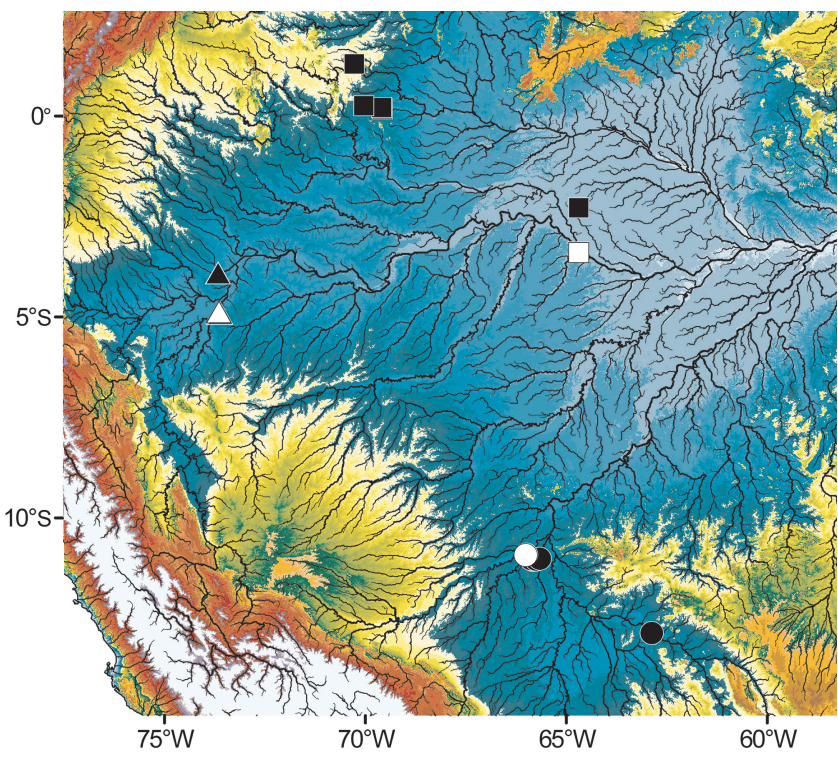

Fig. 5. Collection records for Brachyhypopomus alberti (circles), B. batesi (squares), and B. benjamini (triangles). Holotype locations are marked with open symbols. Elevation data refers to altitude above mean sea level (see Fig. 2 for legend).

Ecological notes. The type series was collected from small low-conductivity clearwater and blackwater terra firme streams in tropical forest and savanna near Riberalta, Bolivia. It was encountered mostly commonly in marginal root mats, and in emergent or submerged aquatic vegetation. The following water parameters were recorded at the sample sites: conductivity $5-15 \mu \mathrm{Scm}^{-1}$, dissolved oxygen $3.0-5.0 \mathrm{mgl}^{-1}$, temperature $22-24^{\circ} \mathrm{C}$, and $\mathrm{pH} 5.2-5.5$. Adults in breeding condition were found during the dry season in June-July 2007. However, the duration and timing of breeding are otherwise unknown. Stomach contents of specimens from the type locality comprise aquatic insect larvae and other small aquatic invertebrates (WGRC unpublished data).

Co-occurring congeners: Brachyhypopomus alberti is known to co-occur in geographical sympatry and ecological syntopy with the following terra firme streamoccurring species: B. brevirostris, B. sullivani, and $B$. walteri. In the region of the type locality it exhibits an allotopic distribution with the following whitewater floodplain occurring species: B. arrayae, B. bombilla, and $B$. pinnicaudatus. Brachyhypopomus alberti cooccurs in geographical sympatry with its sister species $B$. arrayae, but the species exhibit a noteworthy difference in ecological distribution: B. alberti occurs in lowconductivity $\left(\mathrm{ca} .5-15 \mu \mathrm{Scm}^{-1}\right)$ terra firme forest and savanna streams, while $B$. arrayae mainly occurs in higher-conductivity whitewater floodplain systems ( $c a$. $150 \mu \mathrm{Scm}^{-1}$ at the time of sampling). Nonetheless, $B$. alberti was sampled together with $B$. arrayae (and also $B$. pinnicaudatus) in the lower reaches of terra firme streams, at the ecotone with the río Beni floodplain.

Etymology. The specific name is a patronym (noun in the genitive case) in honor of James S. Albert, American (USA) ichthyologist, collector of part of the type series, for his enormous contributions to the systematic biology of gymnotiform fishes.

Local names. Bolivia: cuchillo; Brazil: sarapó.

\section{Brachyhypopomus arrayae, new species}

urn:1sid:zoobank.org:act:836C199C-3EBA-4788-B8D5AB4DEBEABF05

(Fig. 6; Tables 2-6)

Brachyhypopomus sp. "arr". -Crampton, 2011: 176, table 10.2, species list; 179, figs. 10.2-10.3, phylogeny, geographical and ecological distributions (gymnotiform biology).

Brachyhypopomus sp. "arrayae". -Crampton et al., 2016: 1-66, table 1, 3-4, figs. 1-7, 18-20 (phylogeny, biogeography and ecology of Brachyhypopomus).

Holotype. UMSS 7048, male, $77 \mathrm{~mm}$ TL, $66 \mathrm{~mm}$ LEA, Bolivia, Beni, mun. Riberalta, small stream nr. Puerto Hamburgo, affl. río Beni, affl. rio Madeira, Amazonas dr., $11^{\circ} 01^{\prime} 52^{\prime \prime} \mathrm{S} 66^{\circ} 05^{\prime} 39^{\prime \prime} \mathrm{W}, 6$ Jul 2007, W. Crampton, M. Arraya \& J. Albert.

Paratypes. 7 specimens, localities from río Beni dr., aff rio Madeira, Amazonas dr. Bolivia. Beni. ANSP 197574, 1, female, $128 \mathrm{~mm}$; UF 177342, 2, male, 72-92 mm; UMSS 07047, 1, female (CS), $120 \mathrm{~mm}$, mun. Riberalta, small stream nr. Puerto Hamburgo, $11^{\circ} 01^{\prime} 52^{\prime \prime} \mathrm{S}, 066^{\circ} 05^{\prime} 39^{\prime \prime} \mathrm{W}, 6$ Jul 2007, W. Crampton, M. Arraya \& J. Albert. AUM 35556 1, female, $95 \mathrm{~mm}$, mun. Vaca Díez, affl. lago Tumi Chucua, $26 \mathrm{~km}$ S. S. W Riberalta, $c a .11^{\circ} 07^{\prime} \mathrm{S}, 066^{\circ} 11^{\prime} \mathrm{W}, 28 \mathrm{Nov}$ 1981, K. Swing. CAS 81630, 1, 82 mm, lago Rogoagua, río Beni floodplain, $c a .13^{\circ} 58^{\prime} \mathrm{S}, 066^{\circ} 58^{\prime} \mathrm{W}$, Nov $1921, \mathrm{~N}$. Pearson. CBF 10277, 1, immature, $56 \mathrm{~mm}$, mun. Riberalta, stream nr. lago San José, $10^{\circ} 54^{\prime} 47^{\prime \prime} \mathrm{S}, 065^{\circ} 59^{\prime} 49^{\prime \prime} \mathrm{W}, 25$ Jun 2007, W. Crampton, M. Arraya \& J. Albert. 


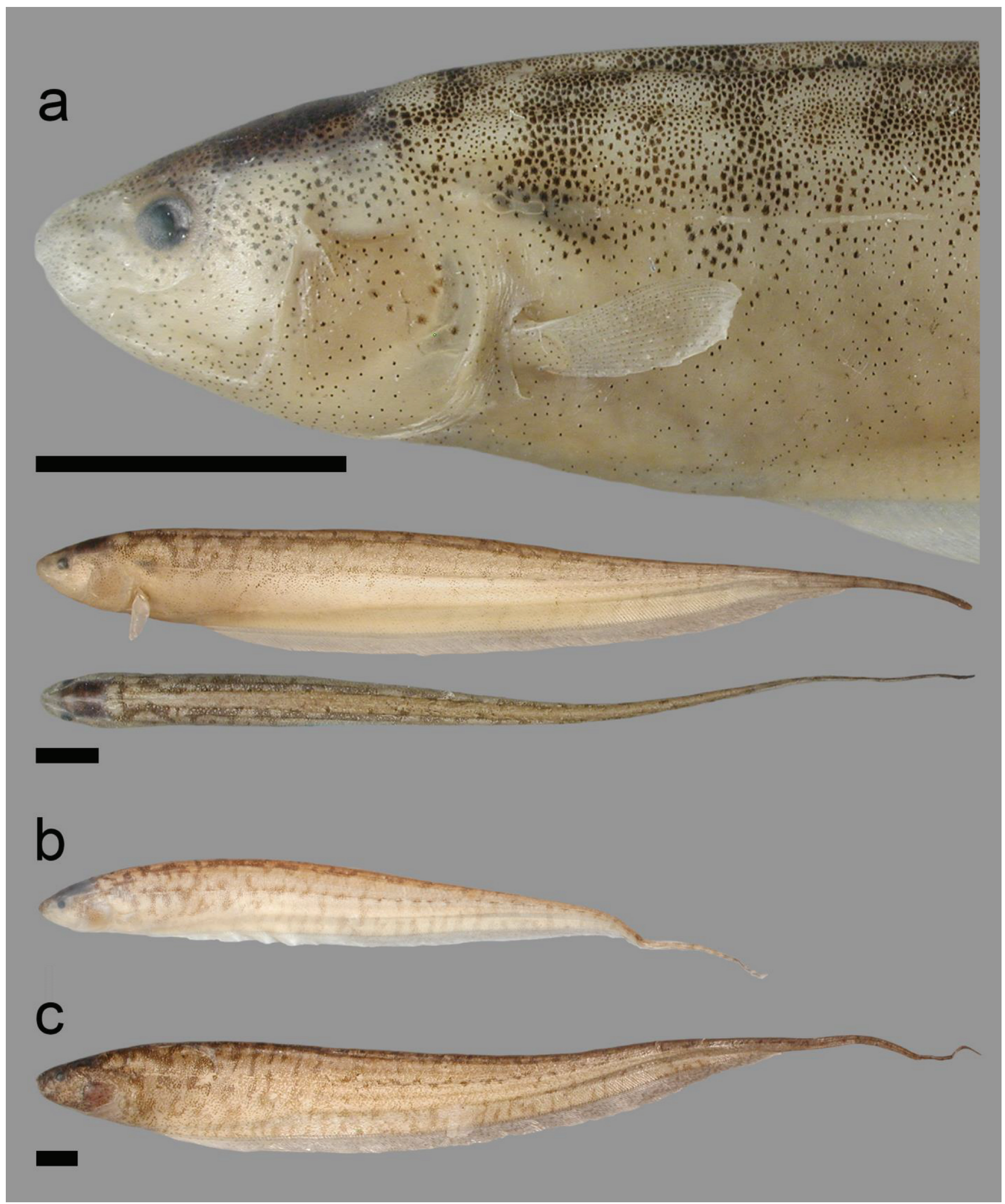

Fig. 6. Brachyhypopomus arrayae. a. UMSS 7048 (WC52.060707), holotype, immature male, $77 \mathrm{~mm}$ TL (head in lateral view, body in lateral and dorsal views); b. UF 177342 (WC54.060707), paratype, male, $97 \mathrm{~mm}$ TL (body in lateral view); c. ANSP 197574 (WC50.060707), paratype, female, $128 \mathrm{~mm}$ TL (body in lateral view); Bolivia, río Beni, Amazonas dr. Note the sexual dimorphism in body pigmentation, in which females are considerably darker. Specimens fixed in formalin and preserved in EtOH. Scale bars $=5 \mathrm{~mm}$. 
Non-types. 68 specimens, localities from rio Madeira dr., Amazonas dr. Brazil. Rondônia. INPA POLO-464, 10, 89-133 mm (3 immature 70-89 mm, 6 female, 90-133, 1 male, $114 \mathrm{~mm}$ ), mun. Porto Velho, rio Jamari, $c a .08^{\circ} 44^{\prime} \mathrm{S}$, $063^{\circ} 27^{\prime} \mathrm{W}$. MCP 41031 (part), 1, $63 \mathrm{~mm}$, mun. Nova Mamoré, rio Ribeirão at hwy. BR-425, ca. $62 \mathrm{~km} \mathrm{~S}$ hwy. BR-364, affl. rio Guaporé, affl. rio Mamoré, $10^{\circ} 13^{\prime} 55^{\prime \prime} \mathrm{S}$, $065^{\circ} 16^{\prime} 55^{\prime \prime} \mathrm{W}$. MCP 41039, 4, 66-77 mm, mun. Porto Velho, affl. río São Sebastião, on hwy. BR-364, affl. rio Abunã, 09 $39^{\prime} 05^{\prime \prime} \mathrm{S} 65^{\circ} 33^{\prime} 53^{\prime \prime} \mathrm{W}$. Peru. Madre de Dios (localities from río Madre de Dios dr., affl. río Beni, aff río Madeira, Amazonas dr.). MUSM 2734, 1, prov. and mun. Tambopata, $3 \mathrm{~km}$. Cocococha, no coordinates. MUSM 9597, 1, MUSM 9621, 1, prov. and mun. Tambopata, Reserva Nacional de Tambopata, Laguna Sandoval, $12^{\circ} 26^{\prime} 30^{\prime \prime} \mathrm{S}, 069^{\circ} 02^{\prime} 18^{\prime \prime} \mathrm{W}$. MUSM 9827, 2, prov. and mun. Tambopata, $27 \mathrm{~km}$ Laberinto, no coordinates. MUSM 9873, 1, $160 \mathrm{~mm}$, lago Tres Chimbadas, Reserva Nacional de Tambopata, río Tambobata, $12^{\circ} 47^{\prime} 26^{\prime \prime} \mathrm{S}, 069^{\circ} 20^{\prime} 31^{\prime \prime} \mathrm{W}$. MUSM 21494, 1, MUSM 21675, 1, prov. Manu, mun. Madre de Dios, Trocha Huangana, río Los Amigos, $12^{\circ} 33^{\prime} 21^{\prime \prime S} 70^{\circ} 06^{\prime} 42^{\prime \prime} \mathrm{W}$. MUSM 21663, 1, prov. Manu, mun. Madre de Dios, Pozo minero, aguajal at "CICRA", no coordinates. MUSM 21683, 3, prov. Tambopata, cocha Huitoto, no coordinates. MUSM 21801, 4, MUSM 21909, 15, MUSM 21910, 1, mun. Tambopata, Aguajal Aguas Negras, Aguajal Oeste, $\mathrm{km} \mathrm{35,} \mathrm{ca} .12^{\circ} 00^{\prime} \mathrm{S}, 070^{\circ} 40^{\prime} \mathrm{W}$. MUSM 21866, 3, mun. Inambari, Mazuco, aguajal, km 180 , río Inambari, $c a .12^{\circ} 30^{\prime} \mathrm{S}, 069^{\circ} 05^{\prime} \mathrm{W}$. MUSM 22004 , 2, mun. Tambopata, Puerto Maldonado, ca. $12^{\circ} 36^{\prime} \mathrm{S}$, $069^{\circ} 11^{\prime} \mathrm{W}$. MUSM 22061, 1, prov. and mun. Tambopata, Aguajal Tripa, no coordinates. MUSM 22142, 1, MUSM 22157, 12, mun. prov. Tambopata, Puerto Maldonado, ca. $12^{\circ} 36^{\prime} \mathrm{S}, 069^{\circ} 11^{\prime} \mathrm{W}$. MUSM 22642 (part), 1, $50 \mathrm{~mm}$, prov. Tambopata, mun. Las Piedras, Planchón las Piedras, quebrada Planchón, affl. rio Tahuamanu [also known as río Las Piedras], ca. $12^{\circ} 30^{\prime} \mathrm{S}, 069^{\circ} 14^{\prime} \mathrm{W}$. MUSM 35347, 1 , prov. Tahuamanu, Mavila, cocha Bote 3, no coordinates. MUSM 37094, 1, $84 \mathrm{~mm}$, quebrada Chorera, río Las Piedras, $12^{\circ} 02^{\prime} 12^{\prime \prime} \mathrm{S}, 069^{\circ} 37^{\prime} 31^{\prime \prime} \mathrm{W}$.

Diagnosis. Brachyhypopomus arrayae is diagnosed from congeners by the following combination of characters: prominent pale uninterrupted middorsal stripe from occipital region to base of caudal filament present, $v s$. absent in all congeners except $B$. beebei, $B$. belindae, $B$. flavipomus, B. gauderio, B. pinnicaudatus, and $B$. verdii; anal-fin rays 174-212 vs. 214-230 in $B$. beebei; precaudal vertebrae 20-23, vs. 24-26 in B. belindae and B. verdii; pectoral-fin rays 16-19 (mode 16), vs. 12-15 (mode 13) for B. gauderio (except populations from Uruguay drainages) and vs. 13-15 (mode 14) for B. pinnicaudatus (except populations from coastal French Guiana). Brachyhypopomus arrayae exhibits similar pigmentation and overlapping meristic counts and morphometric proportions with some populations of $B$. gauderio and $B$. pinnicaudatus from which it can be distinguished by the contact of a small ascending process on the endopterygoid (mesopterygoid of Mago-Leccia, 1978: 10, 57, fig. 8) with the orbitosphenoid (Arratia \& Schultze, 1991: 43, fig. 26; de Santana \& Crampton, 2011: 1104, fig. 2), vs. the lack of a contact between the ascending process on the endopterygoid and the orbitosphenoid in B. gauderio and B. pinnicaudatus.

Description. Head and body shape, and pigmentation illustrated in Fig. 6. Meristic and morphometric data for examined specimens presented in Tables 2-6. Body shallow to moderate in depth. Head short to moderate in length and moderate in depth. Dorsal profile of head slightly convex from occiput to snout, ventral profile of head slightly convex between operculum and snout, snout rounded and bulbous. Eye moderate in size. Upper jaw with slight to moderate sigmoidal angle between premaxillary and maxillary portions in lateral view. No accessory electric organ over operculum. Gill filaments on first gill arch 32-39 (median 36, $\mathrm{n}=6$ ). Pectoral fin moderate in width, pectoral-fin rays 16-19 (mode 17). Precaudal vertebrae 20-23 (mode 22) including 1-2 (mode 2) transitional vertebrae. Anal-fin origin slightly $(<$ $0.25 \mathrm{HL}$ distance) anterior or posterior to, or near, tip of pectoral fin. Anal-fin rays 175-212 (median 201). Dorsal rami of recurrent branch of anterior lateral line nerve not visible. Middorsal region of body scaled. Rows of scales above lateral line 5-6 (mode 5). Lateral line discontinuous in most examined specimens; area in mid-body with no pored lateral-line scales for $c a$. 0.5-1 head lengths. Sparse groove-like depigmented epidermal canals found mainly in posterior half of body - as parallel lines on either side of lateral line, on flank midway from lateral line to dorsal midline, and as a pair of long irregular lines either side of dorsal surface in dorsal portion of flank. Three bilateral horizontal columns of electrocytes at anal-fin terminus and at mid-point between anal-fin terminus and tip of caudal filament in immature specimens, mature females, and mature males. Caudal filament short to moderate in length.

Coloration. (Fig. 6). Immature specimens and males with pale straw to tan background. Several diffuse and poorly defined vertical darker vertical bands comprising higher chromatophore densities located on lateral surface of body, traversing lateral line without disrupting ventrally. Prominent pale stripe along dorsal midline from occipital region to base of caudal filament, flanked by dark borders from which vertical bands extend onto lateral surfaces. Very poorly defined light brown bands also located over analfin pterygiophores. Series of diffuse horizontal dash-like dark markings often present along lateral line in posterior third of body, anterior to anal-fin terminus. Head with evenly scattered dark chromatophores, darker dorsally. Eye without prominent suborbital patch, or stripe, of 
chromatophores/subcutaneous pigmentation. Pectoral and anal-fin membranes hyaline. Pectoral and anal-fin rays hyaline with light scattering of brown chromatophores. Anal-fin ray pigmentation darker in posterior half of fin. Mature female specimens distinctly darker, with much higher density of brown chromatophores over entire body, and head and dorsal regions, background color of lateral surface of body light brown or tan with indistinct oblique darker bands of dark brown. Color in live individuals similar to preserved ones, with opercular region usually rosy due to underlying gills.

Size. Small adult size, largest specimen examined 133 mm TL, $102 \mathrm{~mm}$ LEA $(\mathrm{n}=76)$. Largest male specimen examined $114 \mathrm{~mm}$ TL, $87 \mathrm{~mm}$ LEA $(\mathrm{n}=4)$. Largest female specimen examined $133 \mathrm{~mm}, 102 \mathrm{~mm}$ LEA $(\mathrm{n}=12)$.

Sexual dimorphism. Brachyhypopomus arrayae presents an unusual sexual difference in pigmentation, with sexually mature females exhibiting a much darker overall coloration (a character shared with $B$. alberti) (Figs. 6b-c). Sexually mature males somewhat smaller than mature females, but in the absence of a larger series of mature specimens, sexual size dimorphism is as yet unconfirmed. No other secondary sexually dimorphic characters known.

Geographic distribution. Bolivia, Brazil, and Peru (Fig. 7). Known from the upper rio Madeira (Amazonas dr.), with all but one record (from the rio Jamari, Rondônia, Brazil) occurring above its series of falls beginning at the Cachoeira de Santo Antônio; most records from the río Beni in Bolivia and the río Madre de Dios in Peru.

Ecological notes. The type series was collected from high-conductivity whitewater floodplain sites and from the lower, seasonally inundated reaches of terra firme streams. It was encountered most commonly in marginal root mats, and in emergent or submerged aquatic vegetation. The following water parameters were recorded at the sampling sites: conductivity $150-160 \mu \mathrm{Scm}^{-1}$, dissolved oxygen 5.0-6.0 mgl-1 ${ }^{-1}$, temperature $27-28^{\circ} \mathrm{C}$, and $\mathrm{pH} 7.5$ 7.6. Adults in breeding condition were found during the dry season in June-July 2007. However, the duration and timing of breeding are otherwise unknown. Stomach contents of specimens from the type locality comprise aquatic insect larvae and other small aquatic invertebrates (WGRC unpublished data). Co-occurring congeners: Brachyhypopomus arrayae is known to co-occur in geographical sympatry and ecological syntopy with the following whitewater floodplain-occurring species: $B$. bombilla, $B$. brevirostris, $B$. pinnicaudatus, and $B$. walteri. In the region of the type locality it exhibits a largely allotopic distribution with the following terra firme stream occurring species: $B$. alberti and $B$. sullivani (although $B$. arrayae was found with $B$. alberti at the ecotone between terra firme streams and floodplain systems).

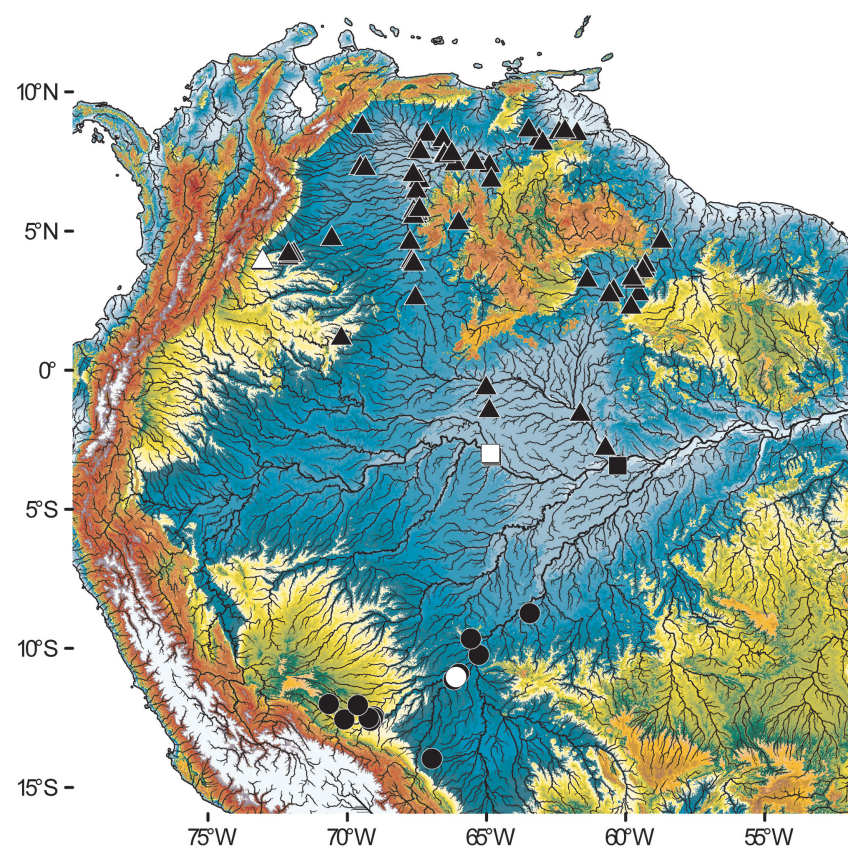

Fig. 7. Collection records for Brachyhypopomus arrayae (circles), B. belindae (squares), and B. bullocki (triangles). Holotype locations are marked with open symbols. Elevation data refers to altitude above mean sea level (see Fig. 2 for legend).

Etymology. The specific name is a patronym (noun in the genitive case) in honor of Bolivian biologist Mariana Arraya for her assistance in collecting the type series in Bolivia.

Local names. Bolivia: Cuchillo; Brazil: sarapó; Peru: macana.

\section{Brachyhypopomus batesi, new species}

urn:1sid:zoobank.org:act:728CA0A5-F5C5-465F-8793D1176B3E7F57

(Figs. 1a, 8; Tables 2-6)

Brachyhypopomus sp. 1. -de Oliveira et al., 2009: fig. 41, color photograph (Brazil, Amazonas, Catuá-Ipixuna Reserve, ca. $\left.03^{\circ} 48^{\prime} \mathrm{S}, 063^{\circ} 59^{\prime} \mathrm{W}\right)$.

Brachyhypopomus sp. "bat". -Crampton, 2011: 176, table 10.2, species list; 179, figs. 10.2-10.3, phylogeny, geographical and ecological distributions (gymnotiform biology).

Brachyhypopomus sp. "batesi”. -Crampton et al., 2016: 1-66, table 1, 3-4, figs. 1-7, 18-20 (phylogeny, biogeography and ecology of Brachyhyроротиs).

Holotype. MCP 47020, female, $104 \mathrm{~mm}$ TL, $82 \mathrm{~mm}$ LEA, Brazil, Amazonas, mun. Tefé, igarapé Xidarini, affl. lago

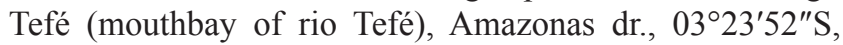
0644ㄴ'17"W, 31 Dec 1996, W. Crampton. 


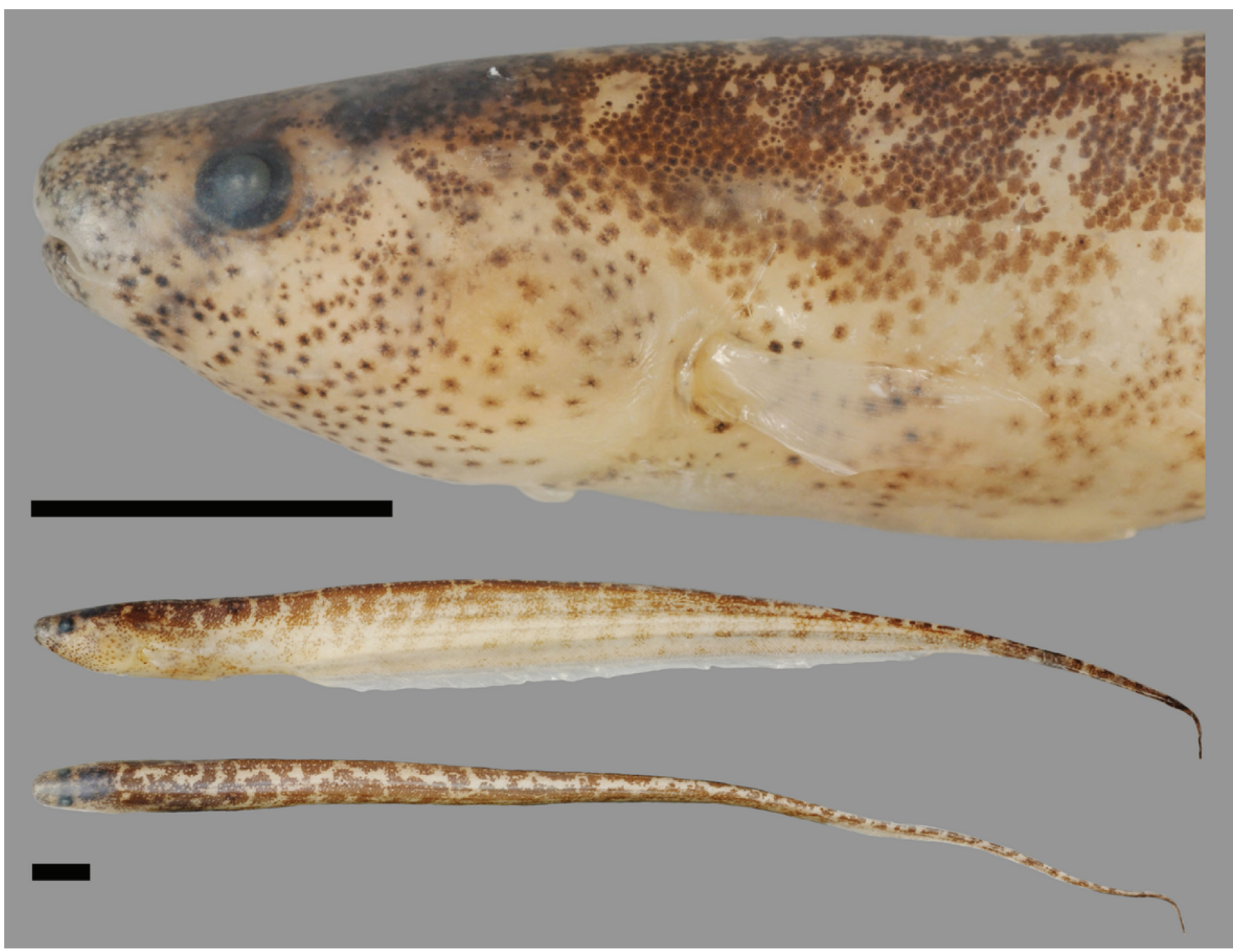

Fig. 8. Brachyhypopomus batesi. MCP 47020 (WC07.291297b), holotype, female, $104 \mathrm{~mm}$ TL (head in lateral view, body in lateral and dorsal views, specimen fixed in formalin and preserved in EtOH); Brazil, rio Tefé, Amazonas dr. Scale bars $=5 \mathrm{~mm}$.

Paratypes. 4 specimens, localities from Amazonas dr., collected by W. Crampton. Brazil. Amazonas. MCP 45308, 1, male, 78 mm, mun. Maraã, igarapé São Sebastião, affl. rio Baré, affl. lago Amanã, affl. rio Japurá, $02^{\circ} 17^{\prime} 05^{\prime \prime} \mathrm{S}$, 06441'25"W, 17 Feb 1993, W. Crampton. MCP 45341, 2, (1 female, $99 \mathrm{~mm}, 1$ male, $100 \mathrm{~mm}$ ), collected with holotype. MCP 45357, immature, 75 mm, mun. Tefé, igarapé Xidarini, affl. lago Tefé, rio Tefé, 032 $3^{\prime} 52^{\prime \prime} \mathrm{S}, 064^{\circ} 41^{\prime} 17^{\prime \prime} \mathrm{W}, 13$ Aug 2001, W. Crampton.

Non-types. (24 specimens). Brazil. Amazonas (localities from Amazonas dr.). MCP 45312, 16 (11 immature, 76$115 \mathrm{~mm}, 4$ female, $83-121 \mathrm{~mm}, 1$ male, $120 \mathrm{~mm})$, mun. Tefé, igarapé Xidarini, affl. lago Tefé, rio Tefé, $03^{\circ} 22^{\prime} 46^{\prime \prime} \mathrm{S}$,

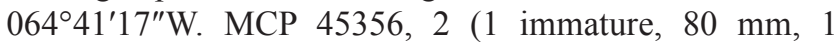
female [CS], $85 \mathrm{~mm})$, mun. Tefé, igarapé Xidarini, affl. lago Tefé, rio Tefé, Amazonas dr., 0323'52"S, 06441'17"W. MZUSP 85041, 1, $104 \mathrm{~mm}$, blackwater affl. rio Tiquié, Comunidade Fronteira, affl. rio Uaupés, affl. rio Negro, $00^{\circ} 15^{\prime} 35^{\prime \prime} \mathrm{N}, 070^{\circ} 02^{\prime} 43^{\prime \prime} \mathrm{W}$. MZUSP 92234, 2, 53-73 mm, mun. São Gabriel da Cachoeira, ca. 500 m downstream São
José II, igarapé Cunuri, affl. rio Tiquié, affl. rio Uaupés, affl. rio Negro, $00^{\circ} 13^{\prime} \mathrm{N}, 069^{\circ} 36^{\prime} \mathrm{W}$. MZUSP 93067, 1, 60, mun. São Gabriel da Cachoeira, igarapé Castanha, nr. Sítio São Pedro, affl. rio Tiquié, affl. rio Uaupés, affl. rio Negro, $00^{\circ} 11^{\prime} \mathrm{N}, 069^{\circ} 35^{\prime} \mathrm{W}$. Colombia. Vuapés: SINCHICIACOL. 1054, 2 (1 immature, $40 \mathrm{~mm}, 1$ female, $101 \mathrm{~mm}$ ), stream affl. río Cuduyarí, affl. río Vuapés, affl. rio Negro, $01^{\circ} 17^{\prime} 36^{\prime \prime} \mathrm{N}, 070^{\circ} 16^{\prime} 53^{\prime \prime} \mathrm{W}$.

Diagnosis. Brachyhypopomus batesi is diagnosed from congeners by the following combination of characters: analfin rays 165-179, vs. 180-293 in B. alberti, B. beebei, $B$. belindae, $B$. bennetti, $B$. brevirostris, $B$. bullocki, $B$. diazi, $B$. gauderio, $B$. hendersoni, $B$. janeiroensis, $B$. jureiae, $B$. occidentalis, $B$. palenque, $B$. verdii, and $B$. walteri; scales present in entire middorsal region (although sparse and hard to see in some specimens), vs. absent in middorsal region of anterior third of body in B. benjamini and B. provenzanoi; accessory electric organ over the opercular region absent, vs. present in B. bombilla, B. menezesi, and B. regani; head depth at occiput $61.0-67.9 \%$ HL, vs. $71.4-94.6 \%$ in 
B. cunia, B. draco, B. flavipomus, B. hamiltoni, and B. pinnicaudatus; absence of scattered conspicuous black or charcoal flecks on flanks, vs. presence in B. sullivani.

Description. Head and body shape, and pigmentation illustrated in Figs. 1a and 8. Meristic and morphometric data for examined specimens presented in Tables 2-6. Body shallow in depth. Head short to moderate in length and shallow in depth. Dorsal profile of head approximately straight from occiput to snout, ventral profile of head approximately straight with convex and concave portions, snout truncated. Eye moderate in size. Upper jaw with slight sigmoidal angle between premaxillary and maxillary portions in lateral view. No accessory electric organ over operculum. Gill filaments on first gill arch $61(\mathrm{n}=1)$. Pectoral fin narrow to moderate in width, pectoral-fin rays 12-13 (mode 13). Precaudal vertebrae 16-18 (mode 18 ) including 1-2 (mode 2) transitional vertebrae. Analfin origin substantially posterior (between 0.25 and 0.75 HL distance) to tip of pectoral fin. Anal-fin rays 159-173 (median 168). Dorsal rami of recurrent branch of anterior lateral line nerve visible. Entire middorsal region of body scaled, although scales in anterior third of body sparse and mostly occluded by skin. Rows of scales above lateral line 5-6 (mode 5). Lateral line complete. Sparse groove-like depigmented epidermal canals found mainly in posterior half of body (mainly in posterior third of body), forming parallel or crisscrossing wavy lines either side of lateral line, as single wavy lines on flank midway from lateral line to dorsal midline, and as a pair of long irregular lines either side of dorsal surface in dorsal portion of flank. Three bilateral horizontal columns of electrocytes at analfin terminus, and at mid-point between anal-fin terminus and tip of caudal filament in immature, mature female, and mature male specimens. Caudal filament short to moderate in length.

Coloration. (Figs. 1a, 8). Pale straw to tan background. Several distinct, truncated, brown vertical bands extending from across upper portion of flank, fading towards lateral line and absent from below lateral line, most prominent for approximately one head length posterior to occiput. Dorsal region comprising irregular brown patches and a pale straw or tan background, with no pale stripe along dorsal midline from occipital region to base of caudal filament. Region over anal-fin pterygiophores straw colored with very faint irregular markings, mostly posteriorly. Head with evenly scattered dark chromatophores, darker dorsally. Eye usually with an indistinct or prominent suborbital patch or stripe of chromatophores and subcutaneous pigmentation extending from anteroventral portion of eye to chin. Pectoral and anal fins with hyaline membranes. Pectoral-fin rays hyaline. Anal-fin rays hyaline in anterior half of body, with light scattering of brown chromatophores in posterior half. Color in live individuals similar to preserved specimens, with opercular region usually rosy due to underlying gills.
Size. Small adult size, largest specimen examined $121 \mathrm{~mm}$ TL, $98 \mathrm{~mm}$ LEA $(\mathrm{n}=28)$. Largest male specimen examined $120 \mathrm{~mm}$ TL, $100 \mathrm{~mm}$ LEA $(\mathrm{n}=3)$. Largest female specimen examined $121 \mathrm{~mm}, 98 \mathrm{~mm}$ LEA $(\mathrm{n}=8)$.

Sexual dimorphism. No known secondary sexual dimorphism.

Geographic distribution. Brazil and Colombia (Fig. 5). Known from the central Amazon, near the main stem of the rio Solimões (Amazon), and from the upper rio Negro.

Ecological notes. The type series was collected from the lower, seasonally flooded reaches of low-conductivity blackwater rainforest streams that flow into lago Tefé, and lago Amanã (large blackwater ria lakes of similar conductivity to the streams). Most specimens were encountered in marginal root mats or submerged leaflitter along stream edges. The following water parameters were recorded at the time of sampling: conductivity 10-30 $\mu \mathrm{Scm}^{-1}$, dissolved oxygen $2.0-5.2 \mathrm{mgl}^{-1}$, temperature 24.3 $27.0^{\circ} \mathrm{C}$, and $\mathrm{pH} 4.5-6.0$. During the high water period this species disappears from the flood-swollen lower stream reaches, probably due to declining dissolved oxygen levels, and apparently moves upstream to terra firme stream reaches above the seasonal river-floodplain flood influence. Breeding occurs during the early rising-water period, which in the Tefé region corresponds to the rainy season (WGRC unpublished data). Records of $B$. batesi outside the area of the type series are all from low-conductivity blackwater rainforest streams. Stomach contents of specimens from the type locality comprise aquatic insect larvae (primarily Chironomidae), and other small aquatic invertebrates (WGRC unpublished data).

Co-occurring congeners: In the region of the type locality Brachyhypopomus batesi co-occurs in geographical sympatry and ecological syntopy with the terra firme stream-occurring species: $B$. beebei, $B$. brevirostris, $B$. sullivani, and $B$. walteri. It also exhibits an allotopic distribution with $B$. belindae, $B$. bennetti, B. flavipomus, $B$. hamiltoni, $B$. hendersoni, B. pinnicaudatus, and B. regani. In the rio Negro, $B$. batesi occurs in geographical sympatry with $B$. beebei, B. brevirostris, B. bullocki, B. hendersoni, $B$. regani, $B$. sullivani, and $B$. walteri.

Etymology. The specific name is a patronym (noun in the genitive case) in honor of Henry Walter Bates (1825-1892), British naturalist and explorer, for his contributions to the natural history of the Tefé region, where the type series was collected.

Local names. Brazil: sarapó (widespread).

Brachyhypopomus beebei (Schultz, 1944)

(Figs. 1b, 9; Tables 2-5, 7) 

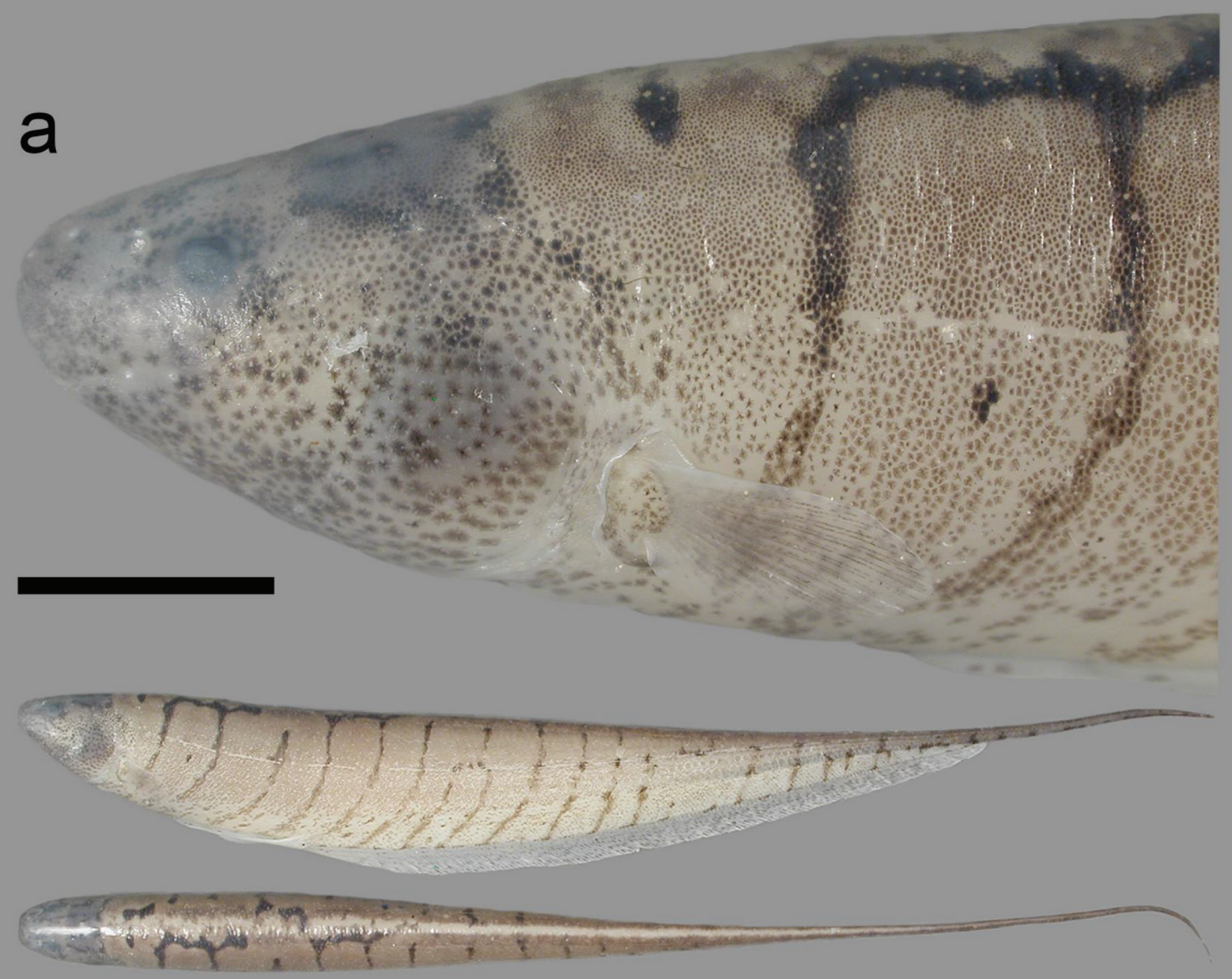

b

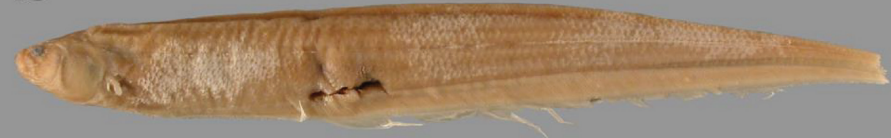

C

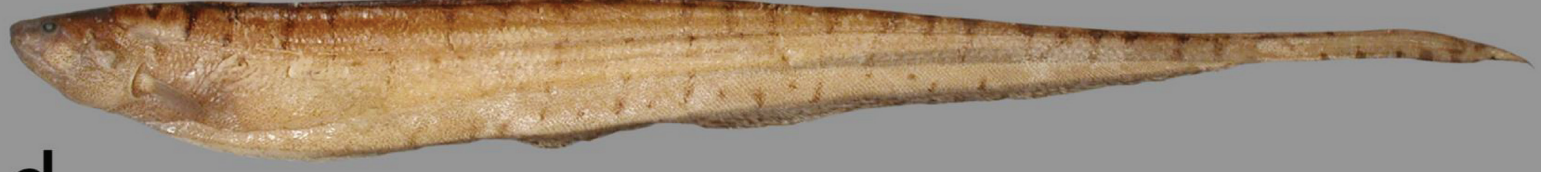

d
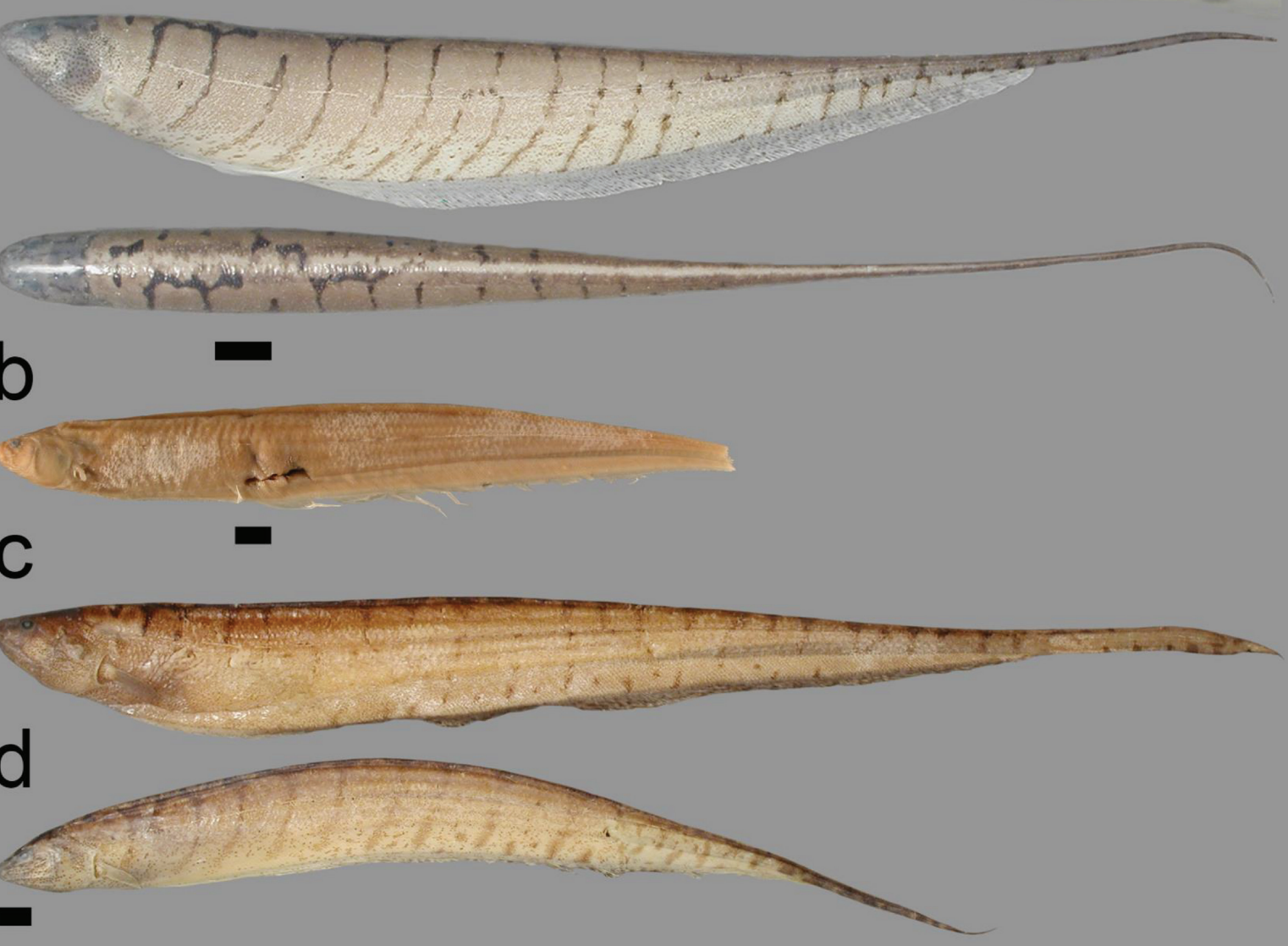

Fig. 9. Brachyhypopomus beebei. a. MCP 45462 (WC04.150801b), immature female, $134 \mathrm{~mm}$ TL, non-type (head in lateral view, body in lateral and dorsal view, specimen fixed in formalin); Brazil, rio Tefé, Amazonas dr. b. USNM 120753, holotype, $103 \mathrm{~mm}$ TL (originally $136 \mathrm{~mm}$ TL, tail now lost) (body in lateral view, specimen preserved in EtOH); Venezuela, río San Juan, Orinoco dr. c. MCP 45424 (WC04.010599), male, 204 mm TL, non-type (body in lateral view, specimen fixed in formalin and preserved in EtOH); d. MCP 45427 (WC01.130300), female, $153 \mathrm{~mm}$ TL, non-type (body in lateral view, specimen fixed in formalin and preserved in EtOH); Brazil, rio Solimões-Japurá confluence, Amazonas dr. Note the sexual dimorphism in caudal filament height. Scale bars $=5 \mathrm{~mm}$. 
Table 7. Morphometrics for Brachyhypopomus beebei, Brachyhypopomus belindae, and Brachyhypopomus benjamini. HT, holotype; SD, standard deviation. Data for holotype of B. beebei (Monagas, Venezuela) are from Schultz (1944). Ranges for $B$. beebei refer to non-type specimens from the vicinity of Tefé, Amazonas, Brazil, and exclude the holotype. Ranges for both $B$. belindae and $B$. benjamini refer to the holotype and to paratype specimens from the type region.

\begin{tabular}{|c|c|c|c|c|c|c|c|c|c|c|c|c|c|c|c|}
\hline & \multicolumn{5}{|c|}{ B. beebei } & \multicolumn{5}{|c|}{ B. belindae } & \multicolumn{5}{|c|}{ B. benjamini } \\
\hline & HT & Range & Mean & $\mathrm{SD}$ & $\mathrm{n}$ & HT & Range & Mean & $\mathrm{SD}$ & $\mathrm{n}$ & HT & Range & Mean & $\mathrm{SD}$ & $\mathrm{n}$ \\
\hline Total length (TL) (mm) & 136 & $64-256$ & 121 & 43.4 & 78 & 110 & $63-110$ & 91.8 & 17.8 & 5 & 126 & $79-136$ & 108 & 14.8 & 47 \\
\hline Length to end of anal fin (LEA) (mm) & 112 & $53-190$ & 99 & 34.5 & 78 & 92 & $52-92$ & 76.8 & 15.0 & 5 & 103 & $65-108$ & 89 & 12.1 & 47 \\
\hline Head length (HL) (mm) & 12.1 & $8.2-21.7$ & 14.4 & 3.2 & 30 & 9.1 & $6.6-9.1$ & 8.2 & 1.0 & 5 & 11.1 & 7.4-11.1 & 9.7 & 0.8 & 30 \\
\hline \multicolumn{16}{|c|}{ Proportion of TL (\%) } \\
\hline Caudal filament length (CF) & 15.4 & $9.0-25.8$ & 18.1 & 3.6 & 78 & 16.4 & $12.2-18.1$ & 16.4 & 2.4 & 5 & 18.3 & $11.3-22.5$ & 18.2 & 2.3 & 47 \\
\hline \multicolumn{16}{|c|}{ Proportion of LEA (\%) } \\
\hline Head length (HL) & 10.8 & $10.5-14.4$ & 11.9 & 0.9 & 27 & 9.9 & $9.9-12.7$ & 10.9 & 1.4 & 5 & 10.7 & $9.6-11.2$ & 10.3 & 0.4 & 30 \\
\hline Snout to occiput (SO) & 7.7 & $8.7-12.3$ & 9.9 & 0.9 & 26 & 7.9 & $7.9-10.5$ & 9.2 & 1.0 & 5 & 9.3 & $7.7-11.7$ & 9.2 & 0.8 & 30 \\
\hline Snout to anal-fin origin (SA) & 15.5 & $17.2-26.0$ & 21.5 & 1.9 & 26 & 16.5 & $16.5-23.6$ & 20.6 & 3.3 & 4 & 20.7 & $18.1-26.9$ & 20.9 & 2.0 & 30 \\
\hline Snout to pectoral-fin base (PP) & 10.0 & $11.9-16.8$ & 13.5 & 1.1 & 26 & 11.0 & $11.0-14.4$ & 12.1 & 1.4 & 5 & 11.5 & $10.4-12.7$ & 11.3 & 0.5 & 30 \\
\hline Longest pectoral-fin ray (PRL) & 4.8 & 4.7-6.1 & 5.4 & 0.3 & 26 & 4.7 & $4.2-5.0$ & 4.5 & 0.3 & 5 & 5.4 & $4.6-6.1$ & 5.2 & 0.3 & 30 \\
\hline Anal fin length (AF) & & $75.0-84.2$ & 80.0 & 2.3 & 26 & 81.5 & $79.2-82.7$ & 81.6 & 1.4 & 5 & 81.6 & $76.5-81.6$ & 79.8 & 1.2 & 30 \\
\hline Longest anal-fin ray (ARL) & 3.6 & $1.6-3.9$ & 2.6 & 0.6 & 26 & 3.7 & $1.9-4.7$ & 3.8 & 1.1 & 5 & 3.5 & $2.0-4.1$ & 3.5 & 0.5 & 30 \\
\hline Body depth at anal-fin origin (BD1) & & $11.0-14.8$ & 13.1 & 1.1 & 26 & 9.7 & $9.5-12.9$ & 10.4 & 1.4 & 5 & 10.0 & $8.3-10.9$ & 9.5 & 0.6 & 30 \\
\hline Body width at anal-fin origin (BW1) & & $5.4-8.0$ & 6.5 & 0.6 & 26 & 5.3 & $5.3-6.0$ & 5.5 & 0.3 & 5 & 5.6 & $3.9-5.6$ & 5.0 & 0.4 & 30 \\
\hline Body depth at anal-fin middle (BD2) & & $8.9-11.8$ & 10.3 & 0.8 & 26 & 9.2 & $9.2-10.4$ & 9.8 & 0.6 & 5 & 8.4 & 7.0-9.4 & 8.3 & 0.5 & 30 \\
\hline Body width at anal-fin middle (BW2) & & $2.2-3.4$ & 2.8 & 0.3 & 26 & 3.1 & $2.4-3.5$ & 3.2 & 0.5 & 5 & 2.8 & $2.2-3.6$ & 3.0 & 0.4 & 30 \\
\hline Caudal filament length (CF) & 18.8 & $10.7-32.4$ & 22.8 & 5.5 & 23 & 19.6 & $13.9-22.1$ & 19.6 & 3.3 & 5 & 22.3 & $14.1-29.0$ & 22.6 & 3.1 & 29 \\
\hline \multicolumn{16}{|c|}{ Proportion of HL (\%) } \\
\hline Preorbital distance (PR) & 27.0 & $25.7-31.9$ & 28.3 & 1.6 & 30 & 22.6 & $22.6-26.4$ & 24.9 & 1.4 & 5 & 25.7 & $22.6-27.2$ & 24.9 & 1.2 & 30 \\
\hline Mouth width (MW) & & $18.1-25.0$ & 20.8 & 1.7 & 30 & 29.2 & $21.1-29.2$ & 25.1 & 3.7 & 5 & 26.4 & $20.7-35.3$ & 29.8 & 2.9 & 30 \\
\hline Internarial distance (AE) & 18.4 & $19.8-26.3$ & 22.9 & 1.6 & 30 & 22.3 & $21.5-26.2$ & 23.8 & 1.9 & 5 & 20.2 & $16.7-25.5$ & 19.0 & 1.6 & 30 \\
\hline Posterior naris to eye (PE) & & $2.3-6.1$ & 4.0 & 0.9 & 30 & 2.3 & $2.2-4.6$ & 3.1 & 1.0 & 5 & 5.3 & $4.5-8.2$ & 6.6 & 0.8 & 30 \\
\hline Orbital diameter (OD) & 10.3 & $9.6-12.7$ & 10.8 & 0.8 & 30 & 10.8 & $9.8-11.9$ & 10.9 & 0.7 & 5 & 13.5 & $10.9-14.6$ & 12.9 & 0.9 & 30 \\
\hline Interorbital distance (IO) & 25.0 & 21.4-31.7 & 25.6 & 2.6 & 30 & 27.8 & $23.3-33.3$ & 27.2 & 3.9 & 5 & 20.7 & $15.5-26.4$ & 22.1 & 2.3 & 30 \\
\hline Head depth at eye (HD1) & & $48.6-58.3$ & 53.3 & 2.8 & 30 & 47.3 & $47.3-55.5$ & 50.9 & 3.1 & 5 & 42.5 & $39.0-49.0$ & 43.9 & 2.3 & 30 \\
\hline Head width at eye (HW1) & 36.9 & $35.7-51.7$ & 43.5 & 4.0 & 30 & 42.9 & $33.3-43.4$ & 40.5 & 4.2 & 5 & 42.5 & $34.6-46.2$ & 40.0 & 3.1 & 30 \\
\hline Head depth at occiput (HD2) & & $71.2-86.7$ & 79.1 & 3.5 & 30 & 76.5 & $75.6-79.1$ & 77.1 & 1.5 & 5 & 69.9 & $63.5-79.3$ & 72.4 & 3.4 & 30 \\
\hline Head width at occiput (HW2) & & $48.8-65.9$ & 58.5 & 3.8 & 30 & 57.1 & $51.1-61.2$ & 57.0 & 3.7 & 5 & 51.8 & $44.8-56.8$ & 51.6 & 3.3 & 30 \\
\hline Postorbital distance (PO) & 70.1 & $61.0-67.6$ & 65.1 & 1.4 & 30 & 65.9 & $60.4-65.9$ & 63.4 & 2.1 & 5 & 59.6 & $56.9-66.9$ & 62.9 & 2.3 & 30 \\
\hline Branchial aperture (BO) & 19.9 & 21.9-32.0 & 26.7 & 2.3 & 30 & 25.8 & $20.3-26.5$ & 24.0 & 2.5 & 5 & 22.6 & $15.2-24.7$ & 20.1 & 2.2 & 30 \\
\hline Pectoral-fin base (PB) & & $10.0-16.4$ & 13.1 & 1.7 & 30 & 9.1 & $9.1-14.1$ & 11.0 & 2.2 & 5 & 9.9 & $7.4-13.3$ & 10.3 & 1.1 & 30 \\
\hline Caudal filament depth (CD) & & $11.5-25.8$ & 16.6 & 4.1 & 29 & 21.1 & $14.5-21.1$ & 16.9 & 2.6 & 5 & 15.9 & $13.7-19.1$ & 16.6 & 1.2 & 30 \\
\hline Caudal filament width (CW) & & 2.9-11.4 & 5.8 & 1.8 & 29 & 7.3 & $4.4-7.3$ & 5.4 & 1.2 & 5 & 7.0 & $4.3-9.2$ & 6.6 & 1.1 & 30 \\
\hline \multicolumn{16}{|c|}{ Other proportions } \\
\hline BW1 (\% of BD1) & & $41.9-67.1$ & 49.8 & 4.9 & 30 & 54.6 & $46.3-57.4$ & 53.6 & 4.0 & 5 & 55.8 & $46.7-59.1$ & 52.4 & 2.9 & 30 \\
\hline BW2 (\% of BD2) & & $22.9-32.1$ & 270.0 & 2.1 & 29 & 33.4 & $26.0-34.1$ & 32.1 & 3.0 & 5 & 33.8 & $29.5-42.7$ & 35.3 & 3.2 & 30 \\
\hline
\end{tabular}

Hypopomus beebei Schultz, 1944: 40, fig. 1, cephalic sensory pore arrangement; 46, pl. 1, fig. 4, black and white photograph of holotype (original description, type locality - Venezuela, Caripito, Monagas, río San Juan, Orinoco dr.). -Schultz, 1949: 66, key to the Hypopomidae, descriptive notes; fig. 9b, cephalic sensory pore arrangement; pl. 2, black and white photograph of holotype (Venezuela, listing of freshwater fish species). -Hoedeman, 1962: 60, fig. 6b, cephalic sensory pores and canals; fig. $7 \mathrm{~b}$, pigmentation (Suriname, listing of new gymnotiform records). -Hopkins \& Heiligenberg, 1978: 132, fig. 10, black and white photograph and EOD (Suriname, species and EOD diversity). -Westby \& Shepherd, 1986: 6, fig. 2; Westby, 1988: 347, fig. 8, black and white photographs of adult male, female and larval specimen, with EODs (French Guiana, species and EOD diversity]. -Hopkins, 1991: 155, fig. 2, black and white photographs of preserved specimens (French Guyana, comparison to Brachyhypopomus pinnicaudatus). -Ferraris \& Vari, 1992: 5 (type catalog). 
Brachyhypopomus beebei. -Mago-Leccia, 1994: 48, 170, fig. 71, black and white photograph of head of holotype (listing of Brachyhypopomus). -Crampton, 1996a: 77, table 6.1.a, species list; 79, fig. 6.1 inset 17-18, color photographs; 85, fig. 6.2; 88, fig. 6.3; 92-94, figs. 6.4a, 6.5; 191, fig. 11.2; 193, fig. 11.3, EOD data; 110 , table $7.5,113$, table 7.7, 115, fig. 7.1, habitats; 136 , table 8.2a,b, diet; 151-160, figs. 9.2, 9.3, table 9.1a,b, hypoxia tolerance; 165-169, table 10.1 reproductive biology (Brazil, central Amazon, ecology and signal diversity). -Planquette et al., 1996: 396, color photograph of live individual (French Guiana, listing of Brachyhypopomus). -Alves-Gomes, 1997: 523 (Brazil, Roraima, listing of gymnotiforms). -Sullivan, 1997: 110 (redescription). -Crampton, 1998a: 821, table 4, 834, fig. 9, drawing of adult specimen, with EOD (Brazil, Amazonas, species and EOD diversity). -Crampton, 1998b: 314, table 2, list of Brachyhypopomus (Brazil, Amazonas, hypoxia tolerance). -Provenzano et al., 1998: 11 (type catalog for MBUCV-V). -Crampton, 1999: 17 (Brazil, Amazonas, Mamirauá Reserve, listing of species). -Stoddard, 1999: 255, fig. 4, color photograph of mature female (EOD evolution). - Stoddard et al., 1999: 610, fig. 1, black and white photograph of mature female with EOD (EOD diversity in Brachyhypopomus). - Ponton \& Mérigoux, 2001: 94, fig. 100, drawings of post-larval morphology (French Guiana, Sinnamary dr. larval ecology). -Albert \& Crampton, 2003: 494 (Bolivia, Brazil, Colombia, Ecuador, Guyana, Paraguay, Peru, Suriname, and Venezuela, catalog of hypopomids).-Triques \& Khamis, 2003: 63 (diagnosis against, and material examined; in description of $B$. jureiae). -Crampton \& Albert, 2006: 672, fig. 23.8, position in phylogenetic tree; 681, notes on EODs (gymnotiform species and EOD diversity). -Galvis et al., 2006: 362, fig. 211, drawing of head; 528 (Colombia, Amazonas, catalog of fishes). -Vari et al., 2009: 46 (Guiana Shield, catalog of fishes). -Crampton, 2011: 176, table 10.2, species list; 179, figs. 10.2-10.3, phylogeny, geographical and ecological distributions (gymnotiform biology). -Mol, 2012: 596, color photograph of preserved specimen, reproduction of photograph from Planquette et al., 1996 (Suriname, catalog of fishes). -Carvalho, 2013: 181-185, figs. 41-43, position in phylogeny (phylogenetic systematics of Rhamphichthyoidea). -Crampton \& Ribeiro, 2013: 236, color photograph (Brazil, rio Madeira, listing of hypopomids). -Tagliacollo et al., 2016: 28, fig. 5 (position in phylogeny of the Gymnotiformes). -Crampton et al., 2016: 1-66, table 1, 3-4, figs. 1-7, 8, 14, 16-17, 18-20 (phylogeny, biogeography and ecology of Brachyhypopomus).

Brachyhypopomus brevirostris. Kirschbaum, 1995: 452, black and white photograph (gymnotiform diversity).

Diagnosis. Brachyhypopomus beebei is diagnosed from congeners by the following combination of characters: prominent pale uninterrupted middorsal stripe from occipital region to base of caudal filament present, vs. absent in all congeners except $B$. arrayae, $B$. belindae, $B$. gauderio, $B$. pinnicaudatus, and $B$. verdii; anal-fin rays 214-230, vs. 174 212 in $B$. arrayae and B. pinnicaudatus; precaudal vertebrae 21-23, vs. 24-26 in B. belindae and B. verdii; longest anal-fin ray $1.6-3.9 \%$ LEA, vs. $3.9-5.4 \%$ in $B$. gauderio.
Description. Head and body shape, and pigmentation illustrated in Figs. $1 \mathrm{~b}$ and 9. Meristic and morphometric data for examined specimens are presented in Tables 2-5, and 7. Data presented here refer to material from the central Amazon of Brazil unless otherwise stated. Body moderate to broad in depth. Head short to long, and shallow to moderate in depth. Dorsal profile of head straight to slightly convex from occiput to snout, ventral profile of head straight to slightly convex between operculum and snout, snout rounded and bulbous. Eye moderate in size. Upper jaw with moderate sigmoidal angle between premaxillary and maxillary portions in lateral view. No accessory electric organ over operculum. Gill filaments on first gill arch 35-55 (median 50, $\mathrm{n}=20$ ). Pectoral fin moderate to broad in width, pectoral-fin rays 15-19 (mode 14). Precaudal vertebrae 21 23 (mode 22) including 1-3 (mode 2) transitional vertebrae. Anal-fin origin slightly $(<0.25 \mathrm{HL}$ distance) anterior or posterior to, or near, tip of pectoral fin. Anal-fin rays 214230 (215-230, median 222 in population from central Amazon; 214 in type region). Dorsal rami of recurrent branch of anterior lateral line nerve not visible. Middorsal region of body scaled. Rows of scales above lateral line 5-7 (mode 6). Lateral line continuous. Groove-like epidermal canals found mainly in posterior half of body - as abundant parallel crisscrossing depigmented grooves either side of lateral line, as isolated horizontal depigmented grooves on flank midway from lateral line to dorsal midline, and as long closely-spaced parallel dark-pigmented lines either side of dorsal surface in dorsal portion of flank. Three bilateral horizontal columns of electrocytes at anal-fin terminus in immature specimens, mature females, and mature males. Three bilateral horizontal columns of electrocytes at midpoint between anal-fin terminus and tip of caudal filament in immature specimens and mature females. Three to four columns of electrocytes at mid-point between anal-fin terminus and tip of caudal filament in mature males (three columns only in specimens from low-conductivity systems; four only in specimens from high-conductivity floodplain systems $>90 \mu \mathrm{Scm}^{-1}$ ). Caudal filament short to moderate in length.

Coloration. (Figs. 1b, 9). Background light tan or grey to dark brown. Head darker, especially dorsally. Dorsal region with prominent depigmented pale stripe extending along midline from occipital region to deep into caudal filament. Pale middorsal stripe flanked in anterior portion of body with very thin dark horizontally oriented lines from which thin wavy lines extend ventrally across dorsal and ventral portions of flank. Pale middorsal stripe flanked in posterior portion of body with approximately vertically oriented thin dark lines which extend down flank, some of which cross lateral line into ventral flank. Ventral region with thin wavy dark lines extending from anal-fin margin towards dorsal margin of pterygiophores, some of which extend dorsally to join lines descending from dorsal flank. In caudal filament dark markings form vertical patches or stripes. 
Head with evenly scattered dark chromatophores, darker dorsally. Eye without prominent suborbital patch, or stripe, of chromatophores/subcutaneous pigmentation. Pectoral and anal-fin membranes hyaline. Pectoral and anal-fin rays hyaline with light scattering of brown chromatophores. Anal-fin ray pigmentation darker in posterior half of fin. Juvenile specimens typically exhibit high contrast dark lines on the flank, set against a pale background pigmentation. In large adults background is typically darker and boundaries of dark lines broken, and less distinctive. Color in live individuals similar to preserved specimens, with opercular region usually rosy due to underlying gills.

Size. Moderate adult size, largest specimen examined 256 $\mathrm{mm}$ TL, $190 \mathrm{~mm}$ LEA $(\mathrm{n}=865)$. Largest male specimen examined $256 \mathrm{~mm}$ TL, $190 \mathrm{~mm} \mathrm{LEA}(\mathrm{n}=36)$. Largest female specimen examined $197 \mathrm{~mm}$ TL, $165 \mathrm{~mm}$ LEA $(\mathrm{n}=62)$.

Sexual dimorphism. Males attain larger sizes than females. Males in breeding condition exhibit longer and deeper caudal filaments than immature individuals and breeding females (Figs. 9c-d), but do not exhibit an elevated number of bilateral horizontal columns or vertical rows of electrocytes (although we noted that mature males in high-conductivity systems have an extra horizontal column of electrocytes at mid caudal filament, Table 5). Breeding males nonetheless exhibit clearly enlarged electrocytes relative to immature specimens and females. Large males often with paddle-like lateral compression at caudal filament tip (Figs. 9c-d). No known sexual dimorphism in pigmentation.

Geographic distribution. Brazil, Colombia, Ecuador, French Guiana, Guyana, Peru, Suriname, and Venezuela (Fig. 10). Widespread through cis-Andean northern South America, including large areas of the Amazonas and Orinoco drainages, and coastal drainages of the Guianas and Amapá State, Brazil. In the Amazon basin apparently absent from the rio Madeira above its series of falls beginning at the Cachoeira de Santo Antônio, near Porto Velho, Rondônia, Brazil. As yet unknown from some major tributaries of the Amazon, including the Juruá, Tocantins, and Xingu. Collection records from tributaries in the northern and western portions of the Orinoco drainage are sparse.

Ecological notes. Brachyhypopomus beebei is an ecologically cosmopolitan species, and is locally common in lentic and slow-flowing freshwater environments throughout its range (Alves-Gomes, 1997; Crampton, 1996a; 1998a; Crampton \& Albert, 2006; Crampton \& Ribeiro, 2013; Hopkins \& Heiligenberg, 1978; Mol, 2012; Westby, 1988). It occurs in seasonally inundated whitewater floodplains (in floating rafts of meadows and shallow newly-flooded forests), along the margins of rivers (in emergent and floating vegetation), in ephemeral or perennial swamps (in vegetation or submerged debris), and in terra firme forest and savanna streams (in emergent and submerged vegetation, leaf litter and debris, and in marginal root mats and holes). In the lowland Amazon, Brachyhypopomus beebei occurs in both low-conductivity blackwater forest streams and swamps $\left(c a .5-30 \mu \mathrm{Scm}^{-1}\right)$ and in high-conductivity whitewater floodplain systems (ca. 90-250 $\mu \mathrm{Scm}^{-1}$ ), as well as in waters of intermediate conductivity (Crampton, 1996a). Brachyhypopomus beebei often occurs in extremely hypoxic swamp and floodplain systems and is able to tolerate protracted periods of hypoxia by undertaking aerial gill respiration and (during periods of anoxia) by reducing activity (Crampton, 1998b). Breeding occurs during the rising and high water period in populations from central Amazonian floodplain floating macrophytes (Crampton, 1996a: 165). Westby (1988) reported the presence of larval $B$. beebei at the beginning of the rainy season in small streams of coastal French Guiana. Westby (1988) also noted that larval B. beebei, estimated to be less than one week from hatching, occurred in "crèches" of 1522 specimens. However, he found no evidence for parental care. Stomach contents in populations from the central Amazon comprise aquatic insect larvae, microcrustacea, and other small aquatic invertebrates - with a predominance of Chironomidae larvae (Crampton, 1996a: 136). Large specimens often consume larger aquatic invertebrates.

Co-occurring congeners: Because of its wide geographical range and occurrence in multiple habitats, $B$. beebei occurs in geographical sympatry and ecological syntopy with multiple congeners: in the Amazonas drainage and coastal drainages of the Guianas: $B$. batesi, B. belindae, $B$. benjamini, $B$. bennetti, $B$. brevirostris, B. cunia, $B$. hamiltoni, $B$. hendersoni, $B$. pinnicaudatus, $B$. regani, $B$. sullivani, $B$. verdii, $B$. walteri; in the Orinoco drainage: $B$. bullocki, B. diazi, B. brevirostris, B. provenzanoi, B. regani, and $B$. sullivani.

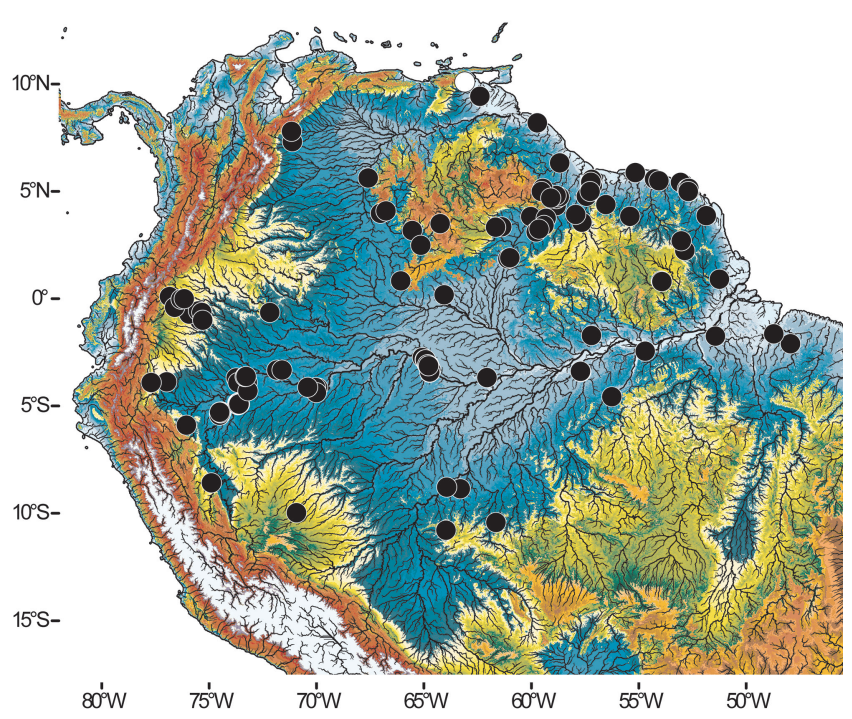

Fig. 10. Collection records for Brachyhypopomus beebei (circles). Holotype location is marked with an open symbol. Elevation data refers to altitude above mean sea level (see Fig. 2 for legend). 
Local names. Brazil: sarapó; Colombia: cuchillo/cuchillo amarillo (Amazon and Orinoco), cuchilleja macana (Putumayo); Ecuador: cuchillo, yayo; French Guiana: poisson couteau (French), and multiple creole/Amerindian names (Planquette et al., 1996); Peru: macana; Suriname: saprapi; Venezuela: cuchillo.

Material examined. 848 specimens. Brazil. Amapá. MNHN 1981-0494, 1 (of 3), $88 \mathrm{~mm}$, rio Ipa, rio Oiapoque drainage, no coordinates. MPEG 3341, 2, 143-170 mm, mun. Ferreira Gomes, rio Cupixi, bridge on hwy. to Serra do Navio, rio Araguari drainage, ca. $00^{\circ} 55^{\prime} \mathrm{N}, 051^{\circ} 15^{\prime} \mathrm{W}$. Amazonas (localities from Amazonas dr., localities listed from the Mamirauá Reserve [Reserva de Desenvolvimento Sustentável Mamirauá] are in rio SolimõesJapurá floodplain, mun. Alvarães). BMNH 1998.3.12.45-53, 9 (6 immature, $122-176 \mathrm{~mm}, 2$ female, $150-190 \mathrm{~mm}, 1$ male, $145 \mathrm{~mm}$ ), IDSM 442, 8, 73-187 mm, MCP 45313, 1, immature, Mamirauá Reserve, cano do lago Rato, $03^{\circ} 02^{\prime} 41^{\prime \prime} \mathrm{S}, 064^{\circ} 51^{\prime} 26^{\prime \prime} \mathrm{W}$. BMNH 1998.3.12.54, 1, immature, $122 \mathrm{~mm}$, mun. Tefé, floodplain lake, Ilha Panamim, rio Solimões, $03^{\circ} 17^{\prime} 38^{\prime \prime} \mathrm{S}, 064^{\circ} 41^{\prime} 16^{\prime \prime} \mathrm{W}$. BMNH 1998.3.12.55, 1, immature, $150 \mathrm{~mm}$, MCP 45381, 5 (2 immature [1CS], 105-140 mm, 2 female, 162-164 mm, 1 male, $220 \mathrm{~mm}$ ), Mamirauá Reserve, Ressaca do Caetono, 02 $50^{\prime} 15^{\prime \prime} \mathrm{S}$, $064^{\circ} 55^{\prime} 50^{\prime \prime} \mathrm{W}$. IDSM 452, 3, 62-98 mm, Mamirauá Reserve, lago Jaraqui, $02^{\circ} 44^{\prime} 07^{\prime \prime} \mathrm{S}, 065^{\circ} 04^{\prime} 38^{\prime \prime} \mathrm{W}$. IDSM 453, 6, 52-129 mm, MCP 45424, 5 (3 immature, 94-132 mm, 1 female, $171 \mathrm{~mm}, 1$ male, $204 \mathrm{~mm}$ ), MCP 45428, 5, 45-194 mm, MCP 45450, 5 (2 immature [2CS], 102-195 mm, 3 female [2CS], 138-178 mm),

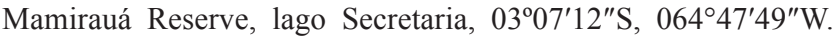
INPA 9943, 15, 85-150 mm, Mamirauá Reserve, lago Mamirauá, ca. $03^{\circ} 07^{\prime} \mathrm{S}, 064^{\circ} 47^{\prime} \mathrm{W}$. INPA 18335,1 , immature, $114 \mathrm{~mm}$, Mamirauá Reserve, cano do lago Arauaé, $03^{\circ} 03^{\prime} 54^{\prime \prime} \mathrm{S} 64^{\circ} 49^{\prime} 04^{\prime \prime} \mathrm{W}$. INPA 18336, 1, immature, 53 mm, MCP 45358, 1, immature, 109 $\mathrm{mm}$, Mamirauá Reserve, lago Araçazinho, $02^{\circ} 59^{\prime} 35^{\prime \prime} \mathrm{S}$, 064 $54^{\prime} 33^{\prime \prime}$ W. INPA 18337, 1, immature, $90 \mathrm{~mm}, \mathrm{MCP} 45361,1$, immature, $63 \mathrm{~mm}$, МСP 45385, 2, immature, $120-146 \mathrm{~mm}$, Mamirauá Reserve, cano do lago Sapucaia, $03^{\circ} 04^{\prime} 07^{\prime \prime} \mathrm{S}$, $064^{\circ} 48^{\prime} 32^{\prime \prime} \mathrm{W}$. INPA 18338, 1, immature, $114 \mathrm{~mm}, \mathrm{MCP} 45343,1$, female, $198 \mathrm{~mm}$, MCP 45380, 1, immature, 65 mm, Mamirauá Reserve, cano do lago Mamirauá, $03^{\circ} 06^{\prime} 40^{\prime \prime} \mathrm{S}, 64^{\circ} 47^{\prime} 52^{\prime \prime} \mathrm{W}$. INPA 18339, 1, immature, $50 \mathrm{~mm}, \mathrm{MCP} 45427,3$ (2 female [1CS], 136$153 \mathrm{~mm}, 1 \mathrm{male}, 170 \mathrm{~mm}$ ), mun. Tefé, igarapé Curupira, Estrada Agrovila rd., affl. lago Tefé, rio Tefé, $03^{\circ} 25^{\prime} 48^{\prime \prime} \mathrm{S}, 064^{\circ} 44^{\prime} 15^{\prime \prime} \mathrm{W}$. INPA 18340, 1, immature, $59 \mathrm{~mm}$, MCP 45488, 1, female, 136 $\mathrm{mm}$, mun. Tefé, terra firme swamp, igarapé Repartimento, Estrada Agrovila rd., affl. lago Tefé, rio Tefé, $03^{\circ} 24^{\prime} 25^{\prime \prime} \mathrm{S}, 04^{\circ} 44^{\prime} 08^{\prime \prime} \mathrm{W}$. INPA 18341, 1, immature, 75 mm, Mamirauá Reserve, Paraná Maiana, $03^{\circ} 06^{\prime} 50^{\prime \prime} \mathrm{S}, 064^{\circ} 47^{\prime} 48^{\prime \prime} \mathrm{W}$. MCP 45342 , 1 , immature, 133 $\mathrm{mm}, \mathrm{MCP} 45344$, 1, immature, $135 \mathrm{~mm}$, Mamirauá Reserve, lago Arauaé, $03^{\circ} 02^{\prime} 52^{\prime \prime} \mathrm{S}, 064^{\circ} 50^{\prime} 04^{\prime \prime} \mathrm{W}$. MCP 45382, 2 , immature, 118 $139 \mathrm{~mm}$, Mamirauá Reserve, Ressaca do Itu, 02 $49^{\prime} 51^{\prime \prime} \mathrm{S}$, $064^{\circ} 57^{\prime} 11^{\prime \prime W}$. MCP 45383, 1, female, 185 mm, Mamirauá Reserve, Ressaca da Vila Alencar, $03^{\circ} 07^{\prime} 41^{\prime \prime} \mathrm{S}, 064^{\circ} 48^{\prime} 04^{\prime \prime} \mathrm{W}$. MCP 45387, 1, immature (CS), $97 \mathrm{~mm}$, Mamirauá Reserve, lago Periquito Comprido, 030. $4^{\prime} 57^{\prime \prime} \mathrm{S}, 064^{\circ} 46^{\prime} 42^{\prime \prime} \mathrm{W}$. MCP 45420, 1, male, 197 mm, Mamirauá Reserve, lago Curuçá Aberto, $03^{\circ} 06^{\prime} 07^{\prime \prime} \mathrm{S}$, $064^{\circ} 49^{\prime} 10^{\prime \prime} \mathrm{W}$. MCP 45421, 2 (1 immature, $132 \mathrm{~mm}, 1$ male [CS], $201 \mathrm{~mm}$ ), Mamirauá Reserve, cano do lago Mamirauá, 03ํำ'29"S, $064^{\circ} 48^{\prime} 29^{\prime \prime}$ W. MCP 45422, 1, male, 226 mm, Mamirauá Reserve, cano do lago Mamirauá, $03^{\circ} 05^{\prime} 15^{\prime \prime} \mathrm{S}, 064^{\circ} 48^{\prime} 03^{\prime \prime} \mathrm{W}$. MCP 45459 , 3, 95-118 mm, Mamirauá Reserve, Ressaca do Caetono, $02^{\circ} 50^{\prime} 15^{\prime \prime} \mathrm{S} 64^{\circ} 55^{\prime} 50^{\prime \prime} \mathrm{W}$. MCP 45460, 1, immature, $72 \mathrm{~mm}$, MCP 45462, 6 (5 not sexed, 83-151 mm, 1 female, $134 \mathrm{~mm}$ ), mun. Tefé, igarapé Repartimento, $1.5 \mathrm{~km}$ downstream bridge Estrada Agrovila rd., affl. lago Tefé, rio Tefé, $03^{\circ} 24^{\prime} 25^{\prime \prime} \mathrm{S}, 064^{\circ} 44^{\prime} 08^{\prime \prime} \mathrm{W}$. MPEG 22741, 1, $108 \mathrm{~mm}$, Mamirauá Reserve, cano do lago Geraldo, $03^{\circ} 07^{\prime} 20^{\prime \prime} \mathrm{S}, 064^{\circ} 48^{\prime} 06^{\prime \prime} \mathrm{W}$. MPEG 22742, 2, 90-145 mm,

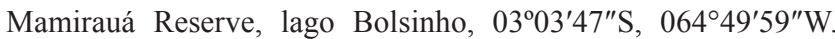
MZUSP 7293, 4, mun. Maués, igarapé do rio Maraú, affl. rio Maués-Açu, $c a .03^{\circ} 24^{\prime} \mathrm{S}, 057^{\circ} 42^{\prime} \mathrm{W}$. MZUSP 23239 , 9, lago Miuá, upstream Codajás, rio Solimões, $c a .03^{\circ} 40^{\prime} \mathrm{S}, 062^{\circ} 04^{\prime} \mathrm{W}$. MZUSP 44164, 2, mun. Maués, igarapé Limãozinho, affl. rio Maués-Açu, ca. $03^{\circ} 23^{\prime} \mathrm{S}, 057^{\circ} 43^{\prime} \mathrm{W}$ [coordinates for Maués]. MZUSP 62069 , $1,53 \mathrm{~mm}$, pool on beach, Tapera, rio Negro, $c a \cdot 00^{\circ} 11^{\prime \prime} \mathrm{N}$, 0640ㄴ"W. UF 177346, 13, 64-211 mm, Mamirauá Reserve, lago Secretaria, $03^{\circ} 07^{\prime} 12^{\prime \prime}$ S, $064^{\circ} 47^{\prime} 49^{\prime \prime W}$. Maranhão. USNM 88283, 1, 140 mm, "Maranhão, Brazil", no coordinates. Pará (localities from Amazonas dr.). INPA 6887, 1, $131 \mathrm{~mm}$, Pimental, rio Tapajós, ca. $04^{\circ} 34^{\prime} \mathrm{S}, 056^{\circ} 15^{\prime} \mathrm{W}$. MCP 23359 (part), 1, $94 \mathrm{~mm}$, Concórdia do Pará, rio Bujarú, affl. rio Guamá, affl. Baía do Guajará, $c a$. $02^{\circ} 06^{\prime} \mathrm{S}, 047^{\circ} 56^{\prime} \mathrm{W}$. MPEG 7140, 1, $95 \mathrm{~mm}$, mun. Barcarena, São Sebastião, rio Arienga, affl. Baía do Guajará, $01^{\circ} 38^{\prime} \mathrm{S}, 048^{\circ} 43^{\prime} \mathrm{W}$. MPEG 10164, 1, 115 mm, mun. Portel, Floresta Nacional Caxiuanã, affl. rio Anapú, affl. rio Pará, affl. Baía do Guajará, $c a$. $01^{\circ} 44^{\prime} \mathrm{S}, 051^{\circ} 26^{\prime} \mathrm{W}$. MPEG 15336, 1, $99 \mathrm{~mm}$, mun. Faro, unnamed stream, affl. rio Nhamundá, $01^{\circ} 42^{\prime} 23^{\prime \prime} \mathrm{S}, 057^{\circ} 12^{\prime} 10^{\prime \prime} \mathrm{W}$. MPEG 15646, 1, $130 \mathrm{~mm}$, mun. Almeirim, rio Ipitinga, affl. rio Jari, $00^{\circ} 49^{\prime} 52^{\prime \prime} \mathrm{N}, 053^{\circ} 57^{\prime} 20^{\prime \prime} \mathrm{W}$. MPEG 15679, 1, $122 \mathrm{~mm}$, mun. Almeirim, rio Ipitinga, affl. rio Jari, $00^{\circ} 48^{\prime} 34^{\prime \prime} \mathrm{N}, 053^{\circ} 55^{\prime} 52^{\prime \prime} \mathrm{W}$. SU 54499, 6, 74-106 mm, SU 66948, 1, $90 \mathrm{~mm}$, Urumari brook into rio Tapajós, Santarém, $c a .02^{\circ} 26^{\prime} \mathrm{S}, 054^{\circ} 42^{\prime} \mathrm{W}$ [coordinates for Santarém]. Rondônia (localities from rio Madeira dr., Amazonas dr.). INPA 818, 2, 118-127 mm, INPA 1154, 2, 121-132 mm, igarapé "13.05", rio Mutum, Serra Pacaás Novos, affl. rio Mamoré [coordinates for Serra Pacaás Novos], ca. $10^{\circ} 47^{\prime} \mathrm{S}$, 0635'ㄴ'W. INPA 19947, 4, 61-153 mm, INPA 19948, 73, 34-131 $\mathrm{mm}$, Lagoa do Feijoal, rio Jamari, upstream UHF Samuel, $c a .15$ $\mathrm{km}, \mathrm{ca} .08^{\circ} 51^{\prime} \mathrm{S}, 063^{\circ} 19^{\prime} \mathrm{W}$. UFRO-I 4470, $1,118 \mathrm{~mm}$, igarapé Lourdes, affl. igarapé Cristal, affl. rio Jí-Paraná, $10^{\circ} 25^{\prime} 13^{\prime \prime} \mathrm{S}$, $61^{\circ} 39^{\prime} 12^{\prime \prime} \mathrm{W}$. UFRO-I 6481, 1, $91 \mathrm{~mm}$, flooded area immediately downstream Cachoeira Santo Antônio, rio Madeira, 0846'30"S $63^{\circ} 56^{\prime} 28^{\prime \prime} \mathrm{W}$. Roraima (localities from rio Branco dr., affl. rio Negro, Amazonas dr.). INPA 6404, 4, 43-108 mm, Ilha de Maracá, stream at end of island, rio Uraricoera, $c a .03^{\circ} 22^{\prime} \mathrm{N}, 061^{\circ} 23^{\prime} \mathrm{W}$. INPA 7387, 1, igarapé Viruaquim [Juruaquim], 48.8 km Normândia on hwy. RR-202, $03^{\circ} 50^{\prime} \mathrm{N}, 60^{\circ} 02^{\prime} \mathrm{W}$ (listed by Sullivan, 1997). MZUSP 23567, 1, stream ca. $1 \mathrm{~km} \mathrm{~N}$ Caracaraí, $01^{\circ} 52^{\prime} \mathrm{N}$, $061^{\circ} 06^{\prime} \mathrm{W}$. MZUSP 30037, 48, MZUSP 30039, 58, igarapé do Cujobim, Ilha de Maracá, rio Uraricoera, $c a .03^{\circ} 20^{\prime} \mathrm{N}, 061^{\circ} 40^{\prime} \mathrm{W}$. MZUSP 30038, 10, igarapé do Bota Panela, nr. Cachoeira do Bem Querer, rio Branco, $c a \cdot 01^{\circ} 55^{\prime} \mathrm{N}, 061^{\circ} 00^{\prime} \mathrm{W}$. Colombia. Amazonas. ROM 56332, 1, $207 \mathrm{~mm}, 5 \mathrm{mi}$. W Leticia, Amazonas dr., 
0409'02"S, 06957'00"W. Caquetá. SINCHI-CIACOL 102, 2, 42-129 mm, Comunidad Aduche (San Miguel), Pueblo Andoke, Poblado Araracuara, affl. río Caquetá, Amazonas dr., 00³8'18"S, $072^{\circ} 10^{\prime} 54^{\prime \prime}$ W. Ecuador (localities from río Napo dr., Amazonas dr.) Francisco de Orellana. FMNH 102272, 1, 60 mm, Laguna Jatuncocha, río Yasuni, $01^{\circ} 00^{\prime} \mathrm{S}, 075^{\circ} 27^{\prime} \mathrm{W}$. QCAZ-I 1369, 1, 123 $\mathrm{mm}$, Chiruisla, río Huiririma, affl. río Tiputini, $00^{\circ} 38^{\prime} 54^{\prime \prime} \mathrm{S}$, 07554'44"W. QCAZ-I 1802, 1, 147 mm, QCAZ-I 1803, 1, 162 $\mathrm{mm}$, Chiruisla, Laguna Muyuna, affl. río Tiputini, $00^{\circ} 42^{\prime} 25^{\prime \prime} \mathrm{S}$, $075^{\circ} 56^{\prime} 08^{\prime \prime} \mathrm{W}$. Sucumbios. ANSP 130488, 5, 37-103 mm, swamp at Santa Cecília, río Aguarico, ca. $00^{\circ} 06^{\prime} \mathrm{N}, 076^{\circ} 51^{\prime} \mathrm{W}$. FMNH 102271, 1, $76 \mathrm{~mm}$, Laguna de Limoncocha, 00²3'42"S, $076^{\circ} 36^{\prime} 49^{\prime \prime}$ W. FMNH 102279, 1, 154 mm, small stream between Laguna Zancudo and Laguna Zancudococha, affl. quebrada Zancudococha, affl. río Aguarico, 00³3'42"S, 075³0'00"W. FMNH 102281, 6, 34-103 mm, ca. 2 km S Marian, río Aguas Negras, about 1-2 km upstream from rd. bridge, affl. río Cuyabeno, affl. río Aguarico, $c a .00^{\circ} 01^{\prime} \mathrm{S}, 076^{\circ} 19^{\prime} \mathrm{W}$. FMNH 102284, 6, 20$93 \mathrm{~mm}$, affl. Laguna Grande de Cuyabeno, affl. río Cuyabeno, affl. río Aguarico, ca. $00^{\circ} 00^{\prime} \mathrm{S}, 076^{\circ} 10^{\prime} \mathrm{W}$. FMNH 102290, 1, $51 \mathrm{~mm}$, affl. río Tarapuy, $\mathrm{N}$ bank just downstream rd. bridge, affl. río Cuyabeno, affl. río Aguarico, no coordinates. KU 12741, 1 (CS), 90 mm; KU 13799, 4, 38-120 mm; KU 13800, 24 (4 CS), 50-145 mm, swamp, S air strip at Santa Cecília, río Aguarico, ca. $03^{\circ} 52^{\prime} \mathrm{S}$, 07659'W. MUSM 5693 (part), 2, 107-117 mm, temporary lake, río Aguarico, $c a .00^{\circ} 38^{\prime} \mathrm{S}, 075^{\circ} 21^{\prime} \mathrm{W}$ [coordinates for mouth of río Aguarico]. French Guiana. Cayenne. BMNH 1926.3.2.658-660, 3, 78-85 mm, crique Iponcin, Approuage River dr., no coordinates. CU 71944, 2, on Rt. 1 Kourou to Sinnamary, km 86 in flooded border of crique Paracou, Kourou River dr., ca. $05^{\circ} 21^{\prime} \mathrm{N}, 052^{\circ} 56^{\prime} \mathrm{W}$ (listed by Sullivan, 1997). CU 71954, 1, Kourou River at Roche Leodate landing, ca. $32 \mathrm{~km}$ upstream from Kourou, Kourou River dr., $05^{\circ} 00^{\prime} \mathrm{N}, 052^{\circ} 39^{\prime} \mathrm{W}$ (listed by Sullivan, 1997). CU 71955, 1, crique Papinabo, ca. 21 mi. from Kourou, Kourou River dr., $05^{\circ} 06^{\prime} \mathrm{N}, 052^{\circ} 40^{\prime} \mathrm{W}$ (listed by Sullivan, 1997). CU 71956, 5, small coastal stream on "CTFT forestry estate" nr. crique Paracou, Kourou River dr., $c a .05^{\circ} 21^{\prime} \mathrm{N}, 052^{\circ} 56^{\prime} \mathrm{W}$ (listed by Sullivan, 1997). MNHN 1981-0495, 1, $78 \mathrm{~mm}$, Trois Sauts (falls) on Oyapock River, rio Oiapoque dr., $c a .02^{\circ} 15^{\prime} \mathrm{N}, 052^{\circ} 51^{\prime} \mathrm{W}$. MNHN 1981-0566, 1, 95 mm, saut Embouchure, Yaroupi River, affl. Oyapock River, rio Oiapoque dr., $c a .02^{\circ} 42^{\prime} \mathrm{N}, 053^{\circ} 01^{\prime} \mathrm{W}$. MNHN 1999-1314, 10 (of 39), 28-49 mm, crique Papinabo, Kourou River dr., $c a .05^{\circ} 06^{\prime} \mathrm{N}, 052^{\circ} 41^{\prime} \mathrm{W}$. MNHN 1999-1321, 1 (of 3), $120 \mathrm{~mm}$, Oyapock River, rio Oiapoque dr., no coordinates. MNHN 19991364, 1 (part), 153 mm, Pont de Papinabo, crique de Papinabo, River Kourou dr., $c a .05^{\circ} 06^{\prime} \mathrm{N}, 052^{\circ} 41^{\prime} \mathrm{W}$. NRM 29482, 1, $60 \mathrm{~mm}$, locality marked "PK12", corresponds to following record from MNHN databases: Pont de Papinabo, crique de Papinabo, Kourou River dr., ca. $05^{\circ} 06^{\prime} \mathrm{N}, 052^{\circ} 41^{\prime} \mathrm{W}$. NRM 32241, 2, 55-59 mm, flooded area along rd. Sinnamary-Saint-Laurent, Sinnamary River dr., ca. $05^{\circ} 25^{\prime} 20^{\prime \prime} \mathrm{N}, 053^{\circ} 03^{\prime} 28^{\prime \prime} \mathrm{W}$. NRM 32242, 1, $51 \mathrm{~mm}$, Oyapock River nr. St. Georges - Maripa Rd crossing, rio Oiapoque dr., ca. 03 $52^{\prime} 13^{\prime \prime} \mathrm{N}, 051^{\circ} 51^{\prime} 17^{\prime \prime} \mathrm{W}$. NRM 32243, 1, $50 \mathrm{~mm}$, crique Soumouro, Kourou River dr., 0507'27"N, 052 $43^{\prime} 52^{\prime \prime}$ W. USNM 301964, 2, 165-176 mm. crique Karouabou, nr. Kourou city, Kourou River dr., ca. $05^{\circ} 09^{\prime} \mathrm{N}, 052^{\circ} 40^{\prime \prime} \mathrm{W}$. USNM 301965, 1, 138 mm. crique Papinabo, 200 m upstream from Kourou River, 20 km Kourou, Kourou river dr., ca. $05^{\circ} 00^{\prime} \mathrm{N}, 052^{\circ} 42^{\prime \prime} \mathrm{W}$. Guyana. Barima-Waini .BMNH 1911.10.31.556, 1, 143 mm, FMNH 53322, 1, $110 \mathrm{~mm}$, Issorora rubber plantation, Mud Creek [probably on Aruka River], Aruka River dr., Barima-Kaituma River dr., $08^{\circ} 12^{\prime} \mathrm{N}, 059^{\circ} 44^{\prime} \mathrm{W}$ [coordinates for lower Aruka]. Cuyuni-Mazaruni. BMNH 1934.9.12.360, 1, 106 mm, Mazaruni River, pond in forest near small community, Essequibo River dr., no precise locality [coordinates for confl. with Essequibo River] $06^{\circ} 20^{\prime} \mathrm{N}, 058^{\circ} 41^{\prime} \mathrm{W}$. Demerara [old division name]. FMNH 85374, 2, 101-110 mm, Dakara Creek, no coordinates. PotaroSiparuni (localities from Essequibo River dr.).ANSP 175956, 4, 67-163 mm, Culvert creek crossing Kurupukari-Surama River rd., $04^{\circ} 19^{\prime} 57^{\prime \prime} \mathrm{N}, 058^{\circ} 51^{\prime} 13^{\prime \prime} \mathrm{W}$. ANSP 175957, 1, $158 \mathrm{~mm}$, blackwater creek, 5 min. upstream from Burro Burro campsite, Iwokroma, $c a$. $04^{\circ} 40^{\prime} \mathrm{N}, 058^{\circ} 50^{\prime} \mathrm{W}$. ANSP 175958, 6, 52-75 mm, small lake, $04^{\circ} 45^{\prime} 15^{\prime \prime} \mathrm{N}, 058^{\circ} 45^{\prime} 12^{\prime \prime} \mathrm{W}$. ANSP 192059, 2, 67-74 mm, creek, Tumble Down Falls, Tumble Down Creek, Siparuni River, 044ㅇ' $59^{\prime \prime} \mathrm{N}, 058^{\circ} 50^{\prime} 56^{\prime \prime} \mathrm{W}$. BMNH 1973.3.29.14-15, 2, 96-101 $\mathrm{mm}$, Kaieteur falls, $2 \mathrm{mi}$. above 'gauge', Potaro River, $05^{\circ} 09^{\prime} \mathrm{N}$, 059 $28^{\prime}$ W. CAS 72220, 1, 59 mm, CAS 72226, 1, 54 mm, Chipoo Creek, affl. Ireng River, ca. $04^{\circ} 42^{\prime} \mathrm{N}, 059^{\circ} 06^{\prime} \mathrm{W}$. FMNH 53325, 1 , $121 \mathrm{~mm}, \mathrm{MCZ} 30175,1,153 \mathrm{~mm}$, Nickaparoo (or Nickaparu) Creek, affl. River Ireng, above the Karona falls, ca. $04^{\circ} 42^{\prime} \mathrm{N}$, $059^{\circ} 06^{\prime} \mathrm{W}$. INHS 49616, 1, $120 \mathrm{~mm}, 2.9 \mathrm{mi}$. E Chenapou, Arnick Creek, affl. Potaro River, 0459'56"N, 059³2'20"W. SU 72222, 2 , 113-119 mm, Nickaparoo Creek, into Ireng River, ca. $04^{\circ} 42^{\prime} \mathrm{N}$, $059^{\circ} 06^{\prime}$ W. Upper Takutu-Upper Essequibo (Localities listed for Takutu River are from the rio Branco dr., affl. rio Negro, Amazonas dr.). AUM 35866, 1, 43 mm, 31.2 km NW Sand Creek village, Sauriwau River, affl. Takutu River, $03^{\circ} 06^{\prime} 51^{\prime \prime} \mathrm{N}, 059^{\circ} 46^{\prime} 31^{\prime \prime} \mathrm{W}$. BMNH 1972.7.27.458-518 (part), 2, 95-102 mm, Karanambo creek, Rupununi River, Essequibo River dr., 03 $45^{\prime} 11^{\prime \prime} \mathrm{N}$, 059 $18^{\prime} 36^{\prime \prime}$ W. FMNH 53327, 1, 112 mm, Twoca Pan, between Rupununi and Pununike, Essequibo River dr., no coordinates. MCZ 165853, 1, 102 mm, Dry creek bed 10 mi. E Nappi village, [probably Moco-Moco River], affl. Takutu River, ca. $03^{\circ} 25^{\prime} \mathrm{N}$, 059²5’W. USNM 209199, 3, 101-127 mm, Rupununi, MocoMoco Creek, affl. Takutu River, ca. $03^{\circ} 15^{\prime} \mathrm{N}, 059^{\circ} 39^{\prime} \mathrm{W}$. Region not specified. AMNH 3247, 1, 65 mm, Guyana, no further locality data, drainage unknown, no coordinates, FMNH 53324, 1, 95 mm, Pacopoo Pan, drainage unknown, no coordinates. USNM 377591 , 2, 115-128 mm, Hassar Creek, no coordinates. Peru (localities are from Amazonas dr.). Amazonas. LACM 41763-2, 1, 110 mm, 1 $\mathrm{km}$ upstream from Caterpiza, río Santiago, affl. río Marañón, Amazonas dr., ca. $03^{\circ} 54^{\prime} \mathrm{S}, 077^{\circ} 42^{\prime} \mathrm{W}$. Loreto (localities listed from nr. Jenaro Herrera are from the río Ucayali dr., mun. Requena). ANSP 167725, 4, 55-78 mm, río Nanay, ca. $6 \mathrm{mi}$. upstream confl. with río Momón, $c a$. $03^{\circ} 44^{\prime} 50^{\prime \prime} \mathrm{S}, 073^{\circ} 17^{\prime} 12^{\prime \prime} \mathrm{W}$. ANSP 167726, 2, 76-90 mm, nr. Mishana on path to Nauta rd., affl. río Nanay, 035' $12^{\prime \prime} \mathrm{S}, 073^{\circ} 28^{\prime} 57^{\prime \prime} \mathrm{W}$. ANSP 167727, 11, 60-85 $\mathrm{mm}$, Laguna Rocafuerte, río Nanay, ca. $6.1 \mathrm{mi}$. upstream confl. with río Momón ca. $03^{\circ} 44^{\prime} \mathrm{S}, 073^{\circ} 17^{\prime} \mathrm{W}$. ANSP 178219, 1, $53 \mathrm{~mm}$, caño Santa Rita, R. bank affl. río Nanay, nr. Pueblo Pampa Chica, $03^{\circ} 45^{\prime} 23^{\prime \prime} \mathrm{S}, 073^{\circ} 17^{\prime} 28^{\prime \prime} \mathrm{W}$. CAS 67701, 4, 87-128 mm, creek nr. Yurimaguas, affl. río Huallaga, affl. río Marañón, ca. $05^{\circ} 53^{\prime} \mathrm{S}$, 
$076^{\circ} 04^{\prime} \mathrm{W}$. CAS 67702, 4, 37-213 mm, stream, affl. río Itaya, affl. río Amazonas, $c a .03^{\circ} 48^{\prime} \mathrm{S}, 073^{\circ} 16^{\prime} \mathrm{W}$. INHS 50010, 2, 102-108 $\mathrm{mm}$, caño Ushpa, $4 \mathrm{~km}$ E Iquitos, affl. río Itaya, affl. río Amazonas, ca. $03^{\circ} 48^{\prime} \mathrm{S}, 073^{\circ} 16^{\prime} \mathrm{W}$. MNRJ 3957, 1, $197 \mathrm{~mm}$, rio Ampiyacu, nr. Pebas, affl. río Amazonas, ca. $03^{\circ} 19^{\prime} \mathrm{S}, 071^{\circ} 51^{\prime} \mathrm{W}$. MUSM 6959 (part), 1, $72 \mathrm{~mm}$, Isla Muyuy, cocha Amazonas, río Amazonas, ca.

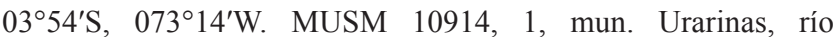
Chambiras nr. San Juan, río Nanay, ca. $03^{\circ} 41^{\prime} \mathrm{S}, 073^{\circ} 45^{\prime} \mathrm{W}$. MUSM 11009, 1, Pijuayal, río Hormiga, affl. rio Amazonas, $04^{\circ} 08^{\prime} \mathrm{S}, 070^{\circ} 03^{\prime} \mathrm{W}$. MUSM 14498 (part), 1, $73 \mathrm{~mm}$, mun. Maynas, Puesto de Vigilancia Arcadia, río Napo, $00^{\circ} 59^{\prime} 37^{\prime \prime} \mathrm{S}, 075^{\circ} 18^{\prime} 30^{\prime \prime} \mathrm{W}$. MUSM 44675, 22 (14 immature, 48-163 mm, 5 female, 157-190 mm, 4 male, 148-222 mm), stream nr. Jenaro Herrera, 04 ${ }^{\circ} 53^{\prime} 59^{\prime \prime} \mathrm{S}$, 0733' $51^{\prime \prime} \mathrm{W}$. MUSM 44681, 4, immature, 50-133 mm, stream nr. Jenaro Herrera, 0453'35"S, 07339'01"W. MUSM 44693, 8 (1 immature, $157 \mathrm{~mm}, 3$ female, 148-185 mm, 4 male, 164-210 mm), stream nr. Jenaro Herrera, 04 $51^{\prime} 51^{\prime \prime} \mathrm{S}, 073^{\circ} 38^{\prime} 45^{\prime \prime} \mathrm{W}$. MUSM 44703, 14 (13 immature, 84-188 mm, 1 female, $169 \mathrm{~mm}$ ), stream nr. cocha Capite, nr. Jenaro Herrera, 04 $52^{\prime} 03^{\prime \prime} \mathrm{S}, 073^{\circ} 40^{\prime} 39^{\prime \prime} \mathrm{W}$. MUSM 44712, 2, immature, $8133 \mathrm{~mm}$, stream nr. Jenaro Herrera, 0453'54"S, 07338'23"W. MUSM 44718, 4 (3 immature 87-196 $\mathrm{mm}, 1 \mathrm{male}, 155 \mathrm{~mm}$ ), stream nr. Jenaro Herrera, 0456'16"S, 07339'20"W. MUSM 44721, 14 (8 immature, 5922 mm, 3 female, $14784 \mathrm{~mm}, 2$ male, 154-192 mm), stream nr. Jenaro Herrera, $04^{\circ} 55^{\prime} 41^{\prime \prime} \mathrm{S}, 073^{\circ} 38^{\prime} 46^{\prime \prime} \mathrm{W}$. MUSM 44762, 18, immature, 58-90

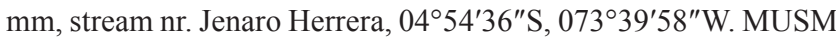
44768, 7 (1 immature, 106 mm, 3 female, 91-155 mm, 3 male, 125-139 mm), cocha Capite, nr. Jenaro Herrera, 04 $54^{\prime} 15^{\prime \prime} \mathrm{S}$, 07336'53"W. NRM 27763, 1, 143 mm, stream nr. Jenaro Herrera, ca. $04^{\circ} 54^{\prime} 23^{\prime \prime} \mathrm{S}, 073^{\circ} 37^{\prime} 27^{\prime \prime} \mathrm{W}$. SU 17230, 1, $204 \mathrm{~mm}$, río Amazonas nr. Pebas, ca. $03^{\circ} 19^{\prime} \mathrm{S}, 071^{\circ} 37^{\prime} \mathrm{W}$. UF 116551, 1, immature, $80 \mathrm{~mm}$, río Nanay at Mishana, $03^{\circ} 52^{\prime} 48^{\prime \prime} \mathrm{S}$, $073^{\circ} 29^{\prime} 30^{\prime \prime} \mathrm{W}$. UF 116555,1 , immature, $86 \mathrm{~mm}$, stream at $\mathrm{km} 23$, Iquitos-Nauta rd., affl. río Nanay, $03^{\circ} 56^{\prime} 38^{\prime \prime} \mathrm{S}, 073^{\circ} 23^{\prime} 54^{\prime \prime} \mathrm{W}$. UF 126211, 1, 112 mm, UF 131121, 1, 67 mm, Reserva Nacional (RN) Pacaya Samiria, río Pacaya, affl. río Ucayali, ca. $05^{\circ} 16^{\prime} \mathrm{S}$, $074^{\circ} 27^{\prime} \mathrm{W}$. UF 126237, 1, $127 \mathrm{~mm}$, UF 126247, 3, immature, $11831 \mathrm{~mm}$, UF 148564, 5 (2 immature, 68-76 mm, 3 female, 7416 $\mathrm{mm})$, RN Pacaya Samiria, cocha Sapote, río Pacaya, affl. río Ucayali, $05^{\circ} 19^{\prime} 06^{\prime \prime} \mathrm{S}, 074^{\circ} 29^{\prime} 18^{\prime \prime} \mathrm{W}$. UF 129052, 1, $76 \mathrm{~mm}, \mathrm{RN}$ Pacaya Samiria, río Pacaya, affl. río Ucayali, ca. $05^{\circ} 25^{\prime} 18^{\prime \prime} \mathrm{S}$, 074³0'45"W. UF 129117, 1, 77 mm, RN Pacaya Samiria, río Pacaya, affl. río Ucayali, ca. $05^{\circ} 18^{\prime} 14^{\prime \prime} \mathrm{S}, 074^{\circ} 30^{\prime} 45^{\prime \prime} \mathrm{W}$. UF 129157, 1, 110 mm, RN Pacaya Samiria, río Pacaya, affl. río Ucayali, ca. $05^{\circ} 23^{\prime} 57^{\prime \prime} \mathrm{S}, 074^{\circ} 32^{\prime} 06^{\prime \prime} \mathrm{W}$. UF 129161, 3, 72-100 $\mathrm{mm}, \mathrm{RN}$ Pacaya Samiria, río Pacaya, affl. río Ucayali, ca. 05²3'51"S, 074³0'23"W. UF 129406, 3, 52-102 mm, RN Pacaya Samiria, río Pacaya, affl. río Ucayali, ca. 05¹9'20"S, 074³0'14"W. UF 148505, 4 (1 male, $130 \mathrm{~mm}, 3$ female, 122-143 mm), stream

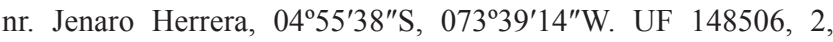
immature, 34-42 mm, quebrada Salomé caño, stream nr. Jenaro Herrera, 04 $4^{\circ} 4^{\prime} 32^{\prime \prime} \mathrm{S}, 073^{\circ} 35^{\prime} 36^{\prime \prime} \mathrm{W}$. UF 148507, 1, immature, 66 $\mathrm{mm}$, affl. quebrada Parnayari, nr. Jenaro Herrera, ca. $04^{\circ} 54^{\prime} \mathrm{S}$, 0733' W. UF 148508, 2 (1 immature $68 \mathrm{~mm}, 1$ female, $164 \mathrm{~mm}$ ), quebrada Parnayari, nr. Jenaro Herrera, $04^{\circ} 55^{\prime} 10^{\prime \prime} \mathrm{S}, 073^{\circ} 38^{\prime} 40^{\prime \prime} \mathrm{W}$. UF 148509, 1, immature, $51 \mathrm{~mm}$, stream, affl. quebrada Chica, nr.

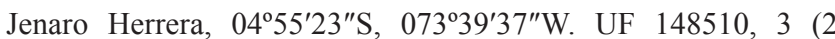
female, 170-197 mm, 1 male, $217 \mathrm{~mm}$ ), quebrada Fierro caño, affl.

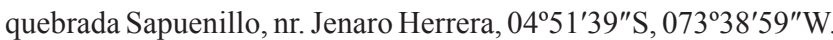
UF 148556, 1, immature, 88 mm, RN Pacaya Samiria, caño Yarina, río Pacaya, affl. río Ucayali, $05^{\circ} 20^{\prime} 08^{\prime \prime} \mathrm{S}, 074^{\circ} 30^{\prime} 05^{\prime \prime} \mathrm{W}$. UF 148557, 1, immature, 7 mm, RN Pacaya Samiria, caño Yarina, río Pacaya, affl. río Ucayali, $05^{\circ} 19^{\prime} 20^{\prime \prime} \mathrm{S}, 074^{\circ} 30^{\prime} 11^{\prime \prime} \mathrm{W}$. UF 148558, 3 ( 1 immature, $70 \mathrm{~mm}, 2$ female, 102-129 mm), RN Pacaya Samiria, caño Yarina, río Pacaya, affl. río Ucayali, $05^{\circ} 18^{\prime} 16^{\prime} \mathrm{S}, 074^{\circ} 30^{\prime} 05^{\prime \prime} \mathrm{W}$. UF 148559, 17 (4 immature, 80-106 mm, 11 female, 90-136 mm, 2 male, 107-125 mm), RN Pacaya Samiria, cocha Yarina, río Pacaya, affl. río Ucayali, $05^{\circ} 23^{\prime} 51^{\prime \prime} \mathrm{S}, 074^{\circ} 30^{\prime} 23^{\prime \prime} \mathrm{W}$. UF 148560 , 3, (1 immature, $44 \mathrm{~mm}, 2$ female, 126-135 mm), RN Pacaya Samiria, cocha Yarina, río Pacaya, affl. río Ucayali, $05^{\circ} 25^{\prime} 20^{\prime \prime} \mathrm{S}$, 074³0'45"W. UF 148561, 5 (4 immature, 104-149 mm, 2 female, 125-161 mm), RN Pacaya Samiria, cocha Yarina, río Pacaya, affl. río Ucayali, $05^{\circ} 23^{\prime} 58^{\prime \prime} \mathrm{S}, 074^{\circ} 32^{\prime} 05^{\prime \prime} \mathrm{W}$. UF 148562, 4 (2 immature, 87-122 mm, 2 female, $160 \mathrm{~mm}$ ), RN Pacaya Samiria, cocha Yarina, río Pacaya, affl. río Ucayali, 05²5'29"S, 074³1'41"W. UF 148563, 1, female, 133 mm, RN Pacaya Samiria, caño Tamara, río Pacaya, affl. río Ucayali, $05^{\circ} 16^{\prime} 40^{\prime \prime} \mathrm{S}, 074^{\circ} 29^{\prime} 38^{\prime \prime} \mathrm{W}$. UF 148565,3 ( 1 immature 73 mm, 2 female, 143-177 mm), RN Pacaya Samiria, río Pacaya, río Ucayali, affl. río Ucayali, $05^{\circ} 16^{\prime} 44^{\prime \prime} \mathrm{S}, 074^{\circ} 31^{\prime} 06^{\prime \prime} \mathrm{W}$. UF 148566, 5 (4 immature, 64-72 mm, 1 female, $109 \mathrm{~mm}$ ), RN Pacaya Samiria, cocha Shitari, affl. río Ucayali, ca. $05^{\circ} 17^{\prime} \mathrm{S}$, $074^{\circ} 31^{\prime} \mathrm{W}$. UF $183770,1,256 \mathrm{~mm}$, río Nanay, 035 $53^{\prime} 50^{\prime \prime} \mathrm{S}$, $073^{\circ} 40^{\prime} 01^{\prime \prime} \mathrm{W}$. UF 184013, 9 (6 immature, 56-199 mm, 2 female, 159-183 mm, 1 male, $96 \mathrm{~mm})$, stream nr. Jenaro Herrera,

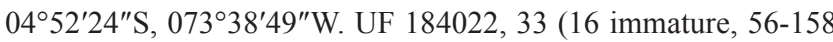
$\mathrm{mm}, 8$ female, $120-170 \mathrm{~mm}, 9$ male, $148-198 \mathrm{~mm})$, stream $\mathrm{nr}$.

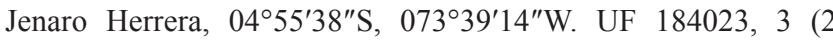
immature, 64-139 mm, 1 female, $164 \mathrm{~mm}$ ), stream nr. Jenaro Herrera, 04 ${ }^{\circ} 54^{\prime} 15^{\prime \prime} \mathrm{S}, 073^{\circ} 36^{\prime} 53^{\prime \prime} \mathrm{W}$. UMMZ 224598, 3, 62-156 $\mathrm{mm}$, río Cayarí, nr. Benjamin Constant, affl. río Yavarí (rio Javari), ca. $04^{\circ} 22^{\prime} \mathrm{S}, 070^{\circ} 01^{\prime} \mathrm{W}$ [coordinates for Benjamin Constant]. UMMZ 224603, 2, 106-153 mm, río Tapira, río Tahwayo [Tahuayo], ca. $04^{\circ} 17^{\prime} \mathrm{S}, 073^{\circ} 12^{\prime} \mathrm{W}$. UMMZ 224604, 1, $111 \mathrm{~mm}$,

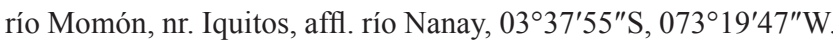
UMMZ 228983, 2, 131-135 mm, quebrada Carana, Buensuceso, rio Javari, $04^{\circ} 07^{\prime} \mathrm{S}, 070^{\circ} 25^{\prime} \mathrm{W}$. UMMZ 228984, 13, 33-119 mm, río Momón, affl. río Nanay, ca. $03^{\circ} 37^{\prime} \mathrm{S}, 073^{\circ} 17^{\prime} \mathrm{W}$. Ucayali. FMNH 84226, 1, $60 \mathrm{~mm}$, first bridge over stream, ca. $34 \mathrm{~km}$ Pucallpa, on Pucallpa-Lima hwy., affl. río Ucayali, 08³4'17"S, $074^{\circ} 54^{\prime} 17^{\prime \prime} \mathrm{W}$. MUSM 39375, 5, 56-61 mm, pools in caño draining Supari cocha, nr. San Miguel village, río Purus, 0958'12"S, $070^{\circ} 56^{\prime} 09^{\prime \prime} \mathrm{W}$. Suriname. Marowijne. UF 177358, 1, male, 161 mm, small unnamed stream on Paramaribo-Albina rd., affl. Cottica

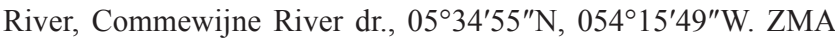
102.377, 1, 83 mm, Albina, Marowijne River dr., ca. $05^{\circ} 29^{\prime} 58^{\prime \prime} \mathrm{N}$, $054^{\circ} 03^{\prime} 44^{\prime \prime} W$. Nickerie. USNM 22588, 21, 32-97 mm, Lana Creek, ca. $4 \mathrm{~km}$ upstream confl. with Corantijn River, Corantijn River dr., ca. $05^{\circ} 27^{\prime} \mathrm{N}, 057^{\circ} 15^{\prime} \mathrm{W}$. USNM 225557, 1, $57 \mathrm{~mm}$, creek at Camp MacClemmen, Dalibane Creek, Corantijn River drainage, $05^{\circ} 34^{\prime} \mathrm{N}, 057^{\circ} 11^{\prime} \mathrm{W}$. USNM 225559, 5, 45-132 mm, Kapoeri Creek, ca. 4 km N confl. with Corantijn River, Corantijn River dr., $05^{\circ} 16^{\prime} \mathrm{N}, 057^{\circ} 10^{\prime} \mathrm{W}$. USNM 225562, 4, 57-70 mm, creek ca. $2 \mathrm{~km}$ 
N Matapi, Corantijn River dr., $05^{\circ} 03^{\prime} \mathrm{N}, 057^{\circ} 17^{\prime} \mathrm{W}$. USNM 225568 , 2, 70-114 mm, small creek affl. Corantijn River, $350 \mathrm{~m}$ below Wilhelm II falls, Corantijn River dr., 033ํ№ $057^{\circ} 39^{\prime} \mathrm{W}$. USNM 225572, 1, $100 \mathrm{~mm}$, Makilikabroe Creek, Corantijn rivier dr., $05^{\circ} 26^{\prime} \mathrm{N}, 057^{\circ} 14^{\prime} \mathrm{W}$. Paramaribo. ZMH 11630, 1, $127 \mathrm{~mm}$, Paramaribo, Suriname River dr., ca. $05^{\circ} 53^{\prime} \mathrm{N}, \quad 055^{\circ} 10^{\prime} \mathrm{W}$. Sipaliwini. AMNH 54831, 1, 67 mm, stream nr. Avanavero Falls, ca. 3 mi. downstream Devis Falls, Corantijn River dr., ca. $04^{\circ} 49^{\prime} \mathrm{N}$, $057^{\circ} 24^{\prime} \mathrm{W}$. AMNH 244582, 2, 68-70 mm, small stream S Tiger Falls, km 405, Corantijn River dr., ca. $03^{\circ} 56^{\prime} \mathrm{N}, 057^{\circ} 56^{\prime} \mathrm{W}$. FMNH 116894, 1, $112 \mathrm{~mm}$, unnamed creek on Linker Coppename, Coppename River dr., 04²3'25"N, 056 31'52"W. USNM 225564 (part), 7, 75-125 mm, small stream $200 \mathrm{~m}$ upriver Dalbana Creek mouth, Corantijn River dr., ca. $04^{\circ} 47^{\prime} \mathrm{N}, 057^{\circ} 26^{\prime} \mathrm{W}$. USNM 302003, 1, 143 mm, Matapi Creek ca. $1 \mathrm{~km}$ confl. with Corantijn rivier, Corantijn River dr., ca. $05^{\circ} 00^{\prime} \mathrm{N}, 057^{\circ} 16^{\prime} \mathrm{W}$. ZMA 107.029, 5, 130-182 mm, stream into left bank Gran rio, $3 \mathrm{~km} \mathrm{NE}$ Awaradam, affl. Gran rio, Suriname River dr., ca. $03^{\circ} 51^{\prime} \mathrm{N}, 055^{\circ} 25^{\prime} \mathrm{W}$. ZMH 11629, 1, 143 mm, "upper Suriname", no coordinates. Venezuela. Amazonas. MBUCV-V 13895, 6 (3 measured: 136-167 mm), caño Niyayowa, W Parima, affl. rio Negro, Amazonas dr., ca. $03^{\circ} 30^{\prime} \mathrm{N}$, $064^{\circ} 15^{\prime} \mathrm{W}$. MBUCV-V 14766, 1, $141 \mathrm{~mm}$, small stream, $1 \mathrm{~km} \mathrm{~S} \mathrm{La}$ Neblina camp, affl. río Baria, aff .río Casiquiare, affl. rio Negro, Amazonas dr., $c a .00^{\circ} 50^{\prime} \mathrm{N}, 066^{\circ} 05^{\prime} \mathrm{W}$. MCNG 23657, 3, Mauritia palm swamp, $1 \mathrm{~km} \mathrm{~N}$ Esmeralda, río Orinoco, Orinoco dr., $03^{\circ} 11^{\prime} \mathrm{N}$, $065^{\circ} 33^{\prime} \mathrm{W}$. MCNG 23894, 1, forest pools, río Ventuari, Orinoco dr., ca. $03^{\circ} 59^{\prime} \mathrm{N}, 067^{\circ} 02^{\prime} \mathrm{W}$ [coordinates for mouth of río Ventuari]. MCNG 23903, 1, morichal, río Guapuche, affl. río Ventuari, río Orinoco drainage, $c a .04^{\circ} 06^{\prime} \mathrm{N}, 066^{\circ} 46^{\prime} \mathrm{W}$ [coordinates for mouth of río Guapuche]. MHNLS 5873, 1, MHNLS 6437, 1, Malvaca, alto río Orinoco, Orinoco dr., $02^{\circ} 30^{\prime} \mathrm{N}, 065^{\circ} 09^{\prime} \mathrm{W}$. UMMZ 228986 , 2, pool of creek nr. Puerto Ayacucho, affl. río Orinoco, Orinoco dr., ca. $05^{\circ} 38^{\prime} \mathrm{N}, 067^{\circ} 36^{\prime} \mathrm{W}$. Apure. MBUCV-V 10776, 1, $101 \mathrm{~mm}$, caño Grande, $3 \mathrm{~km} \mathrm{~S}$ La Ceiba, affl. río Apure, Orinoco dr., $c a$. $07^{\circ} 19^{\prime} \mathrm{N}, 071^{\circ} 08^{\prime} \mathrm{W}$. Barinas. MBUCV-V 5960, 2, 69-70 mm, caño San Antonio, ca. $10 \mathrm{~km}$ Santa Barbara de Barinas, río Anaro, affl. río Suripá, affl. río Apure, Orinoco dr., ca. $07^{\circ} 48^{\prime} \mathrm{N}, 071^{\circ} 10^{\prime} \mathrm{W}$. Monagas. AMNH 15453, 2 (paratypes), 95-146 mm, MBUCV-V 15163, 1 (paratype), 130 mm, USNM 120753, 1 (holotype) (Fig. 9b, herein), $103 \mathrm{~mm}$ (originally $136 \mathrm{~mm}$ TL, $112 \mathrm{~mm}$ LEA(Schultz, 1944), caudal filament now lost), Caripito, río San Juan, Orinoco dr., $c a .10^{\circ} 06^{\prime} \mathrm{N}, 063^{\circ} 06^{\prime} \mathrm{W}, \mathrm{C}$. Beebe, 1942. MBUCV-V 22343, 1, $94 \mathrm{~mm}$, río Morichal Largo, río Orinoco delta, Orinoco dr., $09^{\circ} 26^{\prime} \mathrm{N}, 062^{\circ} 24^{\prime} \mathrm{W}$. Country not specified. USNM 302011, 2, 101-130 mm, no locality, no coordinates.

\section{Brachyhypopomus belindae, new species}

urn:1sid:zoobank.org:act:B20030E3-840F-409D-841FA99F658AA8B0

\section{(Figs. 1c, 11; Tables 2-5, 7)}

Brachyhypopomus sp. "belindae". - Crampton et al., 2016: 1-66, table 1, 3-4, figs. 1-7, 18-20 (phylogeny, biogeography and ecology of Brachyhypopomus).
Holotype. MCP 45360, immature, 110 mm TL, 92 mm LEA, Brazil, Amazonas, mun. Alvarães, Mamirauá Reserve, lago Araçazinho, rio Solimões-Japurá floodplain, Amazonas dr., 02 $59^{\prime} 35^{\prime \prime} \mathrm{S}, 64^{\circ} 51^{\prime} 33^{\prime \prime} \mathrm{W}, 5$ Apr 1997, W. Crampton \& J. Oliveira.

Paratypes. 5 specimens, localities from Amazonas dr., collected by W. Crampton \& J. Oliveira. Brazil. Amazonas (localities listed from Mamirauá Reserve [Reserva de Desenvolvimento Sustentável Mamirauá] are in the rio Solimões-Japurá floodplain, mun. Alvarães). INHS 70253, 1, $74 \mathrm{~mm}$, lago Murumuru, Janauacá, $c a .42 \mathrm{~km}$ SW Manaus, ca. $03^{\circ} 26^{\prime} \mathrm{S}, 060^{\circ} 17^{\prime} \mathrm{W}, 29$ Mar 1978, P. Bayley. MCP 45267, 1, immature, $90 \mathrm{~mm}$, Mamirauá Reserve, cano do lago Mamirauá, 0305'15"S, 06448'03"W, 18 Jun 1999. MCP 47867, 1, immature, $62 \mathrm{~mm}$, Mamirauá Reserve, cano do lago Rato, 0302'41"S, 06451'26"W, Jun 1997. MCP 45430, 1, immature (CS), 102 mm, Mamirauá Reserve, lago Araçazinho, 02059'35"S, 06451'33"W, 15 Jun 1999. MCP 45431, 1, immature, 94 mm, Mamirauá Reserve, cano do lago Mamirauá, 0306'40"S, 06447'52"W, 23 Jul 1999.

Diagnosis. Brachyhypopomus belindae is diagnosed from congeners by the following combination of characters: precaudal vertebrae 24-26, vs. 15-23 in all congeners except $B$. draco, and B. verdii; bilateral columns of electrocytes at the anal-fin terminus 4-5 (no mode, median 4.5), vs. 3 in B. draco and $B$. verdii.

Description. Known only from immature specimens. Head and body shape, and pigmentation illustrated in Figs. 1c and 11. Meristic and morphometric data for examined specimens presented in Tables 2-5 and 7. Body shallow to moderate in depth. Head short to moderate in length and moderate in depth. Dorsal profile of head straight to slightly convex from occiput to snout, ventral profile of head straight gently convex between operculum and snout, snout rounded. Eye moderate in size. Upper jaw with slight to moderate sigmoidal angle between premaxillary and maxillary portions in lateral view. No accessory electric organ over operculum. Pectoral fin narrow to moderate in width, pectoral-fin rays 15-17 (mode 16). Precaudal vertebrae 24-26 (mode 24) including 1-2 (no mode, median 1.5) transitional vertebrae. Anal-fin origin slightly $(<0.25 \mathrm{HL}$ distance) anterior or posterior to, or near, tip of pectoral fin. Anal-fin rays 200-220 (median 209). Dorsal rami of recurrent branch of anterior lateral line nerve not visible. Middorsal region of body scaled. Rows of scales above lateral line 5-6 (mode 6). Lateral line continuous. Sparse groove-like depigmented epidermal canals found mainly in posterior half of the body as parallel crisscrossing lines either side of lateral line, on flank midway from lateral line to dorsal midline, and as a pair of long irregular lines either side of dorsal surface in dorsal portion of flank. 4-5 bilateral horizontal columns of electrocytes at analfin terminus, and at mid-point between anal-fin terminus and tip of caudal filament in immature specimens (mature specimens not available). Caudal filament short to moderate in length. 


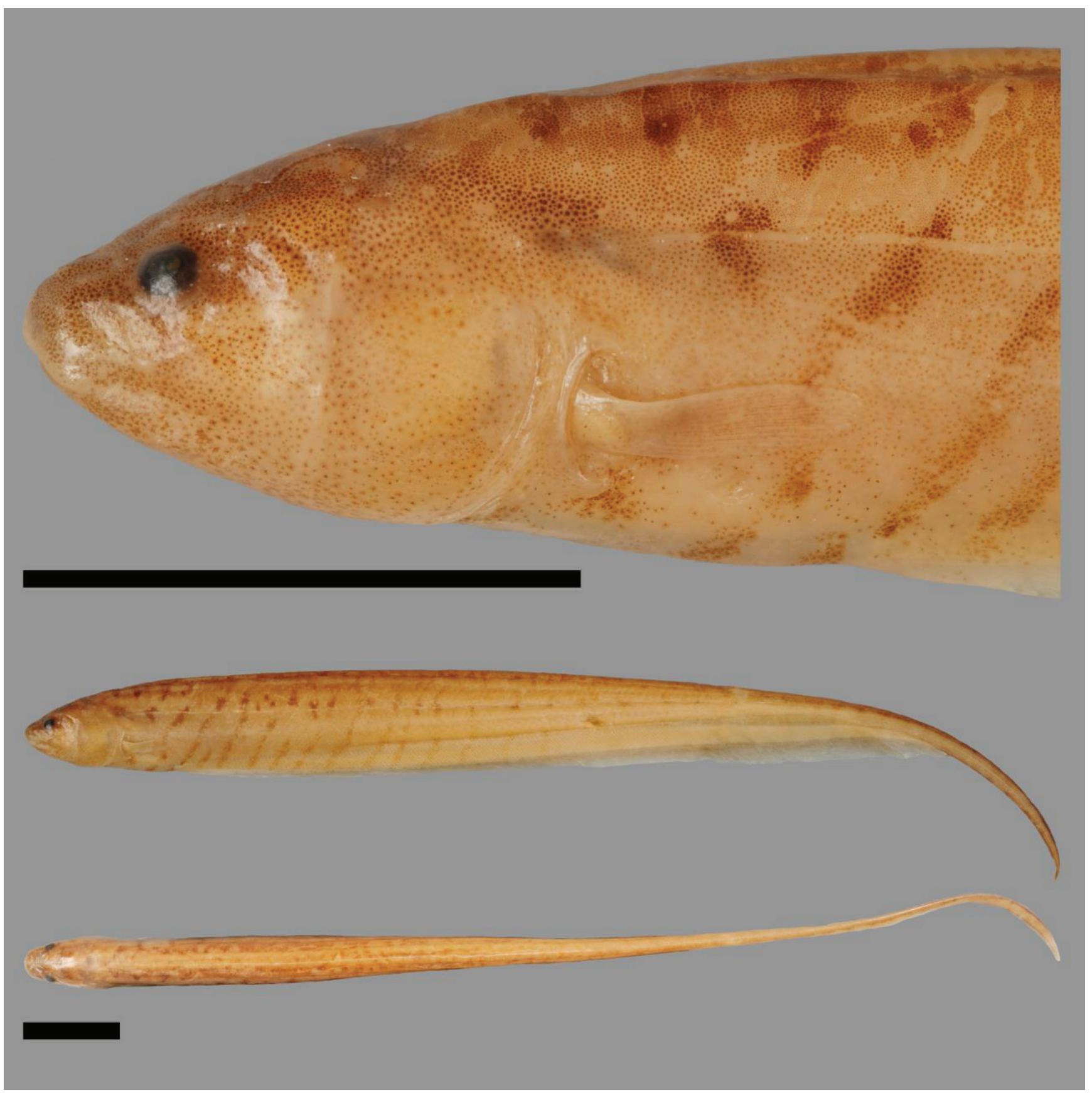

Fig. 11. Brachyhypopomus belindae. MCP 45360 (WC05.050497), holotype, immature, $110 \mathrm{~mm}$ TL (head in lateral view, body in lateral and dorsal views, specimen fixed in formalin and preserved in EtOH); Brazil, rio Solimões-Japurá confluence, Amazonas dr. Scale bars $=5 \mathrm{~mm}$.

Coloration. (Figs. 1c, 11). Background tan to light brown. Dorsal region with prominent depigmented pale stripe extending along midline from occipital region to base of caudal filament. Pale middorsal stripe flanked in anterior portion of body by a thin dark horizontally oriented line from which broken or complete lines extend ventrally in a posterior to anterior direction across dorsal and ventral portions of flank to anal-fin margin. Upper flank in anterior portion of body with additional irregular markings. Markings and dark lines less pronounced in posterior portion of body. Caudal filament darker than body, especially dorsally and ventrally. Head with evenly scattered dark chromatophores, darker dorsally. Eye without prominent suborbital patch, or stripe, of chromatophores/subcutaneous pigmentation. Pectoral and anal-fin membranes hyaline. Pectoral and anal-fin rays hyaline with light scattering of brown chromatophores. Anal-fin ray pigmentation darker in posterior half of fin. Color in live individuals similar to preserved specimens, with opercular region usually rosy due to underlying gills. 
Size. Largest specimen examined $110 \mathrm{~mm}$ TL, $92 \mathrm{~mm}$ LEA $(n=5)$.

Sexual dimorphism. Undetermined. Breeding males and females of this species have not yet been collected.

Geographic distribution. Brazil (Fig. 7). Known only from the central Amazon of Brazil; from the type series near Tefé, Amazonas, Brazil, and from a whitewater floodplain site near Manaus.

Ecological notes. A rare species known only from whitewater floodplain habitats. The type series was collected from floating rafts of macrophytes in high conductivity seasonally hypoxic whitewater floodplains at the confluence of the rio Solimões (Amazonas) and rio Japurá. Water parameters recorded at sites near the type locality, through an entire annual flood cycle were typically within the following ranges: conductivity $54-192 \mu \mathrm{Scm}^{-1}$ dissolved oxygen $0-6.3$ $\mathrm{mgl}^{-1}$, temperature $26.5-34.0^{\circ} \mathrm{C}$, and $\mathrm{pH}$ 6.3-7.3 (Crampton, 1998a). Reproductive biology is unknown. Stomach contents of specimens from the type locality comprise aquatic insect larvae and other small aquatic invertebrates (WGRC unpublished data).

Co-occurring congeners: Brachyhypopomus belindae is known to co-occur in geographical sympatry and ecological syntopy with the following whitewater floodplain-occurring congeners: $B$. beebei, B. bennetti, B. brevirostris, $B$. flavipomus, $B$. hamiltoni, $B$. pinnicaudatus, $B$. regani, and $B$. walteri. It also exhibits an allotopic distribution with $B$. batesi, B. hendersoni, and B. sullivani.

Etymology. The specific name is a patronym (noun in the genitive case) in honor of Belinda Siew-Woon Chang, American (USA) evolutionary biologist, for her inspiration to NRL during the preparation of this work.

Local names. Brazil: sarapó.

\section{Brachyhypopomus benjamini, new species}

urn:1sid:zoobank.org:act:E93 B CBF9-472 A-42EFB4B6-D60937293835

(Fig. 12; Tables 2-5, 7)

Brachyhypopomus sp. I. -Crampton \& Albert, 2006: 672, fig. 23.8, color photograph of head of live individual with EOD, position in phylogenetic tree; 681, notes on EOD (gymnotiform species and EOD diversity).

Brachyhypopomus sp. "benj”. -Crampton, 2011: 176, table 10.2, species list; 179, figs. 10.2-10.3, phylogeny, geographical and ecological distributions (gymnotiform biology).

Brachyhypopomus sp. "benjamini". -Crampton et al., 2016: 1-66, table 1, 3-4, figs. 1-7, 18-20 (phylogeny, biogeography and ecology of Brachyhypopomus).
Holotype. MUSM 39915, male, 126 mm TL, 103 mm LEA, Peru, Loreto, mun. Requena, nr. Jenaro Herrera, quebrada Caño Fierro, affl. río Ucayali, Amazonas dr., $04^{\circ} 51^{\prime} 59^{\prime \prime} \mathrm{S}$, $073^{\circ} 38^{\prime} 48^{\prime \prime} \mathrm{W}, 16$ Jan 2004, B. Crampton, W. Crampton, H. Ortega, R. Reis, F. Lima \& J. Oliveira.

Paratypes. 68 specimens, localities from Amazonas dr., collected by W. Crampton, E Correa Roldán, M. Guevara, J. Waddell, unless otherwise stated. Peru. Loreto (localities listed from nr. Jenaro Herrera are from the río Ucayali dr., mun. Requena). MUSM 44676, 1, female, $115 \mathrm{~mm}$, stream nr. Jenaro Herrera, 04 $53^{\prime} 59^{\prime \prime}$ S, 073 $38^{\prime} 51^{\prime \prime} \mathrm{W}, 28$ Jul 2009. MUSM 44689, 2, immature, 109-131 mm, stream nr. Jenaro Herrera, $04^{\circ} 51^{\prime} 51^{\prime \prime} \mathrm{S}, 073^{\circ} 38^{\prime} 45^{\prime \prime} \mathrm{W}, 13$ Jul 2009. MUSM 44694, 56 (48 immature, 79-135 mm, 8 female, $95-119 \mathrm{~mm}$ ), stream nr. Jenaro Herrera, $04^{\circ} 52^{\prime} 09^{\prime \prime} \mathrm{S}, 073^{\circ} 38^{\prime} 44^{\prime \prime} \mathrm{W}, 15 \mathrm{Jul}-$ 5 Aug 2009. UF 148511, 1, male, $110 \mathrm{~mm}$, stream nr. Jenaro Herrera, $04^{\circ} 53^{\prime} \mathrm{S}, 073^{\circ} 39^{\prime} \mathrm{W}$. UF 148512, 2, $97-98 \mathrm{~mm}(1$ immature $97 \mathrm{~mm}, 1$ male, $98 \mathrm{~mm}$ ), collected with holotype, 16 Jan 2004, W. Crampton, H. Ortega, R. Reis, F. Lima, B. Crampton, J. Oliveira. UF 184015, 4, immature, 89-117 mm, stream nr. Jenaro Herrera, 04 $54^{\prime} 26^{\prime \prime} \mathrm{S}, 073^{\circ} 36^{\prime} 44^{\prime \prime} \mathrm{W}, 17 \mathrm{Jul}$ 2009. USNM 405574, 2, immature (96-96 mm), stream nr.

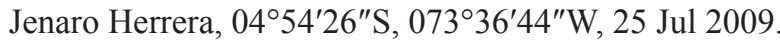

Non-types. 8 specimens. Peru. Loreto. UF 183771, 8, 81$112 \mathrm{~mm}$ (5 immature, $1 \mathrm{CS}, 81-100 \mathrm{~mm}, 2$ female, 90-100 $\mathrm{mm}, 1 \mathrm{male}, 112 \mathrm{~mm}$ ), río Nanay, $50 \mathrm{~km}$ Iquitos, Amazonas dr., $03^{\circ} 53^{\prime} 50^{\prime \prime} \mathrm{S}, 073^{\circ} 40^{\prime} 01^{\prime \prime} \mathrm{W}$.

Diagnosis. Brachyhypopomus benjamini is diagnosed from congeners by the following combination of characters: scales absent in middorsal region of anterior third of body, $v s$. present in all congeners except $B$. provenzanoi; dorsal surface of body speckled with small brown chromatophores on light brown background, $v s$. irregular pattern of distinct dark blotches on depigmented tan-colored background in $B$. provenzanoi.

Description. Head and body shape, and pigmentation illustrated in Fig. 12. Meristic and morphometric data for examined specimens presented in Tables 2-5 and 7. Body shallow to moderate in depth. Head short to moderate in length and shallow to moderate in depth. Dorsal profile of head straight from occiput to snout, ventral profile of head straight anteriorly, gently convex under operculum, snout truncated to rounded. Eye of moderate to large size. Upper jaw with slight sigmoidal angle between premaxillary and maxillary portions in lateral view. No accessory electric organ over operculum. Gill filaments on first gill arch 30-36 (median 34, $\mathrm{n}=5$ ). Pectoral fin narrow to moderate in width, pectoral-fin rays 10-13 (mode 12). Precaudal vertebrae 1617 (mode 17), including 1-2 (mode 2) transitional vertebrae. Anal-fin origin slightly $(<0.25 \mathrm{HL})$ to moderately (reaching $0.33 \mathrm{HL}$ ) posterior to tip of pectoral fin, never anterior to tip of pectoral fin. Anal-fin rays 174-197 (median 186). 
Dorsal rami of recurrent branch of anterior lateral line nerve usually clearly visible. Middorsal region of body without scales in anterior third of body, scales in posterior two thirds of body small and completely occluded by skin. Rows of scales above lateral line 5-7 (mode 6). Lateral line continuous. Sparse groove-like depigmented or darkpigmented epidermal canals found mainly in posterior half of body - parallel to and close to lateral line, mostly dorsal to lateral line. Three bilateral horizontal columns of electrocytes at anal-fin terminus, and at mid-point between anal-fin terminus and tip of caudal filament in immature, mature female and mature male specimens. Caudal filament short to moderate in length.

Coloration. (Fig. 12). Background light tan to dark brown. Dorsal region speckled with small brown chromatophores on light brown background. No pale stripe in middorsal region.
Dorsal areas of flank mottled with dark patches. Distinct stripes or saddles on flank absent. Ventral areas of flank with only sparse, irregular dark blotches. Caudal filament darker than body, with indistinct disrupted vertical bars. Head with evenly scattered dark chromatophores, darker dorsally. Eye without prominent suborbital patch, or stripe, of chromatophores/subcutaneous pigmentation. Pectoral-fin and anal-fin membranes hyaline. Pectoral-fin rays hyaline and unpigmented. Anal-fin rays with light scattering of dark chromatophores. Color in live individuals similar to preserved specimens, with opercular region usually rosy due to underlying gills.

Size. Small adult size, largest specimen examined $135 \mathrm{~mm}$ TL, $107 \mathrm{~mm}$ LEA $(\mathrm{n}=77)$. Largest male specimen examined $126 \mathrm{~mm}$ TL, $103 \mathrm{~mm}$ LEA $(\mathrm{n}=4)$. Largest female specimen examined $119 \mathrm{~mm}$ TL, $109 \mathrm{~mm}$ LEA $(\mathrm{n}=11)$.

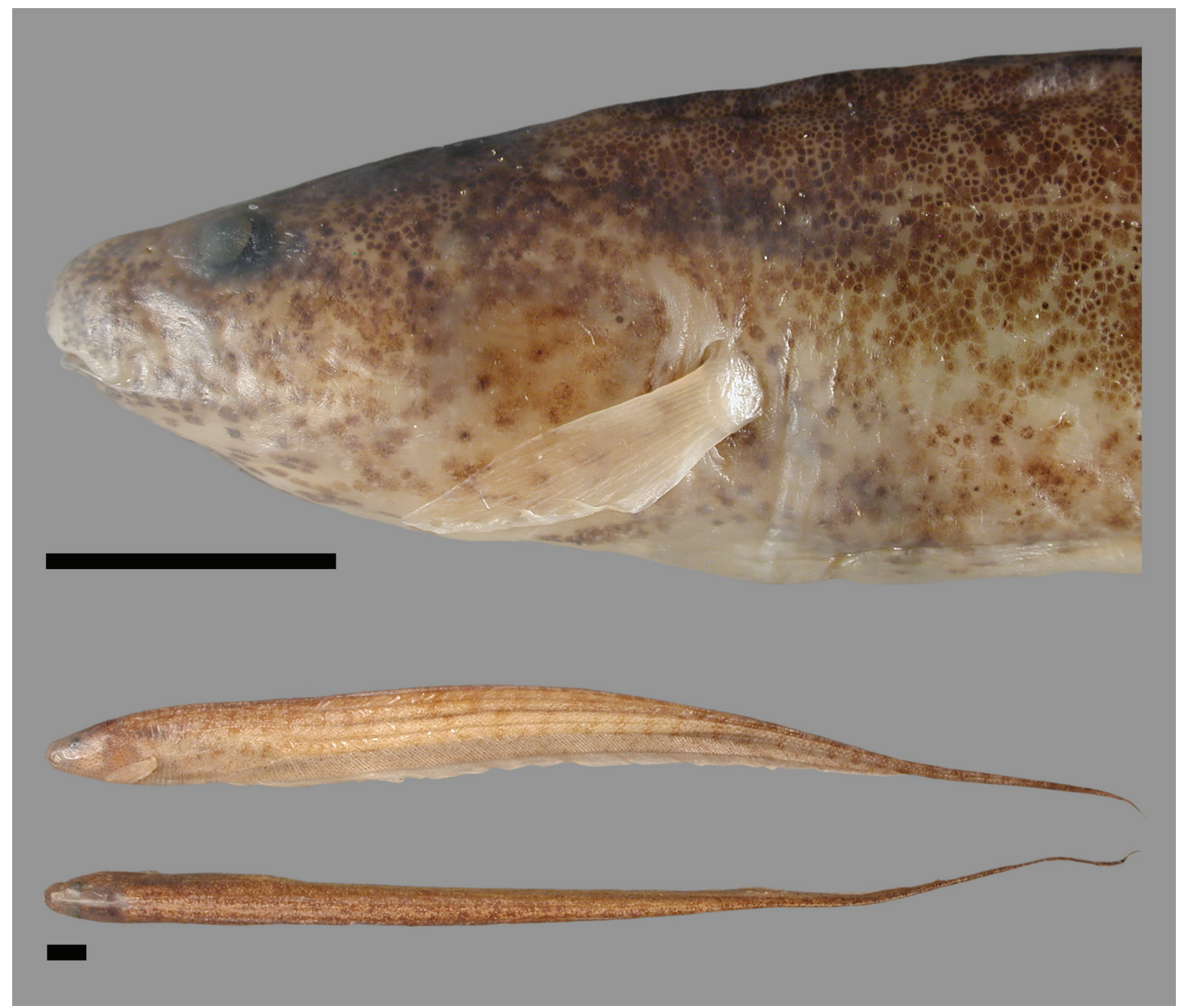

Fig. 12. Brachyhypopomus benjamini. MUSM 39915 (WC10.160104), holotype, male, $126 \mathrm{~mm}$ TL (head, and body in lateral and dorsal view, specimen fixed in formalin and preserved in EtOH); Peru, río Ucayali, Amazonas dr. Scale bars $=5 \mathrm{~mm}$. 
Sexual dimorphism. No known secondary sexual dimorphism.

Geographic distribution. Peru (Fig. 5). Known only from the type series near the town of Jenaro Herrera, Loreto - in forest stream tributaries of the lower río Ucayali, and in forest stream tributaries of the río Nanay, near Iquitos, Loreto.

Ecological notes. Occurs in small low-conductivity blackwater terra firme rainforest streams. In the region of the type locality $B$. benjamini is locally common in streams within undisturbed closed-canopy forest, and absent from streams in open cattle pastures or clearings. Brachyhypopomus benjamini occurs in and around submerged debris and leaf litter, and in root mats and holes along the stream banks. The following water parameters were recorded at the sample sites: conductivity $5-15 \mu \mathrm{Scm}^{-1}$, dissolved oxygen 1.8-6.0 $\mathrm{mgl}^{-1}$, temperature $25-27.5^{\circ} \mathrm{C}$, and $\mathrm{pH}$ 4.5-6.5. Breeding occurs during the rainy season in the area of the type locality (approximately December to April) (JCW unpublished data). Stomach contents of specimens from the type locality comprise small aquatic invertebrates (JCW unpublished data).

Co-occurring congeners: Brachyhypopomus benjamini is known to co-occur in geographical sympatry and ecological syntopy with $B$. beebei, $B$. brevirostris, $B$. sullivani, $B$. verdii, and $B$. walteri. It also exhibits an allotopic distribution with B. bennetti, B. flavipomus, B. hamiltoni, and B. regani.

Etymology. The specific name is a patronym (noun in the genitive case) in honor of Benjamin T. D. Crampton, British diplomat and amateur ornithologist, collector of the holotype.

Local names. Peru: macana.

\section{Brachyhypopomus bennetti Sullivan, Zuanon \& Cox Fernandes, 2013}

\section{(Figs. 1d, 13; Tables 2-5, 8)}

Brachyhypopomus sp. 3. -Crampton, 1996a: 77, table 6.1.a, species list; 79, fig. 6.1 inset 19-21, color photographs; 37, fig. 3.5; 85 , fig. $6.2 ; 88$, fig. 6.3 ; 92-94, figs. 6.4a, 6.5; 191, fig. $11.2 ; 193$, fig. 11.3, EOD data; 110, table 7.5, 113, table 7.7, 115, fig. 7.1, habitats; 136, table 8.2a,b, diet; 151-160, figs. 9.2, 9.3, table 9.1a,b, hypoxia tolerance; 164-169, figs.10.1, 10.2, table 10.1, reproductive biology (Brazil, central Amazon, ecology and signal diversity).

Brachyhypopomus bennetti. -Sullivan, 1997: 100 (description in unpublished thesis with disclaimer stating not available, $a$ nomen nudum).

Brachyhypopomus sp. 2. -Crampton, 1998a, 821, table 4, 834, fig. 9, drawing of adult specimen, with EOD; 837, fig. 10, drawing of caudal filaments of male and female (Brazil, Amazonas, species and EOD diversity). -Crampton, 1998b, 314, table 2, list of Brachyhypopomus (Brazil, Amazonas, hypoxia tolerance).
Brachyhypopomus sp. B. -Crampton, 1999, 17 (Brazil, Amazonas, Mamirauá Reserve, listing of species). -Crampton \& Albert, 2006, 672, fig. 23.8 inset 11, color photograph of head of live individual with EOD, position in phylogenetic tree; 681 , notes on EODs (gymnotiform species and EOD diversity]. -Crampton et al., 2008, 231, fig. 6, black and white photograph of head of live individual (Brazil, adaptations to hypoxia).

Brachyhypopomus sp. 1. -Stoddard, 1999, 255, fig. 4, color photograph of live mature female specimen, with EOD (EOD evolution).

Brachyhypopomus sp. "benn". -Crampton, 2011, 176, table 10.2, species list; 179, figs. 10.2-10.3, phylogeny, geographical and ecological distributions (gymnotiform biology).

Brachyhypopomus (Odontohypopomus) bennetti Sullivan et al., 2013, 14, figs. 6-8, color photographs of holotype and paratypes, backlit electric organ, EODs (original description and assignment to subgenus Odontohypopomus, type locality - Brazil, Amazonas, nr. Manaus, Amazonas dr.). -Carvalho, 2013, 181185 , figs. 41-43, position in phylogeny (phylogenetic systematics of Rhamphichthyoidea). -Crampton et al., 2016: 1-66, table 1, 3-4, figs. 1-7, 9, 18-20 (phylogeny, biogeography and ecology of Brachyhypopomus; Odontohypopomus not recognized).

Diagnosis. Brachyhypopomus bennetti is diagnosed by: bilateral columns of electrocytes at the anal-fin terminus 6 , $v s$. 3-5 in all congeners.

Description. Head and body shape, and pigmentation illustrated in Figs. 1d and 13. Meristic and morphometric data for examined specimens presented in Tables 2-5 and 8. Body moderate to broad in depth. Head moderate to long in length and shallow to moderate in depth. Dorsal profile of head approximately straight from occiput to snout, ventral profile of head approximately straight between operculum and snout, snout rounded. Eye moderate in size. Upper jaw with moderate sigmoidal angle between premaxillary and maxillary portions in lateral view. No accessory electric organ over operculum. Gill filaments on first gill arch 40-51 (median 46, $\mathrm{n}=6$ ). Pectoral fin moderate to broad in width, pectoral-fin rays 14-17 (mode 16). Precaudal vertebrae 17-19 (mode 18), including 1-2 (mode 2) transitional vertebrae. Anal-fin origin substantially $(0.25-0.33 \mathrm{HL}$ distance) anterior to tip of pectoral fin, never posterior. Anal-fin rays 218250 (218-240, median 231 in population from central Amazon). Dorsal rami of recurrent branch of anterior lateral line nerve usually visible. Middorsal region of body scaled. Rows of scales above lateral line 5-7 (mode 6). Lateral line continuous. Very sparse, almost unnoticeable depigmented epidermal canals found mainly in posterior third of body - as parallel wavy lines either side of lateral line. Epidermal canals absent from either side of mid-dorsal region, and absent on flank midway from lateral line to dorsal midline. Hypaxial electric organ very large. Six bilateral electrocyte columns at anal-fin terminus, and 5-6 columns midway between anal-fin terminus and tip of caudal filament in immature, mature female, and mature male specimens. Number of columns of electrocytes in mid-caudal filament sometimes continues to tip, or sometimes dwindles to 2-3 columns. Caudal filament short to moderate in length. 


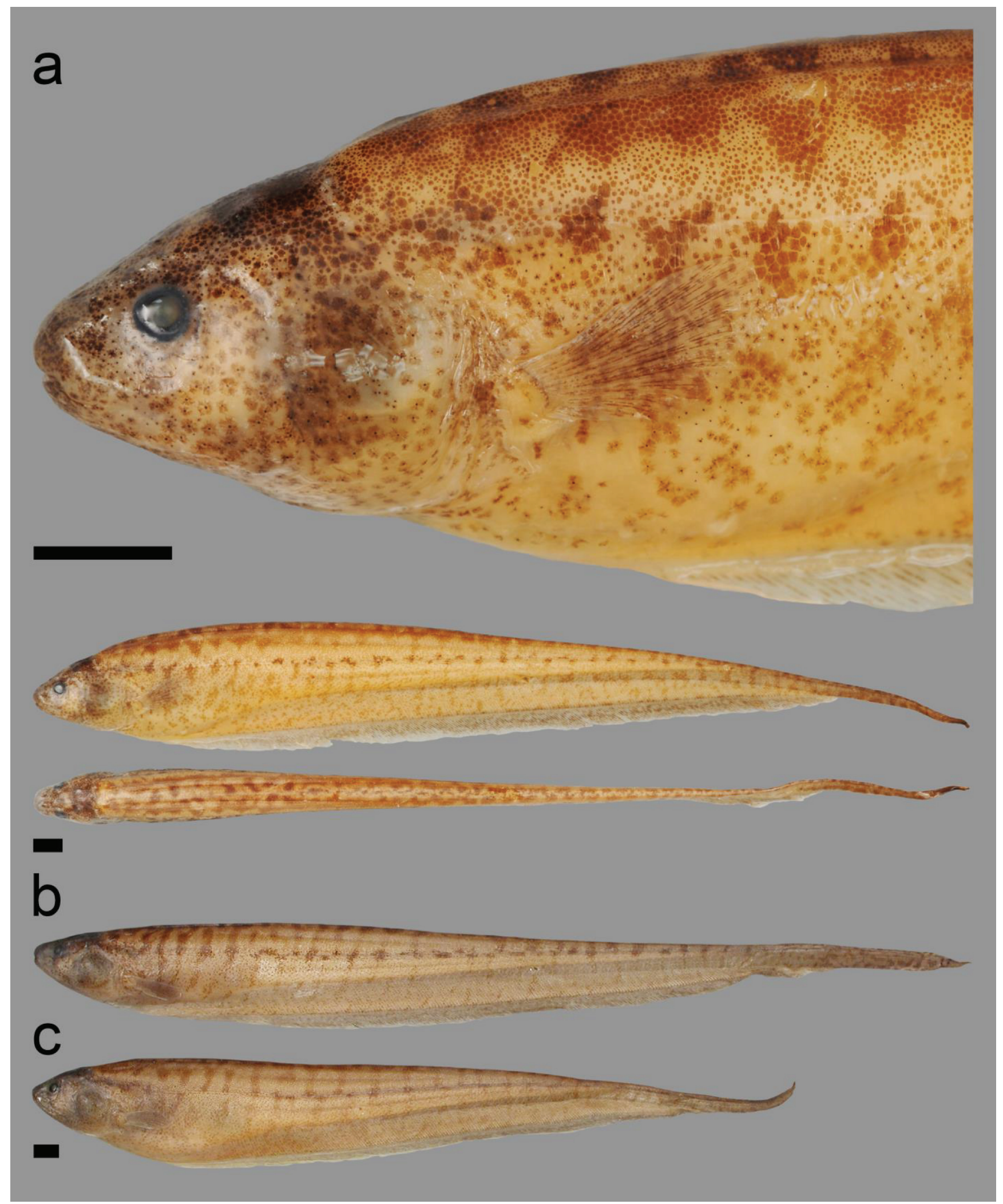

Fig. 13. Brachyhypopomus bennetti. a. MCP 47021 (WC13.160893), female, $158 \mathrm{~mm}$ TL (head in lateral view, and body in lateral and dorsal views, specimen fixed in formalin and preserved in EtOH). b. MCP 45359 (WC18.050497), male, $192 \mathrm{~mm}$ TL (body in lateral view, specimen fixed in formalin and preserved in EtOH). c. MCP 45359 (WC12.050497), female, 169 $\mathrm{mm}$ TL (body in lateral view, specimen fixed in formalin and preserved in EtOH); Brazil, rio Solimões-Japurá confluence, Amazonas dr. Note the sexual dimorphism in caudal filament height. Scale bars $=5 \mathrm{~mm}$. 
Table 8. Morphometrics for Brachyhypopomus bennetti, Brachyhypopomus bullocki, and Brachyhypopomus cunia. HT, holotype; SD, standard deviation. Data for holotype of B. bennetti (near Manaus, Amazonas, Brazil) are from Sullivan et al. (2013). Data for holotype of B. bullocki (Meta, Colombia) are from Sullivan \& Hopkins (2009). Ranges for B. bennetti refer to non-type specimens from the vicinity of Tefé, Amazonas, Brazil. Ranges for $B$. bullocki refer to non-type specimens from the Orinoco drainage of Venezuela and exclude holotype. Ranges for B. cunia refer to holotype, paratype and non-type specimens from the type region.

\begin{tabular}{|c|c|c|c|c|c|c|c|c|c|c|c|c|c|c|c|}
\hline & \multicolumn{5}{|c|}{ B. bennetti } & \multicolumn{5}{|c|}{ B. bullocki } & \multicolumn{5}{|c|}{ B. cunia } \\
\hline & HT & Range & Mean & SD & $\mathrm{n}$ & HT & Range & Mean & SD & $\mathrm{n}$ & HT & Range & Mean & SD & $\mathrm{n}$ \\
\hline Total length (TL) (mm) & 215 & $60-219$ & 129 & 38.1 & 148 & 221 & $110-189$ & 129 & 20.8 & 16 & 142 & $85-146$ & 121 & 56.1 & 17 \\
\hline Length to end of anal fin (LEA) (mm) & 176 & $55-184$ & 110 & 30.2 & 148 & 121 & $78-116$ & 89 & 9.5 & 16 & 104 & $79-104$ & 89 & 7.8 & 16 \\
\hline Head length (HL) (mm) & 18.5 & $10.8-19.4$ & 15.2 & 2.6 & 32 & 11.2 & $7.9-10.7$ & 8.9 & 0.7 & 16 & 10.7 & $7.5-10.7$ & 9.0 & 0.8 & 15 \\
\hline \multicolumn{16}{|c|}{ Proportion of TL $(\%)$} \\
\hline Caudal filament length (CF) & 18.1 & $6.5-20.7$ & 13.7 & 3.1 & 148 & 45.3 & $23.9-45.3$ & 30.3 & 4.4 & 17 & 26.1 & $22.1-33.6$ & 28.6 & 3.6 & 14 \\
\hline \multicolumn{16}{|c|}{ Proportion of LEA (\%) } \\
\hline Head length (HL) & 10.5 & $11.4-15.2$ & 12.7 & 1.0 & 32 & 9.3 & $9.2-10.8$ & 10.1 & 0.4 & 16 & 10.3 & $8.5-10.8$ & 10.0 & 0.6 & 14 \\
\hline Snout to occiput (SO) & 8.2 & $8.4-11.8$ & 9.8 & 0.8 & 32 & 7.2 & $7.2-8.7$ & 8.0 & 0.4 & 16 & 8.8 & $7.6-9.5$ & 8.5 & 0.5 & 14 \\
\hline Snout to anal-fin origin (SA) & 17.0 & $16.4-26.2$ & 20.4 & 2.2 & 32 & 16.3 & $15.7-19.5$ & 18.0 & 1.1 & 16 & 18.3 & $16.0-19.5$ & 17.8 & 1.1 & 14 \\
\hline Snout to pectoral-fin base (PP) & & $12.2-16.1$ & 13.7 & 0.9 & 32 & & $10.5-12.2$ & 11.4 & 0.5 & 16 & 11.4 & $10.5-11.6$ & 11.2 & 0.4 & 14 \\
\hline Longest pectoral-fin ray (PRL) & 5.7 & $5.2-7.9$ & 6.7 & 0.5 & 32 & 5.4 & 4.4-6.2 & 5.6 & 0.4 & 16 & 5.2 & $5.0-6.3$ & 5.5 & 0.4 & 14 \\
\hline Anal fin length (AF) & & $76.1-94.0$ & 86.3 & 4.2 & 32 & & $80.5-86.2$ & 82.8 & 1.4 & 16 & 82.7 & $80.2-84.4$ & 82.7 & 1.0 & 14 \\
\hline Longest anal-fin ray (ARL) & 4.0 & $1.7-3.8$ & 2.4 & 0.5 & 32 & 3.7 & $3.3-4.4$ & 4.0 & 0.3 & 16 & 4.0 & $3.7-4.2$ & 3.9 & 0.1 & 14 \\
\hline Body depth at anal-fin origin (BD1) & & $11.2-15.5$ & 13.2 & 1.2 & 32 & & $9.0-12.4$ & 11.1 & 0.9 & 16 & 10.6 & $9.2-11.8$ & 10.4 & 0.8 & 14 \\
\hline Body width at anal-fin origin (BW1) & & 4.8-7.4 & 6.0 & 0.7 & 32 & & $4.3-6.0$ & 5.4 & 0.5 & 16 & 4.9 & $4.3-5.5$ & 4.8 & 0.3 & 14 \\
\hline Body depth at anal-fin middle (BD2) & & $7.1-10.1$ & 8.7 & 0.7 & 32 & & $8.3-10.2$ & 9.2 & 0.7 & 16 & 8.8 & $8.0-10.1$ & 8.9 & 0.6 & 14 \\
\hline Body width at anal-fin middle (BW2) & & $1.7-3.2$ & 2.3 & 0.3 & 32 & & $2.1-3.5$ & 2.8 & 0.4 & 16 & 2.6 & $2.3-3.2$ & 2.7 & 0.3 & 14 \\
\hline Caudal filament length (CF) & 22.1 & $11.3-26.1$ & 18.6 & 3.5 & 30 & 82.7 & $31.4-62.9$ & 44.0 & 9.4 & 15 & 35.6 & $28.7-50.6$ & 40.5 & 7.0 & 14 \\
\hline \multicolumn{16}{|c|}{ Proportion of HL (\%) } \\
\hline Preorbital distance (PR) & 27.7 & $22.2-28.6$ & 25.4 & 1.5 & 32 & 26.8 & $18.7-23.5$ & 21.2 & 1.3 & 16 & 21.8 & $19.5-25.4$ & 22.5 & 1.5 & 15 \\
\hline Mouth width (MW) & 21.8 & $18.5-23.2$ & 21.2 & 1.3 & 32 & 25.5 & $22.6-35.0$ & 29.8 & 3.0 & 16 & 28.9 & $24.8-38.0$ & 28.6 & 3.5 & 15 \\
\hline Internarial distance $(\mathrm{AE})$ & & $16.5-21.6$ & 18.9 & 1.3 & 32 & & $16.0-21.9$ & 18.5 & 1.5 & 16 & 18.3 & $16.2-21.8$ & 18.7 & 1.5 & 15 \\
\hline Posterior naris to eye (PE) & 4.0 & $3.2-5.1$ & 4.1 & 0.5 & 32 & 2.1 & $2.4-5.8$ & 4.2 & 1.1 & 16 & 4.8 & $2.7-6.0$ & 4.5 & 1.0 & 15 \\
\hline Orbital diameter (OD) & 13.4 & $10.7-14.1$ & 12.3 & 0.9 & 32 & 17.9 & $14.2-18.5$ & 16.2 & 1.3 & 16 & 12.0 & $12.0-20.6$ & 17.1 & 1.9 & 15 \\
\hline Interorbital distance (IO) & 28.4 & $17.6-26.8$ & 23.0 & 2.2 & 32 & 26.8 & $24.0-30.0$ & 27.0 & 1.8 & 16 & 23.0 & $17.6-27.0$ & 23.0 & 2.6 & 15 \\
\hline Head depth at eye (HD1) & & $43.0-55.4$ & 49.4 & 2.4 & 32 & & $49.3-57.1$ & 52.7 & 2.5 & 16 & 53.8 & $47.8-59.9$ & 54.4 & 3.1 & 15 \\
\hline Head width at eye (HW1) & 44.9 & $30.4-48.2$ & 38.0 & 4.5 & 32 & 47.3 & $32.9-52.4$ & 41.9 & 4.4 & 16 & 36.3 & $34.8-48.8$ & 38.4 & 3.5 & 15 \\
\hline Head depth at occiput (HD2) & 80.3 & $69.6-85.4$ & 77.1 & 4.2 & 32 & 83.8 & $72.2-90.7$ & 82.3 & 4.6 & 16 & 82.5 & $75.0-94.6$ & 83.4 & 4.5 & 15 \\
\hline Head width at occiput (HW2) & & $44.4-59.0$ & 52.7 & 4.4 & 32 & & $49.5-64.4$ & 56.3 & 4.2 & 16 & 49.7 & $45.3-56.7$ & 48.8 & 2.9 & 15 \\
\hline Postorbital distance (PO) & & $61.1-66.1$ & 63.6 & 1.1 & 32 & & $60.1-68.2$ & 64.0 & 2.0 & 16 & 60.8 & $57.5-66.7$ & 60.6 & 2.4 & 15 \\
\hline Branchial aperture (BO) & 29.3 & $17.6-33.2$ & 25.3 & 3.6 & 32 & 27.4 & 19.7-30.9 & 26.6 & 3.1 & 16 & 25.5 & $16.9-28.9$ & 23.6 & 2.8 & 15 \\
\hline Pectoral-fin base (PB) & & $11.2-16.1$ & 13.6 & 1.4 & 32 & & $10.0-18.2$ & 13.0 & 1.8 & 16 & 13.3 & $9.5-14.1$ & 11.7 & 1.4 & 15 \\
\hline Caudal filament depth (CD) & 22.8 & $9.8-29.0$ & 15.7 & 4.7 & 32 & 25.0 & $15.2-19.9$ & 17.9 & 1.2 & 16 & 21.0 & $15.4-22.1$ & 18.5 & 2.2 & 14 \\
\hline Caudal filament width (CW) & & $1.9-9.7$ & 4.6 & 1.6 & 32 & & $5.9-9.6$ & 7.8 & 1.0 & 16 & 11.0 & $6.3-11.1$ & 8.8 & 1.6 & 14 \\
\hline \multicolumn{16}{|c|}{ Other proportions } \\
\hline BW1 ( $\%$ of BD1) & & $40.4-54.8$ & 45.5 & 3.1 & 32 & & $38.9-51.8$ & 48.4 & 2.9 & 16 & 46.0 & $42.4-51.6$ & 46.4 & 2.4 & 15 \\
\hline BW2 (\% of BD2) & & $21.7-32.8$ & 26.2 & 2.5 & 32 & & $24.7-34.1$ & 29.8 & 2.6 & 16 & 29.8 & $28.3-32.9$ & 30.8 & 1.3 & 15 \\
\hline
\end{tabular}

Coloration. (Figs. 1d, 13). Background pale straw to tan. Dorsal region with irregular brown blotches which extend to irregular-edged and sometimes divided brown bands which extend towards lateral line. No pale mid-dorsal stripe extending from occiput to base of caudal filament. A series of brown blotches is usually present along lateral line. Flank ventral to lateral line and pterygiophore region with very sparse and indistinct disrupted vertical bands. Caudal filament darker than body, with indistinct dark blotches and disrupted vertical bands. Head darker, especially dorsally. Eye with prominent suborbital patch of chromatophores and subcutaneous pigmentation (sometimes indistinct). Pectoral and anal-fin membranes hyaline. Pectoral and anal-fin rays hyaline with light scattering of brown chromatophores. Analfin ray pigmentation darker in posterior half of fin. Color in live individuals similar to preserved specimens, body sometimes with yellow-green tinge in waters with high sediment load, opercular region usually very rosy due to underlying gills. 
Size. Moderate adult size, largest specimen examined 235 $\mathrm{mm}$ TL, $215 \mathrm{~mm}$ LEA $(\mathrm{n}=1,444)$. Largest male specimen examined $223 \mathrm{~mm}$ TL, $183 \mathrm{~mm}$ LEA $(\mathrm{n}=51)$. Largest female specimen reported $215 \mathrm{~mm}$ TL, $176 \mathrm{~mm}$ LEA (holotype) $(\mathrm{n}=89)$.

Sexual dimorphism. Mature males in breeding condition attain slightly larger sizes, and develop much deeper caudal filaments than immature individuals and breeding females (Figs. 13b-c). Breeding males without an elevated number of bilateral horizontal columns or vertical rows of electrocytes. Instead breeding males exhibit clearly enlarged electrocytes relative to immature specimens and females. Breeding males with paddle-like lateral compression at caudal filament tip, which is partially or sometimes completely free of electrocytes. No known sexual dimorphism in pigmentation.

Geographic distribution. Bolivia, Brazil, Colombia, Ecuador, and Peru (Fig. 14). Restricted to whitewater floodplains of the main stem of the Amazon River and the lower reaches of its major whitewater tributaries, and known also from a single specimen (CAS 81631) from the upper río Beni floodplain, well above the major series of falls on the rio Madeira. This specimen was collected in 1921 by Nathan E. Pearson during the Mulford Expedition, and although faded, exhibits the diagnostic six horizontal electrocyte columns at the anal-fin terminus. One of us (WGRC) made extensive collections in the lower río Beni, near Riberalta, Bolivia, but failed to locate other specimens of $B$. bennetti in this area.

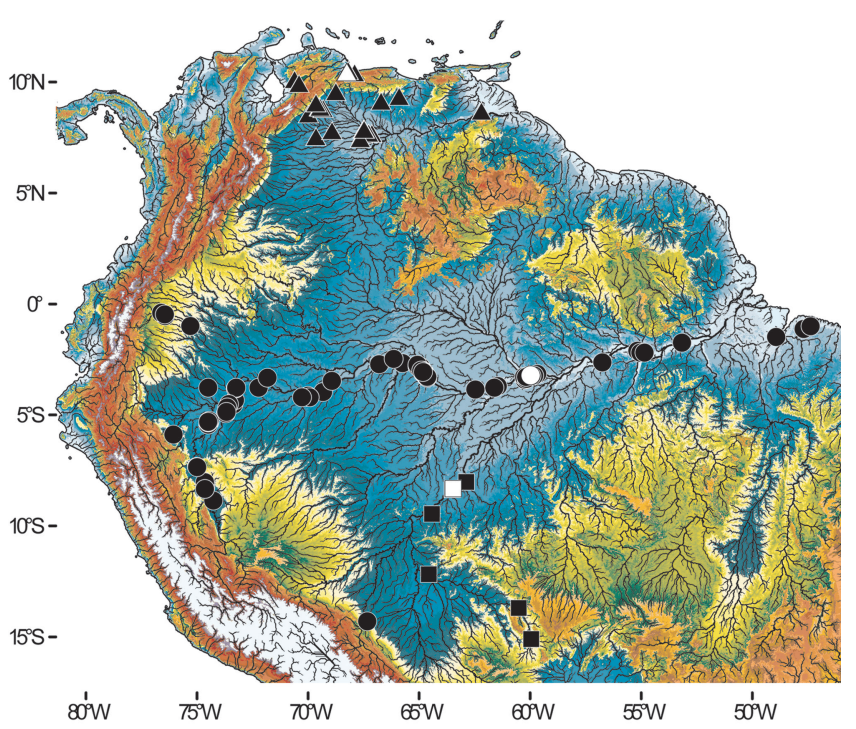

Fig. 14. Collection records for Brachyhypopomus bennetti (circles), B. cunia (squares), and $B$. diazi (triangles). Holotype locations for $B$. bennetti and $B$. cunia, and location of lost holotype for $B$. diazi are marked with open symbols. Elevation data refers to altitude above mean sea level (see Fig. 2 for legend).
Ecological notes. Brachyhypopomus bennetti is exceptionally common (usually the most common gymnotiform) in floating rafts of macrophytes in whitewater floodplains along the main stem of the Amazon, and the lower reaches of its major whitewater tributaries (Crampton, 1996a; 1998a,b; 1999; 2011; Crampton \& Albert, 2006; Crampton \& Ribeiro, 2013; Sullivan et al., 2013). Water parameters for a typical central Amazon whitewater floodplain fluctuate within the following ranges through the annual flood cycle: conductivity 54-192 $\mu \mathrm{Scm}^{-1}$ dissolved oxygen $0-6.3 \mathrm{mgl}^{-1}$, temperature 26.5$34.0^{\circ} \mathrm{C}$, and $\mathrm{pH}$ 6.3-7.3 (Crampton, 1998a). During the low water period $B$. bennetti is found in floating macrophytes along the edges of floodplain channels or lakes, or in smaller pockets of water in low-lying areas of the floodplain. During the rising water period it is common both in floating macrophytes, and in submerged forest leaf-litter at the margin of the advancing flood waters. During the high water period, when the bottom waters are completely deoxygenated, $B$. bennetti is almost completely restricted to floating macrophytes. Reproduction occurs during the rising and high water period in floating macrophytes (Crampton, 1996a: 165). Size class data from floodplain floating macrophytes indicate that $B$. bennetti has an annual life history; it reaches maturity and breeds within one year, and exhibits post-reproductive mortality (Crampton, 1996a: 166, fig. 10.1). The species is also relatively common in rooted and floating macrophytes along the edge of major whitewater river channels. Brachyhypopomus bennetti is absent in blackwater and clearwater systems except very close to their confluences with major whitewater rivers (for example in lago Tefé, near its confluence with the rio Solimões), where there are frequent ingresses of high-conductivity water.

Brachyhypopomus bennetti is a high-conductivity specialist, and its electric organ exhibits impedance matching to systems with low resistance (high conductivity) - with many bilateral columns of electrocytes (Crampton, 1998a; Crampton \& Albert, 2006; Sullivan et al., 2013). Brachyhypopomus bennetti is also adapted to protracted periods of anoxia, by undertaking aerial gill respiration (Crampton, 1996a; 1998b), and its gill lamellae are greatly expanded in comparison to species form normoxic systems, such as terra firme forest streams (Crampton et al., 2008). Brachyhypopomus bennetti reproduces during the late-rising and high water period (Crampton, 1996a). Stomach contents in populations from the central Amazon comprise aquatic insect larvae, microcrustacea, and other small aquatic invertebrates - with a predominance of Chironomidae larvae (Crampton, 1996a: 165).

Co-occurring congeners: Brachyhypopomus bennetti is known to co-occur in geographical sympatry and ecological syntopy with the whitewater floodplain-occurring species: B. beebei, B. belindae, B. brevirostris, B. flavipomus, B. hamiltoni, $B$. pinnicaudatus, $B$. regani, and $B$. walteri (and $B$. arrayae, and B. bombilla in the upper Madeira). It also exhibits an allotopic distribution with: $B$. batesi, $B$. benjamini, $B$. hendersoni, $B$. sullivani, and $B$. verdii (and with $B$. alberti in the upper Madeira). 
Local names. Brazil: sarapó; Colombia: cuchillo; Ecuador: cuchillo, yayo; Peru: macana.

Material examined. 1,466 specimens, localities from Amazonas dr. Bolivia. Beni. CAS 81631, 1, 142 mm (126 mm LEA), Reyes, pampa town, $24 \mathrm{mi}$. NE Rurrenabaque, río Beni, affl. rio Madeira, $14^{\circ} 17^{\prime} 42^{\prime \prime}$ S, $067^{\circ} 20^{\prime} 27^{\prime \prime} \mathrm{W}$. Note: Sullivan et al. (2013) mistakenly duplicated the record for CAS 81631 with CAS 72216 in the nontype list of the original description, CAS 81631 was in fact dismembered from CAS 72216. Brazil. Amazonas (localities listed from Mamirauá Reserve [Reserva de Desenvolvimento Sustentável Mamirauá] are in rio Solimões-Japurá floodplain, mun. Alvarães). ANSP 194026, 4, INPA 39562, 5, rio Camatiã, Monte Sinai, São Paulo de Olivença, 0327'34"S, 068 $56^{\circ} 00^{\prime \prime} \mathrm{W}$ (listed by Sullivan et al., 2013). BMNH 1998.3.12 56-62, 7, immature, INPA 18353, 2, immature, 51-85 mm, MCP 45252, 1, immature (CS), $83 \mathrm{~mm}$, Mamirauá Reserve, lago Juruazinho, $03^{\circ} 02^{\prime} 41^{\prime \prime} \mathrm{S}, 064^{\circ} 51^{\prime} 26^{\prime \prime} \mathrm{W}$. BMNH 1998.3.12 63-64, 2, female, mun. Tefé, lago do Içé, Ilha do Içé, rio Solimões, 03¹6'04"S, 06441'29"W. BMNH 1998.3.12.67-74, 8, BMNH 1998.3.12.65, 1, male, 210 mm, BMNH 1998.3.12.66, 1, male, 192 mm, BMNH 1998.3.12.80, 1, male, 181 mm, BMNH 1998.3.12.81, 1, female, $154 \mathrm{~mm}$, INPA 18352, 1, female, $139 \mathrm{~mm}$, INPA 18356, 1, immature, $50 \mathrm{~mm}$, MCP 33278, 2, 170-178 mm, MCP 45330, 1, male, $200 \mathrm{~mm}$, MCP 45389, 1, female, $165 \mathrm{~mm}$, MCP 46934, 1 (CS) $73 \mathrm{~mm}$, Mamirauá Reserve, cano do lago Rato, $03^{\circ} 02^{\prime} 41^{\prime \prime} \mathrm{S}$, 064'51'26"W. BMNH 1998.3.12.75, 1, immature, $156 \mathrm{~mm}$, BMNH 1998.3.12.76, 1, female, 151 mm, BMNH 1998.3.12.77, 1, male, $210 \mathrm{~mm}$, BMNH 1998.3.12.78, 1, female, $152 \mathrm{~mm}$, Mamirauá Reserve, Ressaca do Caetono, 02050'15"S, 06455'50"W. BMNH 1998.3.12.79, 1, female, 175 mm, Mamirauá

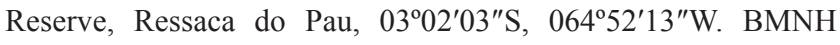
1998.3.12.82, 1, immature, $90 \mathrm{~mm}$, Mamirauá Reserve, lago Sumaumeirinha I, 02 $48^{\prime} 26^{\prime \prime} \mathrm{S}, 065^{\circ} 04^{\prime} 33^{\prime \prime} \mathrm{W}$. Rio Juruá nr. confl. with rio Solimões, CU 97640, 3 (paratypes), ca. $02^{\circ} 40^{\prime} \mathrm{S}$, $065^{\circ} 45^{\prime} \mathrm{W}$. IDSM 454, 3, 97-110 mm, Mamirauá Reserve, lago

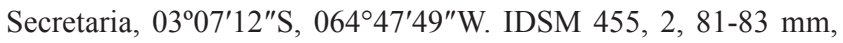
Mamirauá Reserve, lago Jaraqui, 02 $44^{\prime} 07^{\prime \prime S}, 065^{\circ} 04^{\prime} 38^{\prime \prime} \mathrm{W}$. INHS 44485, 5, 110-199 mm, INHS 70542 (part), 5, MZUSP 75571 (part), 6, 106-155 mm, USNM 306929, 6, 71-162 mm, USNM 306947, 1, lago Camaleão, Ilha da Marchantaria, rio Solimões, $03^{\circ} 14^{\prime} 22^{\prime \prime} \mathrm{S}, 059^{\circ} 56^{\prime} 37^{\prime \prime} \mathrm{W}$. INPA 6403, 5 (1 measured, $118 \mathrm{~mm}$ ), lago Janauacá, Ilha Janauacá, rio Amazonas, $03^{\circ} 24^{\prime} \mathrm{S}, 060^{\circ} 15^{\prime} \mathrm{W}$. INPA 39560, 1 (holotype), $215 \mathrm{~mm}$, examined from photograph in Sullivan et al. (2013), Paraná do Paracuúba, nr. mouth of lago Janauari, rio Amazonas, 03²' $12^{\prime} 36^{\prime \prime} \mathrm{S}, 059^{\circ} 59^{\prime} 24^{\prime \prime} \mathrm{W}$. INPA 8940, 3 (paratypes), 1, $190 \mathrm{~mm}$, examined from photograph in Sullivan et al. (2013), INPA 39581, 1 (paratype), $175 \mathrm{~mm}$, examined from photograph in Sullivan et al. (2013), nr. lago Janauari, rio Amazonas, $03^{\circ} 12^{\prime} 36^{\prime \prime} \mathrm{S}, 060^{\circ} 01^{\prime} 54^{\prime \prime} \mathrm{W}$. INPA 9944, 40, 52-175

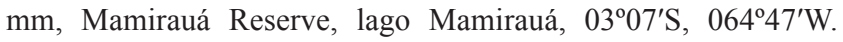
INPA 13569, 2, 45-67 mm, Ilha da Marchantaria, rio Solimões, $03^{\circ} 14^{\prime} 22^{\prime \prime} \mathrm{S}, 059^{\circ} 56^{\prime} 37^{\prime \prime} \mathrm{W}$. INPA 15816, 4 (2 female, $170 \mathrm{~mm}, 2$ male, 160-88 mm), INPA 18243, 1, immature, $192 \mathrm{~mm}$, INPA 18362, 1, female, $139 \mathrm{~mm}$, MCP 33279, 1, $176 \mathrm{~mm}$, MCP 45253, 2 ( 1 female, 179 mm, 1 male, 219 mm), MCP 45255, 8 (4 immature,
119-135 mm, 3 female, 116-145 mm, 1 male, 196 mm), Mamirauá Reserve, lago Secretaria, 0307'12"S, 06447'49"W. INPA 18241, 1, male, $221 \mathrm{~mm}$, MCP 45251, 2 (1 immature, $129 \mathrm{~mm}, 1$ male, $216 \mathrm{~mm}$ ), Mamirauá Reserve, cano do lago Sapucaia, 0304'07"S,

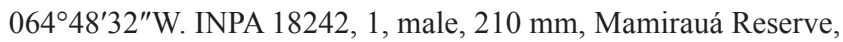
lago Periquito Redondo, 0304'50"S, 06446'35"W. INPA 18244 , 11 (6 female, 121-168 mm, 5 male, 173-223 mm), MCP 45254, 3 (1 female [CS], 141 mm, 2 male, 179-189 mm), Mamirauá Reserve, lago Geraldo, 0306 '53"S, 06449'06"W. INPA 18245, 4 (1 female, 152 mm, 3 male, 190-208 mm), MCP 45392, 2 (1 immature, $103 \mathrm{~mm}, 1$ female, $170 \mathrm{~mm}$ ), Mamirauá Reserve, lago Curuçá Aberto, 0306'07"S, 06449'10"W. INPA 18246, 3 (2 female, 150-166 mm, 1 male, $203 \mathrm{~mm}$ ), MCP 33281, 3, 169-179 mm, MCP 45393, 1, male, $190 \mathrm{~mm}$, Mamirauá Reserve, lago Curuçá Comprido, 0305'31"S, 06448'58"W. INPA 18348, 1, female, $145 \mathrm{~mm}$, INPA 18349, 1, female, $162 \mathrm{~mm}$, MCP 45345, 10 (5 female, 160-170 mm, 5 male, 190-208 mm), Mamirauá Reserve, cano do lago Arauaé, $03^{\circ} 03^{\prime} 54^{\prime \prime} \mathrm{S}, 064^{\circ} 49^{\prime} 04^{\prime \prime} \mathrm{W}$. INPA 18350, 1, female, $161 \mathrm{~mm}$, MCP 45348, 3, female, 156-177 mm, MCP 45394, 1, immature, 132 mm, Mamirauá Reserve, lago Araçazinho, 0259'35"S, 06451'33"W. INPA 18351, 2 (1 female, 139 mm, 1 male, 160mm), MCP 45347, 1, female, 138 mm, MCP 45399, 1, male, $170 \mathrm{~mm}$, Mamirauá Reserve, lago Periquito Comprido, 030' $57^{\prime \prime} \mathrm{S}, 064^{\circ} 46^{\prime} 42^{\prime \prime} \mathrm{W}$. INPA 18354, 3, immature, 46-102 mm, INPA uncat.\$ (ex. 18356), 1, immature, $41 \mathrm{~mm}$, MCP 45346, 3, female (1CS), 142-156 mm, MCP 45390, 1, immature, $107 \mathrm{~mm}$, MCP 45391, 1, female, 110 mm, Mamirauá Reserve, lago Juruá Grande, $03^{\circ} 01^{\prime} 51^{\prime \prime} \mathrm{S}, 064^{\circ} 51^{\prime} 07^{\prime \prime} \mathrm{W}$. INPA 18355, 3, female, 142162 mm, MCP 45401, 1, immature, 77 mm, Mamirauá Reserve, lago Promessa, 0304'29"S, 06447'02"W. INPA 18357, 3 (1 immature, $45 \mathrm{~mm}, 1$ female, $148 \mathrm{~mm}, 1$ male, $206 \mathrm{~mm}$ ), MCP 33280, 1, 76 mm, Mamirauá Reserve, lago Curuçá Redondo, $03^{\circ} 06^{\prime} 13^{\prime \prime} \mathrm{S}, 064^{\circ} 49^{\prime} 06^{\prime \prime} \mathrm{W}$. INPA 18358, 2 (1 immature, $52 \mathrm{~mm}, 1$ female, $156 \mathrm{~mm}$ ), Mamirauá Reserve, lago Miratinin, 030. $04^{\prime} 44^{\prime \prime} \mathrm{S}$, 064 50'17"W. INPA 18359, 2, immature, 50-59 mm, MCP 45256, 1, female, $165 \mathrm{~mm}$, Mamirauá Reserve, cano do lago Mamirauá, $03^{\circ} 05^{\prime} 15^{\prime \prime} \mathrm{S}, 064^{\circ} 48^{\prime} 03^{\prime \prime} \mathrm{W}$. INPA 18360, 4, immature, $42-55 \mathrm{~mm}$, MCP 45257, 2, immature, 87-91 mm, MCP 45465, 2, female, 142$178 \mathrm{~mm}$, Mamirauá Reserve, Ressaca da Vila Alencar, 0307'41"S, 064² $48^{\prime} 04^{\prime \prime W}$. INPA 18361, 2, immature, 54-63 mm, MCP 45429, 1, immature, $78 \mathrm{~mm}$, Mamirauá Reserve, lago Apolônio,

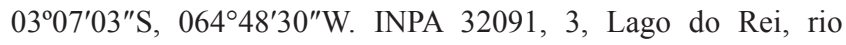
Amazonas floodplain, $c a .03^{\circ} 09^{\prime} \mathrm{S}, 059^{\circ} 41^{\prime} \mathrm{W}$. INPA 33187, 3, Ressaca do Luizinho, rio Jutaí, 02 $42^{\prime} 58^{\prime \prime} \mathrm{S}, 066^{\circ} 48^{\prime} 13^{\prime \prime} \mathrm{W}$ (listed by Sullivan et al., 2013). INPA 33196, 1, Palmares, Tabatinga, rio Solimões, $03^{\circ} 57^{\prime} 53^{\prime \prime} \mathrm{S}, 069^{\circ} 20^{\prime} 11^{\prime \prime} \mathrm{W}$ (listed by Sullivan et al., 2013). INPA 33255, 3, Ilha da Paciência, rio Solimões floodplain, $03^{\circ} 16^{\prime} 41^{\prime \prime} \mathrm{S}, 060^{\circ} 16^{\prime} 35^{\prime \prime} \mathrm{W}$ (listed by Sullivan et al., 2013). INPA 33270, 3, Lago Ressaca Grande, Fonte Boa, 02 $28^{\prime} 26^{\prime \prime} \mathrm{S}$, 06309'17"W (listed by Sullivan et al., 2013). INPA 39563, 4, Coari, 03 $51^{\prime} 10^{\prime \prime} \mathrm{S}, 063^{\circ} 28^{\prime} 07^{\prime \prime} \mathrm{W}$ (listed by Sullivan et al., 2013). MCP 45315, 2, female, 146-164 mm, MCP 45316, 1, male, 156 mm, MCP 45359, 17 (13 female [2 CS], 140-175 mm, 4 male [1 CS], 164-206 mm), Mamirauá Reserve, lago Araçazinho, 0259'35"S, 06451'33"W. MCP 45384, 4 (3 female, 157-162 mm, 1 male, $195 \mathrm{~mm})$, MCP 45388, 1, immature, 152 mm, Mamirauá 
Reserve, lago Arauaé, 0302'52"S, 06450'04"W. MCP 45400, 1, immature, $98 \mathrm{~mm}$, Mamirauá Reserve, lago Tracajá, 0305'43"S, 06446'31"W. MCP 45451, 1, male, 196 mm, Mamirauá Reserve, Paraná Maiana, 0306 $50^{\prime \prime}$ S, 06447'48"W. MCP 45466, 1, female, $136 \mathrm{~mm}$, MCP 45467, 4, immature, 82-125 mm, mun. Tefé, floodplain lake, Ilha Panamim, rio Solimões, 0319'06"S, 0643'ㅇ' $\mathrm{W}$. MCP 45471, 2, immature, 102-122 mm, mun. Tefé, lago Jacaré, 0311'11"S, 06443'05"W. MCP 47021, 1, female, $158 \mathrm{~mm}$, Mamirauá Reserve, lago Pagão, 0302'32"S, 06450'25"W. MCZ 9354, 6, 80-95 mm, mun. Parintins, Villa Bella [Vila Bela], rio Amazonas at Parintins and environs, $c a$. $02^{\circ} 38^{\prime} \mathrm{S}, 056^{\circ} 45^{\prime} \mathrm{W}$. MCZ 78163 (part), 7, 83-122, lago Jacaretinga, nr. Careiro de Várzea, rio Amazonas, 03 $13^{\prime} 00^{\prime \prime} \mathrm{S}, 059^{\circ} 49^{\prime} 41^{\prime \prime} \mathrm{W}$. MPEG 1111, 2, 103-151 mm, mun. Iranduba, rio Solimões, nr. Manaus, ca. $03^{\circ} 17^{\prime} \mathrm{S}, 060^{\circ} 11^{\prime} \mathrm{W}$ [coordinates for Iranduba]. MPEG 1123 (part), 7, 57-82, mun. Tefé, Costa Capivara, rio Solimões, ca. $03^{\circ} 16^{\prime} 38^{\prime \prime} \mathrm{S}, 064^{\circ} 38^{\prime} 09^{\prime \prime} \mathrm{W}$. MPEG 22745, 8, 88-215 $\mathrm{mm}$, Mamirauá Reserve, lago Mamirauá, 0259'38"S, 06454'26"W. MPEG 22746, 2, 96-131 mm, Mamirauá Reserve, lago Bolsinho, 0303'47"S, 06449'59"W. MZUSP 6326 (part), 1, $95 \mathrm{~mm}$, lago Castro, Boca do rio Purus, rio Purus, $03^{\circ} 42^{\prime} \mathrm{S}$, 0612'ㄴ. MZUSP 78954 (part), 1, 120 mm, Costa do Anori, nr. mouth of rio Purus, rio Solimões, $03^{\circ} 46^{\prime} \mathrm{S}, 061^{\circ} 37^{\prime} \mathrm{W}$. USNM 306841, 1, Paraná do lago Janauacá, rio Amazonas, ca. $03^{\circ} 22^{\prime} \mathrm{S}$, $060^{\circ} 10^{\prime} \mathrm{W}$. USNM 306859, 1, lago Terra Preta, lago do Janauari, rio Amazonas, $\mathrm{ca} .03^{\circ} 13^{\prime} \mathrm{S}, 060^{\circ} 00^{\prime} \mathrm{W}$. USNM 306875, 1, lago Janauari, nr. mouth, rio Amazonas, ca. $03^{\circ} 13^{\prime} \mathrm{S}, 060^{\circ} 00^{\prime} \mathrm{W}$. Pará. INPA 39564, 1, mun. Almeirim, Paranaguara, rio Amazonas, $01^{\circ} 44^{\prime} 29^{\prime \prime} \mathrm{S}, 053^{\circ} 10^{\prime} 15^{\prime \prime} \mathrm{W}$ (listed by Sullivan et al., 2013). MPEG 2403, 7, 101-143, MPEG 3722, 1, 109 mm, mun. igarapé-Açu, Pantanal do rio Livramento, nr. iron bridge [on hwy. PA-230], rio Jambu-Açu, affl. rio Marapanin (Amazon estuary), 0107'21"S, 04741'28"W. MCP 49401, 6, mun. Curuá, Lago Preto, Ilha São

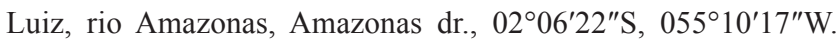
MPEG 7588, 1, 85 mm, MPEG 7597, 1, 59 mm, mun. Ponta de Pedras, Ilha de Marajó, Sitio Castanhal, rio Quiã-Paraná, affl. rio São José, affl. rio Marajó-Açu, affl. Baía do Guajará (Amazon estuary), 0129'09"S, 048 55'24"W. MPEG 12562, 3, 118-147 $\mathrm{mm}$, mun. Peixe Boi, branch of rio Peixe Boi, affl. rio Maracanã, (Amazon estuary), $c a .01^{\circ} 00^{\prime} \mathrm{S}, 047^{\circ} 23^{\prime} \mathrm{W}$ [coordinates for mouth of rio Peixe Boi]. UF 238557, 2, 131-140 mm, mun. Santarém, Lago de Santana, Ilha Marimarituba, rio Amazonas, 02 $11^{\prime} 13^{\prime \prime S}$, $055^{\circ} 02^{\prime} 21^{\prime \prime} \mathrm{W}$. UF 238570, 10, 66-107 mm, UF 238575, 1, 113 mm; UF 238577, 8, 66-104 mm; UF 238580, 3, 100-102 mm; mun. Santarém, lago Pajau, rio Amazonas, 02 $11^{\prime} 29^{\prime \prime} \mathrm{S}$, $054^{\circ} 51^{\prime} 28^{\prime \prime} \mathrm{W}$. Colombia. Amazonas. FMNH 85363, 38, 50-162 $\mathrm{mm}$, río Amazonas 2-3 mi. upstream Leticia, ca. $04^{\circ} 12^{\prime} \mathrm{S}$, $069^{\circ} 57^{\prime} \mathrm{W}$. UF 25560, 1, $121 \mathrm{~mm}$, UF 25561, 1, $66 \mathrm{~mm}$, N shore río Amazonas, just upstream Leticia, $04^{\circ} 08^{\prime} \mathrm{S}, 070^{\circ} 01^{\prime} \mathrm{W}$. UF $33843,1,151 \mathrm{~mm}$, pools on banks of río Amazonas at Leticia, ca. $04^{\circ} 12^{\prime} \mathrm{S}, 069^{\circ} 56^{\prime} \mathrm{W}$. USNM 216870, 1, Leticia, río Amazonas, $c a$. $04^{\circ} 12^{\prime} \mathrm{S}, 069^{\circ} 56^{\prime} \mathrm{W}$. Ecuador. Francisco de Orellana. FMNH 102276, 8, 60-128, Laguna Anangucocha, río Napo, 00³2'00"S, $076^{\circ} 26^{\prime} 42^{\prime \prime}$ W. Sucumbios. FMNH 102270, 2, 63-130, Laguna de Limoncocha, río Napo, 00²3'42"S, 076³6'49"W. QCAZ-I 1960, 1, 149 mm, QCAZ-I 1965 1, 176 mm, QCAZ-I 1968, 1, 138 mm, floodplain lake nr. Sacha Lodge, río Napo, 00²8'24"S, 076 27'24"W. Peru. Loreto. AMNH 78060, 14 (7 examined), 88$119 \mathrm{~mm}$, río Yarapá, affl. río Ucayali, 04³1'S, $073^{\circ} 22^{\prime} \mathrm{W}$. ANSP 178397 , 1, mouth of caño Chincana, río Yanayacu, 04¹9'51"S, $073^{\circ} 18^{\prime} 01^{\prime \prime}$ W. INHS 36829, 3, 72-193 mm, caño Ushpa, affl. río Itaya, affl. río Amazonas, $c a .03^{\circ} 48^{\prime} \mathrm{S}, 073^{\circ} 16^{\prime} \mathrm{W}$. INHS 39732, 1 , 85 mm, INHS 43679, 9, 60-120 mm, INHS 52031, 3, 130-144 mm, INHS 53874, 3, 98-132 mm, caño Moena nr. confl. with caño Ullpa, affl. río Itaya, affl. río Amazonas, 0346'20"S, 073¹4'17"W. INHS 54795, 6, 49-125 mm, floodplain S Nauta, río Marañón, 0430'36"S, 07334'06"W. MCP 26186, 29, 35-166 mm, MCP 26196, 8, 58-98 mm, Reserva Nacional Pacaya Samiria (RN Pacaya Samiria), río Pacaya, affl. río Ucayali, no coordinates. MCP 49413, 3, RN Samiria, Nauta Caño, affl. río Marañón, 04³9'33"S, 073³9'03"W. MUSM 4319, 5, 78-139 mm, quebrada Mondongo, río Huallaga, nr. Yurimaguas, affl. río Marañón, $c a$. $05^{\circ} 53^{\prime} \mathrm{S}, 076^{\circ} 04^{\prime} \mathrm{W}$. MUSM 4499, 4, nr. Contamana, río Ucayali, $07^{\circ} 20^{\prime} \mathrm{S}, 075^{\circ} 01^{\prime} \mathrm{W}$. MUSM 14498 (part), 1, $133 \mathrm{~mm}$, Puesto de Vigiliancia Arcadia, río Napo, 0059'37"S, 075¹8’30"W. MUSM 17333, 8, MUSM 17341, 22, RN Pacaya Samiria, caño Yarina, Puesto de Vigilancia II, río Pacaya, affl. río Ucayali, $05^{\circ} 20^{\prime} \mathrm{S}$, $074^{\circ} 30^{\prime} \mathrm{W}$. MUSM 44757, 1, immature, $117 \mathrm{~mm}$, cocha Supay, nr.

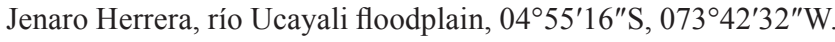
MUSM 44769, 52 (42 immature, 34-131 mm, 7 female, 71-109 mm, 3 male, 115-192 mm), cocha Capite, nr. Jenaro Herrera, río Ucayali floodplain, 0451'21'S, 07340'24"W. NRM 13522 (part), 1, 101 mm, NRM 27700, 1, 86 mm, caño Sacarita, opposite Pebas,

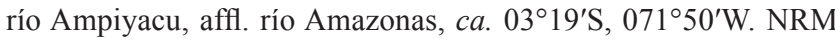
27523 (part), 1, $106 \mathrm{~mm}$, lago Matamata, affl. caño, río Yavarí (rio Javari) upstream from Atalaia do Norte (Brazil), 04²' $\mathrm{S}$, $070^{\circ} 17^{\prime} \mathrm{W}$. UF 114640, 7, 64-90 mm, RN Pacaya Samiria, río Pacaya, affl. río Ucayali, ca. $05^{\circ} 20^{\prime} 53^{\prime \prime} \mathrm{S}, 074^{\circ} 30^{\prime} 18^{\prime \prime} \mathrm{W}$. UF 126151, 10, 60-150 mm, UF 126193, 1, 90 mm, UF 131416, UF 129752, 6, 64-181 mm, 1, immature, 112 mm, RN Pacaya Samiria, caño Yarina, affl. río Ucayali, $05^{\circ} 20^{\prime} 09^{\prime \prime} \mathrm{S}, 074^{\circ} 30^{\prime} 05^{\prime \prime} \mathrm{W}$. UF 126161, 1, 116 mm, UF 129790, 2, 75-85 mm, UF 131417, 1, immature, $108 \mathrm{~mm}$, RN Pacaya Samiria, cocha Sapote, río Pacaya, affl. río Ucayali, $05^{\circ} 19^{\prime} 06^{\prime \prime} \mathrm{S}, 074^{\circ} 29^{\prime} 18^{\prime \prime} \mathrm{W}$. UF 126172, 1,110 mm, UF 128802, 24, 98-183 mm, UF 128870, 13, 95-175 mm, UF 128875, 50, 48-230 mm, UF 128876, 39, 62-155 mm, UF 128913 , 54, 31-130 mm, UF 128915, 35, 55-165 mm, UF 128929, 17, 52 $200 \mathrm{~mm}$, UF 128953, 4, 65-193 mm, UF 128955, 26, 65-185 mm, UF 128966, 14, 33-200 mm, UF 129099, 37, 61-175 mm, UF 129100, 14, 53-190 mm, UF 129122, 4, 65-120 mm, UF 129177, 1, 160 mm, UF 129178, 11, 63-235 mm, UF 129230, 33, 68-170 mm, UF 129240, 11, 57-180 mm, UF 129245, 22, 60-190 mm, UF 129251, 57, 40-190 mm, UF 129273, 62, 37-175 mm, UF 129299 , 18, 80-114 mm, UF 129325, 15, 97-176 mm, UF 129331, 20, 75$140 \mathrm{~mm}$, UF 129333, 35, 90-110 mm, UF 129335, 59, 45-160 mm, UF 129410, 40, 87-207 mm, UF 129814, 4, 35-110 mm, UF 131418 , 12, 71-200 mm, RN Pacaya Samiria, río Pacaya, affl. río Ucayali, $c a .05^{\circ} 16^{\prime} \mathrm{S}, 074^{\circ} 27^{\prime} \mathrm{W}$. UF 126301,2 , 74-87 mm, UF 129800, 26, 58-110 mm, RN Samiria, río Pacaya upstream caño Narina, affl. río Ucayali, $05^{\circ} 25^{\prime} 48^{\prime \prime} \mathrm{S}, 074^{\circ} 30^{\prime} 19^{\prime \prime} \mathrm{W}$. UF 129456 , 42, 45-145 mm, RN Pacaya Samiria, caño Tamara, affl. río Ucayali, $05^{\circ} 16^{\prime} 40^{\prime \prime} \mathrm{S}, 074^{\circ} 29^{\prime} 38^{\prime \prime} \mathrm{W}$. UF $129458,1,160 \mathrm{~mm}$, RN 
Pacaya Samiria, cocha Yanayacu, río Pacaya, affl. río Ucayali, 05¹8'14"S, 074²6'12"W. UF 129751, 2, 138-175 mm, RN Pacaya Samiria, río Pacaya, affl. río Ucayali, ca. 05¹7'37"S, 074²9'53"W. UF 131421, 1, male, 190 mm, RN Pacaya Samiria, caño Yarina, río Pacaya, affl. río Ucayali, $05^{\circ} 18^{\prime} 16^{\prime \prime} \mathrm{S}, 074^{\circ} 30^{\prime} 02^{\prime \prime} \mathrm{W}$. UMMZ 185108, 1, $115 \mathrm{~mm}$, caño Moena, mouth of caño Ullpa, affl. río Itaya, affl. rio Amazonas, 0346 $16^{\prime \prime} \mathrm{S}, 074^{\circ} 30^{\prime} 02^{\prime \prime} \mathrm{W}$. Ucayali. MCP 44238, 2, 87-92 mm, caño Cashibo, Yarinacocha, affl. río Ucayali, $08^{\circ} 17^{\prime} 08^{\prime \prime} \mathrm{S}, 074^{\circ} 37^{\prime} 50^{\prime \prime W}$. MUSM 1550, 1, 143 mm, MUSM 1791, 1, 117 mm, cocha Tacshitea, N Pucallpa, río Ucayali, $08^{\circ} 02^{\prime} \mathrm{S}, 074^{\circ} 39^{\prime} \mathrm{W}$. MUSM 3586, 3 (2 immature, 79-102 mm, 1 male, $118 \mathrm{~mm}$ ), MUSM 3606, 7 (3 immature, 69-113 mm,
1 female, 139 mm, 3 male, 114-164 mm), MUSM 3629, 1, 128 $\mathrm{mm}$, lago Imiria, Bella Flor, Coronel Portillo, affl. río Ucayali, $08^{\circ} 51^{\prime} \mathrm{S}, 074^{\circ} 16^{\prime} \mathrm{W}$. MUSM 6245, 15 (6 immature, 49-111 mm, 6 female, 111-166 mm, 3 male, 127-137 mm), Cashibococha, nr. Pucallpa, río Ucayali, $08^{\circ} 20^{\prime} \mathrm{S}, 074^{\circ} 39^{\prime} \mathrm{W}$. MUSM 9285, 1, immature, $61 \mathrm{~mm}$, MUSM 10874, 3 (2 immature, 93-100 mm, 1 male, $159 \mathrm{~mm})$, floodplain $\mathrm{nr}$. Contamana, río Ucayali, $07^{\circ} 21^{\prime} \mathrm{S}$, $075^{\circ} 00^{\prime} \mathrm{W}$.

\section{Brachyhypopomus bombilla Loureiro \& Silva, 2006}

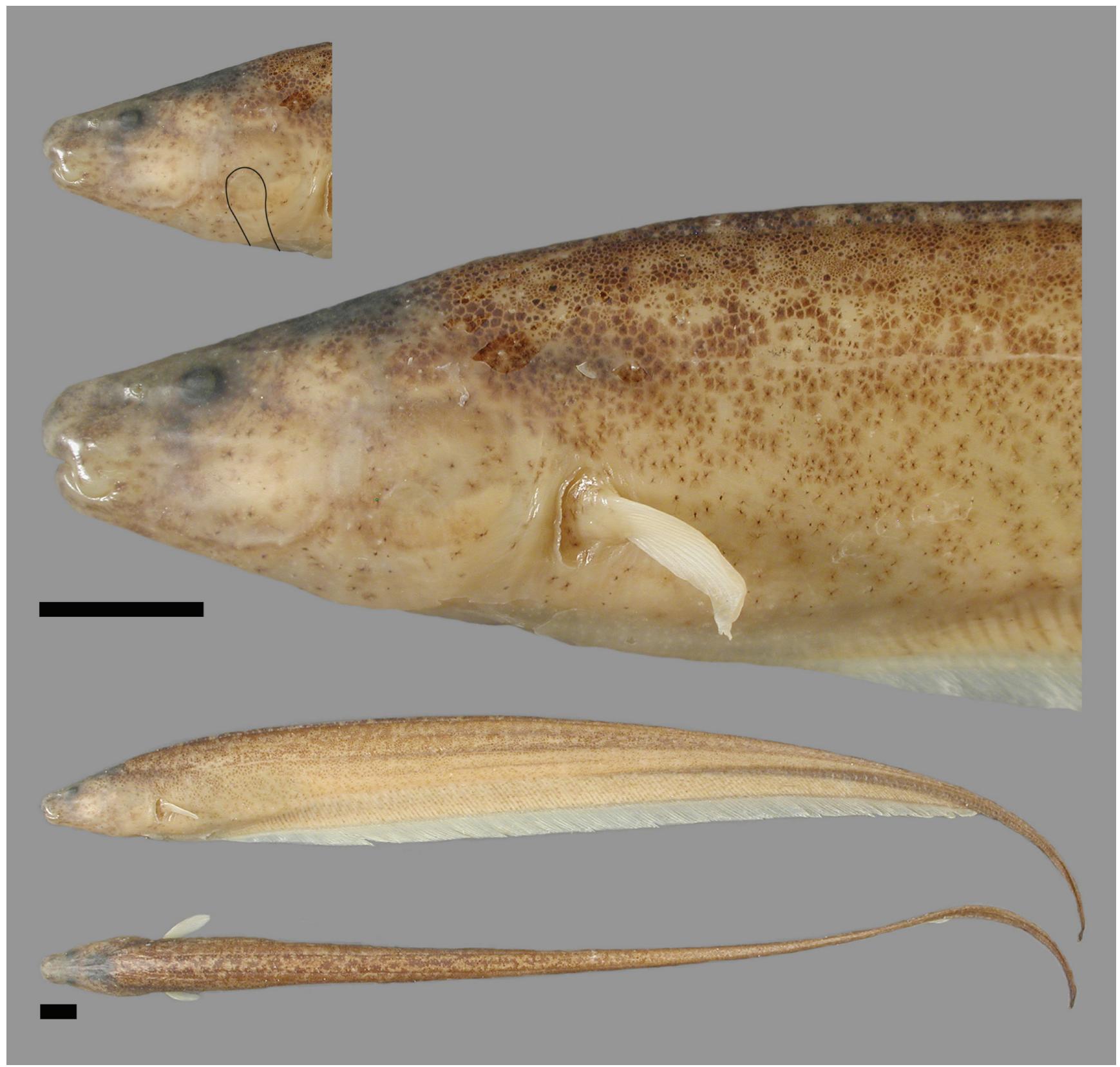

Fig. 15. Brachyhypopomus bombilla. MCP 19847 (part), male, $156 \mathrm{~mm}$ TL, non-type (head in lateral view, and body in lateral and dorsal view, specimen fixed in formalin and preserved in EtOH); Brazil, rio Ibicuí, Uruguay dr. Inset of head emphasizes outline of the opercular accessory electric organ with a black line. Scale bars $=5 \mathrm{~mm}$. 
Table 9. Morphometrics for Brachyhypopomus bombilla. HT., holotype; SD, standard deviation. Data for holotype (Rocha, Uruguay) are from Loureiro \& Silva (2006). Ranges for the type region (laguna dos Patos drainage of Brazil) refer to nontype specimens (data for holotype excluded). Ranges for the other populations refer to non-type specimens from the Paraguay drainage of Brazil and Paraguay (Paraná drainage) and the rio Beni drainage of Bolivia (Upper Madeira drainage).

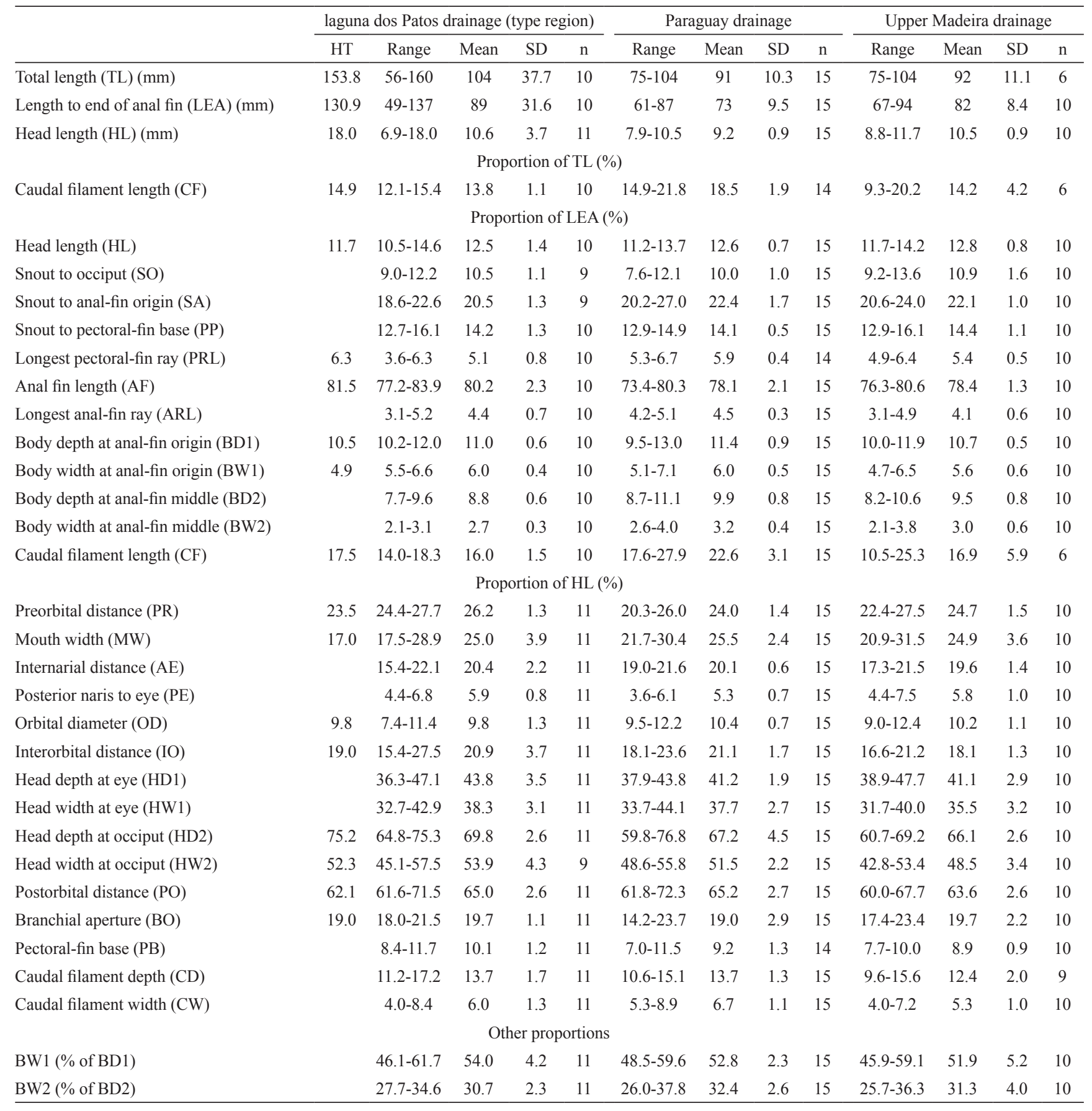

Rhamphichthys brevirostris. -Boulenger, 1896: 38 (Brazil, Mato Grosso, Descalvados, list of fishes collected in the Paraguay river by $\mathrm{C}$. Ternetz, record corresponds to BMNH 1895.5.17.265-268; identified as B. bombilla [part, $\mathrm{n}=3$ ], $B$. gauderio [part, $\mathrm{n}=1]$ ).

Hypopomidae indet. -Hagedorn \& Keller, 1996: 491, fig. 2b, drawing of adult specimen (Peru, Pakitza, Manu Reserve; diversity of gymnotiform species and EODs).
Brachyhypopomus electropomus. -Sullivan, 1997: 122, 322, fig. 54, line drawing of MZUSP 45032, Paraguay drainage (description in unpublished thesis with disclaimer stating nomenclatural acts therein not available, a nomen nudum; name therein refers also to $B$. menezesi and $B$. regani).

Brachyhypopomus sp. N. -Crampton \& Albert, 2006: 672, fig. 23.8, position in phylogenetic tree; 681, notes on EODs (species and EOD diversity). 
Brachyhypopomus bombilla Loureiro \& Silva, 2006: 666, fig. 1, black and white photograph of holotype (original description, type locality - Uruguay, Rocha Department, Cuatro Palmas stream, Patos-Mirim dr.). -Almirón et al., 2010: 573, fig. 2, photograph of live individual (Argentina, Entre Ríos, PreDelta National Park, Paraná dr., listing of Brachyhypopomus). -Crampton, 2011: 176, table 10.2, species list; 179, figs. 10.210.3, phylogeny, geographical and ecological distributions (gymnotiform biology). -Giora et al., 2011 (reproductive and trophic ecology). -Koerber, 2011: 7 (Argentina, species list). -Carvalho, 2013: 177, fig. 37B color photograph of head showing position of accessory electric organ; 472, fig. 1, color photographs of head; 181-185, figs. 41-43, position in phylogeny (phylogenetic systematics of Rhamphichthyoidea). -Mirande \& Koerber, 2015: 48 (Argentina, catalog of fishes). -Crampton et al., 2016: 1-66, table 1, 3-4, figs. 1-7, 18-20 (phylogeny, biogeography and ecology of Brachyhypopomus).

Diagnosis. Brachyhypopomus bombilla is diagnosed from congeners by the following combination of characters: presence of accessory electric organ over the opercular region, $v s$. absence in all congeners except $B$. menezesi and $B$. regani; dorsal surface uniformly speckled with small brown chromatophores on a pale background, $v s$. presence of large dark blotches against a lighter background in $B$. menezesi and B. regani.

Description. Head and body shape, and pigmentation illustrated in Fig. 15 and in the original description. Meristic and morphometric data for examined specimens are presented in Tables 2-5 and 9. Body shallow to moderate in depth. Head short to moderate in length and shallow to moderate in depth. Dorsal profile of head approximately straight from occiput to snout, concave above eye, ventral profile of head straight, snout truncated to rounded. Eye small to moderate in size. Upper jaw with slight sigmoidal angle between premaxillary and maxillary portions in lateral view. Accessory electric organ (AEO) over operculum present; skin over AEO with similar pattern of chromatophores to surrounding area, obscuring margin of $\mathrm{AEO}$ and rendering AEO difficult to see in most specimens. Gill filaments on first gill arch 29-34 (median 32, $\mathrm{n}=6$ ). Pectoral fin narrow to moderate in breadth, pectoral-fin rays 14-17 (mode of 15 in populations from Patos-Mirim lagoon system and upper Madeira, mode of 14 in populations from Uruguay drainages). Precaudal vertebrae 15-18 (mode of 18 from Patos-Mirim, 16 from río Paraguay drainages of Brazil and Paraguay, and 17 from upper Madeira), including 1-2 transitional vertebrae. Anal-fin origin slightly $(<0.25$ HL distance) anterior or posterior to, or near, tip of pectoral fin. Anal-fin rays 155-193 (median of 183 in populations from Patos-Mirim, 171 from río Paraguay, and 176 from upper Madeira). Dorsal rami of recurrent branch of anterior lateral line nerve not visible. Middorsal region of body scaled. Rows of scales above lateral line 5-7 (mode 6). Lateral line continuous. Multiple groove-like depigmented epidermal canals, some with dark borders, form parallel striations mostly dorsal to lateral line in posterior portion of body, beginning $c a$. two thirds of LEA; striations reaching 1-4 scales (including lateral line series) dorsally, and occasionally (with much lower density) 1-2 scales ventrally. Epidermal canals absent in anterior portion of body, never form a pair of long irregular lines either side of dorsal surface in dorsal portion of flank, and do not form isolated horizontal wavy line midway from lateral line to dorsal midline in midbody or anterior portion of body. Three bilateral horizontal columns of electrocytes at anal-fin terminus, and at mid-point between anal-fin terminus and tip of caudal filament in immature, mature female, and mature male specimens. Electrocytes of hypaxial electric organ extend anterior to anal and urogenital pores and appear to extend into opercular area to form paired accessory electric organ. Caudal filament short to moderate in length.

Coloration. (Fig. 15). Background pale straw. Dorsal region with more or less homogenous speckling of small brown chromatophores, declining in density ventrally. Dorsal region without pale stripe extending along midline from occiput to base of caudal filament. Flank ventral to lateral line only sparsely pigmented with irregular small clusters of brown stellate chromatophores. Caudal filament darker. Hypaxial electric organ conspicuous in posterior half to third of body. Head with evenly scattered dark chromatophores, darker dorsally. Eye usually with suborbital patch of subcutaneous pigmentation, of varying darkness and prominence. Region around lips usually depigmented. Presence of chromatophores over entire operculum disrupts borders of AEO, rendering it difficult to see in many specimens. This may account for why the authors of the original description failed to report the presence of this important character (although we inspected the holotype and found the AEO conspicuous). Pectoral and anal-fin membranes hyaline. Pectoral-fin rays hyaline. Analfin rays with uniform light brown pigmentation near bases, hyaline distally. Color in life faintly yellow in individuals from sediment-rich waters, with opercular region usually rosy due to underlying gills.

Size. Small to moderate adult size, largest specimen examined $161 \mathrm{~mm}$ TL, $140 \mathrm{~mm}$ LEA $(\mathrm{n}=537)$. Largest male specimen examined $161 \mathrm{~mm}$ TL, $140 \mathrm{~mm}$ LEA $(\mathrm{n}=13)$. Largest female specimen examined $147 \mathrm{~mm}$ TL, $124 \mathrm{~mm}$ LEA $(\mathrm{n}=43)$.

Sexual dimorphism. No known secondary sexual dimorphism.

Geographic distribution. Argentina, Bolivia, Brazil, Paraguay, Peru, and Uruguay (Fig. 16). Known from the Patos-Mirim lagoon system, Uruguay drainage, Paraná below the former Guaíra Falls, lower and upper Paraguay, and upper Madeira (Amazon drainage) above its major series of rapids beginning at the Salto Santo Antônio (near Porto Velho, Rondônia, Brazil). 
Population variation: We found complete overlap in the range of meristics (Tables 2-5) and morphometric proportions (Table 9), and observed similar pigmentation among populations of $B$. bombilla in the Patos-Mirim system, Paraguay dr., and upper rio Madeira. These observations, in combination with molecular data (Crampton et al., 2016), support the hypothesis that all populations we have assigned to $B$. bombilla are members of a single geographically widespread species, which is morphologically and genetically distinct from all congeners (although we were unable to obtain specimens for genetic analyses from the type locality in the Laguna Merín drainage).

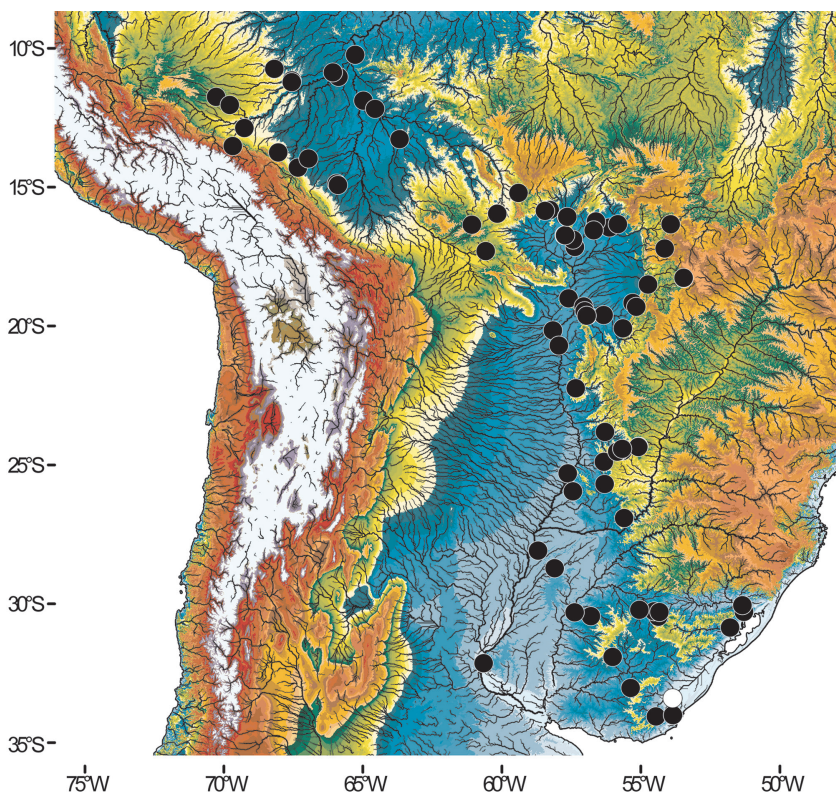

Fig. 16. Collection records for Brachyhypopomus bombilla (circles). Holotype location is marked with an open symbol. Elevation data refers to altitude above mean sea level (see Fig. 2 for legend).

Ecological notes. Brachyhypopomus bombilla occurs in floodplains, swamps and along the edges of slow-moving creeks and rivers - always in association with dense root mats of floating aquatic vegetation (Almirón et al., 2010; Giora et al., 2011; Koerber, 2011; Loureiro \& Silva, 2006). Giora et al. (2011) document the reproductive ecology of $B$. bombilla from southern Brazil. Breeding occurs during the austral spring and summer. In the upper Madeira, WGRC collected $B$. bombilla during the low water period from floating aquatic vegetation in floodplain pools and channelmargins of the río Beni floodplain (conductivity $c a .150$ $\mu \mathrm{Scm}^{-1}, \mathrm{pH}$ 7.3-7.6, dissolved oxygen 5-6 $\mathrm{mgl}^{-1}$, temperature ca. $28-32^{\circ} \mathrm{C}$ ). Hagedorn \& Keller (1996) report B. bombilla (identifications confirmed from voucher specimens at the USNM) from creeks of upper Madeira drainages in Peru. In the Paraná-Paraguay drainage, collection records indicate that $B$. bombilla is found in a variety of habitats, including swamps, riverine floodplains and slow-flowing creeks. Throughout its range $B$. bombilla appears to be adapted to a wide range of temperature, conductivity, and other parameters. In high latitudes it exhibits a period of dormancy in the austral winter, which is apparently unique among congeners (Giora et al., 2011). At this time it burrows in muddy substrates, slows its EOD, ceases to forage, and exhibits arrested gonadal development (Giora et al., 2011). Stomach contents in populations from southern Brazil (Giora et al., 2011) and from the upper Madeira (WGRC pers. obs.) comprise mostly aquatic insect larvae, microcrustacea, and other small aquatic invertebrates.

Co-occurring congeners: In the upper Madeira $B$. bombilla occurs in geographical sympatry and ecological syntopy with $B$. arrayae, $B$. brevirostris, $B$. pinnicaudatus, and $B$. walteri. It also exhibits an allotopic distribution with $B$. alberti and $B$. sullivani. In the upper portions of the Paraguay, $B$. bombilla co-occurs in sympatry and syntopy with $B$. brevirostris, $B$. draco, B. gauderio, and B. walteri. In the lower Paraná, $B$. bombilla co-occurs in sympatry and syntopy with $B$. draco, $B$. gauderio and $B$. walteri. In the lower portions of the Paraguay, and in the Patos-Mirim system, $B$. bombilla co-occurs in sympatry and syntopy with B. draco and B. gauderio.

Local names. Argentina, Paraguay: morenita; Bolivia: Cuchillo; Brazil: tuvira, moreninha (southern Brazil); Peru: macana; Uruguay: morenita, limpiavidrio (Nion et al., 2002).

Material examined. 527 specimens. Argentina. Corrientes. AI 267, 10, 91-132 mm, Esteros del Iberá, Laguna Galarza, Paraná dr., $28^{\circ} 04^{\prime} 56^{\prime \prime} \mathrm{S}, 056^{\circ} 42^{\prime} 04^{\prime \prime} \mathrm{W}$. MACN-ict 9457, 2, 91-96 mm, Iberá wetlands, río Corrientes, Paraná dr., $28^{\circ} 42^{\prime} 45^{\prime \prime} \mathrm{S}, 058^{\circ} 06^{\prime} 40^{\prime \prime} \mathrm{W}$. Entre Ríos. MACN-ict 9458, 1, 65 mm, Arroyo Las Mangas, PreDelta National Park, Paraná dr., 3207'59"S, 060³9'37"W. Bolivia. Beni (localities are from the rio Madeira dr., Amazonas dr.). CAS 81632, 1, $100 \mathrm{~mm}$, Reyes, $24 \mathrm{mi}$. NE Rurrenabaque, río Beni, $14^{\circ} 17^{\prime} 42^{\prime \prime} \mathrm{S}, 067^{\circ} 20^{\prime} 27^{\prime \prime} \mathrm{W}$. CBF 10271, 1, immature, $93 \mathrm{~mm}$, UMSS 7035, 1, female, $106 \mathrm{~mm}$, mun. Riberalta, stream nr. Hormiga, on Riberalta-Guayaramerín rd., affl. río Beni, $11^{\circ} 01^{\prime} 34^{\prime \prime} \mathrm{S}$, 065 52'58"W. CBF 10272, 1, immature, $87 \mathrm{~mm}$, CBF 10273, 1, immature (CS), $91 \mathrm{~mm}$, CBF 10274, 1, male (CS), $96 \mathrm{~mm}$, CBF 10275, 1, immature, $105 \mathrm{~mm}$, CBF 10276, 1, female, $101 \mathrm{~mm}$, UF 177344, 2, immature, 82-90 mm, UMSS 7036, 1, female, $99 \mathrm{~mm}$, UMSS 7037, 1, immature, $90 \mathrm{~mm}$, UMSS 7038, 1, female, $90 \mathrm{~mm}$, UMSS 7039, 1, immature, $87 \mathrm{~mm}$, UMSS 7040, 1, female, $83 \mathrm{~mm}$, mun. Riberalta, arroyo Tres Cuchillos, río Beni floodplain, $10^{\circ} 51^{\prime} 44^{\prime \prime} \mathrm{S}, 066^{\circ} 04^{\prime} 50^{\prime \prime} \mathrm{W}$. INHS $37243,3,74-87 \mathrm{~mm}, 41 \mathrm{~km} \mathrm{E}$ Estación Biológica del Beni, on rd. to San Ignacio [de Moxos], río Chevejecure, affl. río Apere, affl. río Mamoré, $14^{\circ} 52^{\prime} \mathrm{S}, 065^{\circ} 57^{\prime} \mathrm{W}$. INHS 50011, 2, 65-85 mm, 46 km E Estación Ecológica del Beni on rd. to San Ignacio [de Moxos], río Moseruna, affl. río Apere, affl. río Mamoré, $c a$. $14^{\circ} 55^{\prime} \mathrm{S}, 065^{\circ} 54^{\prime} \mathrm{W}$. UF 81921, 2, 102-104 mm, Bella Vista, rio Blanco, affl. rio Guaporé, $13^{\circ} 16^{\prime} \mathrm{S}, 063^{\circ} 41^{\prime} \mathrm{W}$. UMMZ 66420, 1, $89 \mathrm{~mm}$, lago Rogoagua, lake and nearby lagoons, río Beni floodplain, $c a .13^{\circ} 58^{\prime} \mathrm{S}, 066^{\circ} 58^{\prime} \mathrm{W}$. La Paz. CAS 72232, 1, $124 \mathrm{~mm}$, Ixiamas, $60 \mathrm{mi}$. NW Rurrenbaque, río Beni, $c a .13^{\circ} 45^{\prime} \mathrm{S}, 068^{\circ} 03^{\prime} \mathrm{W}$. 
Pando. FMNH 106643, 1, 78 mm, FMNH 106644, 28, 57-75 mm, FMNH 106645, 4, 57-72 mm, río Manuripi, ca. $20 \mathrm{~km}$ upstream Puerto Rico, río Manuripi, affl. río Orthon, affl. río Madre de Dios, affl. río Beni, ca. $11^{\circ} 09^{\prime} 06^{\prime \prime} \mathrm{S}, 067^{\circ} 33^{\prime} 41^{\prime \prime} \mathrm{W}$. FMNH 106646, 11, 53-129 mm, FMNH 106647, 17, 41-68 mm, río Manuripi, ca. 13 $\mathrm{km}$ upstream Puerto Rico, río Manuripi, affl. río Orthon, affl. río Madre de Dios, affl. río Beni, ca. $11^{\circ} 13^{\prime} \mathrm{S}, 067^{\circ} 34^{\prime} \mathrm{W}$. Santa Cruz. UF 82190, 3, 65-79 mm, 71 km N San José de Chiquitos, río San Diablo, affl. río Itonama, affl. río San Martín, affl. río Guaporé, affl. río Mamoré, $17^{\circ} 18^{\prime} \mathrm{S}, 060^{\circ} 35^{\prime} \mathrm{W}$. UF 82309, 1, $65 \mathrm{~mm}$, creek $22 \mathrm{~km}$ W San Javierito and $49 \mathrm{~km}$ W San Ignacio, río Paragua, affl. río Guaporé, affl. río Mamoré, ca. $16^{\circ} 21^{\prime} \mathrm{S}, 061^{\circ} 05^{\prime} \mathrm{W}$. Brazil. Acre. MCP 41034, 2, 66-107 mm, Fazenda Uberaba, ca. 6 km hwy. BR317, rio Iná, affl. rio Xipamanu, affl. rio Abunã, 1044'13"S, $068^{\circ} 11^{\prime} 16^{\prime \prime}$ W. Mato Grosso (localities in rio Paraguai dr., Paraná dr., unless otherwise stated). ANSP 53894, 2, 57-78 mm, BMNH 1895.5.17.265-268 (part), 3, 71-107 mm, mun. Cácares, Descalvados, 1643'59"S, 05744'54"W. BMNH 1900.4.14.91-94 (part), 1, $72 \mathrm{~mm}$, mun. Carandàzinho, rio Cuiabá, ca. $16^{\circ} 27^{\prime} \mathrm{S}$, $056^{\circ} 08^{\prime} \mathrm{W}$. FMNH 54551, 10, 54-127 mm, MCP 15629, 9 (9 immature, 76-110 mm), mun. Cáceres, nr. Cáceres, ca. $16^{\circ} 04^{\prime} \mathrm{S}$, $057^{\circ} 39^{\prime} \mathrm{W}$. FMNH 54553, 1, nr. Conceição [Porto Conceição], ca. $15^{\circ} 57^{\prime} \mathrm{S}, 060^{\circ} 10^{\prime} \mathrm{W}$. FMNH 70397, 1, 88 mm, nr. Conceição, ca. $17^{\circ} 09^{\prime} \mathrm{S}, 057^{\circ} 23^{\prime} \mathrm{W}$. MCP 15646, 1, female, $69 \mathrm{~mm}$, Porto Esperidião, arroio Zé do Cacete, km 70 on Cáceres-Porto Esperidião hwy. BR-174, 1549'S, 058 $17^{\prime} \mathrm{W}$. MCP 41030, 1, $80 \mathrm{~mm}$, mun. Vila Bela da Santíssima Trindade, stream $10 \mathrm{~km}$ rd. between Pontes e Lacerda and Vila Bela da Santíssima Trindade, affl. rio Guaporé, affl. rio Mamoré, affl. rio Madeira, Amazonas dr., 15¹3'58"S, 059²4'30"W. MNRJ 12242, 6, 93-115 mm, mun. Cáceres, Fazenda Pantanalzinho, district of Porto Espiridião, Córrego Carrapato, rio Jauru, $c a$. $15^{\circ} 51^{\prime} \mathrm{S}, 058^{\circ} 27^{\prime} \mathrm{W}$. MZUSP 19243, 1, male, $98 \mathrm{~mm}$, Poconé, ca. $16^{\circ} 14^{\prime} \mathrm{S}, 056^{\circ} 37^{\prime} \mathrm{W}$. MZUSP 25137, 3 (1 immature, 94 mm, 2 female, 87-97 mm), mun. Cáceres, Ilha da Taiamã [Estação Ecológica Taiamã], rio Paraguai, $16^{\circ} 53^{\prime} \mathrm{S}, 057^{\circ} 27^{\prime} \mathrm{W}$ [coordinates from center of island]. MZUSP 25257, 1, $93 \mathrm{~mm}$, Fazenda Santo Antônio do Paraíso, rio Piquiri-Itiquira, $17^{\circ} 12^{\prime} \mathrm{S}, 054^{\circ} 09^{\prime} \mathrm{W}$. MZUSP 43681, 26, MZUSP 45032, 1, 101 mm, mun. Cáceres, Descalvados, ca. $16^{\circ} 44^{\prime} \mathrm{S}, 057^{\circ} 44^{\prime} \mathrm{W}$. MZUSP 44261, 9, mun. Cácares, Cácares and vicinity, ca. $16^{\circ} 04^{\prime} \mathrm{S}, 057^{\circ} 40^{\prime} \mathrm{W}$. USNM 326426, 10, 61-106 mm, nr. Cáceres, ca. 1604'S, 057³9’W. ZUEC 4291, 1, 76 mm, ZUEC 4329, 1, 72 mm, mun. Poconé, rio Piraputanga, affl. rio Vermelho, affl. rio Cuiabá, ca. $16^{\circ} 20^{\prime} \mathrm{S}$, 0535'' W. ZUEC 4351, 1, 94 mm, ZUEC 5449, 3, 73-90 mm, mun. Poconé, km 19, Transpantaneira hwy., rio Bentes Gomes, $c a$. $16^{\circ} 33^{\prime} \mathrm{S}, 56^{\circ} 42^{\prime} \mathrm{W}$. Mato Grosso do Sul (localities in rio Paraguai dr., Paraná dr.). FMNH 54553, 1, 105 mm, FMNH 108540, 16, 38 $88 \mathrm{~mm}$, Corumbá, rio Vermelho, 19³7'13"S, 56 57'16"W. FMNH 108539 , 3, 70-80 mm, rio Vermelho at Fazenda Xarães, $19^{\circ} 36^{\prime} 44^{\prime \prime} \mathrm{S}$, $56^{\circ} 55^{\prime} 25^{\prime \prime} \mathrm{W}$. MUSM 16955, 1, immature, $99 \mathrm{~mm}$, Taquari, rio Bonito, $18^{\circ} 16^{\prime} 23^{\prime \prime} \mathrm{S}, 053^{\circ} 27^{\prime} 27^{\prime \prime} \mathrm{W}$. MUSM 17021, 4, 71-81 mm, Brazo de Santana Sofia, $19^{\circ} 35^{\prime} 59^{\prime \prime}$ S, 056 $20^{\circ} 47^{\prime \prime}$ W. MZUSP 36341 , 2, Ladário, nr. Corumbá, $19^{\circ} 00^{\prime} \mathrm{S}, 057^{\circ} 36^{\prime} \mathrm{W}$. MZUSP 48334, 3, female, 69-80 mm, Pantanal do Paiaguás, Baía da Sede, $18^{\circ} 30^{\prime} \mathrm{S}$, 05445’W. MZUSP 59406, 1, female, 87 mm, Córrego da Furna, riacho Água Clara, $18^{\circ} 15^{\prime} 45^{\prime \prime} \mathrm{S}, 053^{\circ} 27^{\prime} 59^{\prime \prime} \mathrm{W}$. MZUSP 59411, 1 , male, $97 \mathrm{~mm}$, Corumbá, rio Negro on rd. between Nhecolândia and hwy. BR-262, $19^{\circ} 17^{\prime} 16^{\prime \prime} \mathrm{S}, 057^{\circ} 03^{\prime} 39^{\prime \prime} \mathrm{W}$. MZUSP 59412, 1, immature, $49 \mathrm{~mm}$, Corumbá, rio Abobral, 19²7'37"S, 05701'14"W. MZUSP 59441, 33, (15 measured: 2, immature, 61-78 mm, 13 female, 61-100 mm), Corumbá, rio Miranda, bay on left bank, rio Vermelho, 19³7'22"S, 056 ${ }^{\circ} 57^{\prime} 27^{\prime \prime} \mathrm{W} . \quad$ MZUSP 59444, 4 (1 immature, $99 \mathrm{~mm}, 2$ female, $72-93 \mathrm{~mm}, 1 \mathrm{male}, 100 \mathrm{~mm}$ ), Aquidauana, Fazenda Taboco, dead end of rio Taboco, $20^{\circ} 04^{\prime} 38^{\prime \prime} \mathrm{S}$, 055 38'51"W. MZUSP 59445, 2, female, 68-79 mm, rio Verde de Mato Grosso, Anhuma, on rd., rio Negro, $19^{\circ} 09^{\prime} 57^{\prime \prime} \mathrm{S}, 055^{\circ} 17^{\prime} 56^{\prime \prime} \mathrm{W}$. MZUSP 60051 (part), 2, immature, 76-99 mm, rio Verde de Mato Grosso, rio Negro, $19^{\circ} 18^{\prime} 17^{\prime \prime} \mathrm{S}, 055^{\circ} 10^{\prime} 20^{\prime \prime} \mathrm{W}$. MZUSP 95081, 12 (10 immature, 52-83 mm, 2 female, 89-102 mm), mun. Barão de Melgaço, rio Mutum, between Vila de Mimoso and Joselândia, Pantanal de Paiaguás, $16^{\circ} 19^{\prime} 30^{\prime \prime} \mathrm{S}, 055^{\circ} 49^{\prime} 59^{\prime \prime} \mathrm{W}$. Rio Grande do Sul. BMNH 1889.8.24.8, 1, 124 mm, mun. Camaquã, Camaquã, arroio Duro, affl. rio Camaquã, Patos-Mirim dr., ca. $30^{\circ} 51^{\prime} \mathrm{S}$, 051 ${ }^{\circ} 48^{\prime}$ W. BMNH 1891.3.16.103-105, 3, 89-140 mm, "Rio Grande do Sul", drainage unknown, no coordinates. MCP 15326, 1, female, $73 \mathrm{~mm}$, canals along hwy. BR-290 between 1 and $6 \mathrm{~km}$ junction with hwy. BR-116, rio Guaíba, Patos-Mirim dr., between $30^{\circ} 02^{\prime} 39^{\prime \prime} \mathrm{S}, 051^{\circ} 21^{\prime} 20^{\prime \prime} \mathrm{W}$ and $30^{\circ} 02^{\prime} 48^{\prime \prime} \mathrm{S}, 051^{\circ} 23^{\prime} 27^{\prime \prime} \mathrm{W}$. MCP 19545, 1, male, 149 mm, 13.7 km, from São Gabriel, rio Vacacaí, affl. rio Jacuí, affl. rio Guaíba, Patos-Mirim dr., 30²7'18"S, $054^{\circ} 22^{\prime} 26^{\prime \prime} \mathrm{W}$. MCP 19807, 4 (1 immature, 88 mm, 2 female, 81-98 $\mathrm{mm}, 1 \mathrm{male}, 117 \mathrm{~mm}$ ), river at Barra do Ribeiro, affl. rio Guaíba, Patos-Mirim dr., 30¹7'03"S, 051 '18'04"W. MCP 19847 (part), 6 (3 immature, 127-140 mm [2 CS], 2 female, 100-147 mm, 1 male, 156 $\mathrm{mm}$ ), bridge over Banhado do Inhatium, hwy. BR-290, 21 km São Gabriel, affl. rio Ibicuí, Uruguay dr., 30¹5'43"S, 054³1'33"W. MCP 19869, 1, male, $161 \mathrm{~mm}$, MCP 45355, 3, 51-65 mm, bridge on São Gabriel-Tiaraju hwy., affl. rio Vacacaí, affl. rio Jacuí, affl. rio Guaíba, Patos-Mirim dr., 30¹7'29"S, 054²0'18"W. UFRGS 6495, 1, male, 131 mm, UFGRS 6496, 1, male, 133 mm, UFRGS 6498, 1, male, $136 \mathrm{~mm}$, mun. Rosário do Sul, stream at km 82 on hwy. BR290, affl. rio Santa Maria, affl. rio Ibicuí, Uruguay dr., $30^{\circ} 12^{\prime} 42^{\prime \prime} \mathrm{S}$, $055^{\circ} 03^{\prime} 17^{\prime \prime} \mathrm{W}$. UFRGS 6741, 1, $138 \mathrm{~mm}$, UFRGS 6742, 1, $63 \mathrm{~mm}$, UFRGS 6743, 1, 65 mm, UFRGS 6744, 1, 59 mm, UFRGS 6745, 1, $88 \mathrm{~mm}$, UFRGS 6746, 1, UFRGS 6747, 1, 49 mm, UFRGS 9273, 18, 57-126 mm, UFRGS 9282, 15, 58-99 mm, UFRGS 9284, 17 , 61-132 mm, Rosário do Sul, Sanga do Jacaré, stream at BR-290, affl. rio Ibicuí, Uruguay dr., 30² $12^{\prime} 42^{\prime \prime} \mathrm{S}, 055^{\circ} 03^{\prime} 19^{\prime \prime} \mathrm{W}$. UFRGS 10561, 2, 94-108 mm, mun. Rosário do Sul, arroio do Jacaré, affl. rio Ibicuí, Uruguay dr., $30^{\circ} 12^{\prime} 43^{\prime \prime} \mathrm{S}, 055^{\circ} 03^{\prime} 18^{\prime \prime} \mathrm{W}$. Rondônia (localities from rio Madeira, dr., Amazonas dr.). INPA 19944, 5, 71$97 \mathrm{~mm}$, rio Mamoré, upstream Surprêsa, $c a .11^{\circ} 53^{\prime} \mathrm{S}, 064^{\circ} 59^{\prime} \mathrm{W}$. MCP 41031 (part), 2, 87-88 mm, mun. Nova Mamoré, rio Ribeirão at hwy. BR-425, ca. $62 \mathrm{~km} \mathrm{~S}$ hwy. BR-364, affl. rio Guaporé, affl. rio Mamoré, $10^{\circ} 13^{\prime} 55^{\prime \prime} \mathrm{S}, 065^{\circ} 16^{\prime} 55^{\prime \prime} \mathrm{W}$. UFRO-I 6478, 6, 61-76 $\mathrm{mm}$, rio Madeira, no specific locality, no coordinates. UFRO-I 6507, 2, 112-112 mm, UFRO-I uncat. CAU2010010701, 1, 85 mm, mouth of the rio Cautário, affl. rio Guaporé, affl. rio Mamoré, $12^{\circ} 10^{\prime} 51^{\prime \prime} \mathrm{S}, 064^{\circ} 34^{\prime} 00^{\prime \prime} \mathrm{W}$. Paraguay (localities from río Paraguay dr., Paraná dr.). Alto Paraguay. FMNH 108057, 1, 49 mm, MZUSP 54330, 1, $86 \mathrm{~mm}$, Estancia Voluntad, at Puerto Voluntad, 2042'S, $057^{\circ} 57^{\prime} \mathrm{W}$. FMNH 108058, 1, $103 \mathrm{~mm}$, rio Negro, 2009's, 
$058^{\circ} 10^{\prime}$ W. Caaguazú. ANSP 175179, 1, 97 mm, stream crossing rd. N Coronel Oviedo, 2452'59"S, 056 $20^{\circ} 10^{\prime \prime} \mathrm{W}$. Canindeyú. NRM 32529, 1, $116 \mathrm{~mm}$, río Jejui-Guazú, $76 \mathrm{~km}$, rd. to Curuguaty, $24^{\circ} 19^{\prime} 42^{\prime \prime} \mathrm{S}, 055^{\circ} 05^{\prime} 13^{\prime \prime} \mathrm{W}$. NRM 32530, 1, female, $111 \mathrm{~mm}$, stream

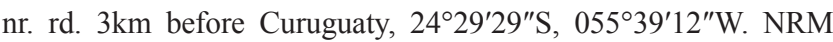
32531 , 1, male, $115 \mathrm{~mm}$, small stream, ca. $12 \mathrm{~km}$ on Curuguaty-

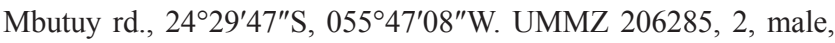
115-142 mm, Arroyo Carimbatay, ca. $15.6 \mathrm{~km}$ WSW Curuguaty, Arroyo Curuguaty, río Jejui-Guazú, 2431'06"S, 056 43'30"W. UMMZ 206470, 1, 84 mm, small stream, affl. río Jegui-Guazú, ca. $79 \mathrm{~km}$ by dirt rd. ENE turnoff to Curuguaty, nr. Santo Domingo, $c a$. $24^{\circ} 21^{\prime} \mathrm{S}, 055^{\circ} 06^{\prime} \mathrm{W}$ [coordinates for Santo Domingo]. UMMZ 206536, 1, 96 mm, small stream, affl. Arroyo Curuguaty, ca. $5.3 \mathrm{~km}$ NNW Curuguaty, río Jejui-Guazu, 242ำ $06^{\prime \prime} \mathrm{S}$, $055^{\circ} 40^{\prime} 48^{\prime \prime} \mathrm{W}$. Central. BMNH 1935.6.4.370-372, 2, 75-85 mm, riacho Caracara, W Asunción, $c a$. $25^{\circ} 18^{\prime} \mathrm{S}, 057^{\circ} 38^{\prime} \mathrm{W}$. Concepción. MZUSP 54331, 1, female, $86 \mathrm{~mm}$, riacho Blandengue, nr. confl. with río Apa, $22^{\circ} 14^{\prime} \mathrm{S}, 057^{\circ} 21^{\prime} \mathrm{W}$. Guairá. NRM 41902, 1, male, $118 \mathrm{~mm}$, stream crossing rd. from Mauricio J. Troche to Independencia, $5 \mathrm{~km} \mathrm{~S}$ río Tebicuary bridge, $25^{\circ} 40^{\prime} 59^{\prime \prime} \mathrm{S}, 056^{\circ} 18^{\prime} 40^{\prime \prime} \mathrm{W}$. Itapúa. NRM 42291 , 1, female, 136 mm, NRM 42294, 1, 75 mm, Bella Vista, Arroyo Posore at $12 \mathrm{~km}$ before Pirapó on rd. from Obligado, 26 54'34"S, 055³6'37"W. Paraguarí. NRM 32533, 1, 98 mm, lago Ypoá, Estancia Ypoá, 2556'43"S, 057²7'03"W. San Pedro. NRM 23195 , 1, female, $122 \mathrm{~mm}$, Estancia Laguna Blanca, Arroyo Mborebi from

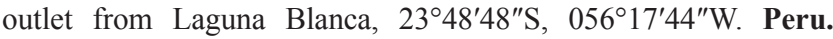
Madre de Dios (localities from río Madre de Dios dr., affl. río Beni, affl. río Madeira, Amazonas dr.). MUSM 1388, 1, 132 mm, MUSM 1428, 1, 126 mm, MUSM 3947, 2, 118-122 mm, MUSM 4524, 4, 120-136 mm, MUSM 4526, 2, 113-118 mm, MUSM 5068, 1, 109 $\mathrm{mm}$, Parque Nacional (PN) Manú, Pakitza Biological Station, nr. Aguajal, ca. $12^{\circ} 52^{\prime} \mathrm{S}, 069^{\circ} 16^{\prime} \mathrm{W}$. MUSM 2553, 1, $105 \mathrm{~mm}$, PN Manú, Pakitza Biological Station, nr. Aguajal, quebrada Fortaleza, ca. $11^{\circ} 45^{\prime} \mathrm{S}, 070^{\circ} 17^{\prime} \mathrm{W}$. MUSM 4520, 8, 84-122 mm, MUSM 14015, 73, 56-133 mm, PN Manú, Pakitza Biological Station, nr. Aguajal, quebrada Carpintero, ca. $12^{\circ} 52^{\prime} \mathrm{S}, 069^{\circ} 16^{\prime} \mathrm{W}$. MUSM 4564, 1, 129 mm, MUSM 14016, 11, 79-140 mm, PN Manú, Pakitza Biological Station, nr. Aguajal, quebrada Trompetero, ca. $12^{\circ} 52^{\prime} \mathrm{S}$, $069^{\circ} 16^{\prime} \mathrm{W}$. MUSM 21691, 1, Huitoto, no coordinates. MUSM 25041, 1, Planchón las Piedras, stream at km. 50, río Manuripe, no coordinates. MUSM 37016, 2, 120-155 mm, stream nr. Soledad Lodge, río Las Piedras, $12^{\circ} 02^{\prime} 03^{\prime \prime S}$, 069 47'47"W. ROM 62201, 5, 74-120 mm, PN Manú, Pakitza Biological Station, stream in aguajal, ca. $12^{\circ} 52^{\prime} \mathrm{S}, 069^{\circ} 16^{\prime} \mathrm{W}$. Puno. MUSM 3692, 2, Zona Reservada (ZR) Tambopata-Candamo, río Tambopata, $c a .13^{\circ} 30^{\prime} \mathrm{S}, 069^{\circ} 34^{\prime} \mathrm{W}$. MUSM 11786, 1, 111 mm, ZR Tambopata-Candamo, río Pacal, affl. río Candamo, ca. $13^{\circ} 31^{\prime} \mathrm{S}, 069^{\circ} 41^{\prime} \mathrm{W}$. MUSM 11186, 2, 106-128 $\mathrm{mm}$, ZR Tambopata-Candamo, río Candamo, ca. $13^{\circ} 31^{\prime} \mathrm{S}$, $069^{\circ} 41^{\prime} \mathrm{W}$. Uruguay. Artigas. ZVC-P 5687, 1, $90 \mathrm{~mm}, 30^{\circ} 26^{\prime} 20^{\prime \prime} \mathrm{S}$, 056 48'14"W, ZVC-P 5916, 2, 133-133 mm, Tres Cruces stream, Uruguay dr., $30^{\circ} 18^{\prime} 09^{\prime \prime} \mathrm{S}, 057^{\circ} 23^{\prime} 21^{\prime \prime} \mathrm{W}$. Durazno. UF183773, 2, immature (1CS), 105-130 mm, Laguna Lavalle, río Negro, Uruguay

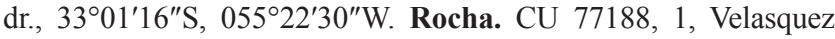
stream, Patos-Mirim dr., $34^{\circ} 03^{\prime} \mathrm{S}, 054^{\circ} 28^{\prime} \mathrm{W}$. CU 77189, 1, Sauces stream, Patos-Mirim dr., ca. $34^{\circ} 00^{\prime} \mathrm{S}, 053^{\circ} 51^{\prime} \mathrm{W}$. ZVC-P 6287, 1 (holotype), $154 \mathrm{~mm}$, Arroyo Cuatro Palmas, Route 15, km 268, nr.
Cebollatí, affl. Arroyo Pelotas, affl. Laguna Merín, Patos-Mirim dr.,

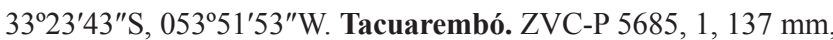
Batoví stream, Uruguay dr., 31 ${ }^{\circ} 54^{\prime} 28^{\prime \prime} \mathrm{S}, 056^{\circ} 01^{\prime} 03^{\prime \prime} \mathrm{W}$.

\section{Brachyhypopomus brevirostris (Steindachner, 1868)}

\author{
(Figs. 1e, 17; Tables 2-5, 10)
}

Rhamphichthys brevirostris Steindachner, 1868b: 177 (original description, no locality given). -Steindachner, 1868a: 254, pl. 2, fig. 2 (redescription, Guaporè [rio Guaporé, Amazonas dr.]).

Rhamphichthys (Brachyrhamphichthys) brevirostris. -Günther, 1870: 6 (Brazil, rio Guaporé, catalog of fishes in British Museum). -Steindachner, 1880: 89 (Brazil, rio Guaporé and rio Amazonas nr. Santarém, catalog of fishes).

Brachyrhamphichthys brevirostris. -Eigenmann \& Eigenmann, 1891: 62 (Brazil, rio Guaporé, Santarém, catalog of South American fishes).-Eigenmann, 1894: 625 (Brazil, Itaituba [lower Tapajós], listing of collections by Frederick C. Hartt).

Hypopomus brevirostris. -Eigenmann \& Ward, 1905: 170 (Amazon and tributaries, Paraguay, catalog of gymnotiforms). -von Ihering, 1907: 281 (Venezuela, lower Amazon, Guaporé, and Paraguay, catalog of Brazilian fishes). -Eigenmann, 1910: 449 (Guyana to Paraguay, catalog of freshwater fishes). -Eigenmann, 1912: 433 (Guyana, multiple locations, catalog of freshwater fishes). -Ellis, 1913: 134: fig. 7, head shape; 189, fig. 32, anatomy of swim bladders (lists distribution as Paraguay, lower Tapajós, lower Amazon, Uruguay basin, Rio Grande do Sul, Brazil, and Guyana [records in Rio Grande do Sul and middle or lower Paraguay basin likely refer to $B$. gauderio, B. bombilla, or B. draco], gymnotiform diversity and anatomy). -Meek \& Hilderbrand, 1916: 310 ("south to the rio Plata" from NW South America [records in Panama refer to B. occidentalis]). -Eigenmann, 1922: 174 ("south to Paraguay" from NW South America [records in Panama refer to B. occidentalis], catalog of fishes in NW South America). -Schindler, 1937: 19, fig. 3, outline of head based on original description; fig, 4, figs. 5-11, outline of heads of syntypes, table with morphometric analysis of syntypes [including designation of lectotype, "Typus", and paralectotype, "Cotypus", specimens], discussion of additional specimens of Brachyhypopomus from Paraguay). -Eigenmann \& Allen, 1942: 314 (Guianas, Paraguay, Peru at Iquitos and Yurimaguas, listing of gymnotiforms). -Schultz, 1944: 40 (diagnosis against by key and morphological data, in description of $B$. beebei). -Fowler, 1945: 179 (Peru at Iquitos and Yurimaguas, Guianas, Brazil, and Paraguay, listing of congeners). -Schultz: 1949, 65 (key to the Hypopomidae]. -Heiligenberg \& Bastian: 1980: 197, fig. 1, black and white photograph (Brazil, Amazonas, rio Negro, EOD diversity in sympatric gymnotiforms). -Ortega \& Vari, 1986: 13 (Peru, listing of members of genus). -Kawasaki \& Heiligenberg, 1989: 732, fig.1A, black and white photograph of male and female (EODs and communication).

Hypopomus artedi. -Hopkins \& Heiligenberg, 1978: 132, fig. 10, black and white photograph (Suriname, EOD evolution in sympatric gymnotiforms). 


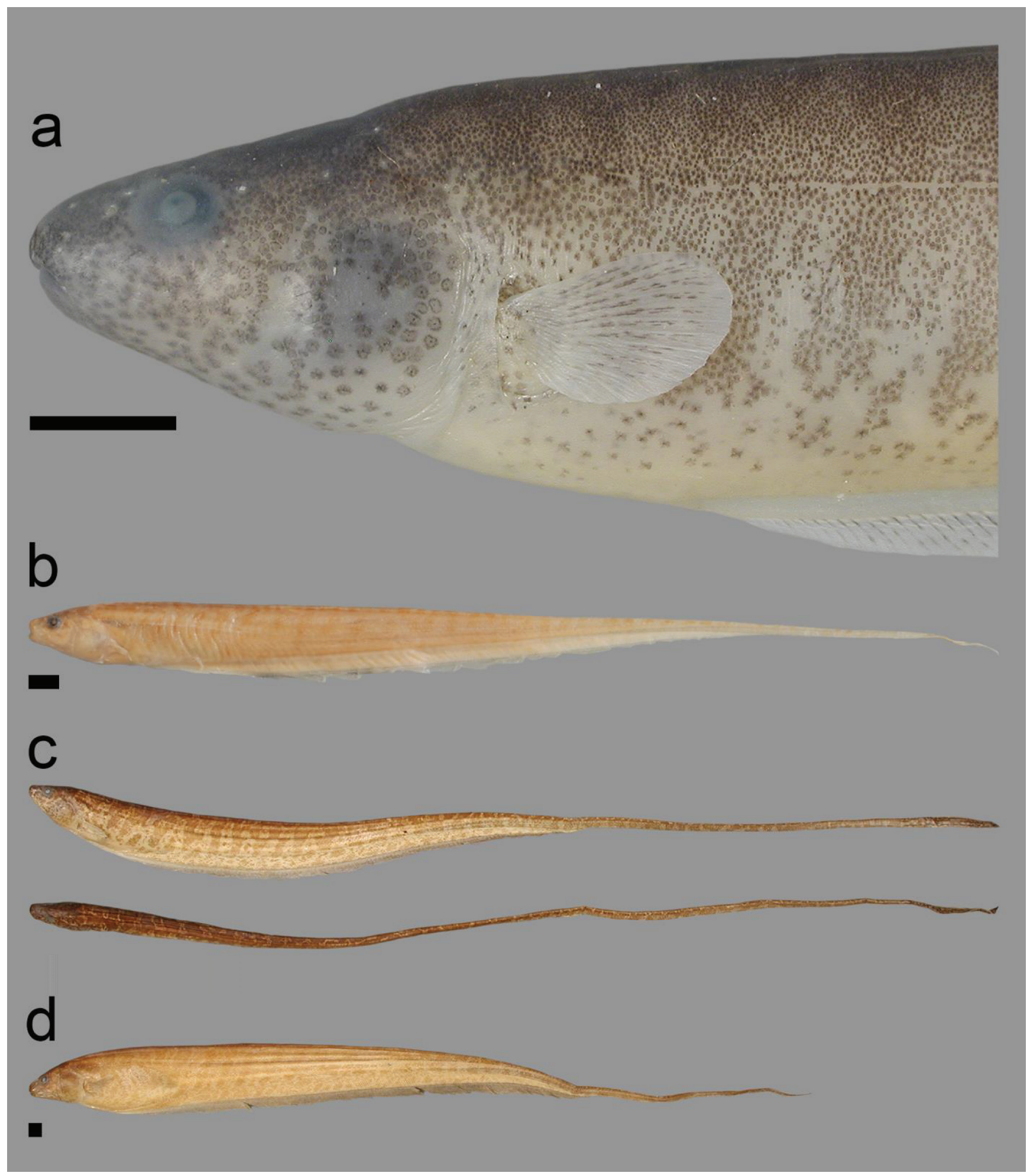

Fig. 17. Brachyhypopomus brevirostris. a. MCP 45470 (WC11.110801b), immature female, $193 \mathrm{~mm}$ TL, (head only, specimen fixed in formalin); Brazil, rio Tefé, Amazonas dr. b. NMW 65040, lectotype, sex not determined, 163 mm TL (body in lateral view, specimen preserved in EtOH), Brazil, rio Guaporé, Amazonas dr. c. MCP 45262 (WCNR06.090600), male, $366 \mathrm{~mm}$ TL, non-type (body in lateral and dorsal view, specimen fixed in formalin and preserved in EtOH); d. MCP 45260 (WC02.180598), female, $305 \mathrm{~mm}$ TL, non-type (body in lateral view; specimen fixed in formalin and preserved in EtOH); Brazil, rio Solimões-Japurá confluence, Amazonas dr. Note the extreme sexual dimorphism in caudal-filament length. Scale bars $=5 \mathrm{~mm}$. 


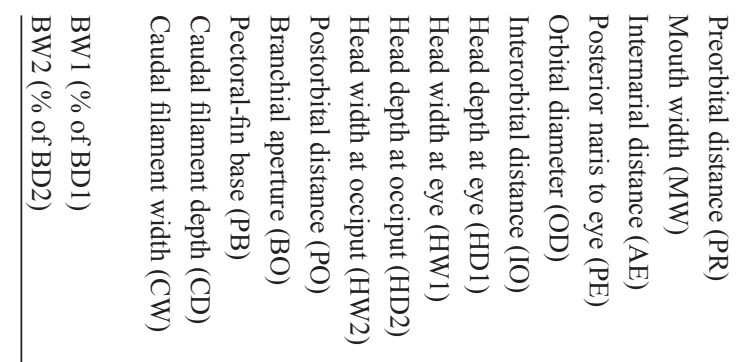

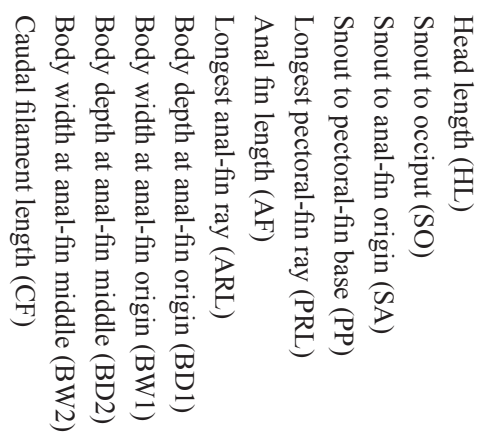

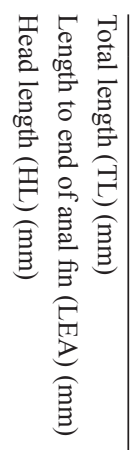

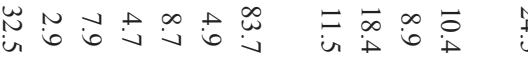

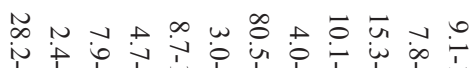

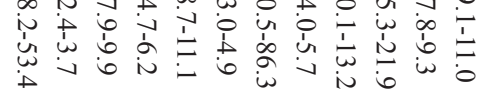

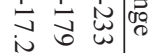

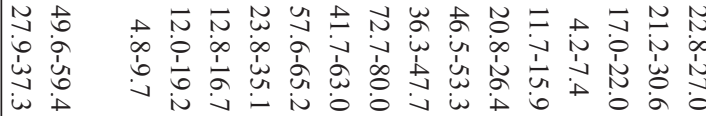

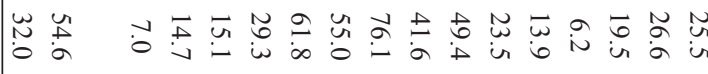

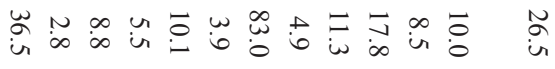

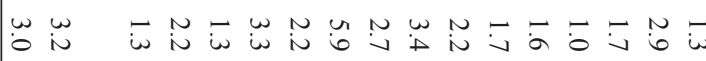

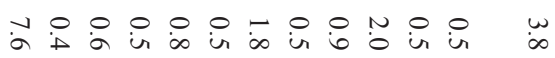

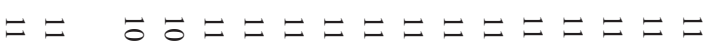

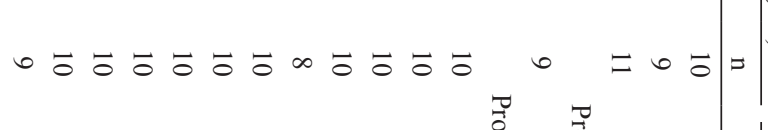

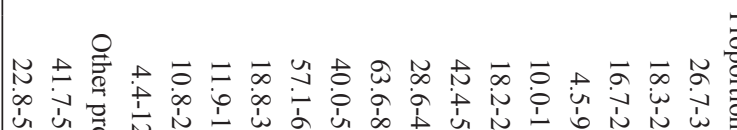

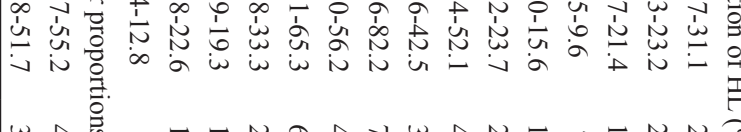

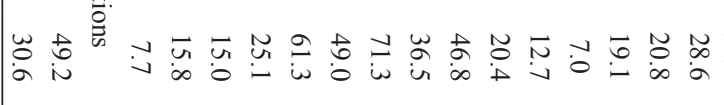
N

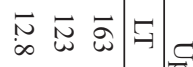

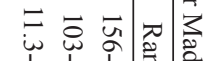

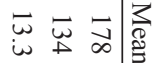
$-n \omega$ ir

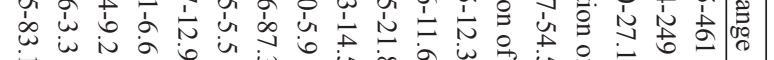
I

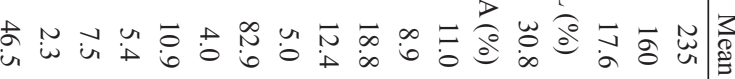

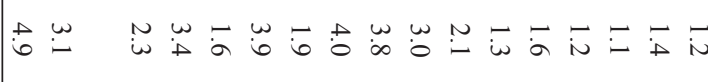

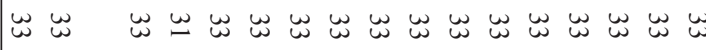

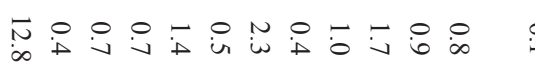

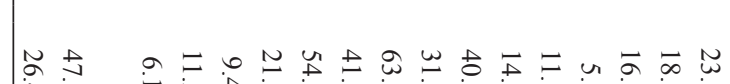

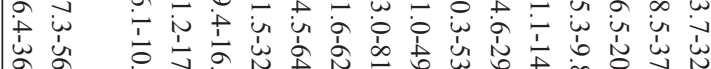
幽

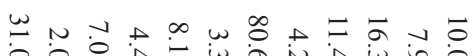

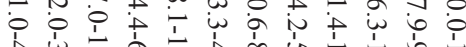

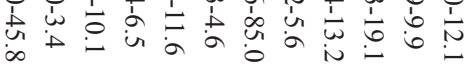

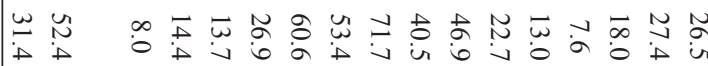

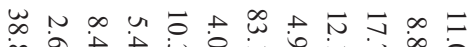

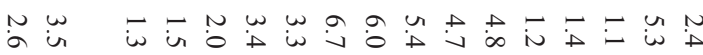

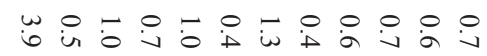

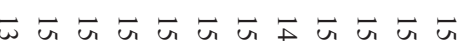

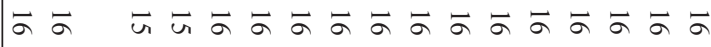

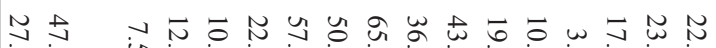

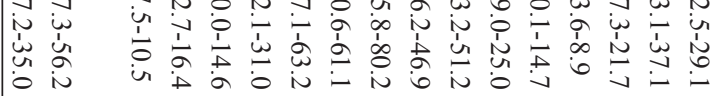

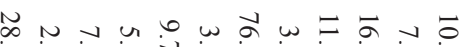

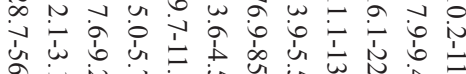

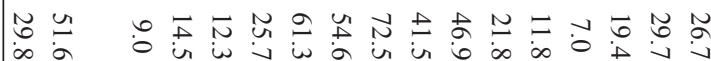

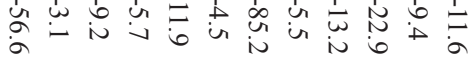

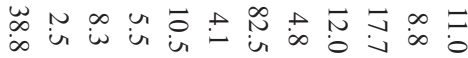

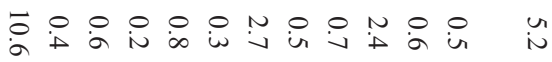

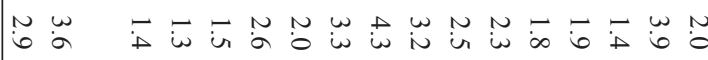

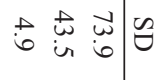
$\underset{\omega}{\omega} \underset{\omega}{\infty}=$ ○一⿻上丨

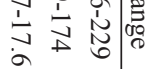

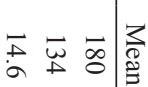

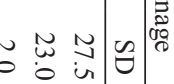
in $\bar{\omega}=$

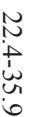

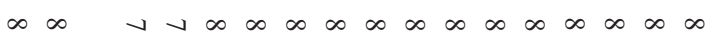


Brachyhypopomus brevirostris. -Mago-Leccia, 1994: 48, 168, fig. 68, reproduction of plate 2, fig. 2 from Steindachner, 1868a (listing of Brachyhypopomus). -Planquette et al., 1996: 396, color photograph of live individual (French Guiana, listing of Brachyhypopomus). -Crampton, 1996a: 77, table 6.1.a, species list; 79, fig. 6.1 inset 27-29, color photographs; 85 , fig. 6.2; 88, fig. 6.3; 92-94, figs. 6.4a, 6.5; 97, fig. 6.7; 191, fig. 11.2; 193, fig. 11.3, EOD data; 110, table 7.5, 113, table 7.7, 115, fig. 7.1, habitats; 136, table 8.2a,b, diet; 151-160, figs. 9.2-9.3, table 9.1a,b, hypoxia tolerance; 165-169, table 10.1, reproductive biology (Brazil, central Amazon, ecology and signal diversity). -Alves-Gomes, 1997: 523 (Brazil, Roraima, listing of gymnotiforms). -Sullivan, 1997: 86 (redescription). -Crampton, 1998a: 821, table 4, 834, fig. 9, drawing of adult specimen, with EOD; 837, fig. 10, drawing of caudal filament of male and female (Brazil, Amazonas, species and EOD diversity). -Crampton, 1998b: 314, table 2, list of Brachyhypopomus (Brazil, Amazonas, hypoxia tolerance). -Crampton, 1999: 17 (Brazil, Amazonas, Mamirauá Reserve, listing of species). -Albert \& Crampton, 2003: 494 (Bolivia, Brazil, Colombia, Ecuador, Guyana, Peru, Suriname, Venezuela, catalog of hypopomids). -Goulding et al., 2003: 177, center photograph, live individual (Peru, upper río Madre de Dios, photographic album of fishes). -MaldonadoOcampo, 2004: table 1 (Colombia, Orinoco dr. and Meta dr., listing of Brachyhypopomus). -Crampton \& Albert, 2006: 672, fig. 23.8, position in phylogenetic tree; 681, notes on EODs (gymnotiform species and EOD diversity). -Galvis et al., 2006: 212, illustration of head and body; 529, plate 104a, photograph of live specimen (Colombia, Amazonas, catalog of fishes). -Ferreira et al., 2007: 164, center left photograph, live specimen (Brazil, Roraima, rio Branco, photographic album of fishes]. -Vari et al., 2009: 46 (Guiana Shield, catalog of fishes). -Crampton, 2011: 176, table 10.2, species list; 179, figs. 10.2-10.3, phylogeny, geographical and ecological distributions (gymnotiform biology). -Mol: 2012, 596 (Suriname, catalog of fishes). -de Queiroz et al., 2013: 545 (Brazil, rio Madeira, lago Cuniã, listing of fish).Carvalho: 2013, 181-185, figs. 41-43, position in phylogeny (phylogenetic systematics of Rhamphichthyoidea). -Crampton \& Ribeiro, 2013: 236, color photograph (Brazil, rio Madeira, listing of hypopomids). -Tagliacollo et al., 2016: 28, fig. 5 (phylogeny of Gymnotiformes). -Crampton et al., 2016: 1-66, table 1, 3-4, figs. 1-7, 9, 14, 16, 18-20 (phylogeny, biogeography and ecology of Brachyhypopomus).

Diagnosis. Brachyhypopomus brevirostris is diagnosed from congeners by the following combination of characters: anal-fin rays 226-293, vs. 143-225 in all congeners except $B$. beebei, B. bennetti, B. bullocki, B. diazi, B. occidentalis, $B$. palenque, and $B$. walteri; bilateral columns of electrocytes at the anal-fin terminus 3, vs. 4-6 in B. bennetti, B. diazi, $B$. occidentalis (except some populations in Colombia and Venezuela, see redescription of $B$. occidentalis), and $B$. palenque; pale stripe along middorsal region of body absent, vs. prominent pale uninterrupted middorsal stripe from occipital region to base of caudal filament present in $B$. beebei; precaudal vertebrae 18-21, vs. $15-17$ in $B$. bullocki; distinct dark suborbital stripe absent, vs. present in $B$. walteri.

Description. Head and body shape, and pigmentation illustrated in Figs. 1e and 17. Meristic and morphometric data for examined specimens presented in Tables 2-5 and 10. Body shallow to moderate in depth. Head short to moderate in length and shallow to moderate in depth. Dorsal profile of head straight to slightly convex from occiput to snout, ventral profile of head approximately straight between operculum and snout. Snout rounded. Eye moderate to large in size. Upper jaw with moderate sigmoidal angle between premaxillary and maxillary portions in lateral view. No accessory electric organ over operculum. Gill filaments on first gill arch 40-63 (median $55, \mathrm{n}=38$ ). Pectoral fin narrow to broad in width, pectoralfin rays 15-20 (mode of 17 in populations from upper Madeira (Guaporé), Orinoco, and upper Paraguay; mode of 18 in a population from central Amazon). Precaudal vertebrae 18-21 (mode of 19 in populations from upper Madeira, central Amazon, and upper Paraguay; mode of 20 in a population from Orinoco), including 1-2 transitional vertebrae. Anal-fin origin slightly $(<0.25 \mathrm{HL}$ distance) anterior or posterior to, or near, tip of pectoral fin. Analfin rays 226-293; range and median values of all examined populations are similar. Dorsal rami of recurrent branch of anterior lateral line nerve visible in most juvenile and some lightly-pigmented adults; mostly in anterior third of body. Middorsal region of body scaled. Rows of scales above lateral line 7-9 (mode 8). Lateral line continuous. Epidermal canals sparse and in some specimens more or less absent; when present located only in posterior two thirds of body. Isolated short sections of depigmented canals midway between the lateral line and dorsal midline in middle third of the body. Sparse crisscrossing epidermal canals close to and either side, of but mostly dorsal to lateral line in posterior third of body, merging near anal-fin terminus with canals midway between the lateral line and dorsal midline. Three bilateral horizontal columns of electrocytes at anal-fin terminus, and at a mid-point between anal-fin terminus and tip of caudal filament in immature, mature female, and mature male specimens. Extreme tip of caudal filament free of electrocytes. Caudal filament moderate in length to long.

Coloration. (Figs. 1e, 17). Background tan to light brown. Dorsal region without prominent depigmented pale stripe extending along midline from occipital region to base of caudal filament. Prominent wide brown saddles, which extend to dark bands that traverse flank, often exhibiting a misalignment in their continuation at lateral line. Dark bands become fainter ventrally, breaking up into indistinct marks over anal-fin pterygiophores. Indistinct dark markings often present in pale interband area. Contrast between 
dark bands and saddles and background is typically greater in juveniles but varies among populations. Some large adults become very dark and bands and saddles become indistinct. Caudal filament darker than body, especially dorsally, ventrally, and near tip; with irregular bands along entire length; darkened tip of caudal filament resembles root tips of water hyacinths when viewed underwater. Head with evenly scattered dark chromatophores, darker dorsally. Eye without prominent suborbital patch, or stripe, of chromatophores/subcutaneous pigmentation. Pectoral and anal-fin membranes hyaline. Pectoral-fin rays hyaline with light scattering of brown chromatophores. Anal-fin rays with scattered or uniform dark pigmentation; darker in posterior half of fin. Color in live specimens similar to preserved ones, with opercular region usually rosy due to underlying gills.

Size. Moderate to large adult size, largest specimen examined $461 \mathrm{~mm}$ TL, $210 \mathrm{~mm}$ LEA ( $=1701)$. No congener reaches greater total length. Largest male specimen examined $461 \mathrm{~mm}$ TL, $210 \mathrm{~mm}$ LEA $(\mathrm{n}=39)$. Largest female specimen examined $315 \mathrm{~mm}$ TL, $230 \mathrm{~mm}$ LEA $(n=84)$.

Sexual dimorphism. Breeding male specimens develop extremely elongated caudal filaments in comparison to immature individuals and breeding females, and consequently reach considerably greater total lengths (Figs. $17 \mathrm{c}-\mathrm{d}$ ), but not lengths as measured to anal-fin terminus. Despite possessing longer caudal filaments, breeding males do not exhibit an elevated number of bilateral horizontal columns or vertical rows of electrocytes. Instead they exhibit clearly enlarged electrocytes relative to immature specimens and females. In breeding males caudal filament tip forms a paddle-like laterally compressed structure, some $15-40 \mathrm{~mm}$ in length, often completely free of electrocytes. In some males the posterior portion of the caudal filament is entirely free of electrocytes for as much as $48 \%$ of caudalfilament length. Some females exhibit a shorter and only slightly laterally compressed caudal filament tip, without electrocytes in terminal portion for up to $10 \%$ of caudalfilament length. No sexual differences in pigmentation.

Geographic distribution. Bolivia, Brazil, Colombia, French Guiana, Guyana, Peru, Suriname, and Venezuela (Fig. 18). Widespread through cis-Andean northern South America, including large areas of the Amazonas and Orinoco drainages, northern portions of the Paraguay drainage, coastal drainages of the Guianas and Amapá state, Brazil, and coastal drainages of Pará state, Brazil. As yet unknown from some major tributaries of the Amazon, including the Juruá, upper Japurá-Caquetá, Napo, and upper Xingu, and many southwestern tributaries of the río Orinoco.

Population variation: We found complete overlap in the range of meristic counts (Tables 2-5) and morphometric proportions (Table 10), and observed similar pigmentation among populations of $B$. brevirostris in the upper Madeira, central Amazon, Orinoco, and upper Paraguay drainages. These observations, in combination with molecular data (Crampton et al., 2016), support the hypothesis that all populations we have assigned to $B$. brevirostris are members of a single geographically widespread species, which is morphologically and genetically distinct from all congeners (although we were unable to obtain specimens for genetic analyses from the Paraguay basin).

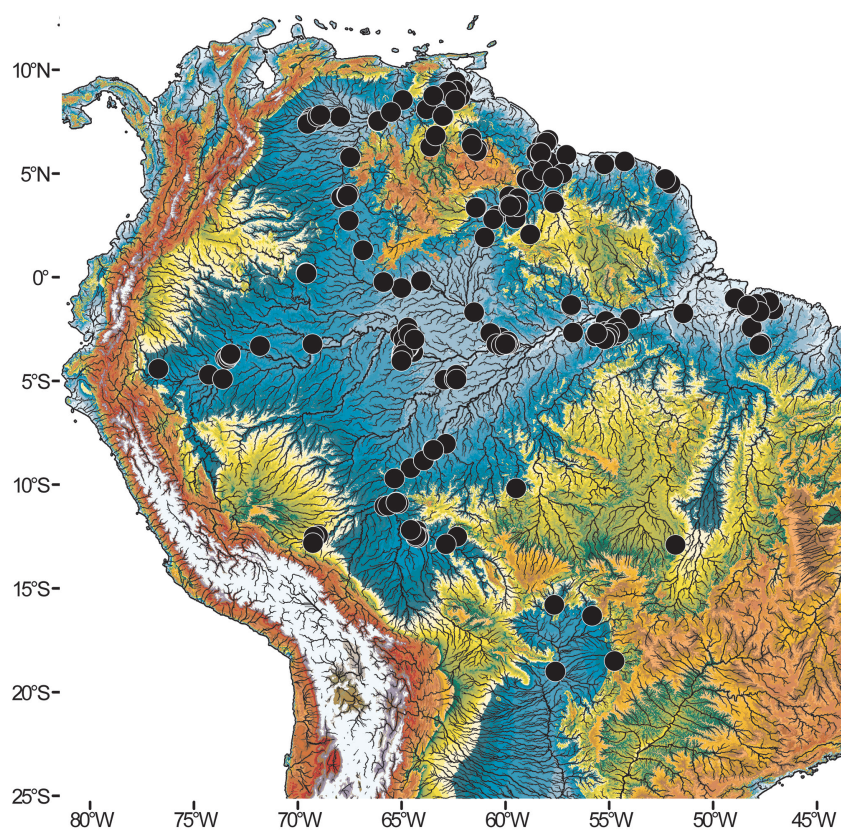

Fig. 18. Collection records for Brachyhypopomus brevirostris (circles). Precise locality of the lectotype is not known. Elevation data refers to altitude above mean sea level (see Fig. 2 for legend).

Ecological notes. Brachyhypopomus brevirostris is an ecologically cosmopolitan species, and varies from being locally rare to exceptionally abundant in lentic and slowflowing environments throughout its range (Alves-Gomes, 1997; Crampton, 1996a; 1998a; Crampton \& Albert, 2006; Crampton \& Ribeiro, 2013; de Queiroz et al., 2013; Hopkins \& Heiligenberg, 1978; Mol, 2012). It occurs in seasonally inundated floodplains (in floating rafts of meadows and shallow newly-flooded forests), along the margins of rivers (in emergent and floating vegetation), in ephemeral or perennial swamps (in vegetation or submerged debris), and in terra firme forest and savanna streams (in emergent and submerged vegetation, leaf litter and debris, and in marginal root mats and holes). In the lowland Amazon, $B$. brevirostris occurs in low-conductivity blackwater and clearwater streams, river margins, and floodplain systems ( $\mathrm{ca}$. 5-30 $\left.\mu \mathrm{Scm}^{-1}\right)$, and also high-conductivity whitewater river margins and floodplain systems ( $c a$. $\left.90-250 \mu \mathrm{Scm}^{-1}\right)$, as well as in waters of intermediate conductivity. Nonetheless, it is most abundant in lowconductivity systems. Brachyhypopomus brevirostris is 
less tolerant of prolonged hypoxia than congeners from the central Amazon, and is unusual among congeners in not exhibiting air-breathing of any kind (Crampton, 1998b). Breeding occurs during the rising and high water period in populations from Amazonian floodplain floating macrophytes (Crampton, 1996a: 165). Stomach contents in populations from the central Amazon comprise aquatic insect larvae, microcrustacea, and other small aquatic invertebrates - with a predominance of Chironomidae larvae (Crampton, 1996a: 136). Large specimens often consume larger aquatic invertebrates.

Co-occurring congeners: Because of its wide geographical range and occurrence in multiple habitats, $B$. brevirostris occurs in geographical sympatry and ecological syntopy with multiple congeners: in the Amazonas drainage and coastal drainages of the Guianas $B$. batesi, $B$. beebei, $B$. belindae, $B$. benjamini, $B$. bennetti, $B$. cunia, $B$. hamiltoni, $B$. hendersoni, $B$. pinnicaudatus, $B$. regani, $B$. sullivani, $B$. verdii, $B$. walteri; in the Orinoco drainage - B. beebei, B. bullocki, B. diazi, B. provenzanoi, $B$. regani, and $B$. sullivani; in the Paraguay drainage $-B$. bombilla, B. draco, B. gauderio, and B. walteri.

Local names. Brazil: sarapó (north), tuvira (Paraguay drainage); Colombia: cuchillo/cuchillo amarillo (Amazon and Orinoco), cuchilleja macana (Putumayo); French Guiana: poisson couteau (French) and multiple creole/ Amerindian names, see Planquette et al. (1996); Peru: macana; Suriname: saprapi; Venezuela: cuchillo.

Remarks. Comments on the type series: In the original brief description, Steindachner (1868b) did not mention specimens, and in the redescription (1868a) he only referred to "several small specimens 2"11" - 71/2" long". The type series consequently consists of several syntypes. Schindler (1937) recognized eight specimens in the NMW as syntypes and designated one of them as (NMW 65040) as "Typus", consequently a lectotype under the International Code of Zoological Nomenclature, Article 74.5. Schindler considered it to be identical with the specimen on plate 2 , fig. 2 in Steindachner (1868a).

Comments on erroneous geographical references: Prior to, and even for several decades after Regan's (1914) description of B. occidentalis (the only species of Brachyhypopomus to occur in trans-Andean Colombia, Panama, and Costa Rica), the name B. brevirostris was repeated in reference to material collected from TransAndean Panama, and Colombia (Eigenmann, 1922; Eigenmann \& Allen, 1942; Eigenmann \& Eigenmann, 1891; Eigenmann \& Fisher, 1914; Eigenmann \& Ward, 1905; Fowler, 1945; Meek \& Hildebrand, 1916; Steindachner, 1880). Several reports prior to ca. 1945 list B. brevirostris as occurring in "Paraguay" although it is unclear whether the country or river is referenced; although present in the upper Paraguay basin of Brazil, B. brevirostris has not yet been collected in Paraguay.
Material examined. 1,787 specimens. Bolivia/Brazil frontier. NMW 65038, 1, $183 \mathrm{~mm}, 152 \mathrm{~mm}$ LEA (paralectotype) corresponds in size to "Co-typus" specimen from first column of table 2 in Schindler (1937), rio Guaporé, affl. rio Mamoré, affl. rio Madeira, Amazonas dr., no coordinates, J. Natterer. NMW 65040, 1, 164 mm TL, 123 mm LEA (lectotype), collected with NMW 65038. Bolivia. Beni (localities from rio Madeira dr., Amazonas dr.). AMNH 40086 (part), 1, $202 \mathrm{~mm}$, rio Baures, $2 \mathrm{~km}$ above mouth, affl. río Guaporé, affl. río Mamoré, 12³0'32"S, 064¹6'46"W. CBF 10259, 1, male, $234 \mathrm{~mm}$, UMSS 07033,1 , immature, $160 \mathrm{~mm}$, stream at $\mathrm{km} 43$ on Riberalta-Guayaramerín rd., affl. río Beni, $11^{\circ} 00^{\prime} 30.2^{\prime \prime} \mathrm{S}$, 065³9'49.4"W. UMMZ 204512, 1, $131 \mathrm{~mm}, 9 \mathrm{~km}$ SE Costa Marquez (Brazil), río Iténez (rio Guaporé), affl. río Mamoré, ca. $12^{\circ} 30^{\prime} 42^{\prime \prime} \mathrm{S}, 64^{\circ} 10^{\prime} 18^{\prime \prime} \mathrm{W}$. UMMZ 204743, 2, 92-167 mm, $6 \mathrm{~km}$ SW Costa Marques, río Iténez at mouth of río Baures, affl. río Mamoré, $12^{\circ} 31^{\prime} \mathrm{S}, 064^{\circ} 19^{\prime} \mathrm{W}$. UMMZ 205163, 3, 155-167 mm, Backwater of río Iténez, $10 \mathrm{~km}$ SE Costa Marques, affl. río Mamoré, $12^{\circ} 33^{\prime} 00^{\prime \prime} \mathrm{S}, 064^{\circ} 12^{\prime} 24^{\prime \prime} \mathrm{W}$. UMMZ 246440, 1, $170 \mathrm{~mm}$, río Baures (río Blanco), ca. $500 \mathrm{~m}$ above mouth on left bank río Iténez, affl. río Mamoré, $12^{\circ} 31^{\prime} 06^{\prime \prime} \mathrm{S}, 064^{\circ} 19^{\prime} 00^{\prime \prime} \mathrm{W}$. UMSS 07024, 1, immature, $229 \mathrm{~mm}$, stream at Bocerón, Riberalta - Guayaramerín rd., affl. río Beni, $11^{\circ} 02^{\prime} 50^{\prime \prime} \mathrm{S}, 065^{\circ} 50^{\prime} 07^{\prime \prime} \mathrm{W}$. Brazil. Amazonas (localities are from the Amazonas dr.; localities listed from Mamirauá Reserve [Reserva de Desenvolvimento Sustentável Mamirauá] are in rio Solimões-Japurá floodplain, mun. Alvarães). BMNH 1985.6.22.51, 1, $270 \mathrm{~mm}$, rio Tarumazinho [rio Tarumã Mirim], affl. rio Negro, $02^{\circ} 54^{\prime} \mathrm{S}, 060^{\circ} 13^{\prime} \mathrm{W}$. BMNH 1998.3.12.133-141, 9, immature, BMNH 1998.3.12.145-156, 12, immature, 2 measured, 200-220 mm, IDSM 444, 7, 90-214 mm, MCP 44605, 1, male (CS), 374 $\mathrm{mm}, \mathrm{MCP} 44759,17$ (2CS), 48-243 mm, MCP 45258, 3, female, 210-264 mm, mun. Tefé, Ressaca do Cachorro, lago Tefé, rio Tefé, 0319'50"S, 6442'15"W. BMNH 1998.3.12.142-144, 3, immature, BMNH 1998.3.12.157, 1, male, mun. Tefé, Cabeceira do lago Tefé, rio Tefé, $c a .03^{\circ} 34^{\prime} \mathrm{S}, 64^{\circ} 58^{\prime} \mathrm{W}$. BMNH 1998.3.12.158, 1, $225 \mathrm{~mm}$, MCP 45332, 1, female, 260 mm, Igarapé Baré, lago Amanã, affl. río Japurá, $02^{\circ} 25^{\prime} 05^{\prime \prime} \mathrm{S}, 64^{\circ} 42^{\prime} 58^{\prime \prime} \mathrm{W}$. IDSM 443, 1, $80 \mathrm{~mm}$, MCP 45331, 3 (1 immature, 272 mm, 1 male, 340 mm, 1 unsexed, 223 $\mathrm{mm})$, Mamirauá Reserve, cano do lago Rato, $03^{\circ} 02^{\prime} 41^{\prime \prime} \mathrm{S}$, $064^{\circ} 51^{\prime} 26^{\prime \prime} \mathrm{W}$. INHS 70542, 1 (part), lago Camaleão, Ilha da Marchantaria, rio Solimões floodplain, $03^{\circ} 14^{\prime} \mathrm{S}, 059^{\circ} 56^{\prime} \mathrm{W}$. INHS 71264, 1, $223 \mathrm{~mm}$, lago do Janauari, rio Amazonas floodplain, $03^{\circ} 13^{\prime} \mathrm{S}, 060^{\circ} 00^{\prime} \mathrm{W}$. INPA 4388, 2, 128-150 mm, INPA 12265, 1 , $200 \mathrm{~mm}$, INPA 19936, 1, $160 \mathrm{~mm}$, INPA 19937, 2, 125-136 mm, Arquipelago das Anavilhanas, rio Negro, $02^{\circ} 41^{\prime} \mathrm{S}, 060^{\circ} 44^{\prime} \mathrm{W}$. INPA 8857,33 , km 55 on Manaus-Manacapuru hwy. AM-070, stream, affl. rio Solimões, $\mathrm{ca}$. $03^{\circ} 13^{\prime} \mathrm{S}, 060^{\circ} 26^{\prime} \mathrm{W}$. INPA 9947, 19, 127-310 mm, Mamirauá Reserve, lago Caetano, 02 $2^{\circ} 50^{\prime} 58^{\prime \prime} \mathrm{S}, 064^{\circ} 55^{\prime} 42^{\prime \prime} \mathrm{W}$. INPA 9948, 3, 290-420 mm, INPA 9949, 3, 218-240 mm, mun. Tefé, Cabeceira do lago Tefé, rio Tefé, $03^{\circ} 34^{\prime} \mathrm{S}, 064^{\circ} 58^{\prime} \mathrm{W}$. INPA 15814, 6 (3 immature, 54-145 mm, 1, female, $253 \mathrm{~mm}, 2$ male, 370-461 mm), MCP 44606, 7 (3 immature [2CS], 124-175 mm, 2 female, 233-270 mm, 2 male [1CS], 295-308 mm), mun. Tefé, Cabeceira do lago Tefé, rio Tefé, 033' $55^{\prime \prime} \mathrm{S}, 064^{\circ} 57^{\prime} 33^{\prime \prime} \mathrm{W}$. INPA 15815, 7, immature, 36-108 mm, INPA 18344, 3, immature, 77-78 mm, MCP 45379, 6 (4 immature, 85-132 mm, 1, female, $203 \mathrm{~mm}$, 1 male, $285 \mathrm{~mm}$ ), MCP 45470, 20, immature, 97-218 mm, mun. 
Tefé, Ressaca do Socorro, lago Tefé, rio Tefé, $03^{\circ} 18^{\prime} 45^{\prime \prime} \mathrm{S}$,

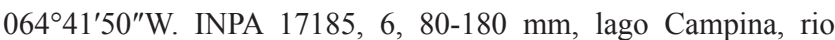
Purus, 04 $55^{\prime} 59^{\prime \prime} \mathrm{S}, 062^{\circ} 56^{\prime} 25^{\prime \prime} \mathrm{W}$. INPA 17186, 5, 90-202 mm, Paraná do Seixo, lago Jari, rio Purus, $04^{\circ} 54^{\prime} 41^{\prime \prime} \mathrm{S}, 062^{\circ} 27^{\prime} 26^{\prime \prime} \mathrm{W}$. INPA 17189, 21, 56-213 mm, lake nr. Sacado da Santa Luzia, rio Purus, $04^{\circ} 42^{\prime} 17^{\prime \prime} \mathrm{S}, 062^{\circ} 22^{\prime} 26^{\prime \prime} \mathrm{W}$. INPA 18247, 1, female, 238 mm, INPA 18345, 2 (1 female, 276, 1 male, 205), MCP 45386, 1, male, $225 \mathrm{~mm}$, Mamirauá Reserve, lago Araçazinho, 0259'35"S, $064^{\circ} 51^{\prime} 33^{\prime \prime} \mathrm{W}$. INPA 18248, 3 (1 immature, $227 \mathrm{~mm}$, 1, female, 230 mm, 1 male, $216 \mathrm{~mm}$ ), Mamirauá Reserve, lago Arauaé, 0302'52"S, 06450'04"W. INPA 18249, 1 male, 279 mm, MCP 45395, 2 (1 immature, $238 \mathrm{~mm}$, 1 female, $240 \mathrm{~mm}$ ), Mamirauá Reserve, cano

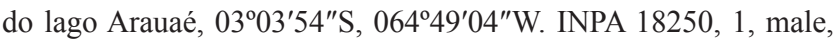
293 mm, INPA 18251, 1, immature, 222 mm, MCP 45260, 2, female, 237-305 mm, Mamirauá Reserve, lago Secretaria, $03^{\circ} 07^{\prime} 12^{\prime \prime} \mathrm{S}, 064^{\circ} 47^{\prime} 49^{\prime \prime} \mathrm{W}$. INPA 18252, 1, female, $305 \mathrm{~mm}$, mun. Tefé, Ilha do Martelo, rio Tefé, 0346'56"S, 06459'39"W. INPA 18342, 1, female, $150 \mathrm{~mm}$, INPA 18346, 1, female, $192 \mathrm{~mm}$, Mamirauá Reserve, lago Periquito Comprido, 0304'57"S, 0644ㄴ $42^{\prime \prime} \mathrm{W}$. INPA 18343, 1, female, $227 \mathrm{~mm}$, mun. Tefé, Cabeceira do lago Tefé, rio Tefé, $03^{\circ} 38^{\prime} 01^{\prime \prime} \mathrm{S}, 064^{\circ} 57^{\prime} 59^{\prime \prime} \mathrm{W}$. INPA 18347, 12 ( 9 female, 180-293 mm, 3 male, 175-220 mm), mun.

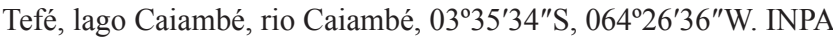
19934, 1, 155 mm, igarapé de Duas Bocas, Paraná do Jari, rio

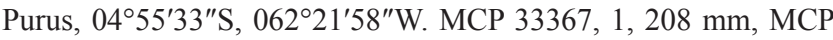
45362, 1, female, $188 \mathrm{~mm}$, Mamirauá Reserve, cano do lago Sapucaia, 030. $04^{\prime}$ "S, 064 $48^{\prime} 32^{\prime \prime} \mathrm{W}$. MCP 44758, 9 (1 immature [CS], $257 \mathrm{~mm}, 4$ female [1CS], 242-265 mm, 4 male, 250-395 $\mathrm{mm}$ ), MCP 45262, 1, male, $366 \mathrm{~mm}, \mathrm{MCP} 45323,25$ (1 immature, 259 mm, 19 female, 138-258 mm, 5 male, 208-311 mm), Mamirauá

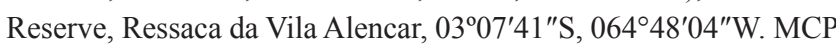
45259, 1, female, 235 mm, Mamirauá Reserve, lago Matá Matá, $03^{\circ} 06^{\prime} 17^{\prime \prime} \mathrm{S}, 064^{\circ} 47^{\prime} 17^{\prime \prime} \mathrm{W}$. MCP 45261, 1, female, $228 \mathrm{~mm}$, Mamirauá Reserve, lago Promessa, 03ํำ'29"S, 064 $47^{\prime} 02^{\prime \prime} \mathrm{W}$. MCP 45263, 2 (1 female, 269 mm, 1 male, 397 mm), MCP 45472, 1, immature, $150 \mathrm{~mm}$, mun. Tefé, rio Tefé, $03^{\circ} 41^{\prime} 23^{\prime \prime} \mathrm{S}$, 0645'인.W. MCP 45317, 7 (6 immature, 86-127 mm, 1 female, $315 \mathrm{~mm}$ ), MCP 46930, 2, immature (2 CS), 110-178 mm, MCP 46935, 1 post-larval (CS), $30 \mathrm{~mm}$, Mamirauá Reserve, cano do lago Mamirauá, $03^{\circ} 03^{\prime} 51^{\prime \prime} \mathrm{S}, 064^{\circ} 51^{\prime} 05^{\prime \prime} \mathrm{W}$. MCP 45318, 3 (2 immature, 149-225 mm, 1 male, 265 mm), MCP 46927, 1, immature (CS), 136 mm, Mamirauá Reserve, lago Bolsinha, 0303'47"S, 06450'03"W. MCP 45319, 1, immature, $161 \mathrm{~mm}, \mathrm{MCP}$ 45404, 1, female, 279

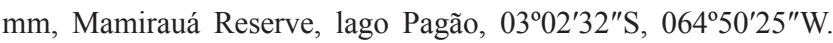
MCP 45322, 2 (1 immature, $111 \mathrm{~mm}, 1$ male, $156 \mathrm{~mm}$ ), MCP 45378, 2, female, 170-215 mm, mun. Tefé, Cabeceira do lago Tefé, rio Tefé, 0335'41"S, 06458'05"W. MCP 45326, 2, immature, 103 $175 \mathrm{~mm}$, Mamirauá Reserve, lago Sumaumeirinha I, 0248'26"S, 0650'3'․ MCP 45363, 1, female, 275 mm, Mamirauá Reserve, cano do lago Mamirauá, 0306 $40^{\prime \prime} \mathrm{S}, 064^{\circ} 47^{\prime} 52^{\prime \prime} \mathrm{W}$. MCP 45396, 2, female, 250-273 mm, Mamirauá Reserve, lago Periquito Redondo, $03^{\circ} 04^{\prime} 50^{\prime \prime} \mathrm{S}, 064^{\circ} 46^{\prime} 35^{\prime \prime} \mathrm{W}$. MCP 45402, 1, immature, $100 \mathrm{~mm}$, Mamirauá Reserve, lago Tracajá, $03^{\circ} 05^{\prime} 43^{\prime \prime} \mathrm{S}, 064^{\circ} 46^{\prime} 31^{\prime \prime} \mathrm{W}$. MCP 45403, 1, female, $230 \mathrm{~mm}$, Mamirauá Reserve, lago Juruazinho, $03^{\circ} 02^{\prime} 34^{\prime \prime} \mathrm{S}, 064^{\circ} 51^{\prime} 00^{\prime \prime} \mathrm{W}$. MCP 45452, 1, immature, $231 \mathrm{~mm}$, mun. Tefé, igarapé Xidarinini, affl. lago Tefé, rio Tefé, $03^{\circ} 22^{\prime} 46^{\prime \prime} \mathrm{S}$,

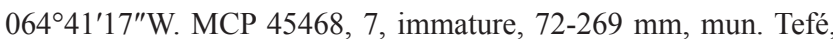
floodplain lake, Ilha Panamim, rio Solimões, 03¹9'29"S, 064³8'37"W. MCZ 9427 (part), 1, 171 mm, mun. Manaus, lago Hyanuary [lago do Janauari], rio Amazonas floodplain, $03^{\circ} 13^{\prime} \mathrm{S}$, $060^{\circ} 00^{\prime} \mathrm{W}$. MCZ 60046, 1, 84 mm, mun. Parintins, lago Maximo [lago do Maximo], nr. Parintins, rio Amazonas floodplain, ca. $02^{\circ} 40^{\prime} \mathrm{S}, 056^{\circ} 45^{\prime} \mathrm{W}$. MCZ 78163 (part), 1, $162 \mathrm{~mm}$, MCZ 78164, 1, 109 mm, mun. Manaus, lago Jacaretinga, nr. Careiro de Várzea, rio Amazonas floodplain, $03^{\circ} 13^{\prime} 00^{\prime \prime} \mathrm{S}, 059^{\circ} 49^{\prime} 41^{\prime \prime} \mathrm{W}$. MCZ 165856, 4, 164-196 mm, mun. Manaus, rio Negro nr. lago Alexo [lago do Aleixo], rio Amazonas floodplain, $03^{\circ} 05^{\prime} 28^{\prime \prime} \mathrm{S}$, 059 $53^{\prime} 09^{\prime \prime} \mathrm{W}$. MPEG 967, 1 (part), 86 mm, MPEG 970 (part), 1, 120 mm, MPEG 978, 1, 202 mm, MZUSP 30044 (part), 98, 82-233 mm, mun. Tefé, lago Jurupari, rio Tefé, $03^{\circ} 48^{\prime} \mathrm{S}, 065^{\circ} 00^{\prime} \mathrm{W}$. MPEG 968, 3, 140-157 mm, MPEG 975, 3, 157-203 mm, MZUSP 30047, 5, 195-250 mm, mun. Tefé, lago Supiã-Puçu, rio Tefé, $03^{\circ} 45^{\prime} \mathrm{S}, 064^{\circ} 57^{\prime} \mathrm{W}$. MPEG 977, 2, 87-225 mm, MZUSP 30046, 6, 110-225 mm, MZUSP 30048, 31, 74-235 mm, mun. Tefé, Arana-tuba, rio Tefé, ca. $04^{\circ} 02^{\prime} \mathrm{S}, 065^{\circ} 01^{\prime} \mathrm{W}$. MPEG 979, 7 (part), 110-200 mm, mun. Tefé, Ipanema da Baixa, rio Tefé, no coordinates. MPEG 980, 23, 84-242 $\mathrm{mm}$, mun. Tefé, Janari-Atuba, rio Tefé, $c a .03^{\circ} 52^{\prime} \mathrm{S}, 065^{\circ} 01^{\prime} \mathrm{W}$. MPEG 1123 (part), 1, $142 \mathrm{~mm}$, mun. Tefé, Costa Capivara, rio Solimões, ca. $03^{\circ} 16^{\prime} 38^{\prime \prime} \mathrm{S}, 064^{\circ} 38^{\prime} 09^{\prime \prime} \mathrm{W}$. MPEG 1128, 2, 226-260 $\mathrm{mm}$, mun. Iranduba, rio Solimões nr. Manaus, ca. $03^{\circ} 17^{\prime} \mathrm{S}$, $060^{\circ} 11^{\prime} \mathrm{W}$ [coordinates for Iranduba]. MPEG 22738, 1, $305 \mathrm{~mm}$, Mamirauá Reserve, lago Mamirauá, 0259'38"S, 06454'26"W. MPEG 22739, 1, 165 mm, mun. Tefé, igarapé Repartimento, affl. lago Tefé, rio Tefé, $03^{\circ} 24^{\prime} 19^{\prime \prime} \mathrm{S}, 064^{\circ} 43^{\prime} 54^{\prime \prime} \mathrm{W}$. MZUSP 30045, 79 (1CS), 59-233 mm, Jauari-atuba, rio Tefé, ca. $03^{\circ} 56^{\prime} \mathrm{S}, 065^{\circ} 01^{\prime} \mathrm{W}$. MZUSP 30049 (part), 2, 55-90 mm, MZUSP 30057, 2, 56-62 mm, Vista Escura, rio Tefé, no coordinates. MZUSP 30061 (part), 17, 85-157 mm, MZUSP 30062, 5, 48-98 mm, lago Mucura, rio Tefé, no coordinates. MZUSP 62060, 2, 87-99 mm, Poça de Praia, Tapera, rio Negro, $00^{\circ} 11^{\prime} \mathrm{S}, 064^{\circ} 04^{\prime} \mathrm{W}$. MZUSP 78032 (part), 1, $181 \mathrm{~mm}$, mun. Maraã, Paraná do Castanho, lago Amanã, affl. rio Japurá, 0244'S, 064³9’W. MZUSP 91651, 1, 164 mm, rio Uapés, affl. rio Negro, no coordinates [listed coordinates invalid]. MZUSP 92250, 65, 123-225 mm, mun. São Gabriel da Cachoeira, igarapé Cunurim, affl. rio Tiquié, ca. $500 \mathrm{~m}$ below São José II, affl. rio Uaupés, affl. rio Negro, $00^{\circ} 13^{\prime \prime} \mathrm{N}, 069^{\circ} 36^{\prime \prime} \mathrm{W}$. MZUSP 92496, 17, 95-205 mm, mun. São Gabriel da Cachoeira, igarapé Castanha, nr. mouth, affl. rio Tiquié, affl. rio Uaupés, affl. rio Negro, $00^{\circ} 12^{\prime \prime} \mathrm{N}$, 069³5"W. MZUSP 93091, 1, 220 mm, mun. São Gabriel da Cachoeira, igarapé Castanha nr. São Pedro, affl. rio Tiquié, affl. rio Uaupés, affl. rio Negro, $00^{\circ} 11^{\prime \prime} \mathrm{N}, 069^{\circ} 35^{\prime \prime} \mathrm{W}$. MZUSP 94034, 7, 105-160 mm, MZUSP 99169, 7, 73-197 mm, canal do lago Amanã, affl. rio Japurá, $c a .03^{\circ} 00^{\prime} \mathrm{S}, 064^{\circ} 23^{\prime} \mathrm{W}$. MZUSP 95226 (part), 1, $158 \mathrm{~mm}$, mun. Santa Isabel do rio Negro, nr. Paricatuba, lagoon on margin of rio Negro, $00^{\circ} 31^{\prime} \mathrm{S}, 065^{\circ} 01^{\prime} \mathrm{W}$. USNM 198081, 2, 138 $181 \mathrm{~mm}$, igarapé da Mãe Joana, lago Grande do Manacapuru, affl. rio Solimões, $c a .03^{\circ} 13^{\prime} \mathrm{S}, 060^{\circ} 44^{\prime} \mathrm{W}$. USNM 229425, 1, $192 \mathrm{~mm}$, USNM 306868, 2, 159-190 mm, mun. Manaus, lago Janauari, nr. mouth, rio Amazonas floodplain, $c a .03^{\circ} 13^{\prime} \mathrm{S}, 060^{\circ} 00^{\prime} \mathrm{W}$. USNM $300973,2,67-120 \mathrm{~mm}$, rio Unini, affl. rio Negro, ca. $01^{\circ} 41^{\prime} \mathrm{S}$, $061^{\circ} 31^{\prime} \mathrm{W}$ [coordinate for mouth of rio Unini]. USNM 306835, 7, 190-259, mun. Manaus, lago do Janauari, rio Amazonas floodplain, 
ca. $03^{\circ} 13^{\prime} \mathrm{S}, 060^{\circ} 00^{\prime} \mathrm{W}$. USNM 306878, 1, $215 \mathrm{~mm}$, lago Camaleão, Ilha da Marchantaria, rio Solimões floodplain, $03^{\circ} 14^{\prime} 22^{\prime \prime} \mathrm{S}$, 059 56'37"W. USNM 306902, 1, 80 mm, USNM 306925, 1, 167 $\mathrm{mm}$, lago Terra Preta, lago do Janauari, rio Amazonas floodplain, ca. $03^{\circ} 13^{\prime} \mathrm{S}, 060^{\circ} 00^{\prime} \mathrm{W}$. USNM 306904, 3, 190-257 mm, USNM 306934, 1, $259 \mathrm{~mm}$, lago Janauari, rio Amazonas floodplain, ca. $03^{\circ} 13^{\prime} \mathrm{S}, 060^{\circ} 00^{\prime} \mathrm{W}$. USNM 306921, 1, $234 \mathrm{~mm}$, lago Canta Galos, lago Janauari, rio Amazonas floodplain, $03^{\circ} 13^{\prime} \mathrm{S}, 060^{\circ} 00^{\prime} \mathrm{W}$. USNM 327307, 1, 96 mm, mun. Barcelos, lago de Caatinga, rio Ererê, affl. rio Negro, $c a .00^{\circ} 14^{\prime} \mathrm{S}, 063^{\circ} 53^{\prime} \mathrm{W}$. Mato Grosso. INPA 4385, 3, 139-169 mm, stream nr. Castanhal, rio Aripuanã, affl. rio Madeira, Amazonas dr., ca. $10^{\circ} 10^{\prime} \mathrm{S}, 059^{\circ} 29^{\prime} \mathrm{W}$ [coordinates for town of Aripuanã]. MNRJ 25152, 4, 62-93 mm, mun. Ribeirão Cascalheira, Ribeirão Manda Brasa, affl. rio Suiá-miçu, affl. rio Xingu, Amazonas dr., ca. 12 ${ }^{\circ} 54^{\prime} \mathrm{S}, 051^{\circ} 49^{\prime} \mathrm{W}$. MZUSP 48311 (part), 1, 122 mm, Fazenda Santo Antônio, Baía da Sede, Pantanal do Paiaguás, nr. Coxim, rio Paraguai, Paraná dr., $18^{\circ} 30^{\prime} \mathrm{S}, 054^{\circ} 45^{\prime} \mathrm{W}$. MZUSP 90178, 3, 129-182 mm, mun. Cáceres, rio Sepotuba (lower course),

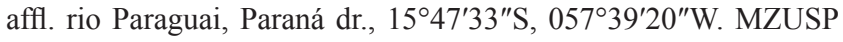
95068 (part), 5, 177-207 mm, mun. Barão de Melgaço. rio Mutum, between Vila de Mimoso and Joselandia (Pantanal de Paiaguás), rio Paraguai, Paraná dr., 16 $19^{\prime} 30^{\prime \prime} \mathrm{S}, 055^{\circ} 49^{\prime} 59^{\prime \prime} \mathrm{W}$. Mato Grosso do Sul. MZUSP 36436, 1, mun. Corumbá, Ladário, rio Paraguai, Paraná dr., $19^{\circ} 00^{\prime} \mathrm{S}, 057^{\circ} 36^{\prime} \mathrm{W}$. Pará (localities from Amazonas dr.). AMNH 12966, 2, 213-327 mm, rio Tocantins, high above waterfall, no coordinates. BMNH 1926.10.27.241-242, 2, 204-207 $\mathrm{mm}$, Monte Alegre, rio Amazonas, $02^{\circ} 00^{\prime} \mathrm{S}, 054^{\circ} 00^{\prime} \mathrm{W}$. INPA 6399 , $1,142 \mathrm{~mm}$, margin of lago Tapagem, rio Trombetas, $01^{\circ} 24^{\prime} \mathrm{S}$, $056^{\circ} 51^{\prime} \mathrm{W}$. MCP 16307, 1, 184 mm, mun. Belém, nr. Belém, Baía de Marajó, ca. $01^{\circ} 26^{\prime} \mathrm{S}, 048^{\circ} 23^{\prime} \mathrm{W}$. MCP 23360, 2, 125-188 mm, Ourém, rio Guamá, affl. Baía de Marajó, ca. $01^{\circ} 33^{\prime} \mathrm{S}, 047^{\circ} 06^{\prime} \mathrm{W}$. MCP 23361, 1, 146 mm, Tomé-Açu, rio Acará-Mirim, affl. rio Acará, affl. Baía de Marajó, $02^{\circ} 25^{\prime} \mathrm{S}, 048^{\circ} 09^{\prime} \mathrm{W}$. MCP 28565, 1, 32 mm, Ihnangapi, São Domingos do Capim, affl. rio Guamá, affl. Baía de Marajó, 01²7'02"S, 4748'26"W. MCP 49397, 1, mun. Santarém, rio Mentai at Cachoeirinha do Mentai, affl. rio Arapiuns, affl. rio Tapajós, $02^{\circ} 43^{\prime} 12^{\prime \prime} \mathrm{S}, 055^{\circ} 36^{\prime} 15^{\prime \prime} \mathrm{W}$. MCP 49399, 2, mun. Santarém, Igarapé do Henrique, affl. Rio Maró, affl. rio Arapiuns, affl. rio Tapajós, $02^{\circ} 51^{\prime} 31^{\prime \prime} \mathrm{S}, 055^{\circ} 41^{\prime} 04^{\prime \prime} \mathrm{W}$. MCP 49402, 1, mun. Curuá, Lago Preto, Ilha São Luiz, rio Amazonas, 0206'22"S, 05510'17"W. MCP 49404, 6; ZUEC 12326, 4, mun. Belterra, stream at Aramanai, affl. rio Tapajós, $02^{\circ} 43^{\prime} 03^{\prime \prime} \mathrm{S}, 055^{\circ} 00^{\prime} 07^{\prime \prime} \mathrm{W}$. MCP 49405, 1, mun. Belterra, rio Tapajós at São Domingos, $02^{\circ} 45^{\prime} 02^{\prime \prime} \mathrm{S}, 055^{\circ} 01^{\prime} 05^{\prime \prime} \mathrm{W}$. MCP 49406, 3, mun. Santarém, stream affl. Lago Anumã, affl. Rio Tapajós, 02³7'27"S, 055¹1'09"W. MCP 49408, 1, mun. Santarém, Igarapé Sucurijú, affl. rio Mentai, affl. rio Arapiuns, affl. rio Tapajós, $02^{\circ} 43^{\prime} 38^{\prime \prime} \mathrm{S}, 055^{\circ} 35^{\prime} 41^{\prime \prime} \mathrm{W}$. MCP 49409, 3, mun. Santarém, rio Maró, nr. Fé em Deus, affl. rio Arapiuns, affl. rio Tapajós, 0250'58"S, 05541'06"W. MCZ 45194, 1, 192 mm, mun. Belém, nr. Icoaraci, igarapé Paracuri, affl. Baía de Marajó, ca. $01^{\circ} 18^{\prime} \mathrm{S}, 048^{\circ} 28^{\prime} \mathrm{W}$. MCZ 165862 (part), 1, 178, mun. Santarém, Santarém and environs, rio Tapajós, ca. $02^{\circ} 26^{\prime} \mathrm{S}$, 54ํㄴ'ㄴ. MPEG 1113, 2, 130-197 mm, MPEG 1114, 6, 75-135 $\mathrm{mm}$, MPEG 1115, 5, 112-230 mm, rio Guamá at Belém, affl. Baía de Marajó, ca. $01^{\circ} 24^{\prime} \mathrm{S}, 048^{\circ} 25^{\prime} \mathrm{W}$. MPEG 1131, 1, $201 \mathrm{~mm}$, MPEG 2397, 8, 150-320 mm, mun. Castanhal, Boa Vista do Apeú, rio
Apeú, affl. rio Guamá, affl. Baía de Marajó, ca. $01^{\circ} 18^{\prime} \mathrm{S}, 047^{\circ} 59^{\prime} \mathrm{W}$. MPEG 1132, 1, 182 mm, MPEG 1133, 1, 173 mm, MPEG 2731, 1, 158 mm, MPEG 6774, 4, 125-192 mm, mun. Cachoeira do Arari, Taperebá, rio Goapi, llha de Marajó, affl. Baía de Marajó, ca. $01^{\circ} 00^{\prime} \mathrm{S}, 048^{\circ} 57^{\prime} \mathrm{W}$ [coordinates for Cachoeira do Arari]. MPEG 3720, 1, 154 mm, MPEG 3736, 1, 135 mm, MPEG 3739, 1, 164 mm, MPEG 4066, 1, 146 mm, mun. Melgaço, Estação Científica Ferreira Pena, rio Puraquequara, affl. rio Anapú, affl. rio Pará, affl. Baía de Marajó, ca. $01^{\circ} 44^{\prime} \mathrm{S}, 051^{\circ} 27^{\prime} \mathrm{W}$. MPEG 4706, 2, 162-177 $\mathrm{mm}$, mun. Ourém, rio Guamá, Furo do Guamá, Lagoa Velha, affl. Baía de Marajó, ca. $01^{\circ} 33^{\prime} \mathrm{S}, 047^{\circ} 06^{\prime} \mathrm{W}$ [coordinates for Ourém]. MPEG 6385, 1, 127 mm, mun. Peixe-Boi, rio Peixe-Boi, affl. rio

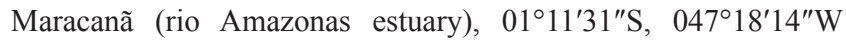
[coordinates for rio Peixe-Boi at Peixe-Boi]. MPEG 6775, 3, 110$147 \mathrm{~mm}$, mun. Cachoeira [do Arari], mouth of rio Goapi, llha de Marajó, affl. Baía de Marajó, 0100'27"S, 04857'44'W. MPEG 6884, 4, 150-270 mm, mun. Paragominas, igarapé Poraquequara, behind camp of Companhia Vale do rio Doce, affl. rio Capim, affl. rio Guamá, affl. Baía de Marajó, 0314'50"S, 04745'40’W. MPEG 6917, 1, 110 mm, MPEG 7295, 6, 110-417 mm, MPEG 7397, 1 (part), 43 mm, mun. Paragominas, igarapé Paraquequara at Fazenda do Sr. Paulo/ Fazenda Monte Santo, affl. rio Capim, affl. rio Guamá, affl. Baía de Marajó, 03 $16^{\prime} 05^{\prime \prime} \mathrm{S}, 047^{\circ} 46^{\prime} 05^{\prime} \mathrm{W}$. MPEG 7310, 1, $180 \mathrm{~mm}$, mun. Paragominas, Vale do rio Doce facility, confl. igarapé Cachoerinha and Paraquequara, affl. rio Capim, affl. rio Guamá, affl. Baía de Marajó, 0309'25"S, 04741'51'W. MPEG 7319, 1 (part), 175 mm, mun. Paragominas, Fazenda Potiritá, igarapé Potiritá, affl. rio Potiritá, affl. rio Capim, affl. rio Guamá, affl. Baía de Marajó, 03²12'13"S, 04739'52'W. MPEG 9884, 2, 63-68 mm, mun. Portel, Floresta Nacional Caxiuanã, affl. rio Anapú, affl. rio Pará, affl. Baía de Marajó, ca. $01^{\circ} 44^{\prime} \mathrm{S}, 051^{\circ} 26^{\prime} \mathrm{W}$. MPEG 12573, 2, 139-188 mm, mun. Benevides, Itá, affl. rio Guamá, affl. Baía de Marajó, ca. 01²1'S, 048¹4’W. MPEG 17818, 1, 92 mm, mun. Benevides, igarapé Taiassuí, affl. rio Guamá, Baía de Marajó, $01^{\circ} 23^{\prime} 46^{\prime \prime} \mathrm{S}$, 048 $14^{\prime} 59^{\prime} \mathrm{W}$. MZUSP 8412, 7, mun. Santarém, igarapé Jacundá, Alter do Chão, affl. rio Tapajós, $02^{\circ} 29^{\prime} \mathrm{S}, 054^{\circ} 47^{\prime} \mathrm{W}$. MZUSP 15567, 1, $165 \mathrm{~mm}$, Cabeceira da Serrinha, lago Jacaré (Reserva Biológica de Trombetas), rio Trombetas, $01^{\circ} 20^{\prime} \mathrm{S}, 056^{\circ} 50^{\prime} \mathrm{W}$. MZUSP 22291, 1, $167 \mathrm{~mm}$, mun. Castanhal, Boa Vista, rio Apeu, ca. $01^{\circ} 17^{\prime} \mathrm{S}$, $047^{\circ} 55^{\prime} \mathrm{W}$. MZUSP 23865, 1, 90 mm, rio Caranandeua, rio Capim, affl. rio Guamá, affl. Baía de Marajó [coordinate for mouth of rio Capim], $01^{\circ} 40^{\prime} \mathrm{S}$, 47ํ'ㄴ'W. MZUSP 23894, 1, rio Capim, lago Jurunundéua, no coordinates. MZUSP 25006, 5, 197-368 mm, rio Curuã-Una, just upstream of dam, $02^{\circ} 49^{\prime} \mathrm{S}, 054^{\circ} 16^{\prime} \mathrm{W}$. SU 54502, 1, $113 \mathrm{~mm}$, SU 54503, 3, SU 64189, 1, Cabeceira lago Grande, nr. Santarém, Ajamury, ca. $02^{\circ} 26^{\prime} \mathrm{S}, 054^{\circ} 41^{\prime} \mathrm{W}$ [coordinates for Santarém]. UF 36600, 1, 54 mm, igarapé Pirelli, affl. rio Maguari, affl. Baía de Marajó, 01 $21^{\prime} 24^{\prime \prime} \mathrm{S}, 048^{\circ} 19^{\prime} 36^{\prime \prime} \mathrm{W}$. UF 238564, 11, 119-237 mm, UF 238565, 11, 106-293 mm, UF 238566, 4, 159-205 mm; UF 238567, 12, 77-234, UF 238568, 12, 91-255, stream at SEPAq station, $39 \mathrm{~km}$ SE Santarém on rd. to Santa Rosa, 02 40'01'S, 054'33'09'W. ZUEC 12327, 6, mun. Santarém, igarapé do Mato Grosso, nr. Jaguarari, affl. rio Tapajós, 0255'01"S, 05503'54"W. ZUEC 12331, 3, mun. Santarém, lago Bravo, nr. Santo Amaro, affl. rio Tapajós, $02^{\circ} 58^{\prime} 22^{\prime \prime S}$, $055^{\circ} 13^{\prime} 42^{\prime \prime} \mathrm{W}$. ZUEC 12333, 5, mun. 
Santarém, igarapé Santo Amaro, nr. Santo Amaro, affl. rio Tapajós, 02 $59^{\prime} 15^{\prime \prime}$ S, 055 $13^{\prime} 59^{\prime \prime}$ W. ZUEC 12334, 6, mun. Santarém, Igarapé do Macaco, affl. rio Mentai, affl. rio Arapiuns, affl. rio Tapajós, 02 $40^{\prime} 28^{\prime \prime} \mathrm{S}, 055^{\circ} 35^{\prime} 55^{\prime \prime} \mathrm{W}$. ZUEC 12335, 1, mun. Santarém, Igarapé Branquinho, affl. rio Mentai, affl. rio Arapiuns, affl. rio Tapajós, $02^{\circ} 41^{\prime} 48.4^{\prime \prime} \mathrm{S}, 055^{\circ} 35^{\prime} 43^{\prime \prime} \mathrm{W}$. Rondônia (localities from rio Madeira dr., Amazonas dr.). FMNH 117761, 1, 158 mm, Maciel, rio Guaporé, affl. rio Mamoré, ca. $12^{\circ} 30^{\prime} \mathrm{S}, 062^{\circ} 20^{\prime} \mathrm{W}$. INPA 9722, 3, 92-212 mm, INPA 9726, 18, 130-287 mm, rio Pacaás Novos, nr. confl. with rio Guaporé, ca. $15 \mathrm{~km}$ Guajará Mirim, affl. rio Mamoré, $10^{\circ} 51^{\prime} \mathrm{S}, 065^{\circ} 16^{\prime} \mathrm{W}$. INPA uncat. (POLO-663), 2, 140$148 \mathrm{~mm}$, rio Guaporé opposite Costa Marquez, affl. rio Mamoré, $12^{\circ} 27^{\prime} \mathrm{S}, 064^{\circ} 20^{\prime} \mathrm{W}$. INPA uncat. (POLO-683), 13, INPA uncat. (POLO-731), 18, rio Pacaás Novos, $c a .15 \mathrm{~km}$ upstream of GuajaráMirim, affl. rio Mamoré, $10^{\circ} 54^{\prime} \mathrm{S}, 065^{\circ} 09^{\prime} \mathrm{W}$. MCP 46938, 2, 100 $200 \mathrm{~mm}$, mun. Calamã, Calamã, rio Madeira floodplains lake, $08^{\circ} 01^{\prime} 37^{\prime \prime} \mathrm{S}, 062^{\circ} 52^{\prime 2} 28^{\prime \prime} \mathrm{W}$. UFRO-I 6476, 38, 59-290 mm, lago Cuniã, rio Madeira floodplain, $08^{\circ} 19^{\prime} 40^{\prime \prime} \mathrm{S}, 063^{\circ} 30^{\prime} 11^{\prime \prime} \mathrm{W}$. UFRO-I 6483, 1, $40 \mathrm{~mm}$, nr. Sítio Santa Helena, in front of Ilha das Pedras, rio Karipunas, $09^{\circ} 11^{\prime} 32^{\prime \prime} \mathrm{S}, 064^{\circ} 34^{\prime} 17^{\prime \prime} \mathrm{W}$. UFRO-I 6485, 42, $53-$ 385 mm, UFRO-I uncat. (field no. CAU2010010701), 3, 180-245 $\mathrm{mm}$, mouth of rio Cautário, $12^{\circ} 10^{\prime} 51^{\prime \prime} \mathrm{S}, 064^{\circ} 34^{\prime} 04^{\prime \prime} \mathrm{W}$. UFRO-I $6487,9,105-215 \mathrm{~mm}$, rio Guaporé upstream of Pedras Negras, affl. rio Mamoré, $12^{\circ} 52^{\prime} 03^{\prime \prime} \mathrm{S}, 62^{\circ} 52^{\prime} 38^{\prime \prime} \mathrm{W}$. UFRO-I 6490, 3, 88-117 mm, UFRO-I 6516, 1, 255 mm, lago Cuniã, 1 km ICMBio base, rio Madeira floodplain, $08^{\circ} 19^{\prime} 20^{\prime \prime} \mathrm{S}, 063^{\circ} 30^{\prime} 14^{\prime \prime} \mathrm{W}$. UFRO-I 6496, 1, $83 \mathrm{~mm}$, mouth of rio Pacaás Novos, affl. rio Mamoré, 1052'07"S, $065^{\circ} 15^{\prime} 42^{\prime \prime}$ W. UFRO-I 6503, 4, 118-160 mm, Furo Variante, lago Cuniã, rio Madeira floodplain, $08^{\circ} 21^{\prime} 18^{\prime \prime} \mathrm{S}, 063^{\circ} 30^{\prime} 11^{\prime \prime} \mathrm{W}$. UFRO-I 6505, 9, 37-122 mm, Arrozal do lago, rio Madeira floodplain, 08¹9'36"S, 06329'59"W. UFRO-I 6512, 2, 195-204 mm, Arrozal do lago, rio Madeira floodplain, $08^{\circ} 21^{\prime} 10^{\prime \prime} \mathrm{S}, 063^{\circ} 30^{\prime} 26^{\prime \prime} \mathrm{W}$. UFRO-I 7924, 1, female, 166 mm, mun. Porto Velho, igarapé Mato Grosso, nr. campus Universidade Federal de Rondônia, nr. Cachoeira Santo Antônio, affl. rio Madeira, 08 $49^{\prime} 41^{\prime \prime}$, $063^{\circ} 56^{\prime} 30^{\prime \prime} \mathrm{W}$. UFRO-I 7925, 11 (5 immature, 136-253 mm, 1 female, $203 \mathrm{~mm}, 5$ male, 181-297 mm), mun. Porto Velho, lago Cuniã, rio Madeira floodplain, $c a .08^{\circ} 19^{\prime} \mathrm{S}, 063^{\circ} 28^{\prime} \mathrm{W}$. UFRO-I uncat. $1,181 \mathrm{~mm}$, rio Madeira at Abunã, ca. $09^{\circ} 42^{\prime} \mathrm{S}, 065^{\circ} 21^{\prime} \mathrm{W}$. Roraima (localities from rio Branco dr., affl. rio Negro, Amazonas dr.). INPA 7358, 1, INPA 7359, 1, INPA 7360, 1, INPA 7361, 1, INPA 73661, 1, INPA 7372, 1, INPA 7381, 1, INPA 7382, 1, INPA 7388, 1, INPA 7392, 1, lago Caracanã, 23 km Normândia on hwy. RR-202, $03^{\circ} 54^{\prime} 12^{\prime \prime} \mathrm{N}, 059^{\circ} 49^{\prime} 17^{\prime \prime} \mathrm{W}$. INPA 7373, 1, INPA 73791, $119 \mathrm{~mm}$, INPA 7380, 1, igarapé do Gentil, $34 \mathrm{~km}$ Boa Vista on Boa Vista to Bonfim hwy., $c a .02^{\circ} 58^{\prime} \mathrm{N}, 060^{\circ} 26^{\prime} \mathrm{W}$. INPA 7413, 3, INPA 7414, 2, Fazenda Santa Cecília, 7 km Boa Vista on Boa VistaBonfim hwy., $02^{\circ} 48^{\prime} \mathrm{N}, 060^{\circ} 36^{\prime} \mathrm{W}$. INPA 7424, 2, 112-159 mm, Fazenda Chico Paraíba, ca. $4 \mathrm{~km}$ Bonfim, rio Tacutu, $c a .03^{\circ} 21^{\prime} \mathrm{N}$, $059^{\circ} 50^{\prime} \mathrm{W}$. INPA 9833, 3, 80-108 mm, Ilha de Maracá, stream at end of island, rio Uraricoera, $03^{\circ} 22^{\prime} \mathrm{N}, 061^{\circ} 23^{\prime} \mathrm{W}$. MZUSP 31775 , 1, $221 \mathrm{~mm}$, igarapé do Cujobim, 'rio Branco' opposite Ilha de Maracá, rio Uraricoera, ca. $03^{\circ} 21^{\prime} \mathrm{N}, 061^{\circ} 26^{\prime} \mathrm{W}$. MZUSP 31777,1 , 176 mm, igarapé do Bota Panela, nr. Cachoeira do Bem Querer on rio Branco, ca. $01^{\circ} 55^{\prime} \mathrm{N}, 061^{\circ} 00^{\prime} \mathrm{W}$. Colombia. Guainía. MBUCV-V 7536, 1, caño Caramacoa, apróx. 45 min de Puerto
Inírida, affl. río Inírida, affl. río Guainía, Orinoco dr., $c a .03^{\circ} 51^{\prime} \mathrm{N}$, $067^{\circ} 55^{\prime} \mathrm{W}$. French Guiana. Cayenne. CU 71942, 4, Kaw River, ca. $3.5 \mathrm{~km}$ NW Kaw, Kaw River dr., $04^{\circ} 31^{\prime} \mathrm{N}, 052^{\circ} 04^{\prime} \mathrm{W}$. MNHN 1999-1325, 3 (of 7), 210-224 mm, Kaw River dr., no coordinates. MNHN 1999-1374, 1, 211 mm, crique Gabrielle, Comté River dr., ca. $04^{\circ} 44^{\prime} \mathrm{N}, 052^{\circ} 19^{\prime} \mathrm{W}$. Guyana. Demerara Mahaica. AUM 27917, 7, 12356, Maduni stop-off, $22.3 \mathrm{mi}$. from Georgetown, Conservancy Canal, affl. Maduni River, Mahaica River dr., $06^{\circ} 30^{\prime} 01^{\prime \prime} \mathrm{N}, 058^{\circ} 02^{\prime} 14^{\prime \prime} \mathrm{W}$. CAS 67700, 1, 164 mm, FMNH 53329 , 1, 52 mm, FMNH 53337, 1, 143 mm, INHS 49037, 1, 270 mm, Lama stop-off, Conservancy Canal, affl. Lama River, Mahaica River dr., 06³7'33"N, 05755'55"W. FMNH 96203, 1, 44 mm, Kuru Kuru creek on Soesdyke-Linden hwy., Demerara River dr. $06^{\circ} 24^{\prime} 12^{\prime \prime} \mathrm{N}, 058^{\circ} 14^{\prime} 26^{\prime \prime} \mathrm{W}$. INHS 49063, 9, 104-158 mm, Maduni stop-off, Conservancy Canal, affl. Maduni River, affl. Mahaica River, $06^{\circ} 30^{\prime} 01^{\prime \prime} \mathrm{N}, 058^{\circ} 02^{\prime} 14^{\prime \prime} \mathrm{W}$. Potaro Siparuni. ANSP 175955 , 1, $70 \mathrm{~mm}$, creek, affl. Essequibo River, ca. $18 \mathrm{~min}$. upstream from Maipuri campsite, Essequibo River dr., ca. $04^{\circ} 34^{\prime} \mathrm{N}, 058^{\circ} 35^{\prime} \mathrm{W}$. ANSP 177494, 1, 147 mm, Siparuni River, Red Hill Creek between Levi Falls and Blackwater camp, Essequibo River dr., 04 $44^{\prime} 14^{\prime \prime} \mathrm{N}$, 058 59'17"W. Upper Demerara-Berbice. AMNH 13421, 1, 169 mm, AMNH 30418, 1, Malali, Mari-Mari River, Demerara River dr., $05^{\circ} 37^{\prime} \mathrm{N}, 058^{\circ} 21^{\prime} \mathrm{W}$. AUM 35812, 1, $127 \mathrm{~mm}$, unnamed river at

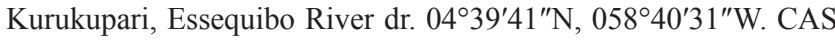
67703, 2, Demerara River at Wisden (formerly Wismar), Demerara River dr. $06^{\circ} 00^{\prime} \mathrm{N}, 058^{\circ} 18^{\prime} \mathrm{W}$. CAS 67705, 2, 156-167 mm, FMNH 53338, 2, Gluck Island, Essequibo River, nr. Rockstone, Essequibo River dr., $05^{\circ} 58^{\prime} \mathrm{N}, 058^{\circ} 31^{\prime} \mathrm{W}$. FMNH 53339, 1, Kumaka, Berbice River dr., $05^{\circ} 35^{\prime} \mathrm{N}, 057^{\circ} 56^{\prime} \mathrm{W}$. INHS 49107, 1, $81 \mathrm{~mm}, 5.05 \mathrm{mi}$. SSW Linden, Demerara River dr., $05^{\circ} 56^{\prime} 00^{\prime \prime} \mathrm{N}, 058^{\circ} 18^{\prime} 22^{\prime \prime} \mathrm{W}$. INHS 49143, 1, 129 mm, 5.05 mi. SSW Linden, Demerara River dr., $05^{\circ} 56^{\prime} 00^{\prime \prime} \mathrm{N}, 058^{\circ} 18^{\prime} 22^{\prime \prime} \mathrm{W}$. ROM 86803, 3, 157-237 mm, ROM 88618 , 5, 143-270 mm, side channel by island, Berbice River, Berbice River dr., 05 $09^{\prime} 03^{\prime \prime} \mathrm{N}, 058^{\circ} 11^{\prime} 46^{\prime \prime} \mathrm{W}$. Upper TakutuUpper Essequibo (localities from Takutu River are in the rio Branco dr., affl. rio Negro, Amazonas dr.). ANSP 179504, 2, $106-$ 133 mm, AUM 35863, 2, 83-104 mm, stream $10.3 \mathrm{~km} \mathrm{NW}$ Karanambo, affl. Rupununi River, Essequibo River dr., $03^{\circ} 48^{\prime} 27^{\prime \prime} \mathrm{N}$, $059^{\circ} 23^{\prime} 06^{\prime \prime}$ W. AUM 38187, 1, 142 mm, Kuyuwini River, 48.4 km E Kuyuwini Landing, Essequibo River dr., $02^{\circ} 03^{\prime} 54^{\prime \prime} \mathrm{N}$, 058 48'33"W. BMNH 1972.7.27.447-450, 4, 133-156 mm, Dadanawa pools, Rupununi River, Essequibo River dr., $c a$. $02^{\circ} 50^{\prime} \mathrm{N}, 059^{\circ} 31^{\prime} \mathrm{W}$. BMNH 1972.7.27.451-455, 6, 64-158 mm, "Guyana", no coordinates. CU 71959, 9, Jawar pond, Manari Ranch, N Lethem, Rupununi savanna, affl. Takutu River, ca. $03^{\circ} 26^{\prime} \mathrm{N}, 059^{\circ} 46^{\prime} \mathrm{W}$ (listed by Sullivan, 1997). MCZ 48553, 1, 204 mm, Agua Branca River, affl. Manari River, affl. Takutu River, $c a$. $03^{\circ} 29^{\prime} \mathrm{N}, 059^{\circ} 48^{\prime} \mathrm{W}$. MCZ 48554, 2, 157-191 mm, dry creek bed, 10 mi. E Nappi, affl. Takutu River, ca. $03^{\circ} 25^{\prime} \mathrm{N}, 059^{\circ} 25^{\prime} \mathrm{W}$. MCZ 165855, 1, $241 \mathrm{~mm}$, small pond $5 \mathrm{mi}$. N Manari Ranch, Takutu River, ca. $03^{\circ} 26^{\prime} \mathrm{N}, 059^{\circ} 46^{\prime} \mathrm{W}$. UMMZ 215583, 1, $244 \mathrm{~mm}$, Shebe pond, Rupununi, no coordinates. UMMZ 215603, 1, $218 \mathrm{~mm}$, pond at Juari Ranch, E Takutu River, no coordinates. Region not specified. UMMZ 187500, 3, 118-184 mm, aquarium specimen, no coordinates. USNM 376799, 1, 183 mm, Waterguage Pond, unknown locality in Guyana, no coordinates. Peru. Loreto (all 
localities are from Amazonas dr.). ANSP 166267, 1, $76 \mathrm{~mm}$, stream of río Yanayacu, W Miraflores, rio Marañón, $c a .04^{\circ} 42^{\prime} \mathrm{S}, 074^{\circ} 17^{\prime} \mathrm{W}$. ANSP 167729, 8, 98-172 mm, stream 0.5 mi. below Santa Clara (R bank), río Nanay, ca. $03^{\circ} 46^{\prime} \mathrm{S}, 073^{\circ} 20^{\prime} \mathrm{W}$. ANSP 178218, 4, 81-156 mm, ANSP 178220, 2, 55-63 mm, caño Santa Rita, R. bank affl. río

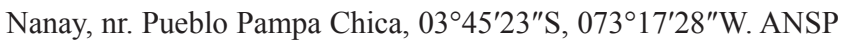
$178655,1,172 \mathrm{~mm}$, río Nanay at Pueblo Pampa Chica, 0345'09"S, $073^{\circ} 17^{\prime} 00^{\prime \prime} \mathrm{W}$. ANSP $180311,1,121 \mathrm{~mm}$, río Nanay, upstream from Santa Clara, 0346'45"S, 073²2'06"W. ANSP 182935, 3, 119-159 $\mathrm{mm}$, caño Shirui (also known as Santa Rita), R. bank affl. río

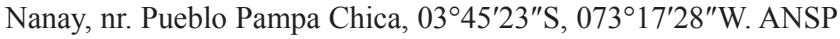
191848, 1, 176 mm, río Nanay, R. bank, downstream mouth caño Shirui, $03^{\circ} 45^{\prime} 06^{\prime \prime} \mathrm{S}, 073^{\circ} 17^{\prime} 14^{\prime \prime} \mathrm{W}$. INHS 36785, 2, 117-137 mm, quebrada Santa Clara, río Nanay, ca. $03^{\circ} 47^{\prime} \mathrm{S}, 073^{\circ} 20^{\prime} \mathrm{W}$. INHS 52490, 3, 99-136 mm, caño Santa Rita, nr. Pampa Chica, affl. río Nanay, $03^{\circ} 45^{\prime} 23^{\prime \prime} \mathrm{S}, 073^{\circ} 17^{\prime} 28^{\prime \prime} \mathrm{W}$. MCP 43159, 1, $183 \mathrm{~mm}$, UF 114499, 4, 128-250 mm, UF 116556, 1, female, $242 \mathrm{~mm}$, stream at $\mathrm{km} 23$ on Iquitos-Nauta hwy., affl. río Nanay, 0356'38"S, $073^{\circ} 23^{\prime} 54^{\prime \prime} \mathrm{W}$. MUSM 1992, 9, 108-331 mm, río Nanay at Iquitos, ca. $03^{\circ} 42^{\prime} \mathrm{S}, 073^{\circ} 15^{\prime} \mathrm{W}$. MUSM 7123 (part), 5, 73-85, Laguna Rimachi, río Pastaza, affl. río Marañón, $04^{\circ} 25^{\prime} \mathrm{S}, 076^{\circ} 43^{\prime} \mathrm{W}$. MUSM 44702, 3 (1 immature, $175 \mathrm{~mm}, 2$ female, 279-296 mm), forest stream nr. Jenaro Herrera, affl. río Ucayali, 04 $54^{\prime} 26^{\prime \prime} \mathrm{S}$, 07336'44"W. SU 66949, 1, 41 mm, río Ampiyacu nr. Pebas, ca. $03^{\circ} 19^{\prime} \mathrm{S}, 071^{\circ} 50^{\prime} \mathrm{W}$. UF 116549 , 1 , immature, $65 \mathrm{~mm}$, río Nanay, $\mathrm{N}$ bank, $1 \mathrm{~km}$ upstream Mishana, 03 $52^{\prime} 12^{\prime \prime} \mathrm{S}, 073^{\circ} 29^{\prime} 38^{\prime \prime} \mathrm{W}$. UF $131127,1,130 \mathrm{~mm}$, Iquitos, procured by ornamental fish catchers, no coordinates. UF 148471, 1, $52 \mathrm{~mm}$, quebrada Sapuenillo, nr. Jenaro Herrera, affl. río Ucayali, $04^{\circ} 54^{\prime} 26^{\prime \prime} \mathrm{S}, 073^{\circ} 36^{\prime} 44^{\prime \prime} \mathrm{W}$. UF 184006, 17 (3 immature, 50-135 mm, 9 female, 157-285 mm),

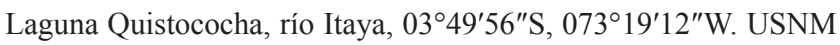
284624, 9, 108-331 mm, río Amazonas, nr. Iquitos, $03^{\circ} 45^{\prime} \mathrm{S}$, $073^{\circ} 15^{\prime} \mathrm{W}$. ZMH 11628 (part), 1, $80 \mathrm{~mm}$, between Iquitos and Leticia, río Amazonas, no coordinates. Madre de Dios (localities from río Madre de Dios dr., affl. Río Beni, affl. Río Madeira, Amazonas dr.). MUSM 9159, 1, 288 mm, MUSM 9605, 4, 158-207 mm, Reserva Nacional (RN) de Tambopata, Laguna Sandoval, $12^{\circ} 26^{\prime} 30^{\prime \prime} \mathrm{S}, 069^{\circ} 02^{\prime} 18^{\prime \prime} \mathrm{W}$. MUSM 25368, 1, Las Piedras, quebrada Malecón, affl. río Manuripe, ca. $12^{\circ} 30^{\prime} \mathrm{S}, 069^{\circ} 14^{\prime} \mathrm{W}$. USNM 264073, 1, 235 mm, RN de Tambopata, Laguna Cocococha, $5.1 \mathrm{~km}$ E Explorer's Inn, 12 $49^{\prime} 00^{\prime \prime} \mathrm{S}, \quad 069^{\circ} 16^{\prime} 30^{\prime \prime} \mathrm{W}$. Suriname. Marowijne. UF 177359, 2 (1 female, $261 \mathrm{~mm}, 1 \mathrm{male}, 210 \mathrm{~mm}$ ), small stream on rd. from Paramaribo to Albina, affl. Cottica River, Commewijne River dr., $05^{\circ} 34^{\prime} 50^{\prime \prime} \mathrm{N}, 054^{\circ} 15^{\prime} 31^{\prime \prime} \mathrm{W}$. Nickerie. USNM 225560, 1, 65 mm, Corantijn River at km 180, side channel, Corantijn River dr., $c a .05^{\circ} 54^{\prime} \mathrm{N}, 57^{\circ} 04^{\prime} \mathrm{W}$. USNM 302005, 1, 91 $\mathrm{mm}$, affl. Sisa Creek, N side, ca. $700 \mathrm{~m}$ downstream of crossing of Amotopo-Camp Geologie rd., Corantijn River dr., $03^{\circ} 42^{\prime} \mathrm{N}$, $057^{\circ} 42^{\prime}$ W. Para. UF 177361, 1, female, 207 mm, mun. Zanderij, Kola kreek, affl. Para River, Suriname River dr., 05²7'09"N, 055¹4'58"S. Sipaliwini. BMNH 1981.6.9.932-986 (part), 1, 190 $\mathrm{mm}$, stream entering Lucie River, Corantijn River dr. $c a .03^{\circ} 35^{\prime} \mathrm{N}$, $057^{\circ} 40^{\prime} \mathrm{W}$. USNM 225561, 1, $191 \mathrm{~mm}$, Matapi creek ca. $1 \mathrm{~km}$ intersection with Corantijn River, Corantijn River dr., $05^{\circ} 00^{\prime} \mathrm{N}$, $057^{\circ} 16^{\prime} \mathrm{W}$. USNM 225563, 2, 95-170 mm, stream ca. $0.5 \mathrm{~km}$ inland of Camp Mataway, Corantijn River dr., ca. $04^{\circ} 48^{\prime} \mathrm{N}, 057^{\circ} 43^{\prime} \mathrm{W}$.
Venezuela. Amazonas. FMNH 94429, 4, 161-183 mm, "upper Orinoco", Orinoco dr., no coordinates. MBUCV-V 8063, 1, 104 $\mathrm{mm}$, caño Moriche, $c a$. $10 \mathrm{~km}$ N San Fernando de Atabapo, affl. río Orinoco, Orinoco dr., ca. $03^{\circ} 57^{\prime} \mathrm{N}, 067^{\circ} 38^{\prime} \mathrm{W}$. MBUCV-V 14710, 1, $95 \mathrm{~mm}$, nr. Santa Lucia, rio Negro, Amazonas dr., ca. $01^{\circ} 18^{\prime} \mathrm{N}$, 066 52’W. MBUCV-V 14734, 1, 204 mm, small affl. río Orinoco, Puerto Ayacucho-El Burro rd., Orinoco dr., ca. $05^{\circ} 47^{\prime} \mathrm{N}, 067^{\circ} 29^{\prime} \mathrm{W}$. MCNG 21516, 3, Maroa, río Guainía, affl. rio Negro, Amazonas dr., ca. $02^{\circ} 43^{\prime \prime} \mathrm{N}, 067^{\circ} 33^{\prime} \mathrm{W}$ (listed by Sullivan, 1997). MCNG 22090, 1, Piaroa San José, río Yureba, Orinoco dr., ca. $04^{\circ} 02^{\prime} \mathrm{N}$, $067^{\circ} 33^{\prime} \mathrm{W}$ (listed by Sullivan, 1997). UF 177350, 7 ( 2 immature, 170-199 mm, 5 female, 167-201 mm), caño Viejita, rd. from San Fernando de Atabapo to Santa Barbara, affl. río Atabapo, Orinoco dr., 0355'59"N, 067³6'34"W. Anzoátegui. MBUCV-V 25751, 1, $325 \mathrm{~mm}$, Hato Morichalito, río Zuata, Orinoco dr., $c a .08^{\circ} 33^{\prime} \mathrm{N}$, $064^{\circ} 57^{\prime} \mathrm{W}$. Apure (localities from Orinoco dr.). ANSP 141616, 4, Jabillal, nr. San Fernando de Apure, affl. río Arauca, ca. $07^{\circ} 44^{\prime} \mathrm{N}$, $067^{\circ} 58^{\prime} \mathrm{W}$. AUM 22561, 1, $130 \mathrm{~mm}$, caño Guarico, affl. río Apure, $07^{\circ} 42^{\prime} 47^{\prime \prime} \mathrm{N}, 069^{\circ} 19^{\prime} 50^{\prime \prime} \mathrm{W}$. AUM 22756, 19, 100-190 mm, INHS 89761, 11, 127-164 mm, caño La Pastora, affl. río Apure, $07^{\circ} 26^{\prime} 40^{\prime \prime} \mathrm{N}, 069^{\circ} 26^{\prime} 05^{\prime \prime} \mathrm{W}$. MBUCV-V 13229, 1, $246 \mathrm{~mm}$, Mantecal, río Arauca, $07^{\circ} 34^{\prime} \mathrm{N}, 069^{\circ} 08^{\prime} \mathrm{W}$. MCNG 2138, 1, MCNG 2489, 22, MCNG 3635, 5, MCNG 3715, 44, MCNG 3799, 6, MCNG 3898, 27, MCNG 4906, 2, MCNG 5134, 4, MCNG 7467, 2, MCNG 28738, 22, Módulo UNELLEZ, río Caicara, affl. río Apure, $07^{\circ} 24^{\prime} \mathrm{N}, 069^{\circ} 32^{\prime} \mathrm{W}$ (listed by Sullivan, 1997). MCNG 3906, 26, Muñoz, Módulo F.C., río Caicara, aff, río Apure, ca. $07^{\circ} 35^{\prime} \mathrm{N}, 069^{\circ} 25^{\prime} \mathrm{W}$ (listed by Sullivan, 1997). MCNG 9777, 3, caño Guaritico, aff río Apure, $c a .07^{\circ} 45^{\prime} \mathrm{N}, 069^{\circ} 03^{\prime} \mathrm{W}$ (listed by Sullivan, 1997). MCNG 10144, 5, MCNG 10344, 1, MCNG 11032 (part), 1, MCNG 12566 (part), 4, MCNG 15744, 2, caño Maporal, nr. módulo UNELLEZ, affl. río Caicara, affl. río Apure, $07^{\circ} 24^{\prime} \mathrm{N}$, $069^{\circ} 32^{\prime} \mathrm{W}$ (listed by Sullivan, 1997). MCNG 28579, 5, affl. of río Caicara, no precise locality, no coordinates (listed by Sullivan, 1997). TNHC 14928, 10, 146-201 mm, caño Maporal, nr. módulo UNELLEZ, affl. río Caicara, affl. río Apure, $07^{\circ} 27^{\prime} \mathrm{N}, 069^{\circ} 32^{\prime} \mathrm{W}$. TNHC 17490, 5, 150-244 mm, TNHC 47217, 1, $212 \mathrm{~mm}$, nr. módulo UNELLEZ, río Caicara, affl. río Apure, $c a \cdot 07^{\circ} 24^{\prime} \mathrm{N}$, 069³2'W. UF 77335, 4, 162-208 mm, ca. $30 \mathrm{~km} \mathrm{SW} \mathrm{La} \mathrm{Ye,} \mathrm{caño}$ Caicara, affl. río Guaratico, affl. río Apure, $07^{\circ} 45^{\prime} \mathrm{N}, 069^{\circ} 03^{\prime} \mathrm{W}$. UF 176887, 10 (1 immature, $197 \mathrm{~mm}, 1$ female, $187 \mathrm{~mm}, 3$ male, 153 $170 \mathrm{~mm}, 5$ unsexed [2 CS], 74-231 mm), MCNG 9698, 1, Hato El Frio, nr. Mantecal, floodplain lake of río Guaratico, affl. río Apure, 0749'32"N, 068'55'23"W. Bolívar. ANSP 166372, 1, 140 mm, lago Maldonado, El Almacén, río Orinoco floodplain, Orinoco dr., ca. $08^{\circ} 06^{\prime} \mathrm{N}, 063^{\circ} 48^{\prime} \mathrm{W}$. AUM 36512, 3, 204-288 mm, northernmost arm of Uraima Falls, río Paragua, affl. río Caroní, Orinoco dr., $06^{\circ} 18^{\prime} 05^{\prime \prime} \mathrm{N}, 063^{\circ} 37^{\prime} 39^{\prime \prime} \mathrm{W}$. MBUCV-V 3971, 38, 10-45 mm, floodplain lake of río Las Claritas, on El Dorado-Santa Elena de Uairén hwy., affl. río Cuyuní, Essequibo River dr., $c a .06^{\circ} 10^{\prime} \mathrm{N}$, $061^{\circ} 25^{\prime} \mathrm{W}$. MBUCV-V 4013, 2, stream at km 67 on El DoradoSanta Elena de Uairén hwy., affl. río Cuyuní, Essequibo River dr., ca. $06^{\circ} 13^{\prime} \mathrm{N}, 061^{\circ} 21^{\prime} \mathrm{W}$. MBUCV-V 7457, 8, 43-66 mm, stream, km 88 on El Dorado-Santa Elena de Uairén hwy., affl. río Cuyuní, River Essequibo dr., $06^{\circ} 05^{\prime} \mathrm{N}, 061^{\circ} 23^{\prime} \mathrm{W}$. MBUCV-V 9490, 1, 337 mm, MBUCV-V 9513, 1, 377 mm, MBUCV-V 9517, 1, 220 mm, 
MBUCV-V 9791, 1, 168 mm, MBUCV-V 14029, 1, 220 mm, 40 km S El Dorado, Isla de Jacobo, nr. raudal de Kinotovaca, affl. río Cuyuní, River Essequibo dr., ca. $06^{\circ} 23^{\prime} \mathrm{N}, 061^{\circ} 37^{\prime} \mathrm{W}$. MBUCV-V 9537, 1, 262 mm, Boca del río Chicanán (at El Dorado), affl. río Cuyuní, River Essequibo dr., ca. 06 $42^{\prime} 24^{\prime \prime} \mathrm{N}, 061^{\circ} 38^{\prime} 05^{\prime \prime} \mathrm{W}$. MBUCV-V 10693, 3 (2 examined), 30-42 mm, La Paragua, río Paragua, aff río Caroní, Orinoco dr., $06^{\circ} 50^{\prime} \mathrm{N}, 63^{\circ} 22^{\prime} \mathrm{W}$. MHNLS 5720, 2, MHNLS 6992, 1, Bandera, lago Guri, rio Caroní, Orinoco dr., $07^{\circ} 46^{\prime} \mathrm{N}, 062^{\circ} 59^{\prime} \mathrm{W}$ (listed by Sullivan, 1997). MZUSP 96453 , 1, $59 \mathrm{~mm}$, mun. Caicara del Orinoco, rio caño, Pelo Ojo, Orinoco

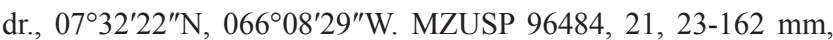
mun. Caicara del Orinoco, Laguna de Castilleros, margin of río Orinoco, Aquaculture Station, Universidad de Oriente, Orinoco dr., 07³0'51"N, 06609'20W. SU 54418, 1, 173 mm, SU 54495, 1, 188 mm, SU 64174, 10, mun. Caicara del Orinoco, caño de Quiribana, Orinoco dr., $c a .07^{\circ} 32^{\prime} \mathrm{N}, 066^{\circ} 08^{\prime} \mathrm{W}$ (listed by Sullivan, 1997). TCWC 8308.18, 2, 106-114 mm, TCWC 8310.24, 2, 182-185 mm, TCWC 8311.24, 2, 158-181 mm, TCWC 8320.05, 1, $297 \mathrm{~mm}$, Campamento del Guri, Guri Reservoir, río Caroní, Orinoco dr., $c a$. $07^{\circ} 46^{\prime} 14^{\prime \prime} \mathrm{N}, 063^{\circ} 00^{\prime} 34^{\prime \prime} \mathrm{W}$. Delta Amacuro. MBUCV-V 10352, 4 (3 examined), 192-209 mm, caño El Chano, Coporito arriba, nr. Tucupita, affl. caño Mamano, río Orinoco delta, Orinoco dr., ca. $09^{\circ} 03^{\prime} \mathrm{N}, 062^{\circ} 03^{\prime} \mathrm{W}$. Guárico (localities from Orinoco dr.). MBUCV-V 25739, 1, $118 \mathrm{~mm}$, S Requena, mouth of río Zuata, $07^{\circ} 58^{\prime} \mathrm{N}, 065^{\circ} 31^{\prime} \mathrm{W}$. MCNG 1760 , 1, Hato Santiago, no coordinates. Monagas (localities from Orinoco dr.). LACM 43349-12, 16, $92-$ 199 mm, MBUCV-V 10696, (part) 20 (4CS), 97-222 mm, Laguna Guatero nr. Barrancas, affl. río Orinoco, ca. $08^{\circ} 42^{\prime} \mathrm{N}, 062^{\circ} 12^{\prime} \mathrm{W}$. MBUCV-V 18905, 1, 43 mm, MBUCV-V 22404, 12, 71-214 mm, río Morichal Largo, río Orinoco delta, $09^{\circ} 26^{\prime} \mathrm{N}, 062^{\circ} 24^{\prime} \mathrm{W}$. MCNG 11201 , 1, río Uracoa, río Orinoco delta, $09^{\circ} 00^{\prime \prime} \mathrm{N}, 062^{\circ} 21^{\prime} \mathrm{W}$. MCNG 15932, 4, río Yabo, at bridge nr. El Pelón, río Orinoco delta, ca. $08^{\circ} 57^{\prime \prime} \mathrm{N}, 062^{\circ} 47^{\prime} \mathrm{W}$. MHNLS 6417, 3, Laguna San José, nr. Coloradito, río Orinoco delta, $c a .08^{\circ} 45^{\prime} \mathrm{N}, 063^{\circ} 29^{\prime} \mathrm{W}$. MHNLS 6621 , 1, lake at Los Cocos, $08^{\circ} 32^{\prime} \mathrm{N}, 062^{\circ} 25^{\prime} \mathrm{W}$. Region not specified. ANSP 85366, 1, 138 mm, La Fria, Pueblo Nueva, no coordinates.

\section{Brachyhypopomus bullocki Sullivan \& Hopkins, 2009}

\section{(Fig. 19; Tables 2-5, 8)}

Hypopomus sp. 4. -Heiligenberg \& Bastian, 1980: 197, fig. 1, black and white photograph (Brazil, Amazonas, rio Negro, species and EOD diversity).

Brachyhypopomus bullocki. -Sullivan, 1997: 91 (description in unpublished thesis with disclaimer stating that nomenclatural acts not available, a nomen nudum).

Brachyhypopomus sp. -Hopkins, 1999: 1218, fig. 1, black and white photographs of breeding male and female (electric organ morphology and impedance matching).

Brachyhypopomus sp. J. -Crampton \& Albert, 2006: 672, fig. 23.8, position in phylogenetic tree; 681 notes on EODs (gymnotiform species and EOD diversity).

Brachyhypopomus bullocki Sullivan \& Hopkins, 2009: 185-186, fig. 1, black and white photograph of holotype (breeding male) and breeding male and female paratypes; 191, fig. 3, EODs (original description, type locality - Venezuela, Meta State, río Metica-Meta dr.). -Crampton, 2011: 176, table 10.2, species list; 179, figs. 10.2-10.3, phylogeny, geographical and ecological distributions (gymnotiform biology). -Carvalho, 2013: 181-185, figs. 41-43, position in phylogeny (phylogenetic systematics of Rhamphichthyoidea). -Tagliacollo et al., 2016: 28, fig. 5 (phylogeny of Gymnotiformes). -Crampton et al., 2016: 1-66, table 1, 3-4, figs. 1-7, 18-20 (phylogeny, biogeography and ecology of Brachyhypopomus).

Diagnosis. Brachyhypopomus bullocki is diagnosed from congeners by the following cobination of characters: orbital diameter $14.2-18.5 \%$ HL, vs. 5.9-14.1\% in all congeners except $B$. batesi, $B$. benjamini, $B$. brevirostris, $B$. cunia, $B$. flavipomus, B. hamiltoni, B. hendersoni, B. janeiroensis, $B$. provenzanoi, and $B$. walteri; caudal filament length 31.4$62.9 \%$ LEA, vs. $14.1-30.0 \%$ in B. batesi, B. benjamini, $B$. hamiltoni, and B. provenzanoi; precaudal vertebrae 15-17, vs. 18-21 in B. brevirostris, B. flavipomus, and B. janeiroensis; distinct dark suborbital stripe absent, vs. present in B. cunia and $B$. walteri; interorbital distance $24.0-30.0 \% \mathrm{HL}$, vs. $16.4-20.4 \%$ in $B$. hendersoni.

Description. Head and body shape, and pigmentation illustrated in Fig. 19, and in original description. Meristic and morphometric data for examined specimens presented in Tables 2-5 and 8. Body shallow to moderate in depth. Head short and moderate to broad in depth. Dorsal profile of head convex from occiput to snout, ventral profile of head slightly concave between operculum and snout, snout rounded. Eye large. Upper jaw with moderate to acute sigmoidal angle between premaxillary and maxillary portions in lateral view. No accessory electric organ over operculum. Gill filaments on first gill arch 33-36 (median 34, $\mathrm{n}=6$ ). Pectoral fin moderate to broad in width, pectoral-fin rays 11-15 (mode 13). Precaudal vertebrae 15-17 (mode 16), including 1-2 transitional vertebrae (mode 2$)$. Anal-fin origin slightly $(<$ $0.25 \mathrm{HL}$ distance) anterior or posterior to, or near, tip of pectoral fin. Anal-fin rays 196-233. Dorsal rami of recurrent branch of anterior lateral line nerve visible in most juvenile and some lightly-pigmented adults - mostly in anterior third of body. Middorsal region of body scaled. Rows of scales above lateral line 4-7 (mode 6). Lateral line continuous. Epidermal canals very sparse and in some specimens more or less absent; when present located only in posterior half of body. Discontinuous meandering horizontal scratch-like epidermal canals in dorsal flank, midway between lateral line and dorsal midline, variably present. Some short epidermal canals as short grooves close to and parallel to lateral line, mainly in posterior third of body. Three bilateral horizontal columns of electrocytes at anal-fin terminus, and at a midpoint between anal-fin terminus and tip of caudal filament in immature, mature female, and mature male specimens. Tip of caudal filament free of electrocytes in some specimens. Caudal filament moderate in length to long. 


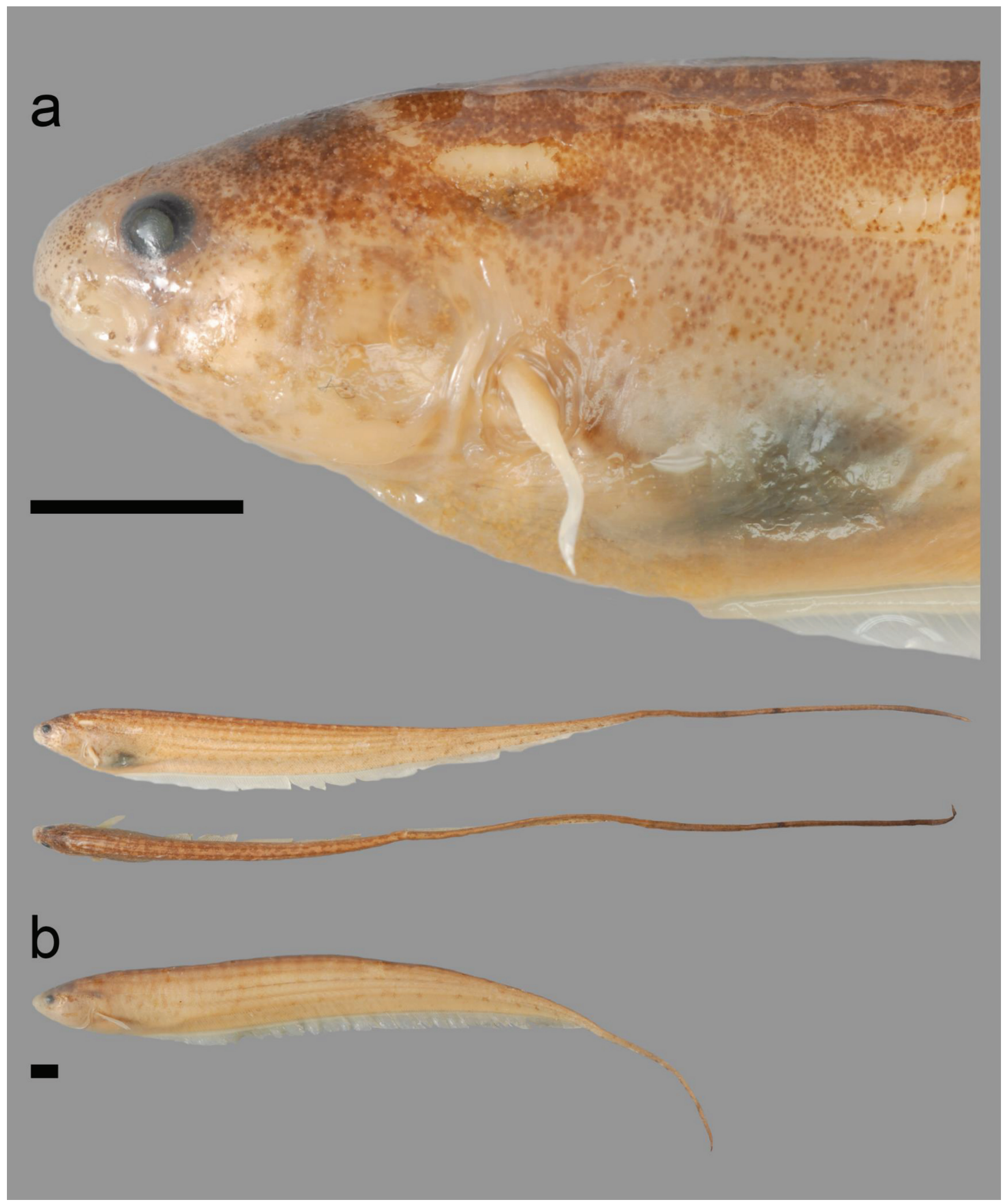

Fig. 19. Brachyhypopomus bullocki. a. MZUSP 96498 , male, $188 \mathrm{~mm}$ TL (head in lateral view and body in lateral and dorsal view); b. TNHC 17486, female, $140 \mathrm{~mm}$ TL (body in lateral view); Venezuela, río Parguaza, Orinoco dr. Specimens fixed in formalin and preserved in EtOH. Scale bars $=5 \mathrm{~mm}$. 
Coloration. (Fig. 19). Background pale straw to dark tan. Dorsal region without prominent depigmented pale stripe extending along midline from occipital region to base of caudal filament. Irregular dark saddles crossing dorsal surface in anterior third of body, extending ventrally as indistinct bands which break up before reaching lateral line. Indistinct bands and irregular patches over dorsal surface in posterior two thirds of body, not forming saddles that cross entire dorsal surface. Banding absent or faint in posterior third of body. Sparse indistinct dark markings over rest of flank. Indistinct series of dark dots form a line along upper margin of anal-fin pterygiophores in posterior third of body. Caudal filament darker with sparse irregular darker bands and dark tip; underwater, tip of caudal filament resembles root tips of water hyacinths. Head with sparse dark chromatophores on cheeks and operculum, darker dorsally. Eye without prominent suborbital patch, or stripe, of chromatophores/subcutaneous pigmentation. Pectoral and anal-fin membranes hyaline. Pectoral-fin rays hyaline with light scattering of brown chromatophores. Anal-fin rays with scattered chromatophores, sometimes fusing to form darker patches. Color in live individuals similar to preserved specimens, with opercular region usually rosy due to underlying gills.

Size. Moderate adult size, largest specimen examined 221 $\mathrm{mm}$ TL, $121 \mathrm{~mm}$ LEA $(\mathrm{n}=363)$. Largest male specimen examined $221 \mathrm{~mm} \mathrm{TL}, 121 \mathrm{~mm}$ LEA $(\mathrm{n}=12)$. Largest female specimen examined $152 \mathrm{~mm}$ TL, $96 \mathrm{~mm}$ LEA ( $\mathrm{n}$ $=11$ ).

Sexual dimorphism. Males attain larger sizes than females. Breeding males develop very elongated caudal filaments in comparison to immature individuals and breeding females, and reach greater lengths (Figs. 19a-b), but do not exhibit an elevated number of bilateral horizontal columns or vertical rows of electrocytes. Instead breeding males exhibit clearly enlarged electrocytes relative to immature specimens and females. Tip of caudal filament in large males often exhibits paddle-like laterally compressed structure. Caudal filament of males often entirely free of electrocytes near tip, with electrocytes sometimes absent in as much as the terminal $25 \%$ of caudal filament. No sexual differences in pigmentation.

Geographic distribution. Brazil, Colombia, Guyana, and Venezuela (Fig. 7). Widespread throughout Orinoco drainage, rio Negro drainage (Amazonas drainage), upper rio Branco (affl. rio Negro), and upper and middle portions of the Essequibo River drainage.

Ecological notes. Sullivan \& Hopkins (2009) report $B$. bullocki from low-conductivity $\left(<25 \mu \mathrm{Scm}^{-1}\right)$ shallow clear water in Mauritia palm swamps and open savanna, and from small pools fed by streams (Orinoco), and from a lowconductivity blackwater stream (rio Negro), but report its absence from high-conductivity sites $\left(>100 \mu \mathrm{Scm}^{-1}\right)$ in the Venezuelan llanos. WGRC and NRL collected $B$. bullocki from low-conductivity streams, Mauritia palm swamps, and savanna gallery forest in the vicinity of San Fernando de Atabapo, Amazonas, Venezuela. Here the following water parameters were recorded at the time of sampling: conductivity 7-13 $\mu \mathrm{Scm}^{-1}$, dissolved oxygen 1.6-3.8 $\mathrm{mgl}^{-1}$, temperature $25.8-28.2^{\circ} \mathrm{C}$, and $\mathrm{pH} 3.1-4.5$. Reproductive biology is unknown. Stomach contents comprise aquatic insect larvae, primarily Chironomidae larvae, and other small aquatic invertebrates (WGRC unpublished data).

Co-occurring congeners: In the Orinoco drainage, we captured $B$. bullocki in ecological syntopy with $B$. brevirostris, $B$. provenzanoi, and $B$. sullivani, and it may also occur in syntopy with $B$. beebei and $B$. regani - both known from low-conductivity systems in the Orinoco. Brachyhypopomus bullocki also occurs in sympatry but ecological allotopy with $B$. diazi, which is restricted to systems with higher conductivity (Sullivan \& Hopkins, 2009). In the rio Negro and Essequibo River drainages, both low-conductivity systems, $B$. bullocki occurs in sympatry and syntopy with $B$. batesi, $B$. beebei, B. brevirostris, $B$. hendersoni, $B$. pinnicaudatus (only near mouth of rio Negro), B. regani, B. sullivani, and $B$. walteri.

Local names. Brazil: sarapó; Colombia and Venezuela: cuchillo.

Material examined. 332 specimens. Brazil. Amazonas (localities from rio Negro dr., Amazonas dr.). CU 94737, 1, stream above mouth rio Branco, rio Branco dr., $c a .01^{\circ} 20^{\prime} \mathrm{S}$, 061 ${ }^{\circ} 54^{\prime} \mathrm{W}$. INPA 19949, 1, $135 \mathrm{~mm}$, INPA 19951, 1, $173 \mathrm{~mm}$, Arquipelago das Anavilhanas, $02^{\circ} 41^{\prime} \mathrm{S}, 060^{\circ} 44^{\prime} \mathrm{W}$. MZUSP 54578, 2 ( 1 immature, $68 \mathrm{~mm}, 1$ female, $117 \mathrm{~mm}$ ), MZUSP 95226 (part), 26 (10 immature, 74-125 mm, 12 female, 98-136 mm, 4 male, 136-148 mm), mun. Santa Isabel do rio Negro, Paricatuba, lake on margin of rio Negro, $00^{\circ} 31^{\prime} \mathrm{S}, 065^{\circ} 01^{\prime} \mathrm{W}$. Roraima (localities from rio Branco dr., affl. rio Negro, Amazonas dr.). INPA 7370, 1, igarapé do Gentil, $34 \mathrm{~km}$ Boa Vista on Boa Vista to Bonfim hwy., $02^{\circ} 58^{\prime} \mathrm{N}, 060^{\circ} 26^{\prime} \mathrm{W}$. INPA 7425, 1, $132 \mathrm{~mm}$, Boa Vista, on rd. to Bonfim, $02^{\circ} 49^{\prime} \mathrm{N}, 060^{\circ} 35^{\prime} \mathrm{W}$. INPA $9829,1,73$ $\mathrm{mm}$, Ilha de Maracá, rio Uraricoera, $03^{\circ} 22^{\prime} \mathrm{N}, 061^{\circ} 23^{\prime} \mathrm{W}$. Colombia (localities from río Meta dr., Orinoco dr.). Meta. ANSP 128100, 2, Hacienda Mozambique, Laguna $\mathrm{N}$ lago Mozambique at outlet to río Metica, affl. río Metica, $03^{\circ} 58^{\prime} \mathrm{N}$, $073^{\circ} 04^{\prime} \mathrm{W}$. ANSP 138024, 17 (paratypes), 150-216 mm, 2 examined from photograph in original description (1 male 215 $\mathrm{mm}$; 1 female, $176 \mathrm{~mm}$ ), ANSP 187477, 1 (holotype), $221 \mathrm{~mm}$, examined from photograph in original description, rancho Llano Grande, N Hacienda Mozambique, affl. río Metica, $04^{\circ} 02^{\prime} \mathrm{N}$, $073^{\circ} 03^{\prime} \mathrm{W}$. AUM 35487, 2, 108-112 mm, 19 km S Puerto Gaitán, Estero $c a .1 \mathrm{~km}$ up caño Pajaro Grande, affl. río Manacacías, $c a$. $04^{\circ} 09 \mathrm{~N}, 072^{\circ} 07^{\prime} \mathrm{W}$. UF $19475,1,114 \mathrm{~mm}$, UF $111998,1,120$ $\mathrm{mm}, c a .10 \mathrm{~km}$ SE Puerto Gaitán, $04^{\circ} 15^{\prime} \mathrm{N}, 072^{\circ} 00^{\prime} \mathrm{W}$. UF 33734 , $1,115 \mathrm{~mm}, c a .15 \mathrm{~km}$ E Puerto Gaitán, $04^{\circ} 18^{\prime} \mathrm{N}, 071^{\circ} 55^{\prime} \mathrm{W}$. UF 148456, 1, $70 \mathrm{~mm}$, UF $148457,1,125 \mathrm{~mm}$, UF $148531,1,85$ 
$\mathrm{mm}, c a .4 .7 \mathrm{~km} \mathrm{~W}$ Puerto Gaitán, affl. río Yucao, $04^{\circ} 18^{\prime} \mathrm{N}$, $072^{\circ} 07^{\prime} \mathrm{W}$. Vichada. IAVH-P uncat. $1,110 \mathrm{~mm}$ (photograph from S. Gavassa, specimen ca. $110 \mathrm{~mm}$ ), mun. Santa Rosalia, El Dera ranch, 46 km Santa Rosalia, small creek, affl. caño Grande, affl. río Tomo, Orinoco dr., 0450'59"N, 070³3'52"W. Vuapés. USNM 320021, 2, 142-146 mm, río Mitu, affl. río Vuapes, affl. rio Negro, Amazonas dr., ca. $01^{\circ} 16^{\prime} \mathrm{N}, 070^{\circ} 13^{\prime} \mathrm{W}$. Guyana. Upper Demerara Berbice. ROM 62548, 1 (of 3), $111 \mathrm{~mm}$, unnamed creek ca. $7 \mathrm{~km}$. SE Tambikabo inlet, Essequibo River, Essequibo River dr., $04^{\circ} 45^{\prime} \mathrm{N}, 058^{\circ} 43^{\prime} \mathrm{W}$. Upper Takutu-Upper Essequibo. AUM 35813, 1, 79 mm, Rupununi River at Massara's Landing, $1.1 \mathrm{~km}$ NE Massara, Essequibo River dr., 0353'41"N, $059^{\circ} 17^{\prime} 37^{\prime \prime} \mathrm{W}$. AUM 50025, 2, 123-133 mm, lake nr. Yukupari, Essequibo River dr., $03^{\circ} 41^{\prime} 38^{\prime \prime} \mathrm{N}, 059^{\circ} 23^{\prime} 40^{\prime \prime} \mathrm{W}$. BMNH 1972.7.27.456-457, 2, 65-95, small ponds in Rupununi River, Essequibo River dr., ca. $02^{\circ} 52^{\prime} \mathrm{N}, \quad 059^{\circ} 32^{\prime} \mathrm{W}$. BMNH 1972.7.27.458-518 (part), 27, 42-100 mm, Karanambo creek, Rupununi River, Essequibo River dr., 03 $45^{\prime} 11^{\prime \prime} \mathrm{N}, 059^{\circ} 18^{\prime} 36^{\prime \prime} \mathrm{W}$. BMNH 1972.7.27.519-525, 1, 110 mm, Rupununi River, Essequibo River dr., no coordinates. CU 71946, 4, to $141 \mathrm{~mm}, 15$ mi. E Lethem, pond nr. Moco-Moco Creek, Takutu River, affl. rio

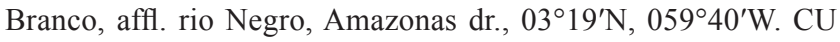
71947, 1, ca. 2 km Lethem, confl. Buru and Arara Creeks, Takutu River, affl. rio Branco, affl. rio Negro, Amazonas dr., $02^{\circ} 23^{\prime} \mathrm{N}$, $059^{\circ} 48^{\prime} \mathrm{W}$. MCZ 48555, 1, male, 149 mm, 5 mi. N Manari Ranch, Takutu River, affl. rio Branco, affl. rio Negro, Amazonas dr., $03^{\circ} 26^{\prime} \mathrm{N}, 059^{\circ} 46^{\prime} \mathrm{W}$. USNM 190552, 2, 93-107 mm, Rupununi, Essequibo River dr., no coordinates. Region not specified. AMNH 35363, 1, 85 mm, Kora-Kora creek, Demerara [Essequibo River dr.?], no coordinates. Venezuela. Amazonas (localities from Orinoco dr.). ANSP 188069, 3, Pozo de Lucas nr. San Fernando de Atabapo, affl. río Orinoco, ca. $03^{\circ} 56^{\prime} \mathrm{N}, 067^{\circ} 36^{\prime} \mathrm{W}$. CU 83825, 13, nr. Puerto Ayacucho, affl. río Orinoco, ca. $05^{\circ} 39^{\prime} \mathrm{N}$, $067^{\circ} 31^{\prime} \mathrm{W}$. CU 83832, 2, nr. San Fernando de Atabapo, affl. río Orinoco, ca. $04^{\circ} 03^{\prime} \mathrm{N}, 067^{\circ} 42^{\prime} \mathrm{W}$. INHS $61558,1,90 \mathrm{~mm}$, caño Pozo Azul, nr. Puerto Ayacucho, affl. río Orinoco, 0545'49"N, 067²9'21"W. MAC-PAY 01237, 2, Laguna Provincial, ca. $20 \mathrm{~km}$ $\mathrm{N}$ Puerto Ayacucho, affl. río Orinoco, ca. $05^{\circ} 50^{\prime} \mathrm{N}, 067^{\circ} 37^{\prime} \mathrm{W}$ (listed in Sullivan \& Hopkins, 2009). MCNG 21515, 4, Maroa, río Guainía, $02^{\circ} 43^{\prime \prime} \mathrm{N}, 067^{\circ} 33^{\prime} \mathrm{W}$. MBUCV-V 15751, 1, female, $152 \mathrm{~mm}$, río Autana, ca. $8 \mathrm{~km}$ confl. with río Sipapo, $04^{\circ} 43^{\prime} \mathrm{N}$, $067^{\circ} 45^{\prime} \mathrm{W}$. UMMZ 228985, 1, $181 \mathrm{~mm}$, creek nr. Puerto Ayacucho, affl. río Orinoco, $c a .05^{\circ} 39^{\prime} \mathrm{N}, 067^{\circ} 37^{\prime} \mathrm{W}$. UF 77949,1 , male, 125 $\mathrm{mm}$, Yavi, río Ventuari, ca. $05^{\circ} 25^{\prime} \mathrm{N}, 066^{\circ} 00^{\prime} \mathrm{W}$. UF 177348,3 , immature (2 CS), 105-183 mm, on rd. from San Fernando de Atabapo to Santa Barbara, affl. río Orinoco, 0358'59"N, 067³8'27"W. UF 177349, 1, female, 132 mm, caño Viejita, on rd. from San Fernando de Atabapo to Santa Barbara, affl. río Orinoco, 035 55'59"N, 067³6'34"W. Anzoátegui. MHNLS 5859, 1, MHNLS 5974, 1, MHNLS 6088, 1, MHNLS 6195, 25, Laguna de Mamo, río Orinoco floodplain, Orinoco dr., ca. $08^{\circ} 25^{\prime} 56^{\prime \prime} \mathrm{N}$, $063^{\circ} 07^{\prime} 10^{\prime \prime} \mathrm{W}$ (listed in Sullivan \& Hopkins, 2009). Apure (localities from Orinoco dr.). ANSP 128106, 1, ca. 13 km N San Fernando de Apure, Camaguán swamp, hwy. to San Fernando de Apure, affl. río Apure, $08^{\circ} 00^{\prime} \mathrm{N}, 067^{\circ} 28^{\prime} \mathrm{W}$. ANSP 165517, 1, female, $112 \mathrm{~mm}$, Morichal along San Fernando de Apure-Puerto
Páez hwy., affl. río Capanaparo, 06 $57^{\prime} \mathrm{N}, 067^{\circ} 28^{\prime} \mathrm{W}$. AUM 22501, 1, 121 mm, Puerto Páez-San Fernando rd., río Claro, affl. río Cunaviche, $07^{\circ} 09^{\prime} 08^{\prime \prime} \mathrm{N}, 067^{\circ} 38^{\prime} 06^{\prime \prime} \mathrm{W}$. CU 94795, 6, CU 94796, 28 (1 CS), CU 94797, 19 (1CS), CU 94798, 4, 10 km. S Santa Juana in Parque Nacional (PN) los Santos Luzardo, affl. río Capanaparo, $06^{\circ} 55^{\prime} \mathrm{N}, 067^{\circ} 27^{\prime} \mathrm{W}$. CU 94799, 1, Caño La Pica, 12 km S Santa Juana, in PN los Santos Luzardo, affl. río Capanaparo, $06^{\circ} 54^{\prime} \mathrm{N}, 067^{\circ} 30^{\prime} \mathrm{W}$. CU 94800 , 8, blackwater lake E of rd. S from Santa Juana, affl. río Capanaparo, $06^{\circ} 56^{\prime} \mathrm{N}, 067^{\circ} 25^{\prime} \mathrm{W}$. INHS 27703, 2 (paratypes), 102-141 mm, on San Fernando de ApurePuerto Páez hwy., río Cinaruco, 06³3'06"N, 067³0'43"W. MBUCV-V 19847, 5 (3 measured: 1 female, $133 \mathrm{~mm}, 2$ male, 141-152 mm), río Capanaparo, no coordinates. MBUCV-V 21369, 1, female, 130 mm, La Montañita, on San Fernando de Apure-Puerto Páez hwy., río Claro, affl. río Cunaviche, $07^{\circ} 09^{\prime} \mathrm{N}$, $67^{\circ} 38^{\prime} \mathrm{W}$. MCNG 2273, 1, MCNG 4842, 3, MCNG 4451, 3, MCNG 10261, 1, MCNG 10754, 1, MCNG 11525, 6, MCNG 28643, 2, TNHC 11833, 1, 115 mm, TNHC 11850, 6, 104-127 mm, TNHC 17535, 1, 108 mm, caño Maporal, nr. módulo de UNELLEZ, río Caicara, affl. río Apure, ca. $07^{\circ} 24^{\prime} \mathrm{N}, 069^{\circ} 32^{\prime} \mathrm{W}$. TNHC 17486, 3 (1 immature, $135 \mathrm{~mm}, 1$ female, $140 \mathrm{~mm}, 1$ male, $179 \mathrm{~mm}$ ), río Caicara, affl. río Apure, $07^{\circ} 24^{\prime} \mathrm{N}, 069^{\circ} 32^{\prime} \mathrm{W}$. UMMZ 217073, 1, $109 \mathrm{~mm}, c a .20 \mathrm{~km} \mathrm{~N}$ Paso de Caballo, río Guariquito, affl. río Apure, $08^{\circ} 36^{\prime} \mathrm{N}, 067^{\circ} 08^{\prime} \mathrm{W}$. USNM 260261 , 2, caño Caicara, at bridge rd. from Montecal, affl. río Apure, $07^{\circ} 22^{\prime} \mathrm{N}, 069^{\circ} 21^{\prime} \mathrm{W}$. Bolívar (localities from Orinoco dr.). ANSP 187476, 3, 93-120 mm, Morichal Zamori on Maripa-Ciudad Bolivar hwy, affl. río Orinoco, $07^{\circ} 28^{\prime} \mathrm{N}, 064^{\circ} 54^{\prime} \mathrm{W}$. ANSP $189185,1,06^{\circ} 57^{\prime} \mathrm{N}, 064^{\circ} 50^{\prime} \mathrm{W}, \mathrm{MBUCV}-\mathrm{V} 24065,4,2$ measured, female, 115-122 mm, Morichal de los Pavones, affl. río Sipapo, affl. río Caura, $07^{\circ} 35^{\prime} \mathrm{N}, 065^{\circ} 25^{\prime} \mathrm{W}$. MHNLS 4897, 1, Laguna Chirere, ca. $30 \mathrm{~km} \mathrm{~W}$ Puerto Ordaz, río Orinoco floodplain, $c a$. $08^{\circ} 17^{\prime} \mathrm{N}, 062^{\circ} 59^{\prime} \mathrm{W}$ (listed in Sullivan \& Hopkins, 2009). MZUSP 96498, 1, male, 189 mm, mun. Cedeño, río Parguaza, Puente Parhuena, affl. río Orinoco, $05^{\circ} 53^{\prime} 30^{\prime \prime} \mathrm{N}, 067^{\circ} 24^{\prime} 14^{\prime \prime} \mathrm{W}$. SU 54496, 2, 97-130 mm, SU 69738, 2, 114-126 mm, Caicara, caño de Quiribana, affl. río Orinoco, ca. $07^{\circ} 32^{\prime} \mathrm{N}, 066^{\circ} 08^{\prime} \mathrm{W}$. Delta Amacuro (localities from río Orinoco delta, Orinoco dr.). CU 94867, 2, 74-88 mm, Caño Anabeta, Isla Portuguesa, $08^{\circ} 37^{\prime} \mathrm{N}$, $061^{\circ} 45^{\prime} \mathrm{W}$. MBUCV-V 10660, 1, female, $94 \mathrm{~mm}$, Los Castillos, ca. $08^{\circ} 40^{\prime} \mathrm{N}, 062^{\circ} 24^{\prime} \mathrm{W}$. Guárico (localities from Orinoco dr.). INHS 34036, 2, 116-118 mm, 20 km ENE La Esperanza, Parque Nacional Aguaro-Guariquito, Morichal Charcote, affl. río Manapire, $08^{\circ} 24^{\prime} 16^{\prime \prime} \mathrm{N}, 066^{\circ} 34^{\prime} 23^{\prime \prime} \mathrm{W}$. INHS 34586, 1, $155 \mathrm{~mm}$, $\mathrm{N}$ Medano de Gomez, río Guariquito, 07 $50^{\prime} 41^{\prime \prime} \mathrm{N}, 066^{\circ} 32^{\prime} 10^{\prime \prime} \mathrm{W}$. MBUCV-V 8992, 1, 115 mm, caño Caracol, on San Fernando de Apure-Guayabal rd., affl. río Guárico, affl. río Portuguesa, affl. río Apure, $07^{\circ} 58^{\prime} \mathrm{N}, 067^{\circ} 23^{\prime} \mathrm{W}$. MBUCV-V 12047, 1, immature, $114 \mathrm{~mm}$, caño Realito, affl. río Guariquito, $c a .07^{\circ} 49^{\prime} \mathrm{N}, 066^{\circ} 31^{\prime} \mathrm{W}$. MBUCV-V 12068, 8 (7 immature, 78-100 mm, 1 male, 118 mm), Cabecera del río Guariquito, no coordinates. MHNLS 2021, 1, lagoon $30 \mathrm{~km} \mathrm{~N}$ Cabruta, $07^{\circ} 54^{\prime} \mathrm{N}, 066^{\circ} 15^{\prime} \mathrm{W}$ (listed in Sullivan \& Hopkins, 2009). Monagas. MBUCV-V 1727, 1, $90 \mathrm{~mm}$, MBUCV-V 14042, 1, 110 mm, USNM 233321, 1, 108 mm, Laguna Guatero, nr. Barrancas, affl. río Orinoco, Orinoco dr., $08^{\circ} 43^{\prime} \mathrm{N}, 062^{\circ} 11^{\prime} \mathrm{W}$. MHNLS 6586, 20, Laguna San José, 
Coloradito, río Orinoco delta, Orinoco dr., ca. $08^{\circ} 45^{\prime} \mathrm{N}, 063^{\circ} 29^{\prime} \mathrm{W}$ (listed in Sullivan \& Hopkins, 2009). Portuguesa. TNHC 14893, 4, 98-134 mm, 35 km SE Guanare, caño (río) Maraca at Urriola Ranch, affl. río Portuguesa, affl. río Apure, Orinoco dr., $08^{\circ} 52^{\prime} 30^{\prime \prime} \mathrm{N}, 069^{\circ} 27^{\prime} 40^{\prime \prime} \mathrm{W}$.

\section{Brachyhypopomus cunia, new species}

urn:1sid:zoobank.org:act:E9E6020F-21D4-42B5-8F2D0F1A1D4E8645

\section{(Fig. 20; Tables 2-5, 8)}

Brachyhypopomus sp. 3. -Crampton \& Ribeiro, 2013: 240, color photograph (Brazil, rio Madeira, listing of hypopomids). -de Queiroz et al., 2013: 545, table 1 (Brazil, rio Madeira, lago Cuniã, listing of fish species).

Brachyhypopomus sp. "cunia". -Crampton et al., 2016: 1-66, table 1, 3-4, figs. 1-7, 18-20 (phylogeny, biogeography and ecology of Brachyhypopomus).
Holotype. INPA 37690, male, 142 mm TL, 104 mm LEA, Brazil, Rondônia, Reserva Extrativista do lago do Cuniã, igarapé do Campo, affl. lago Cuniã, rio Madeira floodplain, Amazonas dr., 08 $19^{\prime} 14^{\prime \prime} \mathrm{S}, 063^{\circ} 28^{\prime} 05^{\prime \prime} \mathrm{W}, 1$ Dec 2008, T. Araújo \& F. Vieira.

Paratypes. 40 specimens, localities from rio Madeira dr., Amazonas dr. Brazil. Rondônia. INPA 37691, 4 (1 immature, $124 \mathrm{~mm}, 3$ female [1 CS], 113-121 mm), MPEG 24297, 10, 99-137 mm, UFRO-I 1323, 3, 80-125 mm, collected with holotype. MCP 46939, 10 (7 immature, 87149 mm, 3 female, 126-136 mm), MZUSP 110994, 5 (1 immature, $90 \mathrm{~mm}, 2$ female, 114-141 mm, 1 male, $117 \mathrm{~mm}$ ), UFRO-I 6398, 1, female, 116 mm, UFRO-I 6399, 1, female, 119 mm, UFRO-I 6400, 1, immature, $112 \mathrm{~mm}$, UFRO-I 6401, 1, immature, $128 \mathrm{~mm}$, UFRO-I 6402, 1, immature, 144 mm, UFRO-I 7928, 4 (2 immature, 107-139 mm, 1 female, $118 \mathrm{~mm}, 1$ male, $154 \mathrm{~mm}$ ), mun. Porto Velho, lago Cuniã, rio Madeira floodplain, $08^{\circ} 19^{\prime} \mathrm{S}, 063^{\circ} 28^{\prime} \mathrm{W}, 6$ Jun 2010, T. Araújo \& F. Vieira.

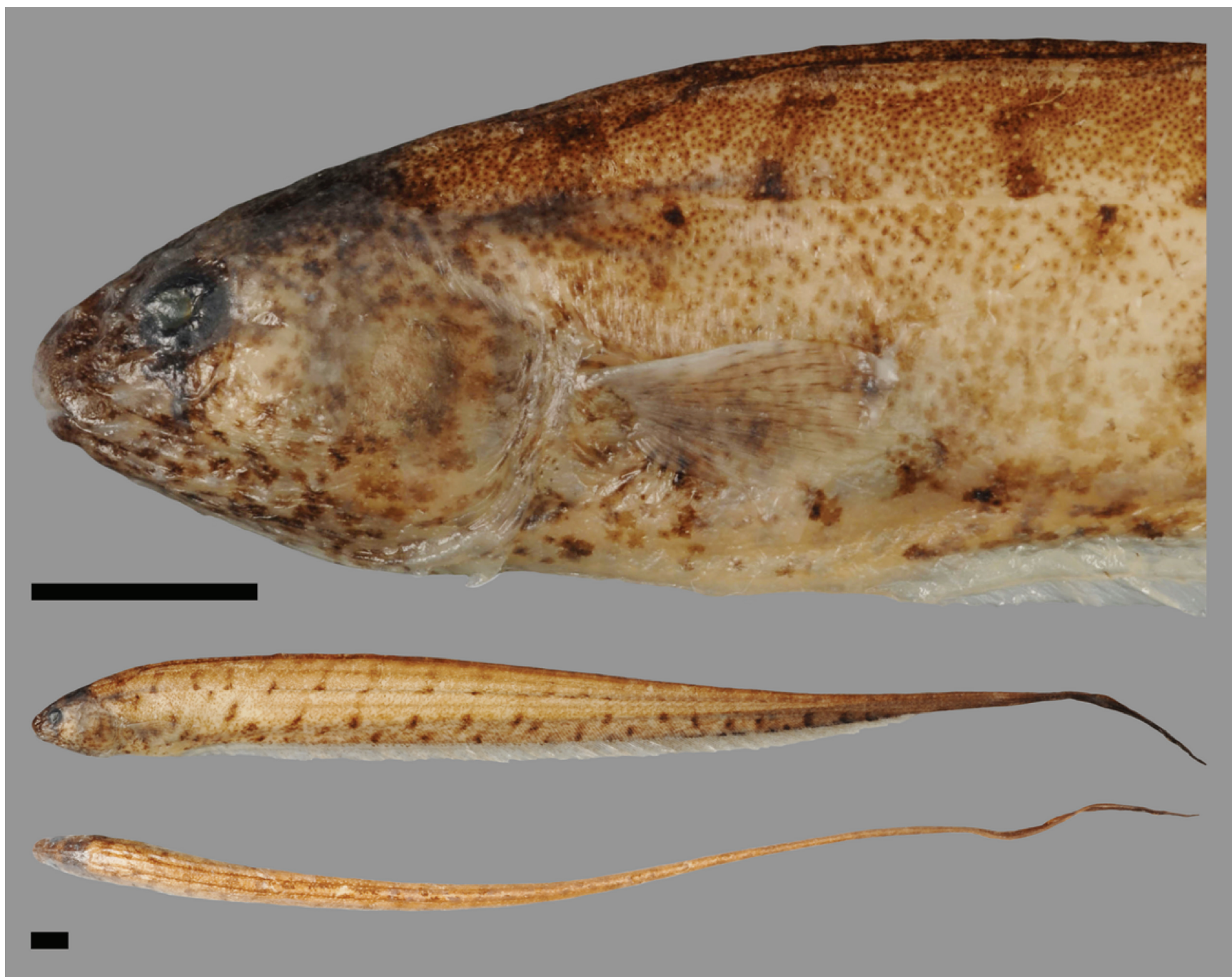

Fig. 20. Brachyhypopomus cunia. INPA 37690, holotype, male, $142 \mathrm{~mm}$ TL (head in lateral view and body in lateral and dorsal view); Brazil, rio Madeira, Amazonas dr. Specimen fixed in formalin and preserved in EtOH. Scale bars $=5 \mathrm{~mm}$. 
Non-types. 216 specimens, localities from rio Madeira dr., Amazonas dr. Brazil. Mato Grosso. FMNH 54552, 7, 48$112 \mathrm{~mm}$, Bastos [Porto Bastos], rio Alegre, affl. rio Guaporé, affl. rio Mamoré, ca. $15^{\circ} 06^{\prime} \mathrm{S}, 059^{\circ} 57^{\prime} \mathrm{W}$. USNM 301979, 3 , 83-135 mm, Brazil-Bolivia border, region between GuajaráMirim and Mato Grosso, rio Guaporé, affl. rio Mamoré, $c a$. $13^{\circ} 42^{\prime} \mathrm{S}, 060^{\circ} 31^{\prime} \mathrm{W}$. Rondônia. MCP 46937, 3 (2 CS), 92$118 \mathrm{~mm}$, mun. Calamã, nr. Calamã, rio Madeira floodplain lake, $08^{\circ} 01^{\prime} 37^{\prime \prime} \mathrm{S}, 062^{\circ} 52^{\prime} 28^{\prime \prime} \mathrm{W}$. UFRO-I 6467, 4,75-95 $\mathrm{mm}$, Colocação Três Praias, rio Jaciparaná, $09^{\circ} 27^{\prime 2} 29^{\prime \prime} \mathrm{S}$, $064^{\circ} 25^{\prime} 22^{\prime \prime} \mathrm{W}$. UFRO-I 6470, 14, 95-146 mm, lago Cuniã, $08^{\circ} 21^{\prime} 10^{\prime \prime} \mathrm{S}, 063^{\circ} 30^{\prime} 26^{\prime \prime} \mathrm{W}$. UFRO-I 6471, 2, 130-147 mm, lago Cuniã, $08^{\circ} 19^{\prime} 45^{\prime \prime} \mathrm{S}, 063^{\circ} 28^{\prime} 06^{\prime \prime} \mathrm{W}$. UFRO-I 6474, 14, 128-176 mm, UFRO-I 6475, 2, 143-148 mm, lago Cuniã, $08^{\circ} 19^{\prime} 36^{\prime \prime} \mathrm{S}, 063^{\circ} 29^{\prime} 59^{\prime \prime} \mathrm{W}$. UFRO-I 6477, 25, 147-165 mm, mouth of rio Cautário, affl. rio Guaporé, affl. rio Mamoré, $12^{\circ} 10^{\prime} 51^{\prime \prime} \mathrm{S}, 064^{\circ} 34^{\prime} 00^{\prime \prime} \mathrm{W}$. UFRO-I 6479, 74, 80-152 mm, nr. igarapé do Campo, lago Cuniã, $08^{\circ} 19^{\prime} 09^{\prime \prime} \mathrm{S}, 063^{\circ} 28^{\prime} 44^{\prime \prime} \mathrm{W}$. UFRO-I 6514, 68, 67-150 mm, nr. ICMBio base, lago Cuniã, $08^{\circ} 19^{\prime} 40^{\prime \prime} \mathrm{S}, 063^{\circ} 30^{\prime} 11^{\prime \prime} \mathrm{W}$.

Diagnosis. Brachyhypopomus cunia is diagnosed from congeners by the following combination of characters: precaudal vertebrae 16-17, vs. 18-26 in B. alberti, B. arrayae, B. beebei, B. belindae, B. brevirostris, B. draco, B. flavipomus, $B$. gauderio, B. hamiltoni, B. janeiroensis, B. jureiae, B. palenque, $B$. pinnicaudatus, and $B$. verdii; head depth at eye 47.8-60.0\% HL, vs. $36.3-47.7 \%$ in B. batesi, B. bombilla, $B$. provenzanoi, $B$. regani, and $B$. sullivani; scales present in middorsal region of anterior third of body, vs. absent in $B$. benjamini; pectoral-fin rays 10-13, vs. 14-20 in B. bennetti, B. diazi, B. menezesi, and B. occidentalis; mouth width 24.8$38.0 \%$ HL, vs. $15.7-22.6 \%$ in B. hendersoni. Brachyhypopomus cunia exhibits overlapping meristic counts and morphological measurements with $B$. bullocki and $B$. walteri, but differs in possessing a very distinctive pigmentation pattern in which the ventral portions of the flank, over the anal-fin pterygiophores, are darkly pigmented, forming a dark stripe along the base of the anal fin (also shared with $B$. hendersoni), and in possessing numerous discrete dark spots (each comprising a cluster of dark chromatophores) which are scattered over the anal-fin pterygiophores, and in some specimens form an irregular dotted line along the lateral line, vs. absence of dark stripe along the anal-fin base and absence of discrete dark spots over the ventral flank in B. bullocki and B. walteri.

Description. Head and body shape, and pigmentation illustrated in Fig. 20. Meristic and morphometric data for examined specimens presented in Tables 2-5 and 8. Body shallow to moderate in depth. Head short and moderate to broad in depth. Dorsal profile of head slightly to moderately convex from occiput to snout, ventral profile of head approximately straight to slightly convex between operculum and snout, snout rounded. Eye moderate to large in size. Upper jaw with moderate sigmoidal angle between premaxillary and maxillary portions in lateral view. No accessory electric organ over operculum. Pectoral fin narrow to moderate in width, pectoralfin rays 10-13 (mode 12). Precaudal vertebrae 16-17 (mode 16), including 1-2 transitional vertebrae (mode 2). Anal-fin origin slightly $(<0.25 \mathrm{HL}$ distance) anterior or posterior to, or near, tip of pectoral fin. Anal-fin rays 177-224 (median 209). Dorsal rami of recurrent branch of anterior lateral line nerve visible in anterior third of body in most specimens. Middorsal region of body scaled. Rows of scales above lateral line 5-7 (mode 6). Lateral line continuous. Sparse depigmented epidermal canals in posterior two thirds of body; present as a meandering and bifurcating single groove in dorsal flank, approximately midway from lateral line to dorsal midline; and as sparse scratch-like marks dorsal to and near lateral line, sometimes connecting to a longer canal in dorsal flank. Three columns at anal-fin terminus and at mid-point between anal-fin terminus and tip of caudal filament in immature, mature female, and mature male specimens. Caudal filament moderate in length to long.

Coloration. (Fig. 20). Background tan to light brown. Dorsal region without prominent depigmented pale stripe extending along midline from occipital region to base of caudal filament. Dorsal surface with irregular patches and vague thin bands; few or none crossing entire dorsal surface, some forming faint oblique bands from dorsal surface to near lateral line which slant in an anteroventral to dorsoposterior direction. No bands on ventral surfaces of flank. Prominent dark flecks scattered over flank and anal-fin pterygiophores, and forming an irregular dotted line along lateral line. Ventral flank over anal-fin pterygiophores, especially in ventral half along anal-fin base with darker background pigmentation forming a dark horizontal band along anal-fin margin (more conspicuous in some specimens than others). Caudal filament darker than body, especially distally; darkened tip of caudal filament resembles root tips of water hyacinths when viewed underwater. Eye always with distinct suborbital patch or stripe of chromatophores and subcutaneous pigmentation. Pectoral and anal-fin membranes hyaline. Pectoral-fin rays and anal-fin rays with light scattering of brown chromatophores. Color in live individuals similar to preserved specimens, with opercular region usually rosy due to underlying gills.

Size. Moderate size, largest specimen examined $176 \mathrm{~mm}$ TL, $121 \mathrm{~mm} \mathrm{LEA}(\mathrm{n}=257)$. Largest male specimen examined $154 \mathrm{~mm}$ TL, $103 \mathrm{~mm}$ LEA $(\mathrm{n}=3)$. Largest female specimen examined $141 \mathrm{~mm}$ TL, $102 \mathrm{~mm}$ LEA $(\mathrm{n}=11)$.

Sexual dimorphism. No known secondary sexual dimorphism.

Geographic distribution. Brazil (Fig. 14). Known only from the rio Madeira; from its lower reaches below its major series of falls and rapids as far downstream as a site near the town of Calamã, Rondônia, Brazil (near the mouth of the rio Machado), and from sites above the major series of falls, along most of the length of the rio Guaporé. Not known from lower reaches of the Madeira downstream of its confluence with the rio Machado. 
Ecological notes. Known from low-conductivity floodplain habitats. The type series was collected from floating macrophytes on the margins of lago Cuniã, a low-conductivity seasonally flooded blackwater lake near the main stem of the lower Madeira. Here the flood season occurs between October and March, and the low-water season from April to September (de Queiroz et al., 2013). Water parameters collected from November 2008 to March 2010 are typical of a lowland blackwater lake: conductivity 2.3-11.2 $\mu \mathrm{Scm}-$ ${ }^{1}$, transparency measured with a Secchi disk $0.35-2.69 \mathrm{~m}$, dissolved oxygen $2.2-5.8 \mathrm{mgl}^{-1}$, temperature $27.1-31.3^{\circ} \mathrm{C}$, and pH 4.52-6.88 (de Queiroz et al., 2013). Brachyhypopomus cunia is also common from low-conductivity clearwater (and some blackwater) floodplain sites along most of the length of the rio Guaporé. Mean water parameters, averaged from multiple sites along the rio Guaporé, and over several seasons, include: conductivity $19.5 \mu \mathrm{Scm}^{-1}$ (SD 16.79), transparency with Secchi disk $1.04 \mathrm{~m}$ (SD 0.44), dissolved oxygen 4.22 $\mathrm{mgl}^{-1}$ (SD 1.98), temperature $27.5^{\circ} \mathrm{C}$ (SD 2.44), and $\mathrm{pH} 7.12$ (SD 1.06) (L. de Queiroz, unpublished data). Reproductive biology is unknown. Stomach contents of specimens from the type locality comprise aquatic insect larvae and other small aquatic invertebrates (WGRC unpublished data).

Co-occurring congeners: Above the major series of rapids and falls in the lower Madeira, Brachyhypopomus cunia co-occurs in geographical sympatry with the following congeners: B. alberti, B. arrayae, B. bombilla, B. brevirostris, $B$. pinnicaudatus, $B$. sullivani, and $B$. walteri. Below the lower Madeira rapids, Brachyhypopomus cunia is known to occur in sympatry and ecological syntopy with $B$. brevirostris, B. pinnicaudatus, and B. walteri (de Queiroz et al., 2013).

Etymology. The specific name refers to the type locality, lago Cuniã, a floodplain lake of the lower rio Madeira inside the Reserva Extrativista do lago do Cuniã. A noun in apposition.

Local names. Brazil: sarapó.

\section{Brachyhypopomus diazi (Fernández-Yépez, 1972)}

(Fig. 21; Tables 2-5, 11)

Hypopomus diazi Fernández-Yépez, 1972: 20, pl. 20, black and white photograph of holotype (original description, type locality - Venezuela, Carabobo, río Alpargatón).

Brachyhypopomus occidentalis. -Mago-Leccia, 1976 (systematics of Gymnotiformes, $B$. diazi listed as junior synonym of $B$. occidentalis).

Brachyhypopomus diazi. -Mago-Leccia, 1994: 48, fig. 72, black and white photograph of MBUCV-V 13443 (listing of Brachyhypopomus). -Sullivan, 1997: 137 (redescription). -Albert \& Crampton, 2003: 495 (Venezuela, listing of Brachyhypopomus). -Crampton \& Albert, 2006: 672, fig. 23.8, position in phylogenetic tree; 681, notes on EODs (gymnotiform species and EOD diversity). -Crampton, 2011: 176, table 10.2, species list; 179, figs. 10.2-10.3, phylogeny, geographical and ecological distributions (gymnotiform biology). -Carvalho, 2013: 181-185, figs. 41-43, position in phylogeny (phylogenetic systematics of Rhamphichthyoidea). -Tagliacollo et al., 2016: 28, fig. 5 (phylogeny of Gymnotiformes). -Crampton et al., 2016: 1-66, table 1, 3-4, figs. 1-7, 11, 18-20 (phylogeny, biogeography and ecology of Brachyhypopomus).

Diagnosis. Brachyhypopomus diazi is diagnosed from congeners by the following combination of characters: bilateral columns at anal-fin terminus $4-5, v s .3$ in all congeners except $B$. belindae, $B$. janeiroensis, $B$. jureiae, $B$. occidentalis, and B. palenque, and vs. 6 columns in $B$. bennetti; pale stripe along middorsal region of body absent, precaudal vertebrae 16-19, vs. 20-26 in B. belindae and $B$. jureiae; dorsal rami of the recurrent branch of the anterior lateral line nerve not visible, vs. visible in B. janeiroensis; continuous or discontinuous dark vertical or diagonally oriented stripes or saddles present on body surface dorsal to lateral line, $v s$. mottled pattern with no stripes in B. palenque (and preopercular sensory canals incised in preopercle, vs. preopercular sensory canals independent of preopercle in B. palenque). Brachyhypopomus diazi exhibits similar pigmentation and overlapping counts and morphometric proportions with $B$. occidentalis. The two species can be distinguished by a more extensive network of depigmented and dark-margin epidermal canals in $B$. diazi (including in the anterior half of the body) than in B. occidentalis, and by the first branchiostegal ray distinctly narrower than the third branchiostegal ray, $v s$. the first branchiostegal ray is approximately as wide as the third in B. occidentalis.

Description. Head and body shape, and pigmentation illustrated in Fig. 21, and in the original description. Meristic and morphometric data for examined specimens presented in Tables 2-5 and 11. Body moderate in depth. Head moderate in length and shallow to broad in depth. Dorsal profile of head slightly convex from occiput to snout, ventral profile of head approximately straight to slightly concave between operculum and snout, snout rounded. Eye moderate in size. Upper jaw with moderate to acute sigmoidal angle between premaxillary and maxillary portions in lateral view. No accessory electric organ over operculum. Gill filaments on first gill arch 37-57 (median 47, $\mathrm{n}=8$ ). Pectoral fin moderate to broad in width, pectoral-fin rays 15-20 (mode of 17 in a population in the río Alpargatón on the Caribbean coast of $\mathrm{N}$ Venezuela, and 16 in populations from the río Apure of the Venezuelan Llanos). Precaudal vertebrae 1619 (mode of 18 from the río Alpargatón, and 17 from the río Apure), including 2-3 (mode 2) transitional vertebrae. Anal-fin origin slightly $(<0.25 \mathrm{HL}$ distance $)$ anterior to, or near, tip of pectoral fin. Anal-fin rays 190-226 (median of 209 from the río Alpargatón and 206 from the río Apure). Dorsal rami of recurrent branch of anterior lateral line nerve not visible. Middorsal region of body scaled. Rows of scales above lateral line 5-7 (mode 6). Lateral line continuous. Extensive network of prominent depigmented epidermal 
canals along much of body length; from approximately one head length posterior to nape, to near anal-fin terminus. In anterior half of body canals occur in low density in upper portions of flank, and curve gently in a shallow anterodorsal to posteroventral slope. In second half of body canals occur as multiple parallel wavy lines in two series: one dorsal to lateral line, and another in upper dorsal flank. A lower density of parallel epidermal canals branch from lateral line over body cavity and extend about half a head length ventrally, in an oblique anterodorsal to posteroventral direction. Region below lateral line completely or almost completely free of epidermal canals in posterior half of body (contrasting with many other congeners in which epidermal canals are found parallel to lateral line on both dorsal and ventral side). 4-5 (mode 4) bilateral horizontal columns of electrocytes at analfin terminus in immature specimens, mature females, and mature males. 4-6 (mode 5) columns at mid-point between anal-fin terminus and tip of caudal filament in immature specimens and mature females and 5-6 (mode 5) in mature males. Caudal filament short to moderate in length.

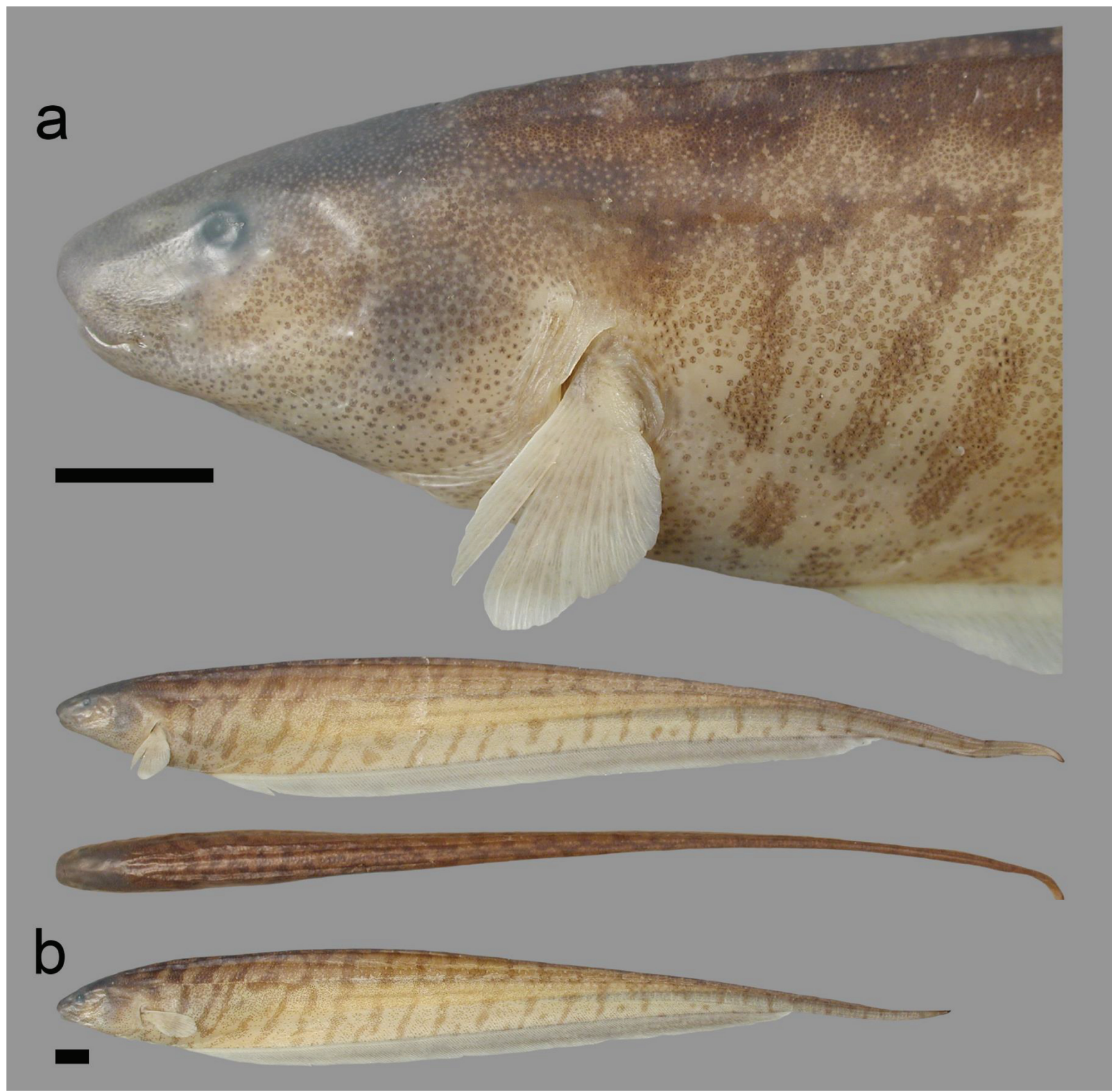

Fig. 21. Brachyhypopomus diazi. a. MBUCV-V 35655 (WC04.210304), male from type locality, $160 \mathrm{~mm}$ TL (head in lateral view, body in lateral and dorsal view); b. UF 174333 (WC03.210304), female from type locality, $141 \mathrm{~mm}$ TL (body in lateral view); Venezuela, río Alpargatón, río Salado dr. Note sexual dimorphism in caudal filament height. Specimens fixed in formalin and preserved in EtOH. Scale bars $=5 \mathrm{~mm}$. 
Table 11. Morphometrics for Brachyhypopomus diazi. SD, standard deviation. Ranges for the type region (río Alpargatón drainage, Venezuela) and for the Orinoco drainage, Venezuela, refer to non-type specimens. No data are available for the (lost) holotype from the original description.

\begin{tabular}{|c|c|c|c|c|c|c|c|c|}
\hline & \multicolumn{4}{|c|}{ Alpargaton drainage (type region) } & \multicolumn{4}{|c|}{ Orinoco drainage } \\
\hline & Range & Mean & SD & $\mathrm{n}$ & Range & Mean & $\mathrm{SD}$ & $\mathrm{n}$ \\
\hline Total length (TL) (mm) & $140-179$ & 162 & 17.3 & 6 & $74-154$ & 124 & 20.7 & 21 \\
\hline Length to end of anal fin (LEA) (mm) & 114-161 & 136 & 17.2 & 7 & $61-131$ & 100 & 18.8 & 22 \\
\hline \multicolumn{9}{|c|}{ Proportion of TL (\%) } \\
\hline Caudal filament length (CF) & 18.6-19.9 & 19.0 & 0.5 & 5 & $12.1-26.6$ & 19.5 & 3.8 & 21 \\
\hline \multicolumn{9}{|c|}{ Proportion of LEA (\%) } \\
\hline Snout to occiput (SO) & $8.7-9.5$ & 9.0 & 0.3 & 6 & $8.6-11.4$ & 9.8 & 0.6 & 22 \\
\hline Snout to anal-fin origin (SA) & $17.6-19.5$ & 18.4 & 0.7 & 7 & $18.4-21.4$ & 19.7 & 0.9 & 22 \\
\hline Snout to pectoral-fin base (PP) & $12.0-13.1$ & 12.3 & 0.4 & 7 & $12.2-15.7$ & 13.5 & 0.9 & 22 \\
\hline Longest pectoral-fin ray (PRL) & $5.2-5.9$ & 5.6 & 0.2 & 7 & 4.7-7.2 & 5.5 & 0.6 & 22 \\
\hline Anal fin length $(\mathrm{AF})$ & $81.5-83.5$ & 82.3 & 0.8 & 7 & $77.0-82.7$ & 80.6 & 1.4 & 22 \\
\hline Longest anal-fin ray (ARL) & $3.9-5.4$ & 4.7 & 0.5 & 7 & $4.0-5.7$ & 4.6 & 0.4 & 22 \\
\hline Body width at anal-fin middle (BW2) & $2.3-3.4$ & 2.9 & 0.4 & 7 & 2.3-3.9 & 3.0 & 0.5 & 22 \\
\hline Caudal filament length (CF) & $22.8-24.6$ & 23.4 & 0.8 & 6 & $13.7-36.2$ & 24.4 & 5.8 & 21 \\
\hline \multicolumn{9}{|c|}{ Proportion of HL (\%) } \\
\hline Preorbital distance (PR) & $23.2-29.5$ & 27.3 & 1.9 & 9 & $22.5-29.0$ & 25.8 & 1.6 & 22 \\
\hline Mouth width (MW) & $24.8-34.4$ & 30.8 & 3.1 & 9 & $23.9-34.4$ & 28.6 & 2.6 & 22 \\
\hline Internarial distance (AE) & $18.7-22.8$ & 20.8 & 1.2 & 9 & $18.8-26.3$ & 20.9 & 1.9 & 22 \\
\hline Posterior naris to eye (PE) & $6.7-8.1$ & 7.5 & 0.5 & 9 & $4.7-8.5$ & 6.9 & 1.0 & 22 \\
\hline Orbital diameter (OD) & $9.6-11.5$ & 10.7 & 0.7 & 9 & $10.2-13.1$ & 11.5 & 0.9 & 22 \\
\hline Interorbital distance (IO) & $25.7-33.6$ & 29.8 & 2.7 & 9 & $23.3-34.8$ & 28.9 & 2.9 & 22 \\
\hline Head depth at eye (HD1) & $47.3-50.5$ & 49.0 & 1.0 & 9 & $48.2-57.4$ & 51.1 & 2.4 & 22 \\
\hline Head width at eye (HW1) & $38.0-47.6$ & 45.1 & 2.9 & 9 & $36.8-54.1$ & 44.6 & 4.6 & 21 \\
\hline \multicolumn{9}{|c|}{ Other proportions } \\
\hline BW1 (\% of BD1) & $50.0-56.3$ & 52.5 & 2.2 & 11 & $47.9-62.6$ & 54.1 & 3.9 & 22 \\
\hline $\mathrm{BW} 2(\%$ of $\mathrm{BD} 2)$ & $23.1-35.2$ & 27.7 & 4.2 & 11 & $23.1-33.2$ & 28.1 & 3.2 & 22 \\
\hline
\end{tabular}

Coloration. (Fig. 21). Background tan to dark brown. Dorsal region without prominent depigmented pale stripe extending along midline from occipital region to base of caudal filament. Prominent narrow brown saddles cross much of dorsal surface in anterior third of body. Dorsal region of posterior portion of body is marked with irregular dark patches, which sometimes form an alternating series of blotches either side of dorsal midline. Thin vertical or anteroventral to posterodorsally oriented brown bands extending from dorsal surface. In anterior third of body bands extend across lateral line and across ventral portion of body, often with a misalignment in their continuation across lateral line. In posterior two thirds of body bands usually reach only to lateral line, where they either peter out or fuse to horizontally-oriented dark lines near lateral line. Thin dark lines or vertically elongated dark patches extend from anal-fin margin to dorsal margin of analfin pterygiophores in posterior two thirds of body. Wide pale patch with no prominent dark bands or spots extending from about third body length behind head to about one head length anterior to anal-fin terminus in many specimens. Caudal filament darker than body, especially dorsally, ventrally, and near tip; with irregular bands along entire length. Head with evenly scattered dark chromatophores, darker dorsally. Eye without prominent suborbital patch, or stripe, of chromatophores/subcutaneous pigmentation. Pectoral and anal-fin membranes hyaline. Pectoral-fin rays hyaline. Analfin rays with uniform light scattering of chromatophores, which fade at distal fin margin, fin rays darker in posterior half. Color in live individuals similar to preserved specimens, with opercular region usually rosy due to underlying gills. 
Size. Moderate adult size, largest specimen examined 193 $\mathrm{mm}$ TL, $184 \mathrm{~mm}$ LEA $(\mathrm{n}=241)$. Largest male specimen examined $193 \mathrm{~mm}$ TL, $184 \mathrm{~mm}$ LEA $(\mathrm{n}=15)$. Largest female specimen examined $143 \mathrm{~mm}$ TL, $115 \mathrm{~mm}$ LEA $(\mathrm{n}=15)$.

Sexual dimorphism. Sexually mature males attain larger sizes than females, and exhibit longer and substantially deeper caudal filaments than immature individuals and breeding females (Figs. 21a-b), but do not exhibit an elevated number of bilateral horizontal columns or vertical rows of electrocytes; although the range of electrocyte columns midway from anal-fin terminus to caudal fin filament is sometimes higher in breeding males (5-6, mode 5) than immature specimens and breeding females (4-6, mode 5). Breeding males nonetheless exhibit clearly enlarged electrocytes relative to immature specimens and females. Breeding males with paddle-like lateral compression at caudal filament tip. No known sexual dimorphism in pigmentation.

Geographic distribution. Venezuela (Fig. 14). Brachyhypopomus diazi is known from the northern Orinoco drainage of Venezuela (with most records from the Llanos wetlands), and from coastal drainages of the Caribbean coast of northern Venezuela, from the río Tocuyo drainage to the río Patanemo drainage.

Population variation: We found complete overlap in the range of meristic counts (Tables 2-5) and morphometric proportions (Table 11), and observed similar pigmentation, between trans-Andean populations of $B$. diazi from the río Alpargatón on the Caribbean coast of N Venezuela, and cisAndean populations from the río Apure of the Venezuelan Llanos. These observations, in combination with molecular data (Crampton et al., 2016), support the hypothesis that all populations we have assigned to $B$. diazi are members of a single geographically widespread species, which is morphologically and genetically distinct from all congeners, and unique among congeners in exhibiting a trans- and cisAndean distribution.

Ecological notes. Locally common in high-conductivity rivers $\left(100-400 \mu \mathrm{Scm}^{-1}\right)$ draining the Andes of the Venezuelan llanos (and their associated floodplains), and from streams and small rivers draining the coastal Andean range of the north Venezuelan Caribbean coast. Brachyhypopomus diazi is typically found in dense marginal emergent aquatic vegetation, or among floating macrophytes (Sullivan \& Hopkins, 2009) (WGRC unpublished data). WGRC and NRL recorded the following water parameters at the type locality in the rio Alpargatón drainage, on March 21 2004: conductivity $340 \mu \mathrm{Scm}^{-1}$, dissolved oxygen 5.2 $\mathrm{mgl}^{-1}$, temperature $27.3^{\circ} \mathrm{C}$, and $\mathrm{pH} 7.1$. Here, reproductively mature male and female specimens were captured along with small juveniles $(20-40 \mathrm{~mm}$ ) in marginal grasses and roots. The duration of the breeding season in the río Alpargatón, and the duration and seasonality of breeding in the Llanos populations, and elsewhere, are unknown. Stomach contents of specimens from the type locality comprise aquatic insect larvae and other small aquatic invertebrates (WGRC unpublished data).

Co-occurring congeners: In the Orinoco drainage $B$. diazi co-occurs in geographical sympatry with $B$. brevirostris, $B$. bullocki $B$. beebei, B. regani, and partially with $B$. sullivani. Of these species, $B$. diazi only occurs in ecological syntopy with $B$. brevirostris, $B$. beebei, and $B$. regani. Sullivan \& Hopkins (2009) note that $B$. diazi and $B$. bullocki exhibit habitat partitioning; the former occurring in systems with high conductivity (conductivity $>100 \mu \mathrm{Scm}^{-1}$ ), and the latter in system with low conductivity $\left(<15 \mu \mathrm{Scm}^{-1}\right)$. Brachyhypopomus sullivani is likewise a low-conductivity specialist throughout its geographical range. In Caribbean coastal drainages of Northern Venezuela, $B$. diazi occurs in the absence of congeners.

Local names. Venezuela: cuchillo.

Remarks. Comments on the type series: The holotype of $B$. diazi is only known only from the photograph in FernándezYépez's original description. Museum catalog numbers were not provided for the holotype and sole paratype listed in the description, and these specimens have subsequently been lost (pers. comm., F. Provenzano, MBUCV). The collection locality of the lost holotype is given as ["Station 138"] from the "rio Yaracuy" hydrogeographic complex (a complex which includes the río Salado). Plate 21 of the description includes photographs of "various views of the collecting sites of the species, all west of Puerto Cabello", one of which features a plaque labeled "139 río Salado". During a 2004 visit to Carabobo, two of us (WGRC and NRL) located a site where the río Alpargatón (a tributary of the río Salado) crosses the main road leading west from Puerto Cabello and Morón $\left(10^{\circ} 27^{\prime} \mathrm{N}, 68^{\circ} 8^{\prime} \mathrm{W}\right)$. The site corresponded clearly to photographs from Plate 21, including a distinctive bridge, and a view towards forested hills. At this site we collected a sizable topotype series (MBUCV-V 35655 and UF 174333).

Material examined. 240 specimens. Venezuela. Apure (localities from río Apure dr., Orinoco dr.). CU 71961, 56, nr. Arichuna, edge of río Apure, $07^{\circ} 42^{\prime} \mathrm{N}, 067^{\circ} 08^{\prime} \mathrm{W}$. INHS 32076 , 8, 96-150 mm, caño Guaritico, $07^{\circ} 33^{\prime} 21^{\prime \prime} \mathrm{N}, 069^{\circ} 38^{\prime} 59^{\prime \prime} \mathrm{W}$. MBUCV-V 10809, 3, 48-77 mm, 4 km San Juan de Payara on Biruaca-San Juan de Payara rd., caño Payara, affl. río La Piedra, affl. río Arichuna, $07^{\circ} 40^{\prime} \mathrm{N}, 067^{\circ} 34^{\prime} \mathrm{W}$. MBUCV-V 11033, 1, $112 \mathrm{~mm}$, El Negro, San Fernando de Apure, Caramacate, affl. río Negro, ca. $07^{\circ} 50^{\prime} \mathrm{N}, 067^{\circ} 28^{\prime} \mathrm{W}$. MBUCV-V 14123, 1, female, $110 \mathrm{~mm}$, San Fernando to Apure-Arichuna hwy., $c a$. $1.5 \mathrm{~km}$ past bridge over río Manglar, $c a .07^{\circ} 50^{\prime} \mathrm{N}, 067^{\circ} 28^{\prime} \mathrm{W}$. MBUCV-V 19923, 7 (4 measured: 2 immature 62-63 mm, 1 female, $107 \mathrm{~mm}, 1$ male, $120 \mathrm{~mm}$ ), Laguna nr. caño Boquerones, nr. Arichuna, affl. río Apure, $c a .07^{\circ} 43^{\prime} \mathrm{N}, 67^{\circ} 17^{\prime} \mathrm{W}$. MBUCV-V 20666, 2 (1 measured, female, $102 \mathrm{~mm}$ ), río Apure, nr. San Fernando de Apure, $07^{\circ} 53^{\prime} \mathrm{N}, 067^{\circ} 28^{\prime} \mathrm{W}$. MCNG 13934, 2, caño 
Las Mercedes, affl. río Apure, $07^{\circ} 53^{\prime} \mathrm{N}, 067^{\circ} 31^{\prime} \mathrm{W} . \mathrm{MCNG}$ 13955, 1, hwy. via San Fernando de Apure, no precise locality/ coordinates. MCNG 24161, 1, on Boquerones-San Fernando de Apure hwy., no coordinates. UF 176888, 12 (2 immature, 74-93 mm, 5 female [3CS], 80-106 mm, 5 male, 91-130 mm), Hato El Frio, nr. Mantecal, floodplain lake of río Guaratico, 07 $49^{\prime} 32^{\prime \prime} \mathrm{N}, 068^{\prime} 55^{\prime} 23^{\prime \prime} \mathrm{W}$. USNM 260254, 2, río El Canito, at rd. from San Fernando to Cunaviche, affl. río Cunaviche, $07^{\circ} 28^{\prime} \mathrm{N}, 067^{\prime} 39^{\prime} \mathrm{W}$. USNM 260263, $1,118 \mathrm{~mm}$, ca. $5 \mathrm{~km} \mathrm{~W}$ San Fernando de Apure, side channel of río Apure, $07^{\circ} 53^{\prime} \mathrm{N}$, $067^{\circ} 29^{\prime}$ W. Barinas. MBUCV-V 7549, 1, 80 mm, quebrada La Yuca, Puente Blanco, ca. $20 \mathrm{~km}$ E Barinas, affl. río Masparro, affl. río Apure, Orinoco dr., ca. $08^{\circ} 35^{\prime} \mathrm{N}, 069^{\circ} 59^{\prime} \mathrm{W}$. Carabobo. MBUCV-V 13443, 5, 47-193 mm (2 sexed, males, 171-193 $\mathrm{mm}$ ), río Patanemo, E Puerto Cabello, río Patanemo dr., ca. $10^{\circ} 26^{\prime} \mathrm{N}, 067^{\circ} 55^{\prime} \mathrm{W}$. MBUCV-V 16986, 3 (2 measured: 1 female, $136 \mathrm{~mm}, 1 \mathrm{male}, 178 \mathrm{~mm}$ ), río Urama, río Urama dr., $10^{\circ} 32^{\prime} \mathrm{N}, 068^{\circ} 12^{\prime} \mathrm{W}$. MBUCV-V 35655, 1 (topotype), male, 160 $\mathrm{mm}$, UF 174333, 16 (topotypes) (7 immature [2CS], 20-132 $\mathrm{mm}, 4$ female [1CS], 100-143 mm, 5 male [1CS], 115-176 mm), mun. Morón, río Alpargatón, at rd. and railway bridge on hwy. 1, $8.4 \mathrm{~km}$ Morón, río Salado dr., $10^{\circ} 27^{\prime} 58^{\prime \prime} \mathrm{N}, 068^{\circ} 15^{\prime} 38^{\prime \prime} \mathrm{W}$. Cojedes. MBUCV-V 10787, 1, 135 mm, quebrada El Pozuelo, bridge on San Carlos-Acarigua rd., affl. río Portuguesa, Orinoco dr., $c a .09^{\circ} 35^{\prime} \mathrm{N}, 068^{\circ} 45^{\prime} \mathrm{W}$. MCNG 13623, 1, caño El Pozuelo, affl. río Portuguesa, affl. río Apure, Orinoco dr., $c a .09^{\circ} 35^{\prime} \mathrm{N}$, $068^{\circ} 45^{\prime} \mathrm{W}$. MCNG 15605, 2, caño Cañafistolo, no coordinates. Guárico (localities from Orinoco dr.). MBUCV-V 14124, 1, female, $123 \mathrm{~mm}$, km 273.6, Calabozo-Camaguan hwy., río Apure, no coordinates. MCNG 14404, 7, stream E Guayabal, affl. río Orituco, affl. río Guárico, affl. río Apure, $09^{\circ} 09^{\prime} \mathrm{N}$, $066^{\circ} 43^{\prime} \mathrm{W}$ [coordinates for Guayabal]. MCNG 14483, 3, stream between hwy. 2 and Uverito, affl. río Manapire, $c a .09^{\circ} 22^{\prime} \mathrm{N}$, $065^{\circ} 54^{\prime} \mathrm{W}$ [coordinates for Uverito] (listed by Sullivan, 1997). Lara. MBUCV-V 10827, 1, female, 69 mm, $60 \mathrm{~km}$ W Carora, río Camoruco, río Todistinctive $0^{\circ} 08^{\prime} \mathrm{N}, 070^{\circ} 37^{\prime} \mathrm{W}$. MBUCV-V 16985, 1, $63 \mathrm{~mm}$, río Los Quediches, headwaters downstream of dam, río Tocuyo dr., ca. $09^{\circ} 57^{\prime} \mathrm{N}, 070^{\circ} 25^{\prime} \mathrm{W}$. MCNG 10854 , 1 , stream at $\mathrm{km} \mathrm{408,} \mathrm{no} \mathrm{precise} \mathrm{locality/coordinates} \mathrm{(listed} \mathrm{by}$ Sullivan, 1997). Monagas. MBUCV-V 10696, (part) 4, 117-142 $\mathrm{mm}$, Barrancas, Laguna el Guatero, affl. río Orinoco, Orinoco dr., ca. $08^{\circ} 42^{\prime} \mathrm{N}, 062^{\circ} 12^{\prime} \mathrm{W}$. Portuguesa (localities from río Apure dr., Orinoco dr.). INHS 61362, 2, 104-112 mm, MCNG 15416, 2, MCNG 23675, 2, MCNG 26821, 3, caño Maracá, affl. caño Igues, affl. río Portuguesa, 08 $53^{\prime} 11^{\prime \prime} \mathrm{N}, 069^{\circ} 29^{\prime} 18^{\prime \prime} \mathrm{W}$ (listed by Sullivan, 1997). MCNG 27290, 1, caño Maracá, at bridge on Guanare-Guanarito hwy., affl. caño Igues, affl. río Portuguesa, $08^{\circ} 49^{\prime} 09^{\prime \prime} \mathrm{N}, 069^{\circ} 19^{\prime} 43^{\prime \prime} \mathrm{W}$ (listed by Sullivan, 1997). MUSM 13406, 1, $58 \mathrm{~mm}$, TNHC 12508, 1, $112 \mathrm{~mm}$, TNHC 12579, 1, $131 \mathrm{~mm}$, TNHC 12663, 3, 95-138 mm, TNHC 12706, 17, 39-141 mm, TNHC, 12774, 2, 149-169 mm, TNHC 12608, 16, 93-135 mm, TNHC 12891, 1, $146 \mathrm{~mm}$, TNHC 12896, 1, $118 \mathrm{~mm}$, TNHC 12938, 4, 68-112 mm, TNHC 13018, 5, 44-124 mm, TNHC 13237, 1, $182 \mathrm{~mm}$, TNHC 13277, 1, $99 \mathrm{~mm}$, TNHC 13313, 3, 129-133 mm, TNHC 13347, 1, 90 mm, TNHC 13367, 4, 110-153 mm, TNHC 15036, 23, 61-174 mm, TNHC 17127, 3, 113-119 mm, 35km SE Guanare, caño (río) Maraca at Urriola Ranch, affl. río Portuguesa, $08^{\circ} 52^{\prime} 30^{\prime \prime} \mathrm{N}, 069^{\circ} 27^{\prime} 40^{\prime \prime} \mathrm{W}$. UF $174334,2,125$ $163 \mathrm{~mm}$, río de las Marias, nr. Guanare, affl. río Portuguesa, $c a$. $09^{\circ} 04^{\prime} \mathrm{N}, 069^{\circ} 39^{\prime} \mathrm{W}$.

\section{Brachyhypopomus draco Giora, Malabarba \& Crampton, 2008}

(Fig. 22; Tables 2-5, 12)

Brachyhypopomus beebei. -Sullivan, 1997: 306, fig. 41; 235-241, table 39 (Paraná-Paraguay basin and coastal drainages of southern Brazil, redescription of $B$. beebei).

Brachyhypopomus sp. -Cognato et al., 2007: 244, fig. 1b, color photograph; fig. 2b, caudal filament damage, fig. 2d caudal filament regeneration (S Brazil, damage and regeneration in gymnotiform fishes).

Brachyhypopomus draco Giora et al., 2008: 161, fig. 1, color photograph of male holotype and female paratype; fig. 2, color photograph of head and tip of caudal filament of holotype (original description, type locality - Brazil, Rio Grande do Sul, Patos-Mirim lagoon system). -Almirón et al., 2010: 573, fig. 5, color photograph of live male (Argentina, Entre Rios, Paraná dr., listing of Brachyhypopomus). -Claudino et al., 2010: 358, fig. 1, color photograph of live female (Brazil, Rio Grande do Sul, Lagoa do Peixe National Park, range extension). -Crampton, 2011: 176, table 10.2, species list; 179, figs. 10.2-10.3, phylogeny, geographical and ecological distributions (gymnotiform biology). -Koerber, 2011: 7 (Argentina, species list]. -Malabarba et al., 2013: 96, color photograph of live individual (Brazil, Rio Grande do Sul, rio Tramandaí, catalog of fishes]. -Carvalho, 2013: 143, fig. 3 C outline of head; $181-185$, figs. 41-43, position in phylogeny (phylogenetic systematics of Rhamphichthyoidea). -Mirande \& Koerber, 2015: 48 (Argentina, catalog of fishes). -Tagliacollo et al., 2016: 28, fig. 5 (phylogeny of Gymnotiformes). -Crampton et al., 2016: 1-66, table 1, 3-4, figs. 1-7, 18-20 (phylogeny, biogeography and ecology of Brachyhypopomus).

Diagnosis. Brachyhypopomus draco is diagnosed from congeners by the following combination of characters: precaudal vertebrae $21-25$, vs. $15-20$ in $B$. batesi, $B$. benjamini, B. bennetti, B. bombilla, B. bullocki, B. cunia, $B$. diazi, B. flavipomus, B. hendersoni, B. janeiroensis, $B$. jureiae, B. menezesi, B. occidentalis, B. provenzanoi, $B$. regani, $B$. sullivani, and $B$. walteri; pale stripe along middorsal region of body absent, vs. prominent pale uninterrupted middorsal stripe from occipital region to base of caudal filament present in $B$. arrayae, $B$. beebei, $B$. belindae, $B$. gauderio, $B$. pinnicaudatus, and $B$. verdii; anal-fin rays 155 204, vs. 226-293 in B. brevirostris; caudal filament length 15.7-35.0\% LEA, vs. $7.4-14.0 \%$ in B. palenque; absence of oblique stripes or saddles on body surface, $v s$. presence of continuous or discontinuous dark vertical or diagonally oriented stripes or saddles absent on body surface dorsal to lateral line, and often extending across lateral line into ventral lateral surface, in B. alberti and B. hamiltoni. 


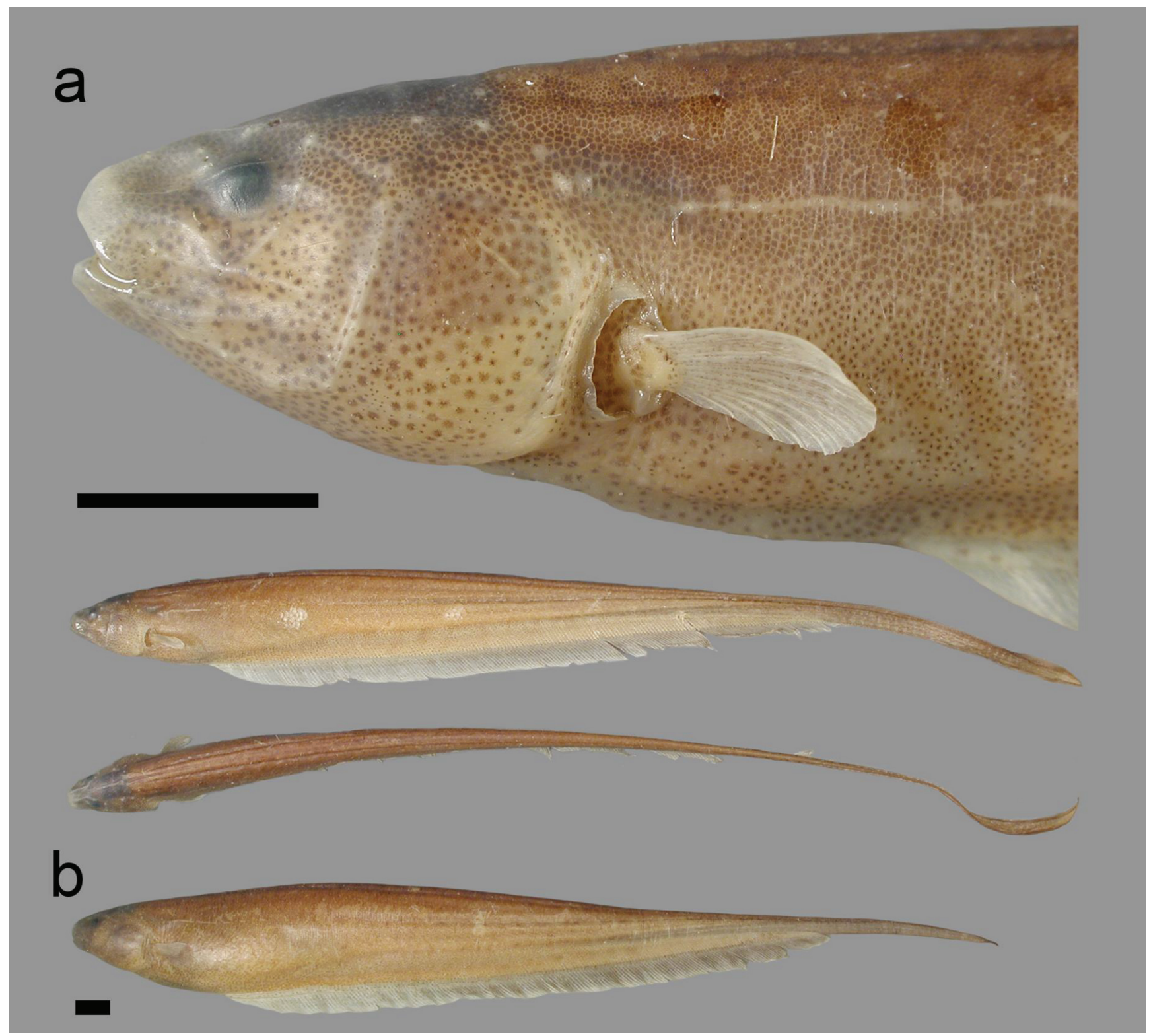

Fig. 22. Brachyhypopomus draco. a. MCP 19847 (part), male, $138 \mathrm{~mm}$ TL (head in lateral view, body in lateral and dorsal view); Brazil, rio Ibicuí, Uruguay dr. b. MCP 13643, female, 132 mm TL (body in lateral view); Brazil, rio Sanga, rio Tramandaí dr. Note sexual dimorphism in caudal filament height. Specimens fixed in formalin and preserved in EtOH. Scale bars $=5 \mathrm{~mm}$.

Description. Head and body shape, and pigmentation illustrated in Fig. 22, and in original description. Meristic and morphometric data for examined specimens are presented in Tables 2-5 and 12. Body moderate in depth. Head short to moderate in length and moderate to broad in depth. Dorsal profile of head straight to slightly convex from occiput to snout, ventral profile of head approximately straight with areas of concavity and convexity between operculum and snout, snout truncate to rounded. Eye small to moderate in size. Upper jaw with moderate to acute sigmoidal angle between premaxillary and maxillary portions in lateral view. No accessory electric organ over operculum. Gill filaments on first gill arch $36(n=1)$. Pectoral fin moderate to broad in width, pectoral-fin rays 14-17 (mode of 16 in populations from Uruguay dr. and coastal drainages of southern Brazil, and 15 in populations from río Paraguay). Precaudal vertebrae 21-25 (mode of 23 in populations from Uruguay dr. and coastal drainages of southern Brazil, and 22 in populations from río Paraguay), including 1-3 (mode 2) transitional vertebrae. Anal-fin origin slightly $(<0.25 \mathrm{HL}$ distance) posterior to, or near, tip of pectoral fin. Anal-fin rays $155-$ 200 , with somewhat higher median value in populations from the río Paraguay (189-197, median 195) than in populations from Uruguay dr. and coastal drainages of southern Brazil (168-200, median 179) (Table 2). Dorsal rami of recurrent branch of anterior lateral line nerve not visible. Middorsal region of body scaled. Rows of scales 
above lateral line 4-6 (mode 5). Lateral line continuous. Sparse depigmented epidermal canals; as an irregular interrupted single groove either side of dorsal midline along most of posterior half body, and as sparse scratch like marks dorsal to and near lateral line in posterior third of body. Three bilateral electrocyte columns at anal-fin terminus in immature, mature female, and mature male specimens. Three columns of electrocytes at or near a mid-point between anal-fin terminus and tip of caudal filament in mature females, and three or four (or three alternating with four) in immature specimens and mature males. Number of columns tapering from 3 or 4 to 2-3 in distal portion of caudal filament. Caudal filament short to moderate in length.

Table 12. Morphometrics for Brachyhypopomus draco. HT, holotype; SD, standard deviation. Data for holotype (Rio Grande do Sul, Brazil) are from Giora et al. (2008). Ranges for the type region refer to non-type specimens from laguna dos Patos, rio Tramandaí, and rio Uruguay drainages, Brazil (holotype data excluded). Specimens from the Paraguay drainage refer to non-type specimens from the río Paraguay of Paraguay.

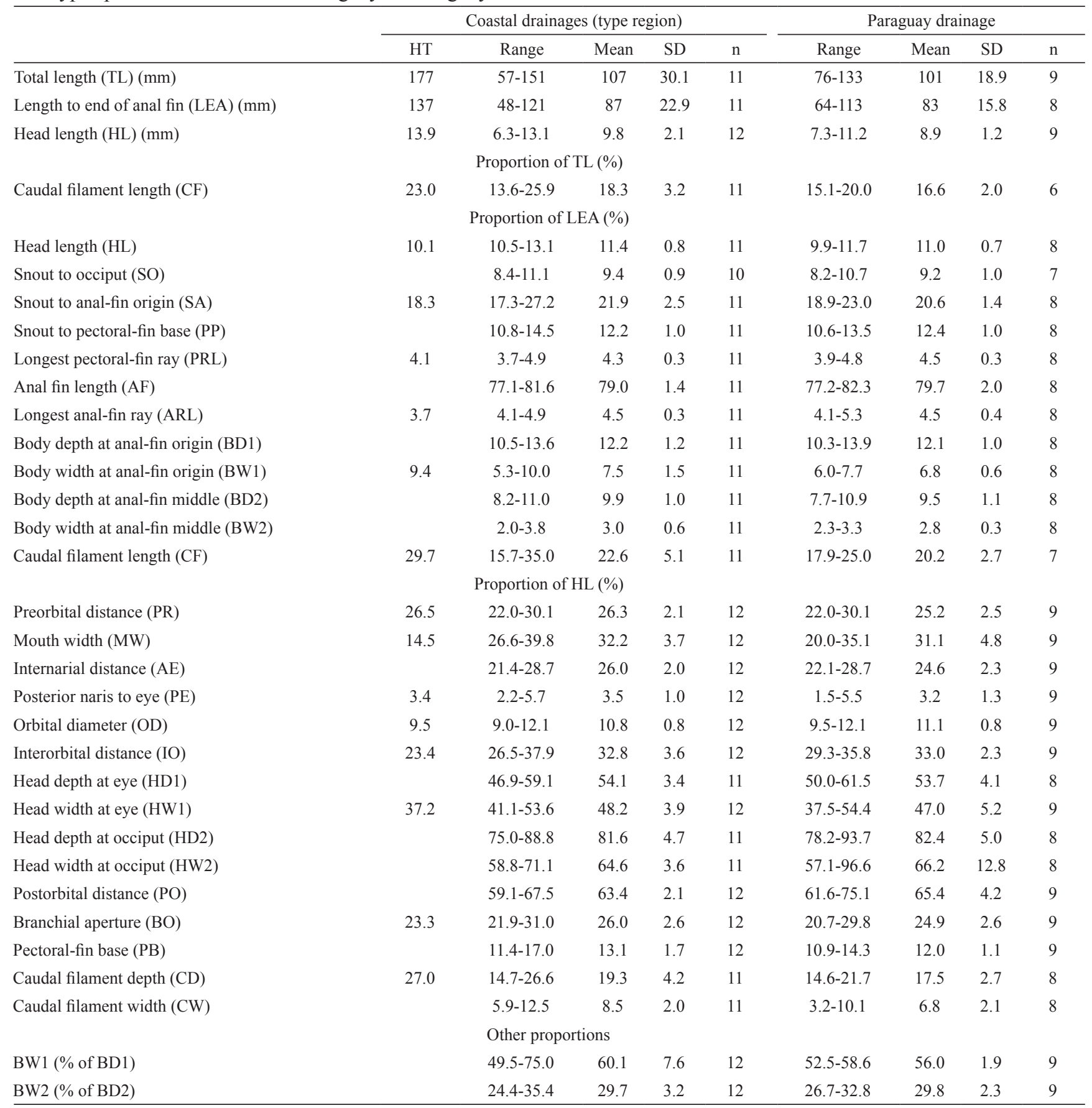


Coloration. (Fig. 22). Background tan to dark brown. Dorsal region without prominent depigmented pale stripe extending along midline from occipital region to base of caudal filament (although in some specimens dorsal midline is somewhat paler than areas adjacent to midline). Dorsal surface without dark markings, or with irregular dark marks or flecks that are sometimes vertically elongated but never form distinct bands. Series of diffuse horizontal dash-like dark markings often present along lateral line in posterior third of body, anterior to anal-fin terminus. Flank below lateral line over body cavity with irregular dark flecks, but rarely vertically elongated bands. Multiple vertically elongated incomplete bands usually, but not always, present over pterygiophores. Caudal filament darker than body, especially dorsally, ventrally, and near tip; with or without irregular, incomplete dark bands. Head with evenly scattered dark chromatophores, darker dorsally. Eye without prominent suborbital patch, or stripe, of chromatophores/subcutaneous pigmentation. Pectoral and anal-fin membranes hyaline. Pectoral-fin rays hyaline with light scattering of brown chromatophores. Analfin rays with scattered or uniform dark pigmentation; darker in posterior half of fin. Color in live individuals similar to preserved specimens, with opercular region usually rosy due to underlying gills.

Size. Moderate adult size, largest specimen examined 182 $\mathrm{mm}$ TL (LEA of this specimen not available) $(\mathrm{n}=264)$. Largest male specimen examined $177 \mathrm{~mm}$ TL, $137 \mathrm{~mm}$ LEA $(\mathrm{n}=27)$. Largest female examined $134 \mathrm{~mm}, 109 \mathrm{~mm}$ LEA ( $\mathrm{n}$ $=14$ ). Maximum male size in type series $151.8 \mathrm{~mm}$ LEA $v s$. $128.4 \mathrm{~mm}$ LEA for females (Giora et al., 2008).

Sexual dimorphism. Sexually mature males attain larger sizes than females. Breeding mature males exhibit longer and deeper caudal filaments than immature individuals and breeding females, and reach slightly larger sizes (Figs. 22a-b) but do not exhibit an elevated number of bilateral horizontal columns or vertical rows of electrocytes. Instead breeding males exhibit clearly enlarged electrocytes relative to immature specimens and females. Tip of caudal filament in breeding males often with paddle-like lateral compression (sometimes very widened), with electrocytes reaching near tip or terminating as far as around half-way along paddle-like structure. No known sexual dimorphism in pigmentation.

Geographic distribution. Argentina, Brazil, Paraguay, and Uruguay (Fig. 23). Widely distributed through lower portions of the Paraguay, Paraná, and Uruguay drainages as far north as $25^{\circ} \mathrm{S}$, the Patos-Mirim lagoon system of southeast Brazil/northeast Uruguay, and some other coastal drainages of Brazil north of Lagoa dos Patos.

Population variation: We found complete overlap in the range of meristics (Tables 2-5) and morphometric proportions (Table 12), and observed similar pigmentation among populations of $B$. draco from Uruguay dr./coastal drainages of southern Brazil, and from the río Paraguay.
These observations, in combination with molecular data (Crampton et al., 2016), support the hypothesis that all populations we have assigned to $B$. draco are members of a single geographically widespread species, which is morphologically and genetically distinct from all congeners.

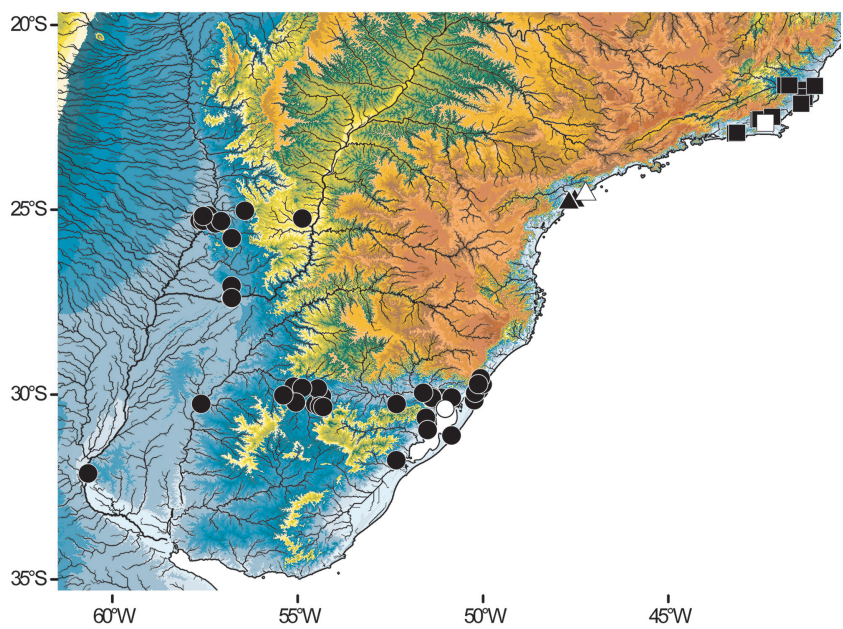

Fig. 23. Collection records for Brachyhypopomus draco (circles), B. janeiroensis (squares), and B. jureiae (triangles). Holotype locations are marked with open symbols. Elevation data refers to altitude above mean sea level (see Fig. 2 for legend).

Ecological notes. The original description of $B$. draco notes that it occurs most commonly in "river edges, slow moving creeks, lagoons and flooded areas with muddy or sandy bottom and abundant emergent or floating vegetation". Schaan et al. (2009) provides a detailed account of the natural history and reproductive biology of $B$. draco. Breeding occurs primarily during the late austral winter and austral summer, which corresponds to a period of higher temperature and rainfall, and substantially longer photoperiod. At this time the sex ratio approximates 1:1. During the austral winter $B$. draco exhibits gonadal quiescence, a general reduction in activity and feeding, and an accompanying reduction of the EOD pulse-rate (Giora et al., 2011; Schaan et al., 2009). Stomach contents of specimens from the type locality comprise aquatic insect larvae and other small aquatic invertebrates (WGRC unpublished data).

Co-occurring congeners: Through most of its range, $B$. draco occurs in geographical sympatry and in ecological syntopy with $B$. bombilla and B. gauderio. Brachyhypopomus draco probably also co-occurs with $B$. walteri in the northern part of the former's distribution in the ParaguayParaná system (see distribution for $B$. walteri, Fig. 47), but not with $B$. brevirostris, which occurs as far south as $19^{\circ} \mathrm{S}$ (Fig. 18) (compared to $25^{\circ} \mathrm{S}$ for the highest known latitude for B. draco, Fig. 23).

Local names. Argentina, Paraguay: morenita; Brazil: tuvira, moreninha, tuvira dragão (Malabarba et al., 2013); Uruguay: morenita, limpiavidrio (Nion et al., 2002). 
Material examined. 264 specimens. Argentina. Entre ríos. AI 268, 2, 118-129 mm, Pre-Delta National Park, Pozo Hondo, Arroyo las Tortugas, Paraná dr., 3208'40"S, 060³9'02"W. Brazil. Rio Grande do Sul. FURG 2186, 3, $42-80$ mm, wetlands draining into Parque Nacional Lagoa do Peixe, $6 \mathrm{~km} \mathrm{~W} \mathrm{Mostardas,}$ Patos-Mirim dr., 31 $06^{\prime} 05^{\prime \prime} \mathrm{S}, 050^{\circ} 51^{\prime} 17^{\prime \prime} \mathrm{W}$. MCP 9487, 3, 64-96 mm, Vila Itapevi, Cecequi-São Francisco de Assis rd., rio Uruguai, Uruguay dr., ca. $29^{\circ} 47^{\prime} \mathrm{S}, 55^{\circ} 07^{\prime} \mathrm{W}$. MCP 13643, 3 (1 immature, $71 \mathrm{~mm}, 1$ female, $134 \mathrm{~mm}, 1$ male, $96 \mathrm{~mm})$, stream, affl. of Lagoa dos Quadros, nr. Praia do Barco, rio Tramandaí dr., ca. $29^{\circ} 42^{\prime} \mathrm{S}, 050^{\circ} 05^{\prime} \mathrm{W}$. MCP 19546, 1, male, $149 \mathrm{~mm}$, Fazenda Pirai rd., 4.5 km São Gabriel, rio Vacacaí, affl. rio Jacuí, affl. rio Guaíba, Patos-Mirim dr., 30¹8'51"S, 054²1'35"W. MCP 19846, 1, female (CS), 130 mm, MCP 19847 (part), 1, male, 139 mm, Banhado do Inhatium, hwy. BR-290, 21 km São Gabriel, affl. rio Ibicuí, Uruguay dr., 30¹5'43"S, 054³1'33"W. MCP 20215, 5, 64-80 mm, Eldorado do Sul, BR-290, affl. rio Jacuí, affl. rio Guaíba, Patos-Mirim dr., 3002'36"S, 051 ${ }^{\circ} 20^{\prime} 56^{\prime \prime} \mathrm{W}$. MCP 20217 , 3, 58-68 mm, arroio Passos dos Carros, rd. linking BR16 to Guaíba, affl. rio Jacuí, affl. rio Guaíba, Patos-Mirim dr., $30^{\circ} 05^{\prime} 55^{\prime \prime} \mathrm{S}, 051^{\circ} 23^{\prime} 15^{\prime \prime} \mathrm{W}$. MCP 20711, 1, 73 mm, Osório, channel linking Lagoa Emboaba and Emboabinha, rio Tramandaí dr., 29 $57^{\prime} 57^{\prime \prime} \mathrm{S}, 050^{\circ} 13^{\prime} 45^{\prime \prime} \mathrm{W}$. MCP 23795, 2 (1 immature, 44 $\mathrm{mm}, 1$ female, $90 \mathrm{~mm}$ ), mun. Tapes, arroio Teixeira on BR-16 E, Patos-Mirim dr., 30³8'34"S, 051 $32^{\prime} 45^{\prime \prime} \mathrm{W}$. MCP 41537 (paratype), 1, female, 108 mm LEA, MCP 41538, 2 (paratypes) (1 female, $92 \mathrm{~mm}$ LEA, 1 male, $88 \mathrm{~mm}$ LEA), MCP 41539, 1 (paratype), male, 147 mm LEA, MCP 41540, 1 (holotype), male, 177 mm TL, 137 mm LEA, MNRJ 30916, 2 (paratypes) (1 female, 128 mm LEA, 1 male, 152 mm LEA), MNRJ 30917, 1 (paratypes) male, $150 \mathrm{~mm}$ LEA, MNRJ 30918, 1 (paratype) male, $138 \mathrm{~mm}$ LEA, MZUSP 94429, 1 (paratype) male, $139 \mathrm{~mm}$ LEA, UFRGS 6515, 1, male, $135 \mathrm{~mm}$, UFRGS 6521, 1, female, $114 \mathrm{~mm}$, UFRGS 6522, 1, female, 127 mm, UFRGS 6523, 1, male, 117 mm, UFRGS 8888, 1 (paratype), male, $126 \mathrm{~mm}$ LEA, mun. Viamão, Lagoa Verde, Parque Estadual de Itapuã, Patos-Mirim dr., $30^{\circ} 22^{\prime} 52^{\prime \prime} \mathrm{S}, 051^{\circ} 01^{\prime} 25^{\prime \prime} \mathrm{W}$. MCP 41938, 2 (1 female, $110 \mathrm{~mm}$, 1 male, $80 \mathrm{~mm}$ ), mun. Cacequi, stream, affl. rio Ibicuízinho, on hwy. Cacequi-Estação São Lucas, Uruguay dr., 2950'12"S, 054²7'423"W. MCP 41961, 2 (1 female, 112 mm,1 male, 95 $\mathrm{mm})$, mun. Cacequi, Fazenda da Convenção, affl. rio Ibicuí, Uruguay dr., 2950'58'S, 05453'02"W. MCP 45352, 1, 134 mm, mun. Pelotas, nr. bridge to Pelotas, rio São Gonçalo, Patos-Mirim

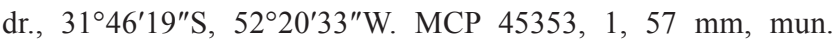
Eldorado do Sul, arroio Passos dos Carros on hwy. connecting hwy. BR-16 to Guaíba, rio Jacuí, affl. rio Guaíba, Patos-Mirim dr., $30^{\circ} 05^{\prime} 55^{\prime \prime} \mathrm{S}, 051^{\circ} 23^{\prime} 15^{\prime \prime} \mathrm{W}$. UFRGS 732, 1 (paratype), female, 86 mm LEA, mun. Viamão, arroio Alexandrina, Patos-Mirim dr., ca. $30^{\circ} 06^{\prime} \mathrm{S}, 050^{\circ} 57^{\prime} \mathrm{W}$. UFRGS 820,1 (paratype), male, $117 \mathrm{~mm}$ LEA, mun. Guaíba, BR-290 hwy., Patos-Mirim dr., ca. $30^{\circ} 07^{\prime} \mathrm{S}$, $051^{\circ} 20^{\prime} \mathrm{W}$. UFRGS 821, 1, $110 \mathrm{~mm}$, UFRGS 822, 1 (paratype), male, 119 mm, mun. Viamão, stream flowing into Praia de Fora, Lagoa dos Patos, Parque Itapuã, Patos-Mirim dr., 30²3'09"S, $051^{\circ} 01^{\prime} 00^{\prime \prime} \mathrm{W}$. UFRGS 3938, 1, $27 \mathrm{~mm}$, mun. Osório, Lagoa Emboaba, rio Tramandaí dr., 29 $57^{\prime} 00^{\prime \prime} \mathrm{S}, 050^{\circ} 13^{\prime} 00^{\prime \prime} \mathrm{W}$. UFRGS 4096, 7, 56-130 mm, mun. Tramandaí, Lagoa do Passo, rio
Tramandaí dr., 2952'01"S, 05006'W. UFRGS 4236, 2, 86-99 mm, mun. Viamão, Lomba do Pinheiro, affl. rio Guaíba, PatosMirim dr., 30 $05^{\prime} 00^{\prime \prime} \mathrm{S}, 051^{\circ} 07^{\prime} 59^{\prime \prime} \mathrm{W}$. UFRGS 4317, 1 (paratype), $59 \mathrm{~mm}$, mun. Viamão, arroio Itapuã, Patos-Mirim dr., $c a \cdot 30^{\circ} 15^{\prime} \mathrm{S}$, $051^{\circ} 02^{\prime} \mathrm{W}$. UFRGS 4503, 1, $50 \mathrm{~mm}$, mun. lago Emboaba, rio Tramandaí dr., $29^{\circ} 57^{\prime} \mathrm{S}, 050^{\circ} 13^{\prime} \mathrm{W}$. UFRGS 4802, 23, $2169 \mathrm{~mm}$, UFRGS 4812, 7, 75-170 mm, UFRGS 4814, 3, 57-82 mm, mun. Viamão, Parque Estadual de Itapuã, Patos-Mirim dr., ca. $30^{\circ} 20^{\prime} \mathrm{S}$, $051^{\circ} 00^{\prime} \mathrm{W}$. UFRGS 5887, 1, $62 \mathrm{~mm}$, mun. Arambaré, unnamed stream, Arambaré - Santa Rita do Sul hwy. Lagoa dos Patos, Patos-Mirim dr., 3057'09"S, 051³0'04"W. UFRGS 6486, 1 (paratype), male, $113 \mathrm{~mm}$, UFRGS 6487, 1, female, $104 \mathrm{~mm}$, UFRGS 6488, 1 (paratype), male, 113 mm, UFGRS 6489, 1 (paratype), male, $112 \mathrm{~mm}$, UFRGS 6490, 1 (paratype), female, 111 mm, UFRGS 6491, 1 (paratype), male, 93 mm, UFRGS 6492, 1, female, 96 mm, mun. Capão da Canoa, stream, Estrada do Mar (hwy. RS-389) km 38, rio Tramandaí dr., 2943'09"S, $050^{\circ} 00^{\prime} 56^{\prime \prime} \mathrm{W}$. UFRGS 6493, 1, male, 100 mm, UFRGS 6494, 1 (paratype), male, $100 \mathrm{~mm}$, UFRGS 6497, 1 (paratype), male, 106 mm, UFRGS 6785, 4 (paratypes) (2 females, 84-85 mm LEA, 2 male, 79-105 mm LEA), mun. Rosário do Sul, stream, hwy. BR290, affl. rio Santa Maria, affl. rio Ibicuí, Uruguay dr., 30¹2'42"S, 055 03'17"W. UFRGS 6507, 1 (paratype), male, $109 \mathrm{~mm}$, UFRGS 6508, 1, male, 126 mm, UFRGS 6509, 1 (paratype), female, 102 mm, UFRGS 6510, 1, female, 86 mm, UFRGS 6511, 1 (paratype), male, $126 \mathrm{~mm}$, UFRGS 6513, 1, female, $113 \mathrm{~mm}$, UFRGS 6514, 1, male, 107 mm, mun. São Gabriel, stream on margin hwy. BR-290, affl. rio Cacequí, affl. rio Santa Maria, affl. rio Ibicuí, Uruguay dr., $30^{\circ} 18^{\prime} 57^{\prime \prime} \mathrm{S}, 054^{\circ} 24^{\prime} 23^{\prime \prime} \mathrm{W}$. UFRGS 6525 , 1, male, $130 \mathrm{~mm}$, UFRGS 6526, 1 (paratype), male, $129 \mathrm{~mm}$, UFRGS 6527, 1, immature, 100 mm, mun. El Dorado do Sul, rd. hwy. BR-290, affl. rio Jacuí, affl. rio Guaíba, Patos-Mirim dr., $30^{\circ} 02^{\prime} 56^{\prime \prime} \mathrm{S}, 051^{\circ} 23^{\prime} 33^{\prime \prime} \mathrm{W}$. UFRGS 6671, 3 (paratypes) (1 female, 96 mm LEA, 2 male, 86-107 mm LEA), mun. Terra de Areia, creek affl. rio Três Forquilhas, hwy. RS 486, rio Tramandaí dr., $29^{\circ} 33^{\prime} 22^{\prime \prime} \mathrm{S}, 050^{\circ} 04^{\prime} 19^{\prime \prime} \mathrm{W}$. UFGRS 6748, 1 (paratype) male, 91 mm LEA, UFRGS 6750, 14, 46-128 mm LEA, mun. Alegrete, Sanga do Jacaré, stream crossing BR-290, affl. rio Ibicuí, Uruguay dr., $30^{\circ} 12^{\prime} 42^{\prime \prime} \mathrm{S}, 055^{\circ} 03^{\prime} 19^{\prime \prime} \mathrm{W}$. MZUSP 94428, 8 (paratypes) (2 male, 96-127 mm LEA, 6 female, 52-104 mm LEA), UFRGS 6752, 1, 100 mm, UFRGS 6753 (paratype), 1 male, 88 mm LEA, UFGRS 6754, 1, 61 mm, UFRGS 6789, 4 (paratypes) (2 females, 72-80 mm LEA, 2 male, 106-126 mm LEA), mun. Rosário do Sul, stream at margin hwy. BR-290, affl. arroio Gueromana, affl. rio Ibirapuitã, affl. rio Ibicuí, Uruguay dr., $30^{\circ} 01^{\prime} 00^{\prime \prime} \mathrm{S}$, $055^{\circ} 23^{\prime} 18^{\prime \prime}$ W. UFRGS 6780, 11, São Gabriel, rio Vacacaí system, Patos-Mirim dr., ca. $30^{\circ} 20^{\prime} \mathrm{S}, 054^{\circ} 19^{\prime} \mathrm{W}$. UFRGS 7686, 30, UFRGS 7707, 3, Viamão, Patos-Mirim dr., ca. $30^{\circ} 05^{\prime} \mathrm{S}, 051^{\circ} 01^{\prime} \mathrm{W}$. UFRGS 8263, 28, Charqueadas, rio Jacuí, Patos-Mirim dr., $c a$. $29^{\circ} 57^{\prime} \mathrm{S}, 051^{\circ} 37^{\prime} \mathrm{W}$. UFRGS 8475, 4, Pantano Grande, rio Pardo, Patos-Mirim dr., ca. $30^{\circ} 15^{\prime} \mathrm{S}, 052^{\circ} 20^{\prime} \mathrm{W}$. UFRGS 8938, 1, rio Tramandaí dr., no coordinates. UFRGS 14562, 2, 136-137 mm, mun. Águas Claras, Refúgio da Vida Silvestre, Banhado dos Pachecos, rio Tramandaí dr., 3004'40"S, 05050'59"W. UFRGS 15292, 2, 103-114 mm, mun. Arambaré, Patos-Mirim dr., no coordinates. UMMZ 143283, 2, 48-100 mm, Conceição do arroio, 
ca. 20 mi. N Tramandaí, rio Maquiné, rio Tramandaí dr., ca. $29^{\circ} 43^{\prime} \mathrm{S}, 050^{\circ} 08^{\prime} \mathrm{W}$. Paraguay (localities from Paraná dr.). Alto Paraná. UMMZ 206908, 1, female, $103 \mathrm{~mm}$, stream, ca. $30 \mathrm{~km}$ NW Hernandarias, rd. to Itakyry, affl. río Paraguay, 2514'12"S, $054^{\circ} 52^{\prime} 48^{\prime \prime W}$. Caaguazú. UMMZ 206252, 5, 33-14 mm, Estancia San Ignacio, $24 \mathrm{~km}$ Carayao, río Hondo, affl. río Paraguay, $25^{\circ} 01^{\prime} 36^{\prime \prime} \mathrm{S}, 056^{\circ} 25^{\prime} 48^{\prime \prime} \mathrm{W}$. Central. UMMZ 205706, 2, 110-115 $\mathrm{mm}$, río Paraguay, NW Asunción, ca. 25¹7'S, 057³9'W. UMMZ 206001 (part), 1, male, $132 \mathrm{~mm}$, río Salado (nr. mouth) nr. N Limpio, affl. río Paraguay, $25^{\circ} 08^{\prime} 42^{\prime \prime} \mathrm{S}, 057^{\circ} 25^{\prime} 12^{\prime \prime} \mathrm{W}$. UMMZ 208106, 2, 84-100 mm, ca. 1.0 km S Puente Remanso bridge, río Paraguay, $25^{\circ} 17^{\prime} 36^{\prime \prime} \mathrm{S}, 057^{\circ} 33^{\prime} 00^{\prime \prime} \mathrm{W}$. Cordillera. ANSP 185112 , 1, female, $105 \mathrm{~mm}$, ANSP 185119, 2, 76-79 mm, stream crossing Caacupe-Tobati rd., $7.0 \mathrm{~km}$ Route 2 in direction of Tobati, affl. río Paraguay, ca. $25^{\circ} 22^{\prime} \mathrm{S}, 057^{\circ} 13^{\prime} \mathrm{W}$. UMMZ 205767, 1, $107 \mathrm{~mm}$, Arroyo Tobati, $1.6 \mathrm{~km} \mathrm{~S}$ Tobati, affl. río Piribebuy, affl. río Paraguay, $25^{\circ} 18^{\prime} 30^{\prime \prime} \mathrm{S}, 057^{\circ} 04^{\prime} 12^{\prime \prime} \mathrm{W}$. Misiones. ANSP 170412, 1, $118 \mathrm{~mm}$, bridge on Ayolas-Asunción-Encarnacíon hwy. (Ruta

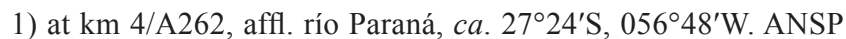
$175180,1,85 \mathrm{~mm}$, stream crossing rd. to Ayolas, affl. río Paraná, $27^{\circ} 03^{\prime} 29^{\prime \prime} \mathrm{S}, 056^{\circ} 47^{\prime} 22^{\prime \prime} \mathrm{W}$. ANSP 185113, 1, $45 \mathrm{~mm}$, marshes, rd. from Ayolas to Asunción-Encarnacíon hwy, affl. río Paraná, ca. $27^{\circ} 23^{\prime} \mathrm{S}, 056^{\circ} 47^{\prime} \mathrm{W}$. Paraguarí. USNM 181483, 5, 88-138 mm, nr. Pueblo Ybytymí, Arroyo Pachongo, affl. río Paraguay, ca. $25^{\circ} 46^{\prime} \mathrm{S}, 056^{\circ} 47^{\prime} \mathrm{W}$. Presidente Hayes. ANSP 185111, 2 (1 female, $103 \mathrm{~mm}, 1 \mathrm{male}, 114 \mathrm{~mm}$ ), Puente Remanso, affl. río Paraguay, $25^{\circ} 10^{\prime} \mathrm{S}, 057^{\circ} 33^{\prime} \mathrm{W}$. Uruguay. Artigas. ZVC-P 2727 , 1, Laguna Redonda on margin of río Uruguay, Franquia, nr. Bella Unión, Uruguay dr., 30¹4'46"S, 057²36'22"W.

\section{Brachyhypopomus flavipomus, new species}

urn:1sid:zoobank.org:act:8368D549-9BE7-45FC-BE0105742AFA0851

(Figs. 1f, 24; Tables 2-5, 13)

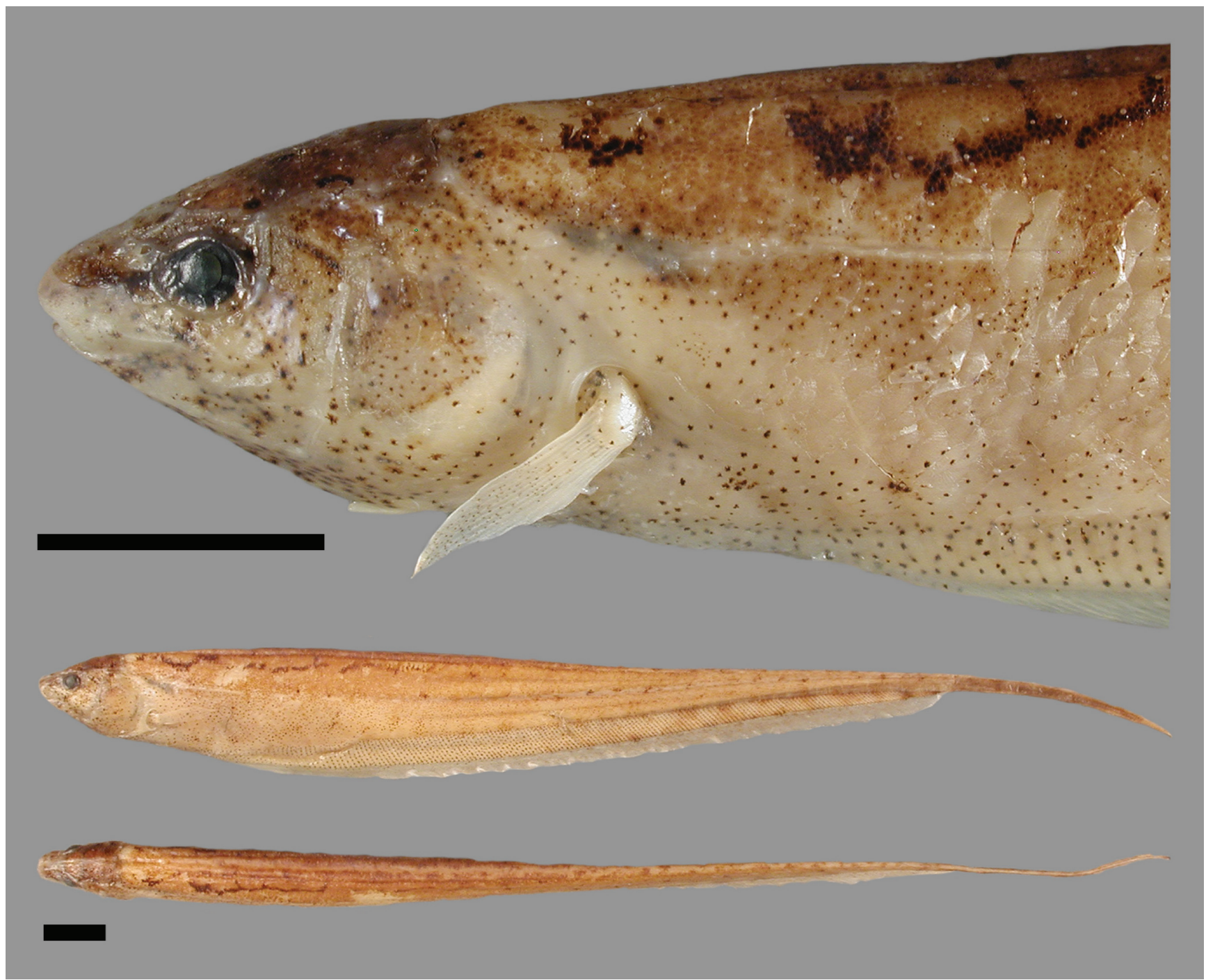

Fig. 24. Brachyhypopomus flavipomus. MCP 45366 (WC19.110597), holotype, immature, $92 \mathrm{~mm}$ TL (head, and body in lateral and dorsal view, specimen fixed in formalin and preserved in EtOH); Brazil, rio Solimões/rio Japurá confluence, Amazonas dr. Scale bars $=5 \mathrm{~mm}$. 
Table 13. Morphometrics for Brachyhypopomus flavipomus, Brachyhypopomus hamiltoni, and Brachyhypopomus hendersoni. HT, holotype; SD, standard deviation. Ranges for each species refer to the holotype and to paratype and non-type specimens from the type regions.

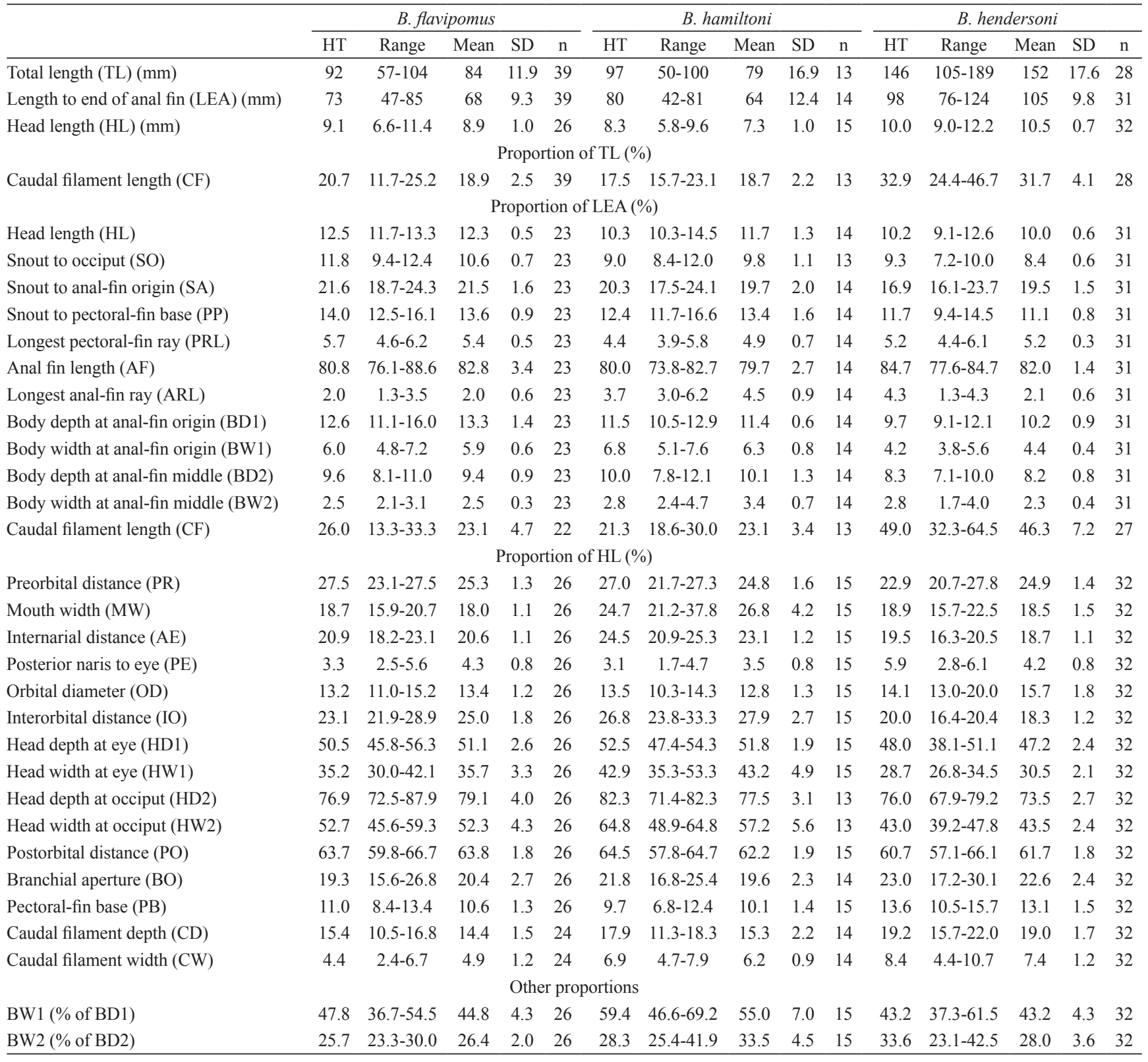

Brachyhypopomus sp. 1. -Crampton, 1996a: 77, table 6.1.a, species list; 79, fig. 6.1 inset 15-16, color photographs; 85, fig. 6.2; 88, fig. 6.3; 92-94, figs. 6.4a, 6.5; 191, fig. 11.2; 193, fig. 11.3, EOD data; 110, table 7.5, 113, table 7.7, 115, fig. 7.1, habitats; 136, table 8.2a,b, diet; 151-160, figs. 9.2-9.3, table 9.1a,b, hypoxia tolerance; 165-169, table 10.1, reproductive biology (Brazil, central Amazon, ecology and signal diversity). -Crampton, 1998b: 314, table 2, list of Brachyhypopomus (Brazil, Amazonas, hypoxia tolerance).

Hypopomidae sp. 1. -Crampton, 1998a: 821, table 4, 834, fig. 9, drawing of adult specimen, with EOD (Brazil, Amazonas, species and EOD diversity).

Brachyhypopomus sp. A. -Crampton, 1999: 17 (Brazil, Amazonas,
Mamirauá Reserve, listing of species). -Crampton \& Albert, 2006: 672, fig. 23.8, position in phylogenetic tree; 681 , notes on EODs (gymnotiform species and EOD diversity). -Crampton et al., 2008: 231, fig. 6, black and white photograph of head of live individual (Brazil, Amazonas, adaptations to hypoxia).

Brachyhypopomus sp. "fla". -Crampton 2011: 176, table 10.2, species list; 179, figs. 10.2-10.3, phylogeny, geographical and ecological distributions (gymnotiform biology).

Brachyhypopomus n. sp. FLAV. -Cardoso et al., 2015: 214 (Brazil, Amazonas, cytogenetics).

Brachyhypopomus sp. "flavipomus". -Crampton et al., 2016: 1-66, table 1, 3-4, figs. 1-7, 18-20 (phylogeny, biogeography and ecology of Brachyhypopomus). 
Holotype. MCP 45366, immature, $92 \mathrm{~mm} \mathrm{TL}, 73 \mathrm{~mm}$ LEA, Brazil, Amazonas, mun. Alvarães, Mamirauá Reserve [Reserva de Desenvolvimento Sustentável Mamirauá], Ressaca do Caetono, rio Solimões-rio Japurá floodplain, Amazonas dr., 02 ${ }^{\circ} 50^{\prime} 15^{\prime \prime} \mathrm{S}, 064^{\circ} 55^{\prime} 50^{\prime \prime} \mathrm{W}, 11$ May 1997, W. Crampton \& J. Oliveira.

Paratypes. 57 specimens, localities from Amazonas dr., collected by W. Crampton \& J. Oliveira. Brazil. Amazonas (localities listed from the Mamirauá Reserve are in rio Solimões-Japurá floodplain, mun. Alvarães). BMNH 1998.3.12.25, 1, female, 113 mm, 20 Jan 1995, BMNH 1998.3.12.26 and 28-41, 15 unsexed, 75-107 mm, 20 Jan 1995, BMNH 1998.3.12.27, 1, immature, 93 mm, 20 Jan 1995, BMNH 1998.3.12.43-44, 2, immature, 90-108 mm, 11 Apr 1994, MCP 45265, 1, female, 98 mm, 14 Mar 2001, Mamirauá Reserve, cano do lago Rato, $03^{\circ} 02^{\prime} 41^{\prime \prime}$, $064^{\circ} 51^{\prime} 26^{\prime \prime} \mathrm{W}$. BMNH 1998.3.12.42, 1, female, $117 \mathrm{~mm}$, 10 Apr 1994, INPA 9942, 21, 58-87 mm, Oct 1993, INPA 18365, 1, immature, $83 \mathrm{~mm}, 11$ May 1997, Mamirauá Reserve, Ressaca do Caetono, 02 ${ }^{\circ} 50^{\prime} 15^{\prime \prime} \mathrm{S}, 064^{\circ} 55^{\prime} 50^{\prime \prime} \mathrm{W}$. INPA 18363, 2, immature, 62-73 mm, 2 Jul 1996, MCP 45349, 1, immature, 85 mm, 4 Jul 1996, Mamirauá Reserve, cano do lago Arauaé, $03^{\circ} 02^{\prime} 52^{\prime \prime} \mathrm{S}, 064^{\circ} 50^{\prime} 04^{\prime \prime} \mathrm{W}$. INPA 18364, 1, immature, 79 mm, 11 Jul 1996, MCP 45350, 2, immature, 80-82 mm, 11 Jul 1996, Mamirauá Reserve, cano do lago Sapucaia, $03^{\circ} 04^{\prime} 07^{\prime \prime}$ S, $064^{\circ} 48^{\prime} 32^{\prime \prime} W$. INPA 18366 , 1, immature, 76 mm, 18 Jun 1999, MCP 45425, 1, immature, 62 mm, 1 May 1999, Mamirauá Reserve, lago Secretaria, $03^{\circ} 07^{\prime} 12^{\prime \prime} \mathrm{S}, 064^{\circ} 47^{\prime} 49^{\prime \prime} \mathrm{W}$. INPA 18367, 1, immature, 70 mm, 24 Jul 1999, MCP 45266, 1, female, $91 \mathrm{~mm}, 19$ Mar 2001, Mamirauá Reserve, cano do lago Mamirauá, $03^{\circ} 06^{\prime} 40^{\prime \prime} \mathrm{S}, 064^{\circ} 47^{\prime} 52^{\prime \prime} \mathrm{W}$. INPA 18368 , 1 , immature, $53 \mathrm{~mm}$, 25 May 1999, MCP 45367, 1, male, 104 mm, 17 May 1997, Mamirauá Reserve, Ressaca da Vila Alencar, $03^{\circ} 07^{\prime} 41^{\prime \prime} \mathrm{S}$, $064^{\circ} 48^{\prime} 04^{\prime \prime} \mathrm{W}$. MCP 45405, 1, immature, $73 \mathrm{~mm}$, Mamirauá Reserve, cano do lago Mamirauá, $03^{\circ} 04^{\prime} 29^{\prime \prime} \mathrm{S}, 064^{\circ} 48^{\prime} 29^{\prime \prime} \mathrm{W}$, 16 Jun 1998. MCP 45406, 1, immature, 48 mm, Mamirauá Reserve, Paraná do Apara, $03^{\circ} 06^{\prime} 50^{\prime \prime} \mathrm{S}, 064^{\circ} 47^{\prime} 48^{\prime \prime} \mathrm{W}, 23$ Jun 1998.

Non-types. 150 specimens, localities from Amazonas dr. Brazil. Amazonas (localities listed from the Mamirauá Reserve are in rio Solimões-Japurá floodplain, mun. Alvarães). IDSM 450, 1, 107 mm, IDSM 455, 5, 64-99 mm, MCP 45368, 14, 58-83 mm, Mamirauá Reserve, cano do lago Rato, $03^{\circ} 02^{\prime} 41^{\prime \prime} \mathrm{S}, 064^{\circ} 51^{\prime} 26^{\prime \prime} \mathrm{W}$. MCP 45264, 1, female,

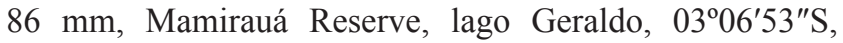
$064^{\circ} 49^{\prime} 06^{\prime \prime} \mathrm{W}$. MCP 45329, 2, immature, 69-74 mm, MCP 45365, 11 (3 immature, 86-97 mm, 5 female [3CS], 85-107 mm, 3 male [1CS], 91-97 mm), Mamirauá Reserve, Ressaca do Caetono, 02 $50^{\prime} 15^{\prime \prime} \mathrm{S}, 064^{\circ} 55^{\prime} 50^{\prime \prime} \mathrm{W}$. MCP 45364, 1, immature, $91 \mathrm{~mm}$, Mamirauá Reserve, cano do lago Rato, $03^{\circ} 02^{\prime} 41^{\prime \prime} \mathrm{S}, 064^{\circ} 51^{\prime} 26^{\prime \prime} \mathrm{W}$. MCP 45453, 1, female, $98 \mathrm{~mm}$, Mamirauá Reserve, lago Secretaria, $03^{\circ} 07^{\prime} 12^{\prime \prime} \mathrm{S}$, 06447'49"W. MPEG 22743, 5, 60-104 mm, Mamirauá
Reserve, cano do lago Rato, $03^{\circ} 02^{\prime} 50^{\prime \prime} \mathrm{S}, 064^{\circ} 51^{\prime} 27^{\prime \prime} \mathrm{W}$. MZUSP 23402, 1, $88 \mathrm{~mm}$, igarapé Tucuxi, affl. rio AtiParaná, NW Fonte Boa, affl. rio Solimões, ca. $02^{\circ} 06^{\prime} \mathrm{S}$, $066^{\circ} 28^{\prime} \mathrm{W}$. MZUSP 23425, 1, $62 \mathrm{~mm}$, lago Buiuçu, affl. rio Ati-Paraná, affl. rio Solimões, $c a .02^{\circ} 06^{\prime} \mathrm{S}, 066^{\circ} 28^{\prime} \mathrm{W}$. Colombia. Amazonas. USNM 216857, 1, 66 mm, Leticia, río Amazonas, $04^{\circ} 13^{\prime} \mathrm{S}, 069^{\circ} 56^{\prime} \mathrm{W}$. Peru. Loreto. ANSP $179835,1,71 \mathrm{~mm}$, caño Moena and mouth of its affl. caño Ullpa, affl. río Itaya, affl. río Amazonas, 0346'19"S, $073^{\circ} 14^{\prime} 16^{\prime \prime} \mathrm{W}$. INHS 39732, 1, $74 \mathrm{~mm}$, INHS 52030, 1, 79 mm, INHS 53873, 2, 76-95 mm, caño Moena, affl. río Itaya, $03^{\circ} 46^{\prime} 20^{\prime \prime} \mathrm{S}, 073^{\circ} 14^{\prime} 17^{\prime \prime} \mathrm{W}$. INHS $94214,3,56-62 \mathrm{~mm}$, caño Ushpa, affl. río Itaya, $c a .03^{\circ} 48^{\prime} \mathrm{S}, 073^{\circ} 16^{\prime} \mathrm{W}$. INHS 56227 , 1, $60 \mathrm{~mm}$, caño Ullpa, 2-3 mi. upstream from mouth and village Ullpa, affl. río Itaya, $03^{\circ} 47^{\prime} 37^{\prime \prime} \mathrm{S}, 073^{\circ} 14^{\prime} 44^{\prime \prime} \mathrm{W}$. MUSM 6959 (part), 3, (1 female, 77 mm, 2 male, 90-92 $\mathrm{mm}$ ), Isla Muyuy, cocha Amazonas, affl. río Amazonas, $c a$. $03^{\circ} 54^{\prime} \mathrm{S}, 073^{\circ} 14^{\prime} \mathrm{W}$. MUSM 44760, 1, immature, $64 \mathrm{~mm}$, nr. Jenaro Herrera, floodplain lake, río Ucayali, $04^{\circ} 54^{\prime} 18^{\prime \prime} \mathrm{S}$, $073^{\circ} 43^{\prime} 12^{\prime \prime} \mathrm{W}$. MUSM 44770, 37 (32 immature, 37-82 mm, 5 female, 54-100 mm), nr. Jenaro Herrera, cocha Capite, floodplain lake, río Ucayali, 04 $51^{\prime} 21^{\prime \prime} \mathrm{S}, 073^{\circ} 40^{\prime} 24^{\prime \prime} \mathrm{W}$. NRM 13522 (part), 1, 69 mm, Sacarita del Tuyé, opposite Pebas, río Ampiyacu, $c a .03^{\circ} 20^{\prime} \mathrm{S}, 071^{\circ} 49^{\prime} \mathrm{W}$. UF 114641,1 , $55 \mathrm{~mm}$, UF 148052, 1, immature, $75 \mathrm{~mm}$, Reserva Nacional (RN) Pacaya Samiria, cocha Yanayacu, affl. río Pacaya, affl. río Ucayali, $05^{\circ} 23^{\prime} 29^{\prime \prime} \mathrm{S}, 074^{\circ} 31^{\prime} 21^{\prime \prime} \mathrm{W}$. UF $126156,1,52$ $\mathrm{mm}$, UF 126327, 1, $69 \mathrm{~mm}$, UF 128883, 2, 40-53 mm, UF $128981,8,47-79 \mathrm{~mm}$, UF $129035,1,76 \mathrm{~mm}$, UF 129047 , 1, $70 \mathrm{~mm}$, UF 129086, 6, 37-74 mm, UF 129144, 8, 47$102 \mathrm{~mm}$, UF 129187, 6, 45-80 mm, UF 129414, 1, $52 \mathrm{~mm}$, UF 129798, 2, 67-82 mm, RN Pacaya Samiria, río Pacaya, affl. río Ucayali, $c a .05^{\circ} 16^{\prime} \mathrm{S}, 074^{\circ} 27^{\prime} \mathrm{W}$. UF 126238,5 , $63-$ $81 \mathrm{~mm}$, UF 129006, 1, immature, $70 \mathrm{~mm}$, UF 129797, 1, $65 \mathrm{~mm}, \mathrm{RN}$ Pacaya Samiria, cocha Sapote, affl. río Pacaya, affl. río Ucayali, $05^{\circ} 20^{\prime} 14^{\prime \prime} \mathrm{S}, 074^{\circ} 29^{\prime} 40^{\prime \prime} \mathrm{W}$. UF 129010 , 1 , immature, $58 \mathrm{~mm}$, RN Pacaya Samiria, río Pacaya, affl. río Ucayali, $05^{\circ} 16^{\prime} 12^{\prime \prime} \mathrm{S}, 074^{\circ} 30^{\prime} 09^{\prime \prime} \mathrm{W}$. UF 129012 , 1 , immature, $53 \mathrm{~mm}, \mathrm{RN}$ Pacaya Samiria, río Pacaya, affl. río Ucayali, $05^{\circ} 16^{\prime} 44^{\prime \prime} \mathrm{S}, 074^{\circ} 31^{\prime} 06^{\prime \prime} \mathrm{W}$. UF 148053, 2 (1 immature, 74 $\mathrm{mm}, 1$ female, $78 \mathrm{~mm})$, RN Pacaya Samiria, caño Tamara, affl. río Pacaya, affl. río Ucayali, $05^{\circ} 16^{\prime} 39^{\prime \prime} \mathrm{S}, 074^{\circ} 29^{\prime} 39^{\prime \prime} \mathrm{W}$. UF 148547, 3 ( 2 immature, 75-76 mm, 1 female, $75 \mathrm{~mm}$ ), NN Pacaya Samiria, caño Yarina, affl. río Pacaya, affl. río Ucayali, $05^{\circ} 19^{\prime} 20^{\prime \prime} \mathrm{S}, 074^{\circ} 30^{\prime} 14^{\prime \prime} \mathrm{W}$. UF 148549 , 1, male, 95 mm, RN Pacaya Samiria, cocha Sapote, affl. río Pacaya, affl. río Ucayali, $05^{\circ} 20^{\prime} 15^{\prime \prime} \mathrm{S}, 074^{\circ} 29^{\prime} 40^{\prime \prime} \mathrm{W}$. UF 148554, 1 , immature, $84 \mathrm{~mm}$, RN Pacaya Samiria, río Pacaya, affl. río Ucayali, $05^{\circ} 18^{\prime} 15^{\prime \prime} \mathrm{S}, 074^{\circ} 30^{\prime} 10^{\prime \prime} \mathrm{W}$. UF 148555 , 2 , female, 84-92 mm, RN Pacaya Samiria, cocha Yanayacu, affl. río Pacaya, affl. río Ucayali, $05^{\circ} 18^{\prime} 14^{\prime \prime} \mathrm{S}, 074^{\circ} 26^{\prime} 12^{\prime \prime} \mathrm{W}$.

Diagnosis. Brachyhypopomus flavipomus is diagnosed by the presence of conspicuous patches of shiny yellow guanine on the operculum and anterior to pectoral-fin base in live individuals, $v s$. absence in all congeners. 
Description. Head and body shape, and pigmentation illustrated in Figs. 1f and 24. Meristic and morphometric data for examined specimens presented in Tables 2-5 and 13 . Body moderate to broad in depth. Head moderate in length and depth. Dorsal profile of head straight to slightly convex from occiput to snout, ventral profile of head approximately straight between operculum and snout, snout triangular. Eye moderate to large in size. Upper jaw with slight sigmoidal angle between premaxillary and maxillary portions in lateral view. No accessory electric organ over operculum. Gill filaments on first gill arch 33-39 (median 34, $\mathrm{n}=5$ ). Pectoral fin narrow to moderate in width, pectoral-fin rays 11-13 (mode 12). Precaudal vertebrae 18-20 (mode 19), with 1-2 (mode 1) transitional vertebrae. Anal-fin origin slightly $(<0.25 \mathrm{HL}$ distance) anterior to, or near, tip of pectoral fin. Anal-fin rays 175-186 (median 179). Dorsal rami of recurrent branch of anterior lateral line nerve not visible. Middorsal region of body scaled. Rows of scales above lateral line 5-6 (mode 5). Lateral line continuous. Sparse depigmented epidermal canals; as an irregular interrupted single groove either side of dorsal midline along most of posterior half body, scratch like marks dorsal to and near lateral line almost absent, present sporadically and sparsely in some specimens. Three bilateral horizontal columns of electrocytes at anal-fin terminus and at or near a midpoint between anal-fin terminus and tip of caudal filament in immature, mature female, and mature male specimens. Number of electrocyte columns sometimes increases to four posterior to mid-point between anal-fin terminus and tip of caudal filament, before tapering to 2-3 distally. Caudal filament short to moderate in length.

Coloration. (Figs. 1f, 24). Background dark brown dorsally fading to pale yellow to tan ventrally. Background of region of body over body cavity below lateral line whitish to yellow in live individuals, pale tan in preserved specimens. Dorsal region without prominent depigmented pale stripe extending along midline from occipital region to base of caudal filament. Prominent pair of irregular horizontal lines formed by aggregations of dark chromatophores either side of dorsal region, visible both dorsally and laterally (in upper part of flank) in anterior third of body, from which short irregular dark patches extend dorsally. Some irregular dark markings along lateral line and over pterygiophores in posterior half of body, but flank otherwise marked only with irregular scatterings of chromatophores; banding absent. Caudal filament darker than body, with irregular dark markings or bands along entire length. Head with very pale background color ventrally, darker dorsally. Irregular dark chromatophores scattered over head, reaching highest density on dorsal surface of head and anterior to eye. Eye without prominent suborbital patch, or stripe, of chromatophores/subcutaneous pigmentation. Pectoral and anal-fin membranes hyaline. Pectoral and anal-fin rays hyaline with light scattering of brown chromatophores. Color in live specimens (Fig. 1f) similar to preserved ones, but with very conspicuous shiny yellow patches of guanine over operculum (forming one or two vertical yellow lines) and anterior to pectoral-fin base (forming a vertically elongated patch) and with conspicuous white or pale yellow color below lateral line over body cavity. Yellow guanine patches and white-yellow background color over body cavity fade soon after fixation. Live individuals with posterior portion of opercular region (posterior to guanine patch) rosy due to underlying gills.

Size. Small adult size, largest specimen examined $117 \mathrm{~mm}$, $89 \mathrm{~mm}$ LEA $(\mathrm{n}=208)$. Largest male specimen examined $104 \mathrm{~mm}$ TL, $77 \mathrm{~mm}$ LEA $(\mathrm{n}=7)$. Largest female specimen examined $117 \mathrm{~mm}$ TL, $89 \mathrm{~mm}$ LEA $(\mathrm{n}=21)$.

Sexual dimorphism. Females reach larger body size than males. Males in breeding condition have somewhat deeper and longer caudal filaments than immature specimens or breeding females but do not exhibit an elevated number of horizontal bilateral columns or vertical rows of electrocytes. Instead breeding males exhibit slightly enlarged electrocytes relative to immature specimens and females. Breeding males without paddle-like lateral compression at caudal filament tip. No known sexual dimorphism in pigmentation.

Geographic distribution. Brazil and Peru (Fig. 25). Known only from whitewater floodplain habitats along the main stem of the Amazon River of the upper and central Amazon, from the río Ucayali-río Marañón confluence in the west to the rio Solimões (Amazonas) - rio Japurá confluence in the east.

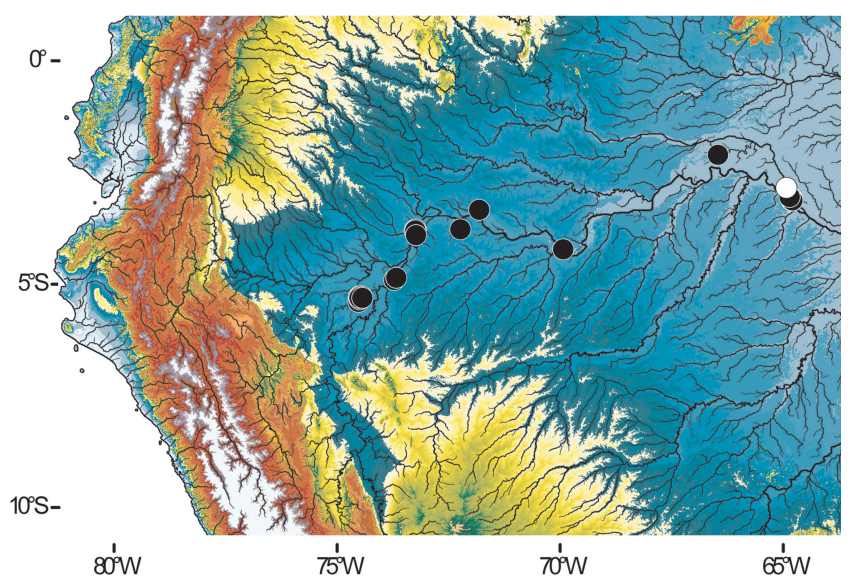

Fig. 25. Collection records for Brachyhypopomus flavipomus (circles). Holotype location is marked with an open symbol. Elevation data refers to altitude above mean sea level (see Fig. 2 for legend).

Ecological notes. Brachyhypopomus flavipomus is restricted to whitewater floodplain habitats where it is encountered throughout the year in floating macrophytes. It is most abundant in sheltered floodplain lakes surrounded by high levee forest, but has a patchy distribution, and varies in abundance at a given site from year to year (WGRC 
unpublished data). See description of $B$. belindae for water quality parameters of the area of the type locality. During the early rising water period B. flavipomus also occurs in leaf litter along the margins of newly flooded forest. Brachyhypopomus flavipomus is able to tolerate protracted periods of hypoxia at high water by aerial gill respiration (Crampton, 1998b), and its gill lamellae are greatly expanded in comparison to species from normoxic system (Crampton et al., 2008). Brachyhypopomus flavipomus breeds during the rising and high water period (Crampton, 1996a, 165). Stomach contents of specimens from the type locality comprise aquatic insect larvae, microcrustacea, and other small aquatic invertebrates - with a predominance of Chironomidae larvae (Crampton, 1996a: 136).

Co-occurring congeners: Brachyhypopomus flavipomus is known to co-occur in geographical sympatry and ecological syntopy with the whitewater floodplain-occurring species $B$. beebei, B. belindae, B. bennetti, B. brevirostris, B. hamiltoni, $B$. pinnicaudatus, $B$. regani, and $B$. walteri. It also exhibits allotopic distributions across parts of its range with: $B$. batesi, $B$. benjamini, $B$. hendersoni, $B$. sullivani, and $B$. verdii.

Etymology. The specific name is an adjective with alternative endings - $u s,-a$, - um, derived from flavus, Latin adjective = yellow; and pomus, latinized from Greek $\pi \tilde{\omega} \mu \alpha=$ lid, cover. In reference to the conspicuous patches of yellow guanine on the operculum of live individuals.

Local names. Brazil: sarapó; Peru: macana.

\section{Brachyhypopomus gauderio Giora \& Malabarba, 2009}

(Fig. 26; Tables 2-5, 14)

Rhamphichthys brevirostris. -Perugia, 1891: 56 (Argentina, Chaco, Resistencia, based on three specimens collected by Spegazzini (MSNG 27458); one of the three was identified as Eigenmannia trilineata, and two, incorrectly, as Brachyhypopomus bombilla by Azpelicueta \& Koerber, 2015: 40-42, fig. 3, color photograph of specimens [in discussion of Perugia listings]). -Ringuelet et al., 1967: 253; Lopez et al., 2003: 65 (repeat listings from Perugia, 1891). -Boulenger, 1896: 38 (Brazil, Mato Grosso, Descalvados, list of fishes collected in río Paraguay by $\mathrm{C}$. Ternetz, record corresponds to BMNH 1895.5.17.265-268; identified herein as B. gauderio (part, $\mathrm{n}=1)$, B. bombilla (part, $\mathrm{n}=3$ ).

Hypopomus brevirostris. -Eigenmann \& Kennedy, 1903: 530 (Paraguay, listing of fishes collected by J. Daniel Anisits, "Four specimens $(108,158,220)$, Mato Grosso or Asunción. Campo Grande and Arroyo Chagalalina."; we identified these four specimens as AMNH 1009, CAS 72215, FMNH 5251, all $B$. gauderio). -Carter \& Beadle, 1931: 337, pl. 21, fig. 7, transverse section of the mouth and gill chamber; pl.31, fig. 8, illustrations of gill-lamellae morphology (Paraguay, ecology with discussion of respiratory adaptations; identified from context as B. gauderio). -Casciotta et al., 2005: 71, line-drawing; 237, fig. 108, color photograph (Argentina, Iberá wetlands, catalog of fishes).
Brachyhypopomus pinnicaudatus. -Franchina, 1997: 115, figs. 7-10, black and white photographs of electrocyte arrangements in caudal filament (electric organ ontogeny). -Stoddard, 1999: 255, fig. 4, color photograph of mature female (EOD evolution). -Stoddard et al., 1999: 610, fig. 1, black and white photograph of mature male and female (EOD evolution). -Albert \& Crampton, 2003: 494 (rio de la Plata basin, Paraguay, Brazil, Uruguay, catalog of hypopomids). -Curtis \& Stoddard, 2003: 330, fig. 1, black and white photographs of mature male and female (communication). -Crampton \& Albert, 2006: 672, fig. 23.8, position in phylogenetic tree; 681, notes on EODs (gymnotiform species and EOD diversity). -Graça \& Pavanelli, 2007: 168, color photograph of live individual (Brazil, upper rio Paraná, species lists - presumed to be an introduced species, see 'Remarks', below).

Brachyhypopomus sp. -Giora et al., 2008: 167 (Brazil, Rio Grande do $\mathrm{Sul}$, listed in comparative material for description of B. draco).

Brachyhypopomus gauderio Giora \& Malabarba, 2009: 62, fig. 1, color photograph of male holotype (original description, type locality - Brazil, Rio Grande do Sul, Palmares do Sul, PatosMirim dr.). -Almirón et al., 2010: 573, fig. 7, color photograph (Argentina, Entre Rios, Pre-Delta Matinal Park, Paraná dr., listing of Brachyhypopomus). -Crampton, 2011: 176, table 10.2, species list; 179, figs. 10.2-10.3, phylogeny, geographical and ecological distributions (gymnotiform biology). -Koerber, 2011: 7 (Argentina, species list, phylogeny, geographical and ecological distributions, gymnotiform biology). -Malabarba et al., 2013: 97, color photograph of live individual (Brazil, Rio Grande do Sul, rio Tramandaí, catalog of fishes). -Carvalho, 2013: 168, fig. 25a, color photograph of head; 181-185, figs. 41-43, position in phylogeny (phylogenetic systematics of Rhamphichthyoidea). -Mirande \& Koerber, 2015: 48 (Argentina, catalog of fishes). -Crampton et al., 2016: 1-66, table 1, 3-4, figs. 1-7, 18-20 (phylogeny, biogeography and ecology of Brachyhypopomus).

Brachyhypopomus bombilla. -Azpelicueta \& Koerber: 2015, 4042, fig. 3, color photograph of two specimens, MSNG 27458 (discussion of fish specimens listed in Perugia, 1891).

Diagnosis. Brachyhypopomus gauderio is diagnosed from congeners by the following combination of characters: prominent pale stripe along middorsal region of present, $v s$. absent in all congeners except $B$. arrayae, $B$. beebei, $B$. belindae, $B$. pinnicaudatus, and $B$. verdii; longest anal-fin ray $3.9-5.4 \%$ LEA, vs. $1.6-3.9 \%$ in $B$. beebei; precaudal vertebrae 18-21, vs. 24-26 in B. belindae and B. verdii. Brachyhypopomus gauderio exhibits similar pigmentation and overlapping meristic counts and morphometric proportions with some populations of $B$. arrayae and $B$. pinnicaudatus. Brachyhyроротиs gauderio can be distinguished from $B$. arrayae by the absence of a contact between a small ascending process on the endopterygoid and the orbitosphenoid $v s$. a contact between these two bones in $B$. arrayae (see diagnosis for $B$. arrayae for details). Brachyhypopomus gauderio can be distinguished from $B$. pinnicaudatus by the absence of a medial bridge on the posterior portion of the basihyal (de Santana \& Vari, 2010: 250. fig. 15; Fernandes et al., 2014: 104, fig. 7), vs. presence in B. pinnicaudatus. 


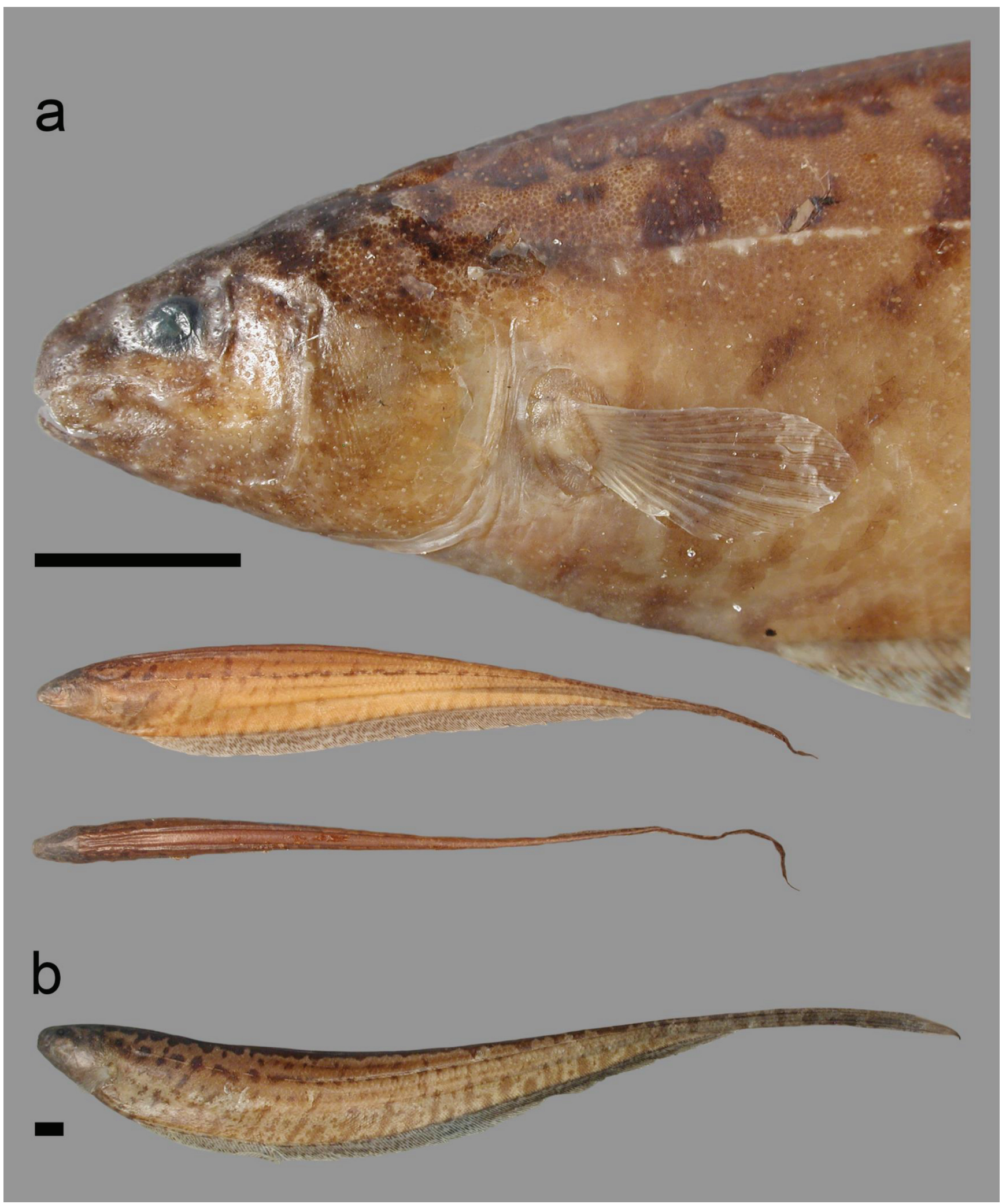

Fig. 26. Brachyhypopomus gauderio. a. MZUSP 59646, female, $138 \mathrm{~mm}$ TL (head in lateral view, body in lateral and dorsal view); Brazil, rio Miranda, Paraguay dr. b. NRM 35349, male, 171 mm TL (body in lateral view); Paraguay, río Negro, Paraguay dr. Note sexual dimorphism in caudal filament height. Specimens fixed in formalin and preserved in EtOH. Scale bars $=5 \mathrm{~mm}$. 
Table 14. Morphometrics for Brachyhypopomus gauderio. HT, holotype; SD, standard deviation. Data for holotype (Rio Grande do Sul, Brazil) are from Giora \& Malabarba (2009). Range for the type region refers to non-type specimens from the lagoa dos Patos and Uruguay drainages in Brazil and Uruguay (holotype data excluded). Specimens from the Paraguay drainage refer to non-type specimens from the río Paraguay of Paraguay.

\begin{tabular}{|c|c|c|c|c|c|c|c|c|c|}
\hline & \multicolumn{5}{|c|}{ Coastal drainages (type region) } & \multicolumn{4}{|c|}{ Paraguay drainage } \\
\hline & HT & Range & Mean & SD & $\mathrm{n}$ & Range & Mean & SD & $\mathrm{n}$ \\
\hline Total length (TL) (mm) & 180 & $63-170$ & 130 & 29.8 & 14 & $113-162$ & 136 & 14.8 & 20 \\
\hline Length to end of anal fin (LEA) (mm) & 149 & $54-130$ & 106 & 23.6 & 15 & $93-135$ & 109 & 11.5 & 20 \\
\hline Head length (HL) (mm) & 16.0 & $7.7-13.4$ & 11.5 & 1.7 & 15 & $9.6-13.4$ & 11.5 & 0.9 & 20 \\
\hline \multicolumn{10}{|c|}{ Proportion of TL (\%) } \\
\hline Caudal filament length (CF) & 17.0 & $14.0-24.7$ & 18.6 & 3.3 & 14 & $13.3-23.8$ & 19.3 & 2.8 & 20 \\
\hline \multicolumn{10}{|c|}{ Proportion of LEA (\%) } \\
\hline Head length (HL) & 10.5 & $9.6-14.2$ & 11.1 & 1.2 & 15 & $9.8-11.4$ & 10.6 & 0.5 & 20 \\
\hline Snout to occiput (SO) & & $7.9-12.1$ & 9.3 & 1.1 & 15 & 7.7-9.9 & 8.9 & 0.5 & 20 \\
\hline Snout to anal-fin origin (SA) & 17.6 & $17.1-24.4$ & 20.1 & 1.7 & 15 & $17.8-20.4$ & 19.3 & 0.8 & 20 \\
\hline Snout to pectoral-fin base (PP) & & $10.6-16.3$ & 12.8 & 1.4 & 15 & $11.5-13.5$ & 12.6 & 0.6 & 20 \\
\hline Longest pectoral-fin ray (PRL) & 5.0 & $4.3-5.5$ & 5.0 & 0.3 & 15 & $5.3-6.4$ & 5.8 & 0.3 & 20 \\
\hline Anal fin length (AF) & & $74.1-83.1$ & 79.7 & 2.9 & 15 & $71.6-83.9$ & 81.4 & 2.5 & 20 \\
\hline Longest anal-fin ray (ARL) & 4.1 & $3.9-5.4$ & 4.4 & 0.4 & 15 & $4.1-5.2$ & 4.6 & 0.3 & 20 \\
\hline Body depth at anal-fin origin (BD1) & 13.1 & $11.1-14.6$ & 13.1 & 1.0 & 15 & $11.3-14.9$ & 13.3 & 1.0 & 20 \\
\hline Body width at anal-fin origin (BW1) & & $5.7-8.2$ & 6.7 & 0.7 & 15 & $5.3-8.0$ & 7.1 & 0.7 & 20 \\
\hline Body depth at anal-fin middle (BD2) & 1.0 & $8.5-12.4$ & 9.9 & 0.9 & 15 & $9.5-14.4$ & 12.5 & 1.2 & 20 \\
\hline Body width at anal-fin middle (BW2) & & $2.3-4.0$ & 3.0 & 0.5 & 15 & $3.0-4.9$ & 4.0 & 0.5 & 20 \\
\hline Caudal filament length (CF) & 20.5 & $16.3-32.8$ & 22.8 & 4.9 & 14 & $16.7-31.2$ & 24.0 & 4.1 & 20 \\
\hline \multicolumn{10}{|c|}{ Proportion of HL (\%) } \\
\hline Preorbital distance (PR) & 25.0 & $24.2-27.8$ & 26.1 & 1.2 & 15 & $25.6-31.3$ & 26.9 & 1.3 & 20 \\
\hline Mouth width (MW) & 18.3 & $24.3-33.7$ & 30.1 & 2.6 & 15 & $22.4-40.1$ & 34.0 & 4.4 & 19 \\
\hline Internarial distance (AE) & & $21.5-25.7$ & 23.2 & 1.3 & 15 & $21.8-26.7$ & 24.0 & 1.5 & 20 \\
\hline Posterior naris to eye (PE) & 6.3 & $3.8-7.4$ & 5.4 & 0.9 & 15 & $4.4-7.7$ & 6.1 & 1.1 & 20 \\
\hline Orbital diameter (OD) & 9.6 & 7.4-11.8 & 10.3 & 1.3 & 15 & $9.9-12.4$ & 11.0 & 0.6 & 20 \\
\hline Interorbital distance (IO) & 30.3 & $29.3-40.6$ & 34.1 & 2.8 & 15 & $27.2-39.6$ & 33.1 & 3.0 & 20 \\
\hline Head depth at eye (HD1) & & $52.0-59.3$ & 55.1 & 2.0 & 15 & $53.7-65.7$ & 58.6 & 3.0 & 20 \\
\hline Head width at eye (HW1) & 46.2 & 42.4-51.4 & 46.9 & 2.7 & 15 & $34.3-58.1$ & 50.6 & 5.1 & 20 \\
\hline Head depth at occiput (HD2) & & $78.6-90.5$ & 83.3 & 2.7 & 15 & $80.6-96.1$ & 87.1 & 3.9 & 20 \\
\hline Head width at occiput (HW2) & & $57.4-66.8$ & 63.0 & 2.9 & 15 & $59.8-75.8$ & 64.7 & 4.1 & 20 \\
\hline Postorbital distance (PO) & & $61.9-67.9$ & 65.1 & 1.7 & 15 & $57.8-70.1$ & 63.6 & 3.0 & 20 \\
\hline Branchial aperture (BO) & 27.8 & $24.6-28.7$ & 26.7 & 1.3 & 15 & $24.1-31.2$ & 27.0 & 2.1 & 20 \\
\hline Pectoral-fin base (PB) & & $10.7-13.9$ & 12.6 & 1.1 & 15 & $10.0-13.7$ & 12.1 & 1.2 & 20 \\
\hline Caudal filament depth (CD) & 20.0 & $12.8-24.9$ & 18.4 & 2.9 & 15 & $15.3-22.1$ & 18.2 & 2.0 & 20 \\
\hline Caudal filament width (CW) & & $5.6-12.1$ & 8.5 & 1.8 & 15 & $6.3-11.8$ & 9.0 & 1.6 & 20 \\
\hline \multicolumn{10}{|c|}{ Other proportions } \\
\hline BW1 (\% of BD1) & & $47.9-56.2$ & 51.1 & 2.6 & 15 & $42.2-58.7$ & 53.5 & 3.8 & 20 \\
\hline BW2 (\% of BD2) & & $25.3-33.6$ & 29.7 & 2.7 & 15 & $27.9-38.5$ & 32.3 & 2.6 & 20 \\
\hline
\end{tabular}

Description. Head and body shape, and pigmentation illustrated in Fig. 26 and in original description. Meristic and morphometric data for examined specimens presented in Tables 2-5 and 14. Body moderate to broad in depth. Head short to moderate in length and moderate to broad in depth. Dorsal profile of head approximately straight from occiput to snout, ventral profile of head approximately straight between operculum and snout, snout rounded. Eye small to moderate in size. Upper jaw with moderate to acute sigmoidal angle between premaxillary and maxillary portions in lateral view. No accessory electric organ over operculum. Gill filaments on first gill arch 35-45 (median
$40, \mathrm{n}=6$ ). Pectoral fin moderate in width, pectoral-fin rays 12-17; range and mode higher in populations from Patos-Mirim lagoon system and Uruguay drainage (1417, mode 15) than from Paraguay drainage (12-15, mode 13). Precaudal vertebrae 18-21; range and mode slightly higher in populations from Lagoa dos Patos and Uruguay drainages (20-21, mode 21) than from Paraguay drainage (18-21, mode 20); transitional vertebrae 1-2 (mode 2) in populations from Lagoa dos Patos and Uruguay drainages and 2-3 (mode 2) in populations from the Paraguay drainage. Anal-fin origin slightly $(<0.25 \mathrm{HL}$ distance $)$ anterior to, or near, tip of pectoral fin. Anal-fin rays 180- 
216; ranges and median values very similar in populations from Lagoa dos Patos and Uruguay drainages (187-206, median 200 in examined specimens) and from Paraguay drainage (183-211, median 201). Dorsal rami of recurrent branch of anterior lateral line nerve not visible. Middorsal region of body scaled. Rows of scales above lateral line 4-7 (mode 5). Lateral line continuous. Epidermal canals not conspicuous, but present in form of single bifurcating and sometimes branched horizontal depigmented groove, with darkened margins, on either side of dorsal surface in dorsal portions of flank, beginning approximately two head lengths posterior to occiput and extending to approximately two head lengths anterior to anal-fin terminus. Epidermal canals also variably present in posterior half of body as very sparse single or sometimes parallel double depigmented grooves either side of, but mostly dorsal to, lateral line. Three bilateral horizontal columns at anal-fin terminus, and three or sometimes three alternating with four at or near a mid-point between anal-fin terminus and tip of caudal filament in immature, mature female, and mature male specimens. Some regenerated specimens exhibit four columns of electrocytes in regenerated portions of caudal filament. Caudal filament short to moderate in length.

Coloration. (Fig. 26). Background pale tan to dark brown. Pigmentation highly variable. Dorsal region with prominent depigmented or lightly pigmented stripe extending along midline from occipital region to base of caudal filament, or deep into caudal filament, bordered by prominent dark horizontal wavy reticulated lines from which short irregular patches extend ventrally. Dorsal pigmentation pattern strongly resembles that of $B$. pinnicaudatus, but pale depigmented stripe is not as conspicuous (and in some specimens the depigmented stripe is obscured). Irregular small dark patches or horizontally elongated spots often extend along lateral line. Ventral flank with irregular broken vertical bands and vertically elongated spots, especially over anal-fin pterygiophores. Caudal filament darker than body, with irregular dark markings or bands along entire length. Head with evenly scattered dark chromatophores, darker dorsally. Eye without prominent suborbital patch, or stripe, of chromatophores/subcutaneous pigmentation. Pectoral and anal-fin membranes hyaline. Pectoral-fin rays with light scattering of brown chromatophores. Analfin rays with light or dark patches of chromatophores, with overall anal-fin pigmentation varying greatly, from almost unpigmented to dark mottled pattern. Color in live individuals similar to preserved specimens, with opercular region usually rosy due to underlying gills.

Size. Moderate adult size, largest specimen examined $200 \mathrm{~mm}(\mathrm{n}=754)$. Largest male specimen examined 181 $\mathrm{mm}$ TL, $139 \mathrm{~mm}$ LEA $(\mathrm{n}=25)$. Largest female specimen examined $172 \mathrm{~mm}$ TL, $137 \mathrm{~mm}$ LEA $(\mathrm{n}=37)$. Maximum male size in type series $149.0 \mathrm{~mm}$ LEA vs. $133.3 \mathrm{~mm}$ LEA for females (Giora \& Malabarba, 2009).
Sexual dimorphism. Sexually mature males attain slightly larger sizes, and develop longer and deeper caudal filaments than immature individuals and breeding females (Figs. 26a-b), but do not exhibit an elevated number of bilateral horizontal columns or vertical rows of electrocytes. Instead breeding males exhibit clearly enlarged electrocytes relative to immature specimens and females. Tip of caudal filament in large breeding males usually with paddle-like compression, sometimes free of electrocytes in tip. No known sexual dimorphism in pigmentation.

Geographic distribution. Argentina, Bolivia, Brazil, Paraguay, and Uruguay (Fig. 27). Widely distributed through Patos-Mirim lagoon system, some coastal drainages of Rio Grande do Sul north of Lagoa dos Patos, Uruguay drainage, Paraná downstream of the former Guaíra Falls, and lower to upper Paraguay to as far north as $14^{\circ} 45^{\prime} \mathrm{S}$.

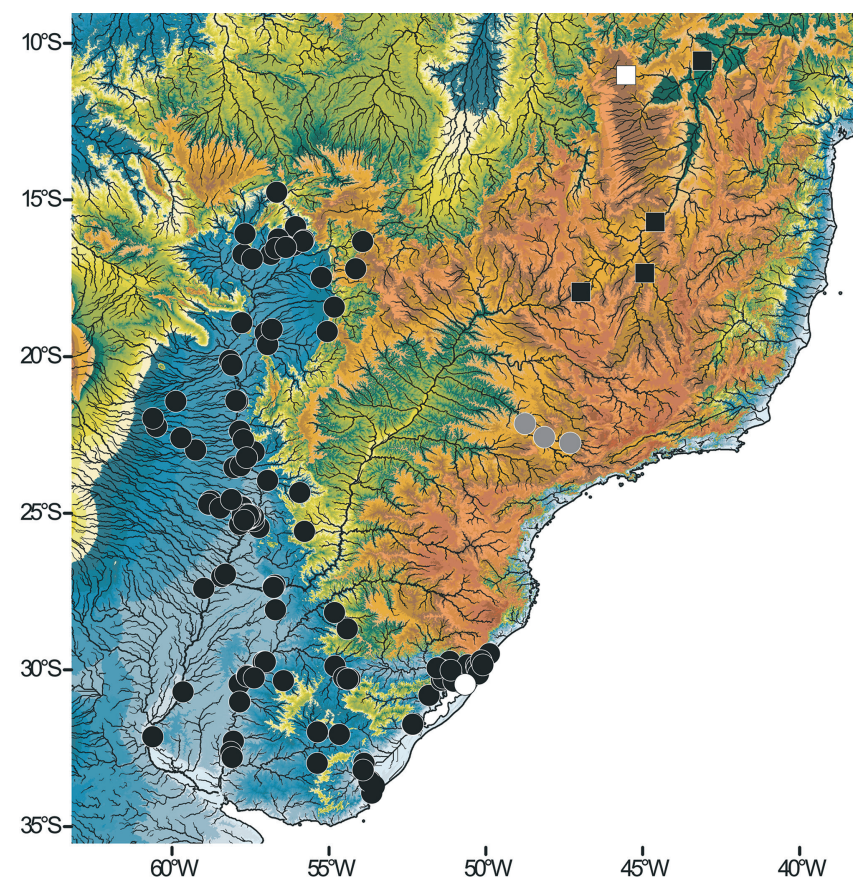

Fig. 27. Collection records for Brachyhypopomus gauderio (circles) and B. menezesi (squares). Holotype locations are marked with open symbols. Grey circles refer to suspected anthropogenic introductions of B. gauderio to upper Paraná dr. (see species redescription in text). Elevation data refers to altitude above mean sea level (see Fig. 2 for legend). Note that the most southerly record for $B$. menezesi is in a headwater of the rio São Francisco, near the drainage divide with rio Paraná headwaters.

Population variation: The osteological diagnostic characters listed in the differential diagnoses that distinguish B. gauderio from B. pinnicaudatus were observed in cleared and stained specimens of B. gauderio from both the Uruguay and Paraná drainages. We also found complete overlap in the range of meristics (Tables 2-5) and morphometric 
proportions (Table 14), and observed similar pigmentation among populations of B. gauderio from the Patos-Mirim lagoon system, Uruguay drainage, and Paraguay drainage. These observations, in combination with molecular data (Crampton et al., 2016), support the hypothesis that all populations we have assigned to $B$. gauderio are members of a single geographically widespread species, which is morphologically and genetically distinct from its allopatric sister species B. pinnicaudatus, known from the Amazon basin and coastal drainages of French Guiana, and from all other congeners.

Ecological notes. Brachyhypopomus gauderio inhabits vegetated river banks, slow-moving creeks, swamps, lagoons, and flooded areas, often with low dissolved oxygen concentrations $\left(<0.5 \mathrm{mgl}^{-1}\right)$ (Giora \& Malabarba, 2009). It is typically the most locally abundant congener throughout its range, and is often one of the most common fishes in aquatic vegetation. Brachyhypopomus gauderio is typically found in systems with relatively high electrical conductivity $\left(50-250 \mu \mathrm{Scm}^{-1}\right)$ and $\mathrm{pH}$ values ranging from 6.5 to 8.5 , but is also found in higher conductivity systems $\left(250-750 \mu \mathrm{Scm}^{-}\right.$ $\left.{ }^{1}\right)$, and less commonly in lower conductivity systems $(<50$ $\mu \mathrm{Scm}^{-1}$ ) (Silva et al., 2003: 333, fig.2). Silva et al. (2003) report large seasonal variations in temperature, conductivity, and $\mathrm{pH}$ from collecting sites in Uruguay. Populations from high latitudes are exposed to a colder, drier austral winter and most foraging and breeding occurs during the warmer summer months (Giora et al., 2014). Respiratory adaptations associated with hypoxia tolerance are discussed by Carter \& Beadle (1931).

Reproductive seasonality, life history, and ecology in B. gauderio are documented by several authors (Gavassa et al., 2012; Giora et al., 2014; Miranda et al., 2008; Quintana et al., 2004; Silva et al., 2003). It breeds during the austral summer, with maturation probably triggered by rising temperature and a change in photoperiod. During the breeding season it lives in dense social groups, and exhibits a sex ratio of mature individuals highly skewed towards females (around 3:1, despite observations of a primary sex ratio close to unity in a long-term laboratory population), possibly due to elevated male mortality related to stress from intrasexual competition (Gavassa et al., 2012; Miranda et al., 2008). Males exhibit a wider spacing, and move less than females - consistent with a polygynous or polygynandrous mating strategy resembling exploded lek polygyny (Miranda et al., 2008). During the austral winter $B$. gauderio exhibits gonadal quiescence, reduced activity and feeding, and a slower EOD rate (Giora et al., 2014; Silva et al., 2002; Silva et al., 2003). Sullivan (1997) cites a personal communication from D. Stewart (State University of New York, Syracuse) reporting that five specimens from FMNH lot 86684 from the Pantanal of Mato Grosso, Brazil (listed below) were "found aestivating in dry soil".

The spawning behavior of $B$. gauderio has been observed from infra-red video recordings in aquaria (pers. comm., P.
Stoddard). Females usually spawn in the presence of one or more males, which fertilize the eggs as they leave the ovipositor. This species also deposits individual fertilized eggs in water hyacinth leaf bracts above the surface. Here trapped pools of rain water presumably form a favorable, predator-free environment for the larva. Parental care of eggs or larvae is unknown in both males and females (Miranda et al., 2008), although see Giora et al. (2014). As in B. occidentalis, adult size classes of B. gauderio more or less disappear subsequent to the breeding season, a pattern consistent with an annual life cycle (Silva et al., 2003).

The diet of $B$. gauderio is dominated by small aquatic insect larvae and microcrustacea, with some (likely incidental) consumption of vegetable matter (Giora et al., 2014).

Co-occurring congeners: Through most of its range, $B$. gauderio occurs in geographical sympatry and in ecological syntopy with $B$. bombilla and B. gauderio. Brachyhypopomus gauderio also co-occurs with $B$. brevirostris and $B$. walteri in the upper río Paraguay.

Local names. Argentina, Paraguay: morenita; Bolivia: cuchillo; Brazil: tuvira, moreninha, tuvira-de-sela (Malabarba et al., 2013) (south), sarapó (north); Uruguay: morenita, limpiavidrio.

Remarks: Identity of the neurobiology model species: $B$. gauderio is a model species for studies of the electrosensory system, and is the subject of multiple papers on neurobiology, neuroethology, EOD plasticity, and ecology - many of which stem from research at the laboratories of Angel Caputi, Omar Macadar, and Ana Silva (Instituto de Investigaciones Biológicas Clemente Estable, Uruguay), Carl Hopkins (Cornell University), Michael Markham (University of Oklahoma), and Philip Stoddard (Florida International University) (see Introduction). Laboratory studies of $B$. gauderio at the Hopkins and Stoddard labs have been based on captive populations that derive from Uruguayan stock (pers. comm., P. Stoddard). We confirmed from cleared and stained specimens that populations from the Stoddard Lab exhibit the osteological characters diagnostic of B. gauderio. Multiple studies prior to $c a .2010$ refer to B. gauderio as B. pinnicaudatus (or Hypopomus pinnicaudatus). Prior to Giora \& Malabarba's (2009) description of B. gauderio, which we confirm here as a valid species, $B$. pinnicaudatus was thought to constitute a single species distributed through much of northern and southern South America.

Invasive populations: We examined three MZUSP lots of B. gauderio collected at sites in the upper Paraná drainage, well upstream of the former Guaíra Falls (drowned by the Itaipu hydroelectric dam since 1982), and also upstream of additional hydroelectric dams (see 'Suspected introduced records' in Material examined). These were collected in 1995 and 2003. Graça \& Pavanelli (2007) and Meschiatti \& Arcifa (2009) list B. gauderio (therein listed as B. pinnicaudatus) as an introduced species in the upper Paraná. 
Citing R. Campos-da-Paz of the Universidade Federal do Rio de Janeiro by personal communication, Graça \& Pavanelli (Graça \& Pavanelli, 2007) suggest that B. gauderio was likely introduced by sport fishermen, who use the species as live bait. They also report that $B$. gauderio is now common in various floodplain lakes in the upper Paraná basin. The sale of live B. pinnicaudatus and species of Gymnotus for sport fishing is very common throughout southern Brazil (pers. comm., F. C. T. Lima, ZUEC), and is also common in the lower Paraguay basin (WGRC pers. obs. from Entre Ríos, Argentina) and upper rio Xingu (pers. comm., R. E. Reis, MCP). Galves et al. (2009), and Meschiatti \& Arcifa (2009) comment on the prevalence of introduced fish species in the upper Paraná. We illustrate the occurrence of these introductions in Fig. 27 (with grey circles) but omit them from the range of the genus presented in Fig. 2.

Material examined. 753 specimens. Argentina (localities from Paraná dr.). Chaco. MSNG 27458, 2, 98-102 mm LEA, Resistencia, Paraná dr., ca. $27^{\circ} 27^{\prime} \mathrm{S}, 058^{\circ} 59^{\prime} \mathrm{W}$ (from photograph). Corrientes. MACN-ict 9459, 9, 109-134 mm, Iberá wetlands, Laguna Galarza, Paraná dr., 28 $04^{\prime} 56^{\prime \prime} \mathrm{S}, 056^{\circ} 42^{\prime} 04^{\prime \prime} \mathrm{W}$. Entre Ríos. MACN-ict 9460, 1, 92 mm, Pre-Delta National Park, Arroyo Los Dorados, río Paraná floodplain, Paraná dr. $32^{\circ} 08^{\prime} 08^{\prime \prime}$ S, 060 37'26"W. UF 177364, 5 (1CS), 135-175 mm, nr. La Paz, río Paraná floodplain, Paraná dr., ca. $30^{\circ} 42^{\prime} \mathrm{S}, 059^{\circ} 39^{\prime} \mathrm{W}$. Bolivia. Santa Cruz. FMNH 54542, 1, $77 \mathrm{~mm}$, Puerto Suárez, río Paraguay, Paraná dr., ca. $18^{\circ} 55^{\prime} \mathrm{S}, 057^{\circ} 47^{\prime} \mathrm{W}$. Brazil. Mato Grosso (localities from rio Paraguai [Paraguay] dr., Paraná dr.). AMNH 97895, 2, 55-117 mm, rio Pixaim [Pixaime], ca. $16^{\circ} 44^{\prime} \mathrm{S}, 056^{\circ} 51^{\prime} \mathrm{W}$. BMNH 1895.5.17.265-268 (part), 1, $64 \mathrm{~mm}$, mun. Cácares, Descalvados, $16^{\circ} 43^{\prime} 59^{\prime \prime} \mathrm{S}, 057^{\circ} 44^{\prime} 54^{\prime \prime} \mathrm{W}$. BMNH 1900.4.14.91-94 (part), 2, 75-79 mm, mun. Carandàzinho, rio Cuiabá, ca. $16^{\circ} 27^{\prime} \mathrm{S}, 056^{\circ} 08^{\prime} \mathrm{W}$. CAS $72225,1,82 \mathrm{~mm}$, "Mato Grosso, Brazil or Asunción, Paraguay [or río Paraguay], no coordinates. FMNH 86684, 5, 79-127 mm, Porto Cercado, side of Poconé-Porto Cercado rd., rio Cuiabá, affl. rio Piquiri, $16^{\circ} 30^{\prime} \mathrm{S}, 056^{\circ} 22^{\prime} \mathrm{W}$. FMNH 108541 , 12, 55-100 mm, mun. Corumbá, on hwy. MS-184, swamp, lower rio Negro, $19^{\circ} 20^{\prime} 17^{\prime \prime} \mathrm{S}, 056^{\circ} 57^{\prime} 42^{\prime \prime} \mathrm{W}$. FMNH 120674, 1, $115 \mathrm{~mm}$, mun. Cáceres, nr. Cáceres, $c a .16^{\circ} 05^{\prime} \mathrm{S}, 057^{\circ} 41^{\prime} \mathrm{W}$. MZUSP 4460, 1, 45 mm, mun. Santo Antônio de Leverger, lake nr. Santo Antônio de Leverger, rio Cuiabá, $c a .15^{\circ} 51^{\prime} \mathrm{S}, 056^{\circ} 04^{\prime} \mathrm{W}$. MZUSP 19224, 1 , Poconé, rio Cuiabá, $c a .16^{\circ} 15^{\prime} \mathrm{S}, 056^{\circ} 37^{\prime} \mathrm{W}$. MZUSP 25149 , 2, 67-70 mm, mun. Cáceres, Ilha da Taiamã [Estação Ecológica Taiamã], $16^{\circ} 53^{\prime} \mathrm{S}, 057^{\circ} 27^{\prime} \mathrm{W}$ [coordinates from center of island]. MZUSP 35910, 1, 67 mm, mun. Itiquira, Fazenda Santo Antônio do Paraíso, lakes between rio Piquiri and rio Itiquiri, $c a .17^{\circ} 12^{\prime} \mathrm{S}, 054^{\circ} 09^{\prime} \mathrm{W}$. MZUSP 77202, 5, 25-134 mm, Nobres Paraguai, Represa da Fazenda Pé de Serra, rio Cuiabá, $14^{\circ} 45^{\prime} \mathrm{S}, 056^{\circ} 40^{\prime} \mathrm{W}$. MZUSP 83562, 3, immature, 55-67 mm, MZUSP 83603, 1, $124 \mathrm{~mm}$, temporary lake, rio Itiquira/Piquiri, $17^{\circ} 28^{\prime} 13^{\prime \prime} \mathrm{S}, 055^{\circ} 14^{\prime} 46^{\prime \prime} \mathrm{W}$. MZUSP 83607 (part), 3, immature, 50-76 mm, mun. Coxim, Paraguai Lagoa, source of Ribeirao dos Veados, affl. rio Coxim (rio Taquari), 18 $25^{\prime} 21^{\prime \prime} \mathrm{S}$, 054 $50^{\prime} 06^{\prime \prime} \mathrm{W}$. MZUSP 96652, 2, 66-67 mm, Pantanal de Paiaguás, swamp $1 \mathrm{~km}$ Vila de Mimoso, $16^{\circ} 17^{\prime} \mathrm{S}, 055^{\circ} 48^{\prime} \mathrm{W}$. MZUSP 96703, 2 , immature, 63-69 mm, mun. Barão de Melgaço, Pantanal de Paiaguás, rio Mutum, between Vila de Mimoso and Joselândia, $16^{\circ} 19^{\prime} 30^{\prime \prime} \mathrm{S}$,
05549'59"W. ZUEC 3697, 2, 86-101 mm, ZUEC 5146, 3, 74-106 $\mathrm{mm}$, ZUEC 5726, 15, 80-115 mm, mun. Poconé, Porto Cercado, rio Cuiabá, $16^{\circ} 31^{\prime} \mathrm{S}, 056^{\circ} 23^{\prime} \mathrm{W}$. ZUEC 4473, 2, $42-49$ mm, mun. Poconé, Transpantaneira hwy, km 24, rio Claro, $16^{\circ} 33^{\prime} \mathrm{S}, 056^{\circ} 42^{\prime} \mathrm{W}$. ZUEC 5466, 1, $74 \mathrm{~mm}$, mun. Poconé, rio Piraputanga, affl. rio Vermelho, affl. rio Cuiabá, ca. $16^{\circ} 20^{\prime} \mathrm{S}, 053^{\circ} 56^{\prime} \mathrm{W}$. Mato Grosso do Sul. MUSM 17129, 2 (1 immature, $80 \mathrm{~mm}, 1$ female, $101 \mathrm{~mm}$ ), rio Negro on rd. between "NHE Colandia and Brazcs", 19 12'09"S, $055^{\circ} 03^{\prime} 23^{\prime \prime} \mathrm{W}$ [longitude reported incorrectly on label as 052 $03^{\prime} 23^{\prime \prime} \mathrm{W}$ ]. MZUSP 36386, 5, 36-98 mm, mun. Corumbá, Fazenda Nhumirim, Nhecolândia, $19^{\circ} 15^{\prime} \mathrm{S}, 057^{\circ} 02^{\prime} \mathrm{W}$. MZUSP 59442 (part), 10 (4 immature, $67-92 \mathrm{~mm}, 6$ female, $62-113 \mathrm{~mm}$ ), rio Vermelho, rio Miranda, $19^{\circ} 37^{\prime} 22^{\prime \prime} \mathrm{S}, 056^{\circ} 57^{\prime} 27^{\prime \prime} \mathrm{W}$. MZUSP 59646, 29 (1CS), 45-172 mm (14 sexed: 2 immature, $67-81 \mathrm{~mm}, 5$ female, 108-172 mm, 7 male, 96-168 mm), Baía da Passagem, at Fazenda Caceres, Nhecolândia, $19^{\circ} 08^{\prime} 17^{\prime \prime} \mathrm{S}, 056^{\circ} 49^{\prime} 14^{\prime \prime} \mathrm{W}$. Rio Grande do Sul. FMNH 54538, 1, $101 \mathrm{~mm}$, Cacequy [Cacequi], rio Ibicuí, Uruguay dr., ca. $29^{\circ} 53^{\prime} \mathrm{S}, 054^{\circ} 49^{\prime} \mathrm{W}$. FMNH 54546, 1, male, 180 $\mathrm{mm}$, Uruguiana, rio Uruguai, Uruguay dr., $c a .29^{\circ} 45^{\prime} \mathrm{S}, 057^{\circ} 05^{\prime} \mathrm{W}$. MCP 9710, 1, male, $164 \mathrm{~mm}$, mouth of the arroio da Faxina, affl. rio Guaíba, Patos-Mirim dr., no coordinates. MCP 11135, 1, male, 144 $\mathrm{mm}$, mun. Santo Angelo, Fazenda dos Hinz, rio Piratini, Uruguay dr., ca. $28^{\circ} 42^{\prime} \mathrm{S}, 054^{\circ} 25^{\prime} \mathrm{W}$. MCP 13956, 2, 83-94 mm, hwy. BR-290, ca. $10 \mathrm{~km}$ BR-116, affl. rio Jacuí, affl. rio Guaíba, Patos-Mirim dr., $30^{\circ} 03^{\prime} 13^{\prime \prime} \mathrm{S}, 051^{\circ} 26^{\prime} 17^{\prime \prime} \mathrm{W}$. MCP 14177, 1, $168 \mathrm{~mm}$, channel, hwy. BR-290, ca. 12 km BR-116, affl. rio Jacuí, affl. rio Guaíba, PatosMirim dr., 30 $03^{\prime} 19^{\prime \prime} \mathrm{S}, 051^{\circ} 27^{\prime} 17^{\prime \prime} \mathrm{W}$. MCP 14459, 2 (1 female, 138 $\mathrm{mm}, 1$ male, $140 \mathrm{~mm}$ ), BR-290, channel, ca. $2 \mathrm{~km} \mathrm{BR-116,} \mathrm{affl.} \mathrm{rio}$ Jacuí, affl. rio Guaíba, Patos-Mirim dr., $30^{\circ} 02^{\prime} 39^{\prime \prime} \mathrm{S}, 051^{\circ} 21^{\prime} 20^{\prime \prime} \mathrm{W}$. MCP 14606, 5 (1 CS) (2 female, 139-147 mm, 3 male, 128-156 mm), channel, hwy. BR-290, $c a .2$ km BR-116, affl. rio Jacuí, affl. rio Guaíba, Patos-Mirim dr., 30 $02^{\prime} 46^{\prime \prime S}, 051^{\circ} 21^{\prime} 07^{\prime \prime} W$. MCP 14775, 2 , male, 142-178 mm, mun. Uruguaiana, Sanga Bárbara, Uruguaiana, affl. rio Uruguai, Uruguay dr., $29^{\circ} 48^{\prime} 00^{\prime \prime} \mathrm{S}, 057^{\circ} 06^{\prime} 00^{\prime \prime} \mathrm{W}$. MCP 15326 (part), 2, 101-114, canals along hwy. BR-290 between 1 and 6 km junction with hwy. BR-116, affl. rio Jacuí, affl. rio Guaíba, PatosMirim dr., between $30^{\circ} 02^{\prime} 39^{\prime \prime} \mathrm{S}, 051^{\circ} 21^{\prime} 20^{\prime \prime} \mathrm{W}$ and $30^{\circ} 02^{\prime} 48^{\prime \prime} \mathrm{S}$, 051 ${ }^{\circ} 23^{\prime} 27^{\prime \prime}$ W. MCP 19547, 2, 140-200 mm, bridge on São GabrielTiaraju hwy., affl. rio Vacacaí, affl. rio Jacuí, affl. rio Guaíba, PatosMirim dr., 30 $0^{\circ} 17^{\prime} 29^{\prime \prime} \mathrm{S}, 054^{\circ} 20^{\prime} 18^{\prime \prime} \mathrm{W}$. MCP 19549, 6, 48-156 mm, MCP 19553, 2, 35-60 mm, bridge over Banhado do Inhatium, hwy. BR-290, 21 km São Gabriel, affl. rio Ibicuí, Uruguay dr., 30¹5'43"S, 054³1'33"W. MCP 20216, 3, 70-100 mm, mun. Eldorado do Sul, on hwy. BR-290, affl. rio Jacuí, affl. rio Guaíba, Patos-Mirim dr., $30^{\circ} 02^{\prime} 36^{\prime \prime} \mathrm{S}, 051^{\circ} 20^{\prime} 56^{\prime \prime} \mathrm{W}$. MCP 20218, 3, 109-157 mm, arroio Passos dos Carros, on rd. linking hwy. BR-116 to Guaíba, affl. rio Jacuí, affl. rio Guaíba, Patos-Mirim dr., $30^{\circ} 05^{\prime} 55^{\prime \prime} \mathrm{S}, 051^{\circ} 23^{\prime} 15^{\prime \prime} \mathrm{W}$. MCP 21168, 1, female, $104 \mathrm{~mm}, \mathrm{MCP} 21169,1,115 \mathrm{~mm}$, channel on margin hwy. BR-290, affl. rio Jacuí, affl. rio Guaíba, Patos-Mirim dr., $30^{\circ} 02^{\prime} 36^{\prime \prime} \mathrm{S}, 051^{\circ} 20^{\prime} 56^{\prime \prime} \mathrm{W}$. MCP 21726, 5, 87-134 mm, mun. Eldorado do Sul, channel on margin hwy. BR-290 ca. $5 \mathrm{~km}$ junction with BR-116, affl. rio Jacuí, affl. rio Guaíba, Patos-Mirim dr., $c a$. $30^{\circ} 03^{\prime} \mathrm{S}, 051^{\circ} 24^{\prime} \mathrm{W}$. MCP 37086, 1, $100 \mathrm{~mm}$, mun. Cerro Largo, arroio Brum Lageado, affl. rio Ijuí, Uruguay dr., $28^{\circ} 12^{\prime} 50^{\prime \prime} \mathrm{S}$, 054 $49^{\prime} 36^{\prime \prime} \mathrm{W}$. MCP 37580, 1, $131 \mathrm{~mm}$, mun. Pelotas, nr. bridge to Pelotas, rio São Gonçalo, Patos-Mirim dr., $31^{\circ} 46^{\prime} 19^{\prime \prime} \mathrm{S}, 52^{\circ} 20^{\prime} 33^{\prime \prime} \mathrm{W}$. 
MCP 43280, 1 (holotype), male, 180 mm TL, 149 mm LEA, mun. Palmares do Sul, channel connecting Lagoa do Casamento and Lagoa dos Gateados, Patos-Mirim dr., ca. 30²8'S, 05040’W. MCP 43281, 1 (paratype), female, $124 \mathrm{~mm}$ LEA, MCP 43282, 1 (paratype), female, 133 mm LEA, mun. Viamão, Lagoa Negra, Parque Estadual de Itapuã, Patos-Mirim dr. MCP 43283, 2 (paratypes) (1 female, 83 mm LEA, 1 male, 92 mm LEA), mun. Capão da Canoa, Lagoa dos Quadros, rio Tramandaí dr., ca. 29² $2^{\prime} \mathrm{S}, 050^{\circ} 06^{\prime} \mathrm{W}$. MNRJ 30915, 2 (paratypes) (1 female, $85 \mathrm{~mm}$ LEA, 1 male, $70 \mathrm{~mm}$ LEA), UFRGS 8874, 1, 154 mm, mun. Cidreira, Lagoa Fortaleza, rio Tramandaí dr., $30^{\circ} 08^{\prime} 58^{\prime \prime} \mathrm{S}, 050^{\circ} 14^{\prime} 30^{\prime \prime} \mathrm{W}$. MNRJ 32792, 5 (paratypes) (2 female, 75-78 mm LEA, 3 male, 74-97 mm LEA), UFRGS 4240, 10, 42-134 mm, mun. Barra do Ribeiro, lake on hwy. linking BR-116 to Barra do Ribeiro, Patos Mirim dr., ca. $30^{\circ} 17^{\prime} \mathrm{S}, 051^{\circ} 21^{\prime} \mathrm{W}$. MNRJ 32793, 1 (paratype) male, $136 \mathrm{~mm}$ LEA, MCP 43284, 1 (paratype) male, 123 mm LEA, MZUSP 100309, 15 (paratypes) (7 female, 78-127 mm, 8 male, 60-127 mm), UFRGS 6499, 1 (paratype), male, $123 \mathrm{~mm}$, UFRGS 6500, 1, female, $123 \mathrm{~mm}$, UFGRS 6501, 1, female, $125 \mathrm{~mm}$, UFRGS 6502, 1, female, $162 \mathrm{~mm}$, UFRGS 6503, 1, (paratype), male, 103 mm, UFRGS 6504, 1, male, 152 mm, UFGRS 6505, 1, male, 178 mm, UFRGS 6506, 1, male, 185 mm, UFRGS 6512, 1, female, 131 mm, mun. São Gabriel, stream, affl. arroio Piraí, on margin hwy. BR290, affl. rio Cacequí, affl. rio Santa Maria, affl. rio Ibicuí, Uruguay

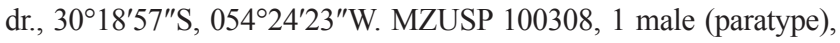
$140 \mathrm{~mm}$ LEA, UFRGS 9581, 2 (paratypes) (1 female, 126 mm LEA, 1 male, 129 mm LEA), UFRGS 10032, 1 (paratype), female, $102 \mathrm{~mm}$ LEA, UFRGS 10035, 1 (paratype), female, 106 mm LEA, UFRGS 10042, 1 (paratype), male (CS), 120 mm LEA, UFRGS 10048, 1 (paratype), male (CS), $118 \mathrm{~mm}$ LEA, mun. Charqueadas, flooded area nr. Arroio dos Ratos, rio Jacuí, Patos-Mirim dr., 2957'32"S, 051³3'10"W. MZUSP 23167, 1, 52 mm, km 124, Camaquá-Pelotas hwy. BR-116, Patos-Mirim dr., ca. $31^{\circ} 45^{\prime} \mathrm{S}, 052^{\circ} 20^{\prime} \mathrm{W}$. MZUSP 23169, 1, $43 \mathrm{~mm}$, Porto Alegre, rio Jacuí, Patos-Mirim dr., ca. $30^{\circ} 02^{\prime} \mathrm{S}, 051^{\circ} 13^{\prime} \mathrm{W}$. MZUSP 23630, 3 (1 immature, $87 \mathrm{~mm}, 1$ female, 127 mm, 1 male, 118 mm), São Leopoldo, Banhado Schreck, affl. rio dos Sinos, affl. rio Jacuí, affl. rio Guaíba, Patos-Mirim dr., ca. $29^{\circ} 45^{\prime} \mathrm{S}, 051^{\circ} 09^{\prime} \mathrm{W}$. MZUSP 25165 (part), 3, 97-132 mm, Camaquã, rio Jacuí, affl. rio Guaíba, Patos-Mirim dr., ca. $30^{\circ} 50^{\prime} \mathrm{S}, 051^{\circ} 49^{\prime} \mathrm{W}$. NRM 27641, 2, 75-103 mm, stream, affl. Lagoa dos Quadros, nr. Praia do Barco, rio Tramandaí dr., ca. $29^{\circ} 42^{\prime} \mathrm{S}, 050^{\circ} 05^{\prime} \mathrm{W}$. UFRGS 187, 1, 97 mm, UFRGS 719, 2, 137-141 mm, mun. Guaíba, Praia de Sans-Souci, rio Guaíba, Patos-Mirim dr., 30 $03^{\prime} 05^{\prime \prime} \mathrm{S}, 051^{\circ} 18^{\prime} 35^{\prime \prime} \mathrm{W}$. UFRGS 733, 1, 53 mm, mun. Guaíba, stream on hwy. BR-290, 2 km junction with BR-116, affl. rio Guaíba, Patos-Mirim dr., $30^{\circ} 03^{\prime} 00^{\prime \prime} \mathrm{S}$, 051²0'59"W. UFRGS 2219, 2 (paratypes), 100-152 mm, mun. Santo Antônio da Patrulha, creek between BR-290 and Santo Antônio da Patrulha, Patos-Mirim dr., ca. $29^{\circ} 52^{\prime} \mathrm{S}, 050^{\circ} 35^{\prime} \mathrm{W}$. UFRGS 5641, 3 (paratypes), 101-158 mm, mun. Viamão, Lagoa do Palácio, PatosMirim dr., 30²4'10"S, 05057'25"W. UFRGS 3845, 1, 84 mm, mun. Palmares do Sul, Lagoa dos Barros, 1st canal, hwy. to Capivarí, Patos-Mirim dr., ca. $29^{\circ} 53^{\prime} \mathrm{S}, 050^{\circ} 18^{\prime} \mathrm{W}$. UFRGS 3895, 2, 78-86 mm, mun. Guaíba, mun. Barra do Ribeiro, arroio Ribeirinho, affl. rio Guaíba, Patos-Mirim dr., 30²1'00"S, 051'24'59"W. UFRGS 4003, 3, 51-72 mm, mun. Cidreira, Lagoa do Lessa, at bridge, rio Tramandaí dr., $29^{\circ} 50^{\prime} 00^{\prime \prime} \mathrm{S}, 050^{\circ} 10^{\prime} 00^{\prime \prime} \mathrm{W}$. UFRGS 4061, 2, 26-85 mm, 2nd canal to Lagoa dos Índios, km 10, rio Tramandaí dr., $c a .29^{\circ} 59^{\prime} \mathrm{S}$, $050^{\circ} 17^{\prime} \mathrm{W}$. UFRGS 5015, 1, 132mm, mun. Arambaré, Patos-Mirim dr., no coordinates. UFRGS 6516, 1, female, $132 \mathrm{~mm}$, UFRGS 6517, 1, female, 143 mm, UFRGS 6518, 1, female, 151 mm, UFRGS 6519, 1, female, 136 mm, UFRGS 6520, 1, female, 144 mm, UFRGS 6524, 1 (paratype) male, $160 \mathrm{~mm}$, UFRGS 10036, 1 (paratype), male (CS), 133 mm LEA, mun. Viamão, Lagoa Negra, Parque Estadual de Itapuã, Patos-Mirim dr., 30²2'52"S, $051^{\circ} 01^{\prime} 25^{\prime \prime}$ W. UFRGS 6765, 1, $141 \mathrm{~mm}$, UFRGS 6768, 1, $149 \mathrm{~mm}$, mun. Uruguiana, stream, affl. Uruguay dr., $29^{\circ} 45^{\prime} 56^{\prime \prime} \mathrm{S}, 057^{\circ} 02^{\prime} 01^{\prime \prime} \mathrm{W}$. UFRGS 8933, 1 (paratype), female, $102 \mathrm{~mm}$ LEA, arroio do Sal, creek on Estrada do Mar rd., rio Tramandaí dr., 29³0'33"S, 04953'34"W. UFRGS 14563, 1, 88 mm, mun. Maquiné, mouth of rio Maquiné, Lagoa dos Quadros, rio Tramandaí dr., 2943'38"S, 05008'36"W.UFRGS 14963, 6, 92-135 $\mathrm{mm}$, mun. Tramandaí, Lagoa do Passo, rio Tramandaí dr., 2952'01"S, 05006'00"W. USNM 191645, 1, 77 mm, Porto Alegre, rio Guaíba, Patos-Mirim dr., $\mathrm{ca} .30^{\circ} 02^{\prime} \mathrm{S}, 051^{\circ} 07^{\prime} \mathrm{W}$. Paraguay (localities from Paraná dr.). Alto Paraguay (localities from río Paraguay dr.). FMNH 108059, 3, 93-130 mm, lagoon nr. Estância Cerrito, 2127's, $057^{\circ} 55^{\prime} \mathrm{W}$. FMNH $108060,1,140 \mathrm{~mm}$, flooded palm forest, riacho

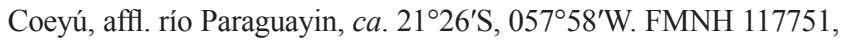
1, $59 \mathrm{~mm}$, río Negro, at confl. with río Paraguay, ca. $20^{\circ} 09^{\prime} \mathrm{S}$, $058^{\circ} 10^{\prime} \mathrm{W}$. MZUSP 54332, 1, female, $104 \mathrm{~mm}$, Puerto 14 de Maio, ca. $20^{\circ} 18^{\prime} \mathrm{S}, 058^{\circ} 06^{\prime} \mathrm{W}$. Boquerón (localities from río Paraguay dr.). ANSP 185115, 1, $132 \mathrm{~mm}$, pond, N side TransChaco hwy. at km 464.1 , ca. $22^{\circ} 15^{\prime} \mathrm{S}, 060^{\circ} 30^{\prime} \mathrm{W}$. ANSP $185116,5,84-134 \mathrm{~mm}$, marsh at Mariscal Estigarribia, ca. $22^{\circ} 00^{\prime} \mathrm{S}, 060^{\circ} 37^{\prime} \mathrm{W}$. NRM 35483, 1, 181 $\mathrm{mm}$, Estancia Água de Sidia, $c a . \mathrm{km} 100$ on Filadelfia-Madrejon hwy. (5.2 km off rd.), ca. $21^{\circ} 27^{\prime} \mathrm{S}, 059^{\circ} 53^{\prime} \mathrm{W}$. Caaguazú. NRM 42920, 1, 47 mm, stream crossing Colonia Sommerfeld-Repatriación rd., affl. río Paraguay, $25^{\circ} 36^{\prime} 02^{\prime \prime} \mathrm{S}, 055^{\circ} 47^{\prime} 11^{\prime \prime} \mathrm{W}$. Central (localities from río Paraguay dr.). AMNH 1009, 1, CAS 72215, 2, 143-152 mm, Campo Grande, lagoons $5 \mathrm{~km}$ Asunción, ca. $25^{\circ} 19^{\prime} \mathrm{S}, 057^{\circ} 31^{\prime} \mathrm{W}$. MZUSP 77990, 1, 34 mm, Balneário Joly, Zona de Pirayú, ca. $25^{\circ} 29^{\prime} \mathrm{S}, 057^{\circ} 14^{\prime} \mathrm{W}$. NRM 42400, 1, $123 \mathrm{~mm}$, swamp nr. lago Ypacaraí, N Areguá, 2516'16"S, 057²3'51"W. UMMZ 205591, 2, 102-103 mm, ca. $1 \mathrm{~km} \mathrm{~S}$ Puente Remanso, E shore río Paraguay, 2512'30"S, 057³3'00"W. UMMZ 205874, 11, 41-125 mm, ca. 1 $\mathrm{km}$ S Puente Remanso, flooded pasture at margin río Paraguay, 2512'30"S, 057³3'00"W. UMMZ 205912, 5, 27-78 mm, Arroyo Pirayú, $13 \mathrm{~km} \mathrm{~W}$ Caacupe, affl. lago Yparcaraí, río Salado, 252'ㄹ' $2^{\prime \prime S}, 057^{\circ} 14^{\prime} 42^{\prime \prime} \mathrm{W}$. UMMZ 206001, 4 (part), 97-144 mm, río Salado (nr. mouth) at bridges 5.4-5.85 km N Limpio, ca. $25^{\circ} 08^{\prime} \mathrm{S}$, $057^{\circ} 25^{\prime} \mathrm{W}$. Concepción (localities from río Paraguay dr.). NRM 43362, 1, 90 mm, NRM 44254, 3, 19-31 mm, NRM 44259, 23, 30$140 \mathrm{~mm}$, Isla Peña Hermosa, río Paraguay, S mouth riacho La Paz, 22²4'09"S, 057 51'13"W. UMMZ 207817, 1, $126 \mathrm{~mm}$, Paso Horqueta, río Aquidabán, $23^{\circ} 03^{\prime} 48^{\prime \prime} \mathrm{S}, 057^{\circ} 23^{\prime 2} 24^{\prime \prime} \mathrm{W}$. ZMH 11631, 1,

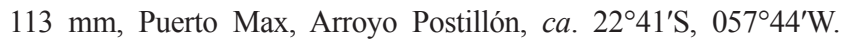
Misiones. ANSP 175178, 3, 110-149 mm, pool (rd. to Ayolas?), affl. río Paraná, $27^{\circ} 19^{\prime} 40^{\prime \prime} \mathrm{S}, 056^{\circ} 43^{\prime} 54^{\prime \prime} \mathrm{W}$. ANSP 185118, 2, 32-79 mm, marshes on rd. from Ayolas to main Asunción-Encarnación hwy.

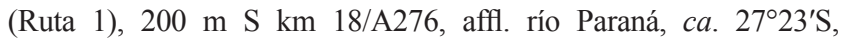
$056^{\circ} 47^{\prime} \mathrm{W}$. UMMZ 207463, 1, $151 \mathrm{~mm}$, río Paraná ca. $2 \mathrm{~km}$ E Ayolas, río Paraná, $27^{\circ} 24^{\prime} 00^{\prime \prime} \mathrm{S}, 056^{\circ} 46^{\prime} 12^{\prime \prime} \mathrm{W}$. Ñeembucú (localities from río Paraguay dr.). NRM 32801, 1, 48 mm, NRM 32829, 9, 53-102 mm, NRM 32852, 1, 42 mm, Paso Cornelio, swamp, Pilar-Humaitá 


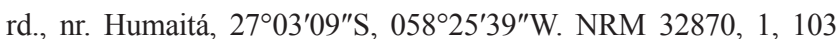

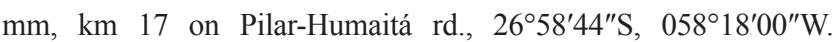
Presidente Hayes (localities from río Paraguay dr.). AMNH 99960, 50, 51-130 mm, AMNH 99977, 1, 154 mm, Estancia la Golondrina, $140 \mathrm{~km}$ NW Asunción, marshlands affl. río Confuso, ca. 24³6'S, $058^{\circ} 07^{\prime} \mathrm{W}$. ANSP $170413,3,85-96 \mathrm{~mm}$, pool, km 15, Ruta General Bruguez, ca. $24^{\circ} 45^{\prime} \mathrm{S}, 058^{\prime} 49^{\prime} \mathrm{W}$ [coordinates for Fortín General Bruguez]. ANSP 185114, 1, 63 mm, pools on Rancho Salmo 23, ca. $8 \mathrm{~km}$ towards Clorinda from Puente Remanso, affl. río Negro, $c a$. $25^{\circ} 11^{\prime} \mathrm{S}, 057^{\prime} 34^{\prime} \mathrm{W}$. ANSP 185117, 1, $129 \mathrm{~mm}$, pools nr. Puente Remanso, ca. $25^{\circ} 10^{\prime} \mathrm{S}, 057^{\circ} 33^{\prime} \mathrm{W}$. FMNH 54540, 2, 66-125 mm, Villa Hayes, $c a .25^{\circ} 05^{\prime} \mathrm{S}, 057^{\circ} 31^{\prime} \mathrm{W}$. NRM $22601,1,32 \mathrm{~mm}$, military

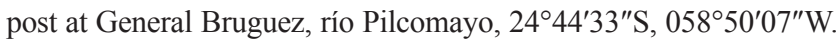
NRM 27919, 1, 140 mm, NRM 32525, 4, 61-135 mm, infantry regiment Pedro Juan Caballero, río Pilcomayo, 24³8'56"S, 058 48'18"W. NRM 32524, 10, 81-105 mm, General Bruguez, ca. 1 km N military camp, río Pilcomayo, 2443'38"S, 058 $49^{\prime} 10^{\prime \prime} \mathrm{W}$. NRM 32526, 11, 57-156 mm, military post at general Bruguez, río Pilcomayo, 244ㄴ $43^{\prime \prime}$ S, 058 50'10"W. NRM 32528, 6, 69-104 mm,

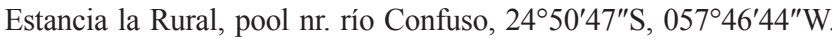
NRM 35257, 39, 92-144 mm, Tajamar, km 78, trans-Chaco rd.,

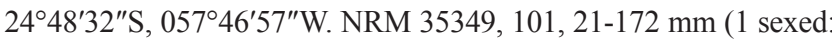
1 male, $172 \mathrm{~mm}$ ), Estero Guazú at Estancia Loma Verde, ca. $10 \mathrm{~km}$ Concepción-Pozo Colorado rd., 2334'53"S, 058 01'53"W. NRM 35447, 5, 26-117 mm, NRM 35830, 8, 38-106 mm, N branch riacho Negro at Makthlawayia, $23^{\circ} 15^{\prime} 58^{\prime \prime} \mathrm{S}, 057^{\circ} 37^{\prime} 42^{\prime \prime} \mathrm{W}$. NRM 35668, 7, 58-106 mm, km 37, Pozo Colorado-Concepción rd., riacho Negro, $23^{\circ} 30^{\prime} 53^{\prime \prime} \mathrm{S}, 057^{\circ} 49^{\prime} 17^{\prime \prime} \mathrm{W}$. UMMZ 206972, 3, 95-120 mm, Puente Remanso bridge [nr. Remansito], ca. $25^{\circ} 10^{\prime} \mathrm{S}, 057^{\circ} 32^{\prime} \mathrm{W}$. UMMZ 207026, 24, 45-145, marsh, $34.3 \mathrm{~km}$ NW toll booth on puente

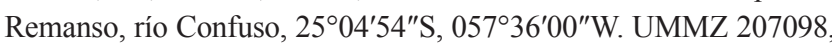
4, 106-138 mm, small stream ca. 33.7 km NW toll booth on Puente Remanso bridge (Estancia La Golondrina), affl. río Confuso, $25^{\circ} 04^{\prime} 54^{\prime \prime} \mathrm{S}, 057^{\circ} 36^{\prime} 00^{\prime \prime} \mathrm{W}$. UMMZ 207369, 6, 127-154 mm, TransChaco hwy., E side, $0.2 \mathrm{~km}, 1.0 \mathrm{~km}$ SE entrance to Estancia Juan de Zalazar, riacho San Carlos, $23^{\circ} 01^{\prime} 36^{\prime \prime} \mathrm{S}$, 059 $15^{\prime} 00^{\prime \prime} \mathrm{W}$. UMMZ 207381, 11, 118-171 mm, pond E rd. ca. 6.6 km N Trans-Chaco

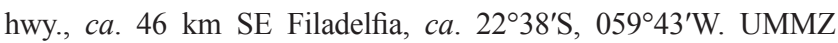
207565, 4, 151-188 mm, UMMZ 207598, 9, 55-140 mm, pools at bridge (Puerto Falcón to Argentina), ca. $12 \mathrm{~km}$ WSW Chaco-I, río Pilcomayo, 25 $5^{\circ} 5^{\prime} 48^{\prime \prime} \mathrm{S}, 057^{\circ} 42^{\prime} 36^{\prime \prime} \mathrm{W}$. UMMZ 207588, 1, $88 \mathrm{~mm}$, pools ca. $83.2 \mathrm{~km}$ Puerto Falcon, 2452'54"S, 058 18'36"W. UMMZ 207620, 1, $120 \mathrm{~mm}$ LEA, riacho Pilco, ca. $25^{\circ} 06^{\prime} \mathrm{S}, 057^{\circ} 46^{\prime} \mathrm{W}$. UMMZ 216612, 4, 72-93 mm, Estancia La Golondrina, marshlands, affl. río Confuso, $c a$. $24^{\circ} 36^{\prime} \mathrm{S}, 058^{\circ} 07^{\prime} \mathrm{W}$. USNM 232235, 4, 78-106 $\mathrm{mm}$, río Confusa [Confuso], ca. $25 \mathrm{~km}$ E Trans-Chaco, ca. $25^{\circ} 06^{\prime} \mathrm{S}$, $057^{\circ} 34^{\prime} \mathrm{W}$. San Pedro (localities from río Paraguay dr.). NRM 33612, 1, $48 \mathrm{~mm}, \mathrm{~km} 20$ on San Pedro-Nuevo Germania rd., 2359'23"S, 056 57'13"W. UMMZ 206572, 1, $122 \mathrm{~mm}$, río Corrientes, affl. río Jejuí Guazú, 24²2'54"S, 05556'24"W. Region not specified. BMNH 1927.11.23.31, 1, $117 \mathrm{~mm}$, swamp at Makthalawaiya, Paraguayan Chaco, no coordinates. CAS 72224, 2, 38-56 mm, FMNH 52591, 1, 140 mm, Arroyo Chagalalina, affl. río Aquido Canigi, no coordinates. NRM 33632, 1, 132 mm, NRM 33634, 1, $27 \mathrm{~mm}$, listed "labels lost, but known to come from S. Kullander's or B. Delling's collection efforts [in Paraguay]".
Uruguay. Artigas (localities from Uruguay dr.). MNHN-Uruguay 1746, 1, río Cuareim, Laguna de las Lavanderas, 30²3'34"S, $056^{\circ} 27^{\prime} 33^{\prime \prime} \mathrm{W}$. MNHN-Uruguay 2823, 1, Arrocera Conti, island in río Uruguay, 30²9'27"S, 057 51'55"W. NRM 56105, 5, 55-79 mm, NRM 56106, 1, $95 \mathrm{~mm}, \mathrm{~N}$ Bella Unión, lagoon on río Uruguay, $30^{\circ} 13^{\prime} 03^{\prime \prime}$ S, $057^{\circ} 37^{\prime} 18^{\prime \prime}$ W. ZVC-P 19, 4, ZVC-P 2925, 14, ZVC-P 3518, 1, ZVC-P 7984, 2, Estancia El Ombú, Barra del Yucutuyá, río Cuareim, 30¹8'09"S, 057²3'21"W. ZVC-P 2923, 1, ZVC-P 7983, 1, Franquia, Bella Unión, Laguna Redonda, 30²146"S, 057 $36^{\prime 2} 22^{\prime \prime} W$. Cerro Largo. ZVC-P 5002, 1, Paso Mazangano, Route 44, río Negro, Uruguay dr., 3206'36"S, 054²40'06"W. Durazno. UF 183774, 2 (CS), 118-155 mm, Laguna Lavalle, río

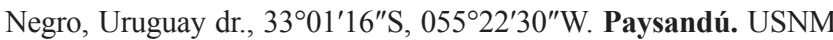

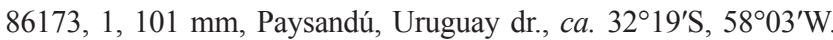
Río Negro (localities from Uruguay dr.). ZVC-P 4658, 1, Estero de Farrapos S side San Javier, 3240'34"S, 058 07'49"W. ZVC-P 4696, 1, Estero de Farrapos, mouth of Arroyo Román Grande, 3250'35"S, $058^{\circ} 05^{\prime} 05^{\prime \prime} \mathrm{W}$. Rocha (localities from Patos-Mirim dr.). CU 77186 , 1, bañado, Route 19, no coordinates. CU 77187, 1, cañada, Route 19, no coordinates. ZVC-P 5917, 1, Potrerillo, Laguna Negra,

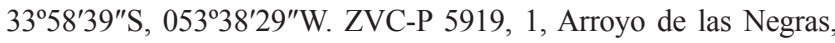
Route 19, km 22, Laguna Mirín, 3335'53"S, 05341'00"W. ZVC-P 5920, 2, río San Luis, Route 19, km 30.65, affl. Laguna Merín, $33^{\circ} 36^{\prime} 06^{\prime \prime} \mathrm{S}, 053^{\circ} 43^{\prime} 32^{\prime \prime} \mathrm{W}$. Salto (localities from Uruguay dr.). NRM $52215,6,106-144 \mathrm{~mm}$, embayment of río Uruguay, Constitución, $31^{\circ} 03^{\prime} 57^{\prime \prime} \mathrm{S}, 057^{\circ} 50^{\prime} 20^{\prime \prime} \mathrm{W}$. NRM 56068, 1, $141 \mathrm{~mm}$, Constitución, río Uruguay, $31^{\circ} 04^{\prime} 03^{\prime \prime} \mathrm{S}, 057^{\circ} 50^{\prime} 45^{\prime \prime} \mathrm{W}$. Tacuarembó. ZVC-P 1992, 4, Paso del Sauce above Arroyo Yaguarí, $29 \mathrm{~km} \mathrm{~S}$ Pueblo Ansina, affl. río Negro, Uruguay dr., $32^{\circ} 02^{\prime} 00^{\prime \prime} \mathrm{S}, 055^{\circ} 22^{\prime} 01^{\prime \prime} \mathrm{W}$. Treinta y Tres (localities from Patos-Mirim dr.). NRM 61393, 1, 86 mm, affl. Arroyo del Parao, Ruta 18, W Vergara, río Olimar Grande, 32 $55^{\prime} 12^{\prime \prime}$ S, 053 $55^{\prime} 18^{\prime \prime} \mathrm{W}$. NRM 61473, 3, 49-63 mm, Arroyo Corrales del Parao, Ruta 91, S Vergara, río Olimar Grande, $33^{\circ} 01^{\prime} 46^{\prime \prime}$ S, 053 52 '57"W. ZVC-P 5918, 2, Arroyo El Tigre, Ruta 17, $\mathrm{km}$ 322, affl. río Olimar, affl. río Cebollati, affl. Laguna Mirim, $33^{\circ} 14^{\prime} 50^{\prime \prime} \mathrm{S}, 053^{\circ} 54^{\prime} 40^{\prime \prime} \mathrm{W}$. Region not specified. UF 183779, 1 (CS), $153 \mathrm{~mm}$, aquarium specimen bred in captivity by P. Stoddard, Florida International University, from specimens exported from Uruguay. Suspected introduced records (15 specimens, grey circles in Fig. 27). Brazil. São Paulo (localities from rio Tietê dr., Paraná dr.). MZUSP 49201, 9, mun. Americana, Represa Santa Maria da Serra, $c a .22^{\circ} 45^{\prime} \mathrm{S}, 047^{\circ} 19^{\prime} \mathrm{W}$ [coordinates for town of Americana], coll. 7 Feb 1995, museum record states: "purchased from fishermen that sell bait in Praia Azul, Americana". MZUSP 53862, 4, 121-176 mm, mun. Santa Maria da Serra, Represa de Barra Bonita, nr. Santa

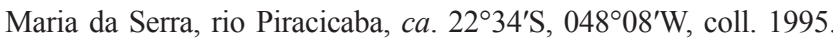
MZUSP 83410, 2, 175-175, mun. Bariri, downstream of hydroelectric barrage UHE Bariri, margin rio Tietê, $c a .22^{\circ} 08^{\prime} \mathrm{S}, 048^{\circ} 45^{\prime} \mathrm{W}$, coll. 4 Apr 2003.

\section{Brachyhypopomus hamiltoni, new species}

urn:1sid:zoobank.org:act:F8BA1881-ABCE-4E3C-A39C09C20739E83E

(Figs. 1g, 28; Tables 2-5, 13) 


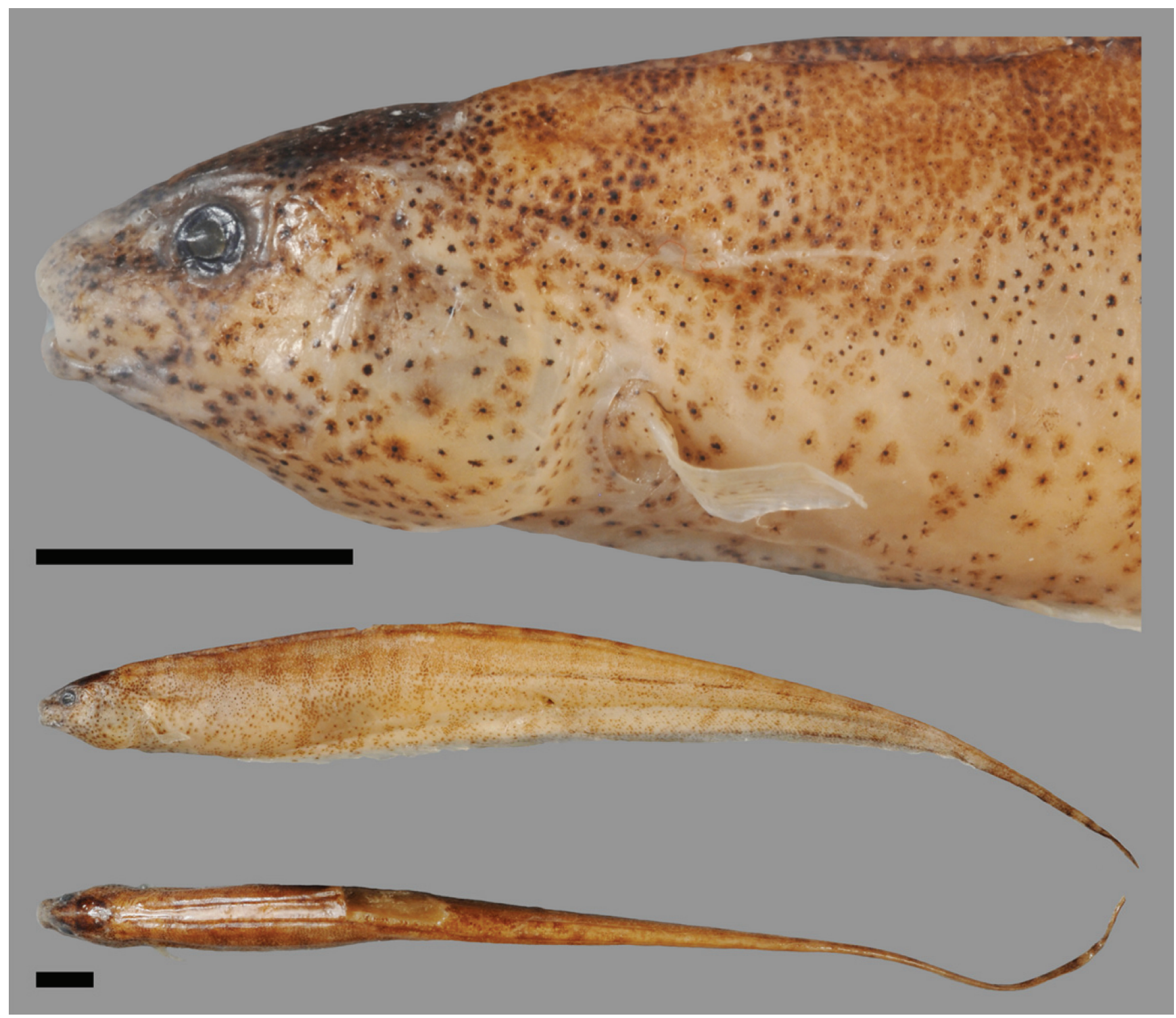

Fig. 28. Brachyhypopomus hamiltoni. MCP 45482 (WC05.080301), holotype, female, $97 \mathrm{~mm}$ TL (head in lateral view, body in lateral and dorsal view); Brazil, rio Solimões-Japurá confluence, Amazonas dr. Specimen fixed in formalin and preserved in EtOH. Scale bars $=5 \mathrm{~mm}$.

Brachyhypopomus sp. G and sp. H. -Crampton \& Albert, 2006: 672, fig. 23.8, position in phylogenetic tree; 681, notes on EODs (gymnotiform species and EOD diversity).

Brachyhypopomus sp. "ayr" and sp. "ham". -Crampton, 2011: 176, table 10.2, species list; 179, figs. 10.2-10.3, phylogeny, geographical and ecological distributions (gymnotiform biology).

Brachyhypopomus sp. "hamiltoni”. -Crampton et al., 2016: 1-66, table 1, 3-4, figs. 1-7, 18-20 (phylogeny, biogeography and ecology of Brachyhypopomus).

Holotype. MCP 45482, female, $97 \mathrm{~mm}$ TL, 80 mm LEA, Brazil, Amazonas, mun. Alvarães, Mamirauá Reserve (Reserva de Desenvolvimento Sustentável), Ressaca da Vila Alencar, rio Solimões-Japurá floodplain, Amazonas dr., 0307'41"S, 06448'04"W, 8 Mar 2001, W. Crampton \& J. Oliveira.
Paratypes. 19 specimens, localities from Amazonas dr., collected by W. Crampton \& J. Oliveira unless otherwise stated; localities listed from the Mamirauá Reserve [Reserva de Desenvolvimento Sustentável Mamirauá] are in rio SolimõesJapurá floodplain, mun. Alvarães). Brazil. Amazonas. MCP 45250, 1 (CS), immature, 80 mm, 26 Nov 1998, MCP 45303, 1, immature, $82 \mathrm{~mm}, 3$ Dec 1998, mun. Maraã, Boca do igarapé Juá Grande, lago Amanã, affl. rio Japurá, 02²7'14"S, 06448'26"W. MCP 45269, 1, male, 104 mm, Mamirauá Reserve, Ressaca do Pau, 0302'03"S, 06452'13"W, 17 Apr 2001. MCP 45302, 11 (3 CS, 2 with gills and brain removed), 75-89 mm (9 immature, 64-89 mm, 1 female, $84 \mathrm{~mm}, 1$ male, 93 mm), 2 Dec 1998, MCP 45307, 1, female, 93 mm, 16 Feb 1993, mun. Maraã, igarapé Juá Grande, lago Amanã, affl. rio Japurá, 02 28 $8^{\prime} 50^{\prime \prime} \mathrm{S}, 064^{\circ} 48^{\prime} 50^{\prime \prime} \mathrm{W}$. MCP 45407, 1, immature, $56 \mathrm{~mm}$, mun. Tefé, lago Jacaré, rio Solimões floodplain, 
0311'11"S, 06443'05"W, 18 Aug 1998. MPEG 27114, 1, 97 mm, lago do Içé, Ilha do Içé, rio Solimões, 6 km NW Tefé, Amazonas dr., 0316'02"S, 06440'50"W, 18 May 2012, A. Cardoso \& J. Oliveira. MPEG 27115, 2, 78-78 mm, Mamirauá Reserve, Paraná do Mamirauá, 0302'58"S, 06450'57"W, 18 May 2012, A. Cardoso \& J. Oliveira.

Non-types. 44 specimens, localities from Amazonas dr. Brazil. Amazonas (localities listed from the Mamirauá Reserve [Reserva de Desenvolvimento Sustentável Mamirauá] are in rio Solimões-Japurá floodplain, mun. Alvarães). IDSM 460, 1, 67 mm, Mamirauá Reserve, cano do lago Sapucaia, 0304'07"S, 06448'32"W. MCP 45268, 1, immature, 84 mm, Mamirauá Reserve, Paraná Maiana, $03^{\circ} 06^{\prime} 50^{\prime \prime} \mathrm{S}, 064^{\circ} 47^{\prime} 48^{\prime \prime} \mathrm{W}$. MCP 45681, 5, immature, 34-56 mm, mun. Tefé, lago Jacaré, rio Solimões floodplain, $03^{\circ} 11^{\prime} 11^{\prime \prime} \mathrm{S}, 064^{\circ} 43^{\prime} 05^{\prime \prime} \mathrm{W}$. MPEG 22744, 2, 54-66 mm, Mamirauá Reserve, lago Bolsinho, 0303'47"S, 06449'59"W. MZUSP 7352 (part), 2, 65-93 $\mathrm{mm}$, mun. Maués, igarapé Limãozinho, affl. rio Maués Açu, ca. $03^{\circ} 24^{\prime} \mathrm{S}, 057^{\circ} 42^{\prime} \mathrm{W}$. MZUSP 30058 (part), 1, immature, $60 \mathrm{~mm}$, lago Amanã, affl. rio Japurá, ca. $02^{\circ} 30^{\prime} \mathrm{S}, 064^{\circ} 42^{\prime} \mathrm{W}$. MZUSP 55112, 1, 65 mm, mun. Santa Isabel do rio Negro, igarapé São João, nr. Santa Isabel do rio Negro (Tapurucuara), affl. rio Negro, ca. $00^{\circ} 24^{\prime} \mathrm{S}, 065^{\circ} 02^{\prime} \mathrm{W}$. MZUSP 79663, 1, 79 mm, mun. Presidente Figueiredo, rd. branch nr. Marcus Freire, km 13 on AM-240 hwy., affl. rio Preto da Eva, 02 $04^{\prime} 06^{\prime \prime S}$, 059 54'33"W. MZUSP 95226 (part), 1, 77 mm, mun. Santa Isabel do rio Negro, Paricatuba, lake, margin of rio Negro, ca. $00^{\circ} 31^{\prime} \mathrm{S}, 065^{\circ} 01^{\prime} \mathrm{W}$. Ecuador (localities from río Napo dr.). Napo. MUSM 5693 (part), 1, 120 mm, río Aguarico, R. bank, temporary lake, ca. $00^{\circ} 38^{\prime} \mathrm{S}, 075^{\circ} 21^{\prime} \mathrm{W}$ [coordinates for mouth of río Aguarico]. Sucumbios. FMNH 102282, 2, $72-84 \mathrm{~mm}$, Laguna Zancudococha, affl. río Aguarico, ca. $00^{\circ} 35^{\prime} \mathrm{S}, 075^{\circ} 29^{\prime} \mathrm{W}$. FMNH $102285,2,73-90 \mathrm{~mm}$, affl. to río Cuyabeno, $\mathrm{N}$ bank ca. $3 \mathrm{~km}$ upstream Laguna Grande de Cuyabeno, affl. río Aguarico, ca. $00^{\circ} 01^{\prime} \mathrm{S}, 076^{\circ} 10^{\prime} \mathrm{W}$. FMNH 102291, 2, 63-68 mm, N bank affl. to río Aguarico, 2nd affl. downstream from Zancudo, no coordinates. FMNH 121058, 8, 21-73 mm, small stream between Laguna Zancudo and Laguna Zancudococha, affl. quebrada Zancudococha, affl. río Aguarico, 00³3'42"S, 075³0'00"W. Peru. Amazonas (localities from río Marañón, affl. río Amazonas). LACM 41774-1, 6, 66-109 mm, Caterpiza, río Santiago, ca. 035'ㄴ, $077^{\circ} 44^{\prime} \mathrm{W}$. LACM 41931-3, 2, 82-91 mm, downstream from Caterpiza, río Santiago, ca. $03^{\circ} 55^{\prime} \mathrm{S}, 077^{\circ} 44^{\prime} \mathrm{W}$. LACM 42012-4, 2, male, 107-125 mm, $1 \mathrm{~km}$ downstream from Caterpiza, río Santiago, ca. $03^{\circ} 55^{\prime} \mathrm{S}, 077^{\circ} 44^{\prime} \mathrm{W}$. Loreto. MUSM 44704, 2, (1 immature, $91 \mathrm{~mm}, 1$ female, $75 \mathrm{~mm}$ ), stream nr. cocha Capite, nr. Jenaro Herrera, affl. río Ucayali,

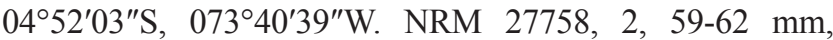
El Estrecho, quebrada de las Granjas, affl. río Putumayo, $02^{\circ} 28^{\prime} 00^{\prime \prime} \mathrm{S}, 072^{\circ} 42^{\prime} 00^{\prime \prime} \mathrm{W}$.

Diagnosis. Brachyhypopomus hamiltoni is diagnosed from congeners by the following combination of characters: precaudal vertebrae $19-21$, vs. $15-18$ in $B$. batesi, $B$. benjamini, B. bullocki, B. cunia, B. hendersoni, B. menezesi, $B$. provenzanoi, B. regani, and B. sullivani, and vs. 24-26 in $B$. belindae and $B$. verdii; pale stripe along middorsal region of body absent, $v s$. prominent pale uninterrupted middorsal stripe from occipital region to base of caudal filament in B. arrayae, B. beebei, B. gauderio, and B. pinnicaudatus; bilateral columns of electrocytes at the anal-fin terminus 3 , vs. 4-6 in B. bennetti, B. diazi and B. occidentalis (except some populations in Colombia and Venezuela, see redescription of B. occidentalis), and B. palenque; dark vertical or diagonally oriented stripes or saddles present, vs. absent in B. bombilla, $B$. draco, and B. jureiae; anal-fin rays 163-208, vs. 226-293 in $B$. brevirostris; absence of conspicuous patches of shiny yellow guanine on the operculum and anterior to pectoralfin base in live individuals, vs. presence in B. flavipomus; caudal filament length 18.6-30.0\% LEA, vs. 31.3-59.1\% in $B$. janeiroensis; dark suborbital stripe absent, vs. present in $B$. walteri. $B$. hamiltoni can be distinguished from $B$. alberti by a lower number of pectoral-fin rays, 12-15 (mode, 13), vs. 1516 (mode 16) in B. alberti (only 2 of 18 measured specimens of $B$. hamiltoni exhibited an overlapping number of pectoralfin rays with $B$. alberti). Brachyhypopomus hamiltoni can be further distinguished from $B$. alberti by the presence of the first of five branchiostegal rays, vs. absence in B. alberti.

Description. Head and body shape, and pigmentation illustrated in Figs. $1 \mathrm{~g}$ and 28. Meristic and morphometric data for examined specimens presented in Tables 2-5 and 13. Body moderate in depth. Head short to moderate in length and shallow to moderate in depth. Dorsal profile of head approximately straight to slightly convex from occiput to snout, ventral profile of head approximately straight to slightly concave between operculum and snout, snout triangular to rounded. Eye moderate to large in size. Upper jaw with moderate sigmoidal angle between premaxillary and maxillary portions in lateral view. No accessory electric organ over operculum. Gill filaments on first gill arch 30-39 (median 33, $\mathrm{n}=10$ ). Pectoral fin narrow to moderate in width, pectoral-fin rays 12-15 (mode 13). Precaudal vertebrae 19-21 (mode 20), with 1-3 (mode 1) transitional vertebrae. Anal-fin origin slightly $(<0.25 \mathrm{HL}$ distance) anterior to, or near, tip of pectoral fin. Anal-fin rays 163-208 (median 180). Dorsal rami of recurrent branch of anterior lateral line nerve not visible. Middorsal region of body scaled. Rows of scales above lateral line 5-7 (mode 5). Lateral line continuous. Depigmented epidermal canals present; as an irregular interrupted single groove either side of dorsal midline along most of posterior half body; and as crisscrossing network of grooves parallel to and dorsal to lateral line, reaching about one third of distance from lateral line to dorsal midline, and mostly restricted to posterior third of body. Three bilateral horizontal columns of electrocytes at anal-fin terminus and at or near a mid-point between analfin terminus and tip of caudal filament in immature, mature female, and mature male specimens, and in populations from both low-conductivity and high-conductivity systems. Caudal filament of moderate length. 
Coloration. (Figs. 1g, 28). Background pale tan to dark brown. Dorsal region without prominent depigmented stripe extending along midline from occipital region to base of caudal filament. Dorsal region with irregular dark patches of dark chromatophores which extend into diffuse, narrow, vertical dark bands which extend across lateral line. Some diffuse bands reach ventral midline or anal-fin margin over body cavity. Vertical bands less distinctive in posterior portion of body and mostly do not cross lateral line. Some diffuse vertically-elongated patches of chromatophores extend dorsally from anal-fin margin over anal-fin pterygiophores. Vertical dark bands much less distinct than in most congeners that possess dark vertical or diagonally oriented stripes or saddles on body surface dorsal to lateral line. Series of elongated diffuse horizontal dash-like dark markings present along lateral line in posterior third of body, anterior to analfin terminus. Caudal filament darker than body, with irregular dark markings or bands along entire length. Head with evenly scattered dark chromatophores, darker dorsally. Eye without prominent suborbital patch, or stripe, of chromatophores/ subcutaneous pigmentation. Pectoral and anal-fin membranes hyaline. Anal-fin rays with light scattering of brown chromatophores, usually anal-fin rays darker than pectoralfin rays. Color in live individuals similar to preserved ones, with opercular region usually rosy due to underlying gills. Individuals from blackwater habitats usually exhibit a darker overall coloration than those in whitewater habitats. Individuals from whitewater habitats often with yellowish tinge to background body color in live individuals.

Size. Small adult size, largest specimen examined $125 \mathrm{~mm}$ TL, 100 mm LEA $(n=61)$. Largest male specimen examined $125 \mathrm{~mm}$ TL, $100 \mathrm{~mm}$ LEA $(\mathrm{n}=4)$. Largest female specimen examined $97 \mathrm{~mm}$ TL, $80 \mathrm{~mm} \operatorname{LEA}(\mathrm{n}=4)$.

Sexual dimorphism. No known secondary sexual dimorphism.

Geographic distribution. Brazil, Ecuador, and Peru (Fig. 29). Widely distributed in the upper, central, and lower Amazon, and from the middle Negro, but with sporadic collection records.

Ecological notes. In the region of the type locality B. hamiltoni is uncommon. It is known from floating macrophytes in whitewater floodplains at the confluence of rio Solimões and Japurá (see description of $B$. belindae for water quality parameters in this area), and from the large low-conductivity (usually $<30 \mu \mathrm{Scm}^{-1}$ ) blackwater floodplain ria lake, lago Amanã; see Crampton (2008) for notes on the water chemistry of lago Amanã. Specimens in reproductive condition were found during the rising and high water period, which is consistent with the reproductive seasonality of sympatric congeners. Stomach contents of specimens from the type locality comprise aquatic insect larvae (primarily Chironomidae), and other small aquatic invertebrates (WGRC unpublished data).
Co-occurring congeners: Brachyhypopomus hamiltoni is known to co-occur in geographical sympatry and ecological syntopy with $B$. beebei, $B$. belindae, B. bennetti, B. brevirostris, $B$. flavipomus, B. hendersoni, B. pinnicaudatus, B. regani, and $B$. walteri. It also exhibits an allotopic distribution across parts of its range with: B. batesi, B. benjamini, B. sullivani, and $B$. verdii.

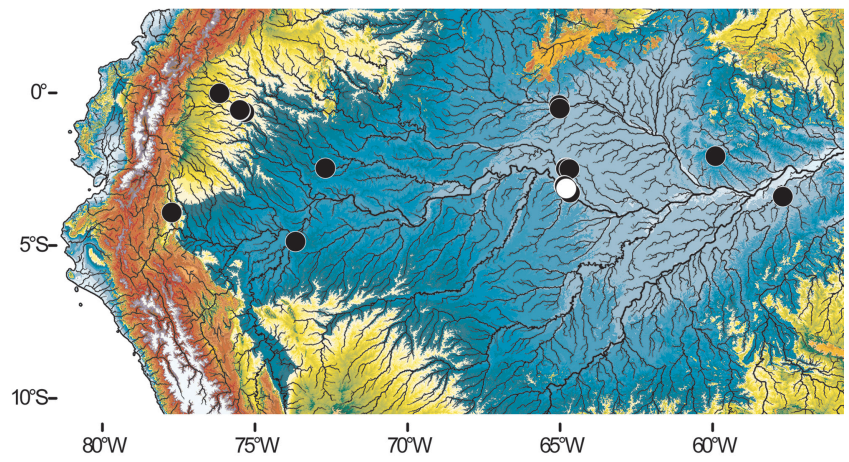

Fig. 29. Collection records for Brachyhypopomus hamiltoni (circles). Holotype location is marked with an open symbol. Elevation data refers to altitude above mean sea level (see Fig. 2 for legend).

Etymology. The specific name is a patronym (noun in the genitive case) in honor of William D. Hamilton (19362000), British evolutionary biologist, and doctoral advisor of WGRC; for his contributions to Amazonian ecology.

Local names. Brazil: sarapó; Ecuador: cuchillo, yayo; Peru: macana.

\section{Brachyhypopomus hendersoni, new species}

urn:1sid:zoobank.org:act:A0D96996-8B4E-4001-A70064F9093BFA4F

(Figs. 1h, 30; Tables 2-5, 13)

Brachyhypopomus beebei ('H2'). -Heiligenberg \& Bastian, 1980: 197, fig. 1, black and white photograph (Brazil, Amazonas, rio Negro, gymnotiform species and EOD diversity).

Brachyhypopomus sp. 8. -Crampton, 1996a: 77, table 6.1.a, species list; 79, fig. 6.1 inset 30-31, color photographs; 85 , fig. 6.2; 88: fig. 6.3; 92-94, figs. 6.4a, 6.5; 191, fig. 11.2; 193, fig. 11.3, EOD data; 110 , table $7.5,113$, table 7.7, 115, fig. 7.1, habitats (Brazil, central Amazon, ecology and signal diversity).

Brachyhypopomus sp. 4. -Crampton, 1998a: 821, list of Brachyhypopomus (Brazil, Amazonas, species and EOD diversity). -Crampton, 1998b: 314, table 2, list of Brachyhypopomus (Brazil, Amazonas, hypoxia tolerance).

Brachyhyроротиs sp. D. -Crampton, 1999: 17 (Brazil, Amazonas, Mamirauá Reserve, listing of species). -Crampton \& Albert, 2006: 672, fig. 23.8, position in phylogenetic tree; 681, notes on EODs (gymnotiform species and EOD diversity). -Crampton et al., 2008: 231, fig. 6, black and white 
photograph of head of live individual (Brazil, Amazonas, adaptations to hypoxia).

Brachyhypopomus sp. 'basepreta'. -Ferreira et al., 2007: 164, bottom left photograph, live individual (Brazil, Roraima, rio Branco, photographic album of fishes).

Brachyhypopomus sp. "hen". -Crampton, 2011: 176, table 10.2, species list; 179, figs. 10.2-10.3, phylogeny, geographical and ecological distributions (gymnotiform biology).
Brachyhypopomus sp. "hendersoni". -Crampton et al., 2016: 1-66, table 1, 3-4, figs. 1-7, 12, 13, 15, 18-20 (phylogeny, biogeography and ecology of Brachyhypopomus).

Holotype. MCP 45305, male, 146 mm TL, 98 mm LEA, Brazil, Amazonas, mun. Maraã, lago Amanã, Igarapé Juá Grande, rio Japurá, Amazonas dr., 02 $28^{\prime} 50^{\prime \prime} \mathrm{S}, 064^{\circ} 48^{\prime} 50^{\prime \prime} \mathrm{W}$, 3 Dec 1998, W. Crampton \& J. Oliveira.

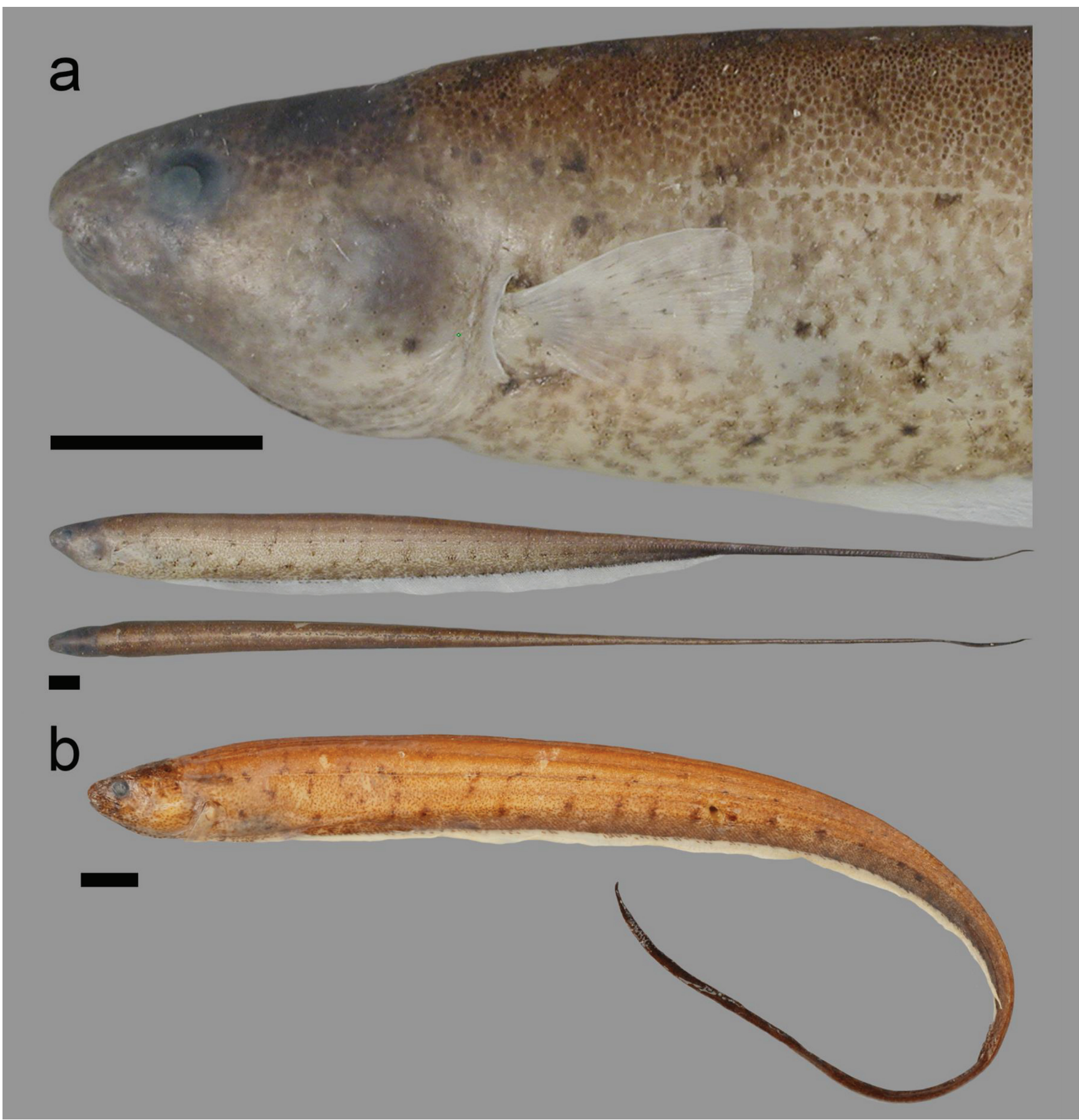

Fig. 30. Brachyhypopomus hendersoni. a. MCP 45408 (WC07.110898b), paratype, female, 162 mm TL, (head, and body in lateral and dorsal view, specimen fixed in formalin); Brazil, rio Tefé, Amazonas dr. b. MCP 45305 (WC13.031298), holotype, male, $146 \mathrm{~mm}$ TL (head in lateral view, body in lateral and dorsal view, specimen fixed in formalin and preserved in EtOH); Brazil, rio Japurá, Amazonas dr. Scale bars $=5 \mathrm{~mm}$. 
Paratypes. 47 specimens, localities from Amazonas dr., collected by W. Crampton \& J. Oliveira unless otherwise stated. Brazil. Amazonas. BMNH 1998.3.12.22-24, 3, immature, 122-140 mm, 7-10 Jan 1997, MCP 45483, 3, 142$142 \mathrm{~mm}, 1997$, mun. Tefé, Ressaca do Socorro, lago Tefé, rio Tefé, $03^{\circ} 18^{\prime} 45^{\prime \prime} \mathrm{S}, 64^{\circ} 41^{\prime} 50^{\prime \prime} \mathrm{W}$. BMNH 1998.3.12.175, 1, female, 159 mm, 27 May 1994, BMNH 1998.3.12.176-177, 2 (1 immature, 132 mm, 1 male, $171 \mathrm{~mm}$ ), 27 Jan 1995, mun. Tefé, Ressaca do Cachorro, lago Tefé, rio Tefé, $03^{\circ} 19^{\prime} 50^{\prime \prime} \mathrm{S}$, $64^{\circ} 42^{\prime} 15^{\prime \prime} \mathrm{W}$. INPA 9950, 10, 125-154 mm, mun. Tefé, Cabeceira do lago Tefé, rio Tefé, $c a .03^{\circ} 34^{\prime} \mathrm{S}, 064^{\circ} 58^{\prime} \mathrm{W}, 11$ Jan 1995. MCP 45272, 1, female, $174 \mathrm{~mm}$, mun. Tefé, Ilha do Martelo, rio Tefé, 0346'56"'S, 06459'39"W, 22 Jan 1999. MCP 45273, 1, male, $121 \mathrm{~mm}$, mun. Tefé, Cabeceira do lago Tefé, rio Tefé, $03^{\circ} 38^{\prime} 01^{\prime \prime} \mathrm{S}, 064^{\circ} 57^{\prime} 59^{\prime \prime} \mathrm{W}, 22$ Jun 1999. MCP 45274 , 1, female, $143 \mathrm{~mm}$, mun. Tefé, rio Tefé, $03^{\circ} 41^{\prime} 23^{\prime \prime} \mathrm{S}$, 064 59'08"W, 16 Feb 2001. MCP 45309, 1, immature, 69 mm, mun. Maraã, igarapé São Sebastião, affl. rio Baré, affl. lago Amanã, affl. rio Japurá, $02^{\circ} 17^{\prime} 05^{\prime \prime} \mathrm{S}, 064^{\circ} 41^{\prime} 25^{\prime \prime} \mathrm{W}$, 17 Feb 1993. MCP 45369, 3 (2 immature, 144-147 mm, 1 female, $175 \mathrm{~mm}$ ), mun. Tefé, mouth of igarapé Açu, Lago Tefé, rio Tefé, 0325'00"S, 06448'23"W, 26-28 Jun 1998. MCP 45397, 13 (3 immature, 158-183 mm, 6 female, 140203 mm, 4 male, 125-190 mm), mun. Tefé, lago Caiambé, rio Caiambé, $03^{\circ} 35^{\prime} 40^{\prime \prime} \mathrm{S}, 064^{\circ} 26^{\prime} 54^{\prime \prime} \mathrm{W}, 28$ Dec 1998. MCP 45408, 1, female, $162 \mathrm{~mm}$, mun. Tefé, Ressaca do Socorro, lago Tefé, rio Tefé, $03^{\circ} 18^{\prime} 45^{\prime \prime} \mathrm{S}, 064^{\circ} 41^{\prime} 50^{\prime \prime} \mathrm{W}, 11$ Aug 1998. MPEG 973, 2, 109-110 mm, MPEG 974, 1, 127 mm, mun. Tefé, lago Jurupari, rio Tefé, $c a .03^{\circ} 48^{\prime} \mathrm{S}, 065^{\circ} 00^{\prime} \mathrm{W}, 1 \mathrm{Jul}$ 1979, M. Goulding. MPEG 976, 1, $102 \mathrm{~mm}$, mun. Tefé, Arana-tuba, rio Tefé, $c a .04^{\circ} 02^{\prime} \mathrm{S}, 065^{\circ} 01^{\prime} \mathrm{W}, 8$ Jul 1979 , M. Goulding. MZUSP 99170, 2, 96-130 mm, canal do lago Amanã, affl. rio Japurá, ca. $02^{\circ} 44^{\prime} \mathrm{S}, 064^{\circ} 39^{\prime} \mathrm{W}, 30$ Sep 1979, R. Barthem.

Non-types. 103 specimens. Brazil. Amazonas (localities from Amazonas dr.). BMNH 1998.3.12.08-10, 3 (2 immature, 153-167 mm, 1 female, $167 \mathrm{~mm})$, BMNH 1998.3.12.13-14, 2, female, 155-167 mm, МСP 45271, 26 (10 immature [1CS], $120-170 \mathrm{~mm}, 8$ female, $145-172$ $\mathrm{mm}, 8$ male, $148-180 \mathrm{~mm})$, mun. Tefé, lago Tefé, rio Tefé, $03^{\circ} 18^{\prime} 45^{\prime \prime} \mathrm{S}, \quad 064^{\circ} 41^{\prime} 50^{\prime \prime} \mathrm{W}$. BMNH 1998.3.12.159-174, 16 unsexed, 108-193 mm, BMNH 1998.3.12.178-182, 5, immature, 102-135 mm, MCP 45270, 3 (2 immature, 130$142 \mathrm{~mm}, 1$ male, $195 \mathrm{~mm}$ ), mun. Tefé, Ressaca do Cachorro, lago Tefé, rio Tefé, $03^{\circ} 19^{\prime} 50^{\prime \prime} \mathrm{S}, 064^{\circ} 42^{\prime} 15^{\prime \prime} \mathrm{W}$. IDSM 456, 1, immature, $130 \mathrm{~mm}$, mun. Tefé, Cabeceira do lago Tefé, rio Tefé, $03^{\circ} 35^{\prime} 41^{\prime \prime} \mathrm{S}, 064^{\circ} 58^{\prime} 05^{\prime \prime} \mathrm{W}$. INPA 9781, 2, 79$140 \mathrm{~mm}$, rio Jaú, affl. rio Negro, $c a .01^{\circ} 57^{\prime} \mathrm{S}, 061^{\circ} 28^{\prime} \mathrm{W}$. MCP 45304, 1, immature (CS), 100 mm, mun. Maraã, lago Amanã, mouth of igarapé Uxi, affl. rio Japurá, $02^{\circ} 32^{\prime} 41^{\prime \prime} \mathrm{S}$, $064^{\circ} 40^{\prime} 12^{\prime \prime} \mathrm{W}$. MCP 45454, 4 (3 female [1CS], 127-153mm, 1 male [CS], $175 \mathrm{~mm}$ ), mun. Tefé, Cabeceira do lago Tefé, rio Tefé, $03^{\circ} 38^{\prime} 01^{\prime \prime} \mathrm{S}, 064^{\circ} 57^{\prime} 59^{\prime \prime} \mathrm{W}$. MCP 45426, 1, female (CS), $166 \mathrm{~mm}$, mun. Tefé, Cabeceira do lago Tefé, rio Tefé, $03^{\circ} 38^{\prime} 01^{\prime \prime} \mathrm{S}, 064^{\circ} 57^{\prime} 59^{\prime \prime} \mathrm{W}$. MCP 45432, 6 (3 immature
[1CS], 134-146 mm, 2 female, 143-163 mm, 1 male [CS], $161 \mathrm{~mm}$ ), mun. Tefé, rio Tefé, $03^{\circ} 37^{\prime} 43^{\prime \prime} \mathrm{S}, 064^{\circ} 59^{\prime} 03^{\prime \prime} \mathrm{W}$. MCP 45489, 2, female, 164-166 mm, mun. Tefé, Ilha do Martelo, rio Tefé, $03^{\circ} 46^{\prime} 56^{\prime \prime} \mathrm{S}$, 064 $59^{\prime} 39^{\prime \prime} \mathrm{W}$. MCP 45491, 5 (4 immature, $128-148 \mathrm{~mm}, 1$ female, $120 \mathrm{~mm}$ ), mun. Tefé, rio Tefé, $03^{\circ} 41^{\prime} 23^{\prime \prime} \mathrm{S}, 064^{\circ} 59^{\prime} 08^{\prime \prime} \mathrm{W}$. MCP 45682, 4 , 123-152 mm, mun. Tefé, Ressaca do Socorro, lago Tefé, rio Tefé, $03^{\circ} 18^{\prime} 45^{\prime \prime} \mathrm{S}, 6^{\circ} 41^{\prime} 50^{\prime \prime} \mathrm{W}$. MPEG 979, 5 (part), 65-147 $\mathrm{mm}$, mun. Tefé, Ipanema da Baixa, rio Tefé, $c a .03^{\circ} 53^{\prime} \mathrm{S}$, $065^{\circ} 00^{\prime} \mathrm{W}$. MPEG 1125, 1, $81 \mathrm{~mm}$, mun. Novo Airão, Anavilhanas archipelago, rio Negro, $c a .02^{\circ} 40^{\prime} \mathrm{S}, 060^{\circ} 44^{\prime} \mathrm{W}$. MZUSP 30044 (part), 1, 127 mm, mun. Tefé, lago Jurupari, rio Tefé, $c a .03^{\circ} 48^{\prime} \mathrm{S}, 065^{\circ} 00^{\prime} \mathrm{W}$. MZUSP $30050,1,106 \mathrm{~mm}$, mun. Tefé, Mastro, rio Tefé, no coordinates. MZUSP 78032 (part), 1, $110 \mathrm{~mm}$, mun. Maraã, Paraná do Castanho, lago Amanã, affl. rio Japurá, ca. $02^{\circ} 44^{\prime} \mathrm{S}, 064^{\circ} 39^{\prime} \mathrm{W}$. Guyana. Upper Demerara-Berbice. INHS 49276, 1, 112 mm, 1.54 mi. SW Rockstone, Essequibo River (Long Lake), Essequibo River dr., $c a .05^{\circ} 58^{\prime} \mathrm{N}, 058^{\circ} 32^{\prime} \mathrm{W}$. Upper Tukutu-Upper Essequibo. ANSP 179500, 2, 70-113 mm, AUM 35818, 2, 84-118 mm, stream at crossing on Massara-Karanambo rd., 10.3 km NW Karanambo, affl. Rupununi River, Essequibo River dr., $03^{\circ} 48^{\prime} 27^{\prime \prime} \mathrm{N}, 059^{\circ} 23^{\prime} 06^{\prime \prime} \mathrm{W}$. AUM 48815, 1, 80 $\mathrm{mm}$, Rupununi River at Massara landing, Essequibo River dr., 035' $42^{\prime \prime} \mathrm{N}, 059^{\circ} 17^{\prime} 37^{\prime \prime} \mathrm{W}$. BMNH 1972.7.519-525, 7, 34-109 mm, Rupununi River, Essequibo River dr., no coordinates.

Diagnosis. Brachyhypopomus hendersoni is diagnosed from congeners by the following combination of characters: caudal filament length $32.3-64.5 \%$ LEA, vs. $7.4-31.6 \%$ in $B$. alberti, B. arrayae, B. batesi, B. belindae, B. benjamini, $B$. bennetti, $B$. bombilla, $B$. hamiltoni, $B$. occidentalis, $B$. palenque, $B$. provenzanoi, $B$. regani, $B$. sullivani, and $B$. verdii; precaudal vertebrae 15-17, vs. $18-25$ in $B$. beebei, B. brevirostris, B. draco, B. flavipomus, B. gauderio, B. janeiroensis, $B$. jureiae, and B. pinnicaudatus; head width at occiput $39.2-47.8 \% \mathrm{HL}, v s .49 .5-70.4 \%$ in B. bullocki, $B$. diazi, and $B$. menezesi; dorsal rami of the recurrent branch of the anterior lateral line nerve not visible, vs. visible in $B$. cunia and B. walteri.

Description. Head and body shape, and pigmentation illustrated in Figs. 1h and 30. Meristic and morphometric data for examined specimens presented in Tables 2-5 and 13. Body shallow to moderate in depth. Head short and shallow to moderate in depth. Dorsal profile of head convex from occiput to snout, ventral profile of head with areas of convexity near mouth and areas of concavity under eye, snout truncate to rounded. Eye moderate to large in size. No accessory electric organ over operculum. Gill filaments on first gill arch 32-34 (median 33, $\mathrm{n}=4$ ). Pectoral fin moderate in width, pectoral-fin rays 12-16 (mode 13). Precaudal vertebrae 15-17 (mode 16), with 1-2 (mode 1) transitional vertebrae. Anal-fin origin slightly $(<0.25$ HL distance) anterior to, or near, tip of pectoral fin. Anal- 
fin rays 192-210 (median 200). Dorsal rami of recurrent branch of anterior lateral line nerve not visible. Middorsal region of body scaled. Rows of scales above lateral line 5-7 (mode 6). Lateral line continuous. Sparse depigmented epidermal canals in posterior two thirds of body, present in three series: as an irregular, interrupted, single groove either side of dorsal midline along most of posterior two thirds of body, as a meandering sometimes bifurcating single groove on each dorsal flank approximately midway from lateral line to dorsal midline, and in posterior third of body as sparse scratch like marks dorsal to and near lateral line. Three bilateral horizontal columns of electrocytes at analfin terminus and at a mid-point between anal-fin terminus and tip of caudal filament in immature, mature female, and mature male specimens. Caudal filament moderate in length to long.

Coloration. (Figs. 1h, 30). Background color tan or light grey to dark brown. Dorsal region with uniform speckling of dark chromatophores, and often with a thin pale line extending along midline from occipital region to deep into caudal filament, but without a prominent depigmented stripe. Flank with narrow diffuse, vague vertical stripes dorsal to lateral line, extending to but not crossing lateral line. Flank in region of body cavity ventral to lateral line with irregular dark flecks and patches, not forming vertical stripes. Prominent horizontal dark band extends from anal-fin margin to near dorsal margin of anal-fin pterygiophores along entire length of anal fin, darkening ventrally and posteriorly. This dark band along anal-fin base is more conspicuous in some specimens than others. Dark spots and diffuse vertically elongated stripes also present over anal-fin pterygiophores, extending dorsally towards lateral line. Caudal filament very dark, with irregular lighter markings, often with black tip; darkened tip resembles root tips of water hyacinths when viewed underwater. Head with evenly scattered dark chromatophores, darker dorsally. Eye with suborbital patch or stripe of chromatophores and subcutaneous pigmentation (more conspicuous in some specimens than others). Pectoral and anal-fin membranes hyaline. Pectoral-fin rays hyaline with light scattering of melanophores. Anal-fin rays with scattered or dark chromatophores, mostly concentrated near anal-fin margin. Color in live individuals similar to preserved specimens, with opercular region usually rosy due to underlying gills and flank over body cavity whitish.

Size. Moderate adult size, largest specimen examined 203 $\mathrm{mm}$ TL, $130 \mathrm{~mm}$ LEA $(\mathrm{n}=150)$. Largest male specimen examined $195 \mathrm{~mm}$ TL, $123 \mathrm{~mm}$ LEA $(\mathrm{n}=18)$. Largest female specimen examined $203 \mathrm{~mm}$ TL, $130 \mathrm{~mm}$ LEA ( $=31$ ).

Sexual dimorphism. Breeding males exhibit slightly enlarged electrocytes relative to immature specimens and females. No other secondary sexually dimorphic characters known.
Geographic distribution. Brazil and Guyana (Fig. 31). Known from the central Amazon from blackwater ria lakes near Tefé, from the lower Negro, and from the Essequibo drainage.

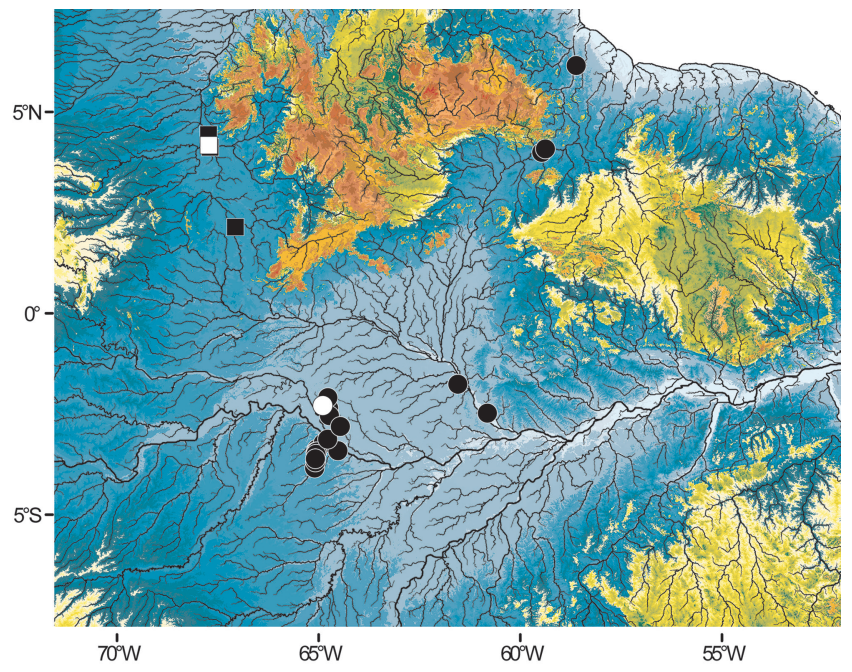

Fig. 31. Collection records for Brachyhypopomus hendersoni (circles) and B. provenzanoi (squares). Holotype locations are marked with open symbols. Elevation data refers to altitude above mean sea level (see Fig. 2 for legend).

Ecological notes. In the region of the type locality $B$. hendersoni is common in marginal emergent plants (mainly wild rices, Oryza spp.), rafts of floating macrophytes (including grasses, e.g., Paspalum, and water hyacinths, Eichhornia spp.), and decaying plant matter along the edges of low-conductivity blackwater floodplain ria lakes (e.g., lago Tefé, lago Amanã, and lago Caiambé), and along river and oxbow lake margins (e.g., in the rio Tefé floodplain). The following water parameters were recorded in the rio Tefé floodplain: conductivity $7-18 \mu \mathrm{Scm}^{-1}$, dissolved oxygen 3.0-6.7 $\mathrm{mgl}^{-1}$, temperature $29.0-33.3^{\circ} \mathrm{C}$, and $\mathrm{pH} 5.3$ 6.7. Brachyhypopomus hendersoni occurs in habitats that are not typically exposed to protracted and severe seasonal hypoxia. Crampton et al. (2008) documented the relatively small gill sizes of $B$. hendersoni relative to congeners that occur in seasonally hypoxic whitewater floodplain systems. Breeding occurs in floating macrophytes during the rising and high water period (WGRC unpublished data). Stomach contents of specimens from the type locality comprise aquatic insect larvae (primarily Chironomidae), and other small aquatic invertebrates (WGRC unpublished data).

Co-occurring congeners: In the region of the type locality Brachyhypopomus hendersoni commonly cooccurs in geographical sympatry and ecological syntopy with $B$. brevirostris, and $B$. walteri, and sometimes with $B$. beebei. It exhibits an allotopic distribution with $B$. batesi, $B$. belindae, B. bennetti, B. flavipomus, B. hamiltoni, $B$. pinnicaudatus, $B$. regani, and $B$. sullivani. In the middle rio Negro $B$. hendersoni co-occurs in geographical sympatry 
with B. batesi, B. bullocki, B. beebei, B. brevirostris, $B$. hamiltoni, B. regani, $B$. sullivani, and $B$. walteri. In the Essequibo River $B$. hendersoni co-occurs in geographical sympatry with $B$. bullocki, B. beebei, B. brevirostris, $B$. regani, B. sullivani, and $B$. walteri.

Etymology. The specific name is a patronym (noun in the genitive case) in honor of Peter A. Henderson, British fish biologist, and doctoral co-advisor of WGRC; for his contributions to Amazonian aquatic ecology.

Local names. Brazil: sarapó; Guyana: knifefish.

\section{Brachyhypopomus janeiroensis (Costa \& Campos-da- Paz, 1992)}

(Fig. 32; Tables 2-5, 15)

Hypopomus janeiroensis Costa \& Campos-da-Paz, 1992: 118, fig. 2, black and white photograph of holotype, fig. 3, color photograph of live paratype (original description, type locality - Brazil, Rio de Janeiro, rio São João dr.).
Brachyhypopomus janeiroensis. -Mago-Leccia, 1994: 48 (listing of Brachyhypopomus). -Sullivan, 1997, 118 (redescription). -Albert \& Crampton, 2003: 495 (Brazil, catalog of hypopomids). -Crampton \& Albert, 2006: 672 fig. 23.8, position in phylogenetic tree; 681, notes on EODs (gymnotiform species and EOD diversity). -Crampton, 2011: 176, table 10.2, species list; 179, figs. 10.2-10.3, phylogeny, geographical and ecological distributions (gymnotiform biology). -Crampton et al., 2016: 1-66, table 1, 3-4, figs. 1-7, 18-20 (phylogeny, biogeography and ecology of Brachyhypopomus).

Diagnosis. Brachyhypopomus janeiroensis is diagnosed from congeners by the following combination of characters: bilateral columns of electrocytes at the anal-fin terminus 4, vs. 6 in $B$. bennetti and vs. 3 in all other congeners except $B$. belindae, $B$. diazi, B. jureiae, B. occidentalis (except some populations in Colombia and Venezuela, see redescription of B. occidentalis), and $B$. palenque; caudal filament length 31.3-59.1\% LEA, vs. $7.4-30.7 \%$ in B. belindae, B. occidentalis, and B. palenque; dorsal rami of the recurrent branch of the anterior lateral line nerve visible, vs. not visible in $B$. diazi; snout to pectoral-fin base $10.7-12.3 \%$ LEA, vs. $12.3-15.7 \%$ in $B$. jureiae.

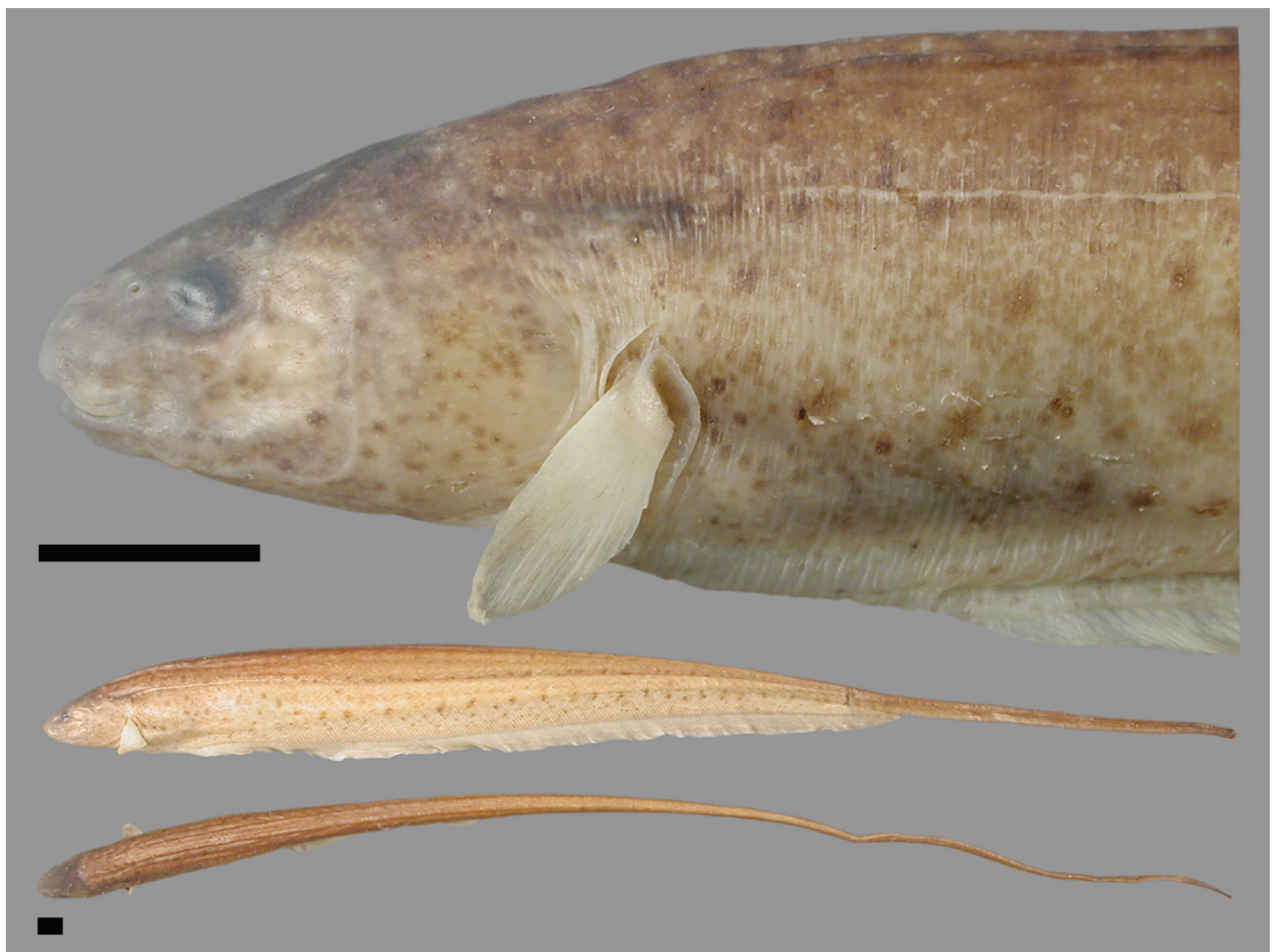

Fig. 32. Brachyhypopomus janeiroensis. MZUSP 80122; male, $174 \mathrm{~mm}$ TL (head in lateral view, and body in lateral and dorsal view, specimen fixed in formalin and preserved in EtOH). Brazil, rio São João dr. Scale bars $=5 \mathrm{~mm}$. 
Table 15. Morphometrics for Brachyhypopomus janeiroensis, Brachyhypopomus jureiae, and Brachyhypopomus menezesi. HT, holotype; SD, standard deviation. Data for holotype of Brachyhypopomus janeiroensis (Rio de Janeiro, Brazil) are from Costa \& Campos-da-Paz (1992). Data for holotype of Brachyhypopomus jureiae (São Paulo, Brazil) are from Triques \& Khamis (2003). Ranges for Brachyhypopomus janeiroensis and Brachyhypopomus jureiae refer to non-type specimens from the type region (holotype data not included except for caudal filament length as \% TL and LEA in $B$. jureiae). Ranges for Brachyhypopomus menezesi refer to the holotype and to paratypes from the type region.

\begin{tabular}{|c|c|c|c|c|c|c|c|c|c|c|c|c|c|c|c|}
\hline & \multicolumn{5}{|c|}{ B. janeiroensis } & \multicolumn{5}{|c|}{ B. jureiae } & \multicolumn{5}{|c|}{ B. menezesi } \\
\hline & HT & Range & Mean & $\mathrm{SD}$ & $\mathrm{n}$ & HT & Range & Mean & SD & $\mathrm{n}$ & HT & Range & Mean & $\mathrm{SD}$ & $\mathrm{n}$ \\
\hline Total length (TL) (mm) & 124 & $103-230$ & 155 & 23.6 & 75 & 292 & $64-169$ & 131 & 47.1 & 5 & 100 & $100-113$ & 106 & 6.1 & 5 \\
\hline Length to end of anal fin (LEA) (mm) & 92 & $67-157$ & 108 & 16.0 & 75 & 194 & $50-117$ & 94 & 30.1 & 5 & 75 & $75-89$ & 82 & 5.7 & 5 \\
\hline Head length (HL) (mm) & 10.3 & $10.4-12.5$ & 11.5 & 0.8 & 20 & & $7.3-13.6$ & 11.2 & 2.4 & 7 & 9.5 & $9.5-12.1$ & 10.8 & 0.9 & 5 \\
\hline \multicolumn{16}{|c|}{ Proportion of TL (\%) } \\
\hline Caudal filament length (CF) & 26.0 & $23.8-39.5$ & 29.8 & 3.8 & 75 & 33.2 & $21.9-33.2$ & 27.1 & 4.1 & 6 & 25.0 & $19.8-25.0$ & 22.6 & 2.1 & 5 \\
\hline Snout to occiput (SO) & 9.7 & $6.9-9.4$ & 8.5 & 0.6 & 20 & & $9.1-11.3$ & 9.9 & 1.0 & 5 & 10.1 & $10.1-11.1$ & 10.5 & 0.4 & 5 \\
\hline Snout to anal-fin origin (SA) & & $14.2-21.4$ & 17.9 & 1.8 & 20 & & $19.1-22.7$ & 20.7 & 1.3 & 5 & 21.6 & $21.6-25.5$ & 23.1 & 1.6 & 5 \\
\hline Snout to pectoral-fin base (PP) & & $10.7-12.3$ & 11.5 & 0.4 & 20 & 10.1 & $12.3-15.7$ & 13.5 & 1.5 & 5 & 13.9 & $13.9-15.5$ & 14.7 & 0.8 & 5 \\
\hline Longest pectoral-fin ray (PRL) & 5.6 & $3.7-4.8$ & 4.4 & 0.3 & 14 & 4.4 & $3.9-5.3$ & 4.8 & 0.5 & 5 & 5.0 & 4.3-5.7 & 5.2 & 0.6 & 5 \\
\hline Anal fin length (AF) & 80.5 & $72.7-85.3$ & 81.9 & 2.6 & 20 & 86.6 & $76.0-81.7$ & 79.5 & 2.2 & 5 & 78.7 & $78.2-82.0$ & 79.8 & 1.6 & 5 \\
\hline Body depth at anal-fin middle (BD2) & & $8.1-11.3$ & 9.4 & 0.9 & 20 & & $6.2-9.3$ & 8.1 & 1.1 & 5 & 9.1 & $9.1-10.7$ & 9.9 & 0.6 & 5 \\
\hline Body width at anal-fin middle (BW2) & & $2.5-4.4$ & 3.1 & 0.6 & 20 & & $1.9-3.4$ & 2.7 & 0.5 & 5 & 2.7 & $2.7-4.2$ & 3.5 & 0.6 & 5 \\
\hline Caudal filament length (CF) & 35.1 & $31.3-59.1$ & 43.1 & 6.2 & 18 & 50.0 & $28.0-50.0$ & 37.6 & 7.7 & 5 & 33.3 & $24.7-33.3$ & 29.3 & 3.6 & 5 \\
\hline \multicolumn{16}{|c|}{ Proportion of HL (\%) } \\
\hline Preorbital distance (PR) & 25.2 & $18.8-26.6$ & 23.1 & 2.1 & 20 & 32.2 & $23.1-26.5$ & 24.9 & 1.2 & 7 & 25.5 & $23.8-25.6$ & 24.8 & 0.9 & 5 \\
\hline Mouth width (MW) & 23.3 & $17.1-33.4$ & 24.3 & 3.5 & 20 & & $23.2-30.0$ & 27.0 & 2.3 & 7 & 26.7 & 21.4-28.7 & 25.5 & 2.7 & 5 \\
\hline Internarial distance (AE) & 27.2 & $17.1-23.0$ & 20.7 & 1.7 & 20 & & $17.5-20.0$ & 18.4 & 0.9 & 7 & 19.9 & $18.9-21.0$ & 19.9 & 0.7 & 5 \\
\hline Posterior naris to eye (PE) & & $2.8-6.1$ & 4.4 & 1.0 & 20 & & $5.5-8.8$ & 7.3 & 1.2 & 7 & 6.5 & $4.9-7.5$ & 6.4 & 0.9 & 5 \\
\hline Orbital diameter (OD) & & $9.6-14.4$ & 12.0 & 1.4 & 20 & 13.0 & $9.0-11.4$ & 10.5 & 0.9 & 7 & 10.1 & $10.1-12.1$ & 11.0 & 0.8 & 5 \\
\hline Interorbital distance (IO) & & $23.2-30.4$ & 26.3 & 2.4 & 20 & 23.7 & $26.8-32.3$ & 29.2 & 2.1 & 7 & 25.0 & $17.6-25.0$ & 22.4 & 2.8 & 5 \\
\hline Head depth at eye (HD1) & 48.5 & $45.7-55.7$ & 49.8 & 2.6 & 20 & & $40.0-51.6$ & 48.0 & 3.8 & 7 & 44.7 & $44.7-50.3$ & 48.2 & 2.3 & 5 \\
\hline Caudal filament depth (CD) & & $17.6-25.0$ & 20.6 & 1.9 & 20 & & $12.5-17.9$ & 15.7 & 2.5 & 5 & 14.0 & $14.0-19.8$ & 17.3 & 2.4 & 5 \\
\hline Caudal filament width $(\mathrm{CW})$ & & $8.3-15.3$ & 10.7 & 1.9 & 20 & & $5.4-9.5$ & 8.1 & 1.7 & 5 & 7.0 & 5.3-8.9 & 7.1 & 1.4 & 5 \\
\hline \multicolumn{16}{|c|}{ Other proportions } \\
\hline BW1 ( $\%$ of BD1) & & $45.3-65.8$ & 52.2 & 4.3 & 19 & & $50.0-58.3$ & 52.7 & 2.9 & 7 & 52.8 & $52.5-54.7$ & 53.4 & 0.9 & 5 \\
\hline BW2 (\% of BD2) & & 27.2-41.9 & 32.8 & 4.1 & 20 & & $25.6-37.0$ & 32.1 & 3.5 & 7 & 29.8 & $29.8-40.7$ & 35.2 & 5.0 & 5 \\
\hline
\end{tabular}

Description. Head and body shape, and pigmentation illustrated in Fig. 32. Meristic and morphometric data for examined specimens presented in Tables 2-5 and 15. Body shallow to moderate in depth. Head short and shallow to moderate in depth. Dorsal profile of head straight to slightly convex from occiput to snout, ventral profile of head straight between operculum and snout, snout truncate to rounded. Eye moderate in size. Upper jaw with moderate to acute sigmoidal angle between premaxillary and maxillary portions in lateral view. No accessory electric organ over operculum. Gill filaments on first gill arch 44-51 (median $48, \mathrm{n}=4)$. Pectoral fin narrow to moderate in width, pectoral-fin rays 13-16 (mode 15). Precaudal vertebrae 19-20 (mode 20), with 1-2 (mode 1) transitional vertebrae. Anal-fin origin slightly $(<0.25 \mathrm{HL}$ distance) anterior to, posterior to, or near, tip of pectoral fin. Anal-fin rays 181-223 (median 203). Dorsal rami of recurrent branch of anterior lateral line nerve visible. Middorsal region of body scaled. Rows of scales above lateral line 4-6 (mode 5). Lateral line continuous. Very sparse and irregular presence of 
depigmented epidermal canal; restricted mostly to posterior half of body, as parallel striations flanking lateral line, and as single irregular meandering lines around midway from lateral line to dorsal midline, or near edges of dorsal surface. Four bilateral columns of electrocytes at anal-fin terminus, and at mid-point between anal-fin terminus and tip of caudal filament in immature, mature female, and mature male specimens. Caudal filament moderate in length to long.

Coloration. (Fig. 32). Background pale tan to brown. Dorsal region with irregular dark markings, without prominent depigmented pale stripe extending along midline from occipital region to base of caudal filament. Diffuse, narrow vertical bands extend from dorsal region to lateral line in some specimens, mainly in anterior third of body. Irregular series of dark spots often present along lateral line. Ventral flank without vertical bands, with irregular dark spots in region of body cavity and over anal-fin pterygiophores. Caudal filament slightly darker than body, usually relatively free of dark markings. Head with evenly scattered dark chromatophores, darker dorsally. Eye without prominent suborbital patch, or stripe, of chromatophores/subcutaneous pigmentation. Pectoral and anal-fin membranes hyaline. Pectoral-fin rays with light, even scattering of dark chromatophores. Anal-fin rays with light scattering of dark chromatophores, which fuse in proximal part of rays. Color in live individuals similar to preserved specimens, with opercular region usually rosy due to underlying gills.

Size. Moderate adult size. Largest specimen examined $230 \mathrm{~mm}$ TL, $148 \mathrm{~mm}$ LEA $(\mathrm{n}=227)$. Largest male specimen examined $199 \mathrm{~mm}$ TL, $135 \mathrm{~mm}$ LEA $(\mathrm{n}=15)$. Largest female specimen examined $230 \mathrm{~mm}$ TL, $148 \mathrm{~mm}$ LEA $(n=13)$.

Sexual dimorphism. Breeding male specimens develop somewhat elongated caudal filaments in comparison to immature individuals and breeding females, but do not exhibit an elevated number of horizontal bilateral columns or vertical rows of electrocytes. Instead breeding males exhibit clearly enlarged electrocytes relative to immature specimens and females. Males not known to reach greater average lengths than females, despite elongated caudal filaments. Tip of caudal filament in large males does not exhibit a paddle-like lateral compression. No sexual differences in pigmentation.

Geographic distribution. Brazil (Fig. 23). Known from the rio São João, rio Paraíba do Sul, and small intervening coastal drainages (e.g., rio Ururaí-Lago Feia dr.), in the state of Rio de Janeiro. Brachyhypopomus janeiroensis was also collected from the area now comprising urban Rio de Janeiro during the 1865-1866 Thayer Expedition, in the presence of the Emperor of Brazil Dom Pedro II (MCZ 9457 and 165863).
Ecological notes. Throughout its range B. janeiroensis occurs in the aquatic vegetation of normoxic, slow-flowing streams, and also lentic, swampy, and periodically hypoxic habitats (pers. comm., R. Campos-da-Paz, UFRJ). The type locality of $B$. janeiroensis is a narrow slow-flowing stream with a sandy bottom and heavily vegetated banks, which traverses forest fragments and pastureland (Costa \& Campos-da-Paz, 1992). In April 2006 the following water parameters were recorded at this site: conductivity $38 \mu \mathrm{Scm}^{-1}$, dissolved oxygen $5.0 \mathrm{mgl}^{-1}$, temperature 23.9 C, transparency slightly silted (WGRC \& R. Campos-daPaz, unpublished data). Reproductive biology is unknown. Stomach contents of specimens from the type locality comprise aquatic insect larvae and other small aquatic invertebrates (WGRC unpublished data).

Co-occurring congeners: None.

Local names. Brazil: tuvira.

Material examined. 227 specimens. Brazil. Rio de Janeiro. FMNH 15201, 6, 125-165 mm, FMNH 54545, 27, 83-210 mm (11 sexed, 3 immature, 148-156 mm, 3 female, 122-157 mm, 5 male, 126-199 mm), Campos, rio Paraíba do Sul dr., ca. $21^{\circ} 45^{\prime} \mathrm{S}, 041^{\circ} 18^{\prime} \mathrm{W}$. FMNH 54547, 4, 131-184 mm (2 sexed: 1 female, $150 \mathrm{~mm}, 1 \mathrm{male}, 184 \mathrm{~mm}$ ), Lagoa Feia, Tocas [Caxia de Tocos?], rio Ururaí-Lagoa Feia dr., ca. $22^{\circ} 00^{\prime} \mathrm{S}, 041^{\circ} 20^{\prime} \mathrm{W}$. MCZ 9457, 25, 105-216 mm, MCZ 165863, 72 (30 examined), 104-202 mm, mun. Rio de Janeiro, Santa Cruz, rio Grande (arroio Fundo), in urban Rio de Janeiro, coastal drainage of urban Rio de Janeiro, $c a .22^{\circ} 56^{\prime} \mathrm{S}, 043^{\circ} 12^{\prime} \mathrm{W}$. MNRJ 12127, 1 (paratype), $166 \mathrm{~mm}$, mun. Silva Jardim, affl. rio Maratuan, rio São João dr., $c a .22^{\circ} 39^{\prime} \mathrm{S}, 042^{\circ} 24^{\prime} \mathrm{W}$. MNRJ 13503, 1, $195 \mathrm{~mm}$, MNRJ 14634, 2, 34-176 mm, mun. São Fidelis, Fazenda Poço d'Antas, rio Dois Rios, aff (R bank), rio Piraíba do Sul dr., $c a$. $21^{\circ} 38^{\prime} \mathrm{S}, 041^{\circ} 51^{\prime} \mathrm{W}$. MNRJ 15593, $1,134 \mathrm{~mm}$, mun. Campos dos Goytacazes, Lagoa Feia at Ponta Grossa dos Fidalgos, rio Ururaí-Lago Feia dr., ca. $21^{\circ} 57^{\prime} \mathrm{S}, 041^{\circ} 20^{\prime} \mathrm{W}$. MNRJ 17509 , 1, $64 \mathrm{~mm}$, mun. Macaé, Lagoa Preta (restinga de Quissamã), boundary of Macaé with Campos, Lagoa Preta dr., ca. $22^{\circ} 08^{\prime} \mathrm{S}$, $041^{\circ} 25^{\prime} \mathrm{W}$. MNRJ 38510, 12 (10 immature, $99-162 \mathrm{~mm}, 1$ female, $153 \mathrm{~mm}, 1 \mathrm{male}, 111 \mathrm{~mm}$ ), mun. Silva Jardim, $c a .5 \mathrm{~km}$ NNW Silva Jardim, bridge over small creek, affl. rio São João dr., $22^{\circ} 35^{\prime} 46^{\prime \prime} \mathrm{S}, 042^{\circ} 24^{\prime} 52^{\prime \prime} \mathrm{W}$. MZUSP 22702, 5 (3 immature, $72-85 \mathrm{~mm}, 1$ female, $121 \mathrm{~mm}, 1 \mathrm{male}, 149 \mathrm{~mm}$ ), MZUSP 22703, 1, female, 114 mm, São João da Barra, rio Paraíba do Sul dr., $c a$. $21^{\circ} 39^{\prime} \mathrm{S}, 041^{\circ} 03^{\prime} \mathrm{W}$. MZUSP 23013 , 1 , São Fidelis, rio Paraíba do Sul dr., $c a .21^{\circ} 38^{\prime} \mathrm{S}, 041^{\circ} 44^{\prime} \mathrm{W}$. MZUSP 43130, 1 (holotype), 124 mm, MZUSP 43131, 7 (paratypes), 75-129 mm LEA, UFRJ 598, 4 (paratypes), 76-131 mm LEA, UFRJ 599, 2 (paratypes), 80-80 mm LEA, collected with holotype. UFRJ 529, 3, 79-91 mm LEA, córrego Salto d'Água $6 \mathrm{~km} N$ Silva Jardim (rd. from Gaviões), rio São João dr., $c a .22^{\circ} 39^{\prime} \mathrm{S}, 042^{\circ} 23^{\prime} \mathrm{W}$. MZUSP 45880,20 , mun. Silva Jardim, riacho Fazenda Nova, $5 \mathrm{~km}$ road to Bananeiras, rio São João dr., $c a .22^{\circ} 40^{\prime} \mathrm{S}, 042^{\circ} 22^{\prime} \mathrm{W}$. MZUSP 80122, 2, male (1 CS), 174-176 mm, mun. Silva Jardim, 28 km N by Boqueirão-Japuiba rd., at Gaviões, rio São João dr., 
ca. $22^{\circ} 34^{\prime} \mathrm{S}, 042^{\circ} 34^{\prime} \mathrm{W}$. NRM 22480, 2, 123-151 mm, mun. Silva Jardim, Japuiba, Gaviões, rio São João dr., ca. $22^{\circ} 33^{\prime}$ S, $042^{\circ} 31^{\prime} \mathrm{W}$. SU 36964, 1, $98 \mathrm{~mm}$, USNM 129915, 6, 85-230 mm ( 3 sexed: 1 immature, $148 \mathrm{~mm}, 2$ female, 138-230 mm), Rio de Janeiro and its vicinity, $c a .22^{\circ} 55^{\prime} \mathrm{S}, 043^{\circ} 09^{\prime} \mathrm{W}$. SU 62742, 4, 44-87 mm, Rio de Janeiro, old Petrópolis rd., no coordinates. UF 183780, 2 (1CS), 120-130 mm, mun. Casimiro de Abreu, rio São João dr., $c a .22^{\circ} 29^{\prime} \mathrm{S}, 042^{\circ} 12^{\prime} \mathrm{W}$. ZMH 11627, 18 (14 measured and sexed: 5 immature, $112-129 \mathrm{~mm}, 4$ female, $120-159 \mathrm{~mm}, 5$ male, 152-185 mm), prov. Rio de Janeiro, no coordinates.

\section{Brachyhypopomus jureiae Triques \& Khamis, 2003}

(Fig. 33; Tables 2-5, 15)
Brachyhypopomus jureiae Triques \& Khamis, 2003: 62, fig. 1, color photograph of paratype (original description, type locality Brazil, São Paulo, rio Una do Prelado, Juréia Ecological Station). -Oyakawa et al., 2006: 74, color photograph of live individual (Brazil, São Paulo, catalog of fishes from Atlantic forest streams). -Menezes et al., 2007: 319 (reproduction of photograph from Oyakawa et al., 2006). -Crampton \& Albert, 2006: 672, fig. 23.8, position in phylogenetic tree; 681, notes on EODs (gymnotiform species and EOD diversity). -Crampton, 2011: 176, table 10.2, species list; 179, figs. 10.210.3, phylogeny, geographical and ecological distributions (gymnotiform biology). -Crampton et al., 2016: 1-66, table 1, 3-4, figs. 1-7, 18-20 (phylogeny, biogeography and ecology of Brachyhypopomus).

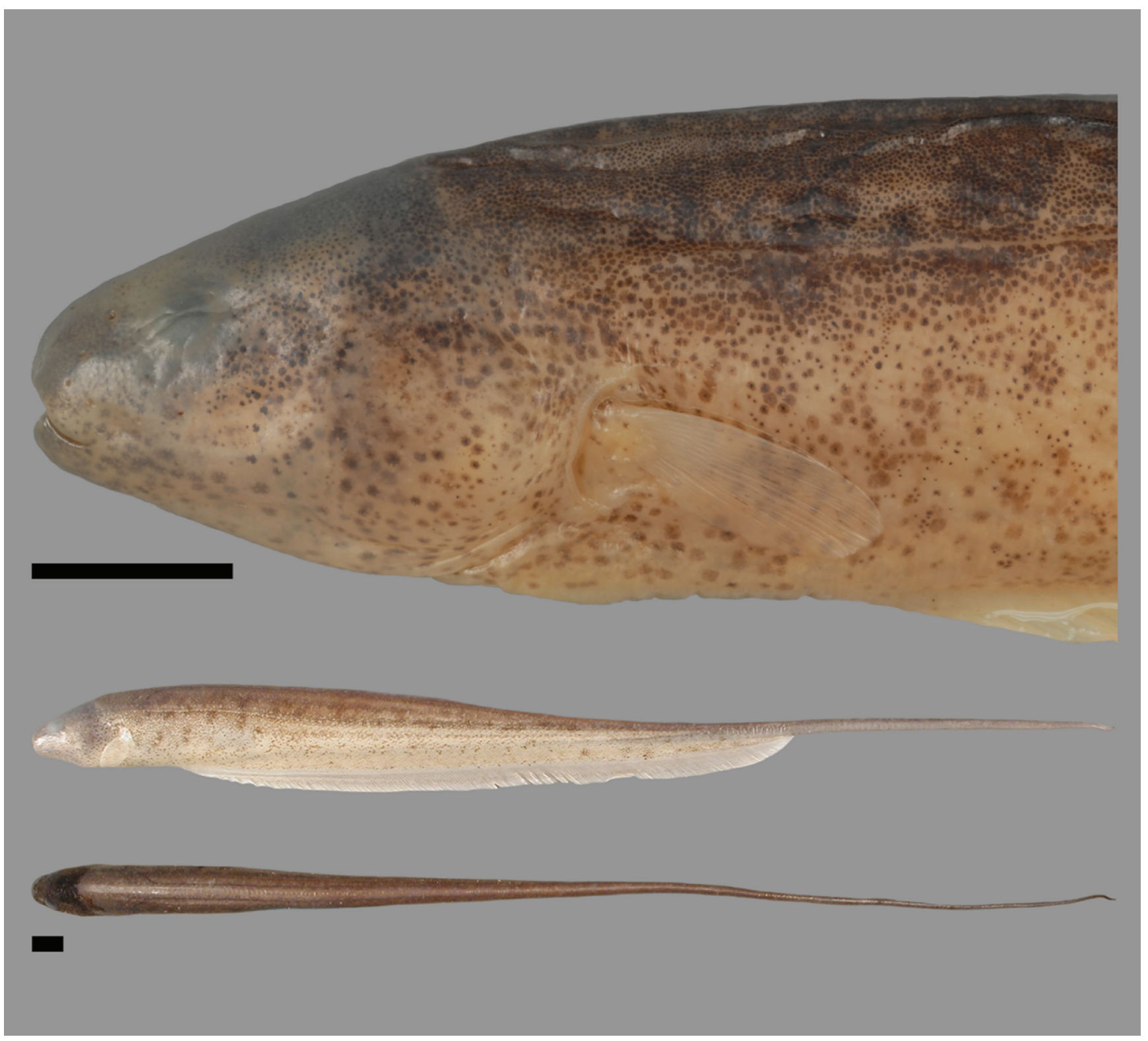

Fig. 33. Brachyhypopomus jureiae. MZUSP 100268, female, $170 \mathrm{~mm}$ TL (head in lateral view, and body in lateral and dorsal view, specimen fixed in formalin and preserved in EtOH); Brazil, rio Bateçaria, rio Ribeira de Iguape dr. Scale bars $=5 \mathrm{~mm}$. 
Diagnosis. Brachyhypopomus jureiae is diagnosed from congeners by the following combination of characters: bilateral columns of electrocytes at the anal-fin terminus 4 , vs. 6 in $B$. bennetti and vs. 3 in all other congeners except B. belindae, B. diazi, B. janeiroensis, B. occidentalis (except some populations in Colombia and Venezuela, see redescription of $B$. occidentalis), and $B$. palenque; precaudal vertebrae 20 , vs. 16-19 in $B$. diazi and vs. 2426 in B. belindae; snout to pectoral-fin base $12.3-15.7 \%$ LEA, vs. $10.7-12.3 \%$ in $B$. janeiroensis; pectoral-fin rays 14-16, vs. 17-21 in B. palenque. Brachyhypopomus jureiae exhibits similar pigmentation and overlapping meristic counts and morphometric proportions with $B$. occidentalis but can be distinguished by absence of the lateral ethmoid absent, vs. presence (Mago-Leccia, 1978: 58, fig. 11) in $B$. occidentalis.

Description. Head and body shape, and pigmentation illustrated in Fig. 33. Meristic and morphometric data for examined specimens presented in Tables 2-5 and 15. Body moderate in depth. Head moderate in length and shallow to moderate in depth. Dorsal profile of head approximately straight from occiput to snout, ventral profile of head approximately straight to slightly concave between operculum and snout, snout rounded. Eye moderate in size. Upper jaw with moderate sigmoidal angle between premaxillary and maxillary portions in lateral view. No accessory electric organ over operculum. Pectoral fin moderate in width, pectoral-fin rays 14-16 (mode 16). Precaudal vertebrae 20, with 1-2 (mode 2) transitional vertebrae. Anal-fin origin slightly $(<0.25 \mathrm{HL}$ distance $)$ posterior to, or near, tip of pectoral fin. Anal-fin rays 188-220 rays (197-220, median 205 in specimens we examined). Dorsal rami of recurrent branch of anterior lateral line nerve visible. Middorsal region of body scaled. Rows of scales above lateral line 6-7 (mode 6). Lateral line continuous. Many specimens with complete absence of groove-like depigmented epidermal canals. Some specimens with very sparse depigmented canals bordered by dark pigment, forming parallel wavy scratch-like marks either side of and near (1-2 scales distance from) lateral line in posterior half of body only (mostly dorsal to lateral line), and some single meandering canals in upper dorsum. Four bilateral horizontal columns of electrocytes at anal-fin terminus, and at a mid-point between anal-fin terminus and tip of caudal filament in immature, mature female, and mature male specimens. Caudal filament moderate to long in length.

Coloration. (Fig. 33). Background pale to dark brown, much darker dorsal to lateral line. Dorsal region without prominent depigmented pale stripe extending along midline from occipital region to base of caudal filament. Dorsal surface speckled with dark pigment and irregular dark flecks or patches over background. Vertical stripes and saddles absent on flank. Dorsal and ventral flank with light, even scattering of chromatophores and with irregular dark brown or charcoal spots. Caudal filament darker dorsally and with irregular dark patches. Head with very pale background color ventrally, darker dorsally. Eye without prominent suborbital patch, or stripe, of chromatophores/subcutaneous pigmentation. Pectoral and anal-fin membranes hyaline. Pectoral and anal-fin rays hyaline with light scattering of brown chromatophores. Color in live individuals similar to preserved specimens, with opercular region usually rosy due to underlying gills.

Size. Moderate adult size, largest specimen examined 292 mm TL, $194 \mathrm{~mm}$ LEA $(\mathrm{n}=14)$. Largest male specimen examined $170 \mathrm{~mm}$ TL, $125 \mathrm{~mm}$ LEA $(\mathrm{n}=1)$. Largest female specimen examined $169 \mathrm{~mm}$ TL, $116 \mathrm{~mm}$ LEA $(n=4)$.

Sexual dimorphism. No known secondary sexual dimorphism.

Geographic distribution. Brazil (Fig. 23). Known only from the rio Una do Prelado and rio Ribeira de Iguape, coastal drainages in southeast São Paulo state.

Ecological notes. Brachyhypopomus jureiae is known from small Atlantic forest and coastal lowland streams. Triques \& Khamis (2003) provide some notes on the ecology of the type locality: "specimens were collected in the rio do Descalvado, a blackwater river of muddy bottom with surface partially covered by water-hyacinth (Eichhornia sp.); grasses are occasionally present on the margins. Water pH 6.0." One of us (WGRC) collected B. jureiae from small very slow-flowing blackwater streams of the rio Ribeira de Iguape drainage in July 2006 and July 2008. The streams traversed forest fragments and pastureland, were $0.5-2 \mathrm{~m}$ in width, up to $0.5 \mathrm{~m}$ deep, and had clay or sandy beds. All specimens were collected from submerged leaf litter or marginal vegetation. The following water parameters were recorded: conductivity 55-62 $\mu \mathrm{Scm}^{-1}$, dissolved oxygen 2.5-3.0 mgl ${ }^{-1}$, temperature $15-23^{\circ} \mathrm{C}, \mathrm{pH} 6.4$. Reproductive biology is unknown. Stomach contents of specimens from the rio Ribeira de Iguape drainage comprise aquatic insect larvae and other small aquatic invertebrates (WGRC unpublished data).

Co-occurring congeners: None.

Local names. Brazil: tuvira.

Material examined. 14 specimens. Brazil. São Paulo. DZUFMG 010, 1 (holotype), $292 \mathrm{~mm}$ TL, $194 \mathrm{~mm}$ LEA, examined from photographs, DZUFMG 011, 2 (paratypes), 142-147 mm LEA, examined from photograph, MZUSP 67491, 1 (paratype), MZUSP 70004, 1 (paratype), 141 mm, Estação Ecológica Juréia, rio do Descalvado, rio Una do Prelado dr., ca. $24^{\circ} 29^{\prime} \mathrm{S}, 047^{\circ} 14^{\prime} \mathrm{W}$. FMNH 54543, 1, 153 mm, Iguape, rio Ribeira de Iguape dr., $c a$. 
$24^{\circ} 40^{\prime} \mathrm{S}, 047^{\circ} 32^{\prime} \mathrm{W}$. MZUSP 83020, 2, immature, 60-65 mm, nr. Vila de Momuna, stream affl. rio Momuna, rio Ribeira de Iguape dr., 244ㄴ $57^{\prime \prime}$ S, 4741'27"W. MZUSP 93118, 1, male (CS), 170 $\mathrm{mm}$, stream, affl. rio Momuna, ca. $1.5 \mathrm{~km}$ Vila de Momuna, rio

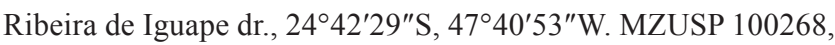
5, (1 immature, $100 \mathrm{~mm}, 4$ female, 149-169 mm), nr. Vila de Momuna, rio Bateçaria, affl. rio Momuna, rio Ribeira de Iguape dr., $24^{\circ} 42^{\prime} 56^{\prime \prime} \mathrm{S}, 047^{\circ} 41^{\prime} 28^{\prime \prime} \mathrm{W}$.

\section{Brachyhypopomus menezesi, new species}

urn:1sid:zoobank.org:act:5C81E7C7-4DCE-4EEA-A56FD0533ACF70E2

\section{(Fig. 34; Tables 2-5, 15)}

Brachyhypopomus sp. "men”. -Crampton, 2011: 176, table 10.2, species list; 179, figs. 10.2-10.3, phylogeny, geographical and ecological distributions (gymnotiform biology).
Brachyhypopomus electropomus. -Sullivan, 1997: 122 (description in unpublished thesis with disclaimer stating that nomenclatural acts not available, a nomen nudum, name refers also to $B$. bombilla and $B$. regani).

Brachyhypopomus sp. "menezesi". -Crampton et al., 2016: 1-66, table 1, 3-4, figs. 1-7, 18-20 (phylogeny, biogeography and ecology of Brachyhypopomus).

Holotype. MZUSP 87147, male, $100 \mathrm{~mm}$ TL, $75 \mathrm{~mm}$ LEA, Brazil, Bahia, 1 km São Marcelo, rio Sapão, affl. rio Preto, rio São Francisco dr., $11^{\circ} 01^{\prime} 02^{\prime \prime} \mathrm{S}, 045^{\circ} 31^{\prime} 50^{\prime \prime} \mathrm{W}$, 11 Nov 2002, C. Moreira \& J. Nolasco.

Paratypes. 6 specimens, localities from rio São Francisco dr. Brazil. Bahia. MZUSP 40190, 1 (CS), 111 mm, mun. Ibiraba, nr. mouth of rio Icatú, ca. $10^{\circ} 33^{\prime} \mathrm{S}, 043^{\circ} 06^{\prime} \mathrm{W}$, Sep 1988, M. Rodrigues. Minas Gerais. MCZ 52124, 5 (2 female, 111-112 $\mathrm{mm}, 3$ male, 112-119 $\mathrm{mm}$ ), rio Pandeiros, ca. $15^{\circ} 42^{\prime} \mathrm{S}, 044^{\circ} 36^{\prime} \mathrm{W}, 1938$, W. Schroeder.

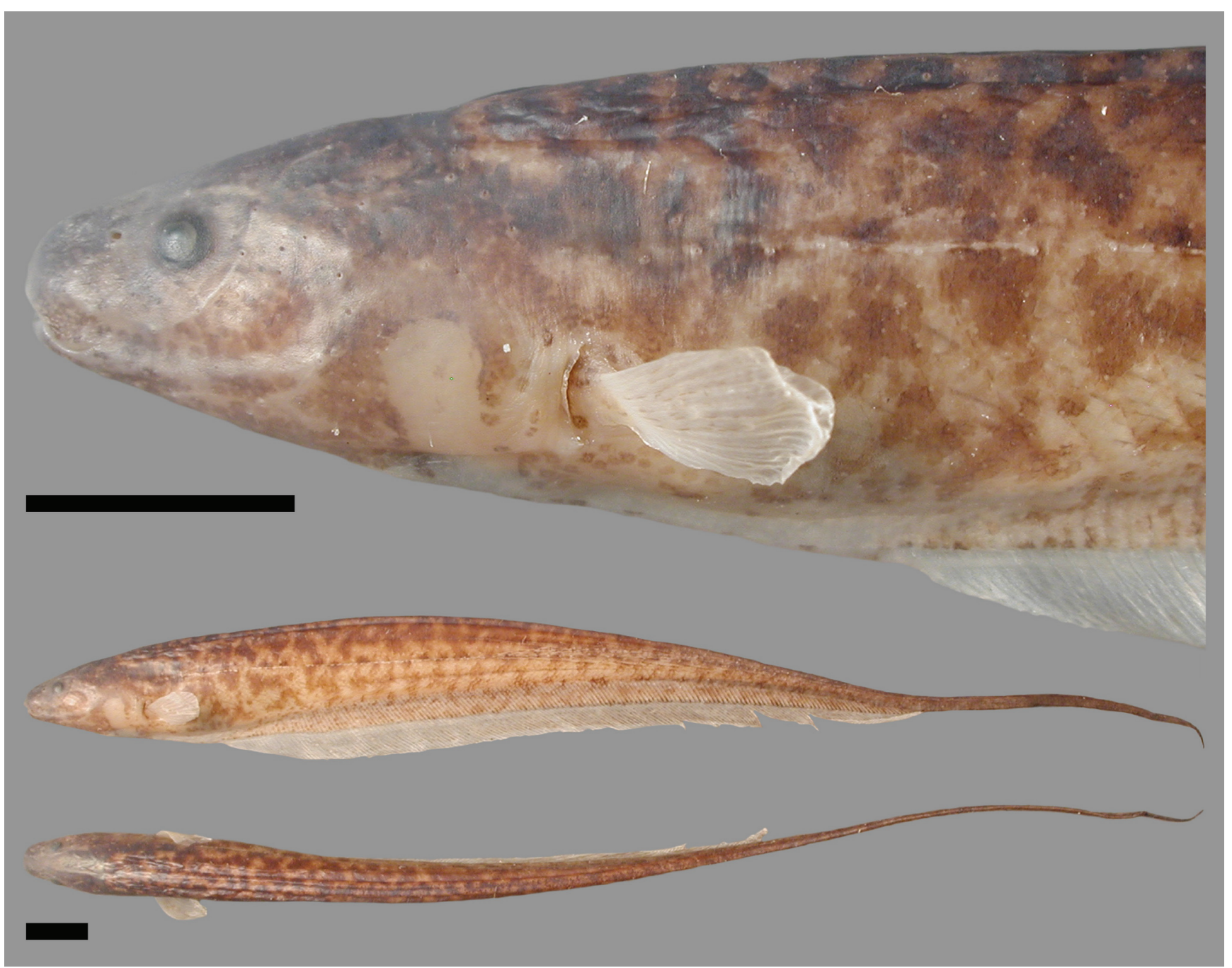

Fig. 34. Brachyhypopomus menezesi. MZUSP 87147, holotype, male, $100 \mathrm{~mm}$ TL (head in lateral view, and body in lateral and dorsal view, specimen fixed in formalin and preserved in EtOH); Brazil, rio Sapão, rio São Francisco dr. Note prominent opercular accessory electric organ (pale structure). Scale bars $=5 \mathrm{~mm}$. 
Non-types. 7 specimens. Brazil. Minas Gerais (localities from rio São Francisco dr.). MCP 28255, 5, 35-56 mm, mun. Claro de Minas, stream at entrance of Claro de Minas, hwy. Guarda-Mor-Vazante, affl. rio Claro, affl. rio Escuro, affl. rio Paracatu, $17^{\circ} 56^{\prime} 07^{\prime \prime} \mathrm{S}, 046^{\circ} 58^{\prime} 28^{\prime \prime} \mathrm{W}$. MZUSP 39420, 2, 68-71 mm LEA, Lagoa do Tacho [nr. Pirapora], L margin rio São Francisco, ca. $17^{\circ} 20^{\prime}$ S, $044^{\circ} 56^{\prime} \mathrm{W}$.

Diagnosis. Brachyhypopomus menezesi is diagnosed from congeners by the following combination of characters: accessory electric organ over the opercular region present, vs. absent in all congeners except $B$. bombilla and $B$. regani; dorsal surface with large dark blotches against a lighter background, vs. dorsal surface speckled with small brown chromatophores on a pale background in B. bombilla; caudal filament length $24.7-33.3 \%$ LEA, vs. $11.1-17.9 \%$ in B. regani.

Description. Head and body shape, and pigmentation illustrated in Fig. 34. Meristic and morphometric data for examined specimens presented in Tables 2-5 and 15. Body moderate in depth. Head moderate in length and shallow to moderate in depth. Dorsal profile of head straight to slightly concave from occiput to snout, ventral profile of head approximately straight to slightly concave between operculum and snout, snout truncate to rounded. Eye moderate in size. Upper jaw with moderate sigmoidal angle between premaxillary and maxillary portions in lateral view. Accessory electric organ (AEO) over operculum present and very prominent; skin over AEO completely depigmented; border rounded and very conspicuous. Pectoral fin narrow to moderate in width, pectoral-fin rays 14-16 (no mode, median 15). Precaudal vertebrae 16-17 (mode 16), with 1-2 (mode 2) transitional vertebrae. Anal-fin origin slightly $(<0.25 \mathrm{HL}$ distance) posterior to, or near, tip of pectoral fin. Rows of scales above lateral line 6-7 (mode 6). Lateral line continuous. Multiple groove-like depigmented and darkly pigmented epidermal canals form parallel striations either side of lateral line in posterior third of body; striations reaching 1-4 scales (including lateral line series) dorsally, and 1-2 scales ventrally. Epidermal canals flanking lateral line not present in anterior portion of body, do not form a pair of long irregular lines either side of dorsal surface in dorsal portion of flank, and do not form isolated horizontal wavy line midway from lateral line to dorsal midline in midbody or anterior portion of body. Additional multiple parallel fine depigmented epidermal canals running in an anterodorsal to posteroventral direction over body cavity, some of which converge posteriorly to two or three canals running posteriorly for approximately third analfin base distance approximately half way from lateral line to proximal edge of anal-fin pterygiophores before fading. Three bilateral columns of electrocytes at anal-fin terminus, and at a mid-point between anal-fin terminus and tip of caudal filament in immature, mature female, and mature male specimens. Electrocytes of hypaxial electric organ extend anterior to anal and urogenital pores and extend into opercular area to form paired accessory electric organ. Caudal filament of moderate length.

Coloration. (Fig. 34). Background light tan to brown. Dorsal region without prominent depigmented pale stripe extending along midline from occipital region to base of caudal filament. Dorsal region mottled with irregular pattern of small, well demarcated, interconnected dark blotches against a homogenous lighter background (contrasting with a uniform speckling of small brown chromatophores against a lighter background in $B$. bombilla). Dark mottling extends ventrally across dorsal and ventral flank, with highest density in dorsal portions of flank. Caudal filament darker than body, with some irregular dark markings. Head with evenly scattered dark chromatophores, darker dorsally. Region over accessory electric organ completely depigmented. Eye without prominent suborbital patch, or stripe, of chromatophores/subcutaneous pigmentation. Pectoral and anal-fin membranes hyaline. Pectoral-fin rays hyaline. Anal-fin rays hyaline with light scattering of brown chromatophores. Color in live individuals never observed, but likely similar to preserved specimens.

Size. Small adult size, largest specimen examined $119 \mathrm{~mm}$ TL, $83 \mathrm{~mm}$ LEA $(\mathrm{n}=14)$. Largest male specimen examined $119 \mathrm{~mm}$ TL, $83 \mathrm{~mm}$ LEA $(\mathrm{n}=4)$. Largest female specimen examined $112 \mathrm{~mm} \mathrm{TL}, 88 \mathrm{~mm}$ LEA $(\mathrm{n}=2)$.

Sexual dimorphism. No known secondary sexual dimorphism.

Geographic distribution. Brazil (Fig. 27). Known only from the middle and upper rio São Francisco drainage, in the states of Bahia and Minas Gerais.

Ecological notes. Geographical coordinates and labels accompanying the examined museum specimens indicate that $B$. menezesi occurs in small rivers and streams, and also lacustrine environments. Stomach contents of one examined specimen comprised aquatic insect larvae - mostly Chironomidae. The ecology of $B$. menezesi is otherwise unknown.

Co-occurring congeners: None.

Etymology. The specific name is a patronym (noun in the genitive case) in honor of Naércio Aquino Menezes, Brazilian ichthyologist, for his important contributions to Neotropical fish systematics.

Local names. Brazil: tuvira.

Brachyhypopomus occidentalis (Regan, 1914) 


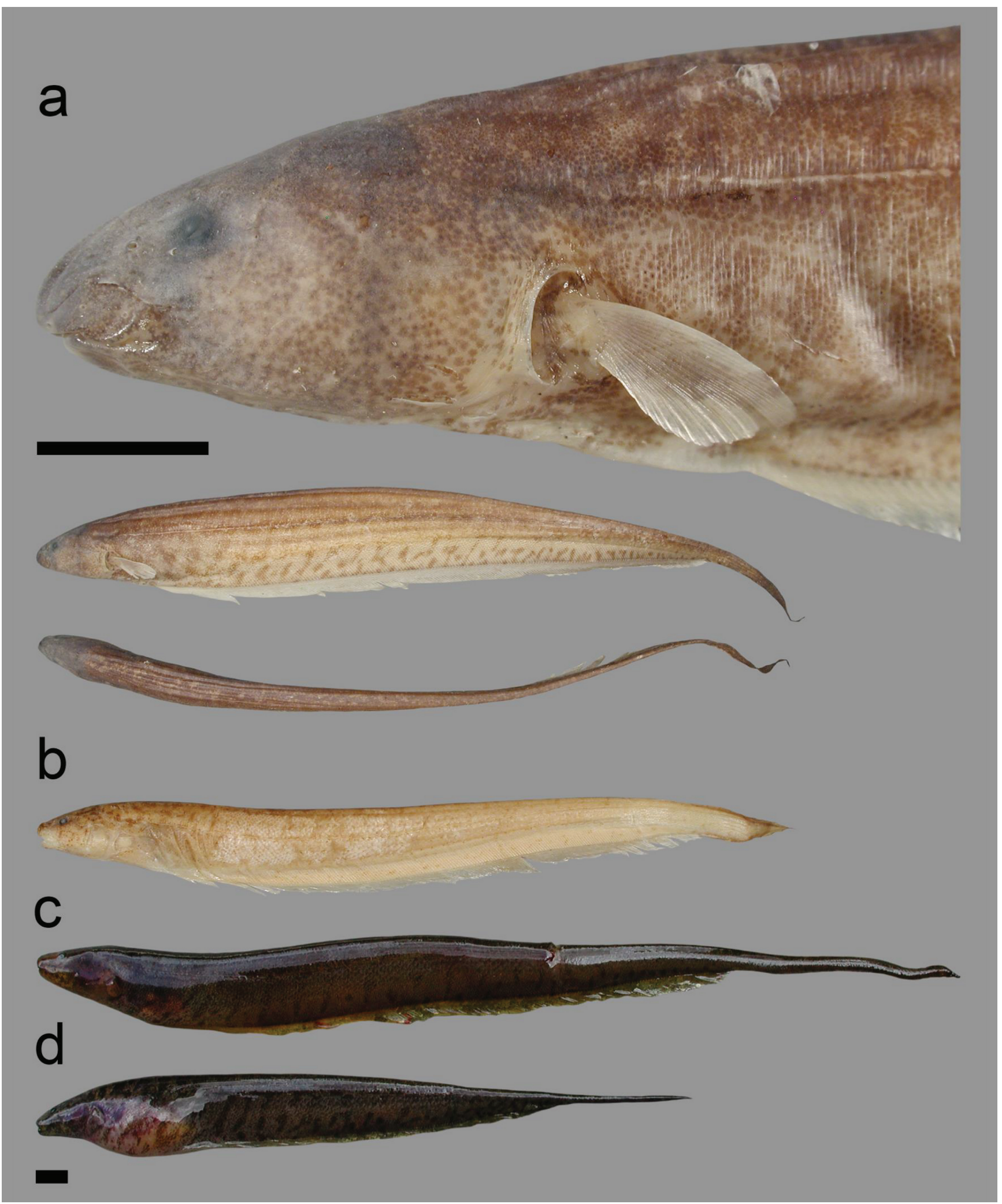

Fig. 35. Brachyhypopomus occidentalis. a. NRM 27736, immature, $131 \mathrm{~mm}$ TL (head in lateral view, and body in lateral and dorsal view, specimen fixed in formalin and preserved in EtOH); Colombia, rio Baudó dr. b. BMNH 2012.6.13.1, largest syntype specimen, sex not determined, $147 \mathrm{~mm}$ TL (body in lateral view, specimen preserved in EtOH, note damage and subsequent regeneration to caudal filament); Colombia, río San Juan dr. c. IAVHP 7022 (WC04.230905), male, 162 mm TL. d. IAvHP 7022 (WC03.230905), female, $112 \mathrm{~mm}$ TL, non-type (bodies in lateral view, live individuals); Colombia, río Atrato dr. Note sexual dimorphism in caudal-filament length and height in c (male) \& d (female). Scale bars $=5 \mathrm{~mm}$. 


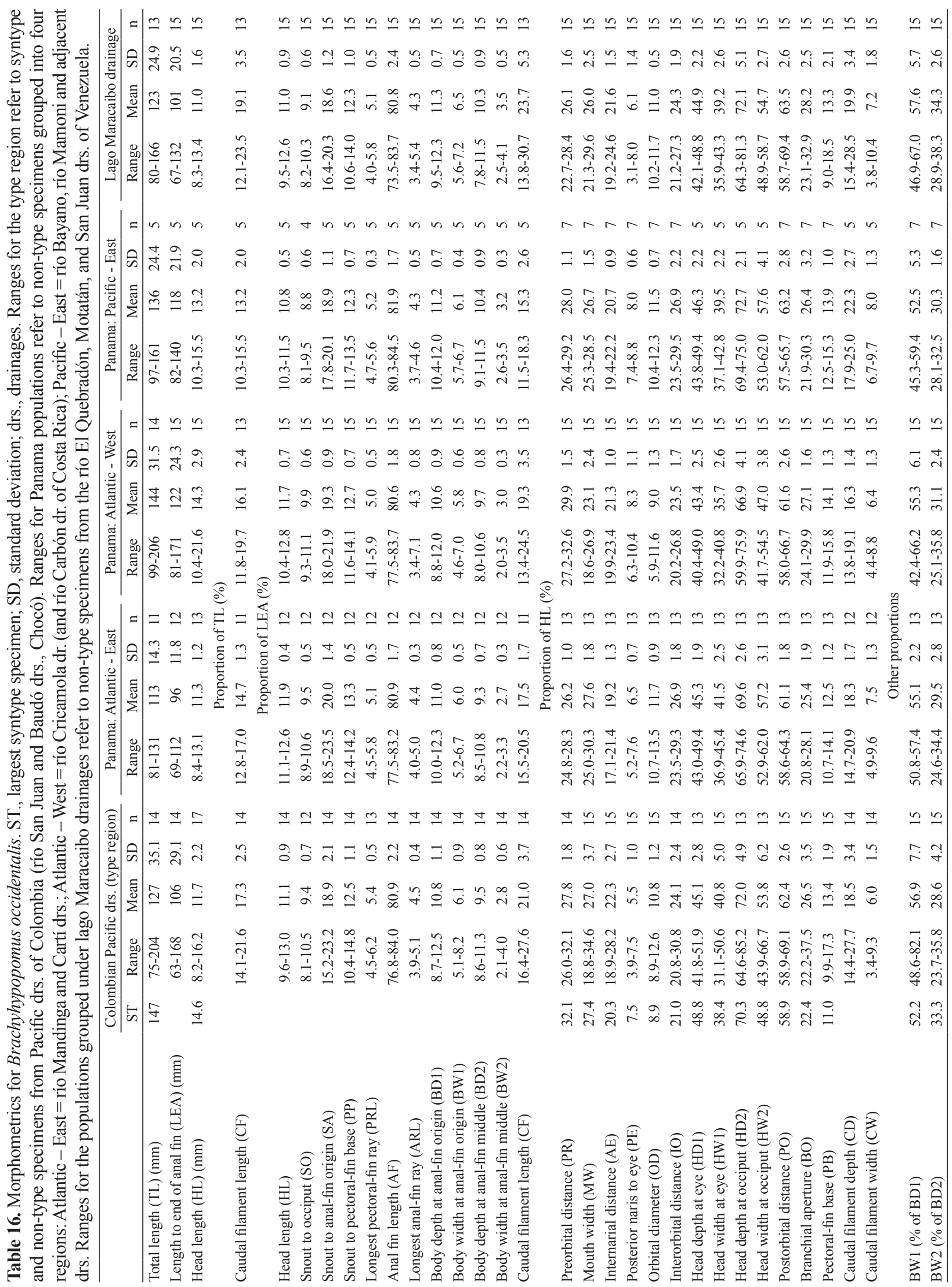


Rhamphichthys (Brachyrhamphichthys) brevirostris. -Steindachner, 1880: 89 (Colombia, Cauca dr., catalog of fishes).

Brachyrhamphichthys brevirostris. -Eigenmann \& Eigenmann, 1891: 62 (Colombia, Cauca dr., catalog of South American fishes).

Hypopomus brevirostris. -Eigenmann \& Ward, 1905: 170, 186. pl. 10, fig. 13, black and white photograph without locality data, photograph is not of $B$. brevirostris; it resembles $B$. occidentalis (Colombia, Cauca dr., listing of gymnotiform species). -Eigenmann, 1910: 449 (Cauca dr., catalog of fishes). -Eigenmann \& Fisher, 1914: 236 (Colombia - Soplaviento [Magdalena dr.], Managru, Truando, Raspadura, Quibdó [Atrato dr.]; Calima [San Juan dr.], Gymnotiformes of trans-Andean Colombia and Ecuador). -Meek \& Hildebrand, 1916: 310 (Panama, río Chagres basin, río Bayano basin, Atlantic and Pacific drainages of Panama and Colombia, catalog of fishes). -Eigenmann, 1922: 174 (Atlantic and Pacific drainages of Panama, Atrato, San Juan and Magdalena drs., catalog of fishes in NW South America). -Eigenmann \& Allen, 1942: 314 (Panama, Chagres, listing of gymnotiforms). -Fowler, 1945: 179 (Panama, catalog of fishes).

Hypopomus occidentalis Regan, 1914: 32 (original description, type locality - Colombia, Chocó, río Condoto, río San Juan dr.). -Eigenmann, 1922: 175 (Colombia, río Condoto, catalog of fishes in NW South America). -Bussing, 1987: 99, fig. 37, black and white photograph (Costa Rica, catalog of fishes). -Schultz, 1944: 40 (diagnosis against B. beebei). -Schultz, 1949: 66, key to hypopomids; fig. 9a., illustration of arrangement of cephalic pores (Venezuela, listings from Motatán and Maracaibo drs.). -Hagedorn \& Carr, 1985: 513, fig. 1, drawing of breeding male and female, with sexual dimorphism of caudal filament (Panama, reproductive ecology - image reprinted in Hagedorn, 1986: 512, fig. 4; Hagedorn: 1988, 330, fig. 2; Kramer: 1990, 14, fig. 1.6). -Galvis et al., 1997: 102, color photograph of live individual (Colombia, río Catatumbo dr., fish list for NW South America). -Bussing, 1998: 134, black and white photograph, note on diet (Costa Rica, catalog of fishes).

Brachyhypopomus occidentalis. -Mago-Leccia, 1994: 48, 170, fig. 70, line drawing of head and anterior portion of body of specimen MBUCV-V 14112, río Guaco, Maracaibo dr., Venezuela (in listing of Brachyhypopomus). -Sullivan, 1997: 133 (redescription). -Bermingham \& Martin, 1998: 502, 505 (Panama - Atlantic and Pacific drs.; Colombia - Atrato and Magdalena drs., molecular phylogeography). -Hopkins, 1999: 1218, fig. 1, black and white photographs of breeding male and female, reproduced from Hagedorn, 1988: 330, fig. 2 (electric organ morphology and impedance matching). -Albert \& Crampton, 2003: 495 (Colombia, Panama, catalog of hypopomids). -Crampton \& Albert 2006: 672, fig. 23.8, position in phylogenetic tree; 681, notes on EODs (gymnotiform species and EOD diversity). -Crampton, 2011: 176, table 10.2, species list; 179, figs. 10.2-10.3, phylogeny, geographical and ecological distributions (gymnotiform biology). -MaldonadoOcampo et al., 2012: 323, color photograph of live individual (Colombia - Atrato, San Juan, Baudó, Calima, Anchicayá, Dagua, Mira drs., listing of fish in Chocó, Colombia). -Carvalho, 2013: 147, fig. 3D, outline of head; 181-185, figs. 41-43, position in phylogeny (phylogenetic systematics of Rhamphichthyoidea). -Picq et al., 2014: 1520-1532 (Panama and Colombia, phylogeography). -Elbassiouny et al., 2016: 402-403, fig. 1 (position in phylogeny of
Ostariophysi, based on mitochondrial genomes). -Picq et al., 2016 (EOD evolution). -Tagliacollo et al., 2016: 28, fig. 5 (phylogeny of Gymnotiformes). -Crampton et al., 2016: 1-66, table 1, 3-4, figs. 1-7, 13, 18-20 (phylogeny, biogeography and ecology of Brachyhyроротиs).

Diagnosis. Brachyhypopomus occidentalis is diagnosed from congeners by the following combination of characters: absence of series of diffuse horizontal dash-like dark markings along lateral line in posterior third of body, anterior to anal-fin terminus, vs. presence in $B$. alberti, $B$. arrayae, $B$. hamiltoni, and $B$. draco; pale stripe along middorsal region of body absent, vs. prominent pale uninterrupted middorsal stripe from occipital region to base of caudal filament in $B$. beebei, $B$. belindae, $B$. gauderio, $B$. pinnicaudatus, and $B$. verdii; analfin rays 191-254, vs. 155-225 in B. batesi, B. flavipomus, $B$. menezesi, and $B$. sullivani; pectoral-fin rays 15-20, vs. 10-13 in $B$. benjamini and B. cunia; bilateral columns of electrocytes at anal-fin terminus 4-5 (or 3 in some populations in Colombia and Venezuela, see below), vs. 6 in B. bennetti; accessory electric organ over opercular region absent, vs. present in B. bombilla and $B$. regani; caudal filament short, $11.5-30.7 \%$ LEA, vs. 31.3-64.5\% in B. bullocki, B. hendersoni, and B. janeiroensis; diagonally oriented bars on flank diffuse and mostly break up ventral to lateral line, vs. vertical bars form distinct saddles which extend across lateral line in B. brevirostris; continuous or discontinuous dark vertical or diagonally oriented stripes or saddles present on body surface dorsal to lateral line, vs. absent in B. jureiae, B. palenque, and B. provenzanoi; dark suborbital stripe absent, vs. present in B. walteri. Brachyhypopomus occidentalis exhibits similar pigmentation and overlapping meristic counts and morphometric proportions with $B$. diazi. These two species can be distinguished by the more extensive network of depigmented and dark-margin epidermal canals in $B$. diazi (including in the anterior half of the body) than in $B$. occidentalis, and by the first branchiostegal ray is approximately as wide as the third branchiostegal ray in $B$. occidentalis, vs. the first branchiostegal ray is distinctly narrower than the third in $B$. diazi.

Description. Head and body shape, and pigmentation illustrated in Fig. 35. Meristic and morphometric data for examined specimens presented in Tables 2-5 and 16. Body shallow to moderate in depth. Head short to moderate in length and shallow to moderate in depth. Dorsal profile of head straight to gently convex from occiput to snout, ventral profile of head slightly concave between operculum and snout, snout rounded. Eye small to moderate in size. Upper jaw slightly to moderately prognathous. Upper jaw with slight sigmoidal angle between premaxillary and maxillary portions in lateral view. No accessory electric organ over operculum. Gill filaments on first gill arch 35-54 (median 48, $\mathrm{n}=10$ ). Pectoral fin narrow to broad in width, pectoral-fin rays 15-20. Modes and ranges similar for specimens from Pacific coastal drainages of Colombia (16-18, mode 17), Maracaibo drainage (15-18, mode 17), eastern Atlantic drainages of Panama (16- 
18, mode 17), and eastern pacific drainages of Panama (1518 , mode 17), but slightly higher for specimens from western Atlantic drainages of Panama (río Cricamola) (18-21, mode 18). Precaudal vertebrae 17-20. Modes and ranges similar for specimens from Pacific coastal drainages of Colombia (19-21, mode 19), eastern Atlantic drainages of Panama (18-19, mode 19), western Atlantic drainages of Panama (18-20, mode 19 ), and eastern pacific drainages of Panama (18-20, mode 19), but slightly lower for specimens from the Maracaibo drainage (17-19, mode 18). 1-3 transitional vertebrae (mode 2 in all examined populations). Anal-fin origin slightly (< $0.25 \mathrm{HL}$ distance) anterior to, or posterior to, or near, tip of pectoral fin. Anal-fin rays 194-254. Ranges and medians for specimens are similar for specimens from the following three regions: eastern Atlantic drainages of Panama, 194234 (216); western Atlantic drainages of Panama, 199-228 (216); eastern Pacific drainages of Panama, 212-229 (216). Ranges and medians are slightly lower for specimens from the Maracaibo drainage, 201-213 (203), and slightly higher for specimens from the Pacific coast of Colombia 207-254 (222). Dorsal rami of recurrent branch of anterior lateral line nerve not visible. Middorsal region of body scaled. Rows of scales above lateral line 5-7 (mode 7). Lateral line continuous. Depigmented epidermal canals sparse, and mostly restricted to posterior half of body. In anterior half of body canals occur in low density in upper portions of flank, usually as single meandering lines in dorsal flank around midway between lateral line and dorsal midline, nearer to lateral line posteriorly. In second half of body canals occur as multiple parallel wavy lines in dorsal and (less commonly) in ventral flank, approximately parallel to lateral line. Density of epidermal canals somewhat higher in populations from the Maracaibo basin, but in all populations considerably less extensive and conspicuous than in B. diazi. Number of bilateral electrocyte columns: in populations from region of type locality (río Condoto): 4 columns at anal-fin terminus in immature specimens and 4-5 (mode 4) in mature females and males, and 4-5 (mode 4) columns at a midpoint from anal-fin terminus to tip of caudal filament in immature specimens, mature females and males. In some specimens extra electrocyte columns appear posterior to mid-point between anal-fin terminus and tip of caudal filament - especially in laterallycompressed paddle-like tip - bringing maximum number of columns to 5-6 (in both males and females). Specimens with regenerated caudal filaments often have more columns of electrocytes in regenerated portions. We noted populationlevel variation in number of bilateral columns of electrocytes in B. occidentalis. For example, number measured at anal-fin terminus was: río Atrato, Colombia (3-4, mode $3, \mathrm{n}=9$ ); río Magdalena, Colombia ( $3, \mathrm{n}=9$ ); río Sinú, Colombia $(3, \mathrm{n}=$ 1), río Motatan, lago Maracaibo, Venezuela (3-4, mode 3, n $=22$ ); río Chagres, Panama (4-6, mode 5; increasing to 6-7 columns in laterally compressed caudal filament tip, $\mathrm{n}=12$ ), río Mandingá, Panama ( $3, \mathrm{n}=5)$; río Cricamola, Panama ( $4, \mathrm{n}$ =12), río Sixaola, Costa Rica (4-5, mode 4, $\mathrm{n}=9$ ). More work is needed to fully characterize population-variation in gross anatomy of the electric organ of B. occidentalis, much of which may be explained by impedance matching to ambient conductivity. Caudal filament short to moderate in length.

Coloration. (Fig. 35). Pigmentation highly variable within and between regions. Eigenmann (1922, 174-175), discussed variation in pigmentation among populations of B. occidentalis from the Magdalena, Atrato, and San Juan drainages of Colombia. Background pale tan to dark brown, usually considerably darker dorsally. Dorsal region without prominent depigmented pale stripe extending along midline from occipital region to base of caudal filament. Dorsal surface with speckling of lighter pigment on dark background, and sometimes with irregular dark patches which may extend into thin vertically oriented or diagonal incomplete bands that extend across flank; banding if present is usually more conspicuous in anterior third of body. Ventral flank over anal-fin pterygiophores with irregular dark patches forming a marbled pattern. Caudal filament darker than body. Head with evenly scattered dark chromatophores, darker dorsally. Eye without prominent suborbital patch, or stripe, of chromatophores/ subcutaneous pigmentation. Pectoral and anal-fin membranes hyaline. Pectoral-fin rays hyaline with light scattering of dark chromatophores. Color in live individuals similar to preserved specimens, often with olive green tinge, opercular region usually rosy due to underlying gills, body cavity of gravid females often yellow due to underlying ripe eggs (Fig. 35d).

Size. Moderate adult size, largest specimen examined 278 $\mathrm{mm}, 216 \mathrm{~mm}$ TL $(\mathrm{n}=892)$. Largest male specimen examined $278 \mathrm{~mm}$ TL, $216 \mathrm{~mm} \mathrm{LEA}(\mathrm{n}=18)$. Largest female specimen examined $162 \mathrm{~mm} \mathrm{TL}, 144 \mathrm{~mm}$ LEA $(\mathrm{n}=33)$.

Sexual dimorphism. We observed population variation in the degree to which caudal morphology differs between breeding males and females, which may be correlated to electrical conductivity. Conductivity varies considerably among drainages occupied by $B$. occidentalis, including lowconductivity systems $\left(c a .5-60 \mu \mathrm{Scm}^{-1}\right)$ and high-conductivity systems (ca. $\left.100-450 \mu \mathrm{Scm}^{-1}\right)$. In low-conductivity systems (e.g. streams of the río Cricamola, río Atrato, conductivity 5-30 $\mu \mathrm{Scm}^{-1}, \mathrm{NRL}$, WGRC pers. obs.), breeding males appear to develop longer and marginally widened caudal filaments. In contrast, in very high conductivity systems (conductivity $300-450 \mu \mathrm{Scm}^{-1}$ in some streams of the río San Juan, río Bayano listed in 'Material examined', and parts of Panama, NRL pers. obs.), breeding males develop very wide but not very elongated caudal filaments. These observations suggest population-level variation in caudal filament morphology associated with impedance matching sensu Hopkins (1999) and merit a more detailed quantitative study.

In addition to exhibiting enlarged caudal filaments, the number of bilateral columns of electrocytes at the anal-fin terminus increases slightly, from invariably 4 in immature specimens and females to 4-5 (mode 4) in breeding males. Breeding males also exhibit clearly enlarged electrocytes 
relative to immature specimens and females. Large breeding males commonly with paddle-like lateral compression at caudal filament tip (Figs. 35c-d). No known sexual dimorphism in pigmentation.

We noted population variation in the maximum size attained by males. Breeding males in museum collections from two drainages, the río San Juan and río Cricamola, were considerably larger than females ( $c a .260-278 \mathrm{~mm}$ in fully mature males, vs. ca. $103-153 \mathrm{~mm}$ in fully mature females). In contrast, mature males were only marginally larger than females in other drainages, including the río Atrato, Atlantic and Pacific drainages of eastern Panama, and Maracaibo drainages. Based on the phylogeny of Picq et al. (2014), these observations suggest multiple independent origins of a prominent secondary sexual dimorphism of body size.

Geographic distribution. Colombia, Costa Rica, Panama, and Venezuela (Fig. 36). Westernmost records from the río Sixaola drainage, an Atlantic drainage of Costa Rica. Known from multiple Atlantic and Pacific drainages throughout Panama, but note gaps in collecting records in Fig. 36. Widespread through Pacific coast of Colombia, including the río Atrato (Atlantic drainage), río San Juan, río Baudó, río San Cipriano and rio Anchicayá (Pacific drainages). Isolated records from the río Sinú (Atlantic drainage) and lower and middle río Magdalena (Atlantic drainage). Widespread in lowland drainages around the entire perimeter of lago de Maracaibo.

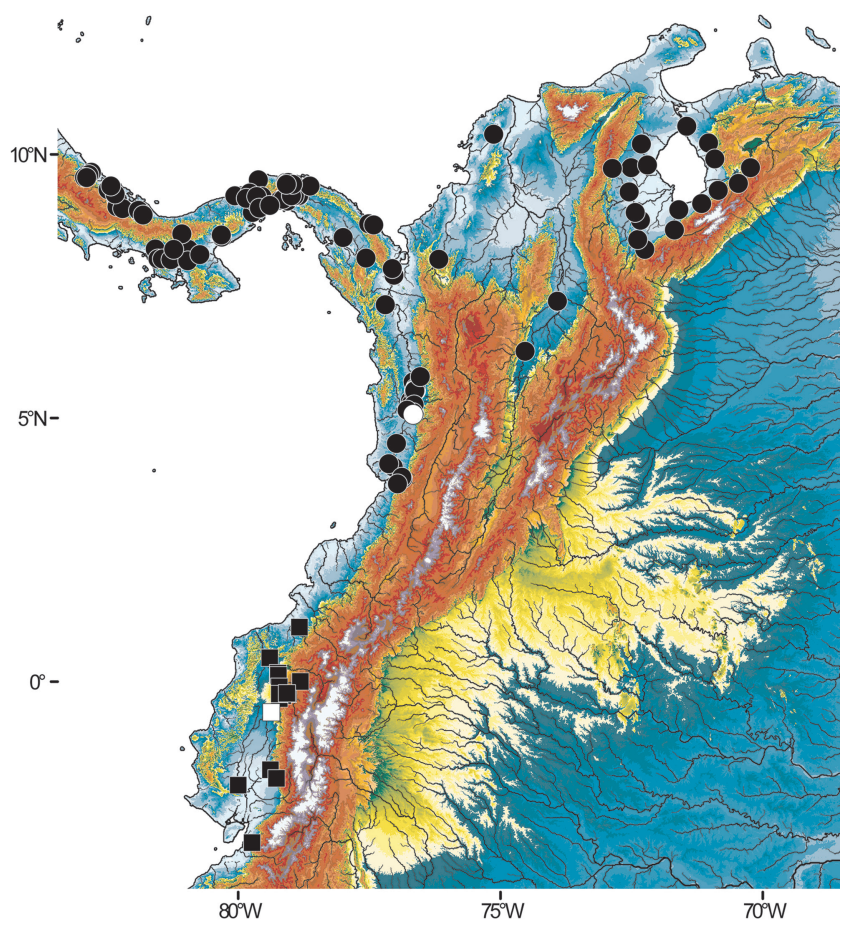

Fig. 36. Collection records for Brachyhypopomus occidentalis (circles) and $B$. palenque (squares). The lectotype location for $B$. occidentalis and holotype location for $B$. palenque are marked with open symbols. Elevation data refers to altitude above mean sea level (see Fig. 2 for legend).
Population variation: We found complete overlap in the range of meristic counts (Tables 2-5) and morphometric proportions (Table 16), and observed similar pigmentation among specimens from five geographical regions. Specimens from no single region exhibited consistently divergent meristic counts or morphometric proportions (see 'Description', and 'Sexual dimorphism'). These observations, in combination with molecular data (Crampton et al., 2016), support the hypothesis that all populations we have assigned to $B$. occidentalis are members of a single geographically widespread species, which is morphologically and genetically distinct from all congeners.

Mirroring the results of an earlier study by Birmingham \& Martin (1998), Picq et al. (2014), presented a population level phylogeny of $B$. occidentalis in which populations from west Panamanian Atlantic drainages (Cricamola, Sixaola, Changuinola, Calovébora) form the sister taxon to populations from all other regions of Panama and northwestern Colombia (including Pacific and Atlantic drainages); both studies also concluded that populations from lago Maracaibo drainages constitute the sister taxon to all Panama-Colombia lineages. Picq et al. (2013; 2016) noted EOD divergences between populations in independent drainages consistent with non-adaptive drift, and observed an unusual divergence of the EOD waveforms of breeding males in the río Cricamola relative to other Panamanian populations. Although we did not note salient morphometric and meristic differences between specimens of $B$. occidentalis from the río Cricamola versus other populations of $B$. occidentalis, we did observe a more extreme form of sexual size dimorphism and larger maximum body sizes in río Cricamola specimens than in populations from other Panamanian drainages, the río Atrato, and lago Maracaibo (see 'Sexual dimorphism').

Ecological notes. Brachyhypopomus occidentalis is common in small, gently-flowing streams and shallow swamps and lagoons throughout its range (Bussing, 1987; Crampton, 2011; Hagedorn, 1988). Water parameters, including $\mathrm{pH}$, conductivity (see 'Sexual dimorphism'), dissolved oxygen, and turbidity are highly variable in the systems inhabited by this species. The reproductive ecology and mating behavior of Panamanian $B$. occidentalis have been documented in detail by M. Hagedorn and co-authors (Hagedorn, 1986; 1988; Hagedorn \& Zelick, 1989; Kawasaki \& Heiligenberg, 1989). Brachyhypopomus occidentalis breeds through the dry season, reaching maturity in its first year and exhibiting post-reproductive mortality indicative of an annual lifecycle. Individuals exhibit a high degree of site fidelity.

Stomach contents of $B$. occidentalis from the Atrato drainage of Colombia comprise aquatic insect larvae (especially Chironomidae) and other small aquatic invertebrates (WGRC unpublished data). Bussing (1987; 1998) also reported a diet of aquatic invertebrates, 
especially Diptera, in populations from Costa Rica. Dunlap et al. (2016) infer predation of Panamanian B. occidentalis by the heptapterid catfish Rhamdia - based on correlations between caudal filament damage and the density of Rhamdia.

Co-occurring congeners: None.

Local names. Colombia: cuchillo, beringo culebra (Atrato), mayupita (Catatumbo) (Galvis et al., 1997); Costa Rica: madre de barbudo (Bussing, 1987); Panama: cuchillo.

Material examined. 773 specimens. Colombia. Antioquia (localities from Atlantic drainages). CAS 72231, 1, $65 \mathrm{~mm}$, quebrada Cristalina at Cristalina, $28 \mathrm{~km}$ above Puerto Berrio, río Magdalena dr., $c a .06^{\circ} 16^{\prime} \mathrm{N}, 074^{\circ} 32^{\prime} \mathrm{W}$. IAVH-P 7278, 4, mun. Turbo, caño Tumarado, last 'cienaga' [swamp], Puerto Plata Vereda, El Cuarenta, río Atrato dr., 07² $2^{\prime} 34^{\prime \prime} \mathrm{N}, 077^{\circ} 01^{\prime} 46^{\prime \prime} \mathrm{W}$. IAVH-P 7411, 1, caño Muerto, last "cienaga" [swamp], Vereda [village] El Cuarenta, río Atrato dr., 0742'31"N, 07701'56"W. Bolívar. FMNH 56788, 5, 88-142 mm, SU 63675, 5, 88-142 mm, Canal de Dique between Cartagena and Calamar, at Soplaviento, affl. río Magdalena, río Magdalena dr. (Atlantic), $c a \cdot 10^{\circ} 23^{\prime} \mathrm{N}$, 07508’W. Chocó. BMNH 1910.7.11.217, 1, 99 mm, río Condoto, río San Juan dr. (Pacific), ca. $05^{\circ} 04^{\prime} \mathrm{N}, 076^{\circ} 40^{\prime} \mathrm{W}$. BMNH 1914.5.18.94-98, 6 (syntypes), 41-105 mm, río Condoto, río San Juan dr. (Pacific), ca. $05^{\circ} 04^{\prime} \mathrm{N}, 076^{\circ} 40^{\prime} \mathrm{W}, 1914$, H. Spurrell. BMNH 1915.10.1.10, 1, 123 mm, Andagoya, río San Juan, río San Juan dr. (Pacific), ca. $05^{\circ} 05^{\prime} \mathrm{N}, 076^{\circ} 43^{\prime} \mathrm{W}$. BMNH 2012.6.13.1, 1 (syntype), $147 \mathrm{~mm}$ (see Fig. 35b), same collection data as BMNH 1914.5.18.94-98. CAS 72219, 3, 85-132 mm, Managru, upper río Quito, río Atrato dr. (Atlantic), ca. $05^{\circ} 20^{\prime} \mathrm{N}, 076^{\circ} 43^{\prime} \mathrm{W}$. CAS 72227, 3, río Truando, río Atrato dr. (Atlantic), ca. $07^{\circ} 09^{\prime} \mathrm{N}$, $077^{\circ} 12^{\prime} \mathrm{W}$. CAS 72229, 2, FMNH 56792, 1, $121 \mathrm{~mm}$, creek nr. mouth of río Guineo, affl. río Calima, río San Juan dr. (Pacific), ca. $04^{\circ} 04^{\prime} 30^{\prime \prime} \mathrm{N}, 077^{\circ} 02^{\prime} 50^{\prime \prime} \mathrm{W}$. FMNH 56779, 3, 80-163 mm, Managrú, rio Atrato dr. (Atlantic), ca. $05^{\circ} 20^{\prime} \mathrm{N}, 076^{\circ} 43^{\prime} \mathrm{W}$. FMNH 56787, 2, 86-128 mm, Truando [río Truando], rio Atrato dr. (Atlantic), ca. $07^{\circ} 09^{\prime} \mathrm{N}, 077^{\circ} 12^{\prime} \mathrm{W}$. FMNH 56789, 2, 90-98 mm, Raspadura [probably Boca de Raspadura and not Raspadura, Nariño because C. E. Wilson 1913-1914 collections were all in vicinity of Quibdó, Chocó], affl. río Paimado, río Atrato dr. (Atlantic), $c a .05^{\circ} 16^{\prime} \mathrm{N}, 076^{\circ} 42^{\prime} \mathrm{W}$. FMNH 56790, 1, $92 \mathrm{~mm}$, Quibdó, río Atrato dr. (Atlantic), ca. $05^{\circ} 41^{\prime} \mathrm{N}, 076^{\circ} 39^{\prime} \mathrm{W}$. FMNH 70885, 1, 117 mm, Pangala, ca. 40 km N Palestina, río San Juan dr. (Pacific), $c a .04^{\circ} 31^{\prime} \mathrm{N}, 076^{\circ} 59^{\prime} \mathrm{W}$. IAVH-P 6812, 2, IAVH-P 6814, 2, IAVH-P 6816, 1, IAVH-P 6817, 1, IAVH-P 6820, 1, IAVH-P 7019, 1, immature, 80 mm, IAVH-P 7020, 13 (8 immature, 38-131 mm, 3 female, 105-134 mm, 2 male, 93-140 mm), mun. Yuto, quebrada Josefa $0.5 \mathrm{~km}$ Yuto, río Atrato dr. (Atlantic), $05^{\circ} 31^{\prime} 58^{\prime \prime} \mathrm{N}$, 076³8'04"W. IAVH-P 6813, 3, IAVH-P 6815, 1, IAVH-P 6818, 3, IAVH-P 6819, 4, IAVH-P 6821, 1, mun. Yuto, río Yuto, río Atrato dr., $c a .05^{\circ} 30^{\prime} \mathrm{N}, 076^{\circ} 39^{\prime} \mathrm{W}$. IAVH-P 7017, 13 (11 immature, 41$120 \mathrm{~mm}, 1$ female, $146 \mathrm{~mm}, 1 \mathrm{male}, 144 \mathrm{~mm})$, mun. Quibdó, quebrada Samurindó, 0-0.5 km upstream bridge on Quibdó-Yuto rd., río Atrato dr. (Atlantic), 05³4'24"N, 076 37'13"W. IAVH-P 7021, 27 (13 immature, 31-98 mm, 10 female, 90-134 mm, 5 male,
105-140 mm), IAVH-P 7022, 12 (9 immature, 46-102 mm, 2 female, 112-160 mm, 1 male, $162 \mathrm{~mm}$ ), quebrada Jigudat, $5 \mathrm{~km} \mathrm{~S}$ Yuto on Quibdó- Certegui rd., río Atrato dr. (Atlantic), $05^{\circ} 30^{\prime} 57^{\prime \prime} \mathrm{N}$, $076^{\circ} 37^{\prime} 48^{\prime \prime W}$. IAVH-P 7410, 1, mun. Riosucio, caño Sautata, río Atrato dr. (Atlantic), ca. $07^{\circ} 50^{\prime} \mathrm{N}, 077^{\circ} 04^{\prime} \mathrm{W}$. IAVH-P uncat. Ict1.1.18, 6, 99-142 mm, IAVH-P uncat. Ict-1.1.19, 2, 130-131 mm, IAVH-P uncat. Ict-1.3.1, 1, $162 \mathrm{~mm}$, IAVH-P uncat. Ict-1.3.2, 9, 95-162 mm, IAVH-P uncat. Ict-1.3.3, 3, 104-173 mm, nr. Yuto, río Atrato dr. (Atlantic), ca. $05^{\circ} 31^{\prime} \mathrm{N}, 076^{\prime} 38^{\prime \prime} \mathrm{W}$. IAVH-P uncat. 12, 155-270 mm, río San Juan dr. (Pacific), no coordinates. NRM 10699, 2, 75-194 mm, headwaters of río San Juan, río San Juan dr. (Pacific), $c a .05^{\circ} 15^{\prime} \mathrm{N}, 076^{\circ} 39^{\prime} \mathrm{W}$. NRM 27728, 4, 74-123 mm, quebrada Tuaró, close to La Victoria on Pan-American hwy., río Baudó dr. (Pacific), no coordinates [listed coordinates $05^{\circ} 32^{\prime} \mathrm{N}$, $076^{\circ} 00^{\prime} \mathrm{W}$, not located in río Baudó dr.]. NRM 27729, 1, $126 \mathrm{~mm}$, NRM 27736, 5, 63-137 mm, NRM 30659, 2, 52-78 mm, Pie de Pepé, río Pepé, affl. río Baudó, río Baudó dr. (Pacific), 0508'59"N, 076 47'00"W. NRM 27735, 5, 56-130 mm, Chaparra Idó, beneath waterfall, Tutunendo-San Francisco de Ichó rd., río Atrato dr. (Atlantic), 05 $46^{\prime} 58^{\prime \prime} \mathrm{N}, 076^{\circ} 32^{\prime} 02^{\prime \prime} \mathrm{W}$. NRM 27747, 2, 58-70 mm, Condoto, quebrada on rd. Condoto-Soledad, $5 \mathrm{~min}$ from airport rd.,

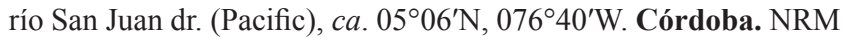
46106, 1, 153 mm, 3 km E Urrá Hydropower Station, quebrada Santa Ana, río Sinú dr. (Atlantic), 0800'40"N, 076¹0'48"W. Santander. CAC-CDMB 129, 1, 132 mm, mun. Barrancabermeja, río Sogamoso, río Magdalena dr. (Atlantic), ca. $07^{\circ} 13^{\prime} \mathrm{N}, 073^{\circ} 55^{\prime} \mathrm{W}$ [coordinate for mouth of río Sogamoso]. Valle del Cauca (localities from Pacific drainages). CAS 72223, 3, río Calima, río San Juan dr., no coordinates. FMNH 56791, 2, 140-174 mm, creek nr. mouth of río Calima, río San Juan dr., ca. $04^{\circ} 08^{\prime} \mathrm{N}, 077^{\circ} 08^{\prime} \mathrm{W}$. IMCN 2248, 2, 132-158 mm, quebrada La Sardiña, affl. río Dagua, río San Cipriano dr., $c a .03^{\circ} 51^{\prime} \mathrm{N}, 076^{\circ} 52^{\prime} \mathrm{W}$. IMCN 4523, 5, quebrada El Venado at Cordoba, río San Cipriano dr., 0352'28"N, 07655'33"W. NRM 27751, 1, $90 \mathrm{~mm}$, Sabaletas, río Sabaletas and streams upstream Sabaletas, rio Anchicayá dr., $03^{\circ} 44^{\prime} \mathrm{N}, 076^{\circ} 58^{\prime} \mathrm{W}$. Region not specified. CU 71934, 4, no locality, no coordinates. Costa Rica (localities from Atlantic drainages). Limón. ANSP 163126, 4, 125-164 mm, creek on rd. from Bribri to Suretka, $6.0 \mathrm{~km} \mathrm{~W}$ Bribri, río Sixaola dr., 09³7'36"N, 082 $52^{\circ} 44^{\prime \prime} \mathrm{W}$. ANSP 163170, 4, $103-$ $131 \mathrm{~mm}$, río Carbón, at Sixaola-Limon rd. $7.0 \mathrm{~km}$ NE Bribri, río Carbón dr., 09 $39^{\prime} 45^{\prime \prime} \mathrm{N}, 082^{\circ} 48^{\prime} 15^{\prime \prime} \mathrm{W}$. ANSP 163176, 10 (5 measured, 107-211 mm), ANSP 163760, 2, 134-156 mm, ANSP $163762,1,133 \mathrm{~mm}$, creek crossing rd. $0.9 \mathrm{~km}$ W Shiroles, affl. río Telire, río Sixaola dr. (Atlantic), 09 $33^{\prime} 56^{\prime \prime} \mathrm{N}, 082^{\circ} 55^{\prime} 59^{\prime \prime} \mathrm{W}$. ANSP 163190, 6, 154-183 mm, ANSP 163205, 1, 129 mm, Suretka, creek crossing old Bribri-Suretka rd., río Sixaola dr., ca. $09^{\circ} 37^{\prime} \mathrm{N}$, $082^{\circ} 51^{\prime} \mathrm{W}$. ANSP $163761,3,113-154 \mathrm{~mm}$, stream at Suretka rd., $1.4 \mathrm{~km} \mathrm{~W}$ Bambu, no coordinates. NCSM 44973, 2, Canton Talamanca, nr. Bratsi, affl. río Telire, río Sixaola dr. (Atlantic), 09³3'41"N, 082 53'27"W. Panama. Bocas del Toro. UF 19727 , 2, female, 121-134 mm, unknown river entering Almirante Bay, mi. 5 along rd. from Almirante (Atlantic), ca. $09^{\circ} 15^{\prime} \mathrm{N}, 082^{\circ} 21^{\prime} \mathrm{W}$. Bocas del Toro: Comarca Ngobe-Buglé (localities from Atlantic drainages). BMNH 1925.3.6.229.238, 10 (3 immature, 137-176 mm, 4 female, 103-153 mm, 3 male, 159-278 mm), FMNH 59196, 5, 109-155 mm, UMMZ 145687, 2, 125-170 mm, UMMZ 145688, 
4, 97-200, UMMZ 145689, 4, 105-200 mm, creek nr. Konkintu, río Cricamola dr., $c a .08^{\circ} 51^{\prime} \mathrm{N}, 081^{\circ} 49^{\prime} \mathrm{W}$. CAS 67704, 4, 116-225 $\mathrm{mm}$, Guibari creek, below Konkintu, río Cricamola dr., ca. $08^{\circ} 51^{\prime} \mathrm{N}, 081^{\circ} 49^{\prime} \mathrm{W}$. CAS 67721, 1, CAS 72230, 7, "Nomormen [label unclear] creek" above Konkintu, Cricamola dr., ca. $08^{\circ} 51^{\prime} \mathrm{N}$, 08149'W. CAS 67722, 1, CAS 67723, 2, creeks nr. Konkintu, Cricamola dr., $c a .08^{\circ} 51^{\prime} \mathrm{N}, 081^{\circ} 49^{\prime} \mathrm{W}$. ROM 89751, 2, 206-214 $\mathrm{mm}$, STRI 7724, 3, 152-203 mm, caño Chalite, stream at mouth of Poman Creek, río Cricamola dr., 08 ${ }^{\circ} 54^{\prime} 13^{\prime \prime} \mathrm{N}, 081^{\circ} 52^{\prime} 53^{\prime \prime} \mathrm{W}$. ROM 89752, 3, 139-207 mm, ROM 89754, 8, 119-222 mm, STRI 7725, 9, 80-202 mm, stream nr. Bisira, río Cricamola dr., $08^{\circ} 54^{\prime} 14^{\prime \prime} \mathrm{N}$, 08151'27"W. ROM 89755, 1, 203 mm, ROM 89756, 2, 72-175 $\mathrm{mm}$, STRI 7727, 2, 118-183 mm, small stream affl. río Uyama, río Uyama dr., $09^{\circ} 08^{\prime} 58^{\prime \prime} \mathrm{N}, 082^{\circ} 19^{\prime} 33^{\prime \prime} \mathrm{W}$. ROM 89757, 3, 102-188 $\mathrm{mm}$, STRI 7728, 4, 55-131 mm $\left(09^{\circ} 08^{\prime} 21^{\prime \prime} \mathrm{N}, 082^{\circ} 18^{\prime} 26^{\prime \prime} \mathrm{W}\right)$, ROM 89758, 3, 64-142 mm (09 $\left.08^{\prime} 17^{\prime \prime} \mathrm{N}, 082^{\circ} 18^{\prime} 31^{\prime \prime} \mathrm{W}\right)$, stream at village (Baisiro?), off rd. from Almirante to Punta Peña, río Uyama dr. ROM 89759, 5, 83-231 mm, STRI 7729, 5, 98-195 mm, stream affl., río Robalo, río Robalo dr., 0900'27"N, 082¹8'49"W. ROM 89760, 4, 119-241 mm, ROM 89786, 1, 182 mm, small creek, N Almirante, río Changuinola dr., $09^{\circ} 20^{\prime} 04^{\prime \prime} \mathrm{N}, 082^{\circ} 28^{\prime} 56^{\prime \prime} \mathrm{W}$. STRI 7730, 4, 81-195 mm, stream on Almirante to Punta Peña rd., $08^{\circ} 58^{\prime} 24^{\prime \prime} \mathrm{N}, 082^{\circ} 12^{\prime} 23^{\prime \prime} \mathrm{W}$. UF 183793, 10, 58-163 mm, Reserva Indígena de Cricamola, río Cricamola dr., $08^{\circ} 56^{\prime} 18^{\prime \prime} \mathrm{N}$, 081 $54^{\prime} 15^{\prime \prime} \mathrm{W}$. USNM 302016, 1, $187 \mathrm{~mm}$, río Changuinola dr., ca. $09^{\circ} 24^{\prime} \mathrm{N}, 082^{\circ} 26^{\prime} \mathrm{W}$. Chiriquí (localities from Pacific drainages). AUM 32356, 1, 202 mm, 10 km ESE Tolé on Pan American hwy., río Tabascara, río Vigui dr., $08^{\circ} 12^{\prime} 27^{\prime \prime} \mathrm{N}, 081^{\circ} 35^{\prime} 07^{\prime \prime} \mathrm{W}$. Coclé. ANSP 151058, 1, $127 \mathrm{~mm}$, río Chorrera at Inter-American hwy., between Penonome and Anton, río Chorrera dr., ca. $08^{\circ} 26^{\prime} \mathrm{N}$, 080¹9'W. AUM 31594, 2, 130-146 mm, Pan American hwy., 3 km SSE Chorrerita, río Hondo dr., Bahia de Parito (Pacific), $08^{\circ} 28^{\prime} 18^{\prime \prime} \mathrm{N}, 080^{\circ} 19^{\prime} 25^{\prime \prime} \mathrm{W}$. Colón. ANSP 99880, 1, 75 mm, Canal Zone, río Agua Salud, creek on pipeline rd. nr. Gamboa, río Gatun dr. (Atlantic), ca. $09^{\circ} 11^{\prime} \mathrm{N}, 079^{\circ} 47^{\prime} \mathrm{W}$. ANSP 104216, 1, $115 \mathrm{~mm}$, coastal stream at Lagarto (Palma Nueva [Palmas Bellas]), W Canal Zone, río Lagarto dr. (Pacific), ca. $09^{\circ} 13^{\prime} \mathrm{N}, 080^{\circ} 04^{\prime} \mathrm{W}$. ANSP 147048, 1, $163 \mathrm{~mm}$, brook $7 \mathrm{~km}$ inland from Portobelo, Pacific coastal dr., $c a .09^{\circ} 31^{\prime} \mathrm{N}, 079^{\circ} 37^{\prime} \mathrm{W}$. AUM 32118, 9, 132-170 mm, Pipeline rd. at km 4.6, Colón, río Frijoles, río Chagres dr. (Atlantic), $09^{\circ} 09^{\prime} 08^{\prime \prime} \mathrm{N}, 079^{\circ} 44^{\prime} 06^{\prime \prime} \mathrm{W}$. FMNH 32034, 1, 69 mm, creek above Monte Líria, río Gatún dr. (Atlantic), ca. $09^{\circ} 16^{\prime} \mathrm{N}, 079^{\circ} 47^{\prime} \mathrm{W}$. INHS 68132, 6, 69-99 mm, Parque Nacional Soberania, Tower Creek, río Chagres dr. (Atlantic), ca. $09^{\circ} 08^{\prime} \mathrm{N}, 079^{\circ} 41^{\prime} \mathrm{W}$. INHS 68142, 1, 68 mm, INHS 68152, 8, Parque Nacional Soberania, río Frijolito, río Chagres dr. (Atlantic), $c a .09^{\circ} 09^{\prime} \mathrm{N}, 079^{\circ} 44^{\prime} \mathrm{W}$. INHS 68180, 17, 33-135 mm, Parque Nacional Soberania, Tayra creek, río Chagres dr. (Atlantic), ca. $09^{\circ} 08^{\prime} \mathrm{N}, 079^{\circ} 41^{\prime} \mathrm{W}$. MCP 27107, 5, 40-131 mm, río Frijoles, $\mathrm{N}$ Gamboa on pipeline rd., río Chagres dr. (Atlantic), $c a .09^{\circ} 12^{\prime} \mathrm{N}, 079^{\circ} 33^{\prime} \mathrm{W}$. MPEG 1103 , 7, to $170 \mathrm{~mm}$, MPEG 1104, 13, 85-160 mm, MPEG 1106, 7, to $150 \mathrm{~mm}$, Canal Zone, Gamboa hwy., nr. " $2^{\text {nd" }}$ bridge, no coordinates. USNM 293085, 7, 55-121 mm, río Pingandi, $1 \mathrm{~km}$ above Pueblo Mandinga, río Mandinga dr. (Atlantic), ca. $09^{\circ} 28^{\prime} \mathrm{N}, 079^{\circ} 06^{\prime} \mathrm{W}$. Darién. INHS 36100, 1, $120 \mathrm{~mm}$, río Iglesia, Gulf of San Miguel (Pacific), $08^{\circ} 25^{\prime} 23^{\prime \prime} \mathrm{N}, 078^{\circ} 00^{\prime} 05^{\prime \prime} \mathrm{W}$. SU 54431, 1, Pito, coastal stream
(Atlantic), ca. $08^{\circ} 41^{\prime} \mathrm{N}, 077^{\circ} 31^{\prime} \mathrm{W}$. USNM 293202, 1, $171 \mathrm{~mm}$, rio Tuira $0.5 \mathrm{~km}$ above community Boca de Cupe, río Tuira dr. (Pacific), ca. $08^{\circ} 02^{\prime} \mathrm{N}, 077^{\circ} 34^{\prime} \mathrm{W}$. Panamá. ANSP 104116, 1, 104 $\mathrm{mm}$, creek at bridge on Interamerican hwy., ca. 9 mi. E río Pacora dr. (Pacific), ca. $09^{\circ} 08^{\prime} \mathrm{N}, 079^{\circ} 09^{\prime} \mathrm{W}$. ANSP 104239, 1, $134 \mathrm{~mm}$, creek (río Cristal) on bridge on Inter-American hwy. between Chorrera and Arraijan (Pacific), ca. $08^{\circ} 54^{\prime} \mathrm{N}, 079^{\circ} 44^{\prime} \mathrm{W}$. ANSP 104436, 1, 142 mm, creek ca. 5 mi. W El Llano rd., río Bayano dr. (Pacific), ca. $09^{\circ} 12^{\prime} \mathrm{N}, 078^{\circ} 51^{\prime} \mathrm{W}$ [coordinates for El Llano]. ANSP 151021, 1, $191 \mathrm{~mm}$, río Canitas at Inter-American hwy., ca. 10.4 $\mathrm{km}$ W Bayano bridge, río Chepes dr. (Pacific), $09^{\circ} 13^{\prime} 05^{\prime \prime} \mathrm{N}$, 078 52'38"W. FMNH 32033, 1, 162 mm, El Capital, río Mamoni, río Mamoni dr. (Pacific), ca. $09^{\circ} 07^{\prime} \mathrm{N}, 079^{\circ} 04^{\prime} \mathrm{W}$. MCZ 45816, 10 (2 immature, 150-164 mm, 2 female, 154-162 mm, 6 male, 148 $209 \mathrm{~mm}$ ), quebrada Jesus Cristo, affl. río Chagres, at Prince Philip rd. río Chagres dr. (Atlantic - now drains to Panama Canal), ca. $09^{\circ} 07^{\prime} \mathrm{N}, 079^{\circ} 42^{\prime} \mathrm{W}$. ROM 89765, 2, 69-192 mm, STRI 7741, 2, 71-75 mm, río Terable, upstream from Inter-American hwy., río Bayano dr. (Pacific), 09¹3'34"N, 078 58'26"W. ROM 89768, 2, 54-58 mm, STRI 7743, 2, 63-66 mm, río Terable, upstream from Inter-American hwy., río Bayano dr. (Pacific), 09² $13^{\prime} 34^{\prime \prime} \mathrm{N}$, 078 58'27"W. ROM 89769, 4, 65-179 mm, STRI 7745, 2, 83-169 $\mathrm{mm}$, río Cañita, upstream from Inter-American hwy., río Bayano dr. (Pacific), 09 $13^{\prime} 10^{\prime \prime} \mathrm{N}, 078^{\circ} 50^{\prime} 54^{\prime \prime} \mathrm{W}$. ROM 89771, 3, 82-186 $\mathrm{mm}$, STRI 7747, 4, 78-110 mm, stream crossing rd. through Tecal forest, off Inter-American hwy., affl. río Chachare, río Bayano dr. (Pacific), 09 $12^{\prime} 41^{\prime \prime} \mathrm{N}, 079^{\circ} 03^{\prime} 14^{\prime \prime} \mathrm{W}$. ROM 89772, 1, $84 \mathrm{~mm}$, small stream crossing rd. to río Mamoni off Inter-American hwy., affl. río Mamoni, río Bayano dr. (Pacific), $09^{\circ} 11^{\prime} 55^{\prime \prime} \mathrm{N}$, 07904'19"W. ROM 89773, 5, 113-146 mm, STRI 7749, 5, 111$132 \mathrm{~mm}$, quebrada Tapagrilla, río Bayano dr. (Pacific), $09^{\circ} 09^{\prime} 00^{\prime \prime} \mathrm{N}$, $079^{\circ} 10^{\prime} 48^{\prime \prime}$ W. STRI 7744, 1, $88 \mathrm{~mm}$, río Cañita below dam, río Bayano dr., 09¹2'35"N, 07853'55"W. SU 24769, 6, Canal Zone, Three Rivers Plantation, Gatun Lake, río Chagres dr. (Atlantic now drains to Panama Canal), ca. $09^{\circ} 10^{\prime} \mathrm{N}, 079^{\circ} 51^{\prime} \mathrm{W}$. UF 12979 , 3, female, 74-109 mm, creek $11 \mathrm{mi}$. S Chepo, on rd. to El Llano [río Pacora dr.?], (Pacific), ca. $09^{\circ} 08^{\prime} \mathrm{N}, 079^{\circ} 12^{\prime} \mathrm{W}$. UF 12995, 1, female, $135 \mathrm{~mm}$, creek $5 \mathrm{mi}$. S Tocumen Airport on Inter-American hwy., small coastal dr. (Pacific), ca. $09^{\circ} 02^{\prime} \mathrm{N}, 079^{\circ} 24^{\prime} \mathrm{W}$. USNM 78764, 1, 150 mm, El Capitán, río Mamoni, río Bayano dr. (Pacific), ca. $09^{\circ} 12^{\prime} \mathrm{N}, 079^{\circ} 03^{\prime} \mathrm{W}$. USNM $78765,1,145 \mathrm{~mm}$, Largataria, rio Chagres dr. (Atlantic), ca. $09^{\circ} 12^{\prime} \mathrm{N}, 079^{\circ} 39^{\prime} \mathrm{W}$. USNM 293144, 3 , 44-140 mm, small stream, Cartí rd., 15 km N Pan-American [InterAmerican hwy.], río Bayano dr. (Pacific), ca. $09^{\circ} 17^{\prime} \mathrm{N}, 078^{\circ} 58^{\prime} \mathrm{W}$. USNM 302009, 4, 82-174 mm, creek about 2 mi. E El Llano rd., río Chepo dr. (Pacific), ca. 09 $13^{\prime} \mathrm{N}, 078^{\circ} 56^{\prime} \mathrm{W}$. USNM 302012, 2, 70 $117 \mathrm{~mm}$, Canal Zone, creeks of río Cocoli, W Canal, río Cocoli dr. (Pacific), ca. $08^{\circ} 59^{\prime} \mathrm{N}, 079^{\circ} 36^{\prime} \mathrm{W}$. USNM 302018, 3, 92-109 mm, creek at bridge on Inter-American hwy., ca. 9 mi. E río Pacora, $c a$. $09^{\circ} 09^{\prime} \mathrm{N}, 079^{\circ} 09^{\prime} \mathrm{W}$. USNM 302019, 1, $120 \mathrm{~mm}$, creek $1 \mathrm{mi}$. W El Llano, río Bayano dr. (Pacific), ca. 09¹2'50"N, 07859'20"W. San Blas - Comarca Guna Yala (locations from Atlantic drainages). ANSP 151073, 1, $91 \mathrm{~mm}$, stream on El Llano-Cartí rd., $15.9 \mathrm{~km}$ Inter-American hwy., no coordinates. INHS 36074, 2, 110-125 $\mathrm{mm}$, Western San Blas Mountains, río Azucar dr., $09^{\circ} 24^{\prime} \mathrm{N}$, $078^{\circ} 38^{\prime} \mathrm{W}$. MCZ 88603, 1, $110 \mathrm{~mm}$, quebrada Sigdi at Pingandi, río 
Mandinga dr., ca. $09^{\circ} 27^{\prime} \mathrm{N}, 079^{\circ} 05^{\prime} \mathrm{W}$. MCZ 88604, 2, 105-110 $\mathrm{mm}$, marsh, río Mandinga, 4-5 km above mouth, río Mandinga dr., ca. $09^{\circ} 28^{\prime} \mathrm{N}, 079^{\circ} 03^{\prime} \mathrm{W}$. USNM 293152, 5 (1CS), 77-160 mm, río Cartí, $3 \mathrm{~km}$ Cartí, río Cartí dr. (Atlantic), ca. $09^{\circ} 26^{\prime} \mathrm{N}, 078^{\circ} 58^{\prime} \mathrm{W}$. USNM 293154, 7, 58-114 mm, río Cangandi, ca. $3 \mathrm{~km}$ above confl. with río Mandinga, río Mandinga dr., $c a .09^{\circ} 26^{\prime} \mathrm{N}, 079^{\circ} 05^{\prime} \mathrm{W}$. USNM 293155, 2, 130-145 mm, 2 km. E Cangandi village, quebrada Dolati, affl. río Cangandi, río Mandinga dr. $c a .09^{\circ} 26^{\prime} \mathrm{N}, 079^{\circ} 05^{\prime} \mathrm{W}$. USNM 302001, 7 (1CS), 102-135 mm, río Chucubti, nr. Obaldía [Puerto Obaldía], río Chucubti dr. ca. $08^{\circ} 39^{\prime} \mathrm{N}, 077^{\circ} 25^{\prime} \mathrm{W}$. Veraguas (locations from Pacific drainages). ANSP 99922, 2, 73-80 mm, creek at bridge $c a .9 \mathrm{mi}$. W El Maria on Sona-Remedios rd., río Tabasara dr., $c a .08^{\circ} 01^{\prime} \mathrm{N}, 081^{\circ} 32^{\prime} \mathrm{W}$. ANSP 104031, 1, bridge $c a$. $17 \mathrm{mi}$. W Santiago on rd. to Sona, río San Pablo dr., ca. $08^{\circ} 03^{\prime} \mathrm{N}$, $081^{\circ} 10^{\prime} \mathrm{W}$. ANSP 104087, 1, $215 \mathrm{~mm}$, stream on Sona-Remedios rd., ca. 2 mi. W El Maria, río Tabasara dr., ca. $08^{\circ} 01^{\prime} \mathrm{N}, 081^{\circ} 27^{\prime} \mathrm{W}$. ANSP 104173, 1, $120 \mathrm{~mm}$, creek at bridge ca. $9 \mathrm{mi}$. from Santiago on rd. to San Francisco, río Santa Maria dr., ca. $08^{\circ} 12^{\prime} \mathrm{N}, 080^{\circ} 57^{\prime} \mathrm{W}$. AUM 32145, 1, $174 \mathrm{~mm}, 1 \mathrm{~km}$ SE Sona on rd. to Santiago, affl. río San Pablo, río San Pablo dr., Golfo de Montijo, 0800'15"N, 081¹7'09"W. USNM 302000, 1, 217 mm, no coordinates (Pacific dr.). USNM 302010, 1, 170 mm, ca. 7 mi. S Santiago de Veraguas, bridge on Ontijo rd., río San Martin Chiquito, río San Pedro dr., ca. $07^{\circ} 59^{\prime} \mathrm{N}, 080^{\circ} 58^{\prime} \mathrm{W}$. USNM 302020, 4 (2 measured), 183-208 mm, creek 1 mile S Santa Fé, río Santa Maria dr., $08^{\circ} 29^{\prime} 40^{\prime \prime} \mathrm{N}$, 081 ${ }^{\circ} 04^{\prime} 33^{\prime \prime}$ W. USNM 302023, 1, $157 \mathrm{~mm}$, creek $20 \mathrm{mi}$. W Santiago de Veraguas, on Inter-American hwy., río San Pablo dr., 08 11'55"N, 081¹3'50"W. USNM 302024, 2, 120-131 mm, río Conaco, ca. 16 mi. E Santiago, río Santa Marta dr., $c a .08^{\circ} 06^{\prime} \mathrm{N}, 080^{\circ} 44^{\prime} \mathrm{W}$. Region not specified. CAS 50274, 1, $53 \mathrm{~mm}$, no locality/coordinates. CU 71935, 7, no locality/coordinates. CU 71936, 27, no locality/ coordinates. MZF-Manitoba 499, 4, 104-110 mm, no locality/ coordinates. Venezuela (localities from Atlantic drainages). Mérida. MBUCV-V 8991, 2, female, 144-146 mm, mun. Alberto Adrianis, río Guayabones, affl. río Chama, lago de Maracaibo dr., ca. $08^{\circ} 34^{\prime} \mathrm{N}, 071^{\circ} 41^{\prime} \mathrm{W}$. Táchira (localities from lago de Maracaibo drainages). MBUCV-V 10877, 2, 90-97 mm, stream between El Vigía and La Fría, affl. río Zulia, affl. río Catatumbo, $c a .08^{\circ} 12^{\prime} \mathrm{N}$, $072^{\circ} 15^{\prime} \mathrm{W}$ [coordinates for La Fría]. MHNLS 1866, 4, 47-105 mm LEA, MHNLS 1867, 4, 62-100 mm LEA, MHNLS 1868, 4, El Vigía hwy., at La Fria, río Zulia, affl. río Catatumbo, $c a .08^{\circ} 12^{\prime} \mathrm{N}$, $072^{\circ} 15^{\prime} \mathrm{W}$ (listed by Sullivan, 1997). Trujillo. INHS 59921, 1, 92 $\mathrm{mm}, 2 \mathrm{~km} \mathrm{~S}$ La Victoria on hwy. 1, río Caus, lago de Maracaibo dr., ca. $09^{\circ} 19^{\prime} \mathrm{N}, 070^{\circ} 51^{\prime} \mathrm{W}$. MBLUZ 3766, 1, Pan American hwy., río Villegas at Puente Villegas, affl. río Bucare, río Tocuyo dr., ca. $09^{\circ} 45^{\prime} \mathrm{N}, 070^{\circ} 14^{\prime} \mathrm{W}$ (listed by Sullivan, 1997). MCNG 24898, 1, $136 \mathrm{~mm}$ LEA, quebrada Catalina, stream in Flor de Pátria, lago de Maracaibo dr., ca. $09^{\circ} 27^{\prime}, 070^{\circ} 28^{\prime} \mathrm{W}$ (listed by Sullivan, 1997). Zulia (localites from lago de Maracaibo drainages). INHS 34708, 1 ,

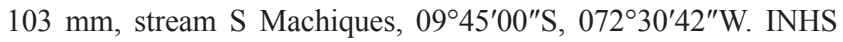
35365, 2, 108-114 mm, $18 \mathrm{~km}$. N Puerto Catatumbo at hwy. 6, bridge over caño Taguara, affl. río Santa Ana, 09²17'22"N, $072^{\circ} 32^{\prime} 49^{\prime} \mathrm{W}$. INHS 60283, 1, $98 \mathrm{~mm}, 12 \mathrm{~km}$ intersection hwy. 6 and hwy. 2, río Zulia, ca. $08^{\circ} 23^{\prime} \mathrm{N}, 072^{\circ} 23^{\prime} \mathrm{W}$. MBLUZ 2645, 1, Mara District, nr. El Sargento, flooded area of río Limon, no coordinates (listed by Sullivan, 1997). MBLUZ 3937, 3, caño
Ictobogui at Aragtoba, Sierra de Perija, no coordinates, $c a .09^{\circ} 44^{\prime} \mathrm{N}$, $072^{\circ} 52^{\prime} \mathrm{W}$ (listed by Sullivan, 1997). MBLUZ 4274, 2, unnamed stream, Parque Sierra de Perija, ca. $09^{\circ} 44^{\prime} \mathrm{N}, 072^{\circ} 52^{\prime} \mathrm{W}$ (listed by Sullivan, 1997). MBUCV-V 2935, 3, female, 155-160 mm, río El Quebradón, between Caja Seca and Aguas Calientes, ca. $09^{\circ} 04^{\prime} \mathrm{N}$, 0711ํW. MBUCV-V 9328, 2, 75-150 mm, mun. Jesús María Semprún, Hacienda El Encantado, stream affl. río Zulia, ca. $08^{\circ} 45^{\prime} \mathrm{N}, 072^{\circ} 20^{\prime} \mathrm{W}$. MBUCV-V 14046, 7, 68-161 mm, Catatumbo region, base of Sierra Azul, río Catatumbo, ca. $08^{\circ} 54^{\prime} \mathrm{N}, 072^{\circ} 26^{\prime} \mathrm{W}$. MBUCV-V 14112, 59, 38-93 mm, MBUCV-V 15067, 18, 33-68 $\mathrm{mm}$, District of Perijá, Cañada Riecito, río Guaco, $c a .09^{\circ} 48^{\prime} \mathrm{N}$, $072^{\circ} 12^{\prime} \mathrm{W}$. MCNG 24884, 1, $125 \mathrm{~mm}$ LEA, río Chiquito, ca. $10^{\circ} 13^{\prime} \mathrm{N}, 071^{\circ} 02^{\prime} \mathrm{W}$ (listed by Sullivan, 1997). UF 21796,1 (CS), $120 \mathrm{~mm}$, stream about $10 \mathrm{~km} \mathrm{~N}$ Mene Grande, $c a .09^{\circ} 55^{\prime} \mathrm{N}$, $070^{\circ} 55^{\prime} \mathrm{W}$. UF $25444,1,179 \mathrm{~mm}$, prov. Libertad, stream, path to $\mathrm{La}$ Misión de los Ángeles, ca. 10³2'N, 071²7’W. UMMZ 56099, 2, 124-176 mm, La Fría, Pueblo Nuevo, ca. $08^{\circ} 57^{\prime} \mathrm{N}, 071^{\circ} 36^{\prime} \mathrm{W}$. USNM 121584, 8, 50-115 mm, 12 km S Rosário, río San Juan, ca. $10^{\circ} 12^{\prime} \mathrm{N}, 072^{\circ} 19^{\prime} \mathrm{W}$. USNM 121585, 11 (2 lost during CS), 69-177 $\mathrm{mm}$, río San Juan at bridge, affl. rio Motatán [presumably río Motatán de los Negros, not río Motatán, listed in Trujillo State], no coordinates. USNM 121586, 17, 62-143 mm, USNM 348532, 15, 93-165 mm, río San Pedro at bridge, affl. río Motatán, no coordinates. Country not specified. ANSP 22980, 2, 118-178 mm, New Grenada [Colombia, Panama and parts of Ecuador and Venezuela prior to 1858], no coordinates.

\section{Brachyhypopomus palenque, new species}

urn:1sid:zoobank.org:act:BA8EFB5D-A00F-42A9-BB1CF7750BCBDF79

\section{(Fig. 37; Tables 2-5, 17)}

Brachyhypopomus occidentalis. -Barriga, 1994: 84, note on diet (NW Ecuador, catalog of fishes). -Jiménez-Prado et al., 2015: 283, color photograph (Ecuador, Pacific drainages - SantiagoCayapas, Esmeraldas, Guayas, Daule, Cóngoma, Güijas, and Baba).

Brachyhypopomus sp. M. -Crampton \& Albert, 2006: 672, fig. 23.8, position in phylogenetic tree, 681; notes on EODs (gymnotiform species and EOD diversity).

Brachyhypopomus sp. 'pal'/PAL. -Lovejoy et al., 2010: 281, table 1; 283, figs. 2-3 (outgroup in phylogenetic analysis of Gymnotus). -Crampton, 2011: 176, table 10.2, species list; 179, figs. 10.2-10.3, phylogeny, geographical and ecological distributions (gymnotiform biology). -Carvalho, 2013: 181-185, figs. 41-43, position in phylogeny (phylogenetic systematics of Rhamphichthyoidea). -Tagliacollo et al., 2016: 28, fig. 5 (phylogeny of Gymnotiformes).

Brachyhypopomus sp. PALE. -Maldonado-Ocampo et al., 2014: 8, fig. 6, position in phylogeny (phylogeny of Rhamphichthyoidea).

Brachyhypopomus sp. "palenque". -Crampton et al., 2016: 1-66, table 1, 3-4, figs. 1-7, 18-20 (phylogeny, biogeography and ecology of Brachyhypopomus). 


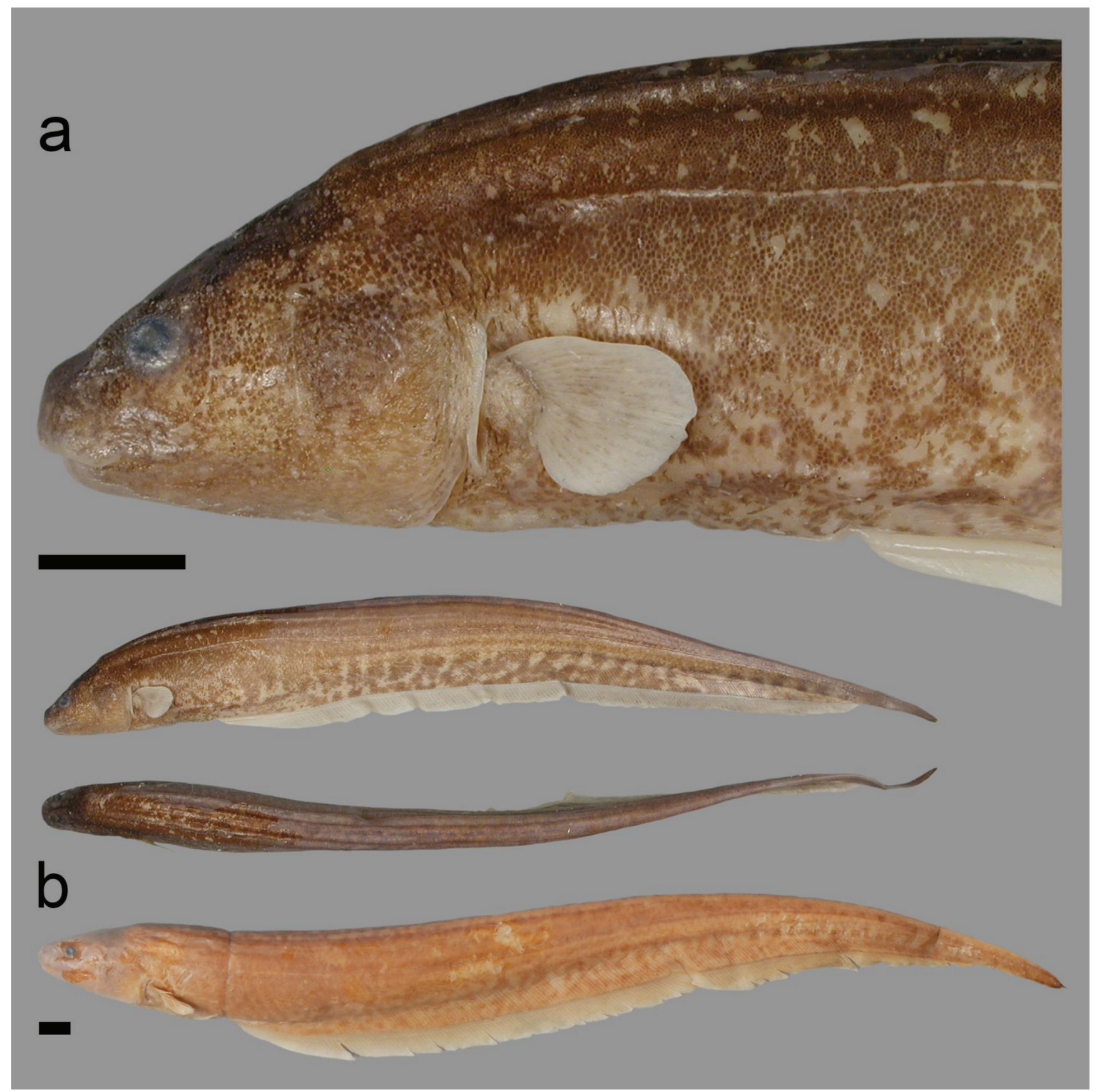

Fig. 37. Brachyhypopomus palenque. a. UF 180270 (WC05.160404), holotype, female, $149 \mathrm{~mm}$ TL (head in lateral view, and body in lateral and dorsal view, specimen fixed in formalin and preserved in EtOH); Ecuador, río Palenque, río Guayas dr. b. USNM 247230, male, $171 \mathrm{~mm}$ TL (body in lateral view, specimen fixed in formalin and preserved in EtOH); Ecuador, río Quinindé, río Esmeraldas dr. Note sexual dimorphism in caudal filament height. Scale bars $=5 \mathrm{~mm}$.

Holotype. UF 180270, female, $149 \mathrm{~mm}$ TL, $135 \mathrm{~mm}$ LEA, Ecuador, Los Ríos, mun. Buena Fé, Parroquia Patricia Pilar, Centro Científico río Palenque, $45 \mathrm{~km} \mathrm{~S}$ Santo Domingo, small forest stream, affl. río Palenque, río Guayas dr., $00^{\circ} 35^{\prime} 01^{\prime \prime} \mathrm{S}, 079^{\circ} 22^{\prime} 13^{\prime \prime} \mathrm{W}, 16$ Apr 2004, W. Crampton, J. Albert, F. Villao, R. Navarrete.

Paratypes. 31 specimens. Ecuador. Los Ríos. FMNH 79125, 3, 56-97 mm, FMNH 79126, 1, 84 mm, FMNH
79209, 1, $114 \mathrm{~mm}$, FMNH 79210, 1, $97 \mathrm{~mm}, \mathrm{FMNH}$ 79211, 1, $104 \mathrm{~mm}$, FMNH 93123, 13, 62-196 mm, FMNH 93133, 7, 86-150 mm, FMNH 93142, 2, 77-83 mm, FMNH 93143, 2, 107-115 mm, Estación Biológica río Palenque, series of small pools at bottom of old $\mathrm{rd}$. to station, and pools nr. the station, affl. río Palenque, affl. río Quevedo, affl. río Babahoyo, río Guayas dr., $c a$. $00^{\circ} 35^{\prime} \mathrm{S}, 079^{\circ} 22^{\prime} \mathrm{W}, 31 \mathrm{Jul} 1974$, G. Glodek, G. Whitmire \& J. Dryan. 
Table 17. Morphometrics for Brachyhypopomus palenque, Brachyhypopomus provenzanoi, and Brachyhypopomus regani. HT, holotype; SD, standard deviation. Ranges for Brachyhypopomus palenque and Brachyhypopomus regani refer to the holotype and to paratype and non-type specimens from the type region. Ranges for Brachyhypopomus provenzanoi refer to the holotype and to paratype specimens from the type region.

\begin{tabular}{|c|c|c|c|c|c|c|c|c|c|c|c|c|c|c|c|}
\hline & \multicolumn{5}{|c|}{ B. palenque } & \multicolumn{5}{|c|}{ B. provenzanoi } & \multicolumn{5}{|c|}{ B. regani } \\
\hline & HT & Range & Mean & $\mathrm{SD}$ & $\mathrm{n}$ & HT & Range & Mean & $\mathrm{SD}$ & $\mathrm{n}$ & HT & Range & Mean & SD & $\mathrm{n}$ \\
\hline Total length (TL) (mm) & 149 & $83-204$ & 118 & 35.1 & 33 & 87 & $68-109$ & 87 & 7.5 & 41 & 128 & $72-138$ & 112 & 18.4 & 26 \\
\hline Length to end of anal fin (LEA) (mm) & 135 & $77-184$ & 109 & 31.7 & 33 & 71 & $59-92$ & 73 & 6.3 & 41 & 115 & $63-127$ & 98 & 16.3 & 30 \\
\hline Head length (HL) (mm) & 14.6 & $9.6-19.2$ & 12.0 & 3.1 & 16 & 7.7 & $7.0-8.8$ & 7.8 & 0.4 & 16 & 13.4 & $9.2-16.8$ & 13.7 & 1.9 & 32 \\
\hline \multicolumn{16}{|c|}{ Proportion of TL $(\%)$} \\
\hline Caudal filament length (CF) & 9.4 & $4.2-12.4$ & 9.1 & 1.9 & 33 & 18.4 & $9.4-20.0$ & 15.8 & 2.3 & 41 & 10.2 & $10.1-15.2$ & 12.6 & 1.8 & 20 \\
\hline \multicolumn{16}{|c|}{ Proportion of LEA (\%) } \\
\hline Head length (HL) & 10.8 & $10.2-12.0$ & 11.3 & 0.5 & 16 & 10.8 & $9.6-11.9$ & 10.7 & 0.6 & 16 & 11.7 & $11.7-17.4$ & 14.0 & 1.0 & 30 \\
\hline Snout to occiput (SO) & 8.8 & $8.0-11.1$ & 9.8 & 0.7 & 16 & 8.3 & $7.8-9.4$ & 8.6 & 0.4 & 16 & 9.3 & $9.3-14.2$ & 11.1 & 0.9 & 30 \\
\hline Snout to anal-fin origin (SA) & 19.6 & $19.4-22.8$ & 20.7 & 1.0 & 16 & 18.4 & $18.2-21.0$ & 19.6 & 0.8 & 16 & 19.7 & $19.6-29.2$ & 23.4 & 2.0 & 30 \\
\hline Snout to pectoral-fin base (PP) & 11.1 & $10.9-13.2$ & 12.4 & 0.6 & 16 & 11.7 & $10.2-12.6$ & 11.6 & 0.5 & 16 & 13.6 & $13.6-20.0$ & 15.9 & 1.2 & 30 \\
\hline Longest pectoral-fin ray (PRL) & 3.6 & $3.2-5.2$ & 4.1 & 0.5 & 16 & 4.7 & $4.2-5.4$ & 4.8 & 0.4 & 16 & 5.0 & $5.0-8.5$ & 6.5 & 0.6 & 30 \\
\hline Anal fin length (AF) & 79.3 & $76.7-83.0$ & 79.7 & 1.3 & 16 & 81.7 & $71.8-82.6$ & 80.0 & 2.7 & 16 & 80.0 & $75.6-83.3$ & 79.9 & 1.5 & 30 \\
\hline Longest anal-fin ray (ARL) & 3.3 & $3.0-4.3$ & 3.7 & 0.3 & 16 & 3.9 & $2.4-4.1$ & 3.5 & 0.5 & 16 & 4.3 & $1.5-4.4$ & 3.1 & 0.7 & 30 \\
\hline Body depth at anal-fin origin (BD1) & 11.9 & $9.4-11.9$ & 10.5 & 0.7 & 16 & 8.5 & $6.7-9.6$ & 7.9 & 0.8 & 16 & 11.8 & $10.6-16.5$ & 13.2 & 1.1 & 30 \\
\hline Body width at anal-fin origin (BW1) & 7.9 & $6.0-7.9$ & 6.6 & 0.4 & 16 & 4.4 & $3.8-4.9$ & 4.2 & 0.3 & 16 & 6.5 & 4.4-7.4 & 6.5 & 0.6 & 30 \\
\hline Body depth at anal-fin middle (BD2) & 10.5 & $8.6-11.7$ & 10.2 & 0.7 & 16 & 7.6 & $5.9-8.2$ & 7.1 & 0.7 & 16 & 11.5 & $9.0-12.3$ & 10.6 & 0.8 & 30 \\
\hline Body width at anal-fin middle (BW2) & 4.0 & $2.7-4.2$ & 3.4 & 0.4 & 16 & 2.6 & $1.9-3.6$ & 2.6 & 0.5 & 16 & 3.3 & $2.2-4.4$ & 2.9 & 0.4 & 30 \\
\hline Caudal filament length (CF) & 9.6 & 7.4-14.0 & 9.9 & 1.8 & 12 & 22.5 & 17.4-24.4 & 19.8 & 2.3 & 15 & 11.3 & 11.1-17.9 & 14.4 & 2.4 & 20 \\
\hline \multicolumn{16}{|c|}{ Proportion of HL $(\%)$} \\
\hline Preorbital distance (PR) & 25.3 & $25.0-30.6$ & 27.1 & 1.6 & 16 & 24.7 & $23.1-26.7$ & 24.5 & 1.1 & 16 & 26.0 & $23.4-29.9$ & 27.1 & 1.5 & 32 \\
\hline Mouth width (MW) & 28.1 & $25.1-37.8$ & 30.9 & 3.9 & 16 & 29.9 & 21.4-29.9 & 25.6 & 2.2 & 16 & 23.0 & $14.9-27.0$ & 19.2 & 2.4 & 32 \\
\hline Internarial distance (AE) & 22.6 & $18.7-23.1$ & 21.4 & 1.3 & 16 & 19.8 & $17.8-21.9$ & 20.1 & 1.3 & 16 & 19.1 & $16.5-21.2$ & 18.6 & 1.1 & 32 \\
\hline Posterior naris to eye (PE) & 5.8 & $4.5-10.1$ & 7.0 & 1.4 & 16 & 6.6 & $3.9-6.6$ & 5.4 & 0.8 & 16 & 7.6 & $4.8-8.3$ & 6.8 & 0.8 & 32 \\
\hline Orbital diameter (OD) & 9.3 & $7.8-11.7$ & 9.8 & 1.1 & 16 & 12.5 & $10.2-14.3$ & 12.2 & 1.0 & 16 & 10.3 & $9.5-13.0$ & 11.1 & 0.9 & 32 \\
\hline Interorbital distance (IO) & 30.1 & $25.0-30.4$ & 28.0 & 2.0 & 16 & 21.2 & $17.8-22.4$ & 20.1 & 1.5 & 16 & 19.7 & $15.0-21.1$ & 18.0 & 1.7 & 32 \\
\hline Head depth at eye (HD1) & 48.2 & $44.5-50.5$ & 46.9 & 1.9 & 16 & 43.8 & $38.3-44.6$ & 41.2 & 1.8 & 16 & 42.3 & $40.0-47.4$ & 43.3 & 1.7 & 32 \\
\hline Head width at eye (HW1) & 38.9 & $37.5-49.9$ & 43.1 & 3.5 & 16 & 39.6 & $28.8-39.6$ & 34.2 & 2.9 & 16 & 36.8 & $30.4-41.8$ & 34.9 & 3.0 & 32 \\
\hline Head depth at occiput (HD2) & 80.4 & $65.6-80.4$ & 73.0 & 3.5 & 16 & 62.5 & $58.0-67.8$ & 64.8 & 2.3 & 16 & 71.6 & $61.3-73.7$ & 67.6 & 2.5 & 32 \\
\hline Head width at occiput (HW2) & 55.7 & $52.5-63.3$ & 56.7 & 2.5 & 16 & 48.8 & $38.9-48.8$ & 44.6 & 2.8 & 16 & 57.5 & $39.1-58.2$ & 50.5 & 4.2 & 32 \\
\hline Postorbital distance (PO) & 63.7 & $61.9-68.5$ & 64.7 & 2.0 & 16 & 61.8 & $60.1-67.5$ & 63.3 & 2.0 & 16 & 64.9 & $60.4-67.4$ & 65.0 & 1.4 & 32 \\
\hline Branchial aperture (BO) & 33.9 & 24.4-33.9 & 29.4 & 3.2 & 16 & 18.7 & $14.1-21.0$ & 17.3 & 1.9 & 16 & 24.6 & $19.0-26.6$ & 23.4 & 2.0 & 32 \\
\hline Pectoral-fin base (PB) & 17.9 & $12.8-17.9$ & 14.8 & 1.4 & 16 & 8.3 & $6.8-10.9$ & 8.9 & 1.2 & 16 & 11.2 & $9.3-13.4$ & 11.7 & 1.0 & 32 \\
\hline Caudal filament depth (CD) & 20.3 & $15.1-21.3$ & 18.1 & 1.8 & 12 & 14.9 & $13.0-19.1$ & 14.3 & 1.5 & 16 & 15.5 & $8.1-15.5$ & 11.9 & 1.6 & 30 \\
\hline Caudal filament width (CW) & 6.0 & $3.8-7.5$ & 5.8 & 1.2 & 12 & 7.3 & 4.6-7.3 & 5.6 & 0.8 & 16 & 3.8 & $3.0-6.1$ & 4.1 & 0.7 & 30 \\
\hline \multicolumn{16}{|c|}{ Other proportions } \\
\hline BW1 (\% of BD1) & 66.3 & $56.8-67.5$ & 63.1 & 3.1 & 16 & 52.2 & $48.4-57.1$ & 53.3 & 3.0 & 16 & 54.7 & $41.3-54.7$ & 49.1 & 3.9 & 32 \\
\hline BW2 (\% of BD2) & 37.7 & $29.9-37.7$ & 33.3 & 2.3 & 16 & 34.1 & 28.4-44.4 & 35.6 & 4.3 & 16 & 29.0 & $22.2-38.1$ & 27.8 & 3.5 & 32 \\
\hline
\end{tabular}

Non-types. 74 specimens. Ecuador. Azuay. ZOOA, FR-PE-003, 1, Ponce Enrique (Cantón), río Fermín, affl. río Siete, río Siete dr. (Gulf of Guayaquil), $03^{\circ} 03^{\prime} 26^{\prime \prime} \mathrm{S}$, $079^{\circ} 44^{\prime} 11^{\prime \prime}$ W. Cotopaxi. USNM 270692, 5 (2 CS), 115-203 $\mathrm{mm}$, "río Sigchos, affl. río Esmeraldo" [interpreted as río Toachi nr. town Sigchos, Cotopaxi, affl. río Esmeraldas], río Esmaraldas dr., no coordinates. Esmeraldas. SU 54426, 2, 142-150 mm, río Cupa confl. with río Blanco at the Guaillabamba junction, río Esmeraldas dr., $c a .00^{\circ} 27^{\prime} \mathrm{N}$, 079²4’ W. USNM 270695, 2, 99-116 mm, río Bogotá, río Santiago dr., $c a .01^{\circ} 02^{\prime} \mathrm{N}, 078^{\circ} 50^{\prime} \mathrm{W}$. Guayas. UF 35330 , $1,150 \mathrm{~mm}, 6.7 \mathrm{~km} \mathrm{~S}$ Nobol, río Daule, rio Guayas dr., $c a$. $01^{\circ} 58^{\prime} \mathrm{S}, 080^{\circ} 00^{\prime} \mathrm{W}$. Los Ríos (localities from río Guayas dr.). ROM 93742, 1, 123 mm, Babahoyo (Cantón), Caracol (Sector), río Clara at La Clara, $01^{\circ} 40^{\prime} 30^{\prime \prime} \mathrm{S}, 079^{\circ} 23^{\prime} 14^{\prime \prime} \mathrm{W}$. ROM 93755, 3, 145-181 mm, Montalvo (Cantón and Sector), creek on San José del Tambo-Juan Montalvo rd., $01^{\circ} 50^{\prime} 16^{\prime \prime} \mathrm{S}, 079^{\circ} 16^{\prime} 14^{\prime \prime} \mathrm{W}$. UF 148572, 4 (3 female, 143$157 \mathrm{~mm}, 1$ male, $203 \mathrm{~mm}$ ), UF 180271, 5, immature (1 CS), 19-40 mm, collected with holotype. FMNH 79122, 9, 74110 mm, FMNH 79124, 9, 55-101 mm, Estación Biológica río Palenque, affl. río Palenque, affl. río Quevedo, affl. río Babahoyo, ca. $00^{\circ} 35^{\prime} \mathrm{S}, 079^{\circ} 22^{\prime} \mathrm{W}$. Pichincha. CAS 72214, 3, 138-170 mm, río Toachi, affl. río Blanco, nr. Santo Domingo de los Colorados, río Esmeraldas dr., $c a$. $00^{\circ} 14^{\prime} \mathrm{S}, 079^{\circ} 03^{\prime} \mathrm{W}$. KU $20008,6,76-119 \mathrm{~mm}$, affl. río 
Baba at Hotel Zaracoyat, E edge Santo Domingo de los Colorados, affl. río Quevedo, río Babahoyo, río Guayas dr., ca. $00^{\circ} 15^{\prime} \mathrm{S}, 079^{\circ} 09^{\prime} \mathrm{W}$. KU 20020, 1, $93 \mathrm{~mm}$, rio Verde, $2 \mathrm{~km}$ SE Santo Domingo, río Baba, affl. río Quevedo, río Babahoyo, río Guayas dr., ca. $00^{\circ} 16^{\prime} \mathrm{S}, 079^{\circ} 09^{\prime} \mathrm{W}$. ROM 93681, 6, 97-173 mm, San Miguel de los Bancos (Cantón), Puerto Quito (Sector), río Silanchi downstream of community of Silanchi, río Esmeraldas dr., $00^{\circ} 09^{\prime} 32^{\prime \prime} \mathrm{N}$, 079 $14^{\prime} 36^{\prime \prime}$ W. ROM 93671, 4, 132-153 mm, San Miguel de los Bancos (Cantón), Puerto Quito (Sector), W Puerto Quito, río Blanco W Puerto Quito, río Esmeraldas dr., $00^{\circ} 07^{\prime} 12^{\prime \prime} \mathrm{N}, 079^{\circ} 14^{\prime} 09^{\prime \prime} \mathrm{W}$. ROM 93707, 5, 128-160 mm, Santo Domingo de los Colorados (Cantón), Santo Domingo (Sector), río Chiguilpe nr. bridge, affl. río Quevedo, río Babahoyo, río Guayas dr., 00¹9'21"S, 079¹3'00"W. SU 54435, 3, 115-121 mm, río Toachi, affl. río Blanco, río Esmeraldas dr., ca. $00^{\circ} 06^{\prime} \mathrm{S}, 079^{\circ} 13^{\prime} \mathrm{W}$. USNM 247230 , 1, male, $168 \mathrm{~mm}, 5 \mathrm{~km}$ Santo Domingo de los Colorados, río Quinindé, affl. río Esmeraldas, río Esmeraldas dr., ca. $00^{\circ} 14^{\prime} \mathrm{S}, 079^{\circ} 13^{\prime} \mathrm{W}$. USNM 270694, 2, 90-111 mm, río Mindó, río Esmeraldas dr., ca. $00^{\circ} 00^{\prime} \mathrm{N}, 078^{\circ} 49^{\prime} W$. Santo Domingo de los Tsáchilas. ZOOA WAM-14-05, 1, Santo Domingo (Cantón), Mimo Chico, nr. Santo Domingo, affl. río Blanco, affl. río Esmeraldas, río Esmeraldas dr., $00^{\circ} 13^{\prime} 26^{\prime \prime} \mathrm{S}, 079^{\circ} 04^{\prime} 20^{\prime \prime} \mathrm{W}$.

Diagnosis. Brachyhypopomus palenque is diagnosed from congeners by the following combination of characters: caudal filament length 7.4-14.0\% LEA, vs. 14.1-83.1\% in B. alberti, B. batesi, B. benjamini, B. brevirostris, $B$. bullocki, B. cunia, B. draco, B. gauderio, B. hamiltoni, $B$. hendersoni, B. janeiroensis, B. jureiae, B. menezesi, $B$. pinnicaudatus, B. provenzanoi, $B$. sullivani, and $B$. walteri; absence of pale stripe along middorsal region, $v s$. presence of prominent pale uninterrupted middorsal stripe from occipital region to base of caudal filament in B. arrayae, B. beebei, and B. belindae; mouth width 25.1-37.8\% HL, vs. $15.9-23.2 \%$ in B. bennetti and B. flavipomus; absence of accessory electric organ over the opercular region, vs. presence in $B$. bombilla and $B$. regani; precaudal vertebrae 19-22, vs. 24-26 in B. verdii; absence of continuous or discontinuous dark vertical or diagonally oriented bars or saddles on body surface dorsal to lateral line, $v s$. presence in B. diazi and B. palenque.

Description. Head and body shape, and pigmentation illustrated in Fig. 37. Meristic and morphometric data for examined specimens presented in Tables 2-5 and 17. Body shallow to moderate in depth. Head short to moderate in length and shallow to moderate in depth. Dorsal profile of head straight to slightly convex from occiput to snout, ventral profile of head approximately straight between operculum and snout, snout bulbous and rounded. Eye small to moderate in size. Upper jaw with slight sigmoidal angle between premaxillary and maxillary portions in lateral view. No accessory electric organ over operculum.
Gill filaments on first gill arch 28-48 (median 38, $\mathrm{n}=8$ ). Pectoral fin broad, pectoral-fin rays 17-21 (no mode, median 18). Precaudal vertebrae 19-22 (mode 21), with 1-3 (mode 2) transitional vertebrae. Anal-fin origin slightly $(<0.25 \mathrm{HL}$ distance) to substantially (0.33-0.5 HL distance) posterior to tip of pectoral fin. Anal-fin rays 180-229 (median 206). Dorsal rami of recurrent branch of anterior lateral line nerve not visible. Middorsal region of body scaled. Rows of scales above lateral line 5-6 (mode 6). Lateral line continuous. Very sparse depigmented epidermal canals; as irregular short curved scratch-mark like grooves in mid dorsal flank in anterior half of body, and sporadically as short scratch marks close to and parallel to lateral line along much of body, but no dense crisscrossing pattern of epidermal canals in posterior third of body as present in many congeners. 4-5 columns (mode 5) bilateral columns of electrocytes at analfin terminus, and 4-6 columns (mode 5) at or near a midpoint between anal-fin terminus and tip of caudal filament in immature, mature female, and mature male specimens. Number of electrocyte columns tapers rapidly to 2-3 at caudal filament tip. Caudal filament very short.

Coloration. (Fig. 37). Background light to dark brown, fading ventrally. Dorsal region without prominent depigmented pale stripe extending along midline from occipital region to base of caudal filament. Dorsal region very dark, with irregular mottling of lighter background pigmentation, no prominent vertically oriented stripes or bands. Ventral flank over body cavity and anal-fin pterygiophores with distinctive irregular marbled pattern of fused dark brown patches over lighter background. Caudal filament darker than body, with or without irregular light patches. Head with evenly scattered dark chromatophores, darker dorsally. Eye without prominent suborbital patch, or stripe, of chromatophores/subcutaneous pigmentation. Pectoral and anal-fin membranes hyaline. Pectoral and anal-fin rays hyaline with light scattering of melanophores. Color in live individuals similar to preserved specimens, with opercular region slightly rosy due to underlying gills.

Size. Moderate adult size, largest specimen examined 203 mm TL, $184 \mathrm{~mm}$ LEA $(\mathrm{n}=85)$. Largest male specimen examined $203 \mathrm{~mm}$ TL, $184 \mathrm{~mm} \operatorname{LEA}(\mathrm{n}=2)$. Largest female specimen examined $157 \mathrm{~mm}$ TL, $151 \mathrm{~mm} \operatorname{LEA}(\mathrm{n}=4)$.

Sexual dimorphism. Sexually mature males attain larger sizes than females, and exhibit substantially deeper caudal filaments than immature individuals and breeding females (Figs. 37a-b), but do not exhibit an elevated number of horizontal bilateral columns or vertical rows of electrocytes. Instead breeding males exhibit clearly enlarged electrocytes relative to immature specimens and females. Large males often with slight paddle-like lateral compression at caudal filament tip (Fig. 37b), which is sometimes free of electrocytes distally. No known sexual dimorphism in pigmentation. 
Geographic distribution. Ecuador (Fig. 36). Known from two large Pacific drainages, the río Esmeraldas and río Guayas. Known also from the río Santiago, a smaller Pacific drainage in northern Ecuador, and from the río Siete, a small drainage of the Gulf of Guayaquil.

Ecological notes. Known from small streams and rivers to an altitude of $c a .650 \mathrm{~m}$. The type series of $B$. palenque was collected in a small clearwater streams (up to about $1 \mathrm{~m}$ wide and 20-30 $\mathrm{cm}$ deep) in protected secondary rainforest. The substrate of these streams comprised clay, pebbles, rocks up to about $30 \mathrm{~cm}$ in diameter, leaf litter, and decaying logs. The following water parameters were recorded: conductivity $52 \mu \mathrm{Scm}^{-1}$, dissolved oxygen $6.8 \mathrm{mgl}^{-1}$, temperature $23.6-23.8^{\circ} \mathrm{C}, \mathrm{pH} 6.5$, and flow $0.05-0.2 \mathrm{~ms}^{-1}$. All specimens were collected from leaf litter or submerged root mats; usually up against or under logs or large rocks. Several fully mature male and female specimens were collected, and all were spaced at least $0.5 \mathrm{~m}$ apart except for a large male and female which were located in very close proximity $(\mathrm{ca} .10 \mathrm{~cm})$ and captured in the same net. Scattered along a $10-15 \mathrm{~m}$ stretch of stream were many ( $c a$. 20 or more estimated from a fish-finder survey) very small specimens in the size range 19-40 $\mathrm{mm}$ (5 vouchered in UF 180271), which were difficult to collect because they passed through the mesh of the dip net. There was no sign of aggregations of post-larval specimens, as observed for B. beebei by Westby (1988). The population at the río Palenque Scientific Center was evidently breeding in midApril 2004, when the type series was collected. However, the full extent of the breeding season is unknown. Stomach contents of specimens from the type locality comprise aquatic invertebrates, predominantly larval Chironomidae and Coleoptera (WGRC unpublished data). Barriga (1994) reports an omnivorous diet.

Co-occurring congeners: None.

Etymology. The specific name derives from the río Palenque, a Pacific Ocean drainage of Ecuador, where the type series was collected. A noun in apposition.

Local names. Ecuador: Cuchillo, bío-bío, anguila (Barriga, 1991).

\section{Brachyhypopomus pinnicaudatus (Hopkins, Comfort, Bastian \& Bass, 1990)}

\author{
(Figs. 1i, 38; Tables 2-5, 18)
}

Hypopomus pinnicaudatus Hopkins et al., 1990: 352, fig. 1, illustration of breeding male and female, showing sexual dimorphism of caudal filament morphology; 360, fig. 9, illustrations of caudal filament with damage and regeneration (original description, type locality - French Guiana, coastal swamp, 'Grand Pripris' [see 'Remarks'], sexual dimorphism of electric organ and EOD). -Hopkins, 1991: 153-154, figs.
1-2, drawings of holotype, black and white photographs of preserved specimens (French Guyana, description of type locality and type series, invalid as original description but includes nomination of holotype, see 'Remarks'). -Costa \& Campos-da-Paz, 1992: 117 (listing in description of $B$. janeiroensis).

Brachyhypopomus pinnicaudatus. -Mago-Leccia, 1994: 48 (listing of Brachyhypopomus). -Planquette et al., 1996: 398, color photograph of live individual (French Guiana, listing of Brachyhypopomus). -Crampton, 1996a: 77, table 6.1.a, species list; 79, fig. 6.1 inset 25-26, color photographs; 85 , fig. 6.2; 88, fig. 6.3; 92-94, figs. 6.4a, 6.5; 191, fig. 11.2; 193, fig. 11.3, EOD data; 110, table 7.5, 113, table 7.7, 115, fig. 7.1, habitats; 136, table 8.2a,b, diet; 151-160, figs. 9.2-9.3, table 9.1a,b, hypoxia tolerance; 165-169, table 10.1 reproductive biology (Brazil, central Amazon, ecology and signal diversity). -Sullivan, 1997: 114 (redescription). -Crampton, 1998a; 821, table 4, 834, fig. 9, illustration of specimen, with EOD (Brazil, Amazonas, species and EOD diversity). -Crampton, 1998b: 314, table 2, list of Brachyhypopomus (Brazil, Amazonas, hypoxia tolerance). -Crampton, 1999: 17 (Brazil, Amazonas, Mamirauá Reserve, listing of species). -Hopkins, 1999: 1218, fig. 1, drawings of breeding male and female, showing sexual dimorphism of caudal filament morphology (electric organ morphology and impedance matching). -Albert \& Crampton, 2003: 495 (Bolivia, Brazil, Colombia, Ecuador, Guyana, Peru, Suriname, catalog of hypopomids). -Goulding et al., 2003: 177, color photograph of live individual (Peru, río Madre de Dios; catalog of fishes). -Triques \& Khamis, 2003: 63 (diagnosis against, in description of B. jureiae). -Loureiro \& Silva, 2006: 667 (diagnosis against, in description of $B$. bombilla). -Giora et al., 2008: 162 (diagnosis against, in description of $B$. draco). -Giora \& Malabarba, 2009: 63 (diagnosis against, in description of B. gauderio). -Sullivan \& Hopkins, 2009: 183 (listing in description of B. bullocki). -Kirschbaum \& Schugardt, 2002: 562 , figs. 3-4, black and white photographs of free embryo after hatching, larva at beginning of exogenous feeding, juvenile, and adult (gymnotiform reproduction). -Crampton \& Albert, 2006: 672, fig. 23.8 (inset 10), color photograph of head of live individual with EOD, position in phylogenetic tree; 681, notes on EODs [survey of gymnotiform species and EOD diversity]. -Crampton, 2011: 176, table 10.2, species list; 179, figs. 10.210.3, phylogeny, geographical and ecological distributions (gymnotiform biology). -Mol, 2012: 598 (Suriname, catalog of fishes). -de Queiroz et al., 2013: 545, table 1 (Brazil, rio Madeira, lago Cuniã, listing of fish species). -Crampton \& Ribeiro, 2013: 238, color photograph of live individual (Brazil, rio Madeira, listing of hypopomids). -Carvalho, 2013: 181-185, figs. 41-43, position in phylogeny (phylogenetic systematics of Rhamphichthyoidea). -Sullivan et al., 2013: 27, fig. 10, color photograph of preserved intact and damaged/regenerated specimen (comparison to B. bennetti and B. walteri). -Cardoso et al., 2015: 214 (Brazil, Amazonas, cytogenetics). -Tagliacollo et al., 2016: 28, fig. 5 (phylogeny of Gymnotiformes). -Crampton et al., 2016: 1-66, table 1, 3-4, figs. 1-7, 18-20 (phylogeny, biogeography and ecology of Brachyhypopomus). 


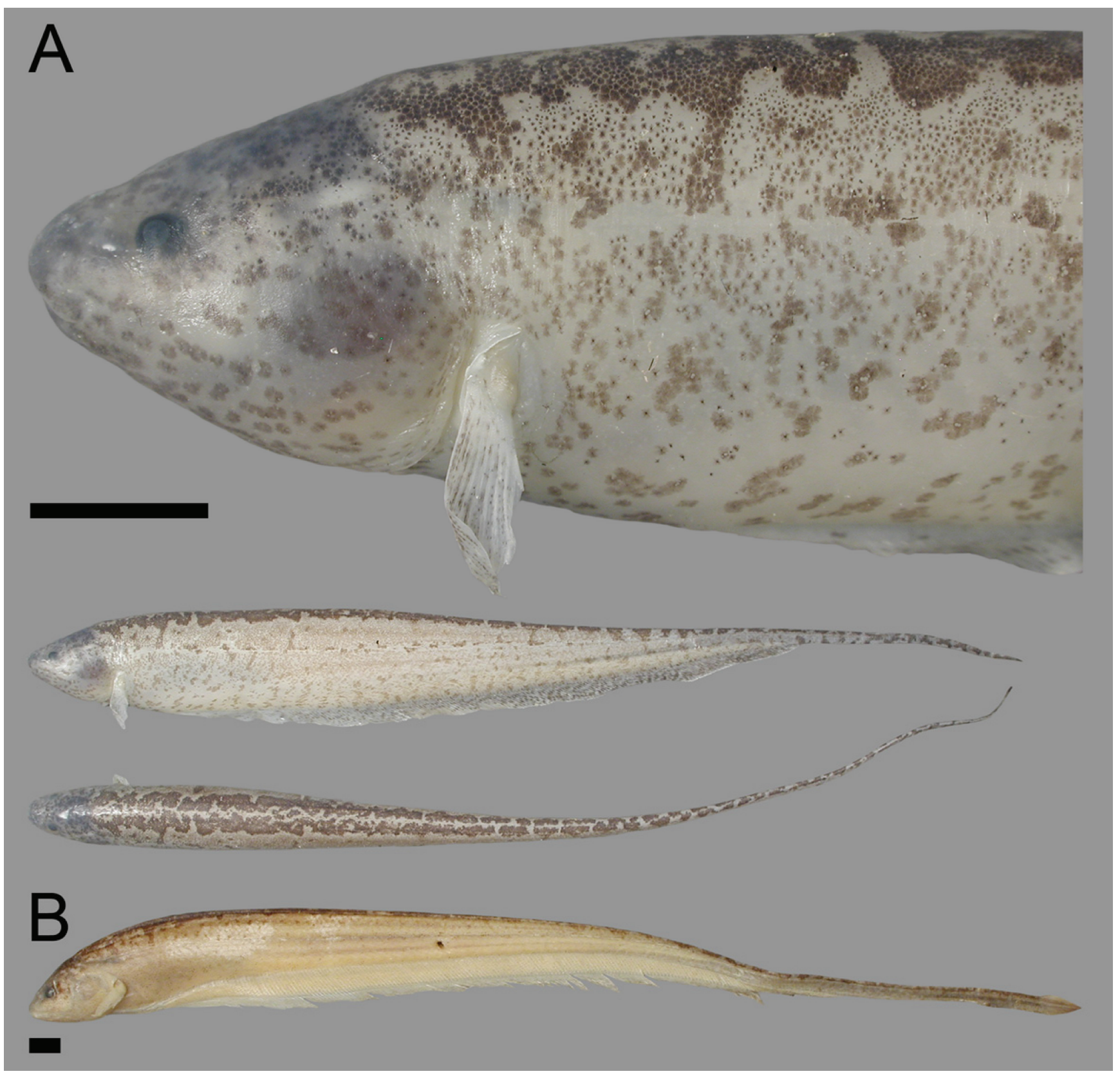

Fig. 38. Brachyhypopomus pinnicaudatus. a. MCP 45469 (WC13.310701b), female, $143 \mathrm{~mm}$ (head in lateral view, and body in lateral and dorsal view, specimen fixed in formalin); b. MCP 45278 (WC13020698), male, $167 \mathrm{~mm}$ TL (body in lateral view, specimen fixed in formalin and preserved in EtOH). Brazil, rio Solimões-Japurá confluence, Amazonas dr. Note sexual dimorphism in caudal-filament length and height, and feather-like structure at caudal filament terminus in male. Scale bars $=5 \mathrm{~mm}$.

Diagnosis. Brachyhypopomus pinnicaudatus is diagnosed from congeners by the following combination of characters: prominent pale stripe along middorsal region present, vs. absent in all congeners except $B$. arrayae, $B$. beebei, B. belindae, B. gauderio, and $B$. verdii); anal-fin rays 176-196, vs. 200-230 in B. beebei and $B$. belindae; precaudal vertebrae 18-21, vs. 24-26 in B. verdii. Brachyhypopomus pinnicaudatus exhibits similar pigmentation and overlapping meristic counts and morphometric proportions with some populations of B. arrayae and B. gauderio. Brachyhypopomus pinnicaudatus can be distinguished from $B$. arrayae by the absence of a contact between a small ascending process on the endopterygoid and the orbitosphenoid $v s$. a contact between these two bones in $B$. arrayae (see diagnosis for $B$. arrayae for further details). Brachyhypopomus pinnicaudatus can be distinguished from $B$. gauderio by the presence of a medial bridge on the posterior portion of the basihyal, vs. absence in B. gauderio (see diagnosis for $B$. gauderio for further details). 
Table 18. Morphometrics for Brachyhypopomus pinnicaudatus. LT, lectotype; SD, standard deviation. Data for lectotype, ANSP 163463 (Cayenne, French Guiana) are from Hopkins (1991). Ranges for the type region refer to type specimens from the "Grand Pripris", French Guiana (lectotype not included in ranges). Ranges for the other population refer to non-type specimens from the vicinity of Tefé, Amazonas, Brazil (Central Amazon basin).

\begin{tabular}{|c|c|c|c|c|c|c|c|c|c|}
\hline & \multicolumn{5}{|c|}{ Coastal French Guiana (type region) } & \multicolumn{4}{|c|}{ Central Amazonas drainage } \\
\hline & LT & Range & Mean & $\mathrm{SD}$ & $\mathrm{n}$ & Range & Mean & SD & $\mathrm{n}$ \\
\hline Total length (TL) (mm) & 125.3 & $110-111$ & 111.0 & 16.0 & 7 & $79-199$ & 132 & 23.8 & 27 \\
\hline Length to end of anal fin (LEA) (mm) & 87.0 & $82-95$ & 88.5 & 12.1 & 7 & $56-137$ & 101 & 18.9 & 29 \\
\hline Head length $(\mathrm{HL})(\mathrm{mm})$ & 11.0 & $8.7-11.9$ & 9.8 & 1.0 & 11 & $8.0-15.0$ & 11.7 & 1.7 & 29 \\
\hline \multicolumn{10}{|c|}{ Proportion of TL (\%) } \\
\hline Caudal filament length (CF) & 31.5 & $13.6-26.1$ & 20.3 & 9.3 & 7 & $12.6-31.2$ & 22.4 & 3.6 & 27 \\
\hline \multicolumn{10}{|c|}{ Proportion of LEA $(\%)$} \\
\hline Head length (HL) & & $10.9-12.0$ & 11.5 & 1.1 & 7 & $10.5-13.1$ & 11.5 & 0.7 & 28 \\
\hline Snout to occiput (SO) & 10.7 & $8.9-10.5$ & 9.7 & 1.3 & 7 & $8.2-12.4$ & 9.7 & 1.0 & 27 \\
\hline Snout to anal-fin origin (SA) & 21.3 & $17.9-21.5$ & 19.7 & 2.0 & 7 & $18.5-24.6$ & 22.0 & 1.7 & 27 \\
\hline Snout to pectoral-fin base (PP) & 13.7 & $12.5-14.3$ & 13.4 & 1.1 & 7 & $11.2-15.2$ & 13.2 & 1.0 & 27 \\
\hline Longest pectoral-fin ray (PRL) & 8.2 & $5.1-6.5$ & 5.8 & 0.9 & 7 & $5.2-7.2$ & 6.2 & 0.5 & 27 \\
\hline Anal fin length (AF) & & $78.0-80.0$ & 79.0 & 1.1 & 7 & $76.9-84.2$ & 81.0 & 1.9 & 28 \\
\hline Longest anal-fin ray (ARL) & 4.2 & $2.6-4.7$ & 4.1 & 0.8 & 7 & $1.5-3.6$ & 2.3 & 0.4 & 27 \\
\hline Body depth at anal-fin origin (BD1) & & $11.9-14.8$ & 13.4 & 1.8 & 7 & $11.1-15.4$ & 13.4 & 1.4 & 27 \\
\hline Body width at anal-fin origin (BW1) & & $6.9-8.2$ & 7.7 & 0.6 & 7 & $4.9-7.1$ & 6.0 & 0.6 & 27 \\
\hline Body depth at anal-fin middle (BD2) & & $10.4-11.2$ & 10.8 & 0.5 & 7 & $7.1-11.5$ & 9.0 & 1.0 & 27 \\
\hline Body width at anal-fin middle (BW2) & & $2.8-3.5$ & 3.1 & 0.6 & 7 & $1.8-3.2$ & 2.4 & 0.3 & 27 \\
\hline Caudal filament length (CF) & 45.3 & $15.8-35.4$ & 25.6 & 12.1 & 7 & $14.6-45.3$ & 29.1 & 6.1 & 27 \\
\hline \multicolumn{10}{|c|}{ Proportion of HL (\%) } \\
\hline Preorbital distance (PR) & 28.6 & $21.8-26.7$ & 23.9 & 1.6 & 11 & $24.2-28.6$ & 27.0 & 1.0 & 29 \\
\hline Mouth width (MW) & & $18.5-30.4$ & 30.8 & 3.2 & 11 & $15.5-21.3$ & 18.8 & 1.5 & 29 \\
\hline Internarial distance (AE) & 22.3 & $20.0-24.6$ & 21.6 & 1.6 & 11 & $19.4-24.0$ & 22.1 & 1.2 & 29 \\
\hline Posterior naris to eye (PE) & 4.6 & $2.0-6.0$ & 4.0 & 1.3 & 11 & $3.8-6.3$ & 4.7 & 0.7 & 29 \\
\hline Orbital diameter (OD) & 12.3 & $7.5-12.3$ & 10.6 & 1.7 & 11 & $9.3-13.1$ & 10.8 & 1.0 & 29 \\
\hline Interorbital distance (IO) & 23.6 & $25.8-39.1$ & 31.0 & 5.1 & 11 & $20.6-28.8$ & 24.2 & 1.9 & 29 \\
\hline Head depth at eye (HD1) & & $51.6-56.5$ & 54.5 & 1.6 & 11 & $48.6-56.5$ & 53.0 & 2.1 & 29 \\
\hline Head width at eye (HW1) & 49.1 & $35.9-51.9$ & 45.7 & 5.2 & 11 & $30.2-42.6$ & 36.8 & 3.0 & 29 \\
\hline Head depth at occiput (HD2) & & $78.3-87.2$ & 83.3 & 3.1 & 11 & $74.3-90.2$ & 82.2 & 4.2 & 29 \\
\hline Head width at occiput (HW2) & & $60.8-74.0$ & 67.2 & 4.6 & 11 & $48.9-66.0$ & 56.3 & 4.3 & 29 \\
\hline Postorbital distance (PO) & 64.6 & $62.8-68.1$ & 65.8 & 1.8 & 11 & $62.5-69.2$ & 65.5 & 1.7 & 29 \\
\hline Branchial aperture (BO) & & $23.6-31.1$ & 27.7 & 3.2 & 11 & $20.3-29.8$ & 24.2 & 2.2 & 29 \\
\hline Pectoral-fin base (PB) & & $10.9-16.9$ & 14.4 & 2.1 & 11 & $10.6-15.5$ & 13.0 & 1.3 & 29 \\
\hline Caudal filament depth (CD) & & $13.1-18.6$ & 5.3 & 4.2 & 7 & $11.3-21.9$ & 16.0 & 2.9 & 29 \\
\hline Caudal filament width (CW) & & $7.4-8.4$ & 2.6 & 3.1 & 7 & $3.4-8.8$ & 5.7 & 1.2 & 29 \\
\hline \multicolumn{10}{|c|}{ Other proportions } \\
\hline BW1 (\% of BD1) & & $52.6-60.0$ & 56.3 & 2.9 & 7 & $36.5-50.7$ & 44.5 & 3.1 & 29 \\
\hline BW2 (\% of BD2) & & $24.5-36.4$ & 33.3 & 4.0 & 7 & $20.0-34.6$ & 26.0 & 2.9 & 29 \\
\hline
\end{tabular}

Description. Head and body shape, and pigmentation illustrated in Figs. 1i and 38. Meristic and morphometric data for examined specimens presented in Tables 2-5 and 18. Body moderate to deep in depth. Head short to moderate in length and moderate to deep in depth. Dorsal profile of head straight to slightly convex from occiput to snout, ventral profile of head approximately straight between operculum and snout, snout rounded. Eye moderate in size. Upper jaw with slight to moderate sigmoidal angle between premaxillary and maxillary portions in lateral view. No accessory electric organ over operculum. Pectoral fin of moderate width, pectoralfin rays 13-16 (median of 15 in populations from coastal drainages of French Guiana; mode of 14 in population from rio Amazon of Brazil). Precaudal vertebrae 18-22 (mode of 20 in populations from coastal drainages of French Guiana; mode of 19 in population from rio Amazon of Brazil), including 1-2 transitional vertebrae. Anal-fin origin slightly $(<0.25 \mathrm{HL}$ distance) anterior to, or near, tip of pectoral fin. Anal-fin rays 177-196 (median of 184 in populations from coastal drainages of French Guiana; mode of 190 in population from rio Amazon of Brazil). Dorsal rami of recurrent branch of anterior lateral line nerve not visible. Middorsal region of body scaled. Rows of scales above lateral line 4-6 (mode 5). Lateral line continuous. Epidermal canals almost entirely absent; present in posterior half of body in form of an irregular, meandering, and sometimes bifurcating horizontal depigmented groove, on 
either side of dorsal surface in dorsal portions of flank; and as very sparse single or sometimes parallel double depigmented grooves either side of, but mostly dorsal to, lateral line. Three bilateral horizontal columns of electrocytes at anal-fin terminus, and at a mid-point between anal-fin terminus and tip of caudal filament in immature, mature female, and mature male specimens. Caudal filament short to moderate in length.

Coloration. (Figs. 1i, 38). Background pale tan to dark brown. Pigmentation highly variable. Dorsal region with prominent depigmented or lightly pigmented stripe extending along midline from occipital region to base of caudal filament, or deep into caudal filament, bordered by prominent dark horizontal wavy reticulated lines from which short irregular patches extend ventrally. Dorsal pigmentation pattern strongly resembles that of $B$. gauderio, but pale depigmented stripe is more conspicuous. Irregular small dark patches or horizontally elongated spots often extend along lateral line. Ventral flank usually without irregular broken vertical bands and vertically elongated spots, especially over anal-fin pterygiophores, as often present in B. gauderio. Caudal filament darker than body, with irregular dark markings or bands along entire length. Head with evenly scattered dark chromatophores, darker dorsally. Eye without prominent suborbital patch, or stripe, of chromatophores/subcutaneous pigmentation. Pectoral and anal-fin membranes hyaline. Pectoral and anal-fin rays with light scattering of brown chromatophores, especially in posterior portion of anal-fin. Color in live individuals similar to preserved specimens, with opercular region usually rosy due to underlying gills.

Size. Moderate adult size, largest specimen examined 208 $\mathrm{mm}$ TL, $142 \mathrm{~mm}$ LEA $(\mathrm{n}=993)$. Largest male specimen examined $208 \mathrm{~mm}$ TL, $142 \mathrm{~mm} \mathrm{LEA}(\mathrm{n}=23)$. Largest female specimen examined $156 \mathrm{~mm}$ TL, $118 \mathrm{~mm}$ LEA $(\mathrm{n}=32)$.

Sexual dimorphism. Sexually mature males attain slightly larger sizes, and develop much broader caudal filaments than immature individuals and breeding females (Figs. 38a-b), but do not exhibit an elevated number of horizontal bilateral columns or vertical rows of electrocytes. Instead breeding males exhibit clearly enlarged electrocytes relative to immature specimens and females. Tip of caudal filament in breeding males often with paddle-like compression, commonly with end free of electrocytes (in some cases with electrocyte-free portion extending anteriorly for up to $20 \%$ of caudal-filament length). Hopkins et al. (1990) and Hopkins (1991) provide detailed notes on secondary sexual dimorphism of EO, electrocyte anatomy, and the EOD. No known sexual dimorphism in pigmentation.

Geographic distribution. Bolivia, Brazil, Peru, and French Guiana (Fig. 39). Brachyhypopomus pinnicaudatus is widespread in eastern portions of the lowland Amazon, as far west as the confluence of the rio Japurá and rio Solimões near Tefé, and throughout the rio Madeira drainage.
Specimens have also been collected from the rio Mearim drainage of Maranhão, Brazil, some $700 \mathrm{~km} \mathrm{SE}$ of the mouth of the Amazon, and (based on photographs from Matavelli et al. 2015), from unspecified localities in the rio Munim basin, further SE (identified incorrectly as Rhabdolichops sp.). Populations are also known from freshwater systems along much of the coast of French Guiana. Museum lots from localities further west along the northern coast of South America are not known although Mol (2012) reports $B$. pinnicaudatus from the Commewijne River in eastern Suriname.

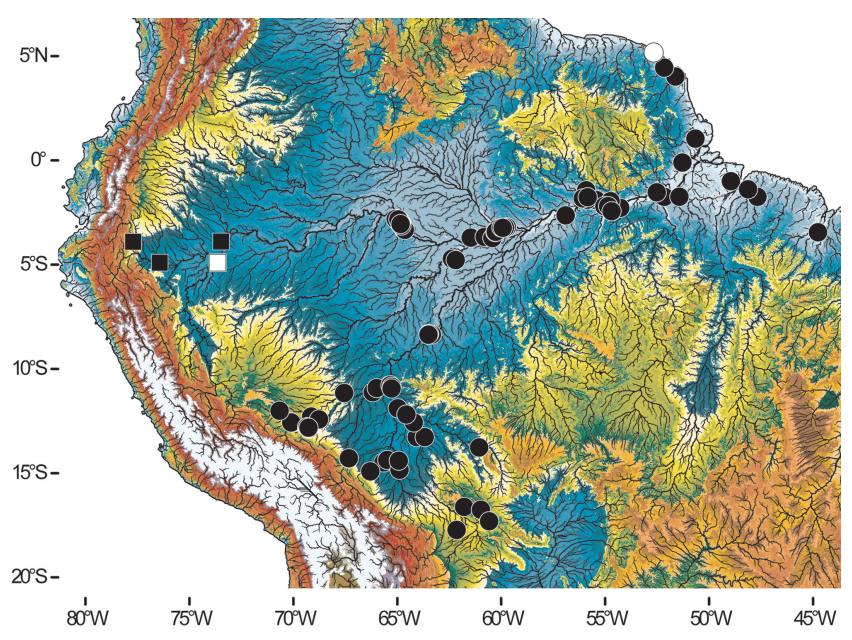

Fig. 39. Collection records for Brachyhypopomus pinnicaudatus (circles) and B. verdii (squares). Holotype locations are marked with open symbols. Elevation data refers to altitude above mean sea level (see Fig. 2 for legend).

Population variation: We found complete overlap in the range of meristic counts (Tables 2-5) and morphometric measurements (Table 18), and observed similar pigmentation among populations of $B$. pinnicaudatus from the eastern portions of the Amazon basin, and from the region of the type locality in coastal swamps of French Guiana. These observations, in combination with molecular data (Crampton et al., 2016), support the hypothesis that all populations we have assigned to $B$. pinnicaudatus are members of a single geographically widespread species, which is morphologically and genetically distinct from its allopatric sister species, B. gauderio, and from all other congeners.

Ecological notes. In the Amazon basin Brachyhypopomus pinnicaudatus occurs primarily in floodplain systems of intermediate conductivity $\left(30-90 \mu \mathrm{Scm}^{-1}\right)$ and high conductivity $\left(90-250 \mu \mathrm{Scm}^{1}\right)$, especially those with turbid water. Records for low-conductivity $\left(5-30 \mu \mathrm{Scm}^{-1}\right)$ clearwater and blackwater rivers are mostly confined to near their confluence with major whitewater rivers (e.g. at the confluence of the rio Negro and rio Tefé with the Amazon River), although specimens are known from the rio Tapajós upstream of its mouthbay (ria) lake. Throughout its range $B$. pinnicaudatus is usually encountered in floating or rooted 
macrophytes along the margins of lakes, channels and swamps (Crampton, 1996a; 1998a; 2011; Crampton \& Albert, 2006; Crampton \& Ribeiro, 2013; de Queiroz et al., 2013; Hopkins, 1991). Brachyhypopomus pinnicaudatus is able to tolerate seasonal or intermittent hypoxia by undertaking aerial gill respiration (Crampton, 1998b; Hopkins, 1991). Hopkins (1991) describes the nature of aerial gill respiration of B. pinnicaudatus: "We noted in containers not oxygenated by bubbling, $H$. pinnicaudatus would rise to the surface every few minutes, take bubbles of air and lodge them in the gill chamber, which became noticeably flared as a result, and return to the bottom. Gentle pressure to the sides of the head would cause the release of several bubbles of air.". Hopkins (1991) also describes the expanded size of the gill chamber in $B$. pinnicaudatus relative to congeners from normoxic systems, which "extends far toward the ventral midline where the two chambers meet at a septum. The secondary lamellae of the respiratory gill are well developed; the respiratory folds are very broad and thin, making them highly efficient in the uptake of oxygen from water". Breeding occurs during the rising and high water period in populations from Amazonian floodplain floating macrophytes (Crampton, 1996a, 165), and during the rainy seasons in populations from coastal French Guiana (Hopkins et al., 1990). Stomach contents in populations from the central Amazon comprise aquatic insect larvae, microcrustacea, and other small aquatic invertebrates - with a predominance of Chironomidae larvae (Crampton, 1996a: 136).

Co-occurring congeners: In the region of the type locality in coastal French Guiana, B. pinnicaudatus occurs in sympatry with $B$. beebei, $B$. brevirostris, and $B$. regani. In the Amazon basin (excluding the rio Madeira), B. pinnicaudatus occurs in sympatry and ecological syntopy with $B$. belindae, B. beebei, B. bennetti, B. brevirostris, B. flavipomus, B. hamiltoni, $B$. regani, and $B$. walteri, and in allotopy with $B$. batesi, B. hendersoni, and B. sullivani (except in terra firme-floodplain ecotones). In the rio Madeira basin $B$. pinnicaudatus is known to occur in sympatry and syntopy with $B$. arrayae, B. beebei, B. bombilla, B. brevirostris, $B$. cunia, and $B$. walteri, and in allotopy with $B$. alberti and $B$. sullivani.

Local names. Bolivia: cuchillo; Brazil: sarapó; French Guiana: poisson couteau (French) and multiple creole/ Amerindian names (Planquette et al., 1996); Peru: macana.

Remarks. Comments on original description: Hopkins (1991), in the journal Copeia, provided a description of $B$. pinnicaudatus (as Hypopomus pinnicaudatus). However, this description was preceded by a paper in the journal Brain, Behavior and Evolution (Hopkins et al., 1990), which uses the name $H$. pinnicaudatus, comments that it is a "new species" (p. 350), specifies a type locality and type series (including repository information) (p. 351), provides an illustration (p. 352), and includes diagnostic notes by way of a comparison of sexual dimorphism to congeners (p. 365).
Consequently, Hopkins et al. (1990), must be considered the valid authority (ICZN, 1999, paragraphs 11.5.1, 13.1.1, and 16.1). Hopkins et al. (1990) evidently intended the Copeia paper to also be published in 1990 and provided a statement on p. 350: "A formal species description can be found in Hopkins [1990]." (Hopkins [1990] is cited "in press" in Copeia in the bibliography). This statement does not in itself constitute a valid disclaimer to availability of the name, but rather makes the name $H$. pinnicaudatus available by indication (ICZN, 1999, paragraph 13.1.2). No holotype was nominated by Hopkins et al. (1990) from a list of what must be considered syntypes. The "holotype" listed by Hopkins (1991) (ANSP 163463) is hereby designated as lectotype. Further, several "paratypes" lots listed by Hopkins (1991) that are not listed among the syntypes listed in Hopkins et al. (1990) must be excluded from the type series.

Material examined. 1,154 specimens. Bolivia. Beni (localities from rio Madeira dr., Amazonas dr.). AMNH 39848, 7, 68-103 mm, Pampa de Meio, ca. $12 \mathrm{~km}$ SE Costa Marques, affl. rio Guaporé, affl. río Mamoré, $12^{\circ} 34^{\prime} 00^{\prime \prime} \mathrm{S}, 064^{\circ} 12^{\prime} 42^{\prime \prime} \mathrm{W}$. AUM 23504, 1, 85 mm, AUM 23643, 3, 124-127 mm, mun. Vaca Díez, affl. lago Tumi Chucua, affl. río Beni, $\mathrm{ca} .11^{\circ} 07^{\prime} \mathrm{S}, 066^{\circ} 11^{\prime} \mathrm{W}$. CAS 72216,3 , $112-$ $165 \mathrm{~mm}$, Reyes, $24 \mathrm{mi}$. NE Rurrenabaque, río Beni, 14²17'42"S, $067^{\circ} 20^{\prime} 27^{\prime \prime} \mathrm{W}$. CBF 10260, 1, immature, $102 \mathrm{~mm}$, CBF 10261, 1, immature, $100 \mathrm{~mm}$, mun. Riberalta, stream nr. lago de San José, floodplain of río Beni, $10^{\circ} 54^{\prime} 47^{\prime \prime S}, 065^{\circ} 59^{\prime} 49^{\prime \prime} \mathrm{W}$. CBF 10262, 1, male, $157 \mathrm{~mm}$, CBF 10263, 1, female, $115 \mathrm{~mm}$, CBF 10264, 1, immature, $115 \mathrm{~mm}$, CBF 10265, 1, immature, $101 \mathrm{~mm}$, CBF 10266, 1, female, $111 \mathrm{~mm}$, CBF 10267, 1, immature, $125 \mathrm{~mm}$, UF 177343, 1, immature, $111 \mathrm{~mm}$, UMSS 7025, 1, male, $119 \mathrm{~mm}$, UMSS 7026, 1, female, $81 \mathrm{~mm}$, UMSS 7027, 1, male, $106 \mathrm{~mm}$, UMSS 7028, 1, female, $108 \mathrm{~mm}$, UMSS 7029, 1, immature, 78 mm, UMSS 7030, 1, female, 95 mm, UMSS 7031, 1, female, 70 mm, UMSS 7032, 1, immature, $86 \mathrm{~mm}$, mun. Riberalta, swamp nr. lago de San José, floodplain of río Beni, $10^{\circ} 54^{\prime} 45^{\prime \prime} \mathrm{S}, 065^{\circ} 59^{\prime} 46^{\prime \prime} \mathrm{W}$. INHS 37118, 1 , $97 \mathrm{~mm}$, borrow pit, río Apere, aff río Mamoré, ca. $14^{\circ} 29^{\prime} \mathrm{S}$, 0654ํW. INHS 37244, 1, 67 mm, 41 km E Estación Biológica del Beni, on rd. to San Ignacio de Moxos, río Chevejecure, affl. río Apere, affl. río Mamoré, ca. $14^{\circ} 22^{\prime} \mathrm{S}, 065^{\circ} 29^{\prime} \mathrm{W}$. MNHN 19981047, 2 (of 5), 123-142 mm, Trinidad, río Beni, ca. 1450’ $\mathrm{S}$, 064 $54^{\prime} \mathrm{W}$. NMW 65039, 1, $102 \mathrm{~mm}$ TL, 80 mm LEA (paralectotype of $B$. brevirostris), rio Guaporé, affl. río Mamoré, no coordinates. UF 81877, 1, 54 mm, Renez prov., stream, $1 \mathrm{~km} \mathrm{~S}$ Magdalena, río Itonomas, affl. río Machupo, affl. río Guaporé, affl. río Mamoré, $c a$. $13^{\circ} 16^{\prime} \mathrm{S}, 064^{\circ} 04^{\prime} \mathrm{W}$. UF 82508, 4, 44-108 mm, pond $25 \mathrm{~km} \mathrm{~N}$ San Javier, río Mamoré, $c a .14^{\circ} 24^{\prime} \mathrm{S}, 064^{\circ} 56^{\prime} \mathrm{W}$. UF 148445, 1, $100 \mathrm{~mm}$, prov. Iténez, río Blanco, nr. Bella Vista, affl. rio Guaporé, affl. río Mamoré, $c a$. $13^{\circ} 16^{\prime} \mathrm{S}, 063^{\circ} 41^{\prime} \mathrm{W}$. UMMZ 66417, 2, 128-139 mm, Reyes and lagoons nr. Reyes, $24 \mathrm{mi}$. NE Rurrenabaque, affl. río Beni, ca. $14^{\circ} 17^{\prime} \mathrm{S}, 067^{\circ} 20^{\prime} \mathrm{W}$. UMMZ 204434, 13, 52-90 mm, pool, Pampa do Meio, ca. $12 \mathrm{~km}$ SE Costa Marques (Brazil), río Iténez (río Guaporé), affl. río Mamoré, $12^{\circ} 34^{\prime} 00^{\prime \prime} \mathrm{S}, 064^{\circ} 12^{\prime} 42^{\prime \prime} \mathrm{W}$. UMMZ 205102, 1, $158 \mathrm{~mm}$, Arroyo Grande at mouth, ca. $1 \mathrm{~km} \mathrm{~N}$ Guayaramerín, affl. río Mamore, $10^{\circ} 48^{\prime} 00^{\prime \prime} \mathrm{S}, 065^{\circ} 24^{\prime} 36^{\prime \prime} \mathrm{W}$. USNM 305593, 1, $85 \mathrm{~mm}$, prov. Ballivia, lago Normandia, $40 \mathrm{~km}$ E San 
Borja, río Matos, affl. río Apere, affl. río Mamoré, $c a$. $14^{\circ} 55^{\prime} \mathrm{S}$, 066 ${ }^{\circ} 18^{\prime}$ W. Pando. FMNH 106648, 1, 75 mm, río Manuripi, ca. 12 $\mathrm{km}$ upstream Puerto Rico, río Manuripi, affl. río Orthon, affl. río Madre de Dios, affl. río Beni, affl. rio Madeira, Amazonas dr., ca. $11^{\circ} 09^{\prime} 06^{\prime \prime} \mathrm{S}, 067^{\circ} 33^{\prime} 41^{\prime \prime} \mathrm{W}$. Santa Cruz (localities from río Mamoré, affl. rio Madeira, Amazonas dr.). UF 82159, 11, 66-139 mm, 41 km SSE Concepción, affl. río San Martin, affl. rio Guaporé, ca. $16^{\circ} 37^{\prime} \mathrm{S}, 061^{\circ} 47^{\prime} \mathrm{W}$. UF 82171, 1, $88 \mathrm{~mm}$, prov. Velasco, $5.5 \mathrm{~km}$ $\mathrm{N}$ San Miguel, affl. río Paraguas, affl. río Iténez (río Guaporé), $c a$. $16^{\circ} 45^{\prime} \mathrm{S}, 060^{\circ} 59^{\prime} \mathrm{W}$. UF 82189, 1, $75 \mathrm{~mm}$, prov. Velasco, $71 \mathrm{~km} \mathrm{~N}$ San Jose de Chiquitos, río San Diablo, affl. río Itonam, affl. río San Martín, affl. río Guaporé, $c a .17^{\circ} 18^{\prime} \mathrm{S}, 060^{\circ} 35^{\prime} \mathrm{W}$. ZUEC 7100, 1, $114 \mathrm{~mm}$, Quimone, $45.4 \mathrm{~km}$ W San José de Chiquitos, río Quimone, affl. río San Pablo, $17^{\circ} 42^{\prime} 48^{\prime \prime} \mathrm{S}, 062^{\circ} 08^{\prime} 56^{\prime \prime} \mathrm{W}$. Brazil. Amapá. MNRJ 12175, 3, 83-191 mm, mun. Mazagão, rio Miri nr. MazagãoMacapá, rio Amazonas estuary, Amazonas dr., ca. $00^{\circ} 06^{\prime} \mathrm{S}$, $051^{\circ} 17^{\prime} \mathrm{W}$. MNRJ 12176, 1, 165 mm, mun. Amapá, Fazenda Modelo do Aporema, rio Aporema, L bank, rio Araguari dr., ca. $01^{\circ} 03^{\prime} \mathrm{N}, 050^{\circ} 40^{\prime} \mathrm{W}$. NRM 32240, 39, 38-85 mm, stream at Aldeia Cunene, rio Oiapoque dr., 04 $01^{\prime} 08^{\prime \prime} \mathrm{N}, 051^{\circ} 37^{\prime} 06^{\prime \prime} \mathrm{W}$. Amazonas (localities from Amazonas dr., localities listed from the Mamirauá Reserve [Reserva de Desenvolvimento Sustentável Mamirauá] are in rio Solimões-Japurá floodplain, mun. Alvarães). BMNH 1998.3.12.108-116, 9 (1 female, 110 mm, 8 immature), mun. Tefé, Ilha Panamim, rio Solimões nr. Tefé, $03^{\circ} 17^{\prime} 38^{\prime \prime S}$, 64 $41^{\prime} 16^{\prime \prime} \mathrm{W}$. BMNH 1998.3.12.117-118, 2, immature, Mamirauá Reserve, cano do lago Rato, 0302'41"S, 06451'26"W. BMNH 1998.3.12.119, 1, immature, 120 mm, MCP 45398, 1, female(CS), 116 mm, Mamirauá Reserve, lago Juruá Grande, 0301'51"S, 064 $51^{\circ} 07^{\prime \prime}$ W. BMNH 1998.3.12.120-122, 3, immature, 111-118 mm, Mamirauá Reserve, cano do lago Mamirauá, $03^{\circ} 03^{\prime} 51^{\prime \prime} \mathrm{S}$, 64 $54^{\circ} 05^{\prime \prime} \mathrm{W}$. BMNH 1998.3.12.123-124, 2 (1 female, $134 \mathrm{~mm}, 1$ male, $163 \mathrm{~mm}$ ), Mamirauá Reserve, lago Pirarara, 02 $57^{\prime} 04^{\prime \prime} \mathrm{S}, 64^{\circ} 50^{\prime} 04^{\prime \prime} \mathrm{W}$. BMNH 1998.3.12.125-126, 2, immature, 150-178 mm, Mamirauá Reserve, lago Saracura, 02²58'34"S, 6455'34"W. BMNH 1998.3.12.127, 1, male, $158 \mathrm{~mm}$, Mamirauá Reserve, cano dos Lago Curuçá, 0304'31"S, 6448'52"W. BMNH 1998.3.12.128, 1, immature, 153 mm, Mamirauá Reserve, Ressaca do Pau, $03^{\circ} 02^{\prime} 03^{\prime \prime} \mathrm{S}, 64^{\circ} 52^{\prime} 13^{\prime \prime} \mathrm{W}$. BMNH 1998.3.12.129, 1, male, 169 mm, Mamirauá Reserve, cano do lago Sapucaia, 0304'07"S, 6448'32"W. BMNH 1998.3.12.130132, 3 male, 154-159 mm, MCP 45328, 1, immature, $89 \mathrm{~mm}$, Mamirauá Reserve, Ressaca do Caetono, $02^{\circ} 50^{\prime} 15^{\prime \prime} \mathrm{S}, 064^{\circ} 55^{\prime} 50^{\prime \prime} \mathrm{W}$. IDSM 445, 1, 116 mm, Mamirauá Reserve, lago Sumaumeirinha I, $02^{\circ} 48^{\prime} 27^{\prime \prime} \mathrm{S}, 065^{\circ} 04^{\prime} 37^{\prime \prime} \mathrm{W}$. IDSM 446, 4, 82-133 mm, MCP 45275 , 2 (1 female, 148 mm, 1 male, 208 mm), MCP 45437, 3 (1 female, 144 mm, 2 male, 139-143 mm), Mamirauá Reserve, lago Secretaria, $03^{\circ} 07^{\prime} 12^{\prime \prime} \mathrm{S}, 064^{\circ} 47^{\prime} 49^{\prime \prime} \mathrm{W}$. INHS 67200, 1, lago Canta Galos, lago Janauari, ca. $03^{\circ} 13^{\prime} \mathrm{S}, 060^{\circ} 00^{\prime} \mathrm{W}$. INHS 70412, 1, $98 \mathrm{~mm}$, INHS $71295,1,141 \mathrm{~mm}$, lago Janauari, $03^{\circ} 13^{\prime} \mathrm{S}, 060^{\circ} 00^{\prime} \mathrm{W}$. INPA 4389, 4, 92-135 mm, INPA 9119, 8, INPA 19939, 39, 23-141 mm, Paraná do Rei, Ilha Careiro, rio Amazonas floodplain, $03^{\circ} 12^{\prime} \mathrm{W}, 059^{\circ} 44^{\prime} \mathrm{W}$. INPA 4738, 1, 113 mm, INPA 4806, 25, 76-142 mm, INPA 4871, 35, 32-135 mm, INPA 19941, 1, $77 \mathrm{~mm}$, INPA 19946, 1, $84 \mathrm{~mm}$, INPA 19955, 6, 75-130 mm, MZUSP 24967, 1, 147 mm, MZUSP 75572, 4, 121-142 mm, lago do Castanho, Ilha Janauacá, rio Solimões floodplain, $03^{\circ} 43^{\prime} \mathrm{S}, 060^{\circ} 26^{\prime} \mathrm{W}$. INPA 9946, 24, 86-140 mm, Reserva Mamirauá, lago Caetano, 02²50'58'S, 06455'42"W. INPA 13568, 2, 33-59 mm, INPA 15771, 2, 77-86 mm, INPA 16700 , 1, $136 \mathrm{~mm}$, lago Comprido, Ilha da Marchantaria, rio Amazonas floodplain, $c a .03^{\circ} 14^{\prime} \mathrm{S}, 059^{\circ} 56^{\prime} \mathrm{W}$. INPA $17119,2,112-138 \mathrm{~mm}$, lake nr. Sacado da Santa Luzía, rio Purus, 04²4'48"S, 062 $22^{\prime} 26^{\prime \prime} \mathrm{W}$. INPA 17120, 2, 71-96 mm, Paranã 2, nr. Arumã, rio Purus, 0446'24"S, 062 11'35"W. INPA 19942, 4, 93-147 mm, rio Negro, Amazonas dr., no coordinates. MCP 45276, 3 (1 immature, 82 mm, 1 female, $129 \mathrm{~mm}, 1$ male, $110 \mathrm{~mm}$ ), Mamirauá Reserve, lago Curuçá Redondo, 0306'13"S, 06449'06"W. MCP 45277, 3 (1 immature, $120 \mathrm{~mm}, 1$ female, $103 \mathrm{~mm}, 1$ male, $104 \mathrm{~mm}$ ), Mamirauá Reserve, lago Curuçá Aberto, 0306 $07^{\prime \prime} \mathrm{S}, 064^{\circ} 49^{\prime} 10^{\prime \prime} \mathrm{W}$. MCP 45278, 4 (1 immature, 120 mm, 1 female, 156 mm, 2 male, 153-177 $\mathrm{mm}$ ), Mamirauá Reserve, lago Curuçá Comprido, 0305'31"S, 06448'58"W. MCP 45279, 1, immature, 134 mm, MCP 45410, 1, immature, 101 mm, MCP 45435, 66, 33-161 mm, Mamirauá

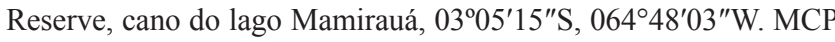
45280, 2, female, 127-136 mm, MCP 45281, 1, male, $158 \mathrm{~mm}$, Mamirauá Reserve, Ressaca da Vila Alencar, 0307'41"S, 06448'04"W. MCP 45351, 2, female, 127-136 mm, Mamirauá Reserve, lago Juruazinho, $03^{\circ} 02.58^{\prime} \mathrm{S}, 064^{\circ} 51.01^{\prime} \mathrm{W}$. MCP 45370, 2 , female (2CS) 122-125 mm, Mamirauá Reserve, lago Araçazinho, 02 $59^{\prime} 35^{\prime \prime} \mathrm{S}, 064^{\circ} 51^{\prime} 33^{\prime \prime} \mathrm{W}$. MCP 45409, 1, immature, $88 \mathrm{~mm}$,

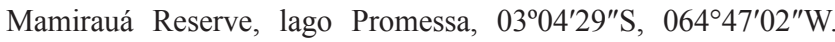
MCP 45433, 2 (1 immature, 115 mm,1 female $145 \mathrm{~mm}$ ), Mamirauá Reserve, lago Araçazinho, 0259'35"S, 06451'33"W. MCP 45436 , 1, male, $173 \mathrm{~mm}$, Mamirauá Reserve, Ressaca do Itu, 0249'51"S, 0645'ㄴ'11"W. MCP 45438, 1, immature, 113 mm, MCP 45455, 3 (1 immature, $67 \mathrm{~mm}, 1$ female, $135 \mathrm{~mm}, 1$ male, $147 \mathrm{~mm})$, MCP 46928, 2, female (2CS), 143-148 mm, Mamirauá Reserve, lago Secretaria, 0307'12"S, 06447'49"W. MCP 45469, 12, 58-143 mm, mun. Tefé, floodplain lake, Ilha Panamim, rio Solimões, nr. Tefé, rio Solimões, 03¹9'29"S, 064³8'37"W. MCZ 8354, 26, 76-83 mm, MCZ 9421, 1, 137 mm, MCZ 9424, 1, 48 mm, mun. Parintins, Villa Bella, rio Amazonas at Parintins, ca. $02^{\circ} 38^{\prime} \mathrm{S}, 056^{\circ} 54^{\prime} \mathrm{W} . \mathrm{MCZ}$ 78165, 1, $95 \mathrm{~mm}$, lago Jacaretinga, nr. Careiro de Várzea, rio Amazonas, 031'00"S, 05949'41"W. MPEG 1110, 11, 67-147 mm, MPEG 1112, 1, 70 mm, MPEG 1117, 6, 53-115 mm, mun. Iranduba, rio Solimões, $c a .03^{\circ} 17^{\prime} \mathrm{S}, 060^{\circ} 11^{\prime} \mathrm{W}$ [coordinates for Iranduba]. MPEG 22740, 1, $86 \mathrm{~mm}$, Mamirauá Reserve, lago do Pagão, 0302'51"S, 06450'23"W. MZUSP 6326 (part), 3, 83-136 $\mathrm{mm}$, lago Castro, Boca do rio Purus, rio Purus, ca. $03^{\circ} 42^{\prime} \mathrm{S}$, 0612으. W. MZUSP 6475, 1, lago Jacaré, upstream Manacapurú, rio Solimões floodplain, $c a .03^{\circ} 40^{\prime} \mathrm{S}, 060^{\circ} 48^{\prime} \mathrm{W}$. MZUSP 165854, 3 , 84-133 mm, mun. Manaus, lago Hyanuary [lago do Janauari], rio Amazonas floodplain, $c a .03^{\circ} 13^{\prime} \mathrm{S}, 060^{\circ} 00^{\prime} \mathrm{W}$. USNM 229915, 2 , 107-135 mm, USNM 306833, 40, 27-137 mm, USNM 306866, 4, 55-150 mm, São José, lago do Castanho, lago Janauacá, rio Amazonas floodplain, $\mathrm{ca} .03^{\circ} 26^{\prime} \mathrm{S}, 060^{\circ} 17^{\prime} \mathrm{W}$ [coordinates taken from center of lago Janauacá]. USNM 306678, 1, 75 mm, USNM 306870, 3, 116-133 mm, lago Murumuru, nr. Curral, lago Janauacá, rio Amazonas floodplain, $c a .03^{\circ} 25^{\prime} \mathrm{S}, 060^{\circ} 16^{\prime} \mathrm{W}$. USNM 306693 , 3, 68-102 mm, USNM 306735, 3, 118-130 mm, USNM 306872, 1, $80 \mathrm{~mm}$, Paraná do lago Janauacá, entrance of lago do Castanho, rio Amazonas floodplain, no coordinates. USNM 306694, 1, $87 \mathrm{~mm}$, Ressaca da Ilha da Marchantaria, rio Amazonas floodplain, ca. 
$03^{\circ} 14^{\prime} \mathrm{S}, 059^{\circ} 56^{\prime} \mathrm{W}$. USNM 306749,1, $116 \mathrm{~mm}$, furo [artificial channel] between lago Murumuru and Paraná de Janauacá, rio Amazonas floodplain, ca. $03^{\circ} 25^{\prime} \mathrm{S}, 060^{\circ} 16^{\prime} \mathrm{W}$. USNM 306785, 1, $116 \mathrm{~mm}$, lago Janauari, rio Amazonas floodplain, ca. $03^{\circ} 13^{\prime} \mathrm{S}$, $060^{\circ} 00^{\prime} \mathrm{W}$. USNM 306789, 5, 48-145 mm, USNM 306811, 7, 100$121 \mathrm{~mm}$, lago Janauari, nr. entrance, rio Amazonas floodplain, $c a$. $03^{\circ} 13^{\prime} \mathrm{S}, 059^{\circ} 59^{\prime} \mathrm{W}$. USNM 306877, 1, 36 mm, lago Canta Galos, lago Janauari, rio Amazonas floodplain, ca. $03^{\circ} 13^{\prime} \mathrm{S}, 060^{\circ} 00^{\prime} \mathrm{W}$. USNM 306948, 1, 54 mm, lago Terra Preta, lago do Janauari, rio Amazonas floodplain, $c a .03^{\circ} 13^{\prime} \mathrm{S}, 060^{\circ} 00^{\prime} \mathrm{W}$. Maranhão. MNRJ 12222, 2, 77-80 mm, mun. Ararí, lago Malhada Grande, rio Mearim dr., ca. $03^{\circ} 27^{\prime} \mathrm{S}, 044^{\circ} 46^{\prime} \mathrm{W}$. MNRJ 12223 , 2, 98-113 mm, mun. Ararí, lago Capivara, rio Mearim dr., ca. $03^{\circ} 27^{\prime} \mathrm{S}, 044^{\circ} 46^{\prime} \mathrm{W}$. Pará. (localities from Amazonas dr.). ANSP 194516, 4, 75-106 mm, mun. Porto de Moz, beach, R bank rio Xingu $3 \mathrm{~km}$ downstream Porto de Moz, rio Xingu, $01^{\circ} 43^{\prime} 54^{\prime \prime} \mathrm{S}, 052^{\circ} 15^{\prime} 16^{\prime \prime} \mathrm{W}$. CAS 33502, 1, 136 $\mathrm{mm}$, SU 54500, 1, $143 \mathrm{~mm}$, Ajamury, lago Grande, rio Amazonas floodplain, $c a .02^{\circ} 16^{\prime} \mathrm{S}, 54^{\circ} 17^{\prime} \mathrm{W}$. FMNH 54550, 3, 84-126 mm, Santarém, rio Amazonas, ca. $02^{\circ} 25^{\prime} \mathrm{S}, 054^{\circ} 42^{\prime} \mathrm{W}$. INPA 19961, 7, 72-107 mm, lago Salgado, rio Cumuní, affl. rio Trombetas, ca. $01^{\circ} 26^{\prime} \mathrm{S}, 055^{\circ} 55^{\prime} \mathrm{W}$. MCP 28564, 1, 64 mm, mun. São Domingos do Capim, igarapé do Onze, ca. $11 \mathrm{~km}$ São Domingos do Capim on Belém-Brasília hwy., affl. rio Guamá, affl. Baía de Marajó, ca. $01^{\circ} 45^{\prime} \mathrm{S}, 047^{\circ} 41^{\prime} \mathrm{W}$. MCP 49403, 11, mun. Curuá, Lago Preto, Ilha São Luiz, rio Amazonas, $02^{\circ} 06^{\prime} 22^{\prime \prime} \mathrm{S}, 055^{\circ} 10^{\prime} 17^{\prime \prime} \mathrm{W}$. MCP 49407 , 15, UF 238558, 28, 116-209 mm, UF 238559, 15, 110-209 mm, UF 238560, 25, 57-155 mm, UF 238561, 1, $68 \mathrm{~mm}$, UF 238562, 46, 48-142 mm, UF 238563, 36, 66-125 mm, mun. Santarém, Lago de Santana, Ilha Marimarituba, rio Amazonas, $02^{\circ} 11^{\prime} 13^{\prime \prime} \mathrm{S}$, $055^{\circ} 02^{\prime} 21^{\prime \prime} \mathrm{W}$. UF $238569,1,58 \mathrm{~mm}$, UF $238571,2,49-57 \mathrm{~mm}$, UF 238572, 23, 131-206, UF 238573, 4, 131-209, UF 238574, 7, 72109, UF 238576, 9, 68-115 m, UF 238578, 1, 16 mm, UF 238579 , 9, 76-91 mm, UF 238581, 19, 67-139 mm, mun. Santarém, lago Pajau, rio Amazonas, $02^{\circ} 11^{\prime} 29^{\prime \prime} \mathrm{S}, 054^{\circ}$ 51'28"W. MCZ 9415, 1, 127 $\mathrm{mm}$, MCZ 45095, 2, 125-132 mm, rio Xingu at Porto de Moz, ca. $01^{\circ} 45^{\prime} \mathrm{S}, 052^{\circ} 10^{\prime} \mathrm{W}$. MCZ 9432, 1, 108 mm, MCZ 9435, 4, 98-137 mm, MCZ 165862, 11 (part), 40-114 mm, mun. Santarém, Santarém and environs, rio Tapajós, ca. $02^{\circ} 26^{\prime} \mathrm{S}, 54^{\circ} 41^{\prime} \mathrm{W}$. MPEG 1130, 2 , 97-107 mm, MPEG 2816, 2, 50-127 mm, MPEG 3723, 1, 88 mm, mun. Cachoeira do Arari, Taperebá, rio Goapi, llha de Marajó, affl. Baía do Marajó, $\mathrm{ca} .01^{\circ} 00^{\prime} \mathrm{S}, 048^{\circ} 57^{\prime} \mathrm{W}$ [coordinates for Cachoeira do Arari]. MPEG 1860, 11, 37-102 mm, mun. Óbidos, rio Amazonas nr. Óbidos, ca. $01^{\circ} 54^{\prime} \mathrm{S}, 055^{\circ} 26^{\prime} \mathrm{W}$. MPEG 2437, 2, 90-112 mm, mun. Cachoeira do Arari, $1 \mathrm{~km}$ upstream Taperebá, rio Goapi, 1lha de Marajó, affl. Baía do Marajó, ca. $01^{\circ} 00^{\prime} \mathrm{S}, 048^{\circ} 57^{\prime} \mathrm{W}$ [coordinates for Cachoeira do Arari]. MPEG 5819, 1, $97 \mathrm{~mm}$, mun. Almeirim, São Raimundo, rio Amazonas, ca. $01^{\circ} 32^{\prime} \mathrm{S}, 052^{\circ} 31^{\prime} \mathrm{W}$ [coordinates for Almeirim]. MPEG 9942, 1, $125 \mathrm{~mm}$, MPEG 9943, 1, $97 \mathrm{~mm}$, MPEG 9958, 6, 47-132 mm, MPEG 9962, 1, 63 mm, MPEG 9987, 1, 125 mm, MPEG 10162, 4, 97-130 mm, mun. Portel, Floresta Nacional Caxiuanã, affl. rio Anapú, affl. rio Pará, affl. Baía de Marajó, ca. $01^{\circ} 44^{\prime} \mathrm{S}, 051^{\circ} 26^{\prime} \mathrm{W}$. MUSM 565, 1, $134 \mathrm{~mm}$, lago Uruira, Oriximinã, rio Trombetas-Amazonas floodplain, $c a$. $01^{\circ} 46^{\prime} \mathrm{S}, 055^{\circ} 51^{\prime} \mathrm{W}$ [coordinate for Oriximinã]. MZUSP 5033, 1, 87 mm, mun. Cachoeira do Arari, Ilha de Marajó, rio Arari, affl. Baía de Marajó, ca. $01^{\circ} 00^{\prime} \mathrm{S}, 048^{\circ} 57^{\prime} \mathrm{W}$. MZUSP 22894, 1, $92 \mathrm{~mm}$, igarapé Caraparú, affl. rio Guamá, aff, Baía de Marajó, ca. 01²3'S, 048 $08^{\prime}$ W. MZUSP 23216, 20, 78-108 mm LEA, lago Ururiá [ L. Urarirá], Oriximiná, rio Amazonas floodplain nr. mouth rio Trombetas, $c a .01^{\circ} 53^{\prime} \mathrm{S}, 055^{\circ} 52^{\prime} \mathrm{W}$. SU $54490,1,57 \mathrm{~mm}$, SU 54498 , $1,88 \mathrm{~mm}$, Uruará brook into rio Tapajós, Santarém, ca. $02^{\circ} 26^{\prime} \mathrm{S}$, $054^{\circ} 42^{\prime} \mathrm{W}$ [coordinates for Santarém]. SU 54493, 1, $109 \mathrm{~mm}$, igarapé do Sapucua [probably affl. lago Sapucuá] at Ayereua, affl. río Trombetas, $c a .01^{\circ} 49^{\prime} \mathrm{S}, 056^{\circ} 05^{\prime} \mathrm{W}$. SU 54501, 1, $130 \mathrm{~mm}$, lago Grande, rio Amazonas floodplain, $c a .02^{\circ} 16^{\prime} \mathrm{S}, 54^{\circ} 17^{\prime} \mathrm{W}$. SU 54614 , 1, $99 \mathrm{~mm}$, Alemquer [Alenquer] pond affl. rio Amazonas, ca. $01^{\circ} 57^{\prime} 30^{\prime \prime} \mathrm{S}, 54^{\circ} 44^{\prime} 18^{\prime \prime} \mathrm{W}$ [coordinates for Alenquer]. Rondônia (localities from rio Madeira dr., Amazonas dr.). INPA 9719, 2, 148155 mm, INPA 9728, 3, 125-145 mm, ca. 15 km Guajará Mirim, rio Pacaás Novos, affl. rio Mamoré, ca. $10^{\circ} 51^{\prime} \mathrm{S}, 065^{\circ} 16^{\prime} \mathrm{W}$. INPA 19943, 15, 53-92 mm, rio Mamoré, upstream Surprêsa, ca. 11²5'S, $064^{\circ} 59^{\prime} \mathrm{W}$. INPA 19953, 3, 34-88 mm, rio Mamoré, just upstream Guajará Mirim, $c a .10^{\circ} 47^{\prime} \mathrm{S}, 065^{\circ} 20^{\prime} \mathrm{W}$. INPA 19957, 1, $148 \mathrm{~mm}$, Surprêsa, at rio Guaporé- Mamoré confl., ca. $11^{\circ} 53^{\prime} \mathrm{S}, 064^{\circ} 59^{\prime} \mathrm{W}$. MPEG 1116, 6, 77-100 mm, rio Pacaás Novas, Guajará Mirim, affl. rio Mamoré, $c a .10^{\circ} 51^{\prime} \mathrm{S}, 065^{\circ} 16^{\prime} \mathrm{W}$. MPEG 1121 (part), 1, $85 \mathrm{~mm}$, rio Pacaás Novos, $20 \mathrm{~km}$ upstream Guajará Mirim, ca.10 56'15"S, $065^{\circ} 16^{\prime} 29^{\prime \prime} \mathrm{W}$. UFRO-I 6484, 34, 43-145 mm, UFRO-I uncat. (field number CUN2009061202), 1, 58 mm, UFRO-I uncat. (field number CUN2009072001), 1, $54 \mathrm{~mm}$, Furo Variante, lago Cuniã, rio Madeira floodplain, $08^{\circ} 21^{\prime} 18^{\prime \prime} \mathrm{S}, 063^{\circ} 30^{\prime} 11^{\prime \prime} \mathrm{W}$. UFRO-I 6486, 11, 68-144 mm, lago Cuniã, nr. ICMBio base, rio Madeira floodplain, 08¹9'40"S, $063^{\circ} 30^{\prime} 11^{\prime \prime} \mathrm{W}$. UFRO-I 6489, 2, 86-161 mm, UFRO-I 6498, 1, $124 \mathrm{~mm}$, lago Cuniã, nr. ICMBio base, rio Madeira floodplain, 08 $19^{\prime} 20^{\prime \prime} \mathrm{S}, 063^{\circ} 30^{\prime} 14^{\prime \prime} \mathrm{W}$. UFRO-I 6491, 4, 38-111 $\mathrm{mm}$, lago Cuniã, nr. igarapé do Campo, rio Madeira floodplain, 08¹9'09"S, $063^{\circ} 28^{\prime} 44^{\prime \prime}$ W. UFRO-I 6493, 3, 141-151 mm, Arrozal do lago, lago Cuniã, rio Madeira floodplain, $08^{\circ} 19^{\prime} 36^{\prime \prime} \mathrm{S}$, $063^{\circ} 29^{\prime} 59^{\prime \prime} \mathrm{W}$. UFRO-I 6500, 3, 55-148 mm, lago Cuniã, rio Madeira floodplain, $08^{\circ} 19^{\prime} 45^{\prime \prime} \mathrm{S}, 063^{\circ} 28^{\prime} 06^{\prime \prime} \mathrm{W}$. UFRO-I 6502, 28, 78-135 mm, lago Cuniã, rio Madeira floodplain, $08^{\circ} 21^{\prime} 10^{\prime \prime} \mathrm{S}$, $063^{\circ} 30^{\prime} 26^{\prime \prime} \mathrm{W}$. UFRO-I 6510, 21, 81-180 mm, mouth of rio Cautário, affl. rio Guaporé, affl. rio Mamoré, $12^{\circ} 10^{\prime} 51^{\prime \prime} \mathrm{S}, 064^{\circ} 34^{\prime} 04^{\prime \prime} \mathrm{W}$. UFRO-I 6511, 1, $113 \mathrm{~mm}$, igarapé do Campo, lago Cuniã, rio Madeira floodplain, $08^{\circ} 19^{\prime} 14^{\prime \prime}$ S, 063 $28^{\prime} 05^{\prime \prime} \mathrm{W}$. UFRO-I 7927, 8 (2 immature, 96-111 mm, 6 female, 90-107 mm), mun. Porto Velho, lago Cuniã, rio Madeira floodplain, $08^{\circ} 19^{\prime} \mathrm{S}, 063^{\circ} 28^{\prime} \mathrm{W}$. USNM 348864, 1, 119 mm, Brazil-Bolivia border region between GuajaráMirim and Mato Grosso, rio Guaporé, affl. rio Mamoré, $c a .13^{\circ} 46^{\prime} \mathrm{S}$, $061^{\circ} 04^{\prime} \mathrm{W}$. French Guiana. Cayenne. ANSP 163463, 1 (lectotype), male, $125 \mathrm{~mm}$, ANSP 163464, 1 (paralectotype), male, $149 \mathrm{~mm}$, ANSP 163465, 1 (paralectotype), female, 119 mm, ANSP 164474, 1 (paralectotype), male, $156 \mathrm{~mm}, \mathrm{BMNH}$ 1990.10.23.1-3, 3), 93$108 \mathrm{~mm}, \mathrm{FMNH} 98817,1$ (paralectotype), $116 \mathrm{~mm}, \mathrm{MBUCV}-\mathrm{V}$ 20221, 3, 82-100 mm, MNHN 1989-1209, 3 (paralectotypes), NMW 90321, 5, 115-139 mm, UMMZ 216032, 3, 48-68 mm [originally 96-106] mm, USNM 301966, 1 (paralectotype), 114 mm, USNM 301967, 1 (paralectotype), female, $114 \mathrm{~mm}$, coastal swamp, 'Grand Pripris', $3.5 \mathrm{~km}$ NW center of old Kourou, ca. $100 \mathrm{~m} \mathrm{~N}$ old Route Nationale 1, Kourou River dr., 05 $10^{\prime} 45^{\prime \prime} \mathrm{N}$, $052^{\circ} 40^{\prime} 00^{\prime \prime} \mathrm{W}$. CU 71933, 1, male, 170 mm, FMNH 98818, 3, 124$161 \mathrm{~mm}$, swampy border, Kaw River, ca. $3.5 \mathrm{~km} \mathrm{SW} \mathrm{Kaw,} \mathrm{River}$ 
Kaw dr., ca. $04^{\circ} 27^{\prime} \mathrm{N}, 052^{\circ} 10^{\prime} \mathrm{W}$. USNM 301968, 1, 64 mm, swamp, old Rn-1 Hwy., nr. city of Kourou, Kourou River dr., ca. $05^{\circ} 10^{\prime} \mathrm{N}$, 052³9’W. Peru. Madre de Dios. (localities from río Madre de Dios, affl. río Beni, affl. rio Madeira, Amazonas dr.). MUSM 2470 , 1, Parque Nacional del Manu, Pakitza, río Manu, ca. $12^{\circ} 52^{\prime} \mathrm{S}$, $069^{\circ} 16^{\prime}$ W. MUSM 2874, 2, Reserva Nacional (RN) Tambopata, cocha Redonda, no coordinates. MUSM 2981, 1, 90 mm, Planchón, $\mathrm{N}$ Puerto Maldonado, río Manuripi, affl. río Orthon, $c a .12^{\circ} 17^{\prime} \mathrm{S}$, 06909'W. MUSM 3521, 2, Reserva Nacional Tambopata, La Colpa, río Tambopata, no coordinates. MUSM 4198, 4, RN Tambopata, no coordinates. MUSM 9265, 10, 94-171 mm, MUSM 9847, 1, 170 mm, MUSM 16004, 1, 123 mm, MUSM 16169, 1, MUSM 16181, 1, 195 mm, lago Tres Chimbadas, RN Tambopata, río Tambobata, 1247'26"S, 069²0'31"W. MUSM 16610, 1, 109 $\mathrm{mm}$, lago Valencia, río Madre de Dios floodplain, ca. $12^{\circ} 25^{\prime} \mathrm{S}$, 068 46'W. MUSM 16712, 2, 142-147 mm, MUSM 16743, 1, 140 $\mathrm{mm}$, Tambopata, lago Copamanu, ca. $12^{\circ} 50^{\prime} \mathrm{S}, 069^{\circ} 17^{\prime} \mathrm{W}$. MUSM 19991, 1, 149 mm, Los Amigos Biostation, río Madre de Dios floodplain, $c a .12^{\circ} 33^{\prime} \mathrm{S}, 070^{\circ} 07^{\prime} \mathrm{W}$. MUSM 20046, $1,150 \mathrm{~mm}$, Manu, no coordinates. MUSM 20184, 4, Mavila, río Los Amigos, no coordinates. MUSM 20588, 2, MUSM 21390, 10, MUSM 21483, 1, Tambopata, Pozo Pedro, río Los Amigos, 12³3'40"S, $070^{\circ} 06^{\prime} 36^{\prime \prime}$ W. MUSM 21731, 2, MUSM 21779, 3, Pozo Santa
Elena, no coordinates. MUSM 22101, 1, Tambopata, Aguajal Oeste, ca. $12^{\circ} 00^{\prime} \mathrm{S}, 070^{\circ} 40^{\prime} \mathrm{W}$. TCWC $13983.01,2,73-87 \mathrm{~mm}$, lago Tres Chimbadas, río Tambopata, $12^{\circ} 47^{\prime} 29^{\prime \prime} \mathrm{S}, 069^{\circ} 20^{\prime} 54^{\prime \prime} \mathrm{W}$. TCWS 13985.02, 2, 86-115 mm, lago Tres Chimbadas, río Tambopata, $12^{\circ} 47^{\prime} 04^{\prime \prime} \mathrm{S}, 069^{\circ} 20^{\prime} 29^{\prime \prime} \mathrm{W}$. USNM 263859, 4, 70-135 mm, RN Tambopata, Laguna Chica, $12^{\circ} 50^{\prime} 30^{\prime \prime} \mathrm{S}, 069^{\circ} 17^{\prime} 30^{\prime \prime} \mathrm{W}$. USNM 264110, 5, 42-102 mm, USNM 387400, 1, 91 mm, NR Tambopata, Laguna Cocococha, $5.1 \mathrm{~km}$ E Explorer's Inn, $12^{\circ} 49^{\prime} 00^{\prime \prime} \mathrm{S}$, $069^{\circ} 16^{\prime} 30^{\prime \prime} \mathrm{W}$

\section{Brachyhypopomus provenzanoi, new species}

urn:1sid:zoobank.org:act:E64D737C-8334-47FA-959879B4F76C89CF

\section{(Fig. 40; Tables 2-5, 17)}

Brachyhypopomus sp. "pro". -Crampton, 2011: 176, table 10.2, species list; 179, figs. 10.2-10.3, phylogeny, geographical and ecological distributions (gymnotiform biology).

Brachyhypopomus sp. "provenzanoi". -Crampton et al., 2016: 1-66, table 1, 3-4, figs. 1-7, 18-20 (phylogeny, biogeography and ecology of Brachyhypopomus).

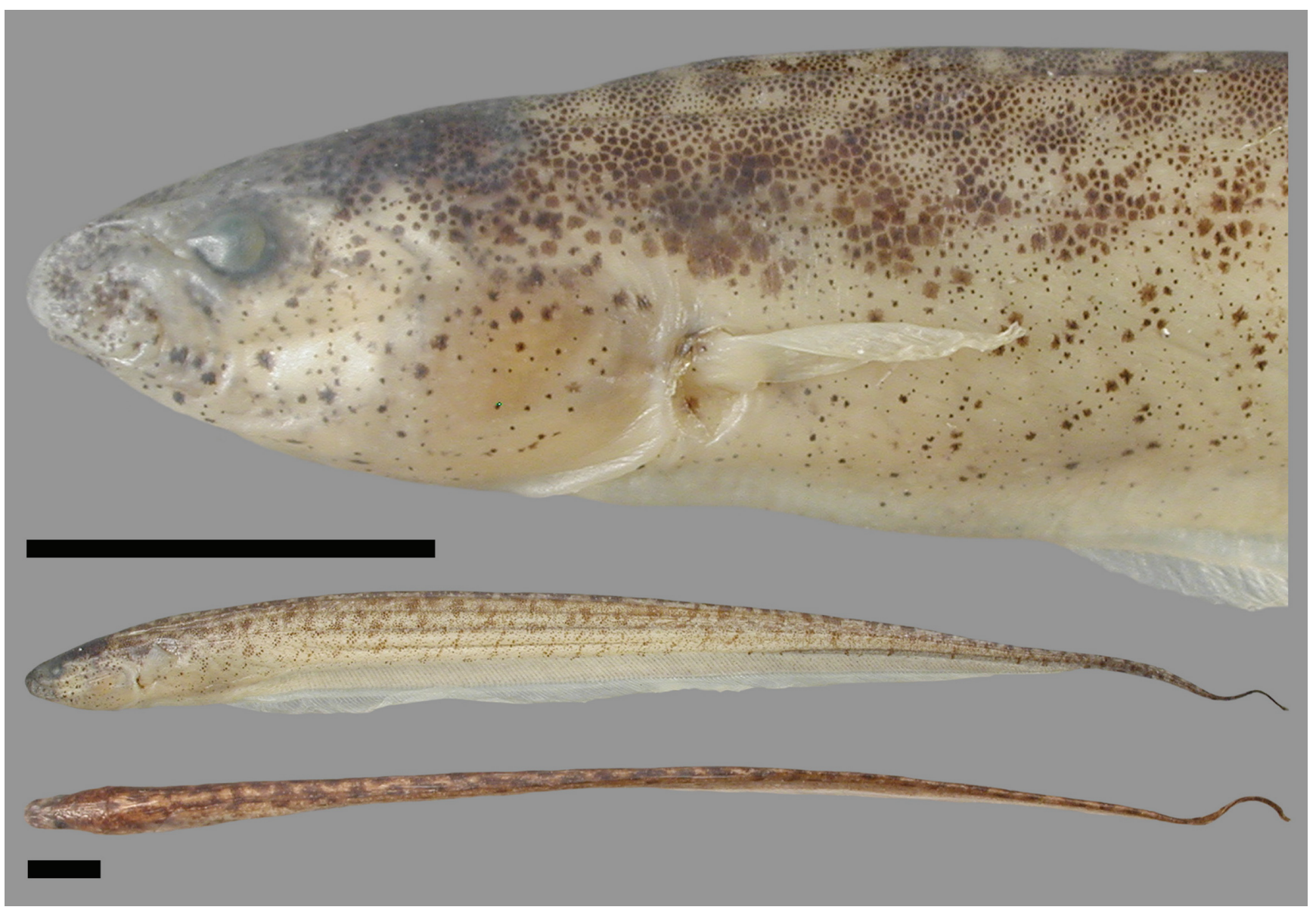

Fig. 40. Brachyhypopomus provenzanoi. MBUCV-V 35650, holotype, immature, $87 \mathrm{~mm}$ TL (head in lateral view, and body in lateral and dorsal view, specimen fixed in formalin and preserved in EtOH); Venezuela, río Orinoco, Orinoco dr. Scale bars $=5 \mathrm{~mm}$. 
Holotype. MBUCV-V 35650, immature, $87 \mathrm{~mm}$ TL, 71 mm LEA, Venezuela, Amazonas, mun. San Fernando de Atabapo, caño "CVG" (Corporacion Venezuelana Guyana), km $10.5 \mathrm{~km}$ on San Fernando de Atabapo-Santa Barbara rd.,

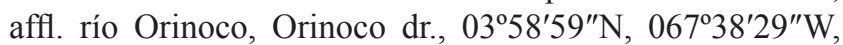
11 Mar 2004, W. Crampton, N. Lovejoy, S. Willis \& C. Montaña.

Paratypes. 59 specimens, all from Orinoco dr. Venezuela. Amazonas. MBUCV-V 12991, 2, 71-72 mm, MBUCV-V 12993, 4 (2 measured), 79-108 mm, Pozo de Lucas, ca. 7 km $\mathrm{S}$ San Fernando de Atabapo, affl. río Orinoco, $c a .04^{\circ} 15^{\prime} \mathrm{N}$, $067^{\circ} 39^{\prime} \mathrm{W}, 8$ Apr 1982, R. Royero \& G. Pereira. MBUCV-V 35651, 16 (14 immature [1 CS], 73-93 mm, 2 male, 92-94 $\mathrm{mm})$, caño Viejita, on rd. from San Fernando de Atabapo to Santa Barbara, affl. río Atabapo, $03^{\circ} 55^{\prime} 59^{\prime \prime} \mathrm{N}, 067^{\circ} 36^{\prime} 34^{\prime \prime} \mathrm{W}$, 13-15 Mar 2004, W. Crampton, N. Lovejoy, S. Willis \& C. Montaña. UF 17734733 (19 immature [3 CS], 67-87 mm, 10 female, $69-110 \mathrm{~mm}, 4$ male, $83-94 \mathrm{~mm}$ ), collected with holotype.

Non-types. 10 specimens. Venezuela. Amazonas. MBUCV-V 11221, 1, 107 mm, caño Chola, rd. to Solano, at $\mathrm{km} 17.7$ from San Carlos de rio Negro, río Negro, upper rio Negro, Amazonas dr., $c a .01^{\circ} 57^{\prime} \mathrm{N}, 066^{\circ} 59^{\prime} \mathrm{W}$. UF 185344 , 9 , immature, collected with holotype.

Diagnosis. Brachyhypopomus provenzanoi is diagnosed from congeners by the following combination of characters: scales absent in middorsal region of anterior third of body ( $v s$. present in all congeners except $B$. benjamini); dorsal surface of body with irregular pattern of distinct dark blotches on depigmented tan-colored background, vs. speckled with small brown chromatophores on light brown background in B. benjamini.

Description. Head and body shape, and pigmentation illustrated in Fig. 40. Meristic and morphometric data for examined specimens presented in Tables 2-5 and 17. Body shallow in depth. Head short to moderate in length and shallow in depth. Dorsal profile of head approximately straight from occiput to snout, ventral profile of head gently convex between operculum and snout, snout rounded. Eye moderate in size. Upper jaw with slight sigmoidal angle between premaxillary and maxillary portions in lateral view. No accessory electric organ over operculum. Gill filaments on first gill arch 26-32 (median 29, $\mathrm{n}=9$ ). Pectoral fin narrow, pectoral-fin rays 12-15 (mode 13). Precaudal vertebrae 15-17 (mode 16), with 1-2 (mode 1) transitional vertebrae. Anal-fin origin slightly $(<0.25 \mathrm{HL}$ distance) to substantially (0.33-0.5 HL distance) posterior to tip of pectoral fin. Anal-fin rays 162-195 (median 184). Dorsal rami of recurrent branch of anterior lateral line nerve visible. Middorsal region of body without scales in anterior third of body. Middorsal region with small scales completely occluded by skin in posterior two thirds of body. Rows of scales above lateral line 4-5 (mode 4); at measuring point in mid-anal fin, scale rows do not reach dorsal midline - instead middorsal region is scaleless, as is condition anterior. Scale rows reach dorsal midline just posterior to this point. Lateral line continuous. Sparse depigmented epidermal canals present as short parallel scratch like marks dorsal to and near lateral line; mostly restricted to posterior third of body. Three bilateral horizontal columns of electrocytes at analfin terminus, and at a mid-point between anal-fin terminus and tip of caudal filament in immature, mature female, and mature male specimens. Caudal filament short to moderate in length.

Coloration. (Fig. 40). Pale straw to tan background. Dorsal region comprising irregular brown patches and a pale straw or tan background, with no pale stripe along dorsal midline from occipital region to base of caudal filament. Distinct stripes or saddles on flank absent. Region over anal-fin pterygiophores straw colored with very faint irregular markings. Caudal filament darker than body, with indistinct, disrupted vertical bars. Head with evenly scattered dark chromatophores, darker dorsally. Eye without prominent suborbital patch, or stripe, of chromatophores/subcutaneous pigmentation. Pectoral-fin and anal-fin membranes hyaline. Pectoral-fin rays hyaline and unpigmented. Anal-fin rays with light scattering of dark chromatophores, in posterior half of fin only. Color in live individuals similar to preserved specimens, with opercular region usually rosy due to underlying gills.

Size. Small adult size, largest specimen examined $110 \mathrm{~mm}$ TL, $91 \mathrm{~mm}$ LEA $(\mathrm{n}=70)$. Largest male specimen examined $94 \mathrm{~mm}$ TL, $83 \mathrm{~mm}$ LEA $(\mathrm{n}=6)$. Largest female specimen examined $110 \mathrm{~mm} \mathrm{TL}, 91 \mathrm{~mm} \mathrm{LEA}(\mathrm{n}=10)$.

Sexual dimorphism. No known secondary sexual dimorphism.

Geographic distribution. Venezuela (Fig. 31). Known from the upper Orinoco and upper Negro (Amazonas dr.).

Ecological notes. Ecology known only for the type series. Brachyhypopomus provenzanoi was collected in small, gently flowing, low-conductivity clearwater terra firme forest and savanna streams, and from static leaf-litter choked blackwater streams and swamps in Mauritia palm swamps. Most specimens in streams were found sheltering in submerged and marginal aquatic vegetation growing in sandy beds, and those in Mauritia palm swamps were collected from dense leaf litter banks and root mats. The following water parameters were recorded at the sampling sites: conductivity 9-10 $\mu \mathrm{Scm}^{-1}$, dissolved oxygen 1.6-3.8 $\mathrm{mgl}^{-1}$, temperature $25.8-28.2^{\circ} \mathrm{C}, \mathrm{pH} 3.1-4.0$. Reproductive biology is unknown. Stomach contents comprise aquatic insect larvae and other small aquatic invertebrates (WGRC unpublished data). 
Co-occurring congeners: In the region of the type locality, Brachyhypopomus provenzanoi was found in sympatry and in ecological syntopy with $B$. bullocki, B. brevirostris, and $B$. sullivani. Its geographical range also overlaps with that of B. beebei and B. regani.

Etymology. The specific name is a patronym (noun in the genitive case) in honor of Francisco Provenzano Rizzi, Venezuelan ichthyologist, for his contributions to Neotropical ichthyology.

Local names. Venezuela: cuchillo.

\section{Brachyhypopomus regani, new species}

urn:1sid:zoobank.org:act:F537AD66-390E-4767-926E0D18CAD199AC

(Figs. 1j, 41; Tables 2-5, 17)

Hypopomus brevirostris. -Hopkins \& Heiligenberg, 1978: 132, fig. 10 , black and white photograph of adult (Suriname, species and EOD diversity).

Hypopomus artedi. -Hopkins \& Westby, 1986: 83, fig. 3, line drawing (Guianas, species and EOD diversity).

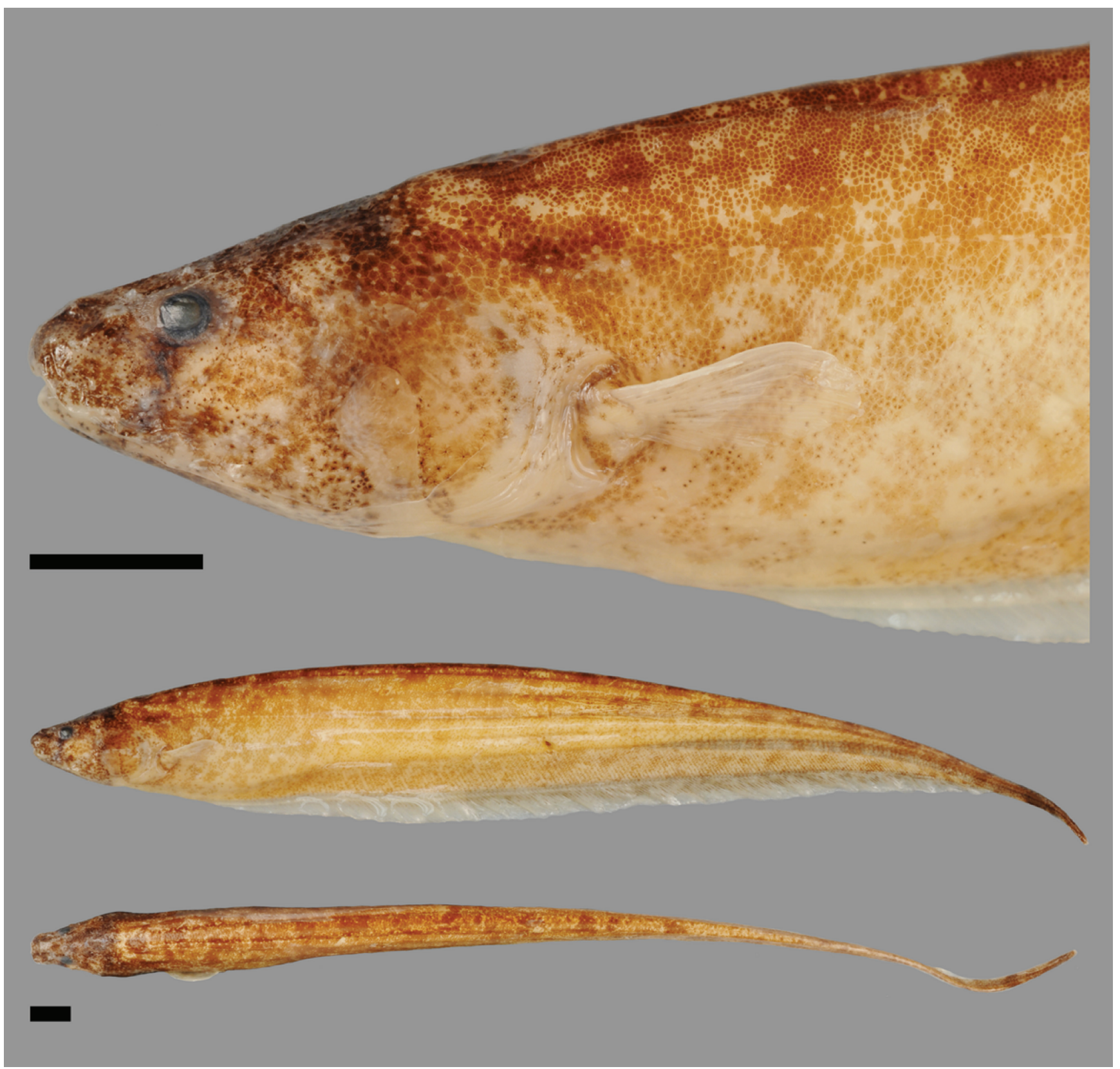

Fig. 41. Brachyhypopomus regani. MCP 47022 (WC04.140301), holotype, female, $128 \mathrm{~mm}$ TL (head in lateral view, and body in lateral and dorsal view, specimen fixed in formalin and preserved in EtOH); Brazil, rio Solimões-Japurá confluence, Amazonas dr. Note prominent opercular accessory electric organ (pale structure). Scale bars $=5 \mathrm{~mm}$. 
Hypopomidae sp. 9. -Crampton, 1996a: 77, table 6.1.a, species list; 79, fig. 6.1 inset 32-33, color photographs; 85, fig. 6.2; 88, fig. 6.3; 92-94, figs. 6.4a, 6.5; 191, fig. 11.2; 193, fig. 11.3, EOD data; 110 , table $7.5,113$, table 7.7, 115, fig. 7.1, habitats; 136 , table 8.2a,b, diet; 151-160, figs. 9.2-9.3, table 9.1a,b, hypoxia tolerance; 165-169, table 10.1 reproductive biology (Brazil, central Amazon, ecology and signal diversity).

Brachyhyроротиs electropomus. -Sullivan, 1997: 91 (description in unpublished thesis with disclaimer stating that nomenclatural acts are not available, a nomen nudum, refers also to B. bombilla and B. menezesi).

Brachyhypopomus sp. 5. -Crampton, 1998a: 821, table 4, 834, fig. 9, illustration of specimen, with EOD (Brazil, Amazonas, species and EOD diversity). -Crampton, 1998b: 314, table 2, list of Brachyhypopomus (Brazil, Amazonas, hypoxia tolerance). -Crampton, 1999: 17 (Brazil, Amazonas, Mamirauá Reserve, listing of species).

Brachyhypopomus sp. E. -Crampton \& Albert, 2006: 672, fig. 23.8, color photograph of head of live individual with EOD, position in phylogenetic tree; 681, notes on EODs (gymnotiform species and EOD diversity).

Brachyhypopomus sp. "reg". -Crampton, 2011: 176, table 10.2, species list; 179, figs. 10.2-10.3, phylogeny, geographical and ecological distributions (gymnotiform biology).

Brachyhypopomus sp. 1. -Mol, 2012: 598 (Suriname, catalog of fishes). -Crampton \& Ribeiro, 2013: 238, color photograph (Brazil, rio Madeira, listing of hypopomids).

Brachyhypopomus sp. 'ele'. -Carvalho, 2013: 177, fig. 37A, color photograph of head showing position of accessory electric organ; 181-185, figs. 41-43, position in phylogeny (phylogenetic systematics of Rhamphichthyoidea).

Brachyhypopomus sp. "regani". -Crampton et al., 2016: 1-66, table 1, 3-4, figs. 1-7, 18-20 (phylogeny, biogeography and ecology of Brachyhypopomus).

Holotype. MCP 47022, female, $128 \mathrm{~mm}$ TL, $115 \mathrm{~mm}$ LEA, Brazil. Amazonas. Mamirauá Reserve, cano do lago Rato, rio Solimões-Japurá floodplain, Amazonas dr., $03^{\circ} 02^{\prime} 41^{\prime \prime} \mathrm{S}$, $064^{\circ} 51^{\prime} 26^{\prime \prime} \mathrm{W}, 14$ Mar 2001, W. Crampton \& J. Oliveira.

Paratypes. 40 specimens. Brazil.Amazonas (collected from Amazonas dr., by W. Crampton \& J. Oliveira; localities from Amazonas dr., localities listed from the Mamirauá Reserve [Reserva de Desenvolvimento Sustentável Mamirauá] are in rio Solimões-Japurá floodplain, mun. Alvarães). BMNH 1998.3.12.183, 1, immature, 117 mm, mun. Tefé, Ressaca do Cachorro, lago Tefé, (rio Tefé), 03 $19^{\prime} 50^{\prime \prime} \mathrm{S}$, 644ㄴ'15"W, 3 Feb 1995. BMNH 1998.3.12.184-188, 6, immature (1 measured, $90 \mathrm{~mm}$ ), Mamirauá Reserve, cano do lago Mamirauá, $03^{\circ} 03^{\prime} 51^{\prime \prime} \mathrm{S}, 64^{\circ} 51^{\prime} 05^{\prime \prime} \mathrm{W}, 12 \mathrm{Apr}$ 1994. BMNH 1998.3.12.189-190, 2 (1 female, 109 mm, 1 male, $134 \mathrm{~mm}$ ), Mamirauá Reserve, cano do lago Rato, $03^{\circ} 02^{\prime} 41^{\prime \prime} \mathrm{S}, 064^{\circ} 51^{\prime} 26^{\prime \prime} \mathrm{W}, 20$ Jan 1995. INPA 9951, 8, 70$108 \mathrm{~mm}$, Mamirauá Reserve, lago Caetano, 02 $50^{\circ} 58^{\prime \prime} \mathrm{S}$, $064^{\circ} 55^{\prime} 42^{\prime \prime} \mathrm{W}$, Oct 1993. INPA 18447, 2, immature, 6679 mm, Mamirauá Reserve, lago Periquito Comprido,

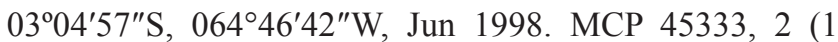
immature, $118 \mathrm{~mm}, 1$, male, $132 \mathrm{~mm}$ ), Mamirauá Reserve,

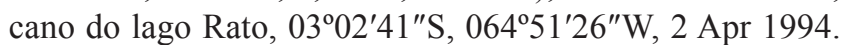
MCP 45334, 1, female, 120 mm, Mamirauá Reserve, lago

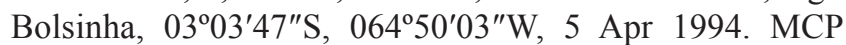
45335, 1, immature, $140 \mathrm{~mm}$, Mamirauá Reserve, cano

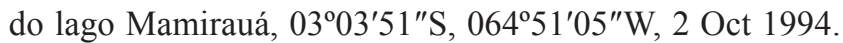
MCP 45411, 1, female, $107 \mathrm{~mm}$, Mamirauá Reserve, cano do lago Sapucaia, 0304'07"S, 06448'32"W, 15 Apr 1998. MCP 45439, 1, immature, $107 \mathrm{~mm}$, Mamirauá Reserve, Paraná do Apara, 0306 $40^{\prime \prime} \mathrm{S}, 064^{\circ} 47^{\prime} 52^{\prime \prime} \mathrm{W}, 23$ Mar 1995. MCP 45446, 4, 57-109 mm, Mamirauá Reserve, cano do lago Rato, 0302'41"S, 06451'26"W, 1997 (no precise date). MCP 45474, 1, $107 \mathrm{~mm}$, mun. Tefé, Ilha Panamim, rio Solimões floodplain, $03^{\circ} 19^{\prime} 29^{\prime \prime} \mathrm{S}, 064^{\circ} 38^{\prime} 37^{\prime \prime} \mathrm{W}, 31 \mathrm{Jul}$ 2001. MCP 45475, 2, 76-86 mm, MCP 45476, 1, female, $141 \mathrm{~mm}$, mun. Tefé, lago Jacaré, rio Solimões floodplain, $03^{\circ} 11^{\prime} 11^{\prime \prime} \mathrm{S}, 064^{\circ} 43^{\prime} 05^{\prime \prime} \mathrm{W}, 18$ Aug 2001. MCP 45484, 8 (1 not sexed [CS], $105 \mathrm{~mm}, 2$ immature [1CS], $84-90 \mathrm{~mm}, 2$ female, $89-117 \mathrm{~mm}, 3$ male, $104-135 \mathrm{~mm}$ [1 with gill and brain removed, $135 \mathrm{~mm}]$ ), Mamirauá Reserve, cano do lago Mamirauá, 0306'40"S, 06447'52"W, 15 Mar 2001.

Non-types. 189 specimens. Brazil (localities from Amazonas dr.). Acre. MCP 41043, 2, mun. Brasiléia, stream, affl. small river, ca. $2 \mathrm{~km}$ hwy. BR-317 on branch 26 , affl. rio Purus, $10^{\circ} 56^{\prime} 10^{\prime \prime} \mathrm{S}, 068^{\circ} 56^{\prime} 01^{\prime \prime} \mathrm{W}$. Amazonas. (localities from Amazonas dr., localities listed from the Mamirauá Reserve [Reserva de Desenvolvimento Sustentável Mamirauá] are in rio Solimões-Japurá floodplain, mun. Alvarães). IDSM 458, 1, $67 \mathrm{~mm}$, MCP 45473, 3 (1 female, $112 \mathrm{~mm}, 2$ male, 104-119 mm), Mamirauá Reserve, lago Periquito Comprido, $03^{\circ} 04^{\prime} 57^{\prime \prime} \mathrm{S}$, $064^{\circ} 46^{\prime} 42^{\prime \prime} \mathrm{W}$. INPA 15443, 1, $143 \mathrm{~mm}$, igarapé Água Viva, nr. Presidente Figueiredo, 12 km on hwy. AM-240, igarapé Mutum, affl. igarapé Urubú, 02 $15^{\prime} 13^{\prime \prime} \mathrm{S}, 060^{\circ} 24^{\prime} 59^{\prime \prime} \mathrm{W}$. INPA 19945, 2, 73-88 mm, Ilha Janauacá, rio Solimões floodplain, ca. $03^{\circ} 43^{\prime} \mathrm{S}, 060^{\circ} 26^{\prime} \mathrm{W}$. MCP 41036 (part), 1, 86 mm, mun. Canutama, igarapé São João on hwy. BR-319, ca. $60 \mathrm{~km} \mathrm{~S}$ Humaitá, affl. rio Ipixuna, affl. rio Purus, $07^{\circ} 56^{\prime} 07^{\prime \prime} \mathrm{S}, 063^{\circ} 20^{\prime} 03^{\prime \prime} \mathrm{W}$. MCP 45282, 1 , immature, 80 $\mathrm{mm}$, rio Japurá, at Nova Colômbia, rio Solimões-Japurá floodplain, 02 $54^{\prime} 47^{\prime \prime} \mathrm{S}, 064^{\circ} 54^{\prime} 19^{\prime \prime} \mathrm{W}$. MCP 45283, 2, immature, 73-76 mm, rio Japurá, nr. Boca do lago Mamirauá, rio Solimões-Japurá floodplain, $03^{\circ} 07^{\prime} 03^{\prime \prime} \mathrm{S}, 064^{\circ} 46^{\prime} 51^{\prime \prime} \mathrm{W}$. MCP 45284, 1, female, 133 mm, Mamirauá Reserve, Ressaca da Vila Alencar, $03^{\circ} 07^{\prime} 41^{\prime \prime} \mathrm{S}, 064^{\circ} 48^{\prime} 04^{\prime \prime} \mathrm{W}$. MCP 45285, 3 ( 2 female, $100-112 \mathrm{~mm}, 1$ male, $123 \mathrm{~mm}$ ), Mamirauá Reserve, cano do lago Rato, $03^{\circ} 02^{\prime} 41^{\prime \prime} \mathrm{S}$, 064 51'26"W. MCP 45286, 2, female, 107-111 mm, Mamirauá Reserve, Ressaca do Pau, $03^{\circ} 02^{\prime} 03^{\prime \prime} \mathrm{S}$, 064 $52^{\prime} 13^{\prime \prime} \mathrm{W}$. MCP 45456, 2 (1 immature, $103 \mathrm{~mm}, 1$ female, $106 \mathrm{~mm}$ ), Mamirauá Reserve, cano do lago Rato, $03^{\circ} 02^{\prime} 41^{\prime \prime} \mathrm{S}, 064^{\circ} 51^{\prime} 26^{\prime \prime} \mathrm{W}$. MCP 45457, 3 (1 not sexed [CS], $130 \mathrm{~mm}, 2$ male, 96-134 mm), Mamirauá Reserve, Ressaca da Vila Alencar, 030. $07^{\prime} 41^{\prime \prime} \mathrm{S}, 064^{\circ} 48^{\prime} 04^{\prime \prime} \mathrm{W}$. 
MZUSP 23403, 1, igarapé Tucuxi, Ati-Paraná, NW Fonte Boa, rio Solimões floodplain, ca. $02^{\circ} 14^{\prime} \mathrm{S}, 066^{\circ} 37^{\prime} \mathrm{W}$. USNM 304962, 1, $82 \mathrm{~mm}$, Paraná do Marauiá, affl. río Negro, $c a .00^{\circ} 23^{\prime} \mathrm{S}, 065^{\circ} 13^{\prime} \mathrm{W}$. Goiás. MZUSP 4887, 1, 53 mm LEA, rio Araguaia at Aruanã, affl. rio Tocantins, Amazonas dr., $14^{\circ} 55^{\prime} \mathrm{S}, 051^{\circ} 04^{\prime} \mathrm{W}$. Mato Grosso (localities from rio Tocantins dr., Amazonas dr.). MCP 40271, 5, mun. Vila Rica, stream on rd. Vila Rica-Confresa rd., affl. Ribeirão Gameleira, affl. rio Araguaia, $10^{\circ} 31^{\prime} 31^{\prime \prime} \mathrm{S}$, 05124'39"W. MCP 40780, 2, 66-90 mm, mun. Confresa, Ribeirão Tucunara, hwy. MT-432 between hwy. BR-158 and Terra Indígena Urubu Branco, affl. rio Araguaia, $10^{\circ} 41^{\prime} 25^{\prime \prime} \mathrm{S}, 051^{\circ} 22^{\prime} 52^{\prime \prime} \mathrm{W}$. MZUSP 88597, 2, 71-80 mm, Cocalinho, ca. $79 \mathrm{~km} \mathrm{~N}$ Cocalinho on hwy. MT-326, Ribeirão Agua Preta, affl. rio Cristalino, affl. rio Araguaia, $14^{\circ} 08^{\prime} 57^{\prime \prime} \mathrm{S}, 051^{\circ} 32^{\prime} 21^{\prime \prime} \mathrm{W}$. Pará (localities from Amazonas dr.). MCP 23358, 3, 76-88 mm, mun. São Miguel do Guamá, stream on rd. from São Miguel do Guamá to Ourem, $c a .10$ km São Miguel do Guamá, affl. rio Guamá, affl. Baía de Marajó, 01³3'18'S, 047²5'41"W. MCP 49412, 2, mun. Alenquer, small stream, affl. rio Curuá, 0136'13"S, 054 55'10"W. MPEG 1127, 1, $115 \mathrm{~mm}$, mun. Castanhal, Boa Vista do Apeú, rio Apeú, affl. rio Guamá, affl. Baía de Marajó, ca. $01^{\circ} 18^{\prime} \mathrm{S}, 047^{\circ} 59^{\prime} \mathrm{W}$. MPEG 2399, 1, $97 \mathrm{~mm}$, mun. Castanhal, Boa Vista do Apeú, rio Apeú, affl. rio Guamá, affl. Baía de Marajó, ca. 01 $18^{\circ} \mathrm{S}, 047^{\circ} 59^{\prime} \mathrm{W}$. MPEG 2402, 1, 103 mm, mun. Peixe-Boi, rio Peixe-Boi, affl. rio Maracanã, rio Amazonas estuary, 01 ${ }^{\circ} 11^{\prime} 31^{\prime \prime} \mathrm{S}$, $047^{\circ} 18^{\prime} 14^{\prime} \mathrm{W}$ [coordinates rio Peixe-boi at Peixe-boi]. MPEG 15005 (part), 1, $82 \mathrm{~mm}$, mun. Itaituba, stream at trail head from BR-163, affl. rio Tapajós., 04²3'41"S, $055^{\circ} 32^{\prime} 20^{\prime \prime} \mathrm{W}$. MPEG 24491, 17, 84-123 mm, mun. Capanema, igarapé Açaiteuazinho nr. Vila de Tauarí, rio Caeté dr., rio Amazonas estuary, $01^{\circ} 11^{\prime} 45^{\prime \prime} \mathrm{S}, 047^{\circ} 10^{\prime} 51^{\prime \prime} \mathrm{W}$. MZUSP 97324, 1, 72 mm, mun. Novo Progresso, nr. Mil, rio Jamanxim, affl. rio Tapajós, $07^{\circ} 43^{\prime} 51^{\prime \prime} \mathrm{S}, 055^{\circ} 16^{\prime} 36^{\prime \prime} \mathrm{W}$. MZUSP 97544, 1, $82 \mathrm{~mm}$, mun. Novo Progresso, affl. rio Jamanxim, bridge on hwy. BR-163, affl. rio Jamanxim, affl. rio Tapajós, $07^{\circ} 21^{\prime} 08^{\prime \prime} \mathrm{S}, 055^{\circ} 17^{\prime} 45^{\prime \prime} \mathrm{W}$. USNM 330451, 1 , $90 \mathrm{~mm}$, Belém, Baía de Marajó, ca. $01^{\circ} 27^{\prime} \mathrm{S}, 048^{\circ} 28^{\prime} \mathrm{W}$. Tocantins (localities from rio Tocantins dr., Amazonas dr.). MZUSP 51366, 2, 75-76 mm, mun. Araguaçu, Fazenda Praia Alta $2.27 \mathrm{~km} \mathrm{~N}$ Araguaçu, on Araguacu-Barreira do Piqui rd., rio Agua Fria, affl. rio Javaés, affl. rio Araguaia, affl. rio Tocantins, $c a .12^{\circ} 45^{\prime} \mathrm{S}^{\circ}, 049^{\circ} 55^{\prime} \mathrm{W}$. MZUSP 52166, 3, 78-90 mm, mun. Araguaçu, Fazenda Praia Alta 2, on Araguaçu-Barreira do Piqui rd., rio Agua Fria, affl. rio Javaés, affl. rio Araguaia, affl. rio Tocantins, $c a .12^{\circ} 43^{\prime} \mathrm{S}$, 049 55'W. MZUSP 52214, 1, 79 mm, mun. Sandolândia, stream on Sandolândia-Barra do rio Verde rd., ca. $5 \mathrm{~km}$ Sandolândia, rio Água Fria, affl. rio Javaés, affl. rio Araguaia, affl. rio Tocantins, ca. $12^{\circ} 32^{\prime} \mathrm{S}, 049^{\circ} 55^{\prime} \mathrm{W}$. Ecuador (localities from río Napo dr., Amazonas dr.). Francisco de Orellana. FMNH 102289, 4, 27-91 mm, affl. río Payamino, downstream from San José de Payamino, N bank, affl. río Payamino, affl. río Napo, $00^{\circ} 30^{\prime} \mathrm{S}, 077^{\circ} 15^{\prime} \mathrm{W}$.
QCAZ-I 1828, 1, $134 \mathrm{~mm}$, QCAZ-I 1829, 1, $132 \mathrm{~mm}$, QCAZ-I 1830, 1, $132 \mathrm{~mm}$, QCAZ-I 1831, 1, $148 \mathrm{~mm}$, QCAZ-I 1832, 1, 136 mm, Parque Nacional Yasuní, km 39 on Pompeya-NPF (oil field) rd., río Tiputini, affl. río Napo, ca. $00^{\circ} 36^{\prime} \mathrm{S}, 076^{\circ} 28^{\prime} \mathrm{W}$. Sucumbios. FMNH 102273, 1, 53 $\mathrm{mm}$, affl. río Aguarico, $\mathrm{N}$ bank, 1-2 km upstream from mouth of río Pushino, affl. río Napo, $00^{\circ} 03^{\prime} 30^{\prime \prime} \mathrm{N}$, $076^{\circ} 56^{\prime} 00^{\prime \prime} \mathrm{W}$. FMNH 120675, 1, $43 \mathrm{~mm}$, small stream between Laguna Zancudo and Laguna Zancudococha, affl. quebrada Zancudococha, affl. río Aguarico, affl. río Napo, ca. $00^{\circ} 26^{\prime} \mathrm{S}, 076^{\circ} 36^{\prime} \mathrm{W}$. KU 13802, 1, $127 \mathrm{~mm}$, río Conejo at Santa Cecília, affl. río Aguarico, affl. río Napo, ca. $00^{\circ} 06^{\prime} \mathrm{N}, 076^{\circ} 51^{\prime} \mathrm{W}$. Guyana. Upper Takutu-Upper Essequibo (localities from Takutu River dr., affl. rio Branco, affl. rio Negro, Amazonas dr.). ANSP 179507, 1, 81 $\mathrm{mm}, 31.2 \mathrm{~km}$ NW Sand Creek village, Sauriwau River, $03^{\circ} 06^{\prime} 52^{\prime \prime} \mathrm{N}, 059^{\circ} 46^{\prime} 32^{\prime \prime} \mathrm{W}$. AUM 48079, 1, $82 \mathrm{~mm}$, creek, 2nd bridge from Moco-Moco power station to Lethem, $03^{\circ} 18^{\prime} 10^{\prime \prime} \mathrm{N}, 059^{\circ} 39^{\prime} 01^{\prime \prime} \mathrm{W}$. CU 71937, 11, Moco-Moco Creek, $15 \mathrm{~km} \mathrm{~W}$ Lethem. ca. $03^{\circ} 15^{\prime} \mathrm{N}, 059^{\circ} 39^{\prime} \mathrm{W}$ (listed by Sullivan, 1997). CU 71939, 3, CU 71940, 1, CU 71941, 2, USNM 209197, 1, 89 mm LEA, overflow of Moco-Moco Creek nr. Moco-Moco village, Kumaka Swamp, $c a .15 \mathrm{~km}$ E Lethem, $c a .03^{\circ} 19^{\prime} \mathrm{N}, 059^{\circ} 40^{\prime} \mathrm{W}$ (listed by Sullivan, 1997). USNM 209196, 1, 135 mm, USNM 209198, 1, 105 mm, Rupununi, Moco-Moco Creek, ca. $03^{\circ} 15^{\prime} \mathrm{N}, 059^{\circ} 39^{\prime} \mathrm{W}$. USNM 209200, 1, $110 \mathrm{~mm}$, Rupununi, creek in swampy area, nr. Moco-Moco Creek, $c a .03^{\circ} 15^{\prime} \mathrm{N}, 059^{\circ} 39^{\prime} \mathrm{W}$. Peru. Amazonas. LACM 41930-5, 1, 129 mm, LACM 41934-4, 2, 116-146 mm, LACM 41935-2, 1, $127 \mathrm{~mm}$, LACM 41936-5, 1, $138 \mathrm{~mm}, 500 \mathrm{~m}$ upstream from Caterpiza, río Santiago, affl. río Marañón, affl. rio Amazonas, Amazonas dr., ca. $03^{\circ} 55^{\prime} \mathrm{S}, 077^{\circ} 44^{\prime} \mathrm{W}$. Loreto (localities from Amazonas dr.). AMNH 78112, 1, $77 \mathrm{~mm}$, río Tahuayo, $c a$. $04^{\circ} 03^{\prime} \mathrm{S}, 073^{\circ} 08^{\prime \prime} \mathrm{W}$. AMNH $78114,1,140 \mathrm{~mm}$, río Yarapá, affl. río Ucayali, $c a .04^{\circ} 31^{\prime} \mathrm{S}, 073^{\circ} 22^{\prime} \mathrm{W}$. INHS 43308 , 2 , 84-87 mm, Maynas, $11 \mathrm{~km} \mathrm{SSW}$ Iquitos, río Itaya, $03^{\circ} 49^{\prime} 48^{\prime \prime} \mathrm{S}, 073^{\circ} 18^{\prime} 03^{\prime \prime} \mathrm{W}$. INHS 52032, 1, $125 \mathrm{~mm}$, Ullpa, caño Moena, nr. confl. with caño Ullpa, SE Iquitos, affl. río Itaya, $03^{\circ} 46^{\prime} 20^{\prime \prime} \mathrm{S}, 073^{\circ} 14^{\prime} 17^{\prime \prime} \mathrm{W}$. INHS 54796, 1, $70 \mathrm{~mm}$, floodplain of río Marañón, S Nauta, 04³0'36"S, $073^{\circ} 34^{\prime} 06^{\prime \prime} \mathrm{W}$. MUSM 4341, 1, $118 \mathrm{~mm}$, MUSM 17308, 2, 91-93 mm, Yurimáguas, San Ramón, quebrada Simay, affl. río Huallaga, affl. río Marañón, ca. $05^{\circ} 56^{\prime} \mathrm{S}, 076^{\circ} 06^{\prime} \mathrm{W}$. MUSM 14494 (part), 2 (1 immature, 104, 1 male, 156 mm), Maynas, Lagartococha, Aguas Negras, río Aguarico, affl. río Napo, 00³1'38"S, 075²15'41"W. MUSM 14497 (part), 6, 91-138 mm, Puesto de Vigiliancia area MH93LO, río Napo, $00^{\circ} 58^{\prime} \mathrm{S}, 075^{\circ} 11^{\prime} \mathrm{W}$. MUSM 14498 (part), 1, 124 $\mathrm{mm}$, mun. Maynas, Puesto de Vigilancia Arcadia, río Napo, $00^{\circ} 59^{\prime} 37^{\prime \prime} \mathrm{S}, 075^{\circ} 18^{\prime} 30^{\prime \prime} \mathrm{W}$. MUSM 18538, 1, $71 \mathrm{~mm}$, nr. Musha Carusha, río Pastaza, affl. río Marañón, 04²6'54"S, $076^{\circ} 39^{\prime} 28^{\prime \prime} \mathrm{W}$. MUSM 21896, 1 (not measured), MUSM 21983, 2, mun. Nauta, Reserva Nacional (RN) Pacaya Samiria, quebrada Llanchama, affl. río Yanayacu-Pucate, río Marañón, ca. $04^{\circ} 39^{\prime} \mathrm{S}, 073^{\circ} 50^{\prime} \mathrm{W}$. MUSM 23141, 1, 
Alto Amazonas, Abanico del Pastaza, stream affl. río Platanoyacu, río Corrientes, río Tigre, río Marañón, $c a$. $03^{\circ} 07^{\prime} \mathrm{S}, 075^{\circ} 40^{\prime} \mathrm{W}$. UF $126192,1,125 \mathrm{~mm}$, RN Pacaya Samiria, río Pacaya, affl. río Ucayali, $c a .05^{\circ} 16^{\prime} \mathrm{S}, 074^{\circ} 27^{\prime} \mathrm{W}$. UF 129816, 1, $62 \mathrm{~mm}$, UF 131404, 1, immature, $100 \mathrm{~mm}$, RN Pacaya Samiria, caño Yarina, río Pacaya, affl. río Ucayali, $05^{\circ} 25^{\prime} 56^{\prime \prime} \mathrm{S}, 074^{\circ} 30^{\prime} 01^{\prime \prime} \mathrm{W}$. UF 131405,1 , immature, $94 \mathrm{~mm}$, RN Pacaya Samiria, cocha Yarina, río Pacaya, affl. río Ucayali, $05^{\circ} 23^{\prime} 58^{\prime \prime} \mathrm{S}, 074^{\circ} 32^{\prime} 05^{\prime \prime} \mathrm{W}$. Suriname. Brokopondo. UF 177362, 7 (5 female, 119-132 mm, 2 male, 133-141 mm), Affobaka rd. from Paranam bauxite refinery to Brokopondo Reservoir, bridge over Marshall kreek, Suriname River dr., $05^{\circ} 14^{\prime} 40^{\prime \prime} \mathrm{N}, 055^{\circ}$ $06^{\prime} 05^{\prime \prime} \mathrm{W}$. Marowijne. UF 177360, 12 (7 female, 105-114 $\mathrm{mm}, 5$ male, $98-129 \mathrm{~mm}$ ), small stream on $\mathrm{rd}$. from Paramaribo to French Guiana, affl. Cottica River, Commewijne River dr., $05^{\circ} 34^{\prime} 51^{\prime \prime} \mathrm{N}, 054^{\circ} 15^{\prime} 31^{\prime \prime} \mathrm{W}$. UF 177363, 2, female, 105-109 mm, small stream on rd. from Paramaribo to French Guiana, affl. Cottica River, Commewijne River dr., $05^{\circ} 35^{\prime} 11^{\prime \prime} \mathrm{N}, 054^{\circ} 17^{\prime} 05^{\prime \prime} \mathrm{W}$. Venezuela (localities from Orinoco dr.). Anzoátegui. MHNLS 9360, 1, $83 \mathrm{~mm}$ LEA, río Morichal Largo, río Orinoco delta, $c a .09^{\circ} 26^{\prime} \mathrm{N}, 062^{\circ} 24^{\prime} \mathrm{W}$ (listed by Sullivan, 1997). Apure. INHS 28179, 4, 90-102 mm, 58 km SSW Bruzual, caño Guaritico, affl. río Apure, $07^{\circ} 33^{\prime} 09^{\prime \prime} \mathrm{N}$, $069^{\circ} 38^{\prime} 40^{\prime \prime} \mathrm{W}$. INHS 28360, 7, 72-91 mm, $62 \mathrm{~km}$ E Elorza on hwy. to La Victoria at Charal, río Orichuma, affl. río Apure, $c a .07^{\circ} 04^{\prime} \mathrm{N}, 070^{\circ} 01^{\prime} \mathrm{W}$. INHS $30112,1,84 \mathrm{~mm}, 58$ $\mathrm{km}$ SSW Bruzual, caño Guaritico, affl. río Apure, $07^{\circ} 33.24^{\prime} \mathrm{N}, 069^{\circ} 38.68^{\prime} \mathrm{W}$. MCNG 11032 (part), 5, 79-98 mm LEA, caño Maporal, río Caicara, affl. río Apure, $c a$. $07^{\circ} 24^{\prime} \mathrm{N}, 069^{\circ} 32^{\prime} \mathrm{W}$ (listed by Sullivan, 1997). Barinas. MCNG 5611, 1, Reserva de Ticoporo, affl. río Suripa, affl. río Apure, ca. $08^{\circ} 09^{\prime} \mathrm{N}, 070^{\circ} 50^{\prime} \mathrm{W}$ (listed by Sullivan, 1997). Bolivar. AUM 22303, 1, $106 \mathrm{~mm}$, río Chaviripa, on Caicara-Puerto Ayacucho rd., $07^{\circ} 07^{\prime} 57^{\prime \prime N}, 066^{\circ} 29^{\prime} 56^{\prime \prime} \mathrm{W}$. FMNH 109967, 1, 38 mm, caño Wani, affl. río Caura, $06^{\circ} 04^{\prime} 47^{\prime \prime} \mathrm{N}, 064^{\circ} 55^{\prime} 20^{\prime \prime} \mathrm{W}$. MCNG 19941, 1, MCNG 19999, 1, stream affl., río Caura, no coordinates. MHNLS 8783, 1, 83 mm LEA, Raudales del Cambur [El Raudal, caño Cambur], $20 \mathrm{~km}$ [SE] bridge at Maripá, ca. $07^{\circ} 22^{\prime} \mathrm{N}$, $065^{\circ} 18^{\prime} \mathrm{W}$ (listed by Sullivan, 1997). Monagas. MBUCV 14122, 1, $97 \mathrm{~mm}$ LEA, bridge over caño Piedritas on Temblador-Barrancas hwy., affl. río Urucoa, río Orinoco delta, $c a .08^{\circ} 49^{\prime} \mathrm{N}, 062^{\circ} 29^{\prime} \mathrm{W}$ (listed by Sullivan, 1997). MHNLS 6512, 1, $76 \mathrm{~mm}$ LEA, inundated area, Chaguaramas, río Urucoa, $c a .08^{\circ} 40^{\prime} \mathrm{N}, 062^{\circ} 45^{\prime} \mathrm{W}$ (listed by Sullivan, 1997). Portuguesa. TNHC 12703, 2, 64-83 $\mathrm{mm}, 35 \mathrm{~km}$ SE Guanare, caño (río) Maraca at Urriola Ranch, affl. río Portuguesa, affl. río Apure, $08^{\circ} 52^{\prime} 30^{\prime \prime} \mathrm{N}$, $069^{\circ} 27^{\prime} 40^{\prime \prime} \mathrm{W}$. Tachira. INHS 35527, 1, $92 \mathrm{~mm}$, [stream] just $\mathrm{W}$ junction between hwy. 5 and hwy. 19, río Caparo,

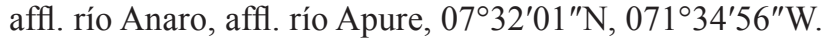

Diagnosis. Brachyhypopomus regani is diagnosed from congeners by the following combination of characters: accessory electric organ over opercular region present, $v s$. absent in all congeners except B. bombilla, and B. menezesi; dorsal surface with large dark blotches against a lighter background, vs. dorsal surface speckled with small brown chromatophores on a pale background in B. bombilla; caudal filament length $11.1-17.9 \%$, vs. $24 .-33.3 \%$ LEA in B. menezesi.

Description. Head and body shape, and pigmentation illustrated in Figs. $1 \mathrm{j}$ and 41. Meristic and morphometric data for examined specimens presented in Tables 2-5 and 17. Body moderate to broad in depth. Head moderate to long in length and shallow to moderate in depth. Dorsal profile of head approximately straight to slightly concave from occiput to snout, ventral profile of head approximately straight between operculum and snout, snout rounded. Eye moderate in size. Upper jaw with moderate sigmoidal angle between premaxillary and maxillary portions in lateral view. Accessory electric organ (AEO) over operculum present and very prominent; skin over AEO completely depigmented; border rounded and very conspicuous. Gill filaments on first gill arch 35-45 (median 40, $\mathrm{n}=9$ ). Pectoral fin narrow to moderate, pectoral-fin rays 14-16 (no mode, median 15). Precaudal vertebrae 16-18 (mode 17), with 1-2 (mode 2) transitional vertebrae. Anal-fin origin slightly $(<0.25$ HL distance) anterior to tip of pectoral fin. Anal-fin rays 165-205 (median 184). Dorsal rami of recurrent branch of anterior lateral line nerve not visible. Middorsal region of body scaled. Rows of scales above lateral line 5-7 (mode 6). Lateral line continuous. Multiple groove-like depigmented epidermal canals, some with dark borders, form parallel striations mostly dorsal to lateral line in posterior portion of body, beginning $c a$. two thirds of LEA; striations reaching 1-4 scales (including lateral line series) dorsally, and occasionally (with much lower density) 1-2 scales ventrally. Epidermal canals absent in anterior portion of body; never form a pair of long irregular lines either side of dorsal surface in dorsal portion of flank; do not form isolated horizontal wavy line midway from lateral line to dorsal midline in midbody or anterior portion of body. Three bilateral horizontal columns of electrocytes at anal-fin terminus and at mid-point between anal-fin terminus and tip of caudal filament in immature, mature female, and mature male specimens. Electrocytes of hypaxial electric organ extend anterior to anal and urogenital pores and appear to extend into opercular area to form paired accessory electric organ. Caudal filament short.

Coloration. (Figs. 1j, 41). Background light tan to brown. Dorsal region without prominent depigmented pale stripe extending along midline from occipital region to base of caudal filament. Dorsal region mottled with irregular, indistinct dark blotches against a homogenous lighter background (contrasting to a speckling with small brown chromatophores in B. bombilla and to much more distinct dark blotches in $B$. menezesi). Dark mottling restricted to dorsal region, extending to around mid-distance from 
dorsal midline to lateral line. No vertical stripes or lines on flank, some vague irregular dark markings over anal-fin pterygiophores, flank otherwise relatively free of markings. Caudal filament darker than body, with some irregular dark markings. Head with evenly scattered dark chromatophores, darker dorsally. Eye with indistinct to very distinct suborbital patch or stripe of chromatophores and subcutaneous pigmentation. Region over AEO completely depigmented. Border of AEO often with very dark chromatophores. Pectoral and anal-fin membranes hyaline. Pectoral and analfin rays hyaline in distal third to half, with scattered or fused light brown chromatophores on proximal half. Color in live individuals similar to preserved specimens, with opercular region usually rosy due to underlying gills.

Size. Small adult size, largest specimen examined $156 \mathrm{~mm}$ TL, $130 \mathrm{~mm}$ LEA $(\mathrm{n}=230)$. Largest male specimen examined $156 \mathrm{~mm}$ TL, $130 \mathrm{~mm}$ LEA $(\mathrm{n}=17)$. Largest female specimen examined $141 \mathrm{~mm}$ TL, $125 \mathrm{~mm}$ LEA $(\mathrm{n}=28)$.

Sexual dimorphism. No known secondary sexual dimorphism.

Geographic distribution. Brazil, Ecuador, Guyana, Peru, Suriname, and Venezuela (Fig. 42). Widely distributed through the Amazon basin, including multiple sites in the upper Amazon, the Negro, Purus, Tapajós, and Araguaia drainages. Absent from the upper Madeira, above its major falls beginning at Porto Velho, where the closely related species B. bombilla occurs. Known also from northern portions of the Orinoco drainage, the Essequibo River, and some coastal drainages of Guyana.

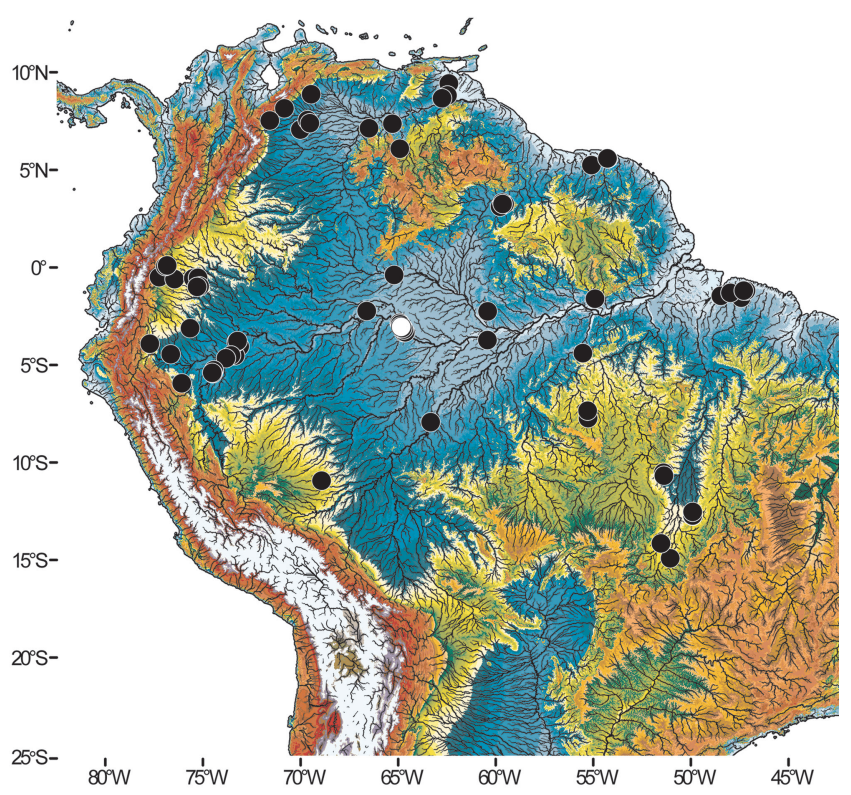

Fig. 42. Collection records for Brachyhypopomus regani (circles). Holotype location is marked with an open symbol. Elevation data refers to altitude above mean sea level (see Fig. 2 for legend).
Ecological notes. In the central and upper Amazon B. regani is restricted to high-conductivity whitewater floodplains occurring in floating rafts of macrophytes, and the leaf litter of newly-inundated forest (Crampton, 1996a; 1998a). Breeding occurs in floating macrophytes during the rising and high water period (Crampton, 1996a, 165). Like other congeners in this habitat, $B$. regani is able tolerate protracted seasonal hypoxia. However, in experimental conditions it is less tolerant of anoxia than syntopic congeners (Crampton, 1998b). Unlike most congeners B. regani does not undertake aerial gill respiration during experimentally-induced hypoxia. Instead it wedges itself into roots near the water surface and exhibits a variant of aquatic surface respiration in which water at the surface meniscus is passed across its gills by buccal pumping (Crampton, 1998b). Populations assigned here to $B$. regani from the lower Amazon in the vicinity of Belém, coastal Suriname, and in the rio Negro (and possibly also the rio Tapajós and rio Araguaia) have a noticeably different ecology, occurring in constantly normoxic rainforest streams and small rivers with relatively low conductivity (10-30 $\left.\mu \mathrm{Scm}^{-1}\right)$ (WGRC unpublished data). We suspect that future studies of ecological, genetic, and EOD variation in this widely distributed species may reveal additional cryptic species. Specimens from whitewater floodplain systems of the central Amazon (Crampton, 1996a: 136), and also terra firme stream from coastal Suriname (WGRC unpublished data), exhibit similar stomach contents, comprising aquatic insect larvae, microcrustacea, and other small aquatic invertebrates with a predominance of Chironomidae larvae.

Co-occurring congeners: In the upper, central, and lower Amazon (excluding the upper Madeira), B. regani occurs in sympatry and ecological syntopy with $B$. arrayae (in lower Madeira), B. beebei, B. belindae, B. bennetti, B. brevirostris, B. flavipomus, B. hamiltoni, B. pinnicaudatus, and $B$. walteri, and exhibits an allotopic distribution with $B$. alberti (in lower Madeira), B. batesi, B. benjamini, B. cunia (in lower Madeira), $B$. hendersoni, $B$. sullivani, and $B$. verdii. In the rio Negro $B$. regani occurs in sympatry (and probably in syntopy) with $B$. batesi, B. beebei, B. brevirostris, B. bullocki, $B$. hamiltoni, $B$. hendersoni, $B$. sullivani, and $B$. walteri. In the río Orinoco basin $B$. regani occurs in sympatry with $B$. beebei, B. brevirostris, B. bullocki, B. diazi, and B. sullivani. In the Essequibo River $B$. regani occurs in sympatry with $B$. beebei, B. brevirostris, B. bullocki, B. hendersoni, $B$. sullivani, and $B$. walteri. In Coastal Suriname and French Guiana $B$. regani occurs in sympatry with $B$. beebei, $B$. brevirostris, and B. pinnicaudatus.

Etymology. The specific name is a patronym (noun in the genitive case) in honor of Charles Tate Regan (1878-1943), British ichthyologist, for his contributions to Neotropical ichthyology.

Local names. Brazil: sarapó; Ecuador: cuchillo, yayo; French Guiana: poisson couteau (French) (and multiple 
creole/Amerindian names), Guyana: knife fish; Peru: macana; Suriname: saprapi; Venezuela: cuchillo.

\section{Brachyhypopomus sullivani, new species}

urn:1sid:zoobank.org:act:A5E0AE79-5691-4FEE-AA8BF9BD8AE86D1C

(Figs. 1k, 43; Tables 2-5, 19)

Hypopomidae sp. 11. -Crampton, 1996a: 77, table 6.1.a, species list; 79, fig. 6.1 inset 36-37, color photographs; 85, fig. 6.2; 88, fig. 6.3 ; 92-94, figs. $6.4 \mathrm{a}$, EOD data; 110 , table 7.5, 113, table 7.7, 115, fig. 7.1, habitats (Brazil, central Amazon, ecology and signal diversity).

Brachyhypopomus royeroi. -Sullivan, 1997: 126 (description in unpublished thesis with disclaimer stating that nomenclatural acts not available, a nomen nudum).

Brachyhypopomus sp. 6. -Crampton, 1998a: 821, table 4 (Brazil, Amazonas, species and EOD diversity). -Crampton, 1998b:
314, table 2, list of Brachyhypopomus (Brazil, Amazonas, hypoxia tolerance).

Brachyhypopomus sp. F. -Crampton, 1999: 17 (Brazil, Amazonas, Mamirauá Reserve, listing of species). -Crampton \& Albert, 2006: 672, fig. 23.8, position in phylogenetic tree; 681 , notes on EODs (gymnotiform species and EOD diversity). -Crampton et al., 2008: 231, fig. 6, black and white photograph of head of live individual (Brazil, Amazonas, adaptations to hypoxia).

Brachyhypopomus beebei. -Galvis, 2006: 528, pl. 104c, color photograph of live individual (Colombia, Amazonas, listing of Brachyhypopomus).

Brachyhypopomus sp. "roy". -Crampton, 2011: 176, table 10.2, species list; 179, figs. 10.2-10.3, phylogeny, geographical and ecological distributions (gymnotiform biology).-Tagliacollo et al., 2016: 28, fig. 5 (phylogeny of Gymnotiformes).

Brachyhypopomus sp. 2. -Crampton \& Ribeiro, 2013: 240, color photograph (Brazil, rio Madeira, listing of hypopomids).

Brachyhypopomus sp. "sullivani". -Crampton et al., 2016: 1-66, table 1, 3-4, figs. 1-7, 14-15, 18-20 (phylogeny, biogeography and ecology of Brachyhypopomus).

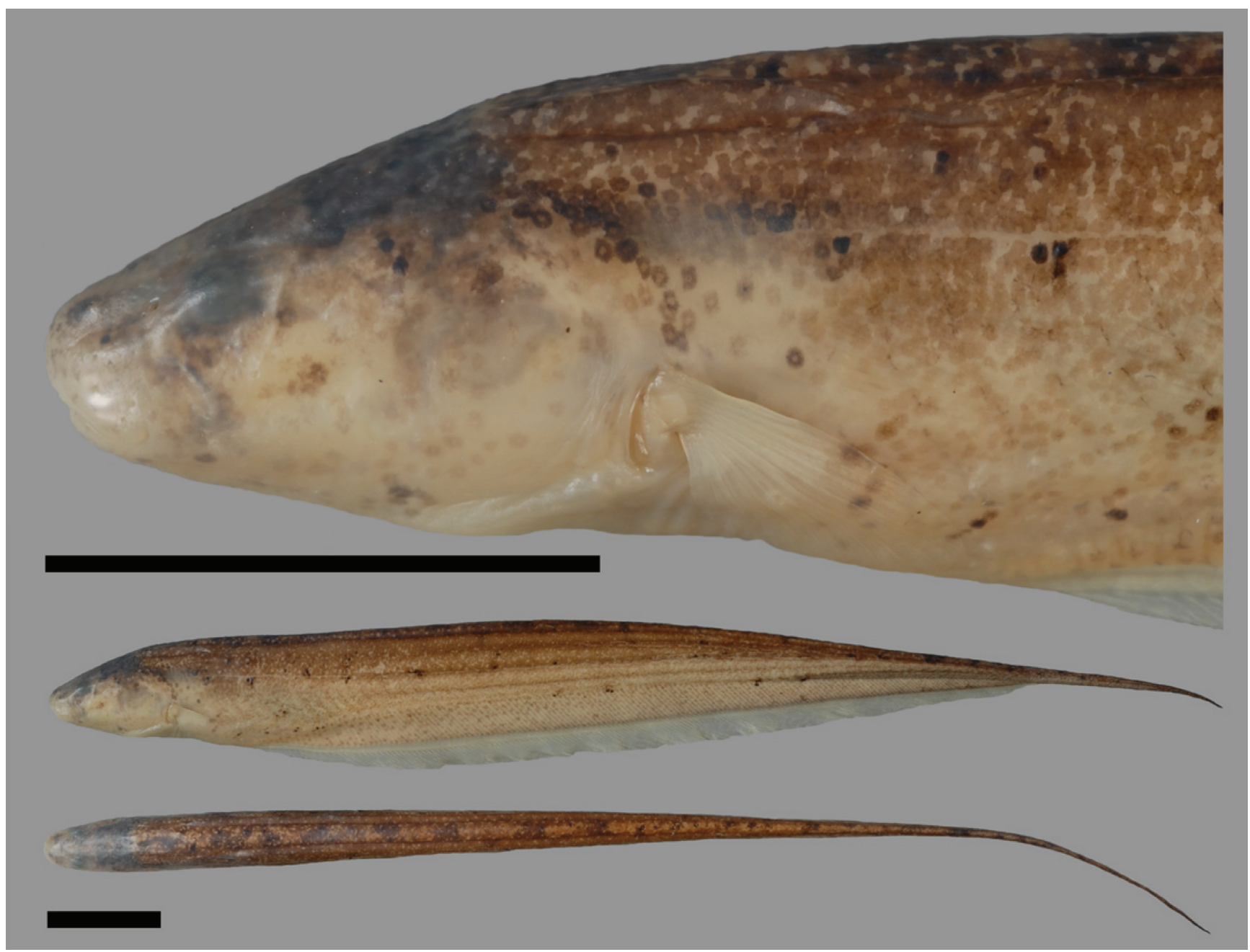

Fig. 43. Brachyhypopomus sullivani. MUSM 39624 (WC48.210709), holotype, female, 108 mm TL (head in lateral view, and body in lateral and dorsal view, specimen fixed in formalin and preserved in EtOH); Peru, río Ucayali, Amazonas dr. Scale bars $=5 \mathrm{~mm}$. 
Table 19. Morphometrics for Brachyhypopomus sullivani. HT, holotype; SD, standard deviation; dr., drainage. Ranges for the type region refer to the holotype and to paratype specimens (and also non-types for caudal filament length as \% of TL). Ranges for the other populations refer to non-type specimens from the lower río Beni of Bolivia, and the upper río Orinoco of Venezuela, respectively.

\begin{tabular}{|c|c|c|c|c|c|c|c|c|c|c|c|c|c|}
\hline & \multicolumn{5}{|c|}{ Upper Amazonas dr. (type region) } & \multicolumn{4}{|c|}{ Upper Madeira drainage } & \multicolumn{4}{|c|}{ Upper Orinoco drainage } \\
\hline & HT & Range & Mean & SD & $\mathrm{n}$ & Range & Mean & $\mathrm{SD}$ & $\mathrm{n}$ & Range & Mean & SD & $\mathrm{n}$ \\
\hline Total length (TL) (mm) & 108 & $62-123$ & 92 & 16.4 & 111 & $65-127$ & 105 & 27.9 & 6 & $75-126$ & 94 & 17.1 & 9 \\
\hline Length to end of anal fin (LEA) (mm) & 91 & $51-106$ & 77 & 13.7 & 111 & $56-98$ & 80 & 18.4 & 7 & $60-123$ & 84 & 19.3 & 14 \\
\hline \multicolumn{14}{|c|}{ Proportion of TL (\%) } \\
\hline Caudal filament length (CF) & 15.7 & $12.7-23.9$ & 16.5 & 1.8 & 111 & $15.0-20.7$ & 17.3 & 2.7 & 6 & $14.6-22.8$ & 18.8 & 2.6 & 9 \\
\hline Snout to occiput (SO) & 9.2 & $8.5-10.2$ & 9.2 & 0.4 & 16 & $8.9-10.0$ & 9.5 & 0.4 & 7 & $6.7-10.7$ & 9.7 & 1.2 & 14 \\
\hline Snout to anal-fin origin (SA) & 20.9 & $19.1-22.2$ & 21.0 & 0.9 & 16 & $20.0-23.3$ & 21.3 & 1.3 & 7 & $19.2-22.7$ & 20.7 & 1.1 & 14 \\
\hline Snout to pectoral-fin base (PP) & 12.6 & $11.9-13.2$ & 12.6 & 0.4 & 16 & $12.2-13.9$ & 13.0 & 0.7 & 7 & $11.4-15.0$ & 13.2 & 0.8 & 14 \\
\hline Longest pectoral-fin ray (PRL) & 6.4 & $5.8-7.0$ & 6.4 & 0.3 & 16 & $5.1-6.1$ & 5.6 & 0.5 & 7 & $5.1-6.5$ & 5.8 & 0.3 & 13 \\
\hline Anal fin length (AF) & 79.1 & $78.6-81.9$ & 80.1 & 1.0 & 16 & $75.0-80.6$ & 78.6 & 2.1 & 7 & $76.3-81.3$ & 79.2 & 1.5 & 13 \\
\hline Body width at anal-fin middle (BW2) & 2.9 & 2.6-3.9 & 3.0 & 0.4 & 16 & $2.9-3.8$ & 3.5 & 0.4 & 7 & $2.6-4.0$ & 3.3 & 0.5 & 13 \\
\hline Caudal filament length $(\mathrm{CF})$ & 18.7 & $16.2-24.2$ & 19.6 & 2.3 & 16 & $17.9-26.0$ & 21.4 & 3.6 & 6 & $17.1-30.7$ & 23.9 & 4.0 & 13 \\
\hline \multicolumn{14}{|c|}{ Proportion of HL (\%) } \\
\hline Preorbital distance (PR) & 28.5 & $20.5-28.5$ & 25.7 & 2.1 & 16 & $24.4-27.9$ & 25.8 & 1.2 & 8 & $23.6-30.6$ & 27.4 & 1.3 & 15 \\
\hline Mouth width (MW) & 29.1 & $22.0-30.7$ & 27.1 & 2.4 & 16 & $27.0-33.3$ & 29.7 & 2.5 & 8 & $20.3-30.4$ & 25.6 & 2.9 & 15 \\
\hline Internarial distance (AE) & 20.8 & $18.5-22.9$ & 20.6 & 1.2 & 16 & $19.8-21.7$ & 20.9 & 0.8 & 8 & $19.1-24.5$ & 22.2 & 1.8 & 15 \\
\hline Posterior naris to eye (PE) & 5.5 & 4.3-6.6 & 5.6 & 0.5 & 16 & $3.5-6.0$ & 4.7 & 0.9 & 8 & $3.1-7.3$ & 5.2 & 0.8 & 15 \\
\hline Orbital diameter (OD) & 10.3 & $6.6-12.6$ & 10.3 & 1.5 & 16 & $9.1-11.4$ & 10.2 & 1.0 & 8 & $9.5-11.5$ & 10.4 & 0.7 & 15 \\
\hline Interorbital distance (IO) & 20.3 & $15.6-22.9$ & 19.2 & 1.8 & 16 & $20.5-27.1$ & 24.1 & 2.7 & 8 & $17.4-23.5$ & 21.0 & 1.7 & 15 \\
\hline Head depth at eye (HD1) & 45.5 & $39.8-47.6$ & 43.9 & 2.4 & 16 & $43.0-47.0$ & 45.2 & 1.5 & 8 & $38.5-43.9$ & 41.1 & 1.7 & 15 \\
\hline Caudal filament width (CW) & 4.3 & $3.6-7.5$ & 5.2 & 1.1 & 16 & $5.9-7.2$ & 6.6 & 0.5 & 7 & 4.4-6.9 & 5.6 & 0.6 & 12 \\
\hline \multicolumn{14}{|c|}{ Other proportions } \\
\hline BW1 (\% of BD1) & 47.3 & $41.8-51.1$ & 46.5 & 2.2 & 16 & $51.5-57.6$ & 54.8 & 2.1 & 7 & $51.7-86.4$ & 59.0 & 10.5 & 15 \\
\hline $\mathrm{BW} 2(\%$ of BD2) & 29.3 & $26.7-37.0$ & 31.9 & 3.0 & 16 & $30.8-34.5$ & 32.6 & 1.6 & 7 & $27.4-40.7$ & 34.5 & 3.4 & 15 \\
\hline
\end{tabular}

Holotype. MUSM 39624, 108 mm TL, 91 mm LEA, Peru, Loreto. mun. Requena, stream nr. Jenaro Herrera, affl. río

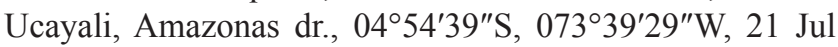
2009, W. Crampton, E Correa Roldán, M. Aldea Guevara \& J. Waddell.

Paratypes. 59 specimens. Peru. Loreto (localities are from Amazonas dr.; localities listed from nr. Jenaro Herrera are from the río Ucayali dr., mun. Requena; collected by W. Crampton, E. Correa Roldán, M. Aldea Guevara \& J. Waddell unless stated otherwise). MUSM 44682, 9 (6 immature, $72-94 \mathrm{~mm}, 2$, female, 96-113 mm, 1 male, 107 $\mathrm{mm})$, stream nr. Jenaro Herrera, $04^{\circ} 53^{\prime} 35^{\prime \prime} \mathrm{S}, 073^{\circ} 39^{\prime} 01^{\prime \prime} \mathrm{W}$,
11-20 Jul 2009. MUSM 44731, 1, immature, $110 \mathrm{~mm}$, stream nr. Jenaro Herrera, $04^{\circ} 54^{\prime} 49^{\prime \prime} \mathrm{S}, 073^{\circ} 39^{\prime} 43^{\prime \prime} \mathrm{W}, 4$ Aug 2009. MZUSP 91427, 2, 45-87 mm, affl. quebrada Chica, nr. Jenaro Herrera, $04^{\circ} 55^{\prime}$ S, $073^{\circ} 40^{\prime} \mathrm{W}$, Jan 2004 , W. Crampton, R. Reis, F. Lima, H. Ortega \& B. Crampton. UF 148513, 1, female, $112 \mathrm{~mm}$, quebrada Chupiai nr. Jenaro Herrera, ca. $04^{\circ} 53^{\prime} \mathrm{S}, 073^{\circ} 39^{\prime} \mathrm{W}$, 9 Jan 2004 , W. Crampton, R. Reis, F. Lima, H. Ortega \& B. Crampton. UF 148515 , 2 (1 immature, $87 \mathrm{~mm}, 1$ male, $94 \mathrm{~mm}$ ), quebrada Salomé nr. Jenaro Herrera, 04 $54^{\prime} 32^{\prime \prime} \mathrm{S}, 073^{\circ} 35^{\prime} 37^{\prime \prime} \mathrm{W}, 13$ Jan 2004, W. Crampton, R. Reis, F. Lima, H. Ortega \& B. Crampton. UF 148516, 9, 98-120 mm, affl. quebrada Chica, nr. Jenaro Herrera, $c a .04^{\circ} 55^{\prime} \mathrm{S}, 073^{\circ} 40^{\prime} \mathrm{W}, 15$ Jan 2004 , W. Crampton, 
R. Reis, F. Lima, H. Ortega \& B. Crampton. UF 148517 , 5 (1 immature, $46 \mathrm{~mm}, 3$ female, $88-105 \mathrm{~mm}, 1$ male, 108 $\mathrm{mm})$, affl. quebrada Chica, nr. Jenaro Herrera, $04^{\circ} 55^{\prime} 22^{\prime \prime} \mathrm{S}$, $073^{\circ} 39^{\prime} 45^{\prime \prime} \mathrm{W}, 15$ Jan 2004, W. Crampton, R. Reis, F. Lima, H. Ortega \& B. Crampton. UF 148518, 5, 95-104 mm, quebrada Chica, nr. Jenaro Herrera, ca. $04^{\circ} 55^{\prime} \mathrm{S}, 073^{\circ} 40^{\prime} \mathrm{W}$, 15 Jan 2004, W. Crampton, R. Reis, F. Lima, H. Ortega \& B. Crampton. UF 148519, 3 (1 immature, $103 \mathrm{~mm}, 1$ female, $101 \mathrm{~mm}, 1$ male, $125 \mathrm{~mm}$ ), quebrada Fierro caño, affl. quebrada Sapuenillo, nr. Jenaro Herrera, 04 $51^{\prime} 39^{\prime \prime}$ S,

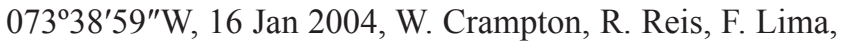
H. Ortega, B. Crampton. UF 184008, 6 (5 immature, 52$118 \mathrm{~mm}, 1$ female, $103 \mathrm{~mm}$ ), stream near Jenaro Herrera, $04^{\circ} 52^{\prime} 42^{\prime \prime} \mathrm{S}, \quad 073^{\circ} 38^{\prime} 51^{\prime \prime} \mathrm{W}, 12-16$ Jul 2009. USNM 405575, 5, immature, 58-70 mm, stream nr. Jenaro Herrera, $04^{\circ} 52^{\prime} 24^{\prime \prime} \mathrm{S}, 073^{\circ} 38^{\prime} 49^{\prime \prime} \mathrm{W}, 14 \mathrm{Jul} 2009$. USNM 405576, 3 (2 immature, 89-108 mm, 1 male, $101 \mathrm{~mm}$ ), stream nr. Jenaro Herrera, 04 54'40"S, 07339'29"W, 21 Jul 2009. USNM 405577, 8 (4 immature, 74-94 mm, 2 female, 101$104 \mathrm{~mm}, 2$ male, 114-123 mm), stream nr. Jenaro Herrera, $04^{\circ} 56^{\prime} 16^{\prime \prime} \mathrm{S}, 073^{\circ} 39^{\prime} 20^{\prime \prime} \mathrm{W}, 27 \mathrm{Jul} 2009$.

Non-types. 326 specimens. Bolivia. Beni (localities from rio Madeira dr., Amazonas dr.). AMNH 39824, 4, 60-71 mm LEA, $10 \mathrm{~km}$ SE Costa Marques (Brazil), río Iténez floodplain (río Guaporé), affl. rio Mamoré, $12^{\circ} 30^{\prime} 37^{\prime \prime} \mathrm{S}, 064^{\circ} 12^{\prime} 59^{\prime \prime} \mathrm{W}$. CBF 10253, 1, immature, $90 \mathrm{~mm}$, mun. Riberalta, stream nr. lago San José, affl. río Beni, $10^{\circ} 54^{\prime} 47^{\prime \prime} \mathrm{S}, 065^{\circ} 59^{\prime} 49^{\prime \prime} \mathrm{W}$. CBF 10254, 1, female, $127 \mathrm{~mm}$, CBF 102455, 1, female, $122 \mathrm{~mm}$, UMSS 7018, 1, immature, $66 \mathrm{~mm}$, mun. Riberalta, stream nr. San José, nr. Riberalta, affl. río Beni, $10^{\circ} 55^{\prime} 32^{\prime \prime} \mathrm{S}$, $066^{\circ} 00^{\prime} 36^{\prime \prime} \mathrm{W}$. UF 177341, 1, female, $105 \mathrm{~mm}$, UMSS 7019, 1, female, $136 \mathrm{~mm}$, mun. Riberalta, Riberalta-Guayaramerín rd., nr. km 43, affl. río Beni, $1^{\circ} 01^{\prime} 58^{\prime \prime} \mathrm{S}, 065^{\circ} 44^{\prime} 53^{\prime \prime} \mathrm{W}$. Brazil (localities from Amazonas dr.). Acre. MCP 35527, 1, $85 \mathrm{~mm}$, mun. rio Branco, igarapé Quinoa, affl. rio Acre, affl. rio Purus, $c a .10^{\circ} 03^{\prime} \mathrm{S}, 067^{\circ} 37^{\prime} \mathrm{W}$. Amazonas. INPA 6611, 1 , Santa Isabel do rio Negro, igarapé de Santo Antônio, nr. Nova Esperança, $c a .00^{\circ} 24^{\prime} \mathrm{S}, 065^{\circ} 01^{\prime} \mathrm{W}$ [coordinates for Santa Isabel do rio Negro]. BMNH 1998.3.12.193, 1, female, 84 mm, BMNH 1998.3.12.210, 1, immature, $46 \mathrm{~mm}$, INPA 9954, 2 (1 immature, $78 \mathrm{~mm}, 1$ female, $94 \mathrm{~mm}$ ), MCP 45288, 1, female, $92 \mathrm{~mm}$, mun. Tefé, igarapé Curupira, at bridge of Estrada Agrovila rd., affl. lago Tefé (rio Tefé), $03^{\circ} 25^{\prime} 48^{\prime \prime} \mathrm{S}$, $064^{\circ} 44^{\prime} 15^{\prime \prime} \mathrm{W}$. BMNH 1998.3.12.208-209, 1, female, $91 \mathrm{~mm}$, 1 male, $97 \mathrm{~mm}$, MCP 45287, 3 (2 immature, $60-84 \mathrm{~mm}, 1$ female, $100 \mathrm{~mm}$ ), MCP 45336, 16 (10 female, $81-100 \mathrm{~mm}, 6$ male, 98-113 mm), MCP 45464, 1, immature, $79 \mathrm{~mm}$, MCP 45486, 10 (2 immature, 84-85 mm, 3 female [1CS], 83-91 $\mathrm{mm}, 5$ male [2CS], 91-101 mm), MCP 45492, 2 (1 female, 85 mm, 1 male, $99 \mathrm{~mm}$ ), UF 177357, 2, 86-88 mm, mun. Tefé, igarapé Repartimento, $1.5 \mathrm{~km}$ downstream from Estrada Agrovila rd., affl. lago Tefé (rio Tefé), 03⒉ $24^{\prime} 25^{\prime \prime} \mathrm{S}$, $064^{\circ} 44^{\prime} 08^{\prime \prime} \mathrm{W}$. INPA 8886, 1 , rio Preto da Eva, downstream rio Preta da Eva, $c a .02^{\circ} 40^{\prime} \mathrm{S}, 059^{\circ} 40^{\prime} \mathrm{W}$. MCP 41032 (part), 1, mun. Humaitá, stream on BR-319, ca. 96 km S Humaitá, affl. rio Pixuna [Ipixuna], affl. rio Purus, $08^{\circ} 05^{\prime} 31^{\prime \prime} \mathrm{S}$, $063^{\circ} 35^{\prime} 47^{\prime \prime} \mathrm{W}$. MCP 41033 (part), 1, 56 mm, MCP 41036 (part), 6, 43-85 mm, mun. Canutama, igarapé São João on hwy. BR-319, ca. $60 \mathrm{~km} \mathrm{~S}$ Humaitá, affl. rio Ipixuna, affl. rio Purus, $07^{\circ} 56^{\prime} 07^{\prime \prime} \mathrm{S}, 063^{\circ} 20^{\prime} 03^{\prime \prime} \mathrm{W}$. MCP 41040, 1, $59 \mathrm{~mm}$, mun. Humaitá, stream ca. $43 \mathrm{~km} \mathrm{E}$ rio Madeira on Transamazon hwy., affl. rio Madeira, 0 0 $38^{\prime} 49^{\prime \prime} \mathrm{S}$, 062 40'57"W. MCP 41042 (part), 2, 59-69 mm, mun. Humaitá, stream ca. $68 \mathrm{~km}$ E rio Madeira on Transamazon hwy., affl. rio Madeira, $07^{\circ} 44^{\prime} 02^{\prime \prime} \mathrm{S}, 062^{\circ} 29^{\prime} 40^{\prime \prime} \mathrm{W}$. MCP 41045 (part), 1, 68 mm, mun. Humaitá, rio Maicimirim, ca. $45 \mathrm{~km}$ rio Madeira on Transamazon hwy., affl. rio Madeira, $07^{\circ} 38^{\prime} 04^{\prime \prime} \mathrm{S}, 062^{\circ} 39^{\prime} 44^{\prime \prime} \mathrm{W}$. MCP 45310, 5 (1 immature, 61 mm, 2 female, 65-67 mm, 2 male, 76-97 mm), mun. Maraã, lago Amanã, rio Baré, igarapé São Sebastião, rio Japurá, $02^{\circ} 17^{\prime} 05^{\prime \prime} \mathrm{S}, 064^{\circ} 41^{\prime} 25^{\prime \prime} \mathrm{W}$. MCP 45325, 1 , immature, $88 \mathrm{~mm}$, mun. Alvarães, igarapé Jarauá, Nogueira-Alvarães rd., affl. Lago Tefé (rio Tefé), $03^{\circ} 15^{\prime} 06^{\prime \prime} \mathrm{S}, 064^{\circ} 48^{\prime} 55^{\prime \prime} \mathrm{W}$. MCP 45434, 1, female, $76 \mathrm{~mm}$, mun. Tefé, igarapé Xidarinini, affl. lago Tefé (rio Tefé), 0322'46"S, 064²1'17"W. MCP 45449, 1, male, $109 \mathrm{~mm}$, mun. Tefé, swamp, margin of igarapé Repartimento, at Estrada Agrovila rd., affl. lago Tefé (rio Tefé), 03⒉ $4^{\prime} 25^{\prime \prime} \mathrm{S}, 064^{\circ} 44^{\prime} 08^{\prime \prime} \mathrm{W}$. MPEG 12450, 3, 79-85 $\mathrm{mm}$, mun. Coari, stream in Urucu oil field, affl. rio Urucu, affl. lago de Coarí, Amazonas dr., 04 ${ }^{\circ} 52^{\prime} 19^{\prime \prime} \mathrm{S}, 065^{\circ} 15^{\prime} 02^{\prime} \mathrm{W}$. MPEG 12458, 2, 73-82 mm, mun. Coari, igarapé Tartaruga, affl. rio Urucu, affl. lago de Coarí, affl. Amazonas dr., $04^{\circ} 53^{\prime} 55^{\prime \prime} \mathrm{S}, 065^{\circ} 19^{\prime} 18^{\prime} \mathrm{W}$. MPEG 12459, 2, 74-85 mm, MPEG 13110, 1, 62 mm, mun. Coari, igarapé Tamanduá, affl. rio Urucu, affl. lago de Coarí, 04 $51^{\prime} 43^{\prime \prime} \mathrm{S}, 065^{\circ} 15^{\prime} 54^{\prime} \mathrm{W}$. MPEG 12504, 1, 74 mm, MPEG 12523, 4, 76-122 mm, mun. Coari, igarapé Lontra, affl. rio Urucu, affl. lago de Coarí, $04^{\circ} 52^{\prime} 47^{\prime \prime} \mathrm{S}, 065^{\circ} 08^{\prime} 58^{\prime} \mathrm{W}$. MCP 13670, $1,96 \mathrm{~mm}$, mun. Juriti, nr. Juriti, igarapé da Ponte, $02^{\circ} 10^{\prime} 47^{\prime \prime} \mathrm{S}, 056^{\circ} 04^{\prime} 38^{\prime \prime} \mathrm{W}$. MZUSP 59227, 1, $41 \mathrm{~mm}$, pools in beach, Tapera, rio Negro, ca. $00^{\circ} 12^{\prime} \mathrm{S}, 064^{\circ} 04^{\prime} \mathrm{W}$. Mato Grosso. INPA 11585,2 , 103 119, INPA 11593, 2, 113-141 mm, INPA 11595, 5 (3CS), 85$121 \mathrm{~mm}$, INPA 11606, 2, 100-104 mm, INPA 11607, 1, 98 mm, INPA 11692, 3, 88-149 mm, INPA 11693, 1, 88 mm, INPA 11722, 2, 130-130 mm, Igarapé do Aeroporto, Humboldt [Aripuanã], rio Aripuanã, affl. rio Madeira, $10^{\circ} 11^{\prime} 18^{\prime \prime} \mathrm{S}$, 059 $27^{\prime} 47^{\prime \prime} \mathrm{W}$. MPEG 1313, 1, 74 mm, mun. Juína, Reserva Ecológica Iquê, affl. rio Iquê, on AR 1, Vilhena-Juína hwy., affl. rio Juruena, affl. rio Tapajós, $11^{\circ} 25^{\prime} \mathrm{S}, 058^{\circ} 45^{\prime} \mathrm{W}$ [coordinates for Juína]. Pará. FMNH 70075, 13 (2 CS), 60$112 \mathrm{~mm}$, Missão do Cururu, rio Cururu, affl. rio Tapajós, 07³6'50"S, 057 38'58"W. MPEG 3039, 1, 87 mm, mun. igarapé-Açu, bridge over rio Cajueiro, affl. rio Marapanim, ca. $01^{\circ} 07^{\prime} \mathrm{S}, 047^{\circ} 36^{\prime} \mathrm{W}$. MPEG 7177, 2, 68-77 mm, mun. Barcarena, igarapé Tauá, nr. hwy. PA 483, affl. Furo do Arrozal, affl. Baía de Marajó, ca. $01^{\circ} 34^{\prime} \mathrm{S}, 048^{\circ} 42^{\prime} \mathrm{W}$. MPEG 7468, 1, 71 mm, MPEG 7471, 1, 73 mm, mun. Paragominas, igarapé Cachoeirinha, Fazenda Potiritá, affl. rio Potiritá, affl. rio Capim, affl. rio Guamá, affl. Baía de Marajó, $03^{\circ} 12^{\prime} 13^{\prime \prime} \mathrm{S}$, $047^{\circ} 39^{\prime} 52^{\prime} \mathrm{W}$. MPEG 15623, 1, $103 \mathrm{~mm}$, mun. Almeirim, rio Ipitinga, affl. rio Jari, $00^{\circ} 49^{\prime} 02^{\prime \prime} \mathrm{S}, 053^{\circ} 56^{\prime} 27^{\prime \prime} \mathrm{W}$. UF 36599 , 
1, 109 mm, igarapé Pirelli, affl. rio Maguari, affl. Baía de Marajó, 01²1'24"S, 048 19'36"W. Rondônia (localities from rio Madeira dr.). FMNH 54544, 1, 59 mm LEA, Maciel, rio Guaporé, affl. rio Mamoré, $c a .12^{\circ} 30^{\prime} \mathrm{S}, 062^{\circ} 20^{\prime} \mathrm{W}$. MCP 41041, 1, $77 \mathrm{~mm}$, mun. rio Crespo, stream ca. $20 \mathrm{~km} \mathrm{E} \mathrm{hwy.}$ BR-364 in direction of rio Crespo, affl. rio Preto do Crespo, affl. rio Jamari, $09^{\circ} 42^{\prime} 21^{\prime \prime} \mathrm{S}, 062^{\circ} 55^{\prime} 38^{\prime \prime} \mathrm{W}$. UFRO-I 6494, 2 , 72-93 mm, stream, Estação Ecológica do Cuniã (ESEC), $08^{\circ} 04^{\prime} 30^{\prime \prime} \mathrm{S}, 063^{\circ} 28^{\prime} 34^{\prime \prime} \mathrm{W}$. UFRO-I 6495, 1, $73 \mathrm{~mm}$, stream, ESEC, $08^{\circ} 04^{\prime} 07^{\prime \prime} \mathrm{S}, 063^{\circ} 28^{\prime} 34^{\prime \prime} \mathrm{W}$. UFRO-I 6506, 4, 62-75 $\mathrm{mm}$, UFRO-I 6508, 1, $67 \mathrm{~mm}$, stream, ESEC, $08^{\circ} 05^{\prime} 30^{\prime \prime} \mathrm{S}$, $063^{\circ} 28^{\prime} 35^{\prime \prime} \mathrm{W}$. UFRO-I 6509, 1, $73 \mathrm{~mm}$, stream, ESEC $08^{\circ} 04^{\prime} 09^{\prime \prime} \mathrm{S}, 063^{\circ} 28^{\prime} 34^{\prime \prime} \mathrm{W}$. Roraima. INPA 7362, 1, INPA 7363,1 , INPA 7367, 1, igarapé do Cajú, 21 km Bonfim on old rd. to Normândia, rio Tacatú, affl. rio Branco, affl. rio Negro, ca. $03^{\circ} 32^{\prime} \mathrm{N}, 059^{\circ} 53^{\prime} \mathrm{W}$. Colombia. Meta (localities from Orinoco dr.). MPEG 1105, 9, 90-122 mm, 7 km SE Puerto Lopez, affl. río Meta, $c a .04^{\circ} 03^{\prime} \mathrm{N}, 072^{\circ} 54^{\prime} \mathrm{W}$. SU 50348, 1 , $77 \mathrm{~mm}$, nr. Los Micos, N end Cordillera Macarena, río Guaviare, río Meta, $c a .03^{\circ} 19^{\prime} \mathrm{N}, 073^{\circ} 54^{\prime} \mathrm{W}$. SU 53762, 1,67 mm LEA, stream affl. río Guayabero, ca. 5 mi. below El Refugio, affl. río Guayabero, affl. río Guaviare, $c a .02^{\circ} 17^{\prime} \mathrm{N}$, $073^{\circ} 44^{\prime} \mathrm{W}$. UF $19472,1,68 \mathrm{~mm}$, UF 26012, 3, 66-67 mm, UF 33466 (part), 2, 83-89 mm, UF 33467, 1, $66 \mathrm{~mm}, 4.7 \mathrm{~km} \mathrm{~W}$ Puerto Gaitán, affl. río Yucao, affl. río Manacacias, affl. río Meta, $c a .04^{\circ} 18^{\prime} \mathrm{N}, 072^{\circ} 07^{\prime} \mathrm{W}$. UF $33373,1,102 \mathrm{~mm}, 47 \mathrm{~km}$ ENE Villavicencio, affl. río Humea, ca. $04^{\circ} 18^{\prime} \mathrm{N}, 073^{\circ} 11^{\prime} \mathrm{W}$. UF 36603, 2, 93-98 mm, río Guatiguia nr. Villavicencio, affl. río Meta, $c a .04^{\circ} 09^{\prime} \mathrm{N}, 073^{\circ} 38^{\prime} \mathrm{W}$. UF $80991,1,99 \mathrm{~mm}$, small creek $c a .70 \mathrm{~km}$ E Villavicencio on rd. to Puerto López, $c a$. $04^{\circ} 03^{\prime} \mathrm{N}, 073^{\circ} 04^{\prime} \mathrm{W}$. Ecuador. Sucumbios (localities from Río Napo dr.). FMNH 102278, 1, $121 \mathrm{~mm}, c a .10 \mathrm{~km} \mathrm{~N}$ Marian and $1 \mathrm{~km} \mathrm{~S}$ río Cuyabeno below rd., affl. to río Cuyabeno, affl. río Aguarico, $c a .00^{\circ} 07^{\prime} \mathrm{N}, 076^{\circ} 17^{\prime} \mathrm{W}$. FMNH 102283, 1, 103 mm, headwater río Aguas Negras, 1-2 km N Marian, affl. río Cuyabeno, affl. río Aguarico, $c a .00^{\circ} 46^{\prime} \mathrm{S}$, $076^{\circ} 20^{\prime} \mathrm{W}$. Guyana. Potaro-Siparuni (localities from Essequibo River dr.). ANSP 175954, 4, 57-70 mm, small creek crossing Kurupukari-Surama River rd. ca. 2.0 mi. from Kurupukari [Iwokroma] field station, $04^{\circ} 30^{\prime} 12^{\prime \prime} \mathrm{N}$, $058^{\circ} 42^{\prime} 32^{\prime \prime}$ W. FMNH 53323, 1, 99 mm, Chipoo Creek, affl. Ireng River, $c a .04^{\circ} 42^{\prime} \mathrm{N}, 059^{\circ} 06^{\prime} \mathrm{W}$. ROM $62556,1,83 \mathrm{~mm}$, stream affl. Siparuni River, affl. Siparuni River, $04^{\circ} 49^{\prime} 11^{\prime \prime} \mathrm{N}$, $058^{\circ} 51^{\prime} 01^{\prime \prime} \mathrm{W}$. Peru. Loreto. ANSP 178620, 4, 50-123 mm, caño Sabolito, terra firme stream, $c a$. $25 \mathrm{mi}$. S Iquitos, affl. río Amazonas, $04^{\circ} 14^{\prime} 45^{\prime \prime} \mathrm{S}, 073^{\circ} 24^{\prime} 57^{\prime \prime} \mathrm{W}$. INHS 43903, 3, 64-79 $\mathrm{mm}$, quebrada Shushuna, on rd. to Corrientillos, affl. rio Nanay, $03^{\circ} 49^{\prime} 48^{\prime \prime} \mathrm{S}, 073^{\circ} 20^{\prime} 14^{\prime \prime} \mathrm{W}$. MUSM 5402, 2, 113-126 $\mathrm{mm}$, stream nr. Jenaro Herrera, affl. río Ucayali, $c a .04^{\circ} 54^{\prime} \mathrm{S}$, $073^{\circ} 37^{\prime} \mathrm{W}$. MUSM 14494 (part), 1, $131 \mathrm{~mm}$, Lagartococha, Aguas Negras, río Aguarico, affl. río Napo, $00^{\circ} 31^{\prime} 22^{\prime \prime} \mathrm{S}$, $075^{\circ} 15^{\prime} 25^{\prime \prime} \mathrm{W}$. MUSM 44695, 40 (25 immature, 62-113 mm, 13 female, 76-112 mm, 2 male, 84-112 mm), stream nr. Jenaro Herrera, 04 $51^{\prime} 51^{\prime \prime} \mathrm{S}, 073^{\circ} 38^{\prime} 45^{\prime \prime} \mathrm{W}$. MUSM 44752, 4 (3 immature, $17-85 \mathrm{~mm}, 1$ female, $104 \mathrm{~mm}$ ), stream nr. Jenaro Herrera, affl. río Ucayali, $04^{\circ} 54^{\prime} 54^{\prime \prime} \mathrm{S}, 073^{\circ} 39^{\prime} 25^{\prime \prime} \mathrm{W}$. MUSM
44763, 1, immature, $55 \mathrm{~mm}$, stream nr. Jenaro Herrera, affl. río Ucayali, $04^{\circ} 54^{\prime} 36^{\prime \prime} \mathrm{S}, 073^{\circ} 39^{\prime} 58^{\prime \prime} \mathrm{W}$. UF 148514,7 (1 immature, $92 \mathrm{~mm}, 3$ female, 98-107 mm, 3 male, 103-125

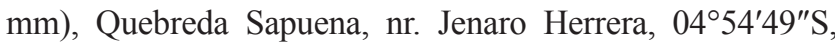
$073^{\circ} 34^{\prime} 59^{\prime \prime} \mathrm{W}$. UF $183772,4,29-79 \mathrm{~mm}$, río Nanay, $03^{\circ} 53^{\prime} 50^{\prime \prime} \mathrm{S}, 073^{\circ} 40^{\prime} 01^{\prime \prime} \mathrm{W}$. UF 184012, 10 (8 immature, 66$118 \mathrm{~mm}, 1$ female, $98 \mathrm{~mm}, 1$ male, $104 \mathrm{~mm}$ ), stream nr. Jenaro Herrera, $04^{\circ} 53^{\prime} 59^{\prime \prime} \mathrm{S}, 073^{\circ} 38^{\prime} 51^{\prime \prime} \mathrm{W}$. UF 184019 , 11 (8 immature, $62-89 \mathrm{~mm}, 2$ female, $108-110 \mathrm{~mm}, 1$ male, 102 $\mathrm{mm})$, stream nr. Jenaro Herrera, $04^{\circ} 55^{\prime} 19^{\prime \prime} \mathrm{S}, 073^{\circ} 38^{\prime} 57^{\prime \prime} \mathrm{W}$. UF 184024, 21 (11 immature, 56-114 mm, 6 female, 90-109 $\mathrm{mm}, 3$ male, 113-116 mm), stream nr. Jenaro Herrera, $04^{\circ} 54^{\prime} 15^{\prime \prime} \mathrm{S}, 073^{\circ} 36^{\prime} 53^{\prime \prime} \mathrm{W}$. Venezuela. Amazonas. AMNH 230873, 1, $85 \mathrm{~mm}$, MBUCV-V 29177, 1, $86 \mathrm{~mm}, 1 \mathrm{hr}$. downstream Campamento Yutaje, río Coracora, affl. río Manapiari, affl. río Ventuari, Orinoco dr., $05^{\circ} 36^{\prime} 25^{\prime \prime} \mathrm{N}$, 066 07'03"W. MBUCV-V 228 (part), 2, 84-106 mm, Caños Parú and Canna, upper río Ventuari, Orinoco dr., no coordinates. MBUCV-V 3460, 3, 78-83 mm, caño Iguapo, E La Esmeralda, $c a .03^{\circ} 10^{\prime} \mathrm{N}, 065^{\circ} 33^{\prime} \mathrm{W}$. MBUCV-V 12986, 1, $143 \mathrm{~mm}$, Tobogan de la Selva, $c a .36 \mathrm{~km}$ de Puerto Ayacucho, affl. río Orinoco, Orinoco dr., $05^{\circ} 23^{\prime} 13^{\prime \prime} \mathrm{N}, 07^{\circ} 37^{\prime} 02^{\prime \prime} \mathrm{W}$. MBUCV-V 14330, 1, $131 \mathrm{~mm}$, small stream nr. Sarama Sota, upper río Cataniapo, Orinoco dr., $05^{\circ} 38^{\prime} 00^{\prime \prime} \mathrm{N}, 067^{\circ} 07^{\prime} 40^{\prime \prime} \mathrm{W}$. MBUCV-V 14678, 1, $132 \mathrm{~mm}, 0.5-1 \mathrm{~km}$ upstream La Neblina basecamp, río Baria, affl. río Casiquiare, affl. rio Negro, Amazonas dr., $c a .00^{\circ} 50^{\prime} \mathrm{N}, 066^{\circ} 05^{\prime} \mathrm{W}$. MBUCV-V 14713, 5 (2 measured, 120-122 mm), stream nr. La Neblina basecamp, río Baria, affl. río Casiquiare, affl. rio Negro, Amazonas dr., ca. $00^{\circ} 50^{\prime} \mathrm{N}, 066^{\circ} 05^{\prime} \mathrm{W}$. MBUCV-V 14720, 1, male, $152 \mathrm{~mm}$, La Neblina basecamp, río Baria, aff río Casiquiare, affl. rio Negro, Amazonas dr., ca. $00^{\circ} 50^{\prime} \mathrm{N}, 066^{\circ} 05^{\prime} \mathrm{W}$. MBUCV-V $14760,1,82 \mathrm{~mm}$, stream, $100 \mathrm{~m}$ before intersection of Gavilán and Las Palmas, affl. río Cataniapo, Orinoco dr., $05^{\circ} 32^{\prime} 45^{\prime \prime} \mathrm{N}, 67^{\circ} 23^{\prime} 36^{\prime \prime} \mathrm{W}$. MBUCV-V 14768, 1, $78 \mathrm{~mm}$, Raudal Yahui, $5 \mathrm{~km}$ upstream La Reforma, río Cataniapo, affl. río Orinoco, Orinoco dr., $c a .05^{\circ} 33^{\prime} \mathrm{N}, 067^{\circ} 31^{\prime} \mathrm{W}$. UF 177354, 11 (8 immature, 73-104 mm, 4 female, 73-127 mm, 2 male, $89-117 \mathrm{~mm}$, on rd. from Puerto Ayacucho to Gavilan, caño Culebra, affl. río Cataniapo, Orinoco dr., $05^{\circ} 32^{\prime} 48^{\prime \prime} \mathrm{N}$, 067²6'55"W. UF 177355, 6, 60-117 mm (3 CS), on rd. from Puerto Ayacucho to Gavilan, caño Moia, affl. río Cataniapo, Orinoco dr., $05^{\circ} 32^{\prime} 26^{\prime \prime} \mathrm{N}, 067^{\circ} 23^{\prime} 02^{\prime \prime} \mathrm{W}$. UF 177356, 8, 61-83 $\mathrm{mm}$, rd. from Puerto Ayacucho to Gavilan, caño Samaria, affl. rio Cataniapo, Orinoco dr., $05^{\circ} 33^{\prime} 52^{\prime \prime} \mathrm{N}, 067^{\circ} 28^{\prime} 15^{\prime \prime} \mathrm{W}$. USNM 266718, 3, 80-119 mm, "Cãno Igapo" [probably río Iguapo], E La Esmeraldas [La Esmeralda], affl. upper río Orinoco, Orinoco dr., $c a .03^{\circ} 07^{\prime} \mathrm{N}, 065^{\circ} 28^{\prime} \mathrm{W}$. Apure. MCNG 26553, 2, 62-63 mm LEA, stream W Parque Nacional Galeras del Cinaruco, affl. río Cinaruco, Orinoco dr., $c a .06^{\circ} 34^{\prime} \mathrm{N}$, $067^{\circ} 17^{\prime} \mathrm{W}$. Bolívar. SU 54492, 1, 73 mm LEA, SU 54612, 1, 65 mm LEA, mun. Caicara del Orinoco, caño de Quiribana, Orinoco dr., $c a .07^{\circ} 32^{\prime} \mathrm{N}, 066^{\circ} 08^{\prime} \mathrm{W}$.

Diagnosis. Brachyhypopomus sullivani is diagnosed from congeners by the following combination of characters: 
precaudal vertebrae 15-18, vs. 19-26 in B. alberti, B. arrayae, B. beebei, B. belindae, B. draco, B. hamiltoni, B. janeiroensis, $B$. jureiae, $B$. palenque, and $B$. verdii; anal-fin rays 143-185, vs. 190-293 in B. bennetti, B. brevirostris, B. bullocki, B. diazi, $B$. hendersoni, $B$. occidentalis, and $B$. walteri; scales present in middorsal region, vs. absent in B. benjamini and $B$. provenzanoi; accessory electric organ over the opercular region absent, vs. present in $B$. bombilla, B. menezesi, and B. regani; head depth at eye 38.5-47.6\% HL, vs. $47.8-59.9 \%$ in B. cunia, B. gauderio, and B. pinnicaudatus; presence of scattered conspicuous black or charcoal flecks on flank, vs. absence in B. batesi and B. flavipomus.

Description. Head and body shape, and pigmentation illustrated in Figs. $1 \mathrm{k}$ and 43. Meristic and morphometric data for examined specimens presented in Tables 2-5 and 19. Body shallow to moderate in depth. Head short to moderate in length and shallow to moderate in depth. Dorsal profile of head approximately straight from occiput to snout, ventral profile of head approximately straight between operculum and snout, snout rounded. Eye small to moderate in size. Upper jaw with moderate sigmoidal angle between premaxillary and maxillary portions in lateral view. Accessory electric organ (AEO) over operculum absent. Gill filaments on first gill arch 29-33 (median 31, n=6). Pectoral fin of moderate width, pectoral-fin rays 11-16 (median of 11 in populations from the upper Amazon of Peru; mode of 14 in populations from upper rio Madeira, and from río Orinoco). Precaudal vertebrae 15-17 (mode of 16 in all populations), including 1-2 transitional vertebrae. Anal-fin origin slightly $(<0.25 \mathrm{HL}$ distance) posterior to, or near, tip of pectoral fin. Anal-fin rays 143-184 (median of 171 in populations from the upper Amazon of Peru; 159 from the upper rio Madeira; and 171 from the río Orinoco). Dorsal rami of recurrent branch of anterior lateral line nerve not visible. Middorsal region of body scaled. Rows of scales above lateral line 5-7 (mode 6). Lateral line continuous. Extensive network of epidermal canals dorsal to lateral line in posterior half of body, extending to about midway from lateral line to dorsal midline and forming a crisscrossing pattern of posteriorly converging chevrons. Groove-like canals are depigmented or almost depigmented, sometimes with chromatophores along margins, and are filled with a translucent material covered with skin (overlain by scales). Groove-like canals with apparent partitioning into rectangular segments and with pores to exterior located at posterior ends in many cases (possibly tuberous electroreceptors). Three bilateral horizontal columns of electrocytes at anal-fin terminus and at mid-point between anal-fin terminus and tip of caudal filament in immature, mature female, and mature male specimens. Caudal filament short to moderate in length.

Coloration. (Figs. 1k, 43). Background pale tan to dark brown. Dorsal region without prominent depigmented pale stripe extending along midline from occipital region to base of caudal filament, although in some individuals there is an indistinct lighter middorsal stripe in posterior third to half of body. Dorsal region variably pigmented, often with irregular dark blotches against a homogenous lighter background, sometimes with dark blotches forming a single intermittent series along dorsal midline. Dark blotches restricted to dorsal region, never extending far ventrally and only just visible in lateral view. No vertical stripes or lines on flank; flank with evenly scattered chromatophores, darker dorsally and lighter ventrally, especially over pterygiophores. Characteristic prominent dark brown or black flecks scattered irregularly over flank. Caudal filament darker than body, with some irregular dark markings. Head with evenly scattered dark chromatophores, darker dorsally. Eye with indistinct to very distinct suborbital patch or stripe of chromatophores and subcutaneous pigmentation. Pectoral and anal-fin membranes and rays hyaline. Color in live individuals similar to preserved specimens, with opercular region usually rosy due to underlying gills.

Size. A Brachyhypopomus of small adult size, largest specimen examined $152 \mathrm{~mm}$ TL, $123 \mathrm{~mm}$ LEA ( $\mathrm{n}=390)$. Largest male specimen examined $152 \mathrm{~mm}$ TL, $123 \mathrm{~mm}$ LEA $(\mathrm{n}=36)$. Largest female specimen examined $127 \mathrm{~mm} \mathrm{TL}$, $100 \mathrm{~mm} \operatorname{LEA}(\mathrm{n}=67)$.

Sexual dimorphism. No known secondary sexual dimorphism.

Geographic distribution. Bolivia, Brazil, Colombia, Ecuador, Guyana, Peru, and Venezuela (Fig. 44). Widely distributed through the Amazon basin including multiple sites in the upper, central, and lower Amazon, the Negro, Purus, lower and upper Madeira, and Tapajós drainages. Known from some coastal drainages in Pará state, near the mouth of the rio Tocantins. Known also from parts of the Orinoco basin, and from the Essequibo River.

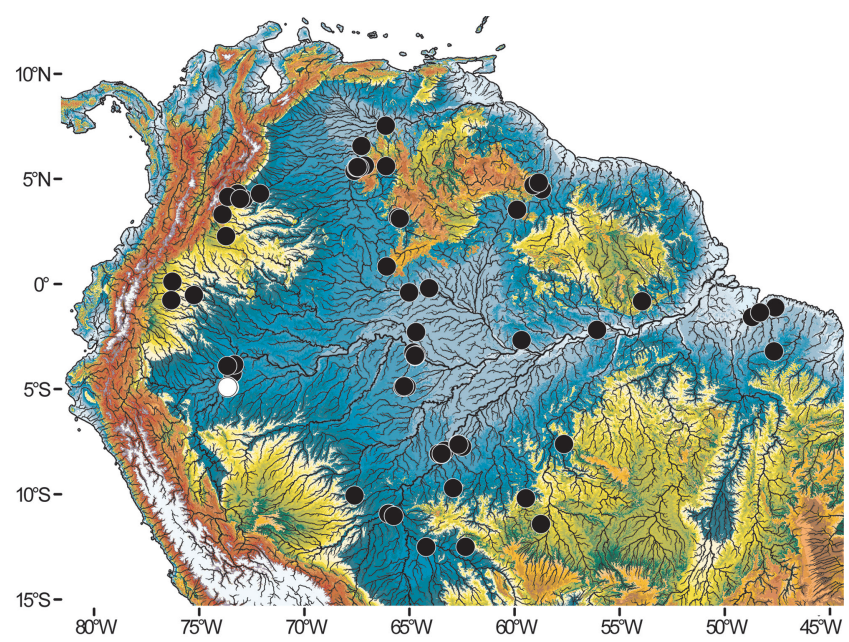

Fig. 44. Collection records for Brachyhypopomus sullivani (circles). Holotype location is marked with an open symbol. Elevation data refers to altitude above mean sea level (see Fig. 2 for legend). 
Population variation: We found overlap in the range of meristic counts (Tables 2-5) and morphometric measurements (Table 19), and observed similar pigmentation among populations of $B$. sullivani across its entire distribution. One meristic character, the number of pectoral-fin rays, is somewhat divergent between populations in the upper Amazon (11-13, mode 11) vs. the upper Madeira (13-14, mode 14) and Orinoco (13-15, mode 14). These observations, in combination with molecular data (Crampton et al., 2016), support the hypothesis that all populations we have assigned to $B$. sullivani are members of a single geographically widespread species, which is morphologically and genetically distinct from all congeners.

Ecological notes. Brachyhypopomus sullivani is restricted to gently flowing, normoxic, low-conductivity streams and small rivers in closed canopy rainforest and savanna, where it is typically encountered in or near aquatic vegetation, root mass, or leaf litter. See description of B. benjamini for water quality parameters of the area of the type locality. Crampton et al. (2008) documented the relatively small gill sizes of $B$. sullivani relative to congeners that occur in seasonally hypoxic whitewater floodplain systems. Breeding occurs during the rainy season; both in the type locality, and in the vicinity of Tefé, Brazil (approximately December to April in both regions) (JCW, WGRC unpublished data). Stomach contents of specimens from the type locality comprise aquatic insect larvae (primarily Chironomidae), and other small aquatic invertebrates (JCW, WGRC unpublished data).

Co-occurring congeners: In the upper, central, and lower Amazon (including lower Madeira, but excluding upper Madeira), B. sullivani occurs in sympatry and ecological syntopy with $B$. batesi, $B$. beebei, $B$. benjamini, B. brevirostris, $B$. verdii, and $B$. walteri, and exhibits an allotopic distribution to $B$. belindae, B. bennetti, B. cunia, B. flavipomus, $B$. hamiltoni, $B$. hendersoni, $B$. pinnicaudatus, and $B$. regani (note: in the lower Amazon populations of $B$. regani occur in low-conductivity systems in syntopy with $B$. sullivani). In the upper Madeira, $B$. sullivani is known to occur in sympatry with $B$. alberti, $B$. brevirostris, and $B$. walteri, and in allotopy with $B$. arrayae, $B$. bombilla, and $B$. cunia. In the rio Negro $B$. sullivani occurs in sympatry with $B$. batesi, B. beebei, $B$. brevirostris, $B$. bullocki, $B$. hendersoni, $B$. regani, and $B$. walteri (likely in syntopy with all these species except $B$. hendersoni, which is a floodplain species). In the río Orinoco $B$. sullivani occurs in sympatry and syntopy with $B$. beebei, $B$. brevirostris, B. bullocki, B. diazi, B. provenzanoi, and $B$. regani (likely in syntopy with all these species except $B$. diazi which is restricted to high-conductivity systems). In the Essequibo River, B. sullivani occurs in sympatry with $B$. beebei, B. brevirostris, B. bullocki, B. hendersoni, B. regani, and $B$. walteri (likely in syntopy with all these species except $B$. hendersoni, which is a floodplain endemic).

Etymology. The specific name is a patronym (noun in the genitive case) in honor of John P. Sullivan, American (USA) ichthyologist, for his contributions to gymnotiform biology.
Local names. Bolivia: cuchillo; Brazil: sarapó; Colombia: cuchillo; Ecuador: cuchillo, yayo; Guyana: knife fish; Peru: macana; Venezuela: cuchillo.

\section{Brachyhypopomus verdii, new species}

urn:1sid:zoobank.org:act:3FC61943-7A01-40A4-A5D6C7E296167CE0

(Fig. 45; Tables 2-5, 20)

Brachyhypopomus pinnicaudatus. -Chang \& Ortega, 1995: 3, (Peru, catalog of fishes).

Brachyhypopomus sp. 'ver'. -Crampton, 2011: 176, table 10.2, species list; 179, figs. 10.2-10.3, phylogeny, geographical and ecological distributions (gymnotiform biology).

Brachyhypopomus n. sp. VERD. -Elbassiouny et al., 2016: 401403, fig. 1 (description of mitogenome, position in phylogeny of Ostariophysi based on mitochondrial genomes).

Brachyhypopomus sp. "verdii". -Crampton et al., 2016: 1-66, table 1, 3-4, figs. 1-7, 18-20 (phylogeny, biogeography and ecology of Brachyhypopomus).

Holotype. MUSM 35307, female, $79 \mathrm{~mm}$ TL, $67 \mathrm{~mm}$ LEA, Peru, Loreto, mun. Maynas, Jenaro Herrera, stream, nr. Jenaro Herrera-Colonia Angamos rd., affl. río Ucayali, Amazonas dr., 0453'54"S,07338'22"W, 16 Jan 2004, W. Crampton, H. Ortega, R. Reis, F. Lima \& J. Oliveira.

Paratypes. 41 specimens, localities from Amazonas dr. Peru. Loreto (localities listed from nr. Jenaro Herrera, are from the río Ucayali dr., mun. Requena). MUSM 44683, 4 (2 immature, 89-103 mm, 1 female, $79 \mathrm{~mm}, 1$ male, $107 \mathrm{~mm}$ ),

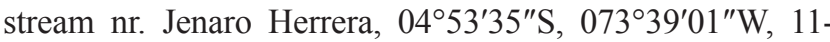
30 Jul 2009, W. Crampton, E. Correa Roldán, M. Guevara \& J. Waddell. MUSM 44726, 1, female, $76 \mathrm{~mm}$, forest stream $1.65 \mathrm{~km}$ Jenaro Herrera, affl. río Ucayali, Amazonas dr., 0454'33"S, 073³9'26"W, 30 Jul 2009, W. Crampton, E. Correa Roldán, M. Guevara \& J. Waddell. UF 148522, 2, immature, 82-106 mm, nr. Jenaro Herrera, stream in agricultural field, affl. quebrada Parnayari, ca. 04 $54^{\prime} \mathrm{S}$, $073^{\circ} 37^{\prime}$ W, 14 Jan 2004, W. Crampton, H. Ortega, R. Reis, F. Lima \& J. Oliveira. UF 148523, 7, immature, 84-109 mm, stream nr. Jenaro Herrera, 04 $53^{\prime} 54^{\prime \prime} \mathrm{S}, 073^{\circ} 38^{\prime} 21^{\prime \prime} \mathrm{W}$, 16 Jan 2004, W. Crampton, H. Ortega, R. Reis, F. Lima \& J. Oliveira. UF 184011, 1, female, $107 \mathrm{~mm}$, stream nr.

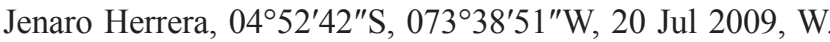
Crampton, E. Correa Roldán, M. Guevara \& J. Waddell. UF 184021, 1, immature, $66 \mathrm{~mm}$, stream nr. Jenaro Herrera,

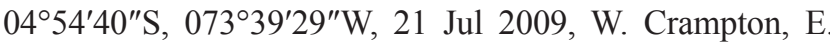
Correa Roldán, M. Guevara \& J. Waddell. UF 184026, 19 (4 immature, 67-87 mm, 6 female, $67-100 \mathrm{~mm}, 9$ male, $79-120$

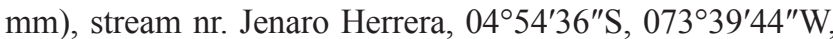
31 Jul 2009. W. Crampton, E. Correa Roldán, M. Guevara \& J. Waddell. USNM 405580, 3, immature, 74-78 mm, stream nr. Jenaro Herrera, 04 $54^{\prime} 15^{\prime \prime} \mathrm{S}, 073^{\circ} 36^{\prime} 53^{\prime \prime} \mathrm{W}, 25$ Jul 2009 , 
W. Crampton, E. Correa Roldán, M. Guevara \& J. Waddell. USNM 405582, 3 (2 immature, 83-104 mm, 1 female, 91 $\mathrm{mm}$ ), stream nr. Jenaro Herrera, 04 $54^{\prime} 49^{\prime \prime} \mathrm{S}, 073^{\circ} 39^{\prime} 43^{\prime \prime} \mathrm{W}$, 31 Jul 2009, W. Crampton, E. Correa Roldán, M. Guevara \& J. Waddell.

Non-types. 185 specimens, localities from Amazonas dr. Peru. Amazonas. FMNH 96997, 1, 125 mm, Caterpiza, affl. río Santiago, affl. río Marañón, ca. $03^{\circ} 54^{\prime} \mathrm{S}, 077^{\circ} 42^{\prime} \mathrm{W}$. Loreto (localities listed from nr. Jenaro Herrera, are from the río Ucayali dr., mun. Requena). ANSP 167724, 4, 65$109 \mathrm{~mm}$, swamp on path from Mishana village to Nauta rd., affl. río Nanay, 035'12"S, 073²8'57"W. MUSM 3229, 3,

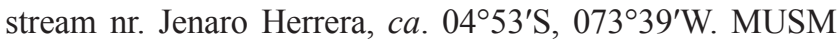
4449, 2, mun. Yurimaguas, San Ramón, río Marañón, ca. $04^{\circ} 54^{\prime} \mathrm{S}, 076^{\circ} 26^{\prime} \mathrm{W}$. MUSM 44677, 30 (14 immature, 33-110 mm, 10 female, 77-104 mm, 6 male, 88-111 mm), stream nr. Jenaro Herrera, 0453'59"S, 073³8'51"W. MUSM 44764, 27 (18 immature, 26-107 mm, 9 female, 61-109 mm), stream

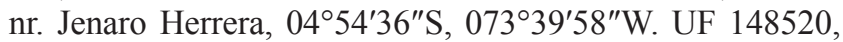
19 (16 immature [3 CS], 38-105 mm, 2 female, 83-96 mm, 1 male, $106 \mathrm{~mm})$, stream nr. Jenaro Herrera, ca. $04^{\circ} 53^{\prime} \mathrm{S}$, $073^{\circ} 39^{\prime} \mathrm{W}$. UF 148521, 32 (28 immature, 45-109 mm, 3 female, 80-97 mm, 1 male, $109 \mathrm{~mm}$ ), stream $\mathrm{nr}$. Jenaro

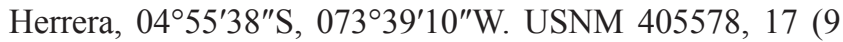
immature, 49-114 mm, 6 female, $82-101 \mathrm{~mm}, 2$ male, $98-114$

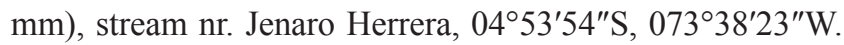
USNM 405579, 24 (14 immature, 44-100 mm, 4 female, 92-112 mm, 6 male, 92-115 mm), stream nr. Jenaro Herrera, 04 $55^{\prime} 38^{\prime \prime} \mathrm{S}, 073^{\circ} 39^{\prime} 14^{\prime \prime} \mathrm{W}$. USNM 405581, 25 (15 immature, 55-95 mm, 4 female, 69-95 mm, 6 male, 93-110 mm), stream nr. Jenaro Herrera, 045' $41^{\prime \prime} \mathrm{S}, 073^{\circ} 38^{\prime} 46^{\prime \prime} \mathrm{W}$.

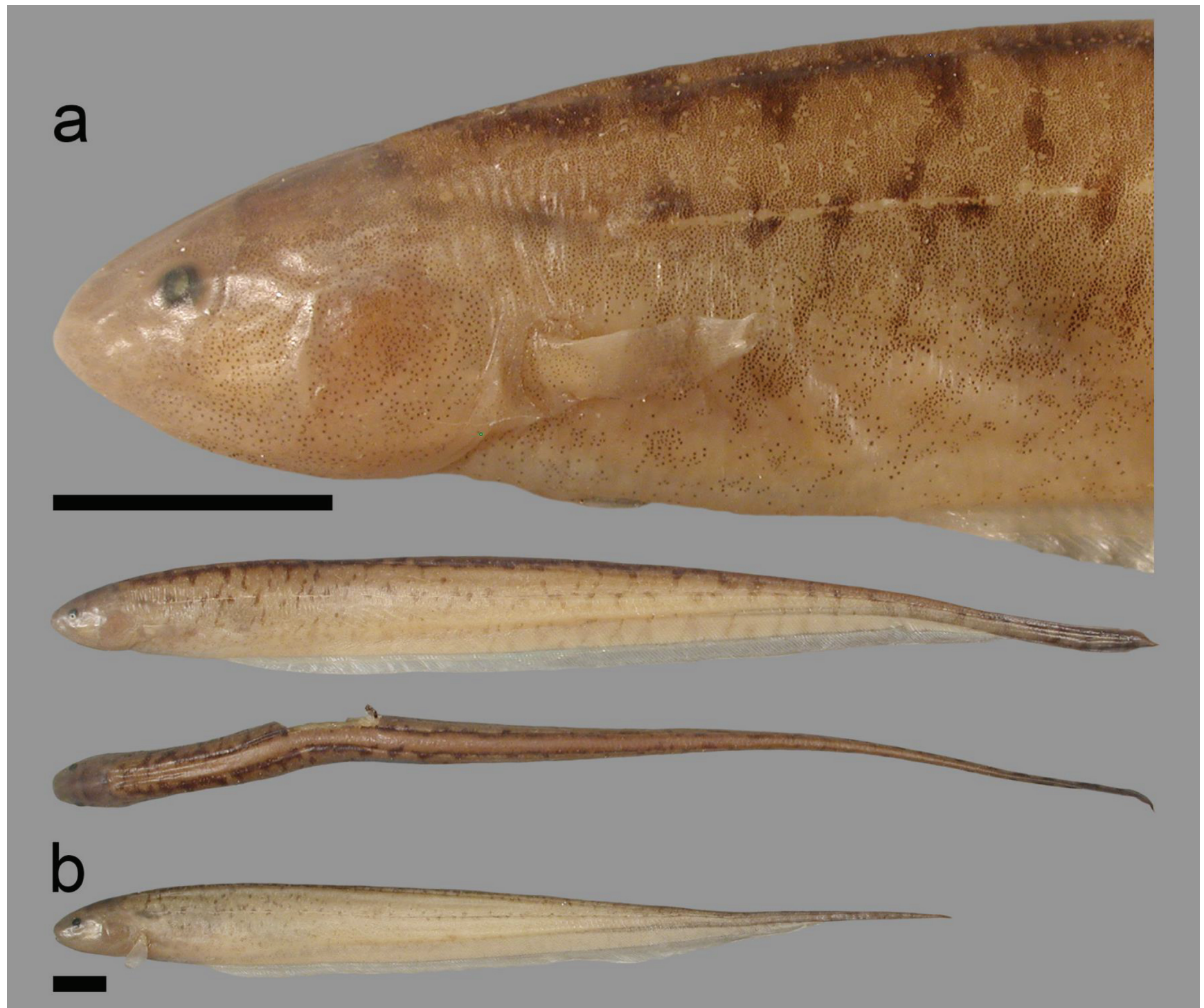

Fig. 45. Brachyhypopomus verdii. a. UF 148520 (WC24.090104), paratype, male, $106 \mathrm{~mm}$ TL (head in lateral view, and body in lateral and dorsal view); b. MUSM 35307 (WC06.160104), holotype, female, 79 mm TL (body in lateral view). Peru, río Ucayali, Amazonas dr. Note sexual dimorphism in caudal-filament length and height. Specimens fixed in formalin and preserved in EtOH. Scale bars $=5 \mathrm{~mm}$. 
Table 20. Morphometrics for Brachyhypopomus verdii. HT, holotype; SD, standard deviation. Ranges refer to the holotype and to paratype specimens from the type region.

\begin{tabular}{|c|c|c|c|c|c|}
\hline & \multicolumn{5}{|c|}{ B. verdii } \\
\hline & HT & Range & Mean & SD & $\mathrm{n}$ \\
\hline Total length (TL) (mm) & 79 & $59-115$ & 87 & 14.5 & 106 \\
\hline Length to end of anal fin (LEA) (mm) & 67 & $52-102$ & 74 & 12.1 & 106 \\
\hline Head length (HL) (mm) & 7.3 & $6.9-9.1$ & 8.2 & 0.7 & 17 \\
\hline \multicolumn{6}{|c|}{ Proportion of TL $(\%)$} \\
\hline Caudal filament length (CF) & 15.2 & $10.1-21.9$ & 16.8 & 2.1 & 106 \\
\hline Snout to occiput (SO) & 8.9 & $7.0-10.5$ & 8.1 & 0.8 & 17 \\
\hline Snout to anal-fin origin (SA) & 19.7 & $16.1-20.7$ & 18.2 & 1.2 & 17 \\
\hline Snout to pectoral-fin base (PP) & 11.7 & $10.1-11.8$ & 10.9 & 0.5 & 17 \\
\hline Longest pectoral-fin ray (PRL) & 4.2 & $3.7-4.6$ & 4.0 & 0.2 & 17 \\
\hline Anal fin length (AF) & 80.6 & $79.5-84.1$ & 81.5 & 1.1 & 17 \\
\hline Body depth at anal-fin middle (BD2) & 8.2 & $7.3-10.0$ & 8.6 & 0.9 & 17 \\
\hline Body width at anal-fin middle (BW2) & 3.1 & $2.5-4.2$ & 3.2 & 0.5 & 17 \\
\hline Caudal filament length (CF) & 17.9 & $11.8-25.3$ & 17.2 & 2.9 & 17 \\
\hline \multicolumn{6}{|c|}{ Proportion of HL $(\%)$} \\
\hline Preorbital distance (PR) & 23.4 & $22.3-28.1$ & 25.2 & 1.7 & 17 \\
\hline Mouth width (MW) & 23.4 & $23.1-33.5$ & 27.9 & 3.1 & 17 \\
\hline Internarial distance (AE) & 24.2 & $22.6-27.0$ & 24.5 & 1.4 & 17 \\
\hline Posterior naris to eye (PE) & 2.9 & $1.2-3.0$ & 2.1 & 0.6 & 17 \\
\hline Orbital diameter (OD) & 11.0 & $8.9-12.0$ & 10.3 & 0.9 & 17 \\
\hline Interorbital distance (IO) & 28.9 & $24.5-30.6$ & 27.5 & 1.7 & 17 \\
\hline Head depth at eye (HD1) & 47.8 & $43.1-54.5$ & 48.2 & 3.0 & 17 \\
\hline Caudal filament depth (CD) & 12.5 & $11.2-19.9$ & 15.6 & 2.3 & 17 \\
\hline Caudal filament width (CW) & 5.5 & 4.6-7.6 & 6.0 & 1.0 & 17 \\
\hline \multicolumn{6}{|c|}{ Other proportions } \\
\hline BW1 (\% of BD1) & 52.9 & $49.2-62.5$ & 55.1 & 3.7 & 17 \\
\hline $\mathrm{BW} 2(\%$ of $\mathrm{BD} 2)$ & 38.0 & $30.5-46.8$ & 36.9 & 3.8 & 17 \\
\hline
\end{tabular}

Diagnosis. Brachyhypopomus verdii is diagnosed from congeners by the following combination of characters: precaudal vertebrae 24-26, vs. 15-23 in all congeners except $B$. belindae and B. draco; bilateral columns of electrocytes at the anal-fin terminus 3, vs. 4-5 in B. belindae; continuous or discontinuous dark vertical or diagonally oriented bands or saddles present on body surface dorsal to lateral line, $v s$. absence of oblique bands or saddles on body surface dorsal to lateral line in B. draco.

Description. Head and body shape, and pigmentation illustrated in Fig. 45. Meristic and morphometric data for examined specimens presented in Tables 2-5 and 20. Body shallow in depth. Head short and shallow to moderate in depth. Dorsal profile of head convex from occiput to snout, ventral profile of head convex between operculum and snout, snout rounded. Eye small to moderate in size. Upper jaw with moderate sigmoidal angle between premaxillary and maxillary portions in lateral view. Accessory electric organ (AEO) over operculum absent. Gill filaments on first gill arch 35-40 (median 37, $\mathrm{n}=6$ ). Pectoral fin narrow to moderate, pectoral-fin rays 13-17 (mode 15). Precaudal vertebrae 24-26 (mode 25), with 1-2 (mode 2) transitional vertebrae. Anal-fin origin slightly $(<0.25 \mathrm{HL}$ distance) posterior to tip of pectoral fin. Anal-fin rays 194-225 (median 208). Dorsal rami of recurrent branch of anterior lateral line nerve not visible. Middorsal region of body scaled. Rows of scales above lateral line 5-6 (mode 5). 
Lateral line continuous. Sparse groove-like depigmented epidermal canals - as parallel crisscrossing lines either side of lateral line (mainly in posterior third of body), or branching ventrally from lateral line (mainly in mid-third of body); and as a pair of long irregular lines either side of dorsal surface in dorsal portion of flank, along posterior two thirds of body. Three bilateral horizontal columns of electrocytes at anal-fin terminus and at mid-point between anal-fin terminus and tip of caudal filament in immature, mature female, and mature male specimens. Caudal filament short to moderate in length.

Size. Small adult size, largest specimen examined 125 $\mathrm{mm}$ TL, $107 \mathrm{~mm}$ LEA $(\mathrm{n}=222)$. Largest male specimen examined $120 \mathrm{~mm}$ TL, $102 \mathrm{~mm}$ LEA $(\mathrm{n}=32)$. Largest female specimen examined $112 \mathrm{~mm}$ TL, $95 \mathrm{~mm}$ LEA ( $\mathrm{n}$ $=52$ ).

Coloration. (Fig. 45). Background light tan to brown. Dorsal region with prominent depigmented or lightly pigmented stripe extending along midline from occipital region to base of caudal filament, or deep into caudal filament, bordered by prominent dark horizontal wavy reticulated lines from which short irregular patches extend ventrally - reaching or traversing lateral line in anterior third of body but reaching less than half distance from dorsal midline to lateral line posteriorly. Dorsal pigmentation pattern resembles that of B. gauderio and $B$. pinnicaudatus, but reticulated lines extending ventrally from either side of depigmented dorsal stripe are shorter and often only faintly visible in lateral view in posterior two thirds of body. Ventral flank otherwise marked only with small irregular dark spots. Very faint short vertical dark markings sometimes present over anal-fin pterygiophores in posterior half of body. Caudal filament darker than body, with irregular dark markings or bands along entire length. Head with evenly scattered dark chromatophores, darker dorsally. Eye without prominent suborbital patch or stripe of chromatophores/subcutaneous pigmentation. Pectoral and anal-fin membranes hyaline. Pectoral and anal-fin rays with light scattering of brown chromatophores, accentuated in posterior portion of anal-fin. Color in live individuals similar to preserved specimens, with opercular region usually rosy due to underlying gills.

Sexual dimorphism. Sexually mature males attain slightly larger sizes. Sexually mature males develop broader caudal filaments than immature individuals and breeding females (Figs. 45a-b) but do not exhibit an elevated number of horizontal bilateral columns or vertical rows of electrocytes. Instead breeding males exhibit clearly enlarged electrocytes relative to immature specimens and females. Breeding males with paddle-like lateral compression at caudal filament tip. No known sexual dimorphism in pigmentation.
Geographic distribution. Peru (Fig. 39). Known only from tributaries of the río Marañón and río Ucayali in the upper Amazon basin.

Ecological notes. Known only from low-conductivity blackwater terra firme rainforest streams and swamps, where it is found in association with submerged roots and leaf litter. See description of $B$. benjamini for water quality parameters of the area of the type locality. Breeding occurs during the rainy season in the area of the type locality (approximately December to April) (JCW unpublished data). Stomach contents of specimens from the type locality comprise small aquatic invertebrates (JCW unpublished data).

Co-occurring congeners: In the region of the type locality in the lower río Ucayali, $B$. verdii occurs in sympatry and syntopy with $B$. beebei, $B$. benjamini, $B$. brevirostris, $B$. sullivani, and $B$. walteri. It exhibits an allotopic distribution to $B$. bennetti, B. flavipomus, B. hamiltoni, and $B$. regani.

Etymology. The specific name is a patronym (noun in the genitive case) in honor of Lorgio Verdi Olivares, Peruvian conservation biologist, for his support to WGRC and NRL.

Local name. Peru: macana.

\section{Brachyhypopomus walteri Sullivan, Zuanon \& Cox Fernandes, 2013}

(Figs. 11, 46; Tables 2-5, 21)

Brachyhypopomus sp. 4. -Crampton, 1996a: 77, table 6.1.a, species list; 79, fig. 6.1 inset 22-24, color photographs; 85, fig. 6.2; 88, fig. 6.3; 92-94, figs. 6.4a, 6.5; 191, fig. 11.2; 193, fig. 11.3, EOD data; 110 , table 7.5, 113, table 7.7, 115, fig. 7.1, habitats; 136, table 8.2a,b, diet; 151-160, figs. 9.2-9.3, table 9.1a,b, hypoxia tolerance; $165-169$, table 10.1 reproductive biology (Brazil, central Amazon, ecology and signal diversity).

Brachyhypopomus walteri. -Sullivan, 1997: 105 (description in unpublished thesis with disclaimer stating that nomenclatural acts not available, a nomen nudum).

Brachyhypopomus sp. 3. -Crampton, 1998a: 821, table 4, 834, fig. 9 , illustration of specimen, with EOD (Brazil, Amazonas, species and EOD diversity). -Crampton, 1998b: 314, table 2, list of Brachyhypopomus (Brazil, Amazonas, hypoxia tolerance).

Brachyhypopomus sp. C. -Crampton, 1999: 17 (Brazil, Amazonas, Mamirauá Reserve, listing of species). -Crampton \& Albert, 2006: 672, fig. 23.8 (inset 11), color photograph of head of live individual with EOD, position in phylogenetic tree; 681, notes on EODs (gymnotiform species and EOD diversity).

Brachyhypopomus sp. 2. -Stoddard, 1999: 255, fig. 4, color photograph of live mature female specimen, with EOD (EOD evolution). 
Brachyhypopomus sp. "walteri". -Stoddard et al., 1999: 610, fig. 1, black and white photograph of female specimen, with EOD (EOD evolution).

Brachyhypopomus sp. 2. -Ferreira et al., 2007: 164, center right and bottom right photographs, live individuals, (Brazil, Roraima, rio Branco, photographic album of fishes).

Brachyhypopomus sp. 'wal'. -Crampton, 2011: 176, table 10.2, species list; 179, figs. 10.2-10.3, phylogeny, geographical and ecological distributions (gymnotiform biology).

Brachyhypopomus sp. 4. -de Queiroz et al., 2013: 545, table 1, (Brazil, rio Madeira, lago Cuniã, listing of fish species].
-Crampton \& Ribeiro, 2013: 242, color photograph (Brazil, rio Madeira, listing of hypopomids).

Brachyhypopomus (Odontohypopomus) walteri Sullivan et al., 2013: 8, figs. 3-4, 8, color photographs of holotype, paratypes, backlit electric organs (original description and assignment to subgenus Odontohypopomus, type locality - Brazil, Amazonas, nr. Manaus, Amazonas dr.). -Carvalho, 2013: 181-185, figs. 41-43, position in phylogeny (phylogenetic systematics of Rhamphichthyoidea). -Crampton et al., 2016: 1-66, table 1, 3-4, figs. 1-7, 12, 18-20 (phylogeny, biogeography and ecology of Brachyhypopomus; Odontohypopomus not recognized).

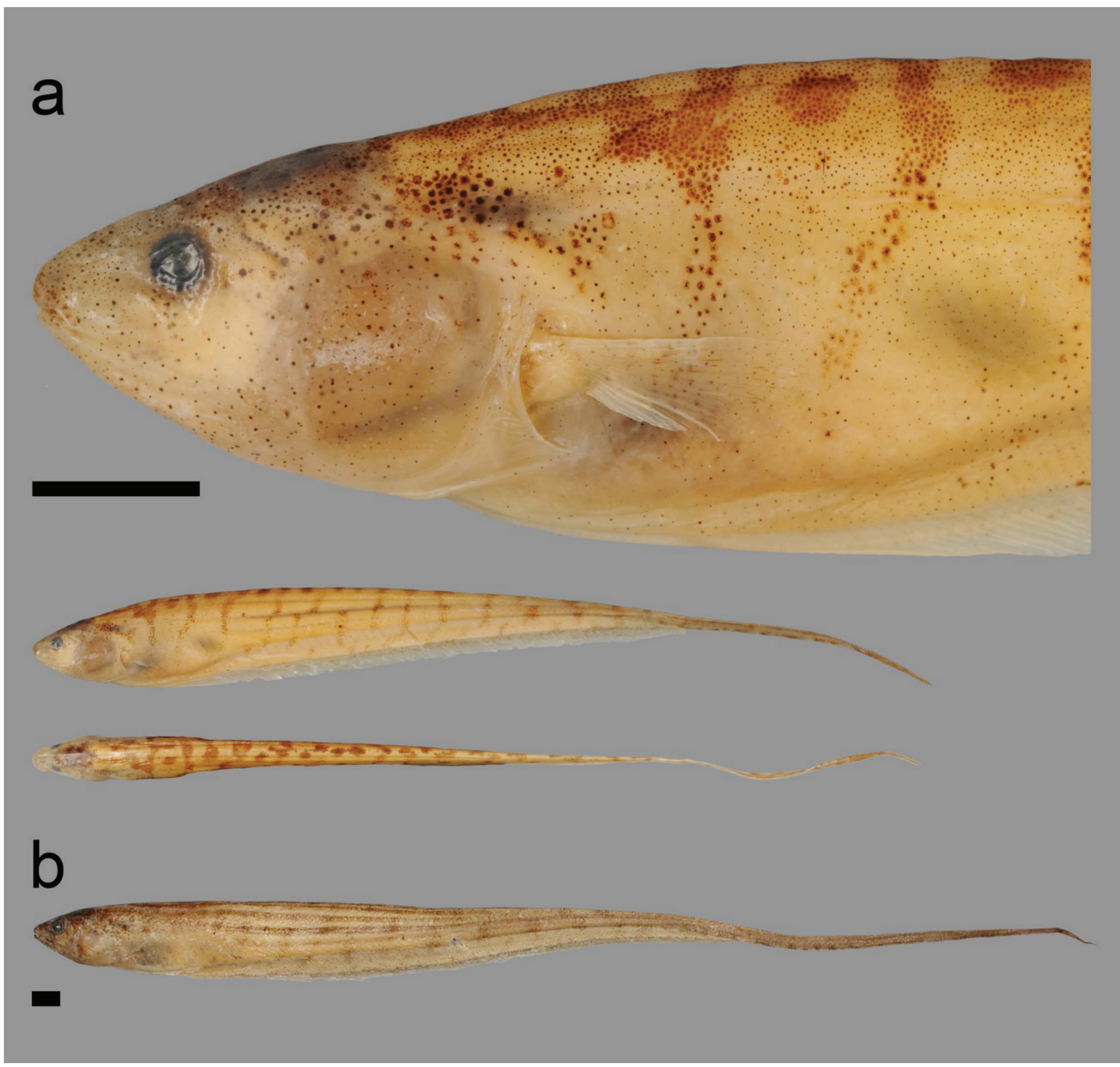

Fig. 46. Brachyhypopomus walteri. a. MCP 45291 (WC16.180598), female, $145 \mathrm{~mm}$ TL (head, and body in lateral and dorsal view); b. MCP 45292 (WC05.190598), male, 168 mm TL (body in lateral view); Brazil, rio Solimões-Japurá confluence, Amazonas dr. Note sexual dimorphism in caudal-filament length. Specimen fixed in formalin and preserved in EtOH. Scale bars $=5 \mathrm{~mm}$. 
Table 21. Morphometrics for Brachyhypopomus walteri. HT, holotype; SD, standard deviation; dr., drainage. Data for holotype (nr. Manaus, Amazonas, Brazil) from Sullivan et al. (2013). Range for type region (Central Amazonas drainage) refers to non-type specimens from the vicinity of Tefé, Amazonas, Brazil. Specimens from the Upper rio Paraguay drainage are from Mato Grosso and Mato Grosso do Sul, Brazil.

\begin{tabular}{|c|c|c|c|c|c|c|c|c|c|}
\hline & \multicolumn{5}{|c|}{ Central Amazonas dr. (type region) } & \multicolumn{4}{|c|}{ Upper Paraguay drainage } \\
\hline & HT & Range & Mean & $\mathrm{SD}$ & $\mathrm{n}$ & Range & Mean & SD & $\mathrm{n}$ \\
\hline Total length (TL) (mm) & 163 & $75-180$ & 127 & 24.8 & 86 & $106-154$ & 128 & 16.6 & 14 \\
\hline Length to end of anal fin (LEA) (mm) & 126 & $55-131$ & 92 & 16.8 & 86 & $72-106$ & 87 & 12.0 & 15 \\
\hline Head length (HL) (mm) & 14.4 & $9.0-16.0$ & 11.7 & 2.2 & 30 & $8.5-11.9$ & 10.0 & 1.0 & 15 \\
\hline \multicolumn{10}{|c|}{ Proportion of TL (\%) } \\
\hline Caudal filament length (CF) & 22.7 & $18.2-35.0$ & 27.5 & 3.6 & 86 & $29.0-36.1$ & 32.6 & 1.9 & 14 \\
\hline \multicolumn{10}{|c|}{ Proportion of LEA $(\%)$} \\
\hline Head length (HL) & 11.4 & $10.9-13.8$ & 12.1 & 0.8 & 29 & $10.2-12.2$ & 11.4 & 0.6 & 15 \\
\hline Snout to occiput (SO) & 8.5 & $8.9-11.8$ & 9.9 & 0.6 & 29 & 7.8-11.4 & 9.4 & 1.0 & 15 \\
\hline Snout to anal-fin origin (SA) & 19.9 & $17.8-26.6$ & 20.5 & 2.0 & 29 & $14.7-18.9$ & 16.7 & 1.2 & 15 \\
\hline Snout to pectoral-fin base (PP) & & $12.7-15.2$ & 13.7 & 0.7 & 29 & $9.7-14.0$ & 12.8 & 1.2 & 15 \\
\hline Longest pectoral-fin ray (PRL) & 5.4 & $4.5-7.7$ & 6.5 & 0.7 & 29 & $4.9-6.5$ & 5.7 & 0.5 & 15 \\
\hline Anal fin length (AF) & & $75.0-83.2$ & 80.3 & 2.1 & 27 & $80.8-85.9$ & 83.6 & 1.6 & 15 \\
\hline Longest anal-fin ray (ARL) & 4.6 & $1.3-3.5$ & 2.1 & 0.5 & 28 & $3.1-5.2$ & 4.1 & 0.5 & 15 \\
\hline Body depth at anal-fin origin (BD1) & & $9.2-15.2$ & 12.5 & 1.6 & 29 & $9.3-12.2$ & 10.6 & 0.8 & 15 \\
\hline Body width at anal-fin origin (BW1) & & $3.9-7.0$ & 5.7 & 0.7 & 29 & $4.5-5.6$ & 5.2 & 0.4 & 15 \\
\hline Body depth at anal-fin middle (BD2) & & $6.6-11.2$ & 8.9 & 0.9 & 27 & $6.7-9.4$ & 8.3 & 0.7 & 15 \\
\hline Body width at anal-fin middle (BW2) & & $1.6-2.9$ & 2.2 & 0.3 & 27 & $1.6-3.3$ & 2.4 & 0.4 & 15 \\
\hline Caudal filament length (CF) & 29.4 & $27.9-52.8$ & 38.8 & 6.4 & 18 & $40.8-56.4$ & 48.6 & 4.1 & 14 \\
\hline \multicolumn{10}{|c|}{ Proportion of HL (\%) } \\
\hline Preorbital distance (PR) & 28.5 & $23.3-27.1$ & 25.4 & 1.1 & 30 & $20.3-31.6$ & 23.7 & 2.6 & 15 \\
\hline Mouth width (MW) & 22.9 & $17.1-23.3$ & 20.8 & 1.6 & 30 & $22.9-32.2$ & 28.7 & 2.7 & 15 \\
\hline Internarial distance (AE) & & $17.5-22.2$ & 19.7 & 1.2 & 30 & $17.7-21.2$ & 19.4 & 1.0 & 15 \\
\hline Posterior naris to eye (PE) & 3.4 & $2.0-5.0$ & 3.4 & 0.9 & 30 & $2.3-4.9$ & 3.8 & 0.7 & 15 \\
\hline Orbital diameter (OD) & 12.5 & $11.0-16.3$ & 13.8 & 1.5 & 30 & $12.0-15.6$ & 13.9 & 1.2 & 15 \\
\hline Interorbital distance (IO) & 23.9 & $18.8-24.4$ & 21.8 & 1.4 & 30 & $21.5-27.6$ & 24.3 & 2.2 & 15 \\
\hline Head depth at eye (HD1) & & $44.4-53.2$ & 49.7 & 2.0 & 30 & $45.2-51.7$ & 48.9 & 1.7 & 15 \\
\hline Head width at eye (HW1) & 42.2 & $28.9-42.9$ & 35.9 & 3.4 & 30 & $33.3-46.0$ & 39.1 & 3.5 & 15 \\
\hline Head depth at occiput (HD2) & 79.0 & $67.1-84.7$ & 76.3 & 3.7 & 30 & $72.9-84.4$ & 78.3 & 3.4 & 15 \\
\hline Head width at occiput (HW2) & & $46.2-60.5$ & 52.2 & 4.1 & 30 & $47.3-57.1$ & 52.6 & 2.6 & 15 \\
\hline Postorbital distance (PO) & & $60.0-67.1$ & 63.3 & 1.6 & 30 & $60.4-68.0$ & 65.1 & 2.2 & 15 \\
\hline Branchial aperture (BO) & 29.7 & $16.3-35.4$ & 26.3 & 4.3 & 30 & $19.0-30.3$ & 22.6 & 2.9 & 15 \\
\hline Pectoral-fin base (PB) & & $10.3-15.6$ & 13.4 & 1.4 & 30 & $10.3-14.1$ & 11.6 & 1.1 & 15 \\
\hline Caudal filament depth (CD) & 23.2 & $7.9-19.0$ & 14.6 & 2.4 & 29 & $10.3-16.6$ & 12.9 & 1.9 & 15 \\
\hline Caudal filament width (CW) & & $3.3-6.7$ & 4.6 & 0.9 & 29 & 4.3-7.0 & 6.0 & 0.8 & 15 \\
\hline \multicolumn{10}{|c|}{ Other proportions } \\
\hline BW1 (\% of BD1) & & $39.7-52.5$ & 46.1 & 3.4 & 30 & $42.3-53.2$ & 48.6 & 2.9 & 15 \\
\hline BW2 (\% of BD2) & & $18.9-28.1$ & 24.1 & 2.1 & 27 & $19.0-34.5$ & 28.7 & 3.7 & 15 \\
\hline
\end{tabular}

Diagnosis. Brachyhypopomus walteri is diagnosed from congeners by the following combination of characters: caudal filament length $27.8-56.4 \%$ LEA, vs. $7.4-26.8 \%$ in $B$. batesi, B. belindae, B. bennetti, B. palenque, B. provenzanoi, $B$. regani, and $B$. verdii; distinct dark suborbital stripe present, $v s$. absent in $B$. alberti, $B$. arrayae, $B$. beebei, $B$. benjamini, B. brevirostris, $B$. bullocki, B. diazi, $B$. draco, B. flavipomus, B. gauderio, B. janeiroensis, B. jureiae, B. menezesi, B. occidentalis, and B. pinnicaudatus; accessory electric organ over the opercular region absent, $v$ s. present in $B$. bombilla; absence of scattered conspicuous black or charcoal flecks on flanks, vs. presence in B. cunia, $B$. hendersoni, and B. sullivani.
Description. Head and body shape, and pigmentation illustrated in Figs. 11 and 46. Meristic and morphometric data for examined specimens presented in Tables 2-5 and 21. Body shallow to moderate in depth. Head short to moderate in length and shallow to moderate in depth. Dorsal profile of head approximately straight from occiput to snout, ventral profile of head gently convex between operculum and snout, snout rounded. Eye moderate in size. Upper jaw with moderate sigmoidal angle between premaxillary and maxillary portions in lateral view. No accessory electric organ over operculum. Gill filaments on first gill arch 3448 (median $39, \mathrm{n}=29$ ). Pectoral fin moderate to broad in width, pectoral-fin rays 12-17 (mode of 15 in populations 
from the region of the type localities in the central Amazon basin; 14 in populations from the Paraguay basin). Precaudal vertebrae 16-20 (mode of 18 in populations from the region of the type localities in the central Amazon basin, and from the Paraguay basin), including 1-2 transitional vertebrae. Anal-fin origin substantially (0.25-0.33 HL distance) anterior to tip of pectoral fin, never posterior. Anal-fin rays 192-235 (median of 210 in populations from the region of the type localities in the central Amazon basin; 226 in populations from the Paraguay basin). Dorsal rami of recurrent branch of anterior lateral line nerve usually visible. Middorsal region of body scaled. Rows of scales above lateral line 5-7 (mode 7). Lateral line continuous. Depigmented epidermal canals very sparse, almost unnoticeable, found mainly in middle section of body - as curved short lines (usually no more than one) either side of lateral line. Epidermal canals absent from either side of mid-dorsal region, and absent on flank midway from lateral line to dorsal midline. 3-4 bilateral columns at anal-fin terminus and at mid-point between anal-fin terminus and tip of caudal filament in immature, mature female, and mature male specimens. Three or four electrocyte columns sometimes alternate in middle of caudal filament. Caudal filament moderate in length to long.

Coloration. (Figs. 11, 46). Pigmentation strongly resembles that of $B$. bennetti. Background pale straw to tan. Dorsal region with irregular brown blotches which extend to irregular-edged and sometimes divided brown bands which extend towards lateral line. No pale mid-dorsal stripe extending from occiput to base of caudal filament. A series of brown blotches is usually present along lateral line. Flank ventral to lateral line and pterygiophore region with very sparse and indistinct disrupted vertical bands. Caudal filament darker than body, with indistinct dark blotches and disrupted vertical bands, often with darkened tip; darkened tip of caudal filament resembles root tips of water hyacinths when viewed underwater. Head darker, especially dorsally. Eye with prominent suborbital patch of chromatophores and subcutaneous pigmentation (rarely indistinct, never absent). Pectoral and anal-fin membranes hyaline. Pectoral and anal-fin rays hyaline with light scattering of brown chromatophores. Anal-fin ray pigmentation darker in posterior half of fin. Color in live individuals similar to preserved specimens, body sometimes with yellow-green tinge in waters with high sediment load, opercular region usually very rosy due to underlying gills.

Size. A Brachyhypopomus of moderate adult size, largest specimen examined $215 \mathrm{~mm}$ TL, $c a .150 \mathrm{~mm}$ LEA ( $\mathrm{n}=$ 1,400). Largest male specimen examined $195 \mathrm{~mm}$ TL, 136 mm LEA $(\mathrm{n}=25)$. Largest female specimen examined 201 $\mathrm{mm}$ TL, $140 \mathrm{~mm}$ LEA $(\mathrm{n}=55)$.

Sexual dimorphism. No salient sexual dimorphism in size. Breeding males develop elongated caudal filaments in comparison to immature individuals and breeding females
(Fig. 46a,b), but do not exhibit an elevated number of horizontal bilateral columns or vertical rows of electrocytes. Instead breeding males exhibit clearly enlarged electrocytes relative to immature specimens and females. Tip of caudal filament in large males never exhibits a paddle-like lateral compression. No sexual differences in pigmentation.

Geographic distribution. Bolivia, Brazil, Ecuador, Guyana, Paraguay, and Peru (Fig. 47). Widely distributed through the Amazon basin, including whitewater, clearwater and blackwater systems. Also known from the middle Essequibo, and from the Paraguay-Paraná drainage. Records from the Paraná-drainage are mostly from collections in the Pantanal Matogrossense in the northern río Paraguay, but a single specimen was collected from the río Paraná of Paraguay, at a latitude of $27^{\circ} 17^{\prime} 03^{\prime \prime}$ S. Sullivan et al. (2013) did not report $B$. walteri from the Paraná-Paraguay drainage.

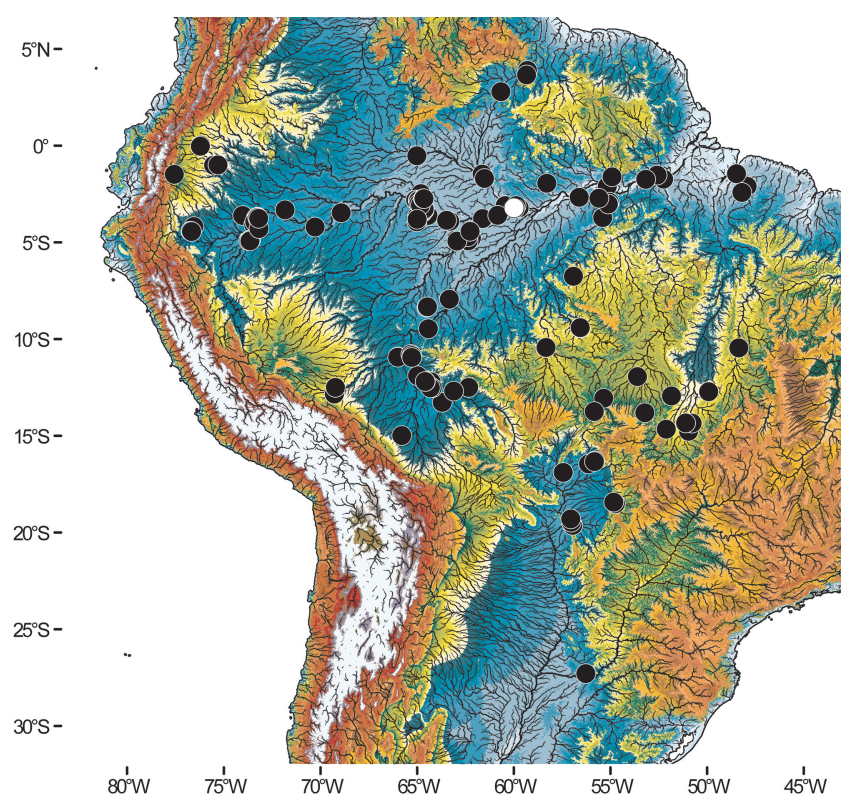

Fig. 47. Collection records for Brachyhypopomus walteri (circles). Holotype location is marked with an open symbol. Elevation data refers to altitude above mean sea level (see Fig. 2 for legend).

Population variation: We found overlap in the range of meristic counts (Tables 2-5) and morphometric measurements (Table 21), and observed similar pigmentation among populations of $B$. walteri from the central Amazon and from the upper Paraguay. These observations, in combination with molecular data (Crampton et al., 2016), support the hypothesis that all populations we have assigned to $B$. walteri are members of a single geographically widespread species, which is morphologically and genetically distinct from all congeners.

Ecological notes. Brachyhypopomus walteri is an ecologically cosmopolitan species that occurs in both high-conductivity and low-conductivity systems, but is 
usually more abundant in low-conductivity systems. It is a common component of floating meadow habitats in the high-conductivity whitewater floodplains of the Amazon River and its whitewater tributaries, and also along the margins of low-conductivity blackwater rivers such as the rio Negro, and rio Tefé. In the central and upper Amazon, in the vicinity of Tefé, Brazil, and Iquitos, Peru, $B$. walteri is much more common in floating meadow communities of normoxic low-conductivity blackwater systems than in seasonally hypoxic high-conductivity whitewater systems. Populations from central Amazon whitewater floodplains are able to tolerate protracted periods of experimentally induced hypoxia by undertaking aerial gill respiration (Crampton, 1998b). Brachyhypopomus walteri is also abundant in small low-conductivity terra firme rivers and streams, where it is associated with aquatic vegetation and submerged root masses and leaf litter. Sullivan et al. (2013) infer, incorrectly, that $B$. walteri is predominantly a white-water floodplain specialist that occurs in blackwater systems only near their confluence with whitewater systems. For instance, contrary to Sullivan et al. (2013), B. walteri is known from the rio Negro far from its confluence with the Amazon River near Manaus (Fig. 47). Breeding occurs during the rising and high water period in populations from Amazonian floodplain floating macrophytes (Crampton, 1996a: 165). Stomach contents in populations from the central Amazon comprise aquatic insect larvae, microcrustacea, and other small aquatic invertebrates - with a predominance of Chironomidae larvae (Crampton, 1996a: 136).

Co-occurring congeners: Because of its wide geographical range and occurrence in multiple habitats, $B$. walteri occurs in geographical sympatry and ecological syntopy with multiple congeners: in the Amazonas drainage, rio Essequibo, and coastal drainages of the Guianas: $B$. alberti, B. arrayae, B. batesi, B. beebei, B. belindae, $B$. benjamini, B. bennetti, B. bombilla, B. brevirostris, B. bullocki, B. cunia, B. flavipomus, B. hamiltoni, B. hendersoni, B. pinnicaudatus, B. regani, $B$. sullivani, and $B$. verdii; and in the río Paraná-Paraguay drainage with $B$. bombilla, B. brevirostris, B. draco, and B. gauderio.

Local names. Bolivia: cuchillo; Brazil: sarapó (north), tuvira (south); Ecuador: cuchillo, yayo; Guyana: knife fish; Paraguay: morenita; Peru: macana.

Material examined. 1,032 specimens. Bolivia. Beni (localities from rio Madeira dr., Amazonas dr.) AMNH 39773, 5, 96-113 mm, $6 \mathrm{~km}$ SW Costa Marques (Brazil), río Baures (río Blanco) at confluence with río Iténez (río Guaporé), affl. río Mamoré, $12^{\circ} 30^{\prime} 10^{\prime \prime} \mathrm{S}, 064^{\circ} 16^{\prime} 47^{\prime \prime} \mathrm{W}$. AMNH 40086 (part), 1, $121 \mathrm{~mm}$, rio Baures, $2 \mathrm{~km}$ above mouth, affl. rio Guaporé, affl. río Mamoré, $12^{\circ} 30^{\prime} 32^{\prime \prime} \mathrm{S}, 064^{\circ} 16^{\prime} 46^{\prime \prime} \mathrm{W}$. CBF 10256, 1, immature, $117 \mathrm{~mm}$, CBF 10257, 1, immature, $116 \mathrm{~mm}$, mun. Riberalta, stream nr. lago San José, affl. río Beni, 1054'47"S, 06559'49"W. CBF 10258, 1, immature, $115 \mathrm{~mm}$, UMSS 7020, 1, immature, $137 \mathrm{~mm}$, UMSS 7021, 1, female, $201 \mathrm{~mm}$, UMSS 7022, 1, immature, $102 \mathrm{~mm}$,
UMSS 7023, 1, immature, $126 \mathrm{~mm}$, mun. Riberalta, stream nr. San José, affl. río Beni, $10^{\circ} 55^{\prime} 32^{\prime \prime} \mathrm{S}, 066^{\circ} 00^{\prime} 36^{\prime \prime} \mathrm{W}$. INHS 37193, 1, 89 $\mathrm{mm}, 46 \mathrm{~km}$ E Estación Biológica del Beni on rd. to San Ignacio, río Moseruna, affl. río Apere, affl. río Mamoré, ca. $14^{\circ} 59^{\prime} \mathrm{S}$, 06548’W. UF 82009, 2, 96-142 mm, Bella Vista, río Blanco (río Baures), affl. río San Martín, affl. río Guaporé, affl. río Mamoré, $13^{\circ} 16^{\prime} \mathrm{S}, 063^{\circ} 42^{\prime} \mathrm{W}$. UMMZ 204297, 2, 89-105 mm, $2 \mathrm{~km} \mathrm{SW}$ Costa Marques (Brazil), río Iténez (río Guaporé), affl. río Mamoré, 12²9'30"S, 064²15'24"W. UMMZ 204632, 6, 90-142 mm, 5 km SW Costa Marques, río Iténez, nr. mouth of río Baures (río Blanco), affl. río Guaporé, affl. río Mamoré, $12^{\circ} 30^{\prime} 30^{\prime \prime} \mathrm{S}$, 064¹9'00"W. UMMZ 204829, 1, 80 mm, UMMZ 246439, 3, 78$114 \mathrm{~mm}$, río Baures (río Blanco), nr. mouth, L bank río Iténez (río Guaporé), affl. río Mamoré, 12³1'06"S, 064¹9'00"W. UMMZ 205163, 3, 87-108 mm, $10 \mathrm{~km}$ SE Costa Marques (Brazil), backwater of río Iténez (río Guaporé), affl. río Mamoré, $12^{\circ} 30^{\prime} 37^{\prime \prime} \mathrm{S}, 064^{\circ} 12^{\prime} 59^{\prime \prime} \mathrm{W}$. Brazil. Amazonas (localities from Amazonas dr.; localities listed from the Mamirauá Reserve [Reserva de Desenvolvimento Sustentável Mamirauá] are in rio Solimões-Japurá floodplain, mun. Alvarães). ANSP 194025, 6 (1CS), 60-126 mm LEA, INPA 30241, 8, 64-114 mm LEA, mun. São Paulo de Olivença, Monte Sinai, Rio Camatiã, affl. rio Solimões, $03^{\circ} 27^{\prime} 34^{\prime \prime} \mathrm{S}, 068^{\circ} 56^{\prime} 00^{\prime \prime} \mathrm{W}$ [coordinates for São Paulo de Olivença] (listed in Sullivan et al., 2013). BMNH 1998.3.12.83101, 19 (3 immature, 136-184 mm, 1 male, 142 mm, 15 unsexed/ measured), MCP 46933, 1 (CS), 70 mm, Mamirauá Reserve, lago Rato, 0303'01"S, 06452'23"W. BMNH 1998.3.12.102, 1, male, $186 \mathrm{~mm}$, mun. Tefé, Cabeceira do lago Tefé, rio Tefé, $03^{\circ} 38^{\prime} 01^{\prime \prime} \mathrm{S}$, 06457'59"W. BMNH 1998.3.12.103-104, 2, female, 150-160

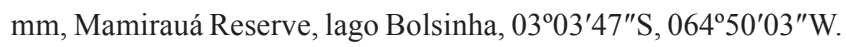
BMNH 1998.3.12.105, 1, female, 166 mm, MCP 45445, 33, 79$160 \mathrm{~mm}$, Mamirauá Reserve, cano do lago Rato, $03^{\circ} 02^{\prime} 41^{\prime \prime} \mathrm{S}$, 064'51'26"W. BMNH 1998.3.12.106-107, 2, female, 163-191 mm, MCP 45490, 2 (1 female, $152 \mathrm{~mm}, 1$ male, $167 \mathrm{~mm}$ ), Mamirauá Reserve, Ressaca do Caetono, 02 $50^{\prime} 15^{\prime \prime} \mathrm{S}$, $064^{\circ} 55^{\prime} 50^{\prime \prime} \mathrm{W}$. CU 97641, 1 (paratype), $80 \mathrm{~mm}$, rio Negro upstream of Manaus, rio Negro, $03^{\circ} 05^{\prime} 23^{\prime \prime} \mathrm{S}, 060^{\circ} 27^{\prime} 01^{\prime \prime} \mathrm{W}$. CU 97642, 5 (paratypes), 95-104 mm LEA, rio Negro upstream of Manaus, rio Negro, $03^{\circ} 05^{\prime} 35^{\prime \prime} \mathrm{S}, 060^{\circ} 26^{\prime} 50^{\prime \prime} \mathrm{W}$. IDSM 457, 6, 72-130 mm, MCP 45297, 1, female, $138 \mathrm{~mm}$, mun. Tefé, lago Martelo, rio Tefé, 0346'56"S, 06459'39"W. INHS 44484, 4, 101-140 mm, mun. Manaus, inlet to lago Camaleão, Ilha da Marchantaria, rio Solimões floodplain, Amazonas dr., 03¹4'22"S, 059 56'37"W. INHS 70542 (part), 4, 71-162 mm LEA, lago Camaleão, Ilha da Marchantaria, rio Solimões floodplain, $03^{\circ} 14^{\prime} 22^{\prime \prime} \mathrm{S}, 059^{\circ} 56^{\prime} 37^{\prime \prime} \mathrm{W}$. INPA 8880, 3 (paratypes), 108-116 mm LEA, 1 examined from photograph in original description (164 mm), INPA 8939, 1 (paratype), $125 \mathrm{~mm}$ LEA, examined from photograph in original description, INPA 8941, 1 (holotype), 163 mm TL, 126 mm LEA, examined from photograph in original description, Paraná do Paracuúba, nr. mouth of rio Negro and entrance to lago Januari, rio Solimões floodplain, 0312'36"S, 059 59'24"W. INPA 9945, 31, 51-143 mm, Mamirauá Reserve, lago Caetano, 02 ${ }^{\circ} 50^{\prime} 58^{\prime \prime} \mathrm{S}$, $064^{\circ} 55^{\prime} 42^{\prime \prime} \mathrm{W}$. INPA 17112, 20, 58-132 mm, Paraná do Seixo, lago Jari, rio Purus, 0454'41"S, 062 27'26"W. INPA 17121, 1, 92 mm, Paraná 2, nr. Arumã, rio Purus, 0446'24"S, 062 ${ }^{\circ} 11^{\prime} 35^{\prime \prime} \mathrm{W}$. INPA 
17161, 2, 92-108 mm, INPA 19935, 2, 72-102 mm, lake nr. Sacado da Santa Luzia, rio Purus, $04^{\circ} 42^{\prime} 17^{\prime \prime} \mathrm{S}, 062^{\circ} 22^{\prime} 26^{\prime \prime} \mathrm{W}$. INPA 17170 , 1, $82 \mathrm{~mm}$, lago Sacado, rio Purus, 04 $42^{\prime} 49^{\prime \prime} \mathrm{S}, 062^{\circ} 23^{\prime} 55^{\prime \prime} \mathrm{W}$. INPA 17192, 26, 72-127 mm, igarapé de Duas Bocas, Paraná do Jari, rio Purus, $04^{\circ} 55^{\prime} 33^{\prime \prime} \mathrm{S}, 062^{\circ} 21^{\prime} 58^{\prime \prime} \mathrm{W}$. INPA 19938, 2, 70-94 $\mathrm{mm}$, lago Campina, rio Purus, 0455'59"S, 062 56'25"W. INPA 19940, 1, 58 mm, Paraná do Rei, Ilha Careiro, rio Amazonas, Amazonas dr., $03^{\circ} 12^{\prime} \mathrm{S}, 059^{\circ} 44^{\prime} \mathrm{W}$. INPA 29259, 12, mun. Beruri, igarapé Ajará, lago Ayapá, affl. rio Purus, 04²5'07"S, 062 ${ }^{\circ} 15^{\prime} 36^{\prime \prime} \mathrm{W}$ (listed in Sullivan et al., 2013). INPA 33253, 3, mun. Manacapuru,

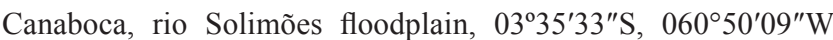
(listed in Sullivan et al., 2013). INPA 33268, 3, mun. Coari, rio Solimões floodplain, 0351'10"S, 062 $28^{\circ} 07^{\prime \prime} \mathrm{W}$ (listed in Sullivan et al., 2013). INPA 39074, 1, mun. São Sebastião do Uatamã, São José do Jabote, rio Uatamã, $01^{\circ} 56^{\prime} 12^{\prime \prime} \mathrm{S}, 058^{\circ} 17^{\prime} 47^{\prime \prime} \mathrm{W}$ (listed in Sullivan et al., 2013). MCP 33368, 1, 124 mm, MCP 44649, 2 (1 female [CS], $140 \mathrm{~mm}, 1$ male [CS], $185 \mathrm{~mm}$ ), Mamirauá Reserve, lago Curuçá Comprido, 0305'31"S, 06448'58"W. MCP 33369, 1, $141 \mathrm{~mm}$, Mamirauá Reserve, lago Curuçá Redondo, 0306'13"S, 0644'06"W. MCP 41033 (part), 1, 109 mm, mun. Canutama, igarapé São João on hwy. BR-319, ca. 60 km S Humaitá, affl. rio Ipixuna, affl. rio Purus, 0756'07"S, 06320'03"W. MCP 44607, 1, female (CS), 150 mm, MCP 45488, 1, female, 116 mm, Mamirauá Reserve, lago Periquito Comprido, 030' $57^{\prime \prime} \mathrm{S}, 064^{\circ} 46^{\prime} 42^{\prime \prime} \mathrm{W}$. MCP 44741, 2, immature (1CS), 126-146 mm, MCP 45296, 3, immature, 110-123 mm, MCP 45372, 2, male, 137-146 mm, MCP 45415, 4, 82-129 mm, MCP 45440, 2, immature, 100-136 mm, MCP 45478, 1, female, 141 mm, mun. Tefé, igarapé Repartimento, $1.5 \mathrm{~km}$ downstream Estrada Agrovila rd., affl. Lago Tefé (rio Tefé), 032 $4^{\prime} 25^{\prime \prime} \mathrm{S}, 064^{\circ} 44^{\prime} 08^{\prime \prime} \mathrm{W}$. MCP 44742, 6 (2 immature, 125-142 mm, 4 female [1CS], 104-149 mm), Mamirauá Reserve, lago Periquito Comprido, 0304'57"S, 06446'42"W. MCP 44743, 11 (10 immature [2CS], 86-124 mm, 1 female, $100 \mathrm{~mm}$ ), mun. Tefé, rio Tefé, $03^{\circ} 37^{\prime} 43^{\prime \prime} \mathrm{S}$, 064 $59^{\prime} 03^{\prime \prime} \mathrm{W}$. MCP 45289, 1, immature, 122 mm, MCP 45298, 1, female, 123 mm, MCP 45477, 1, male, $155 \mathrm{~mm}$, mun. Tefé, igarapé Curupira, Estrada Agrovila

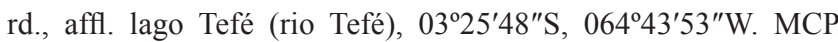
45290, 1, male, 152 mm, MCP 45442, 1, male, 165 mm, Mamirauá Reserve, cano do lago Sapucaia, 030.'07"S, 06448'32"W. MCP 45292, 6 (5 female, 120-153 mm, 1 male, $168 \mathrm{~mm}$ ), Mamirauá Reserve, lago Promessa, 0304'29"S, 064²7'02"W. MCP 45293, 3 (2 female, 135-168 mm, 1, male, $135 \mathrm{~mm}$ ), Mamirauá Reserve, lago Curuçá Aberto, 0306'07"S, 06449'10"W. MCP 45294, 1, female, $156 \mathrm{~mm}$, mun. Tefé, lago Caiambé, rio Caiambé, $03^{\circ} 35^{\prime} 40^{\prime \prime} \mathrm{S}, 064^{\circ} 26^{\prime} 58^{\prime \prime} \mathrm{W}$. MCP 45291, 1, female, $145 \mathrm{~mm}$, MCP 45295, 1, female, $176 \mathrm{~mm}$, MCP 45458, 1, male, $161 \mathrm{~mm}$, Mamirauá Reserve, lago Secretaria, $03^{\circ} 07^{\prime} 12^{\prime \prime} \mathrm{S}, 064^{\circ} 47^{\prime} 49^{\prime \prime} \mathrm{W}$. MCP 45306, 1, immature, 132 mm, mun. Maraã, lago Amanã, Igarapé Juá Grande, rio Japurá, $02^{\circ} 28^{\prime} 50^{\prime \prime} \mathrm{S}, 064^{\circ} 48^{\prime} 50^{\prime \prime} \mathrm{W}$. MCP 45314, 5 (4 immature, 95-116 mm, 1 male, $138 \mathrm{~mm}$ ), MCP 45417, 1, 98 mm, MCP 45371, 1, female, 96 mm, MCP 45373, 3 (1 immature, $94 \mathrm{~mm}, 2$ male, 123-125 mm), mun. Tefé, igarapé Xidarinini, affl. lago Tefé (rio Tefé), 0322'46"S, 064²41'17"W. MCP 45320, 1, immature, $130 \mathrm{~mm}$, Mamirauá Reserve, cano do lago Mamirauá, 0302'41"S, 064 $51^{\prime} 26^{\prime \prime} \mathrm{W}$. MCP 45327, 1, immature, $105 \mathrm{~mm}$, Mamirauá Reserve, lago Sumaumeirinha I, $02^{\circ} 48^{\prime} 26^{\prime \prime} \mathrm{S}, 065^{\circ} 04^{\prime} 33^{\prime \prime} \mathrm{W}$. MCP 45412, 1, female, $153 \mathrm{~mm}, \mathrm{MCP}$ 45444, 2 (1 female, 166 mm, 1 male, 165 mm), Mamirauá Reserve, Ressaca da Vila Alencar, 0307'41"S, 06448'04"W. MCP 45413, 1, male, $181 \mathrm{~mm}$, Mamirauá Reserve, cano do lago Mamirauá, 0304'29"S, 06448'29"W. MCP 45418, 23, 54-150 mm, mun. Tefé, lago Jacaré, rio Solimões floodplain, $03^{\circ} 11^{\prime} 11^{\prime \prime} \mathrm{S}$, $064^{\circ} 43^{\prime} 05^{\prime \prime} \mathrm{W}$. MCP 45441, 1, female, $142 \mathrm{~mm}$, Mamirauá Reserve, lago Pirarara, 0257'04"S, 06450'04"W. MCP 45443, 1, female, $181 \mathrm{~mm}$, Mamirauá Reserve, Ressaca do Itu, 0249'51"S, 06457'11"W. MCP 45479, 1, 124 mm, mun. Tefé, rio Solimões, nr. mouth Paraná Capivara, 0316 $37^{\prime \prime S}$, 064³7'42"W. MCP 45321, 1, immature, $141 \mathrm{~mm}$, MCP 45487, 1, male, $184 \mathrm{~mm}$, Mamirauá Reserve, lago Bolsinha, 030. $03^{\prime} 47^{\prime \prime} \mathrm{S}, 064^{\circ} 50^{\prime} 03^{\prime \prime} \mathrm{W}$. MCP 45414, 1, female, $173 \mathrm{~mm}$, MCP 45683, 20, 86-162 mm, mun. Tefé, Ilha Panamim, rio Solimões floodplain, 03¹9'29"S, 064³8'37"W. MCZ 2769, 4, 99-160 mm, mun. Manaus, rio Negro nr. lago Alexo [lago do Aleixo, E Manaus], 0305'28"S, 059 53'09"W. MCZ 9427 (part), 1, 85 mm, mun. Manaus, lago Hyanuary [lago do Janauari], rio Amazonas floodplain, 03ำ' $13^{\prime} \mathrm{S}$, 06000’W. MPEG 967 (part), 29, 42-137 mm, MPEG 970 (part), 3, 57-105 mm, MPEG 971 (part), 1, 47 mm, MZUSP 30063 (part), 1, 50 mm, MZUSP 30065 (part), 1, 32 mm, MZUSP 44060 (part), 3, 52-62 mm, MZUSP 85816, 4, 77-133 mm, mun. Tefé, lago Jurupari, rio Tefé, $03^{\circ} 48^{\prime} \mathrm{S}, 065^{\circ} 00^{\prime} \mathrm{W}$. MPEG 1123 (part), 5, 65$85 \mathrm{~mm}$, mun. Tefé, Costa Capivara, rio Solimões, ca. 03¹6'38"S, 064³8'09"W. MPEG 22747, 3, 113-151 mm, Mamirauá Reserve, lago Mamirauá, 0259'38"S, 06454'26"W. MPEG 22748, 3, 90 $145 \mathrm{~mm}$, Mamirauá Reserve, lago Bolsinho, 0303'47"S, 0644'ㄷ'ㄱ. MZUSP 7803, 1, 123 mm, mun. Parintins, stream affl. lago José Açu, $02^{\circ} 40^{\prime} \mathrm{S}, 056^{\circ} 37^{\prime} \mathrm{W}$. MZUSP 23282, 1, 107 $\mathrm{mm}$, mun. Coari, Ilha Surubim, upstream Coari, branch of rio Solimões, 03 ${ }^{\circ} 54^{\prime} \mathrm{S}, 063^{\circ} 19^{\prime} \mathrm{W}$. MZUSP 28054 (part), 1, $78 \mathrm{~mm}$, MZUSP 28055, 1, 96 mm, Moura, Pedra do Gavião, rio Negro, $01^{\circ} 28^{\prime} \mathrm{S}, 061^{\circ} 38^{\prime} \mathrm{W}$. MZUSP 30049 (part), 8, 42-60 mm, MZUSP 30054, 1, 58 mm, mun. Tefé, Vista Escura, rio Tefé, no coordinates. MZUSP 30051, 1, 68 mm, MZUSP 30056, 1, 50 mm, MZUSP 30061 (part), 28, 37-103 mm, mun. Tefé, lago Mucura, rio Tefé, no coordinates. MZUSP 30052 (part), 1, 78 mm, mun. Tefé, Mastro, rio Tefé, no coordinates. MZUSP 30058 (part), 2, 114-115 mm, mun. Maraã, lago Amanã, canal do lago Amanã, affl. rio Japurá, $02^{\circ} 44^{\prime} \mathrm{S}, 6^{\circ} 41^{\prime} \mathrm{W}$. MZUSP 75571 (part), 4, 106-152 mm, USNM 306903, 1, 136 mm, USNM 306919, 1, 75 mm LEA, USNM 374466, 4, 135-155 mm, lago Camaleão, Ilha da Marchantaria, rio Solimões floodplain, 03¹4'22"S, 059 56'37"W. MZUSP 78032 (part), 1, 110, mun. Maraã, Paraná do Castanho, lago Amanã, affl. rio Japurá, 0244'S, 064³9’W. MZUSP 78954 (part), 1, 92 mm, Costa do Anori, nr. mouth of rio Purus, rio Solimões, $03^{\circ} 46^{\prime} \mathrm{S}$, $061^{\circ} 37^{\prime} \mathrm{W}$. MZUSP 85814, 1, $55 \mathrm{~mm}$, mun. Tefé, Jauari-atuba, rio Tefé, $c a .03^{\circ} 56^{\prime} \mathrm{S}, 065^{\circ} 01^{\prime} \mathrm{W}$. MZUSP 94038, 1, $100 \mathrm{~mm}$, MZUSP 99170, 7, 60-135 mm, canal do lago Amanã, affl. rio Japurá, ca. $02^{\circ} 44^{\prime} \mathrm{S}, 064^{\circ} 39^{\prime} \mathrm{W}$. MZUSP 95226 (part), 1, $100 \mathrm{~mm}$, mun. Santa Isabel do rio Negro, Paricatuba, lake on margin of rio Negro, $c a$. $00^{\circ} 31^{\prime} \mathrm{S}, 065^{\circ} 01^{\prime} \mathrm{W}$. USNM 300978, 1, $94 \mathrm{~mm}$, rio Unini, affl. rio Negro, $01^{\circ} 41^{\prime} \mathrm{S}, 061^{\circ} 31^{\prime} \mathrm{W}$ [coordinates for mouth of rio Unini]. USNM 306874, 2, 91-100 mm LEA, Paraná da Ilha da Marchantaria, rio Solimões floodplain, $c a$. $03^{\circ} 14^{\prime} 22^{\prime \prime} \mathrm{S}$, 
059 $56^{\prime} 37^{\prime \prime}$ W. Goiás (localities from rio Araguaia dr., affl. rio Tocantins, Amazonas dr.). AUM 45436, 9, 107-187 mm, mun. Aruanã, Fazenda Sobrado, margin Aruanã-Cocalinho rd., 1445'51"S, 05057'50"W. MZUSP 89218 (part), 1, $114 \mathrm{~mm}$, mun. Aruanã, Fazenda Viplan, on hwy. GO 334, floodplain of rio do Peixe, $14^{\circ} 20^{\prime} 11^{\prime \prime} \mathrm{S}, 050^{\circ} 46^{\prime} 31^{\prime \prime} \mathrm{W}$. Mato Grosso. BMNH 1900.4.14.91-94 (part), 1, 98 mm, mun. Carandàzinho, rio Cuiabá, affl. rio Paraguai, Paraná dr., ca. $16^{\circ} 27^{\prime} \mathrm{S}, 056^{\circ} 08^{\prime} \mathrm{W}$. BMNH 1982.9.24.132, 1, $78 \mathrm{~mm}$, Corgo do Gato [córrego do Gato], on hwy. BR-158, affl. rio Suiazinho, affl. rio Suiá Missu, affl. rio Xingu, Amazonas dr., ca. 12 ${ }^{\circ} 56^{\prime} \mathrm{S}, 051^{\circ} 51^{\prime} \mathrm{W}$. MCP 30786, 1, 106 mm, mun. Nova Ubiratã, rio Celeste $c a .9 \mathrm{~km}$ W Nova Ubiratã on rd. to Sorriso, affl. rio Tapajós, Amazonas dr., $13^{\circ} 03^{\prime} 08^{\prime \prime} \mathrm{S}$, $055^{\circ} 21^{\prime} 13^{\prime \prime W}$. MCP 44451, 1, 87 mm, mun. Nova Mutum, Córrego Ranchão on Nova Mutum-Santa Rita do Trivelato hwy., MT-235, affl. rio Tapajós, Amazonas dr., $13^{\circ} 44^{\prime} 35^{\prime \prime} \mathrm{S}, 055^{\circ} 50^{\prime} 08^{\prime \prime} \mathrm{W}$. MZUSP 25138, 2, 102-111 mm, MZUSP 25143, 1, 147 mm, mun. Cáceres, Ilha da Taiamã [Estação Ecológica Taiamã], rio Paraguai, Paraná dr., $16^{\circ} 53^{\prime} \mathrm{S}, 057^{\circ} 27^{\prime} \mathrm{W}$ [coordinates from center Ilha da Taiamã]. MZUSP 57478, 1, 67 mm, mun. Xavantina, rio Areões, affl. rio das Mortes, affl. rio Araguaia, affl. rio Tocantins, Amazonas dr., $14^{\circ} 39^{\prime} \mathrm{S}, 052^{\circ} 07^{\prime} \mathrm{W}$. MZUSP $77724,6,108-147 \mathrm{~mm}$, rio Juruena, ca. $1 \mathrm{~km}$ upstream mouth rio Arinos, affl. rio Tapajós, Amazonas dr., ca. $10^{\circ} 26^{\prime} \mathrm{S}, 58^{\circ} 20^{\prime} \mathrm{W}$. MZUSP 88593, 15, 83-215 mm, mun. Cocalinho, stream, affl. Corixo da Saudade, ca. $17 \mathrm{~km}$ NW Cocalinho on hwy. MT-326, affl. rio Araguaia, aff rio Tocantins, Amazonas dr., 14 $19^{\prime} 35^{\prime \prime} \mathrm{N}, 051^{\circ} 06^{\prime} 25^{\prime \prime} \mathrm{W}$. MZUSP 95068 (part), 18, 93-155 mm, MZUSP 100307, 18, 89-152 mm, mun. Barão de Melgaço, rio Mutum, between Vila de Mimoso and Joselândia (Pantanal de Paiaguás), rio Paraguai, Paraná dr., $16^{\circ} 19^{\prime} 30^{\prime \prime} \mathrm{S}, 055^{\circ} 49^{\prime} 59^{\prime \prime} \mathrm{W}$. MZUSP 95736, 40, 72-179 mm, stream, affl. rio Culuene, on rd. to Cachoeira do Adelino, affl. rio Xingu, Amazonas dr., 134ㅇ' $12^{\prime \prime} \mathrm{S}, 053^{\circ} 13^{\prime} 35^{\prime \prime} \mathrm{W}$. MZUSP 96252 , 3, 94-106 mm, mun. Paranaíta, rio Teles Pires, affl. rio Tapajós, Amazonas dr., 09²5'44"S, 056³2'36"W. MZUSP 96667, 1, 87 mm, mun. Barão de Melgaço, swamp, 1 km Vila de Mimoso, Pantanal de Paiaguás, affl. rio Cuiabá, affl. rio Paraguai, Paraná dr., ca. $16^{\circ} 17^{\prime} \mathrm{S}, 055^{\circ} 48^{\prime} \mathrm{W}$. MZUSP 100084,2 , 75-110 mm, mun. Paranaíta, just upstream of Sete Quedas, rio Teles Pires, affl. rio Tapajós, Amazonas dr., 09²4'05"S, 056³3'49"W. USNM 301981, 2, 103-124 mm, rio Batovi, affl. rio Xingu, Amazonas dr., ca. $11^{\circ} 56^{\prime} \mathrm{S}, 053^{\circ} 36^{\prime} \mathrm{W}$. Mato Grosso do Sul (localities from rio Paraguai dr., Paraná dr.). FMNH 108538, 8, 55-91 mm, Corumbá, bay on left margin rio Vermelho, affl. rio Miranda, 19³7'13"S, $056^{\circ} 57^{\prime} 16^{\prime \prime} \mathrm{W}$. MUSM 17103, 2, 84-95 mm, side channel, rio Abobral, 19²7'22"S, 05701'38"W. MZUSP 48311 (part), 1, 98 mm, MZUSP 48312, 3, 85-95 mm, mun. Coxim, Fazenda Santo Antonio (Baía da Sede), Pantanal do Paiaguás, affl. rio Coxim (rio Taquari), ca. $18^{\circ} 30^{\prime} \mathrm{S}, 054^{\circ} 45^{\prime} \mathrm{W}$. MZUSP 59443, 25, 83-133 mm, mun. Corumbá, on rd. from Nhecolândia to BR-262, rio Negro, 19¹7'16"S, 05703'39"W. MZUSP 83607 (part), 1, 87 mm, mun. Coxim, Paraguai Lagoa, source of Ribeirao dos Veados, affl. rio Coxim (rio Taquari), 18²5'21"S, 054 ${ }^{\circ} 50^{\prime} 06^{\prime \prime} \mathrm{W}$. Pará (localities from Amazonas dr.). ANSP 194517, 32, 74-140 mm, mun. Porto de Moz, beach, $3 \mathrm{~km}$ downstream Porto de Moz, rio Xingu, $01^{\circ} 43^{\prime} 54^{\prime \prime} \mathrm{S}, 052^{\circ} 15^{\prime} 16^{\prime \prime} \mathrm{W}$. INPA 4209, 2, 103-155, Ilha de
Babaguara, rio Xingu (no coordinates). INPA 7267, 2, 108-110 $\mathrm{mm}$, rio Tapajós, nr. mouth rio Cupari, ca. $03^{\circ} 43^{\prime} \mathrm{S}, 055^{\circ} 24^{\prime} \mathrm{W}$. INPA 32703, 1, Jacareacanga, igarapé do Cocho, rio Crepori, affl. rio Tapajós, 06 $44^{\prime} 54^{\prime \prime} \mathrm{S}$, 056 $6^{\circ} 4^{\prime} 43^{\prime \prime} \mathrm{W}$ (listed in Sullivan et al., 2013). INPA 33192, 1, mun. Almeirim, Paranaguara, rio Amazonas floodplain, $01^{\circ} 44^{\prime} 29^{\prime \prime} \mathrm{S}, 053^{\circ} 10^{\prime} 15^{\prime \prime} \mathrm{W}$ (listed in Sullivan et al., 2013). MCP 23359 (part), 1, 107 mm, Concórdia do Pará, rio Bujarú, affl. rio Guamá, affl. Baía de Marajó, ca. $02^{\circ} 06^{\prime} \mathrm{S}$, 04756'W. MCZ 9440, 1, 52 mm, Belém and environs., Báia de Marajó, ca. $01^{\circ} 27^{\prime} \mathrm{S}, 048^{\circ} 29^{\prime} \mathrm{W}$. MCP 49398, 3, mun. Santarém, Cachoerinha do Mentai, rio Mentai, affl. Rio Arapiuns, affl. Rio Tapajós, 0243'12"S, 055³6'15"W. MCP 49400, 7, mun. Curuá, Lago Preto, Ilha São Luiz, rio Amazonas floodplain, $02^{\circ} 06^{\prime} 22^{\prime \prime} \mathrm{S}$, $055^{\circ} 10^{\prime} 17^{\prime \prime} \mathrm{W}$. MCP 49411, 4, mun. Alenquer, small stream affl.

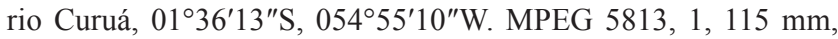
mun. Almeirim, Pesqueiro da Ilha, no precise locality, rio Amazonas, $01^{\circ} 31^{\prime} \mathrm{S}, 052^{\circ} 35^{\prime} \mathrm{W}$ [coordinates for Almeirim]. MPEG 9758, 10, 46-105 mm, mun. Tomé-Açu, igarapé Arrainha, affl. rio Acará-Mirim, affl. rio Acará, affl. Baía de Marajó, $02^{\circ} 25^{\prime} 11^{\prime \prime} \mathrm{S}, 048^{\circ} 12^{\prime} 13^{\prime \prime} \mathrm{W}$. ZUEC 12328, 1, mun. Belterra, Igarapé do Mato Grosso, nr. Jaguarari, affl. rio Tapajós, $02^{\circ} 55^{\prime} 01^{\prime \prime} \mathrm{S}$, $055^{\circ} 03^{\prime} 54^{\prime \prime} \mathrm{W}$. ZUEC 12329, 1, Igarapé Jandá, nr. Piquiatuba, affl. rio Tapajós, $03^{\circ} 00^{\prime} 37^{\prime \prime S}$, 05506' $16^{\prime \prime} \mathrm{W}$. ZUEC 12330, 1, mun. Belterra, Igarapé Dominguinho, nr. Piquiatuba, affl. rio Tapajós, $03^{\circ} 00^{\prime} 48^{\prime \prime} \mathrm{S}, 055^{\circ} 06^{\prime} 22^{\prime \prime} \mathrm{W}$. ZUEC 12336, 5, mun. Santarém, rio Mentai at Cachoeirinha do Mentai, affl. rio Arapiuns, affl. rio Tapajós, $02^{\circ} 43^{\prime} 12^{\prime \prime} \mathrm{S}, 055^{\circ} 36^{\prime} 15^{\prime \prime} \mathrm{W}$. Rondônia (localities from rio Madeira dr., Amazonas dr.). FMNH 117760, 4, 82-104 mm, Maciel, rio Guaporé, affl. rio Mamoré, $12^{\circ} 30^{\prime} \mathrm{S}, 062^{\circ} 20^{\prime} \mathrm{W}$. INPA 9721, 19, 100-146 mm, INPA 9727, 18, 56-148 mm, rio Pacaás Novos, nr. confluence with rio Mamoré, $c a$. $15 \mathrm{~km}$ Guajará Mirim, $10^{\circ} 51^{\prime} \mathrm{S}, 065^{\circ} 16^{\prime} \mathrm{W}$. INPA 9724, 2, 49-131 mm, rio Mamoré, just upstream Guajará Mirim, ca. $10^{\circ} 47^{\prime} \mathrm{S}, 065^{\circ} 20^{\prime} \mathrm{W}$. INPA 9729 (part) $1,127 \mathrm{~mm}$, mouth of rio Pacaás Novos, ca. $15 \mathrm{~km}$ Guajará Mirim, rio Mamoré, $10^{\circ} 51^{\prime} \mathrm{S}, 065^{\circ} 16^{\prime} \mathrm{W}$. INPA 19958, 1, $105 \mathrm{~mm}$, Surprêsa, at rio Mamoré-Madeira confl., ca. $11^{\circ} 53^{\prime} \mathrm{S}, 064^{\circ} 59^{\prime} \mathrm{W}$. MPEG 1120 (part), 1, 95 mm, rio Pacaás Novos, Guajará Mirim, affl. rio Mamoré, $c a .10^{\circ} 51^{\prime} \mathrm{S}, 065^{\circ} 16^{\prime} \mathrm{W}$. MPEG 1121 (part), 2, 111-120, rio Pacaás Novos, $20 \mathrm{~km}$ upstream Guajará Mirim, affl. rio Mamoré, ca. $10^{\circ} 56^{\prime} 15^{\prime \prime} \mathrm{S}, 065^{\circ} 16^{\prime} 29^{\prime \prime} \mathrm{W}$. UFRO-I 6463, 30, 125-160 mm, mouth rio Cautário, affl. rio Guaporé, affl. rio Mamoré, $12^{\circ} 10^{\prime} 51^{\prime \prime} \mathrm{S}, 064^{\circ} 34^{\prime} 00^{\prime \prime} \mathrm{W}$. UFRO-I 6465, $1,130 \mathrm{~mm}$, UFRO-I uncat. (field number CAU2010010701), 2, 141-145 mm, mouth of rio Cautário, affl. rio Guaporé, affl. rio Mamoré, $12^{\circ} 11^{\prime} 22^{\prime \prime} \mathrm{S}, 064^{\circ} 35^{\prime} 20^{\prime \prime} \mathrm{W}$. UFRO-I 6466, 5, 75-112 mm, UFRO-I 6469, 5, 93-105 mm, Colocação Três Praias, rio Jaciparaná, 09²7'29"S, 064²5'22"W. UFRO-1 6468, 26, 85-155 $\mathrm{mm}$, Furo Variante, lago Cuniã, rio Madeira floodplain, 0821'17"S, 064³0'11"W. UFRO-I uncat. (field number CUN2008120102), 4, 115-153 mm, igarapé do Campo, lago Cuniã, rio Madeira floodplain, $08^{\circ} 19^{\prime} 14^{\prime \prime} \mathrm{S}, 064^{\circ} 28^{\prime} 05^{\prime \prime} \mathrm{W}$. USNM 301980, 10, 98-132 mm, Brazil-Bolivia border, region between Guajará-Mirim and Mato Grosso, rio Guaporé, affl. rio Mamoré, affl. rio Madeira, Amazonas dr., $12^{\circ} 41^{\prime} \mathrm{S}, 063^{\circ} 07^{\prime} \mathrm{W}$ [coordinate taken half-way from Guajará-Mirim to Mato Grosso, listed coordinate is in Bolivian territory nr. Guayara-Mirín]. Roraima. 
INPA 30748, 11, INPA 30749, 8, rio Branco, near Boa Vista, affl. rio Negro, Amazonas dr., 02 $47^{\prime} 31^{\prime \prime} \mathrm{N}, 060^{\circ} 40^{\prime} 09^{\prime \prime} \mathrm{W}$. Tocantins (localities in rio Tocantins dr., Amazonas dr.). MCP 40909, 1, 156 mm, MCP 40912, 1, 165 mm, mun. Porto Nacional, Córrego da Prata in area of PFI São João, $10^{\circ} 26^{\prime} 43^{\prime \prime}$ S, $048^{\circ} 21^{\prime} 51^{\prime \prime} \mathrm{W}$. MZUSP 52139, 3, 67-111 mm, MZUSP 73436, 3, 85-124 mm, mun. AraguAçu, Fazenda Praia Alta 2, Araguaçu - Barreira do Piqui hwy., 27 km N AraguAçu, rio Água Fria, affl. rio Javaés, affl. rio Araguaia, $12^{\circ} 43^{\prime} \mathrm{S}, 049^{\circ} 55^{\prime} \mathrm{W}$. Ecuador (localities from río Napo dr., Amazonas dr.). Sucumbios. FMNH 102275, 2, 113-122 mm, stream affl. río Juntuncocha, $c a$. $1 \mathrm{~km}$ upstream Laguna Jatuncocha, affl. río Yasuni, affl. $01^{\circ} 00^{\prime} 06^{\prime \prime} \mathrm{S}, 075^{\circ} 31^{\prime} 36^{\prime \prime} \mathrm{W}$. FMNH 102276, 8, 69-107 mm LEA, lago Anangucocha, 01²8'29"S, 077³3'44"W. FMNH 102277, 1, 118 mm, stream affl. río Juntuncocha, ca. 2 km upstream Laguna Jatuncocha, affl. río Yasuni, $01^{\circ} 00^{\prime} 18^{\prime \prime} \mathrm{S}$, 075³1'24"W. FMNH 102286, 1, 117 mm, Laguna Augacocha, affl. río Cuyabeno, affl. río Aguarico, $c a .00^{\circ} 02^{\prime} \mathrm{N}, 076^{\circ} 12^{\prime} \mathrm{W}$. FMNH 102287, 2, 90-107 mm, río Cuyabano, ca. $3 \mathrm{~km}$ Laguna Grande de Cuyabeno, affl. río Aguarico, $00^{\circ} 01^{\prime} 30^{\prime \prime} \mathrm{N}, 076^{\circ} 13^{\prime} 12^{\prime \prime} \mathrm{W}$. Guyana (localities from Essequibo River dr.). Upper TukutuUpper Essequibo. ANSP 185107, 1, 109 mm, 1.1 km NE Massara, Massara's Landing, Rupununi River, 03 $53^{\prime} 41^{\prime \prime} \mathrm{N}, 059^{\circ} 17^{\prime} 37^{\prime \prime} \mathrm{W}$. AUM 47959, 1, 150 mm, Rupunini River, upstream Yupukari landing, on beach, $03^{\circ} 39^{\prime} 45^{\prime \prime} \mathrm{N}, 059^{\circ} 30^{\prime} 32^{\prime \prime} \mathrm{W}$. Paraguay. Itapúa. NRM 42254, 1, 107 mm, Arroyo Aguapey, affl. rio Paraná, Paraná dr., $27^{\circ} 17^{\prime} 03^{\prime \prime} \mathrm{S}, 056^{\circ} 16^{\prime} 35^{\prime \prime} \mathrm{W}$. Peru (localities from Amazonas dr.). Loreto (localities listed from nr. Jenaro Herrera are from the río Ucayali dr., mun. Requena). ANSP 167728, 2, 99-103 mm, small floodplain lake, río Nanay, ca. $1 \mathrm{~km}$ upstream Minchana [Mishana], ca. $03^{\circ} 52^{\prime} \mathrm{S}, 073^{\circ} 29^{\prime} \mathrm{W}$. ANSP $192030,1,88 \mathrm{~mm}$, caño Santa Rita, R bank affl. río Nanay, nr. pueblo Pampa Chica, $03^{\circ} 45^{\prime} 23^{\prime \prime} \mathrm{S}, 073^{\circ} 17^{\prime} 28^{\prime \prime} \mathrm{W}$. CAS 98605, 1, $107 \mathrm{~mm}$, stream, affl. río Itaya, $c a .03^{\circ} 48^{\prime} \mathrm{S}, 073^{\circ} 16^{\prime} \mathrm{W}$. FMNH $84277,1,115 \mathrm{~mm}$, lake, affl. río Mamon [Momón], affl. río Nanay, ca. $03^{\circ} 41^{\prime} \mathrm{S}, 073^{\circ} 16^{\prime} \mathrm{W}$. FMNH 96035, 3, 82-102 mm, quebrada Michana [Mishana], affl. río Nanay, ca. $03^{\circ} 53^{\prime} \mathrm{S}, 073^{\circ} 29^{\prime} \mathrm{W}$. FMNH 113876, 2, 95-100 mm, cocha Huanayo, affl. río Pastaza, affl. río Marañón, $04^{\circ} 10^{\prime} 30^{\prime \prime} \mathrm{S}$, 076³3'03"W. INHS 36934, 2, 64-95 mm, Felipe cocha, nr. 25 de Enero, $12 \mathrm{~km}$ Iquitos on Iquitos-Quistococha rd., affl. río Itaya, $c a$. $03^{\circ} 49^{\prime} \mathrm{S}, 073^{\circ} 19^{\prime} \mathrm{W}$. INHS 39773, 2, 60-93 mm, lago Pabellon, upstream Paucarillo base camp, río Oroso, affl. río Nanay, ca. $03^{\circ} 36^{\prime} \mathrm{S}, 074^{\circ} 02^{\prime} \mathrm{W}$. INHS 39908, 1, $102 \mathrm{~mm}$, nr. Corrientillos, rio Nanay upstream Santa Clara, ca. $03^{\circ} 46^{\prime} \mathrm{S}, 073^{\circ} 20^{\prime} \mathrm{W}$. INHS 43902, 1, $119 \mathrm{~mm}$, quebrada Shushuna, rd. to Corrientillos, affl.

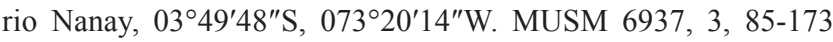
$\mathrm{mm}$, Padre Cocha, quebrada Chancacayo, affl. río Nanay, 0341'55"S, 07316'59"W. MUSM 7123 (part), 1, $161 \mathrm{~mm}$, Laguna Rimachi, río Pastaza, affl. río Marañón, $04^{\circ} 25^{\prime} \mathrm{S}$, 076²4'W. MUSM 14498 (part), 7, 68-142 mm, Puesto de Vigiliancia Arcadia, río Napo, 0059'37"S, 075²18'30"W. MUSM 16062, 1, $101 \mathrm{~mm}$, stream, Jeberos, km 44, Iquitos-Nauta rd., ca. $04^{\circ} 05^{\prime} \mathrm{S}, 073^{\circ} 27^{\prime} \mathrm{W}$. MUSM 18553, 1, 102 mm, Laguna Rimachi, nr. caño Rimachi, affl. río Pastaza, affl. río Marañón, 04²5'49"S, 076 40'20"W. MUSM 44678, 16 (8 immature, 85-178 mm, 8, female, 126-175 mm), stream nr. Jenaro Herrera, 045' $59^{\prime \prime} \mathrm{S}$, $073^{\circ} 38^{\prime} 51^{\prime \prime} \mathrm{W}$. MUSM 44684, 1, female, $127 \mathrm{~mm}$, stream nr.

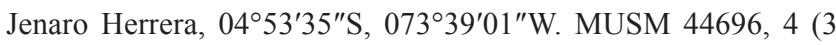
immature, $150-159 \mathrm{~mm}, 1$ male, $167 \mathrm{~mm}$ ), stream nr. Jenaro Herrera, $04^{\circ} 51^{\prime} 51^{\prime \prime} \mathrm{S}, 073^{\circ} 38^{\prime} 45^{\prime \prime} \mathrm{W}$. MUSM 44707, 4, immature, 99-130 mm, stream nr. Jenaro Herrera, 0454'40"S, 073³9'29"W. MUSM 44730, 31 (19 immature, 61-132 mm, 8 female, 118-148 mm, 4 male, 150-163 mm), stream nr. Jenaro Herrera, 0454'49"S, $073^{\circ} 39^{\prime} 43^{\prime \prime} \mathrm{W}$. MUSM 44749, 1, immature, $84 \mathrm{~mm}$, stream nr.

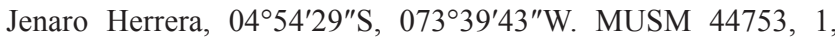
immature, $88 \mathrm{~mm}$, stream nr. Jenaro Herrera, 0454'54"S, 07339'25"W. MUSM 44758, 4 (3 immature, 108-118 mm, 1 female, $93 \mathrm{~mm})$, cocha Supay, nr. Jenaro Herrera, río Ucayali

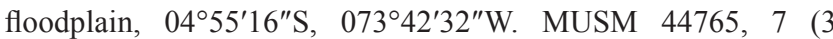
immature, 60-149 mm, 3 female, 107-169 mm, 1 male, $195 \mathrm{~mm}$ ), stream nr. Jenaro Herrera, 0454'36"S, 07339'58”W. NRM 13522 (part), 1, $90 \mathrm{~mm}$, caño Sacarita, opposite Pebas, río Ampiyacu, $c a$. $03^{\circ} 19^{\prime} \mathrm{S}, 071^{\circ} 50^{\prime} \mathrm{W}$. NRM 27523 (part), 1, 69 mm, lago Matamata, affl. caño, río Yavarí (rio Javari), upstream Atalaia do Norte (Brazil), 04 ${ }^{\circ} 12^{\prime} \mathrm{S}, 070^{\circ} 17^{\prime} \mathrm{W}$. UF 114499, 4, 110-120 mm, stream, $\mathrm{km} 23$ on Iquitos-Nauta rd., affl. río Nanay, $03^{\circ} 56^{\prime} 38^{\prime \prime} \mathrm{S}$, $073^{\circ} 23^{\prime} 54^{\prime \prime} \mathrm{W}$. UF $117124,7,53-105 \mathrm{~mm}$, río Nanay, 0343'S, $073^{\circ} 15^{\prime} \mathrm{W}$. UF $131122,1,120 \mathrm{~mm}$, Iquitos, specimen collected by ornamental fish catchers, no coordinates. UF 148472, 1, male, 192 mm, small stream, nr. Jenaro Herrera, affl. quebrada Parnayari, $c a$. $04^{\circ} 54^{\prime} \mathrm{S}, 073^{\circ} 37^{\prime} \mathrm{W}$. UF 184010 , 1 , immature, $105 \mathrm{~mm}$, stream nr.

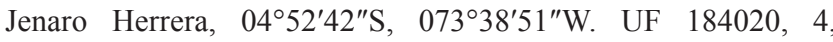
immature, 86-125 mm, stream nr. Jenaro Herrera, 045'19"S, 073³8'57"W. UMMZ 224602, 1, 137 mm, nr. Santa Ana, río Tahwayo [Tahuayo], 04 $17^{\prime} \mathrm{S}, 073^{\circ} 11^{\prime} \mathrm{W}$. UMMZ 224606, 1, 131 $\mathrm{mm}$, nr. Pebas, río Ampiyacu, ca. $03^{\circ} 19^{\prime} \mathrm{S}, 071^{\circ} 50^{\prime} \mathrm{W}$. USNM $86845,1,90 \mathrm{~mm}$, Iquitos, ca. $03^{\circ} 45^{\prime} \mathrm{S}, 073^{\circ} 12^{\prime} \mathrm{W}$. ZMH 9637, 5, 102-132 mm, region between Iquitos and Leticia, río Amazonas, no coordinates. ZMH 11628 (part), 5, 100-123 mm, between Iquitos and Leticia, río Amazonas, no coordinates. Madre de Dios (localities from río Madre de Dios, affl. río Beni, affl. rio Madeira, Amazonas dr.). MUSM 21754, 1, Pozo Santa Elena, ca. $12^{\circ} 50^{\prime} \mathrm{S}$, $069^{\circ} 17^{\prime} \mathrm{W}$. MUSM 22642, 1 (part), Las Piedras, Planchon las Piedras, quebrada Planchón, affl. río Tahuamanu [also known as río Las Piedras], ca. $12^{\circ} 30^{\prime} \mathrm{S}, 069^{\circ} 14^{\prime} \mathrm{W}$.

\section{Additional undescribed species}

Of the 12,279 specimens and 2,287 lots of Brachyhypopomus we examined, 930 specimens from 197 lots were not allocated to any of the 28 species herein. These records are included in Fig. 2 (where geographical coordinates were available). Many of these specimens represented very small juveniles, or poorly preserved material that were impossible to reliably identify. However, we are aware of some ten additional species of Brachyhypopomus represented within these lots, for which we are compiling additional information and will describe elsewhere. These include a single lot of an unidentified species from near the city of Salvador, Bahia, Brazil (MCZ 9462, collected in July 1865 during the Thayer Expedition). This lot, visible as the most easterly record for the genus in Fig. 2, occupies a location distant from the ranges of all congeners. 


\section{Acknowledgements}

The following provided access to museum specimens and associated data: Adriana Almirón (AI, MACN-ict); Barbara Brown, Scott Schaeffer (AMNH); Kyle Luckenbill, John Lundberg, Mark Sabaj-Pérez (ANSP); Jonathan Armbruster, David Werneke (AUM); Oliver Crimmen, James Maclaine (BMNH); David Catania, Mysi Hoang (CAS/SU); Mariana Arraya Borges, Mabel Maldonado (CBF, UMSS); John Friel, Charles Dardia (CU); Mauro Triques (DZUFMG); Susan Mochel, W. Leo Smith, Mark Westneat, Philip Willink (FMNH); Javier MaldonadoOcampo (CAC-CDMB/IAVHP/IMCN); Michael Retzer, Chris Taylor, Rachel Vinsel (INHS); Carlos Araujo-Lima, Lana Brasil, Efrem Ferreira, Lúcia Rapp Py-Daniel, Jansen Zuanon (INPA); Sabrina Owens, Andrew Bentley (KU); Rick Feeney (LACM); Francisco Provenzano, Alberto Marcano (MBUCV-V); Donald Taphorn (MCNG); Carlos de Lucena, Margarete Seixas de Lucena, José Pezzi da Silva, Roberto Reis (MCP); Karsten Hartel, Andrew Williston (MCZ); Carlos Lasso (MHNLS); Romain Causse, Philippe Keith, Beatrice Parisi, P. Pruvost (MNHN); Marcelo Loureiro, Ana Silva (MNHN-Uruguay, ZVC-P); Marcelo Britto, Paulo Buckup (MNRJ); Wolmar Wosiacki (MPEG); Ericka Correa Roldán, Hernán Ortega (MUSM); Margaret Docker (MZF-Manitoba); José Figueiredo, Flávio Lima, Osvaldo Oyakawa, Mario de Pinna (MZUSP); Gabriela Hogue (NCSM); Anja Palandacic, Helmut Wellendorf (NMW); Erik Åhlander, Bodil Kajrup, Sven Kullander, Anders Silfvergrip (NRM); Erika Carrera Garcia (QCAZ-I); Martien van Oijen (RMNH); Mary Burridge, Erling Holm, Hernán López-Fernández, Richard Winterbottom (ROM); Juan Bogotá (SINCHI-CIACOL); Eldredge Birmingham (STRI); Kevin Conway, Heather Prestridge (TCWC); Dean Hendrickson, Jessica Rosales (TNHC); Larry Page, Robert Robins (UF); Júlia Giora, Luiz Malabarba (UFRGS); Ricardo Campos-da-Paz (UFRJ); Luiz de Queiroz, Willian O'Hara, Adriana Ribeiro (UFRO-I); William Fink, Douglas Nelson (UMMZ); Susan Jewett, Sandra Raredon, Shirleen Smith, Erika Wilbur, Jeff Williams, Richard Vari (USNM); Flora Fernandes, Lurdes Almeida-Toledo (Instituto de Biociências, Universidade de São Paulo); Isaäc Isbrücker, Hielke Praagman (ZMA); Fredy Nugra (ZOOA); Flávio Lima (ZUEC); Horst Wilkens (ZMH). W. Crampton was funded by the United States National Science Foundation (grants DEB-0215388, DEB-0614334, DEB-1146374, and supplements), Conselho Nacional de Desenvolvimento Científico e Tecnológico - Brazil (grants CNPq 38062/96-2 \& 381597/97-0), Ministério da Ciência, Tecnologia, Inovações e Comunicações - Brazil, Instituto de Desenvolvimento Sustentável Mamirauá - Brazil, Fisheries Society of the British Isles, The University of Oxford, and the University of Central Florida (UCF). C. de Santana was funded by a post-doctoral fellowship at the Crampton Lab (UCF). N. Lovejoy was funded by Canadian National Science and Engineering Research Council (NSERC) Discovery grants and by NSF grant DEB-0614334. We are greatly indebted to the following for providing assistance with field collections or for providing specimens: Pedro Aguilera, James Albert, Jonas Alves Oliveira, Carlos Araujo-Lima, Mariana Arraya, José Márcio Ayres, Maria Aldea Guevara, João Bastos, Waldecy Bentes Monteiro, Eldredge Birmingham, Devin Bloom, Juan Bogotá, Kristen Brochu, Ricardo Campos-daPaz, Angel Caputi, Adauto Cardoso, Gilberto Castro, Wilmar Chaverra Salazar, Diego Cognato, Ericka Correa Roldán, Ben Crampton, Danillo dos Santos, Flora Fernandes, Sat Gavassa, Júlia Giora, William Hamilton, Peter Henderson, Francesco Janzen, Matthew Kolmann, Jeff Lambert, Kristy Lester, Flávio Lima, Hernán López-Fernández, Nathan Lujan, Javier Maldonado-Ocampo, Jan Mol, Carmen Montaña, Cleusa Nagamachi, National Geographic Television, Ronald Navarrette-Amaya, Fredy Nugra, Alex Orfinger, Hernán Ortega, Osvaldo Oyakawa, Luiz Queiroz, Jonathan Ready, Roberto Reis, Mathilde Richer-de-Forges, Alejo Rodríguez-Cattaneo, Mark Sabaj-Pérez, Ana Silva, Charles Smyth, John Stark, Phillip Stoddard, Lorgio Verdi, Kenneth Wang Tong You, Stuart Willis. Richard Crampton translated taxonomic papers from German. Adrian Urquiola built the GIS system for the maps. For fruitful discussions we thank James Albert, Gloria Arratia, Angel Caputi, Hernán Fernández-López, Júlia Giora, Carl Hopkins, Flávio Lima, Javier Maldonado-Ocampo, Cleusa Nagamachi, Roberto Reis, Phillip Stoddard, John Sullivan, Mauro Triques, Richard Vari, and Harold Zakon.

\section{References}

Albert, J. S. 2001. Species diversity and phylogenetic systematics of American knifefishes (Gymnotiformes, Teleostei). Miscellaneous Publications Museum of Zoology University of Michigan, 190: 1-127.

Albert, J. S. \& R. Campos-da-Paz. 1998. Phylogenetic systematics of Gymnotiformes with diagnoses of 58 clades: A review of available data. Pp. 419-446. In: Malabarba L. R., R. E. Reis, R. P. Vari, Z. M. S. Lucena \& C. A. S. Lucena (Eds.). Phylogeny and classification of neotropical fishes. Porto Alegre, Edipucrs.

Albert, J. S. \& W. G. R. Crampton. 2003. Family Hypopomidae (bluntnose knifefishes). Pp. 494-496. In: Reis R. E., S. O. Kullander \& C. J. Ferraris (Eds.). Checklist of the freshwater fishes of South and Central America. Porto Alegre, Edipucrs.

Albert, J. S. \& W. G. R. Crampton. 2005. Electroreception and electrogenesis. Pp. 431-472. In: Evans D. (Ed.) The physiology of fishes. 3rd edition. New York, C.R.C. Press.

Albert, J. S. \& W. G. R. Crampton. 2009. A new species of electric knifefish, genus Compsaraia (Gymnotiformes: Apteronotidae) from the Amazon River, with extreme sexual dimorphism in snout and jaw length. Systematics and Biodiversity, 7: 81-92.

Almeida-Toledo, L. F., M. F. Z. Daniel-Silva, C. E. Lopes \& S. Toledo-Filho. 2000. Sex chromosome evolution in fish. II. Second occurrence of an X1 X2 Y sex chromosome system in Gymnotiformes. Chromosome Research, 8: 335-340. 
Almirón, A., J. Casciotta, L. Ciotek, P. Giorgis, P. Soneira \& F. Ruíz Díaz. 2010. Pisces, Gymnotiformes, Hypopomidae, Brachyhypopomus Mago-Leccia, 1994: First country record of three species of the genus, Argentina. Check List: Journal of Species Lists and Distribution, 6: 572-575.

Alves-Gomes, J. A. 1997. Informações preliminares sobre a bioecologia de peixes elétricos (Ordem Gymnotiformes) em Roraima. Pp. 47-55. In: Barbosa R. I., E. J. G. Ferreira \& E. G. Castellón (Eds.). Homen, ambiente e ecologia no estado do Roraima. Manaus, INPA.

Alves-Gomes, J. A., G. Ortí, M. Haygood, W. Heiligenberg \& A. Meyer. 1995. Phylogenetic analysis of the South American electric fishes (Order Gymnotiformes) and the evolution of their electrogenic system: A synthesis based on morphology, electrophysiology, and mitochondrial sequence data. Molecular Biology and Evolution, 12: 298-318.

Arratia, G. \& H. Schultze. 1991. Palatoquadrate and its ossifications: Development and homology within Osteichthyans. Journal of Morphology, 208: 1-81.

Assad, C., B. Rasnow \& P. K. Stoddard. 1999. Electric organ discharges and electric images during electrolocation. Journal of Experimental Biology, 202: 1185-1193.

Azpelicueta, M. \& S. Koerber. 2015. On some freshwater fish species reported by Perugia (1891) from Argentina. Aqua Journal of Ichthyology and Aquatic Biology, 21: 39-46.

Barriga, R. 1991. Peces de agua dulce. Politécnica, 16: 7-88.

Barriga, R. 1994. Peces del noroeste del Ecuador. Politécnica, 19: 43-154.

Bass, A. H. 1986. Electric organs revisited: evolution of a vertebrate communication and orientation organ. Pp. 13-70. In: Bullock T. H. \& W. Heiligenberg (Eds.). Electroreception. New York, Wiley.

Bastian, J. 1976. Frequency response characteristics of electroreceptors in weakly electric fish (Gymnotoidei) with a pulse discharge. Journal of Comparative Physiology a-Neuroethology Sensory Neural and Behavioral Physiology, 112: $165-180$.

Bastian, J. 1977. Variations in the frequency response of electroreceptors dependent on receptor location in weakly electric fish (Gymnotoidei) with a pulse discharge. Journal of Comparative Physiology a-Neuroethology Sensory Neural and Behavioral Physiology, 121: 53-64.

Bennett, M. V. L. 1961. Modes of operation of electric organs. Annals of the New York Academy of Sciences, 94: 458-509.

Bennett, M. V. L. 1971a. Electric organs. Pp. 347-484. In: Hoar W. S. \& D. J. Randall (Eds.). Fish physiology. New York, Academic Press.

Bennett, M. V. L. 1971b. Electroreception. Pp. 493-574. In: Hoar W. S. \& D. J. Randall (Eds.). Fish physiology. New York, Academic Press.

Bermingham, E. \& E. P. Martins. 1998. Comparative mtDNA phylogeography of neotropical freshwater fishes: Testing shared history to infer the evolutionary landscape of lower Central America. Molecular Ecology, 7: 499-517.

Boulenger, G. A. 1896. On a collection of fishes from the Rio Paraguay. Transactions of the Zoological Society of London, 14 (part II): 1-39, pls. 31-38.
Boulenger, G. A. 1898. On a collection of fishes from the rio Rio Jurua. Transactions of the Zoological Society of London, 14 (part VII): 421-428, pls. 39-42.

Bullock, T. H., S. Hagiwara, K. Kusano \& K. Negishi. 1961. Evidence for a category of electroreceptors in the lateral line of gymnotid fishes. Science, 134: 1426-1427.

Bullock, T. H., C. D. Hopkins, A. N. Popper \& R. R. Fay (Eds.). 2005. Electroreception. New York, Springer, 467 pp.

Bussing, W. A. 1987. Peces de las aguas continentales de Costa Rica. San José, Editorial de la Universidad de Costa Rica, 271 $\mathrm{pp}$

Bussing, W. A. 1998. Peces de las aguas continentales de Costa Rica/Freshwater fishes of Costa Rica, 2nd. edition. San José, Editorial Universidad de Costa Rica, 468 pp.

Campos-da-Paz, R. 2000. On Sternarchorhynchus Castelnau: a South American electric knifefish, with descriptions of two new species (Ostariophysi: Gymnotiformes: Apteronotidae). Copeia, 2: 521-535.

Caputi, A. A. 1999. The electric organ discharge of pulse gymnotiforms: The transformation of a simple impulse into a complex spatiotemporal electromotor pattern. Journal of Experimental Biology, 202: 1229-1241.

Caputi, A. A., A. C. Silva \& O. Macadar. 1998. The electric organ discharge of Brachyhypopomus pinnicaudatus: The effects of environmental variables on waveform generation. Brain Behavior and Evolution, 52: 148-158.

Cardoso, A., J. C. Pieczarka \& C. Y. Nagamachi. 2015. $\mathrm{X}_{1} \mathrm{X}_{1} \mathrm{X}_{2} \mathrm{X}_{2} /$ $\mathrm{X} 1 \mathrm{X}_{2} \mathrm{Y}$ sex chromosome systems in the Neotropical Gymnotiformes electric fish of the genus Brachyhypopomus. Genetics and Molecular Biology, 38: 213-219.

Cardoso, A. L., J. C. Pieczarka, E. Feldberg, S. S. R. Milhomem, T. Moreira-Almeida, D. dos Santos Silva, P. Corrêa da Silva \& C. Y. Nagamachi. 2011. Chromosomal characterization of two species of genus Steatogenys (Gymnotiformes: Rhamphichthyoidea: Steatogenini) from the Amazon basin: sex chromosomes and correlations with Gymnotiformes phylogeny. Review of Fish Biology and Fisheries, 21: 613-621.

Carter, G. S. \& L. C. Beadle. 1931. The fauna of the swamps of the Paraguayan Chaco in relation to its environment. II. Respiratory adaptations in the fishes. Journal of the Linnean Society of London, Zoology, 37: 327-366.

Carvalho, T. P. 2013 Systematics and evolution of the toothless knifefishes Rhamphichthyoidea Mago-Leccia (Actinopterygii: Gymnotiformes): Diversification in South American freshwaters. Unpublished Ph.D. Dissertation, University of Louisiana at Lafayette, Lafayette, 516 pp.

Carvalho, T. P. \& J. S. Albert. 2011. Redescription and phylogenetic position of the enigmatic Neotropical electric fish Iracema caiana Triques (Gymnotiformes: Rhamphichthyidae) using x-ray computed tomography. Neotropical Ichthyology, 9: 457-469.

Carvalho, T. P., C. S. Ramos \& J. S. Albert. 2011. A new species of Gymnorhamphichthys (Gymnotiformes: Rhamphichthyidae) from the Paraná-Paraguay basin. Copeia, 2011: 400-406.

Casciotta, J., A. Almirón \& J. Bechara. 2005. Peces del Iberá: Hábitat y diversidad. La Plata, Argentina, Grafikar, 244 pp. 
Chang, F. \& H. Ortega. 1995. Additions and corrections to the list of freshwater fishes of Peru. Publicaciones del Museo de Historia Natural, Universidad Nacional Mayor de San Marcos, Serie A - Zoology, 5: 1-11.

Chao, N. L. 2001. Fisheries, diversity and conservation of ornamental fishes of the Rio Negro basin, Brazil - a review of Project Piaba (1989-1999). Pp. 161-204. In: Chao N. L., P. Petry, G. Prang, L. Lonneschien \& M. Tlusty (Eds.). Conservation and management of ornamental fish resources of the rio Negro basin, Amazonia, Brazil - Project Piaba. Manaus, Editora da Universidade do Amazonas.

Claudino, M. C., F. Corrêa, R. F. Bastos \& A. Miranda Garcia. 2010. Pisces, Gymnotiformes, Hypopomidae, Brachyhypopomus draco (Giora, Malabarba and Crampton, 2008): New species record at Lagoa do Peixe National Park, state of Rio Grande do Sul, Brazil. Check List: Journal of Species Lists and Distribution, 6: 358-359.

Cognato, D. D. P., J. Giora \& C. B. Fialho. 2007. Análise da ocorrência de lesões corporais em três espécies de peixe elétrico (Pisces: Gymnotiformes) do sul do Brasil. Pan-American Journal of Aquatic Sciences, 2: 242-246.

Costa, W. J. E. M. \& R. Campos-da-Paz. 1992. Description d'une nouvelle espèce de poisson électrique du genre néotropical Hypopomus (Siluriformes : Gymnotoidei : Hypopomidae) du Sud-Est du Brésil. Revue Française d'AquariologieHerpétologie, 18: 117-120.

Crampton, W. G. R. 1996a The electric fish of the upper Amazon: ecology and signal diversity. Unpublished D.Phil. Dissertation, The University of Oxford, Oxford. 223p.

Crampton, W. G. R. 1996b. Gymnotiform fish: an important component of Amazonian floodplain fish communities. Journal of Fish Biology, 48: 298-301.

Crampton, W. G. R. 1998a. Electric signal design and habitat preferences in a species rich assemblage of gymnotiform fishes from the upper Amazon basin. Anais da Academia Brasileira de Ciencias, 70: 805-847.

Crampton, W. G. R. 1998b. Effects of anoxia on the distribution, respiratory strategies and electric signal diversity of gymnotiform fishes. Journal of Fish Biology, 53 (Suppl. A): 307-330.

Crampton, W. G. R. 1999. Os peixes da Reserva Mamirauá: diversidade e história natural na planície alagável da Amazônia. Pp. 10-36. In: Queiroz H. L. \& W. G. R. Crampton (Eds.). Estratégias para manejo de recursos pesqueiros em Mamirauá. Brasília, Sociedade Civil Mamirauá/CNPq.

Crampton, W. G. R. 2007. Diversity and adaptation in deep channel Neotropical electric fishes Pp. 283-339. In: Sebert P., D. W. Onyango \& B. G. Kapoor (Eds.). Fish life in special environments. Enfield, NH, Science Publishers.

Crampton, W. G. R. 2008. Ecology and life history of an Amazon floodplain cichlid: the discus fish Symphysodon (Perciformes: Cichlidae). Neotropical Ichthyology, 6: 599-612.

Crampton, W. G. R. 2011. An ecological perspective on diversity and distributions Pp. 165-189. In: Albert J. S. \& R. E. Reis (Eds.). Historical biogeography of neotropical freshwater fishes. Berkeley, University of California Press.
Crampton, W. G. R. \& J. S. Albert. 2006. Evolution of electric signal diversity in gymnotiform fishes. I. Phylogenetic systematics, ecology and biogeography. Pp. 647-696; 718-731. In: Ladich F., S. P. Collin, P. Moller \& B. G. Kapoor (Eds.). Communication in fishes. Enfield, NH, Science Publishers.

Crampton, W. G. R., L. J. Chapman \& J. Bell. 2008. Interspecific variation in gill size is correlated to ambient dissolved oxygen in the Amazonian electric fish Brachyhypopomus (Gymnotiformes: Hypopomidae). Environmental Biology of Fishes, 83: 223-235.

Crampton, W. G. R., C. D. de Santana, J. C. Waddell \& N. R. Lovejoy. 2016. Phylogenetic systematics, biogeography, and ecology of the electric fish genus Brachyhypopomus (Ostariophysi: Gymnotiformes). PLoS One, 11(10): 1-63: e0161680. doi:10.1371/journal.pone.0161680.

Crampton, W. G. R., N. R. Lovejoy \& J. C. Waddell. 2011. Reproductive character displacement and signal ontogeny in a sympatric assemblage of electric fishes. Evolution, 65: 1650-1666.

Crampton, W. G. R. \& A. C. Ribeiro. 2013. Hypopomidae. Pp. 232249. In: Torrente-Vilara G., L. Queiroz \& J. Zuanon (Eds.). Peixes do rio Madeira. Porto Velho, Universidade Federal de Rondônia.

Crampton, W. G. R., J. K. Wells, C. Smyth \& S. A. Walz. 2007. Design and construction of an electric fish finder. Neotropical Ichthyology, 5: 425-428.

Curtis, C. C. \& P. K. Stoddard. 2003. Mate preference in female electric fish, Brachyhypopomus pinnicaudatus. Animal Behaviour, 66: 329-336.

Dunlap, K. D., A. Tran, M. A. Ragazzi \& R. Krahe. 2016. Predators inhibit brain cell proliferation in natural populations of electric fish Brachyhypopomus occidentalis. Proceedings of the Royal Society B-Biological Sciences, 283: 283 20152113; DOI: 10.1098/rspb.2015.2113

Dutra, G. M., F. C. Jerep, R. P. Vari \& C. D. De Santana. 2015. The pseudotympanum in the Gymnotiformes (Teleostei, Ostariophysi, Otophysi): homology and evolution of a previously unexplored system in Neotropical electric fishes. Zoological Journal of the Linnean Society, 174: 114-129.

Eigenmann, C. H. 1894. Notes on some South American fishes. A. Fishes collected by Frederick C. Hartt. Annals of the New York Academy of Sciences, 7 (art. 5): 625-637.

Eigenmann, C. H. 1910. Catalogue of the freshwater fishes of tropical and south temperate America. Pp. 375-511. In: Scott W. B. (Ed.) Reports of the Princeton University expeditions to Patagonia 1896-1899, Volume III, Part IV. Princeton, University of Princeton.

Eigenmann, C. H. 1912. The freshwater fishes of British Guiana, including a study of the ecological groupings of species and the relation of the fauna of the plateau to that of the lowlands. Memoirs of the Carnegie Museum, 5: i-xxii,1-578, pls. 571-103.

Eigenmann, C. H. 1922. The fishes of western South America. Part I: The fresh-water fishes of northwestern South America, including Colombia, Panama, and the Pacific slopes of Ecuador and Peru, together with an appendix upon the fishes of the Rio Meta in Colombia. Memoirs of the Carnegie Museum, 9: 1-346, pls. 341-338. 
Eigenmann, C. H. \& W. R. Allen. 1942. Fishes of western South America. Lexington, University of Kentucky, 494 pp.

Eigenmann, C. H. \& R. S. Eigenmann. 1891. A catalogue of the freshwater fishes of South America. Proceedings of the United States National Museum, 14: 1-81.

Eigenmann, C. H. \& H. G. Fisher. 1914. The Gymnotidae of Trans-Andean Colombia and Ecuador (Contributions from the Zoological Laboratory of Indiana University, No. 141). Indiana University Studies, 25: 235-237.

Eigenmann, C. H. \& C. H. Kennedy. 1903. On a collection of fishes from Paraguay, with a synopsis of the American genera of cichlids. Proceedings of the Academy of Natural Sciences of Philadelphia, 55: 497-537

Eigenmann, C. H. \& D. P. Ward. 1905. The Gymnotidae. Proceedings of the Washington Academy of Sciences, 7: 158-188.

Elbassiouny, A. A., R. K. Schottt, J. C. Waddell, M. A. Kolmann, E. S. Lehmberg, A. Van Nyatten, W. G. R. Crampton, B. S. W. Chang \& N. R. Lovejoy. 2016. Mitochondrial genomes of the South American electric knifefishes (Order Gymnotiformes). Mitochondrial DNA. Part B: Resources, 1: 401-403.

Ellis, M. M. 1913. The gymnotid eels of tropical America. Memoirs of the Carnegie Museum, 6: 109-195.

Fernandes, C. C., J. G. Lundberg \& C. Riginos. 2002. Largest of all electric-fish snouts: hypermorphic facial growth in Apteronotus hasemani and the identity of Apteronotus anas (Gymnotiformes: Apteronotidae). Copeia, 2002: 52-61.

Fernandes, C. C., A. Nogueira \& J. A. Alves-Gomes. 2014. Procerusternarchus pixuna, a new genus and species of electric knifefish (Gymnotiformes: Hypopomidae, Microsternarchini) from the Negro River, South America. Proceedings of the Academy of Natural Sciences of Philadelphia, 163: 95-118.

Fernandes, C. C., A. Nogueira, A. Williston \& J. A. AlvesGomes. 2015. A new species of electric knifefish from the rio Negro, Amazon basin (Gymnotiformes: Hypopomidae, Microsternarchini). Proceedings of the Academy of Natural Sciences of Philadelphia, 164: 213-227.

Fernández-Yépez, A. 1972. Analisis ictiológico del complejo hidrográfico (04) río Yaracuy Caracas, Dirección de Obras Hidraulicas: Ministerio de Obras Públicas. República de Venezuela, 25p.

Ferraris, C. J. J. \& R. P. Vari. 1992. Catalog of type specimens of Recent fishes in the National Museum of Natural History, Smithsonian Institution, 4: Gonorhynchiformes, Gymnotiformes, and Siluriformes (Teleostei: Ostariophysi). Smithsonian Contributions to Zoology, 535: 1-52.

Ferreira, E. J. G., J. Zuanon, B. Forsberg, M. Goulding \& BrigliaFerreira. S. R. 2007. Rio Branco: peixes, ecologia e conservação de Roraima. Manaus, Amazon Conservation Association, Instituto Nacional de Pesquisas da Amazônia, Sociedade Civil Mamirauá, 201pp.

Fink, S. V. \& W. L. Fink. 1981. Interrelationships of the ostariophysan fishes (teleostei). Zoological Journal of the Linnean Society, 72: 297-353.

Fowler, H. W. 1945. Colombian Zoological Survey. Part I. The freshwater fishes obtained in 1945. Proceedings of the Academy of Natural Sciences of Philadelpia, 97: 93-135.
França, G. F., C. Oliveira \& I. Quagio-Grassiotto. 2007. Ultrastructure of spermiogenesis and spermatozoa of Gymnotus cf. anguillaris and Brachyhypopomus cf. pinnicaudatus (Teleostei: Gymnotiformes). Tissue and Cell, 39: 131-139.

Franchina, C. R. 1997. Ontogeny of the electric organ discharge and the electric organ in the weakly electric pulse fish Brachyhypopomus pinnicaudatus (Hypopomidae, Gymnotiformes). Journal of Comparative Physiology a-Neuroethology Sensory Neural and Behavioral Physiology, 181: 111-119.

Galves, W., O. A. Shibatta \& F. C. Jerep. 2009. Estudos sobre diversidade de peixes da bacia do alto rio Paraná: uma revisão histórica. Semina: Ciências Biológicas e da Saúde, Londrina, 30: 141-154.

Galvis, G., J. I. Mojica \& M. Camargo. 1997. Peces del Catatumbo. Bogotá, Asociación Cravo Norte, 118 pp.

Galvis, G., J. I. Mojica, S. R. Duque, C. Castellanos, P. SánchezDuarte, M. Arce, Á. Gutiérrez, L. F. Jiménez, M. Santos, S. Vejerano, F. Arbelaez, E. Prieto \& M. Leiva. 2006. Peces del medio Amazonas - Región de Leticia. Bogotá, Conservation International, 548p.

Gavassa, S., A. Goldina, A. C. Silva \& P. K. Stoddard. 2013. Behavioral ecology, endocrinology and signal reliability of electric communication. Journal of Experimental Biology, 216: 2403-2411.

Gavassa, S., A. C. Silva, E. Gonzalez, J. Molina \& P. K. Stoddard. 2012. Social competition masculinizes the communication signals of female electric fish. Behavioral Ecology and Sociobiology, 66: 1057-1066.

Géry, J. \& T. T. Vu. 1964. Gymnorhamphichthys hypostomus petiti ssp. nov. Un curieux poisson Gymnotoïde arénicole. Vie et Milieu, 17 (Suppl.): 485-498.

Gill, T. N. 1864. Several points in ichthyology and conchology. Proceedings of the Academy of Natural Sciences of Philadelphia, 16: 151-152.

Giora, J. \& J. R. Burns. 2011. Sperm ultrastructure in three different families of weakly electric fishes (Teleostei: Gymnotiformes). Neotropical Ichthyology, 9: 881-888.

Giora, J. \& L. R. Malabarba. 2009. Brachyhypopomus gauderio, new species, a new example of underestimated species diversity of electric fishes in the southern South America (Gymnotiformes: Hypopomidae). Zootaxa: 60-68.

Giora, J., L. R. Malabarba \& W. Crampton. 2008. Brachyhypopomus draco, a new sexually dimorphic species of neotropical electric fish from southern South America (Gymnotiformes: Hypopomidae). Neotropical Ichthyology, 6: 159-168.

Giora, J., H. M. Tarasconi \& C. B. Fialho. 2011. Reproduction and feeding habits of the highly seasonal Brachyhypopomus bombilla (Gymnotiformes: Hypopomidae) from southern Brazil, with evidence for a domancy period. Environmental Biology of Fishes, 94: 649-662.

Giora, J., H. M. Tarasconi \& C. B. Fialho. 2014. Reproduction and feeding of the electric fish Brachyhypopomus gauderio (Gymnotiformes: Hypopomidae) and the discussion of a life history pattern for gymnotiforms from high latitudes. PLoS One, 9 (e106515): 1-11. 
Goulding, M., C. Cañas, B. R., B. Forsberg \& H. Ortega. 2003. Amazon headwaters: rivers, life and conservation of the Madre de Dios river basin. Lima, Asociación para la Conservación de la Cuenca Amazónica, Amazon Conservation Association, Gráfica Biblos S.A., 198p.

Graça, W. J. \& C. S. Pavanelli. 2007. Peixes da planície de inundação do alto rio Paraná e áreas adjacentes. Maringá, Universidade de Maringá, 241p.

Günther, A. 1870. Catalogue of the fishes in the British Museum. London, British Museum of Natural History, 549 pp.

Hagedorn, M. 1986. The ecology, courtship and mating of gymnotiform electric fish. Pp. 495-525. In: Bullock T. H. \& W. Heiligenberg (Eds.). Electroreception. New York, John Wiley and Sons.

Hagedorn, M. 1988. Ecology and behaviour of a pulse-type electric fish, Hypopomus occidentalis (Gymnotiformes, Hypopomidae), in a fresh-water stream in Panama. Copeia, 1988: 324-335.

Hagedorn, M. \& C. Carr. 1985. Single electrocytes produce a sexually dimorphic signal in South American electric fish, Hypopomus occidentalis (Gymnotiformes, Hypopomidae). Journal of Comparative Physiology a-Neuroethology Sensory Neural and Behavioral Physiology, 156: 511-523.

Hagedorn, M. \& C. H. Keller. 1996. Species diversity of gymnotiform fishes in Manu Bioreserve, Pakitza, Perú. Pp. 483-502. In: Wilson D. E. \& A. Sandoval (Eds.). Manu: the biodiversity of southeastern Peru. Washington DC, Smithsonian Institution Press.

Hagedorn, M. \& R. Zelick. 1989. Relative dominance among males is expressed in the electric organ discharge characteristics of a weakly electric fish. Animal Behavior, 38: 520-525.

Hagiwara, S., K. Kusano \& K. Negishi. 1962. Physiological properties of electroreceptors of some gymnotids. Journal of Neurophysiology, 25: 430-449.

Heiligenberg, W. \& J. Bastian. 1980. Species specificity of electric organ discharges in sympatric gymnotoid fish of the Rio Negro. Acta Biológica Venezuélica, 10: 187-203.

Hoedeman, J. J. 1962. Notes on the ichthyology of Surinam and other Guianas. 9. New records of gymnotid fishes. Bulletin of Aquatic Biology, Amsterdam, 3: 53-60.

Hopkins, C. D. 1991. Hypopomus pinnicaudatus (Hypopomidae), a new species of gymnotiform fish from French Guiana. Copeia, 1: 151-161.

Hopkins, C. D. 1999. Design features for electric communication. Journal of Experimental Biology, 202: 1217-1228.

Hopkins, C. D. 2005. Passive electrolocation. Pp. 264-289. In: Bullock T. H., C. D. Hopkins, A. N. Popper \& R. R. Fay (Eds.). Electroreception. New York, Springer.

Hopkins, C. D., N. C. Comfort, J. Bastian \& A. H. Bass. 1990. Functional analysis of sexual dimorphism in an electric fish, Hypopomus pinnicaudatus, order Gymnotiformes. Brain Behavior and Evolution, 35: 350-367.

Hopkins, C. D. \& W. Heiligenberg. 1978. Evolutionary designs for electric signals and electroreceptors in gymnotoid fishes of Surinam. Behavioral Ecology and Sociobiology, 3: 113-134.

Hopkins, C. D., K. Shieh, D. W. J. McBride \& M. Winslow. 1997. A quantitative analysis of passive electrolocation behavior in electric fish. Brain Behavior and Evolution, 50 (suppl 1): 32-59.
Hopkins, C. D. \& G. W. M. Westby. 1986. Time domain processing of electric organ discharge waveforms by pulse type electric fish. Brain Behavior and Evolution, 29: 77-104.

Hulen, K. G., W. G. R. Crampton \& J. S. Albert. 2005. Phylogenetic systematics and historical biogeography of the Neotropical electric fish Sternopygus (Teleostei : Gymnotiformes). Systematics and Biodiversity, 3: 407-432.

ICZN. 1999. International Code of Zoological Nomenclature. London, U.K., The International Trust for Zoological Nomenclature, 306p.

Jiménez-Prado, P., W. Aguirre, R. Laaz-Moncayo, R. NavaretteAmaya, F. Nugra-Salazar, E. Rebolledo-Monsalve, E. ZárateHugo, A. Torres-Noboa \& J. Valdiviezo-Rivera. 2015. Gúia de peces para aguas continentales en la vertiente occidental del Ecuador. Esmeraldas, Ecuador, PUCESE, UDA, MECN, 416p.

Julian, D., W. G. R. Crampton, S. E. Wohlgemuth \& J. S. Albert. 2003. Oxygen consumption in weakly electric Neotropical fishes. Oecologia, 137: 502-511.

Kaup, J. J. 1856. Family Gymnotidae. Pp. 124-142. In: Kaup J. J. (Ed.) Catalogue of apodal fishes. London, British Museum of Natural History.

Kawasaki, M. \& W. Heiligenberg. 1989. Distinct mechanisms of modulation in a neuronal oscillator generate different social signals in the electric fish Hypopomus. Journal of Comparative Physiology a-Neuroethology Sensory Neural and Behavioral Physiology, 165: 731-741.

Kirschbaum, F. 1995. Taxonomy, zoogeography and general ecology of South American knifefishes. Pp. 446-454. In: P. M. (Ed.) Electric fishes: history and behaviour. London, Chapman \& Hall.

Kirschbaum, F., U. Leyendecker, B. Nyonge, C. Schulz, H. Weitkamp, S. Didhiou, M. Thomas \& C. Schugardt. 2008. Environmental control of cyclical reproduction of tropical freshwater fish: Evidence from comparative experimental data. Cybium, 32: 294-296.

Kirschbaum, F. \& C. Schugardt. 2002. Reproductive strategies and developmental aspects in mormyrid and gymnotiform fishes. Journal of Physiology-Paris, 96: 557-566.

Koerber, S. 2011. List of Freshwater Fishes from Argentina - Update 14. Ichthyological Contributions of Peces Criollos, 26: 1-11.

Kramer, B. 1990. Electro-communication in teleost fishes: behaviour and experiments. New York, Springer, 240p.

Kramer, B. 1995. Electroreception and communication in fishes. Stuttgart, Georg Fischer Verlag, 119p.

Lannoo, M. J. \& S. J. Lannoo. 1993. Why do electric fishes swim backwards? An hypothesis based on gymnotiform foraging behaviour interpreted through sensory constraints. Environmental Biology of Fishes, 36: 157-165.

Lázár, G. P., P. Tóth \& T. Szabo. 1987. Retinal projections in gymnotiform fishes. Journal für Hirnforschung, 28: 13-26.

Lehner, B., K. Verdin \& A. Jarvis. 2006. HydroSHEDS technical documentation. Version 1.0 (http://hydrosheds.cr.usgs.gov). Washington, DC, World Wide Fund for Nature, US, 27p.

Lissmann, H. W. 1961. Ecological studies on gymnotids. Pp. 215-226. In: Chagas C. \& A. Paes de Carvalho (Eds.). Bioelectrogenesis. Amsterdam, Elsevier. 
López, H. L., A. M. Miquelarena \& R. C. Menni. 2003. Lista comentada de los peces continentales de la Argentina. ProBiota - Serie Técnica y Didáctica, 5: 1-85.

Loureiro, M. \& A. Silva. 2006. A new species of Brachyhypopomus (Gymnotiformes, Hypopomidae) from northeastern Uruguay. Copeia, 2006: 665-673.

Lovejoy, N. R., K. Lester, W. G. R. Crampton, F. P. L. Marques \& J. S. Albert. 2010. Phylogeny, biogeography, and electric signal evolution of Neotropical knifefishes of the genus Gymnotus (Osteichthyes: Gymnotidae). Molecular Phylogeny and Evolution, 54: 278-290.

Mago-Leccia, F. 1976 Los peces Gymnotiformes de Venezuela: un estudio preliminar para la revisión del grupo en la América del Sur. Unpublished Ph.D. dissertation, Universidad Central de Venezuela, Caracas. 376p.

Mago-Leccia, F. 1978. Los peces de la familia Sternopygidae de Venezuela. Acta Científica Venezolana, 29: 1-89.

Mago-Leccia, F. 1994. Electric fishes of the continental waters of America. Caracas, Biblioteca de la Academia de Ciencias Fisicas, Matematicas y Naturales, Caracas, 206p.

Malabarba, L. R., P. C. Neto, V. A. Bertaco, T. P. Carvalho, J. F. dos Santos \& L. G. S. Artioli. 2013. Guia de identificação dos peixes da bacia do rio Tramandaí. Porto Alegre, Editora Via Sapiens, 140 pp.

Maldonado-Ocampo, J.A. 2004. Peces de la Orinoquia Colombiana: una aproximacíon al estudo actual de su conocimento. Pp. 303-368. In: Diazgranados M. C. \& F. Trujillo (Eds.). Fauna acuática en la Orinoquia Colombiana. Pontificia Universidad Javeriana, Bogotá.

Maldonado-Ocampo, J. A., H. López-Fernández, D. C. Taphorn, C. B. Bernard, W. G. R. Crampton \& N. R. Lovejoy. 2014. Akawaio penak, a new genus and species of Neotropical electric fish (Gymnotiformes, Hypopomidae) endemic to the upper Mazaruni River in the Guiana Shield. Zoologica Scripta, 43: 24-33.

Maldonado-Ocampo, J. A., J. S. U. Oviedo, F. A. Vila-Navarro, A. Ortega-Lara, S. Prada-Pedreros, L. F. Jiménez S, U. JaramilloVilla, A. Arango, T. S. Rivas \& G. C. S. Garcés. 2012. Peces dulceacuícolas del Chocó biogeográfico de Colombia. Bogotá, World Wide Fund for Nature, Colombia, 400p.

Markham, M. R. 2013. Electrocyte physiology: 50 years later. Journal of Experimental Biology, 216: 2451-2458.

Matavelli, R., A. M. Campos, J. do Vale, N. M. Piorski \& P. dos Santos Pompeu. 2015. Ichthyofauna sampled with tadpoles in northeastern Maranhão state, Brazil. Check List: Journal of Species Lists and Distribution, 11: 1-16.

McKibben, J. R., C. D. Hopkins \& D. D. Yager. 1993. Directional sensitivity of tuberous electroreceptors- polarity preferences and frequency tuning. Journal of Comparative Physiology a-Sensory Neural and Behavioral Physiology, 173: 415-424.

Meek, S. E. \& S. F. Hildebrand. 1916. The fishes of the freshwaters of Panama. Field Museum Natural History Publications, Zoölogical Series, 10: 1-374, pls. 376-332.

Mendes, V. P., A. L. D. Portela-Castro \& H. F. Julio. 2012. First record of supernumary (B) chromosomes in electric fish (Gymnotiformes) and the karyotypic structure of three species of the same order from the upper Paraná River basin. Comparative Cytogenetics, 6: 1-16.
Menezes, N. A., S. H. Weitzman, O. T. Oyakawa, F. C. T. Lima, R. M. C. Castro \& M. J. Weitzman. 2007. Peixes de água doce da mata atlântica: lista preliminar das espécies e comentários sobre conservação de peixes de água doce neotropicais. São Paulo, Museu de Zoologia da Universidade de São Paulo, 407 pp.

Meschiatti, A. J. \& M. S. Arcifa. 2009. A review on the fishfauna of Mogi-Guaçu River basin: a century of studies. Acta Limnologica Brasileira, 21: 135-159.

Milhomem, S. S. R., J. C. Pieczarka, W. G. R. Crampton, D. S. Silva, A. C. P. de Souza \& J. R. Carvalho. 2008. Chromosomal evidence for a putative cryptic species in the Gymnotus carapo species-complex (Gymnotiformes, Gymnotidae). BMC Genetics, 9: 75.

Miranda, M., A. C. Silva \& P. K. Stoddard. 2008. Use of space as an indicator of social behavior and breeding systems in the gymnotiform electric fish Brachyhypopomus pinnicaudatus. Environmental Biology of Fishes, 83: 379-389.

Mirande, J. M. \& S. Koerber. 2015. Checklist of the freshwater fishes of Argentina (CLOFFAR). Ichthyological Contributions of Peces Criollos, 36: 1-68.

Mol, J. H. 2012. The freshwater fishes of Suriname. Leiden, Brill Academic Publishers, 890 pp.

Moller, P. 1995. Electric fish: history and behavior. London, Chapman \& Hall, 584 pp.

Nagamachi, C. Y., J. C. Pieczarka, S. S. R. Milhomem, P. C. M. O’Brien, A. C. P. de Souza \& M. A. Ferguson-Smith. 2010. Multiple rearrangements in cryptic species of electric knifefish, Gymnotus carapo (Gymnotidae, Gymnotiformes) revealed by chromosome painting. BMC Genetics, 11: 28.

Nanjappa, P., L. Brand \& M. Lannoo. 2000. Swimming patterns associated with foraging in phylogenetically and ecologically diverse American weakly electric teleosts (Gymnotiformes). Environmental Biology of Fishes, 58: 97-104.

Nijssen, H., I. J. H. Isbrücker \& J. Géry. 1976. On the species of Gymnorhamphichthys Ellis 1912, translucent sand-dwelling gymnotid fishes from South America (Pisces, Cypriniformes, Gymnotoidei). Studies On Neotropical Fauna and Environment, 11: 37-63.

Nion, H., C. Ríos \& P. Meneses. 2002. Peces del Uruguay: lista sistemática y nombres comunes. Montevideo, Dirección Nacional de Recursos Acuáticos/InfoPesca, 105 pp.

Oliveira, C., F. Foresti \& A. W. S. Hilsdorf. 2009. Genetics of neotropical fish: from chromosomes to populations. Fish Physiology and Biochemistry, 35: 81-100.

De Oliveira, R. R., M. S. Rocha, M. B. dos Anjos, J. Zuanon \& L. H. Rapp Py-Daniel. 2009. Fish fauna of small streams of the Catua-Ipixuna Extractive Reserve, State of Amazonas, Brazil. Check List: Journal of Species Lists and Distribution, 5: 154-172.

Ortega, H. \& R. P. Vari. 1986. Annotated checklist of the freshwater fishes of Peru. Smithsonian Contributions to Zoology, 437: 1-25.

Oyakawa, O. T., A. Akama, K. C. Mautari \& J. C. Nolasco. 2006. Peixes de riachos da mata atlântica nas unidades de conservação do vale do rio Ribeira de Iguape no Estado de São Paulo. São Paulo, Editora Neotrópica, 201pp. 
Perugia, A. 1891. Appunti sopra alcuni pesci sud-americani conservati nel Museo Civico di Storia Naturale di Genova. Annali del Museo Civico di Storia Naturale di Genova (Serie 2), 10: 605-657.

Picq, S., F. Alda, E. Bermingham \& R. Krahe. 2013. Phylogeny and geographical variation in the electric signals of the primary neotropical knifefish Brachyhypopomus occidentalis. Frontiers in Behavioral Neuroscience Conference Abstract: Tenth International Congress of Neuroethology.: doi: 10.3389/ conf.fnbeh.2012.3327.00283.

Picq, S., F. Alda, R. Krahe \& E. Bermingham. 2014. Miocene and Pliocene colonization of the Central American isthmus by the weakly electric fish Brachyhypopomus occidentalis (Hypopomidae, Gymnotiformes). Journal of Biogeography, 41: 1520-1532.

Picq, S., F. Alda, E. Bermingham \& R. Krahe. 2016. Driftdriven evolution of electric signals in Neotropical knifefish. Evolution, doi: 10.1111/evo.13010.

Planquette, P., P. Keith \& P. Y. Le Bail. 1996. Atlas des poissons d'eau douce de Guyane. Vol. I. Paris, Muséum National d'Histoire Naturelle, 431pp.

Ponton, D. \& S. Mérigoux. 2001. Description and ecology of some early life stages of fishes in the River Sinnamary (French Guiana, South America). Folia Zoologica, 50, Monograph 1: 1-116.

Provenzano, F., A. Marcano \& P. Mondaca. 1998. Catálogo de ejemplares tipos en la coleccíon de peces del Museo de Biología de la Universidad Central de Venezuela (MBUCV-V). Acta Biológica Venezuélica, 18: 1-24.

de Queiroz, L. J., G. Torrente-Vilara, F. G. Vieira, W. M. Ohara, J. Zuanon \& C. R. C. Doria. 2013. Fishes of Cuniã Lake, Madeira River Basin, Brazil. Check List: Journal of Species Lists and Distribution, 9: 540-548.

Quintana, L., A. Silva, N. Berois \& O. Macadar. 2004. Temperature induces gonadal maturation and affects electrophysiological sexual maturity indicators in Brachyhypopomus pinnicaudatus from a temperate climate. Journal of Experimental Biology, 207: 1843-1853.

Regan, C. T. 1914. Fishes from the Condoto river, Colombia collected by Dr. H.G.F. Spurrell. Annals and Magazine of Natural History, (8) 14: 13-32.

Ringuelet, R. A., R. H. Arámburu \& A. Alonso de Arámburu. 1967. Los peces argentinos de agua dulce. La Plata, Argentina, Gobernación de la provincia de Buenos Aires, Comisión de Investigacíon Científica.

Sabaj Pérez, M. H. 2014. Standard symbolic codes for institutional resource collections in herpetology and ichthyology: an Online Reference. Version 5.0 (22 September 2014). http://www.asih. org, Washington, DC., American Society of Ichthyologists, 60 pp.

Salazar, V. L., R. Krahe \& J. E. Lewis. 2013. The energetics of electric organ discharge in gymnotiform weakly electric fish. Journal of Experimental Biology, 216: 2459-2468.

de Santana, C. D. \& R. P. Vari. 2010. Electric fishes of the genus Sternarchorhynchus (Teleostei, Ostariophysi, Gymnotiformes); phylogenetic and revisionary studies. Zoological Journal of the Linnean Society, 159: 223-371. de Santana, C. D., R. P. Vari \& W. B. Wosiacki. 2013. The untold story of the caudal skeleton in the electric eel (Ostariophysi: Gymnotiformes: Electrophorus). PLoS One, 8: e68719.

de Santana, C. D. \& W. G. R. Crampton. 2011. Phylogenetic interrelationships, taxonomy, and reductive evolution in the Neotropical electric fish genus Hypopygus (Teleostei, Ostariophysi, Gymnotiformes). Zoological Journal of the Linnean Society, 163: 1096-1156.

Schaan, A. B., J. Giora \& C. B. Fialho. 2009. Reproductive biology of the Neotropical electric fish Brachyhypopomus draco (Teleostei: Hypopomidae) from southern Brazil. Neotropical Ichthyology, 7: 737-744.

Schindler, O. 1937. Bemerkungen zu Hypopomus brevirostris (Steind.). Zoologischer Anzeiger, 119: 19-25.

Schlesinger, G. 1910. Die gymnonoten. Eine phylogenetischethologische Studie. Zoologische Jahrbuecher, 29: 613-640.

Schultz, L. P. 1944. Two new species of fishes (Gymnotidae, Loricariidae) from Caripito, Venezuela. Zoologica, Scientific Contributions of the New York Zoological Society, 29: 39-44.

Schultz, L. P. 1949. A further contribution to the ichthyology of Venezuela. Proceedings of the United States National Museum, 99: 1-211.

Schwassmann, H. O. 1976. Ecology and taxonomic status of different geographic populations of Gymnorhamphichthys hypostomus Ellis (Pisces, Cypriniformes, Gymnotoidei). Biotropica, 8: 25-40.

Schwassmann, H. O. 1978. Ecological aspects of electroreception. Pp. 521-533. In: Ali M. A. (Ed.) Sensory ecology. New York, Plenum.

Schwassmann, H. O. 1989. Gymnorhamphichthys rosamariae, a new species of knife fish (Rhamphichthyidae, Gymnotiformes) from the upper Rio Negro, Brazil. Studies on Neotropical Fauna and Environment, 24: 57-167.

Silva, A., R. Perrone \& O. Macadar. 2007. Environmental, seasonal, and social modulations of basal activity in a weakly electric fish. Physiology and Behavior, 90: 525-536.

Silva, A., L. Quintana, J. L. Ardanaz \& O. Macadar. 2002. Environmental and hormonal influences upon EOD waveform in gymnotiform pulse fish. Journal of Physiology-Paris, 96: 473-484.

Silva, A., L. Quintana, M. Galeano \& P. Errandonea. 2003. Biogeography and breeding in Gymnotiformes from Uruguay. Environmental Biology of Fishes, 66: 329-338.

Silva, A., L. Quintana, R. Perrone \& F. Sierra. 2008. Sexual and seasonal plasticity in the emission of social electric signals. Behavioral approach and neural bases. Journal of PhysiologyParis, 102: 272-278.

Silva, A. C., R. Perrone, L. Zubizarreta, G. Batista \& P. K. Stoddard. 2013. Neuromodulation of the agonistic behavior in two species of weakly electric fish that display different types of agression. Journal of Experimental Biology, 216: 2412-2420.

Springer, V. G. \& G. D. Johnson. 2000. Use and advantages of ethanol solution of alizarin red $\mathrm{S}$ dye for staining bone in fishes. Copeia, 2000: 300-301.

Steindachner, F. 1868a. Die Gymnotidae des K.K.HofNaturaliencabinetes zu Wien. Sitzungsberichte der Kaiserlichen Akademie der Wissenschaften. MathematischNaturwissenschaftliche Classe, 58: 249-264, pls. 241-242). 
Steindachner, F. 1868b. Abhandlung über die Gymnotiden des Wiener Museums. Anzeiger der Akademie der Wissenschaften in Wien, 5: 176-177.

Steindachner, F. 1880. Zur Fisch-fauna des Cauca und der Flüsse bei Guayaquil. Denkschriften der Kaiserlichen Akademie der Wissenschaften in Wien, Mathematisch-Naturwissenschaftliche Classe, 42: 55-104, pls. 1-9. [Also published as a separate (1880), pp. 1-51, pls. 1-9.].

Stoddard, P. K. 1999. Predation enhances complexity in the evolution of electric fish signals. Nature, 400: 254-256.

Stoddard, P. K. 2006. Plasticity of the electric organ discharge waveform: contexts, mechanisms, and implications for electrocommunication. Pp. 623-646. In: Ladich F., S. P. Collin, P. Moller \& B. G. Kapoor (Eds.). Communication in fishes. Enfield, NJ., Science Publishers.

Stoddard, P. K., B. Rasnow \& C. Assad. 1999. Electric organ discharges of the gymnotiform fishes: III. Brachyhypopomus. Journal of Comparative Physiology a-Sensory Neural and Behavioral Physiology, 184: 609-630.

Sullivan, J. P. 1997 A phylogenetic study of the neotropical hypopomid electric fishes (Gymnotiformes, Rhamphichthyoidea). Unpublished Ph.D Dissertation, Duke University, Durham, NC. 335p.

Sullivan, J. P. \& C. D. Hopkins. 2009. Brachyhypopomus bullocki, a new species of electric knifefish (Gymnotiformes: Hypopomidae) from northern South America. Proceedings of the Academy of Natural Sciences of Philadelphia, 158: 18392.

Sullivan, J. P., J. Zuanon \& C. C. Fernandes. 2013. Two new species and a new subgenus of toothed Brachyhypopomus electric knifefishes (Gymnotiformes, Hypopomidae) from the central Amazon and considerations pertaining to the evolution of a monophasic electric organ discharge. ZooKeys, 327: 1-34.

Szabo, T. 1974. Anatomy of the specialized lateral line organs of electroreception. Pp. 13-58. In: Fessard A. (Ed.) Handbook of sensory physiology, Vol. III. Electroreceptors and other specialized receptors in lower vertebrates. Berlin, Springer.

Szamier, R. B. \& A. W. Wachtel. 1970. Special cutaneous receptor organs of fish. VI. Ampullary and tuberous organs of Hypopomus. Journal of Ultrastructure Research, 30: 450-471.

Tagliacollo, V. A., M. J. Bernt, J. M. Craig, C. Oliveira \& J. S. Albert. 2016. Model-based total evidence phylogeny of Neotropical electric knifefishes (Teleostei, Gymnotiformes). Molecular Phylogenetics and Evolution, 95: 20-33.
Taylor, W. R. \& G. C. Van Dyke. 1985. Revised procedures for staining and clearing small fishes and other vertebrates for bone and cartilage study. Cybium, 9: 10719.

Triques, M. L. 1999. Three new species of Rhamphichthys Müller et Troschel, 1846. Revue Française d'AquariologieHerpétologie, 26: 1-6.

Triques, M. L. \& D. K. Khamis. 2003. Brachyhypopomus jureiae, a new species of freshwater Neotropical electric fish (Teleostei: Gymnotiformes: Hypopomidae) from a coastal stream of Southeastern Brazil. Lundiana, 4: 61-64.

Vari, R. P., C. D. De Santana \& W. B. Wosiacki. 2012. South American electric knifefishes of the genus Archolaemus (Ostariophysi, Gymnotiformes): undetected diversity in a clade of rheophiles. Zoological Journal of the Linnean Society, 165: 670-699.

Vari, R. P., C. J. Ferraris, A. Radosavljevic \& V. A. Funk. 2009. Checklist of Freshwater Fishes of the Guiana Shield. Bulletin of the Biological Society of Washington, 17: 1-93.

von Ihering, R. 1907. Os peixes de água doce do Brasil. Part 1. A. Revista do Museu Paulista, 7: 258-336.

Waddell, J. C., A. Rodríguez-Cattaneo, A. A. Caputi \& W. G. R. Crampton. 2016. Electric organ discharges and near-field spatiotemporal patterns of the electromotive force in a sympatric assemblage of Neotropical electric fish. Journal of Physiology (Paris), in press DOI: 10.1016/j.jphysparis.2016.10.004.

Westby, G. W. M. 1988. The ecology, discharge diversity and predatory behaviour of gymnotiform electric fish in the coastal streams of French Guiana. Behavioral Ecology and Sociobiology, 22: 341-354.

Westby, G. W. M. \& D. K. Shepherd. 1986. Waveform recognition in weakly electric fish. SERC (Science and Engineering Research Council) Bulletin, 3: 6-7.

Yager, D. D. \& C. D. Hopkins. 1993. Directional characteristics of tuberous electroreceptors in the weakly electric fish, Hypopomus (Gymnotiformes). Journal of Comparative Physiology a-Sensory Neural and Behavioral Physiology, 173: 401-414.
Submitted September 21, 2015 Accepted August 24, 2016 by Sven Kullander 